\title{
GCC Consolidated Feedback to ICH on the 2019 ICH M10 Bioanalytical Method Validation Draft Guideline
}

Corey Nehls ${ }^{1}$, Michael Buonarati ${ }^{2}$, Stephanie Cape ${ }^{3}$, Rafiq Islam ${ }^{4}$, Christina Satterwhite ${ }^{5}$, Chad Briscoe $^{6}$, Roger Hayes ${ }^{7}$, Andrew Dinan ${ }^{8}$, Kurt Sales ${ }^{8}$, Shelby Anderson ${ }^{9}$, Jennifer Vance ${ }^{9}$, John Lindsay ${ }^{10}$, Jennifer Zimmer ${ }^{11}$, Philippe Couerbe ${ }^{12}$, Ardeshir Khadang ${ }^{13}$, Sarah Maasjo ${ }^{13}$, Orlando Bravo ${ }^{14}$, Kathie Lindley ${ }^{15}$, Liam Moran ${ }^{16}$, Timothy Sangster ${ }^{17}$, Jenifer $\mathrm{Vija}^{18}$, John Stamatopoulos ${ }^{19}$, Jenny Lin ${ }^{20}$, Scott Reuschel ${ }^{21}$, Angie Underberg ${ }^{3}$, Robert MacNeill22, Luca Matassa ${ }^{23}$, Adriane Spytko7, Wei Garofolo*,24, Natasha Savoie ${ }^{24}$, Paul Rhyne $^{25}$, Jason Ambrosius ${ }^{25}$, Valentine Barra ${ }^{2}$, Dawn Dufield ${ }^{26}$, Marsha Luna 26 , Franklin Spriggs $^{26}$, Evgueni Fedorov ${ }^{27}$, Hanna Ritzén ${ }^{28}$, Saadya Fatmi ${ }^{29}$, Cheikh Kane ${ }^{30}$, Mohammed Bouhajib $^{31}$, Chantal DiMarco 31 , Adriana lordachescu ${ }^{32}$, Ariana Tudoroniu ${ }^{32}$, Esme Farley ${ }^{33}$, Bruce Stouffer ${ }^{1}$, Edward Tabler ${ }^{1}$, Stacie McCown ${ }^{6}$, Joseph Bower ${ }^{34}$, Travis Harrison ${ }^{35}$, Shane Karnik ${ }^{36}$, Michael Brown ${ }^{37}$, Philip Joyce ${ }^{38}$, Ira DuBey ${ }^{39}$, Brian Hoffpauir ${ }^{39}$, Nadine Boudreau $^{40}$, Jerome Bergeron ${ }^{40}$, Hao Wang ${ }^{41}$, Hao Feng ${ }^{42}$, Shaolian Zhou ${ }^{43}$, Edward Wells ${ }^{43}$, John Pirro ${ }^{44}$, Xinping Fang ${ }^{44}$, Jing Shi ${ }^{45}$ \& Changming Yang ${ }^{45}$

\footnotetext{
${ }^{1}$ PPD Laboratories, Richmond, Virginia, USA

${ }^{2}$ Intertek, San Diego, California, USA

${ }^{3}$ Covance, Madison, Wisconsin, USA

${ }^{4}$ Celerion, Lincoln, Nebraska, USA

${ }^{5}$ Charles River Laboratories, Reno, Nevada, USA

${ }^{6}$ PRA Health Sciences, Lenexa, Kansas, USA

${ }^{7}$ ICON Laboratory Services, Whitesboro, New York, USA

${ }^{8}$ Agilex Biolabs, Adelaide, Australia

${ }^{9}$ AIT Bioscience, Indianapolis, Indiana, USA

${ }^{10}$ Altasciences, Everett, Washington, USA

${ }^{11}$ Alturas Analytics, Moscow, Idaho, USA

${ }^{12}$ Atlanbio, Saint-Nazaire, France

${ }^{13}$ Axis Clinicals, Dilworth, Minnesota, USA

${ }^{14} \mathrm{BASi}$, West Lafayette, Indiana, USA

${ }^{15}$ BioAgilytix, Durham, North Carolina, USA

${ }^{16}$ Charles River Laboratories, Ashland, Ohio, USA

${ }^{17}$ Charles River Laboratories, Edinburgh, UK

${ }^{18}$ Charles River Laboratories, Skokie, Illinois, USA

${ }^{19}$ CiToxLAB North America, Laval, Quebec, Canada

${ }^{20} \mathrm{CMIC}$, Hoffman Estates, Illinois, USA

${ }^{21}$ Covance, Salt Lake City, Utah, USA

${ }^{22}$ Envigo, Somerset, New Jersey, USA

${ }^{23}$ Frontage Lab, Exton, Pennsylvania, USA

${ }^{24}$ GCC, Toronto, Ontario, Canada
}

\author{
${ }^{25}$ Immunologix Laboratories, Tampa, Florida, USA \\ ${ }^{26}$ KCAS Bioanalytical \& Biomarker Services, Shawnee \\ Mission, Kansas, USA \\ ${ }^{27}$ Lambda Therapeutic Research, Toronto, Ontario, \\ Canada \\ ${ }^{28}$ Mercodia AB, Uppsala, Sweden \\ ${ }^{29}$ NEOMED-LABS, Laval, Quebec, Canada \\ ${ }^{30}$ Pacific Biomarkers, Seattle, Washington, USA \\ ${ }^{31}$ Pharma Medica Research Inc., Mississauga, Ontario, \\ Canadian \\ ${ }^{32}$ Pharma Serv International, Bucharest, Romania \\ ${ }^{33}$ Pharmaron, Germantown, Maryland, USA \\ ${ }^{34}$ Precision for Medicine, Frederick, Maryland, USA \\ ${ }^{35}$ Precision for Medicine, Redwood City, California, USA \\ ${ }^{36}$ Pyxant Labs, Colorado Springs, Colorado, USA \\ ${ }^{37} \mathrm{Q}^{2}$ Solutions, Marietta, Georgia, USA \\ ${ }^{38} \mathrm{Q}^{2}$ Solutions, Ithaca, New York, USA \\ ${ }^{39}$ Smithers Avanza, Gaithersburg, Maryland, USA \\ ${ }^{40}$ Syneos Health, Quebec City, Quebec, Canada \\ ${ }^{41}$ TETRAQ, Brisbane, Australia \\ ${ }^{42}$ Value pharmaceutical service, Nanjing, China \\ ${ }^{43}$ Worldwide Clinical Trials, Austin, Texas, USA \\ ${ }^{44}$ WuXi Apptec, Plainsboro, New Jersey, USA \\ ${ }^{45}$ WuXi Apptec, Shanghai, China
}

*Author for correspondence: Global CRO Council for Bioanalysis (GCC), Toronto, Ontario, Canada; wei@global-cro-council.org; Website: www.global-cro-council.org

Note:

Due to the equality principles of Global CRO Council in Bioanalysis (GCC), the authors are presented in alphabetical order of company name, with the exception of the first to seventh authors who provided major contributions as the team leaders ( 7 teams were built to work on the 2019 ICH M10 Bioanalytical Method Validation Draft Guideline).

Disclaimers:

- This article is based on ICH Harmonised Guideline Bioanalytical Method Validation Draft version Endorsed on 26 February 2019: https://www.ich.org/fileadmin/Public_Web_Site//CH_Products/Guidelines/Multidisciplinary/M10/M10EWG_Step2_DraftGuideline_2 019_0226.pdf

- The data was collected using SurveyMonkey Inc. San Mateo, California, USA: www.surveymonkey.com

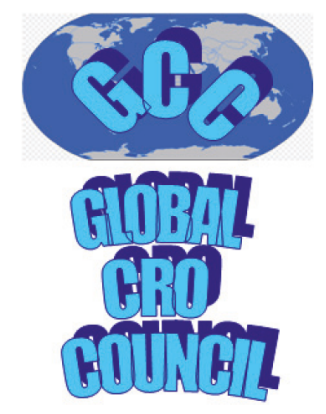

newlands 
The $13^{\text {th }}$ GCC Closed Forum for Bioanalysis was held in New Orleans, Louisiana, USA on April $5^{\text {th }}, 2019$. This GCC meeting was organized to discuss the contents of the 2019 ICH M10 Bioanalytical Method Validation Draft Guideline published in February 2019 and consolidate the feedback of the GCC members. In attendance were 63 senior-level participants from eight countries representing 44 bioanalytical CRO companies/sites. This event represented a unique opportunity for CRO bioanalytical experts to share their opinions and concerns regarding the ICH M10 Bioanalytical Method Validation Draft Guideline and to build unified comments to be provided to the $\mathrm{ICH}$.

First draft submitted: 31 July 2019; Accepted for publication: 16 August 2019; Published online: TBC

Index

- Introduction

- Team 1 - Introduction, Objective, Background, Scope, Partial, Cross Validation, Incurred Sample Reanalysis (ISR)

- Team 2 - Chromatography I - Key Validation Parameters: Selectivity, Specificity, Matrix Effect, Calibration Curve, Accuracy, Precision, Quality Control, Carry-over, Stability, Dilution Integrity, Reinjections, Extraction Recovery

- Team 3 - Chromatography II - Study Sample Analysis, Acceptance Criteria for an Analytical Run, Calibration Range Reanalysis, Reinjection of Study Samples, Reintegration of Chromatograms

- Team 4 - LBA I - Key Validation parameters: Selectivity, Specificity, Calibration Curve, Accuracy, Precision, Quality Controls, Carryover, Stability, Dilution Linearity, Hook Effect, including Parallelism, Minimum Required Dilution

- Team 5 - LBA II - Study Sample Analysis, Acceptance Criteria for an Analytical Run, Calibration Range, Reanalysis of Study Samples including Critical Reagents

- Team 6 - Reference Standards, Dried Matrix Methods, New or Alternative Technologies, Commercial and Diagnostic Kits, Analytes that are also Endogenous Compounds

- Team 7 - General Principles of Method Development/Validation, Documentation for Validation and Bioanalytical Reports

- Future Perspective

- Acknowledgments

- References

\section{Introduction}

In 2010, the GCC was created as a global independent consortium bringing together many senior-level CRO representatives to openly discuss and share opinions on scientific and regulatory issues related to bioanalysis [1]. As part of regular meetings, the GCC members discuss various topics and challenges specific to the bioanalytical CRO community. The dialogue held as part of past GCC meetings is documented in numerous published GCC conference reports, which serve to share GCC's discussions and opinions raised during these events [2-9]. Several influential White Papers on specific topics of widespread interest in bioanalysis have also been published by the GCC [10-16]. These White Papers provide unified GCC recommendations that are helpful to the global bioanalytical community.

The $13^{\text {th }}$ GCC Closed Forum was specifically dedicated to performing an in-depth review of the 2019 ICH M10 Bioanalytical Method Validation (BMV) Draft Guideline, endorsed in February 2019 [17]. Considering the importance of this Guideline, which will replace the regional ones such as US FDA [18], EMA [19], MHLW [20], the GCC went through the draft guideline line by line to provide feedback on every point (Agree, Partially Agree, or Disagree) to ensure the CROs' opinions are sent to the participating Regulatory Agencies for completing the final version of this document.

\section{Formation of specific GCC teams}

Prior to the $13^{\text {th }}$ GCC Closed Forum, seven teams were formed within the GCC to thoroughly review designated portions of the ICH M10 BMV draft Guidance: 
Team 1 (led by Corey Nehls):

Introduction, Objective, Background, Scope, Partial Validation, Cross Validation, Incurred Sample Reanalysis (ISR)

Team 2 (led by Mike Buonarati):

Chromatography I - Key Validation Parameters: Selectivity, Specificity, Matrix Effect, Calibration Curve, Accuracy, Precision, Quality Controls, Carry-over, Stability, Dilution Integrity, Reinjections, Extraction Recovery

Team 3 (led by Stephanie Cape):

Chromatography II - Study Sample Analysis, Acceptance Criteria for an Analytical Run, Calibration Range Reanalysis, Reinjection of Study Samples, Integration of Chromatograms

Team 4 (led by Rafiq Islam):

LBA I - Key Validation parameters: Selectivity, Specificity, Calibration Curve, Accuracy, Precision, Quality Controls, Carry-over, Stability, Dilution Linearity, Hook Effect, Parallelism, Minimum Required Dilution

Team 5 (led by Christina Satterwhite):

LBA II - Study Sample Analysis, Acceptance Criteria for an Analytical Run, Calibration Range, Reanalysis of Study Samples, Critical Reagents

Team 6 (led by Chad Briscoe):

Reference Standards, Dried Matrix Methods, New or Alternative Technologies, Commercial and Diagnostic Kits, Analytes that are also Endogenous Compounds

Team 7 (led by Roger Hayes):

General Principles of Method Development/Validation, Documentation for Validation and Bioanalytical Reports

Seven surveys were developed [22] based on each line of the guideline and divided into seven parts based on the seven teams built. For each question in each survey, respondents were asked to select one of the three options below:

- Agreement with ICH M10 - no changes proposed

- Partial Agreement - minor changes proposed

- Disagreement or missing parts - additions/major changes requested

The results and main trends in the comments of the survey and the additional comments from the $13^{\text {th }}$ GCC Closed Forum are summarized below per team and line by line in the following order:

- Line number \& excerpt from ICH M10 BMV draft Guideline [17]

- Statistical data from the survey (graph \& table) for Agreement, Partial Agreement and Disagreement

- Summary of the most important comments from Disagreement or missing parts

- Summary of the most important comments from Partial Agreement

- Summary of the additional most important comments from the $13^{\text {th }}$ GCC only if different from the survey comments

Note: The summary of the most important comments is based on specific trends, where multiple CROs expressed the same concern. Isolated comments were not reported. 
Team 1 Introduction, Objective, Background, Scope, Partial, Cross Validation, Incurred Sample Reanalysis (ISR)

T1Q1: Objectives/Intention; Lines 81-87: "This guideline is intended to provide recommendations for the validation of bioanalytical assays for chemical and biological drug quantification and their application in the analysis of study samples. Adherence to the principles presented in this guideline will improve the quality and consistency of the bioanalytical data in support of the development and market approval of both chemical and biological drugs."

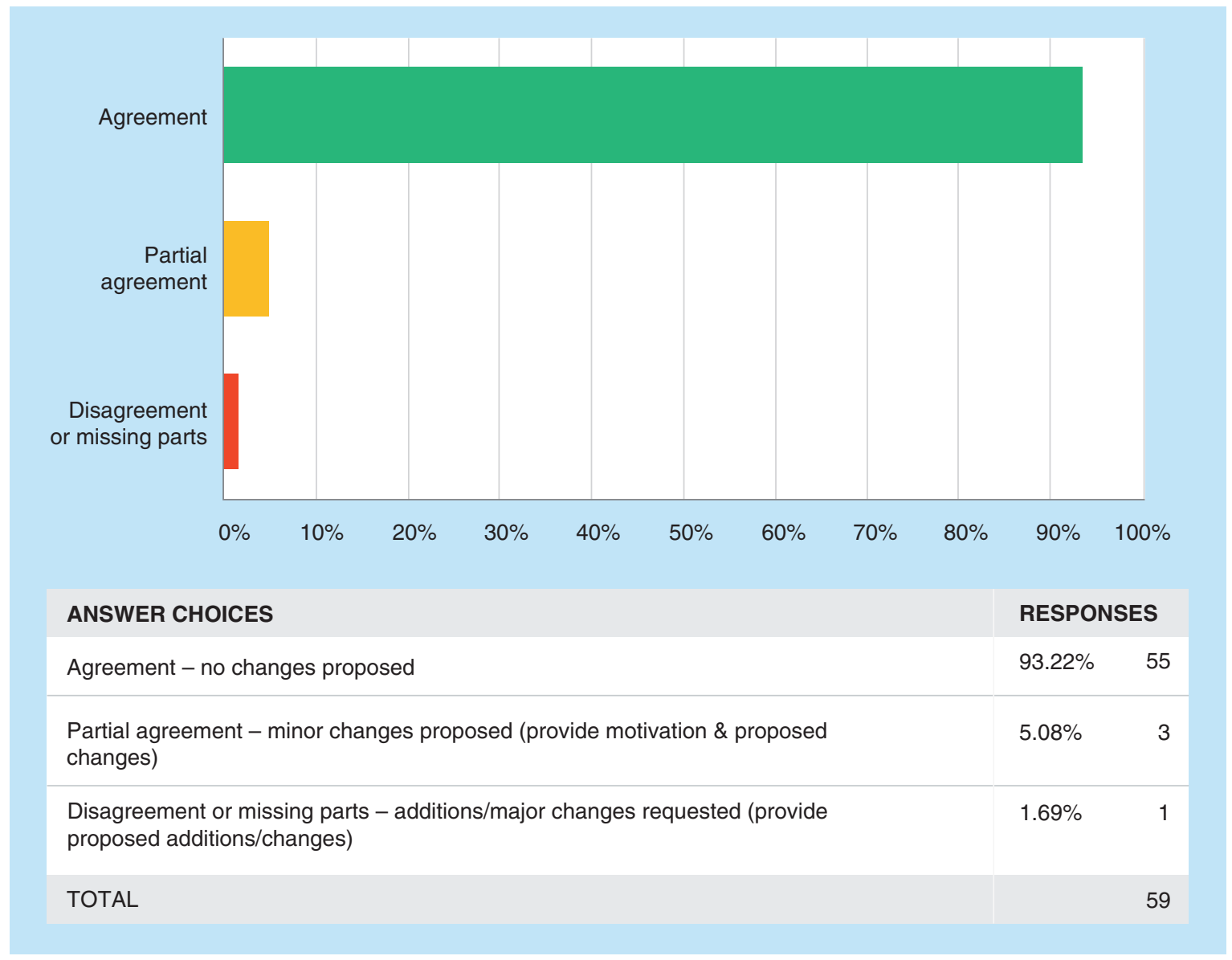

Partial agreement

No trend was found in these comments. 
T1Q2: Objectives; Lines 88-89: "The objective of the validation of a bioanalytical assay is to demonstrate that it is suitable for its intended purpose."

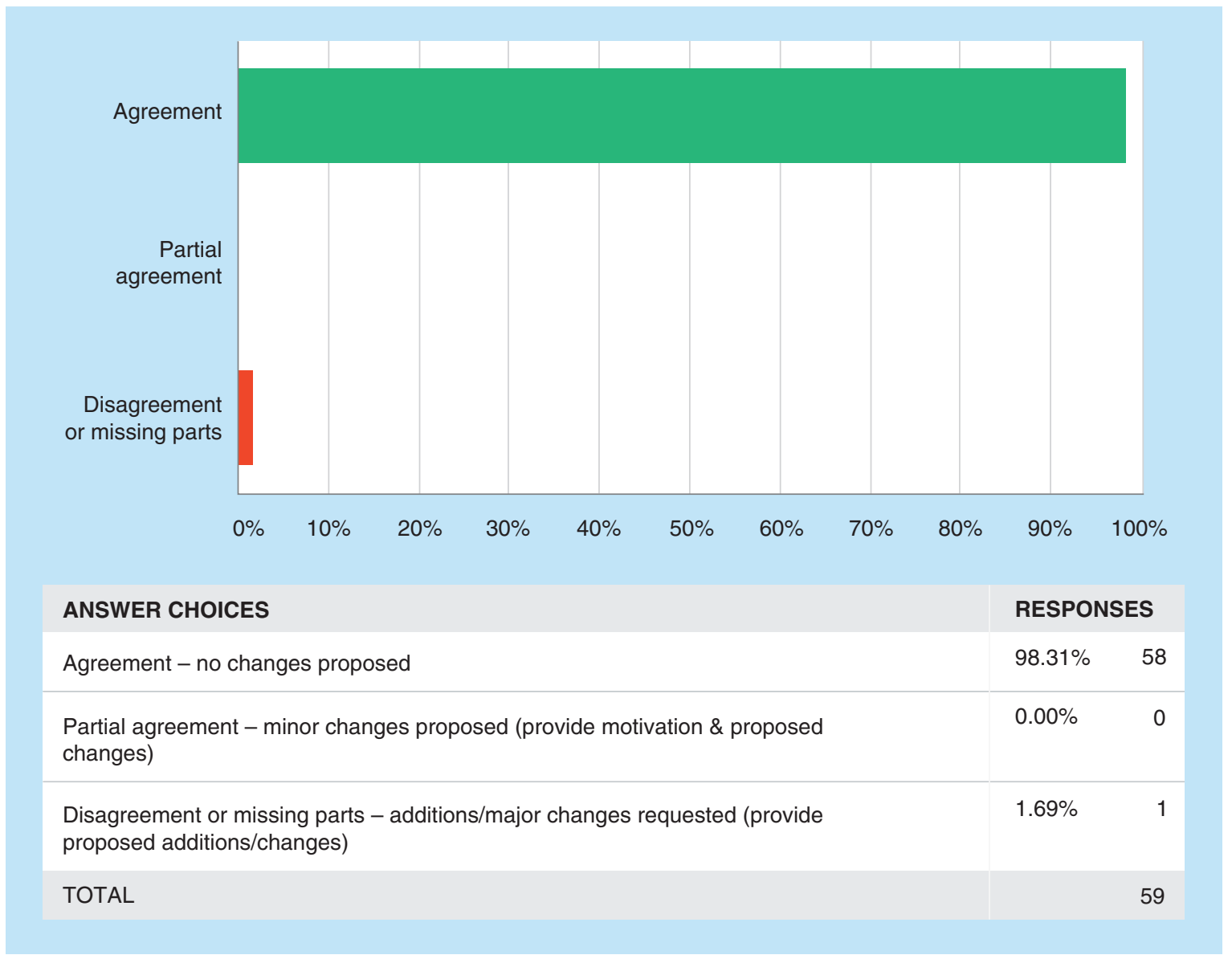


T1Q3: Objectives/Changes; Lines 89-92: "Changes from the recommendations in this guideline may be acceptable if appropriate scientific justification is provided. Applicants are encouraged to consult the regulatory authority(ies) regarding significant changes in method validation approaches when an alternate approach is proposed or taken."

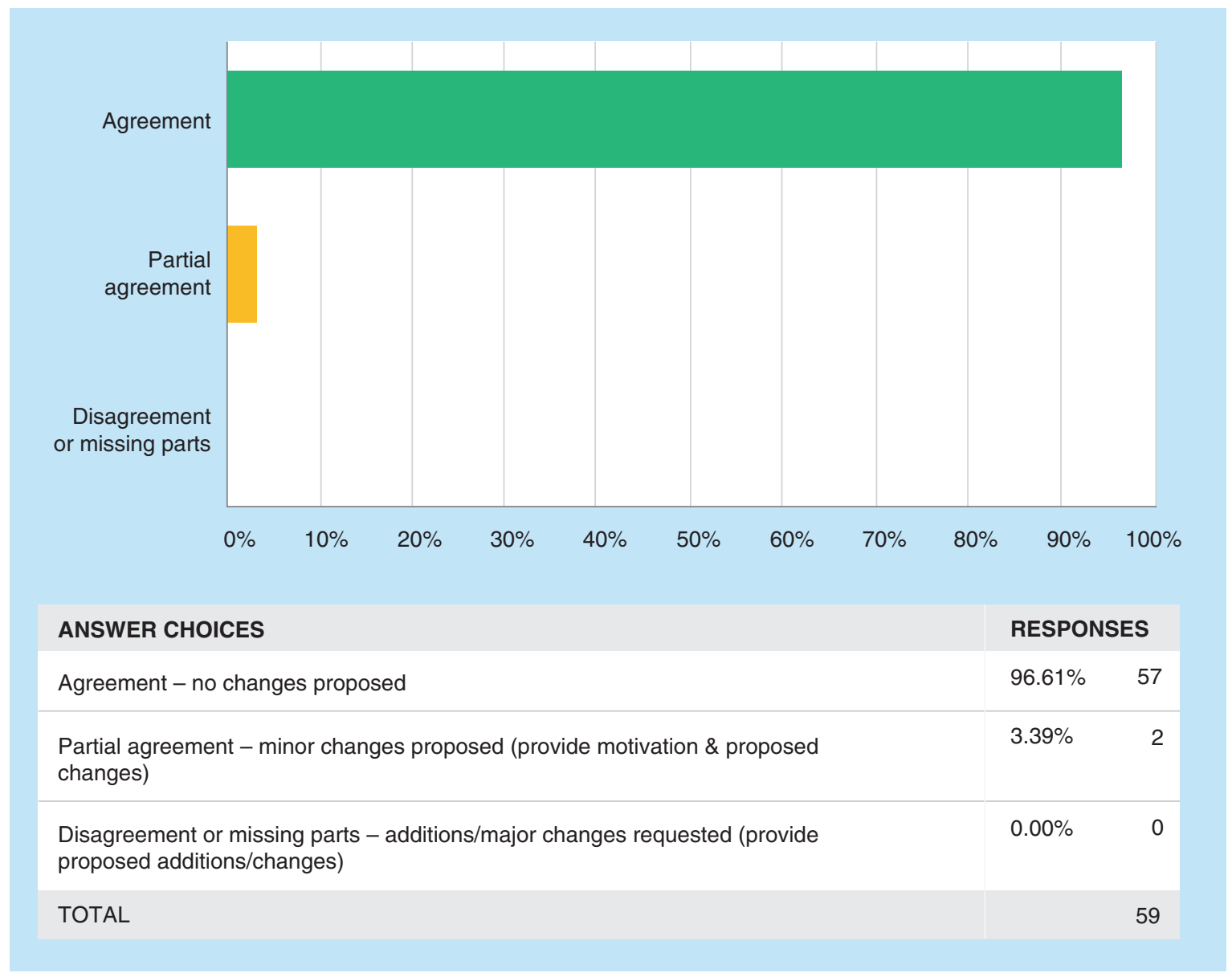

Partial agreement

No trend was found in these comments. 
T1Q4: Background; Lines 93-95: "Concentration measurements of chemical and biological drug(s) and their metabolite(s) in biological matrices are an important aspect of drug development."

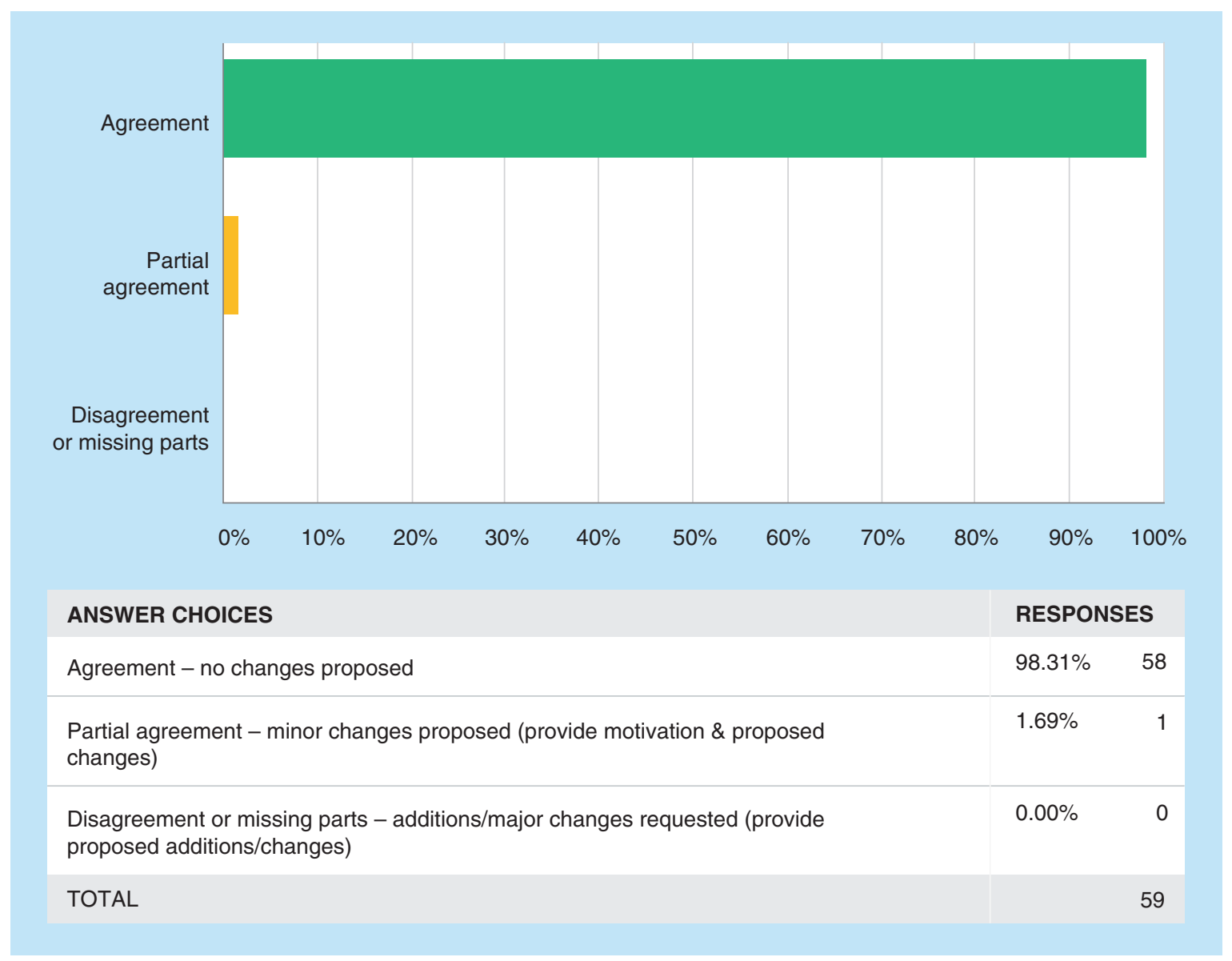


T1Q5: Background; Lines 95-100: "The results of pivotal nonclinical toxicokinetic (TK)/ pharmacokinetic (PK) studies and of clinical trials, including comparative bioavailability/ bioequivalence (BA/BE) studies, are used to make regulatory decisions regarding the safety and efficacy of drug products. It is therefore critical that the bioanalytical methods used are well characterised, appropriately validated and documented in order to ensure reliable data to support regulatory decisions."

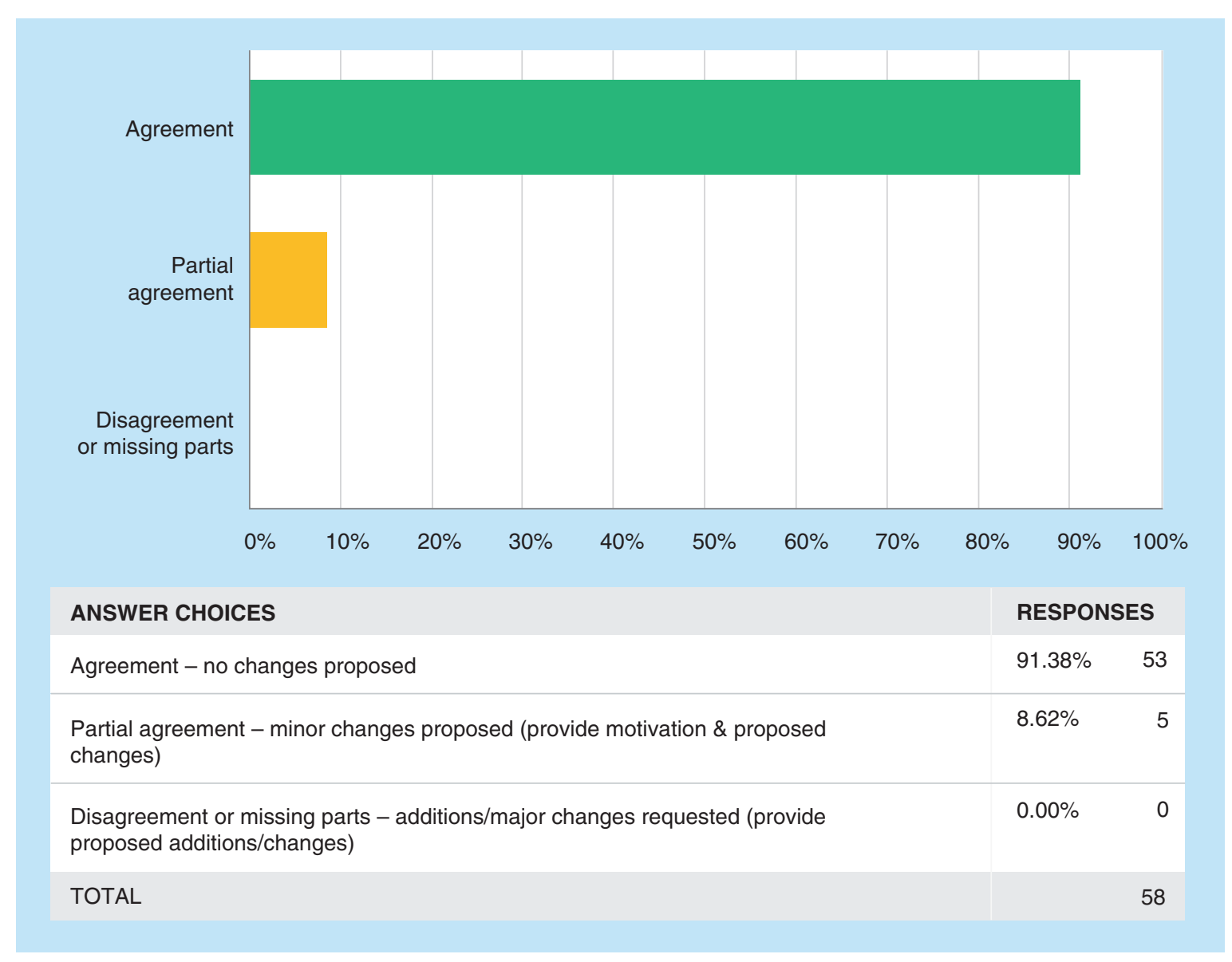

Partial agreement

It is suggested to clarify what constitutes a "pivotal nonclinical" study, perhaps in the form of examples. 
T1Q6: In Scope; Lines 102-109: "This guideline describes the method validation that is expected for bioanalytical assays that are submitted to support regulatory submissions. The guideline is applicable to the validation of bioanalytical methods used to measure concentrations of chemical and biological drug(s) and their metabolite(s) in biological samples (e.g., blood, plasma, serum, other body fluids or tissues) obtained in pivotal nonclinical TK/PK studies that are used to make regulatory decisions and all phases of clinical trials in regulatory submissions. Full method validation is expected for the primary matrix (ces) intended to support regulatory submissions. Additional matrices should be partially validated as necessary."

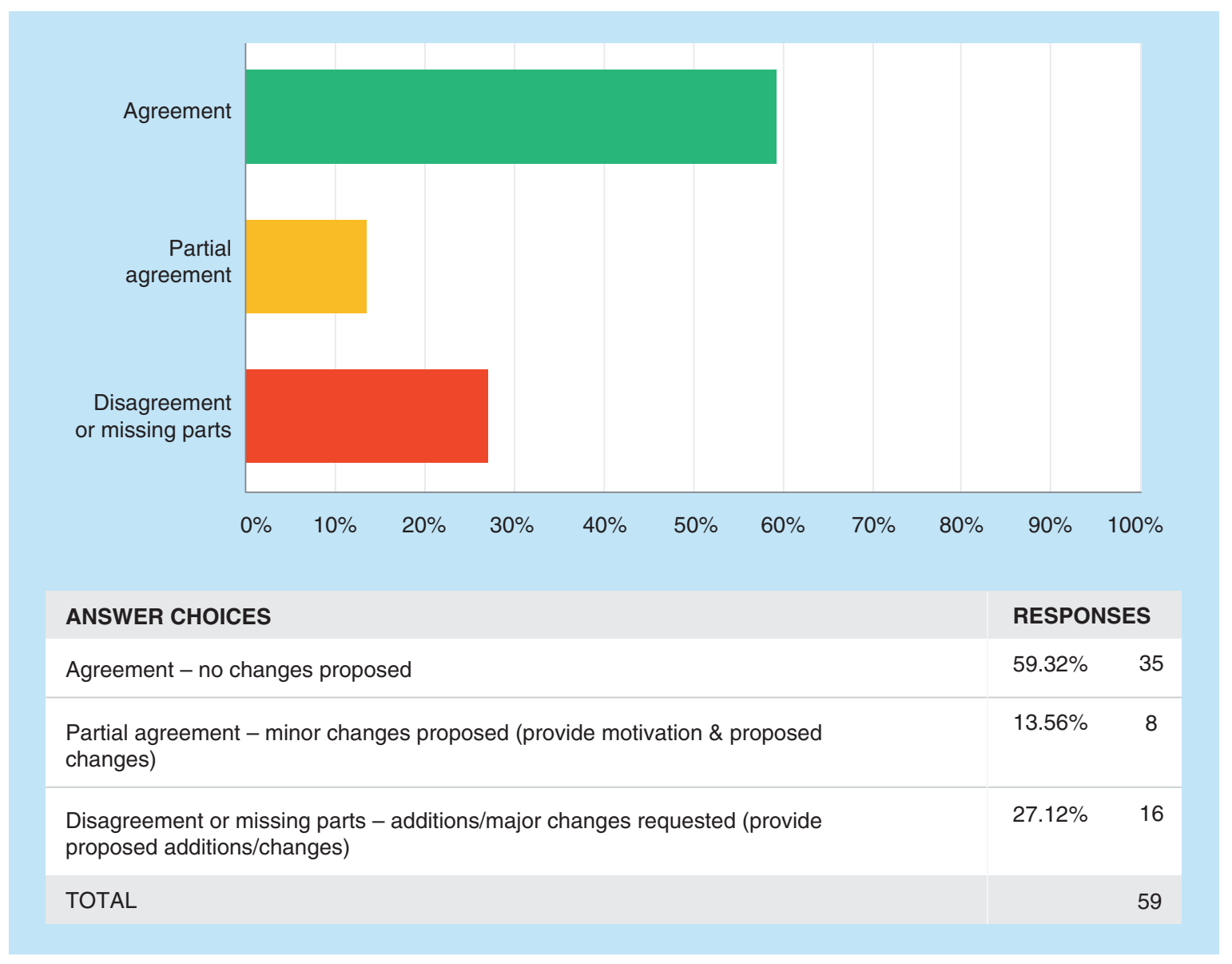

Disagreement or missing parts

- The addition of hybrid LBA/LCMS assays for large molecules, as an established technology in bioanalysis, is strongly encouraged.

- Additional clarification when using new technologies is recommended.

- As in T1Q5, it is suggested that clarity be provided for what constitutes a pivotal study, perhaps in the form of examples.

- For bioanalysis of tissues, the guidance does not acknowledge the challenge of fully validating methods in tissues given that they are typically homogenized prior to analysis. The issue of stability in tissue should also be addressed in the guideline.

\section{Partial agreement}

Additional clarification for partial validations is suggested, since many changes in matrices require full validation.

\section{Additional comments from the 13th GCC:}

- It was confirmed that a definition of "pivotal" for the glossary is recommended.

- It is also recommended to add a statement that if secondary matrices such as urine are validated, a full validation is needed. 
T1Q7: In Scope; Lines 109-111: "The analytes that should be measured in nonclinical and clinical studies and the types of studies necessary to support a regulatory submission are described in other ICH and regional regulatory documents."

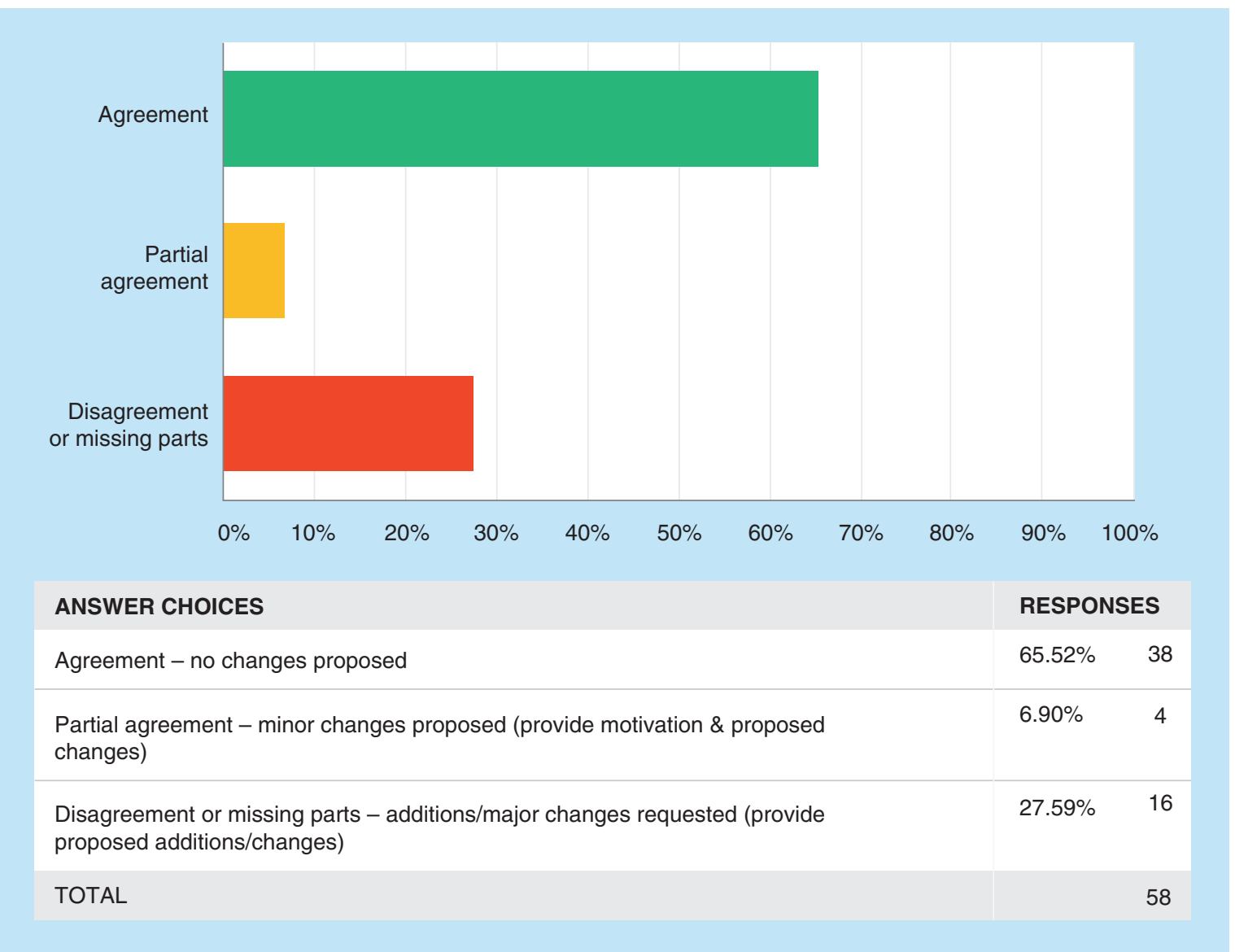

Disagreement or missing parts

All comments clearly requested that references for "other ICH and regional regulatory documents" be provided.

Partial agreement

No trend was found in these comments. 
T1Q8: Fit-for-Purpose (FFP); Lines 112-114: "For studies that are not submitted for regulatory approval or not considered for regulatory decisions regarding safety, efficacy or labelling (e.g., exploratory investigations), applicants may decide on the level of qualification that supports their own internal decision making."

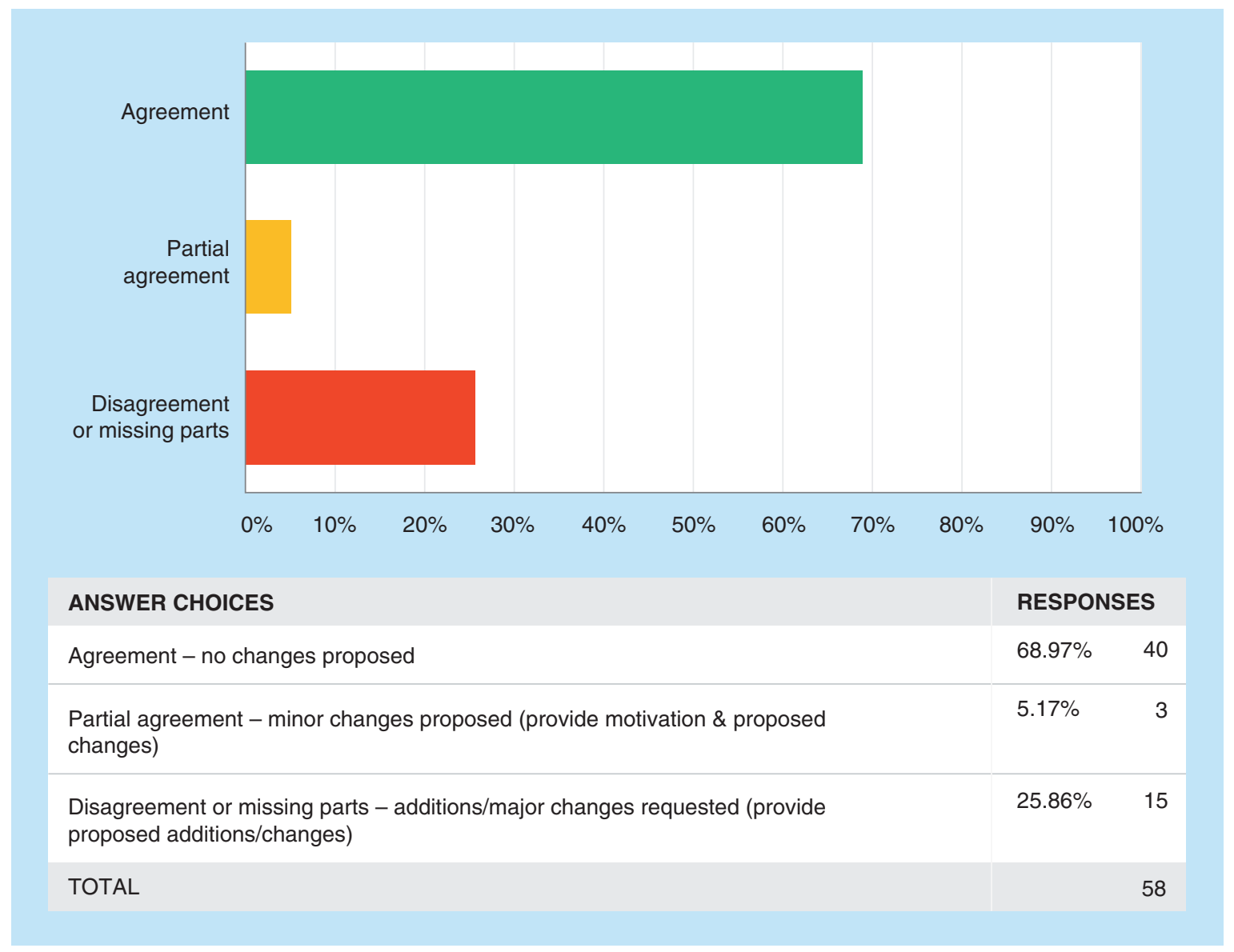

Disagreement or missing parts

All the comments clearly pointed out that the language "level of qualification" is confusing, misleading and must be changed with "fit-for-purpose" since this is the harmonized term currently in use across the industry.

Partial agreement

No trend was found in these comments. 
T1Q9: In Scope; Lines 115-120: "The information in this guideline applies to the quantitative analysis by ligand binding assays (LBAs) and chromatographic methods such as liquid chromatography (LC) or gas chromatography (GC), which are typically used in combination with mass spectrometry (MS) detection and occasionally with other detectors. For studies that are subject to Good Laboratory Practice (GLP) or Good Clinical Practice (GCP) the bioanalysis of study samples should also conform to their requirements."

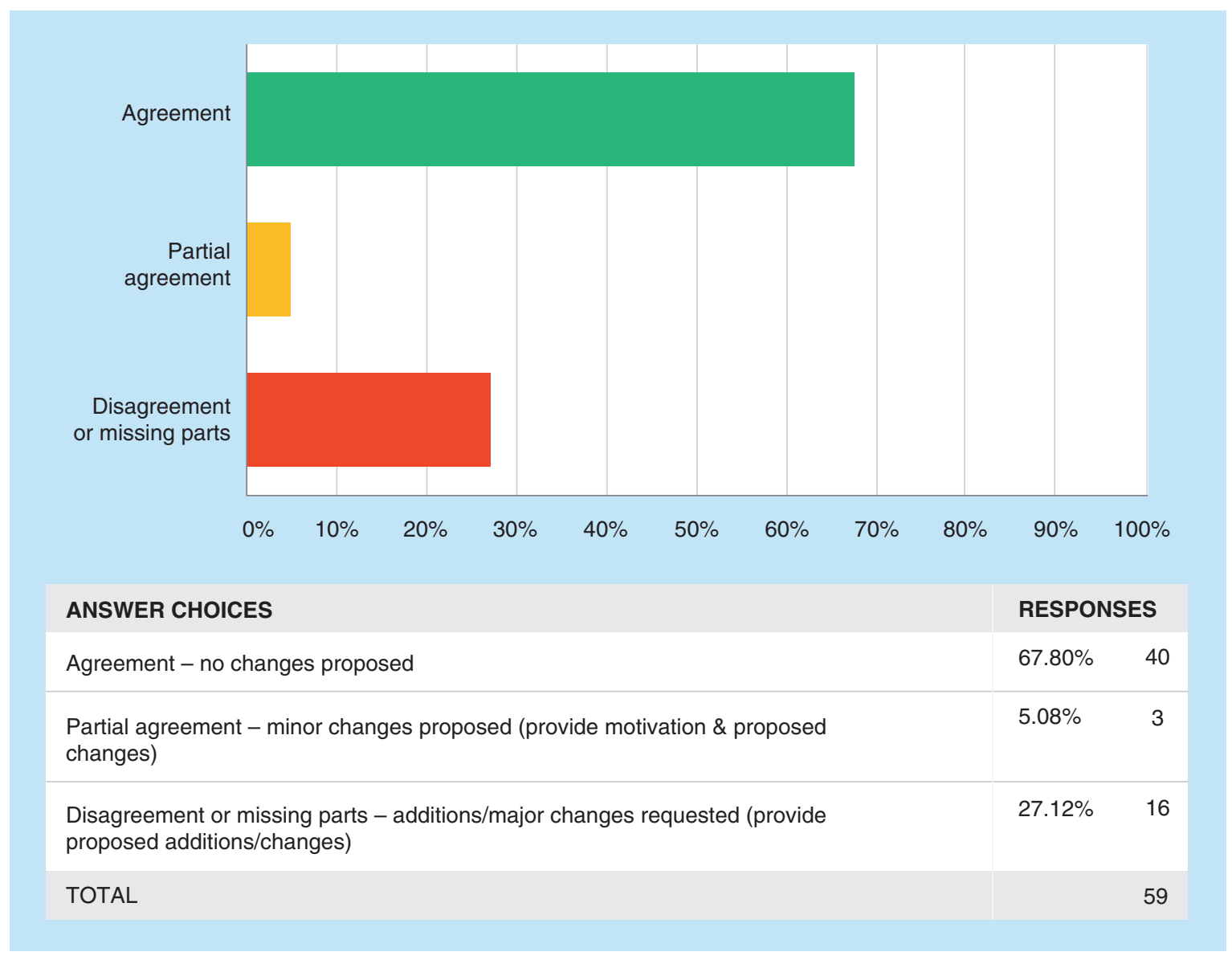

Disagreement or missing parts

The majority of the comments requested a guideline for hybrid LBA/LCMS for biotherapeutics. Hence, it is strongly recommended to add the bold wording to the first sentence: "The information in this guideline applies to the quantitative analysis by ligand binding assays (LBAs) and chromatographic methods such as liquid chromatography $(L C)$, or gas chromatography $(G C)$, which are typically used in combination with mass spectrometry (MS) detection and occasionally with other detectors. The information in this guideline also applies to hybrid assays (bybrid LBA/LCMS)."

Partial agreement

No trend was found in these comments. 
T1Q10: Out of Scope; Lines 121-122: "The bioanalysis of biomarkers and bioanalytical methods used for the assessment of immunogenicity are not within the scope of this guideline."

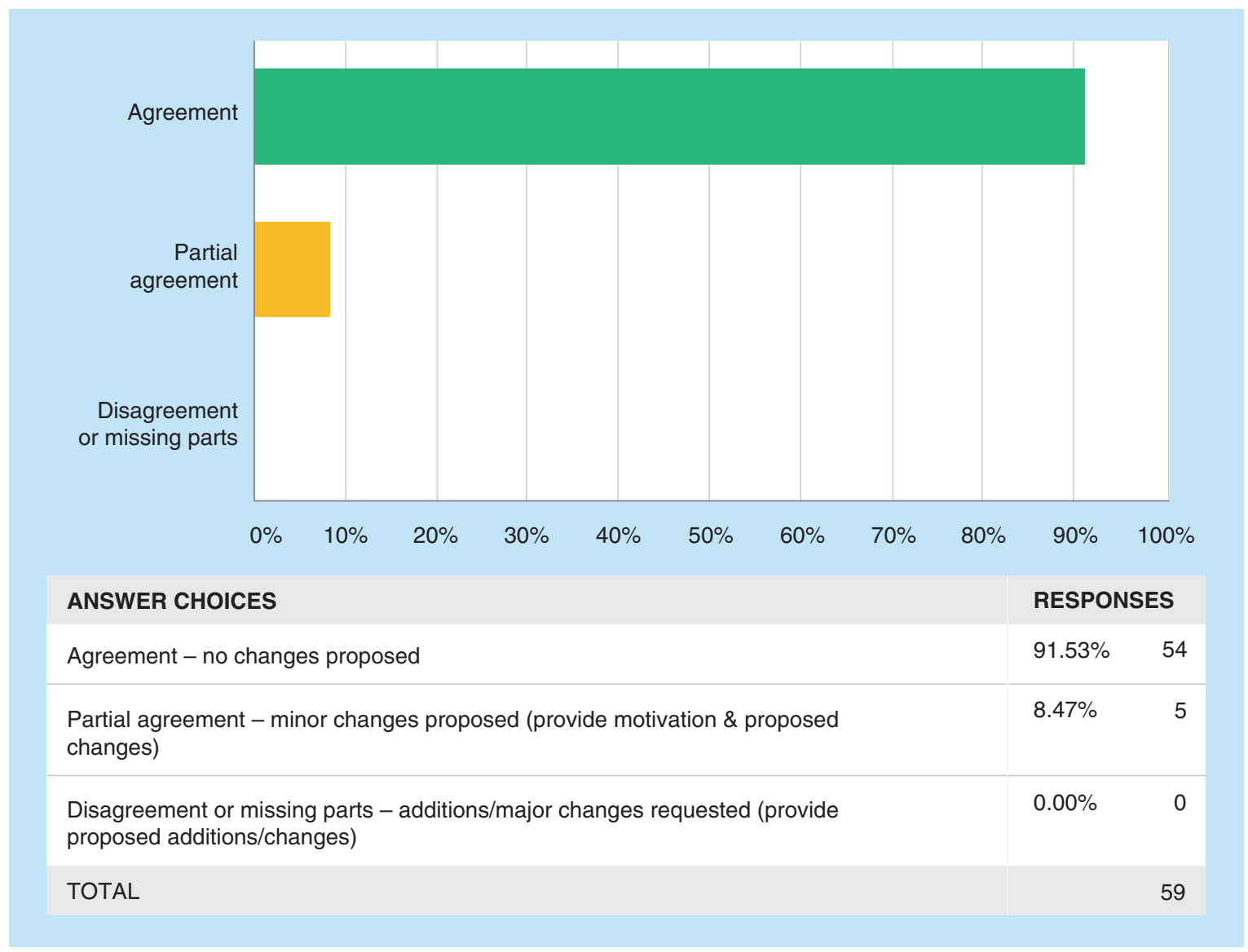

\section{Partial agreement}

It may be useful to mention that three CROs commented on the exclusion of biomarkers in this guideline versus the 2018 FDA BMV guidance [18] where they are included and proposed the addition of a reference to biomarkers in the "fit-for-purpose" section. 
T1Q11: ISR Background/Purpose; Lines 917-925: "The performance of study samples may differ from that of the calibration standards and QCs used during method validation, which are prepared by spiking blank matrix. Differences in protein binding, back-conversion of known and unknown metabolites, sample inhomogeneity, concomitant medications or biological components unique to the study samples may affect the accuracy and precision of analysis of the analyte in study samples. Therefore, ISR is a necessary component of bioanalytical method validation. It is intended to verify the reliability of the reported sample analyte concentrations and to critically support the precision and accuracy measurements established with spiked QCs."

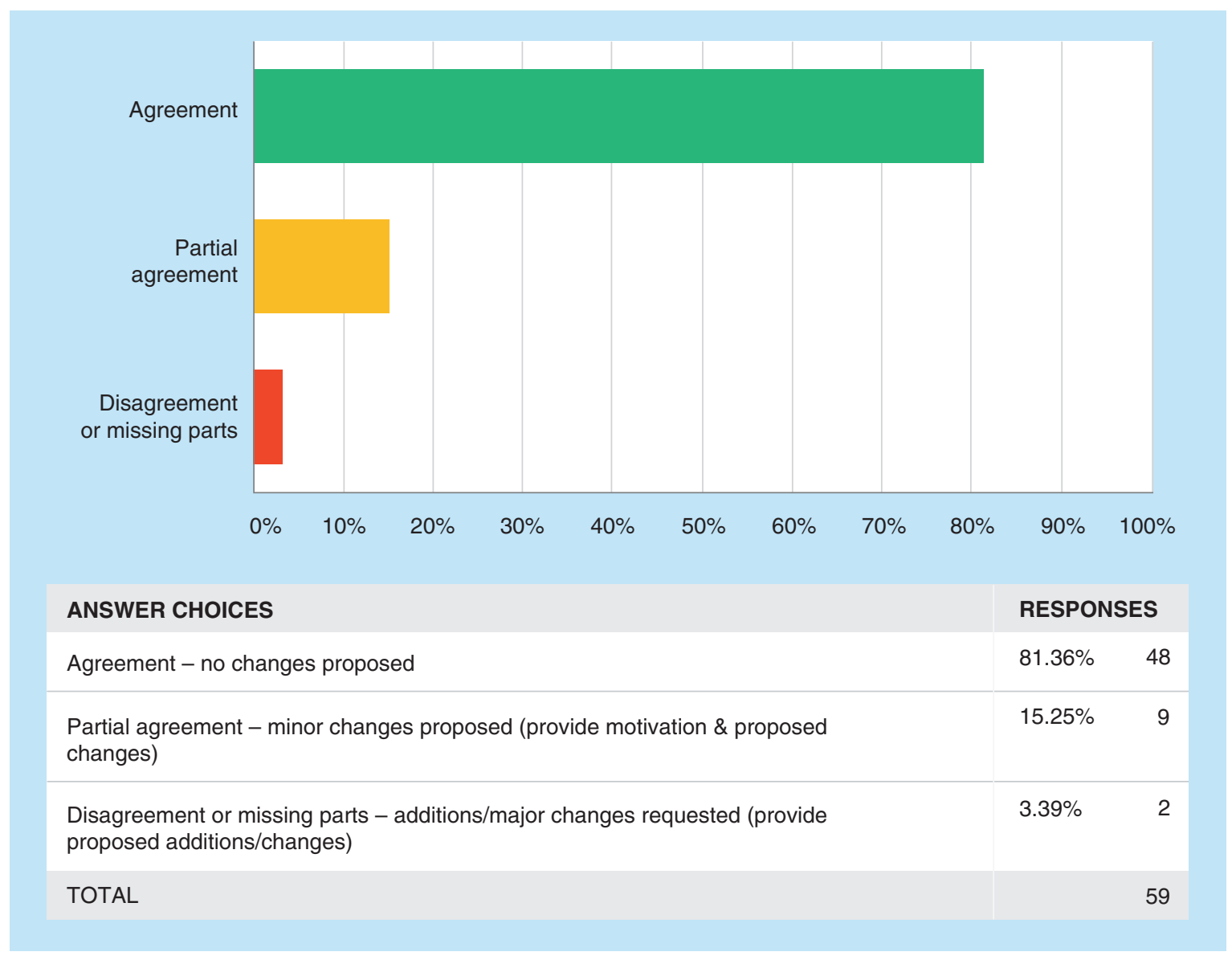

Disagreement or missing parts

No trend was found in these comments.

\section{Partial agreement}

- The majority of the comments were concerned with the language that ISR is a component of method validation. The ISR evaluation is typically performed and reported as part of sample analysis for clinical studies and it is unclear if this language is suggesting ISR information should be included in validation reports (e.g., as an addendum).

- The remaining comments were primarily focused on the notion that ISR demonstrates reliability and accuracy. ISR can demonstrate the reproducibility of assay (instead of reliability) after method validation is complete. The reproducibility of the ISR may not necessarily show bias attributed to any of the issues mentioned in the text, therefore it is recommended to remove "accuracy" and replace "reliability" with "reproducibility." 
T1Q12: ISR Studies; Lines 926-934: "ISR should be performed at least in the following situations:

- For preclinical studies, ISR should, in general, be performed for the main nonclinical TK studies once per species. However, ISR in a PK study instead of a TK study might also be acceptable, as long as the respective study has been conducted as a pivotal study, used to make regulatory decisions.

- All pivotal comparative BA/BE studies

- First clinical trial in subjects

- Pivotal early patient trial(s), once per patient population

- First or pivotal trial in patients with impaired hepatic and/or renal function"

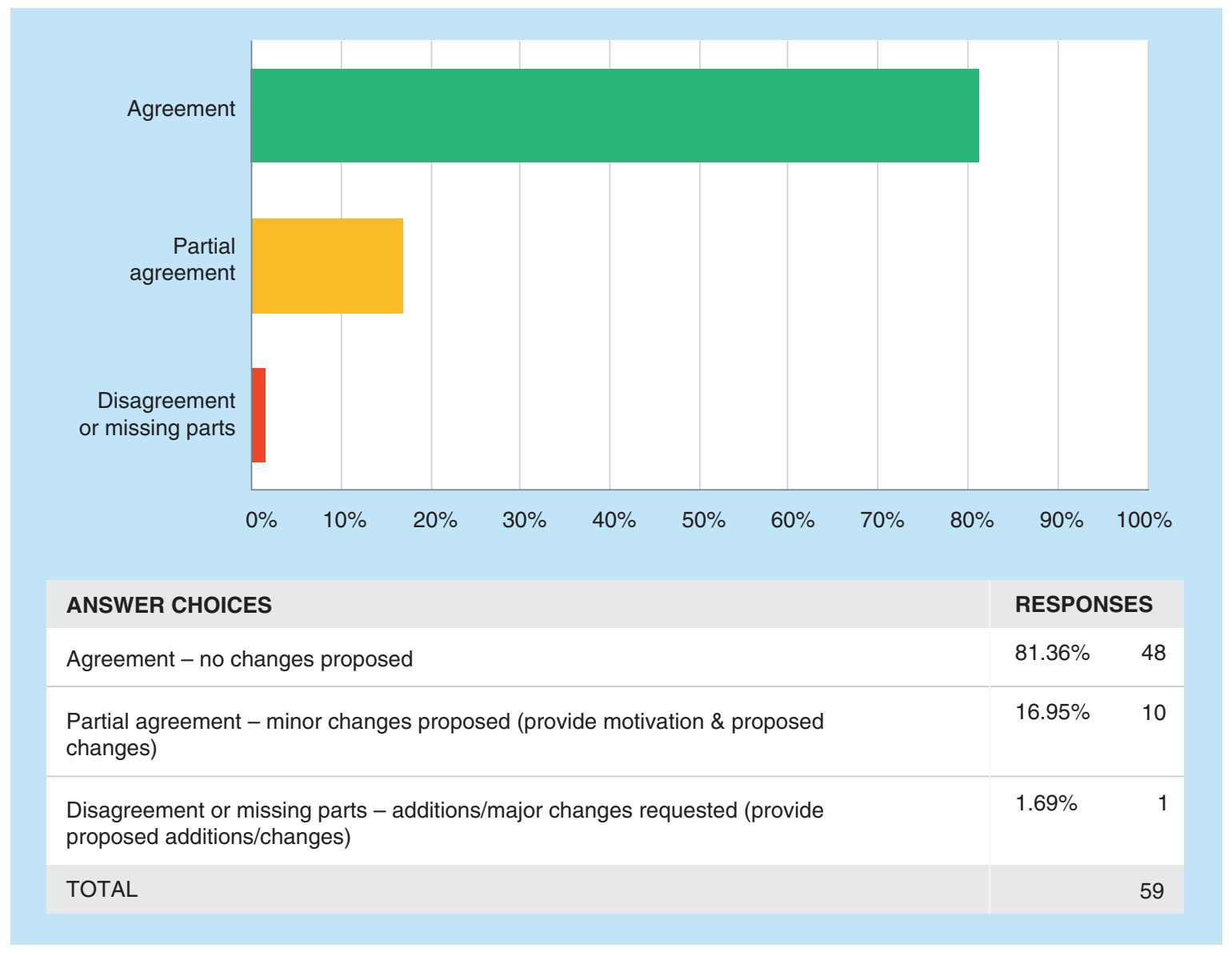

\section{Partial agreement}

It was suggested to clarify:

- if "main study" refers to a pivotal study;

- if "first clinical trial in subjects" refers to healthy volunteers or patients, or both;

- the definition of "early patient trials." 
T1Q13: ISR Procedure; Lines 935-936: "ISR is conducted by repeating the analysis of a subset of samples from a given study in separate (i.e., different to the original) runs on different days using the same bioanalytical method."

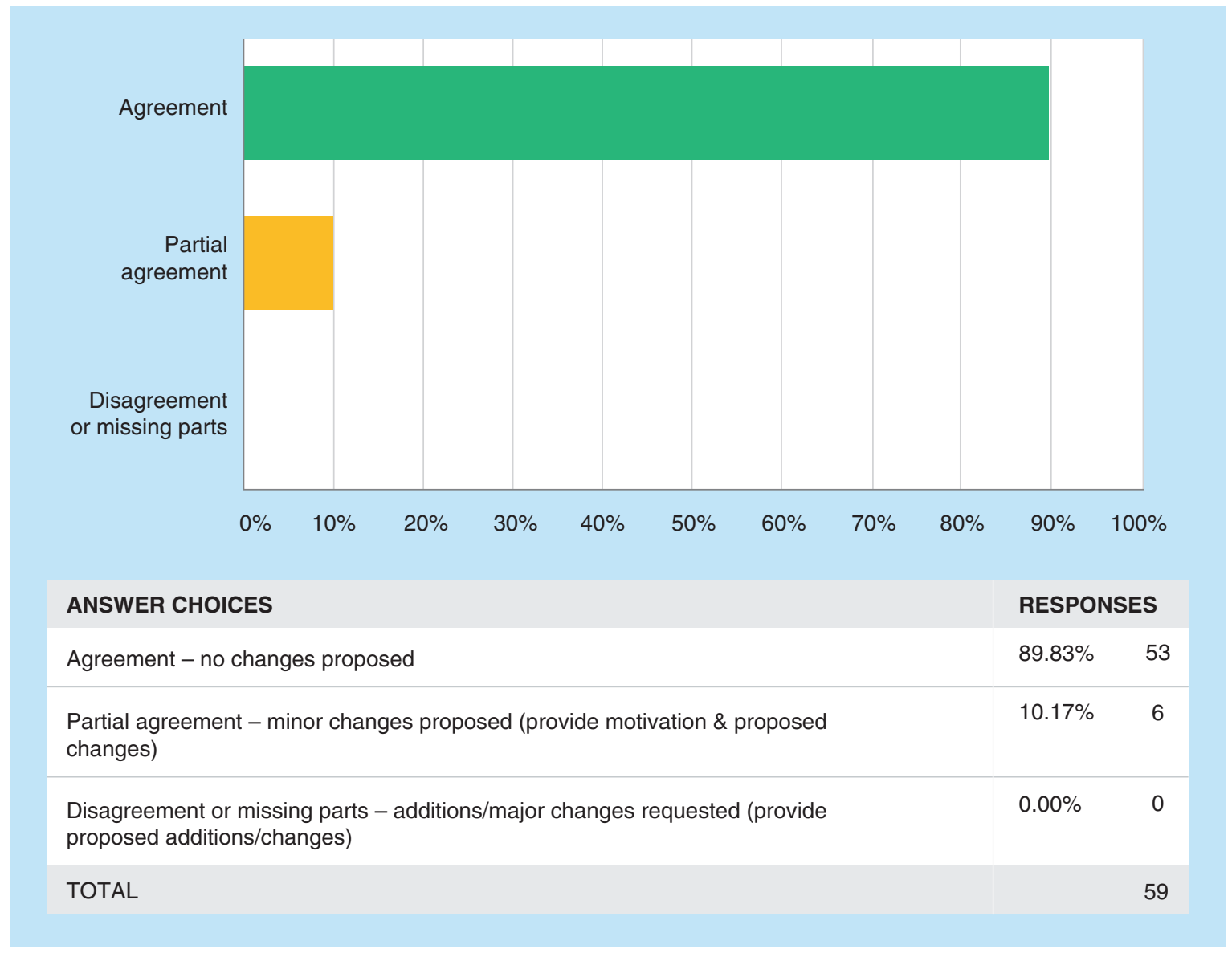

\section{Partial agreement}

All comments suggested replacing the requirement for different days to independent analytical occasions so as not to artificially increase timelines. 
T1Q14: ISR Number of Samples; Lines 937-940: "The extent of ISR depends upon the analyte and the study samples and should be based upon an in-depth understanding of the analytical method and analyte. However, as a minimum, if the total number of study samples is less than 1000, then 10\% of the samples should be reanalysed; if the total number of samples is greater than 1000, then 10\% of the first 1000 samples (100) plus 5\% of the number of samples that exceed 1000 samples should be assessed."

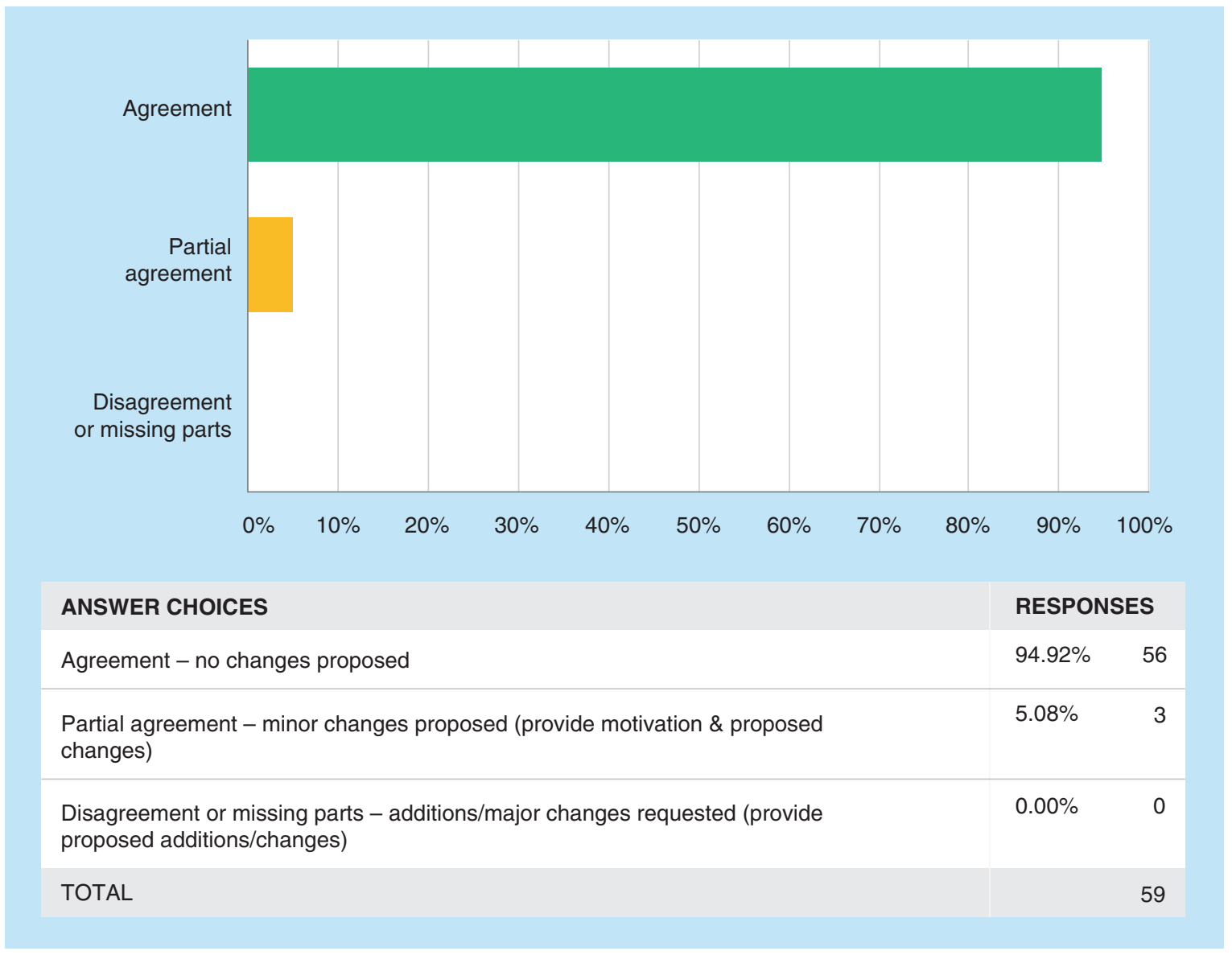

\section{Partial agreement}

The three comments suggested that a minimum number of samples be proposed for very small studies (e.g., below 1000 samples). Additionally, a proposed maximum number of samples would reduce the needless repetition of samples. 
T1Q15: ISR Samples Selection/Requirements; Lines 941-950: "Objective criteria for choosing the subset of study samples for ISR should be predefined in the protocol, study plan or an SOP. While the subjects should be picked as randomly as possible from the dosed study population, adequate coverage of the PK profile in its entirety is important. Therefore, it is recommended that the samples for ISR be chosen around the maximum concentration (Cmax) and some in the elimination phase. Additionally, the samples chosen should be representative of the whole study. Samples should not be pooled, as pooling may limit anomalous findings. ISR samples and QCS should be prepared in the same manner as in the original analysis. ISR should be performed within the stability window of the analyte, but not on the same day as the original analysis."

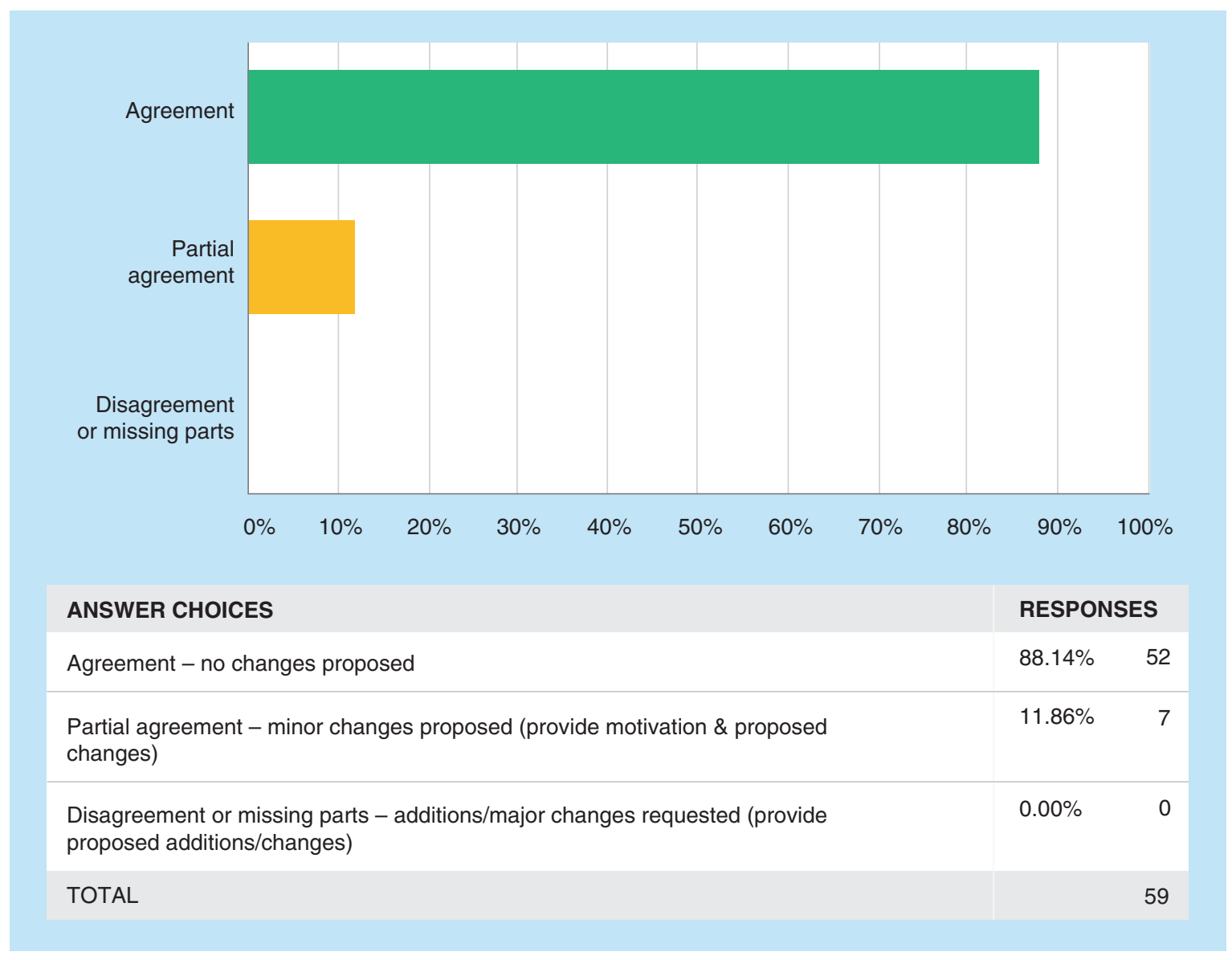

\section{Partial agreement}

- As for T1Q13, a few comments suggested to replace the requirement for different days to independent analytical occasions.

- A few other comments requested more clarification on the language stating that samples should be randomly selected since it may be confusing with the next statement to select samples near the Cmax and elimination phase (i.e., cannot both randomly select samples and specify specific samples for selection). 
T1Q16: ISR Formula; Lines 951-954: "The percent difference between the initial concentration and the concentration measured during the repeat analysis should be calculated in relation to their mean value using the following equation:

$\%$ difference $=($ repeat value - initial value $) /$ mean value $\times 100^{\prime \prime}$

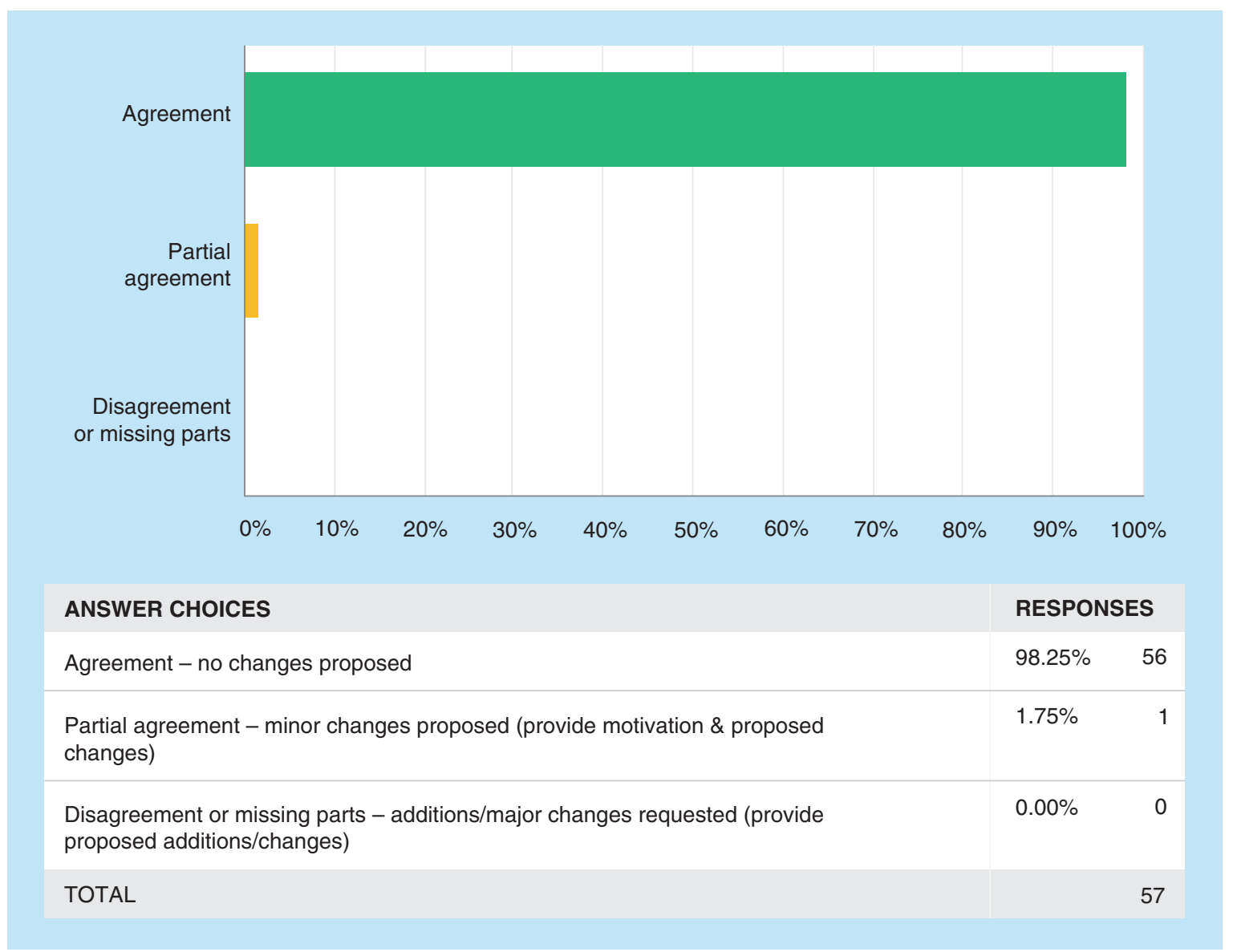


T1Q17: ISR Criteria; Lines 955-956: "For chromatographic methods, the percent difference should be $\leq 20 \%$ for at least 2/3 of the repeats. For $L B A s$, the percent difference should be $\leq 30 \%$ for at least $2 / 3$ of the repeats."

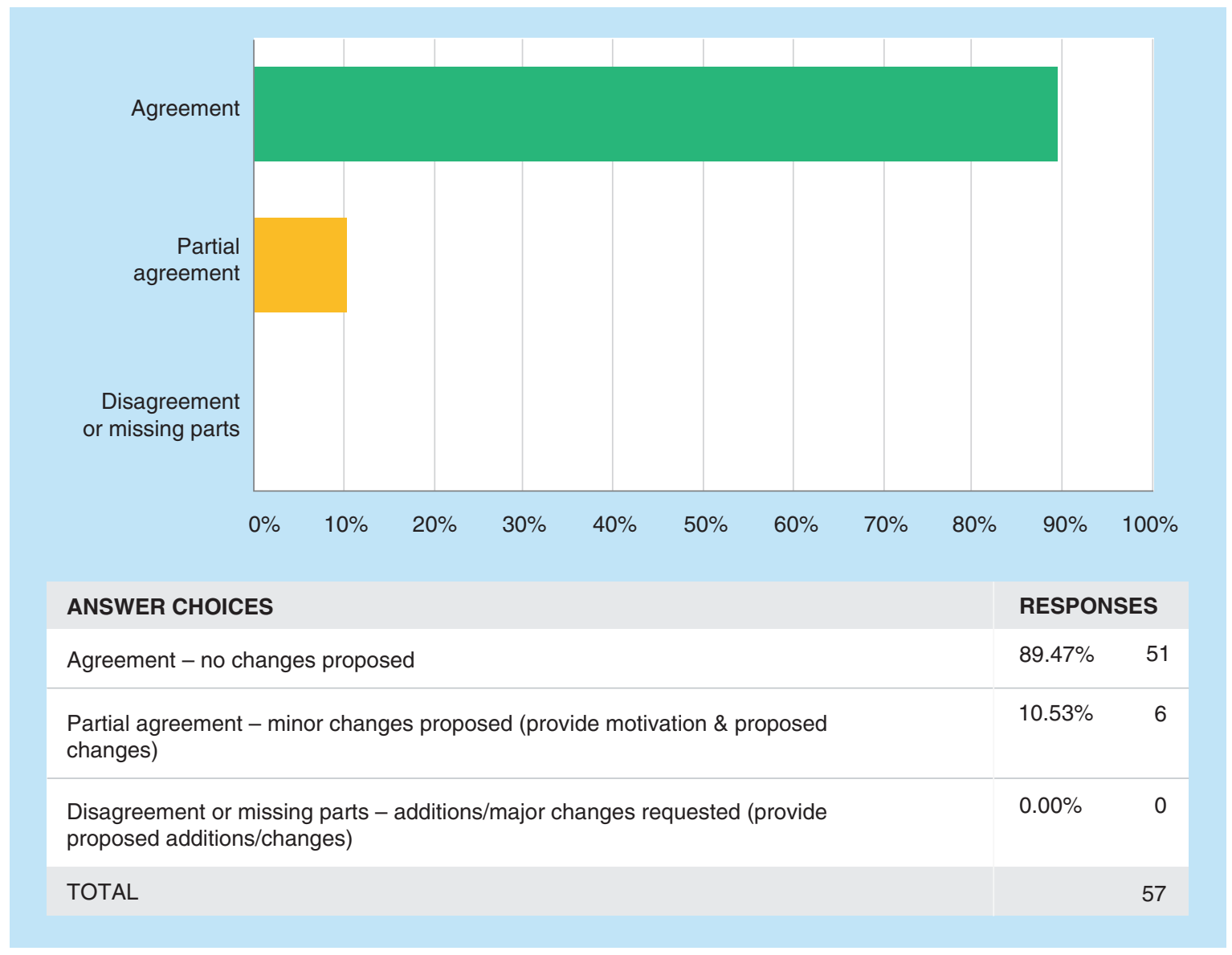

Partial agreement

No trend was found in these comments. 
T1Q18: ISR Failure/Reporting; Lines 957-969: "If the overall ISR results fail the acceptance criteria, an investigation should be conducted and the causes remediated. There should be an SOP that directs how investigations are triggered and conducted. If an investigation does not identify the cause of the failure, the potential impact of an ISR failure on study validity should also be provided in the Bioanalytical Report. If ISR meets the acceptance criteria yet shows large or systemic differences between results for multiple samples, this may indicate analytical issues and it is advisable to investigate this further. Examples of trends that are of concern include:

- All samples from one subject fail

- All of samples from one run fail

All aspects of ISR evaluations should be documented to allow reconstruction of the study and any investigations. Individual samples that are quite different from the original value (e.g., > 50\%, "flyers") should not trigger reanalysis of the original sample and do not need to be investigated. ISR sample data should not replace the original study sample data."

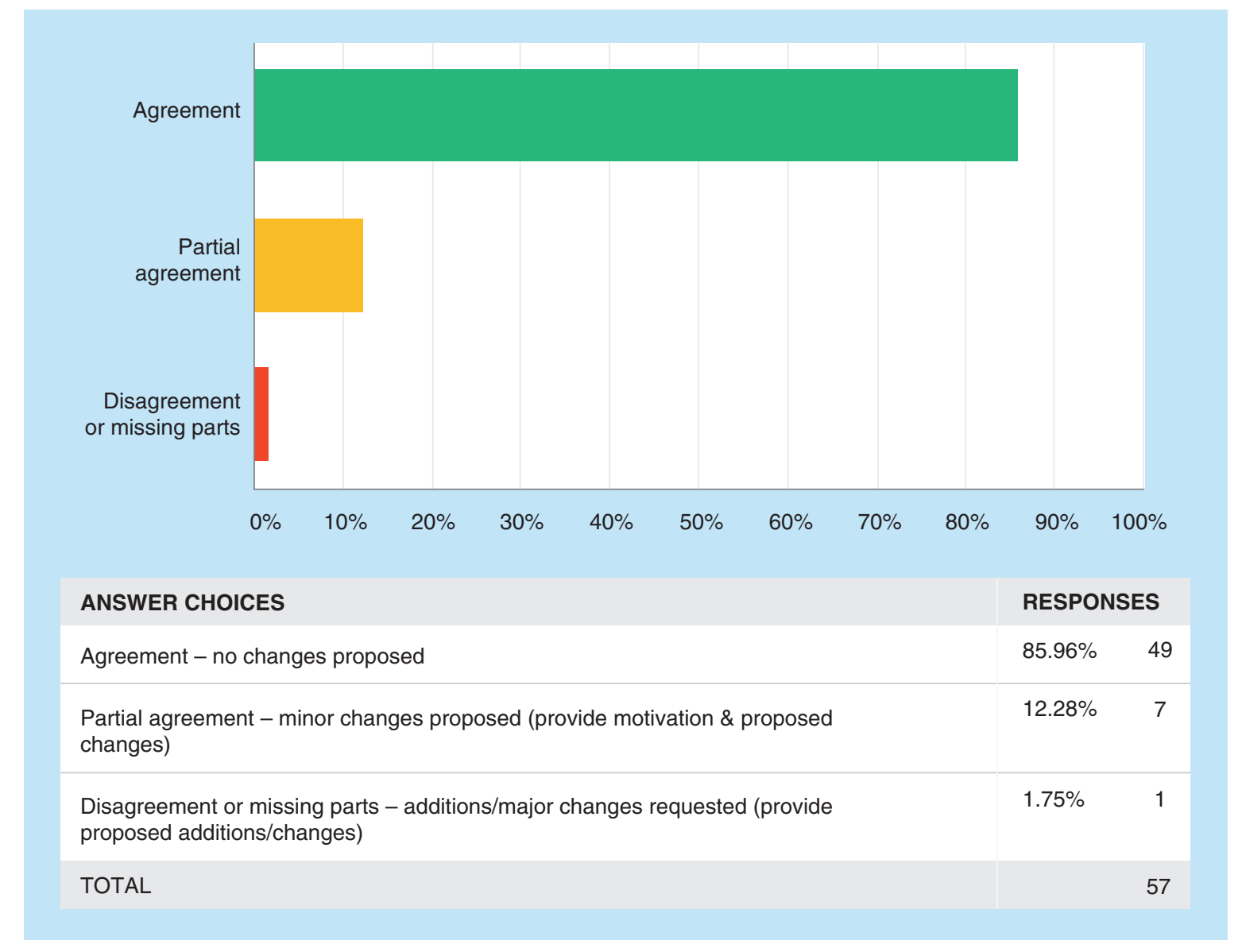

\section{Partial agreement}

The majority of the comments suggested that the statement should be updated to indicate that the results of the investigation, regardless of outcome, should be provided in the bioanalytical report.

\section{Additional comments from the 13th GCC:}

It was acknowledged that it was difficult to know at what point in an investigation it is permissible to reanalyze samples. It was suggested to add the bold text to the sentence (as well as remove the word "flyers"): "Individual samples that are quite different from the original value (e.g., >50\%) but do not correlate with a trend or analytical pattern, should not automatically trigger reanalysis of the original sample and may need to be investigated." 
T1Q19: Partial Validation; Lines 972-974: "Partial validations evaluate modifications to already fully validated bioanalytical methods. Partial validation can range from as little as one within-run accuracy and precision determination, to a nearly full validation."

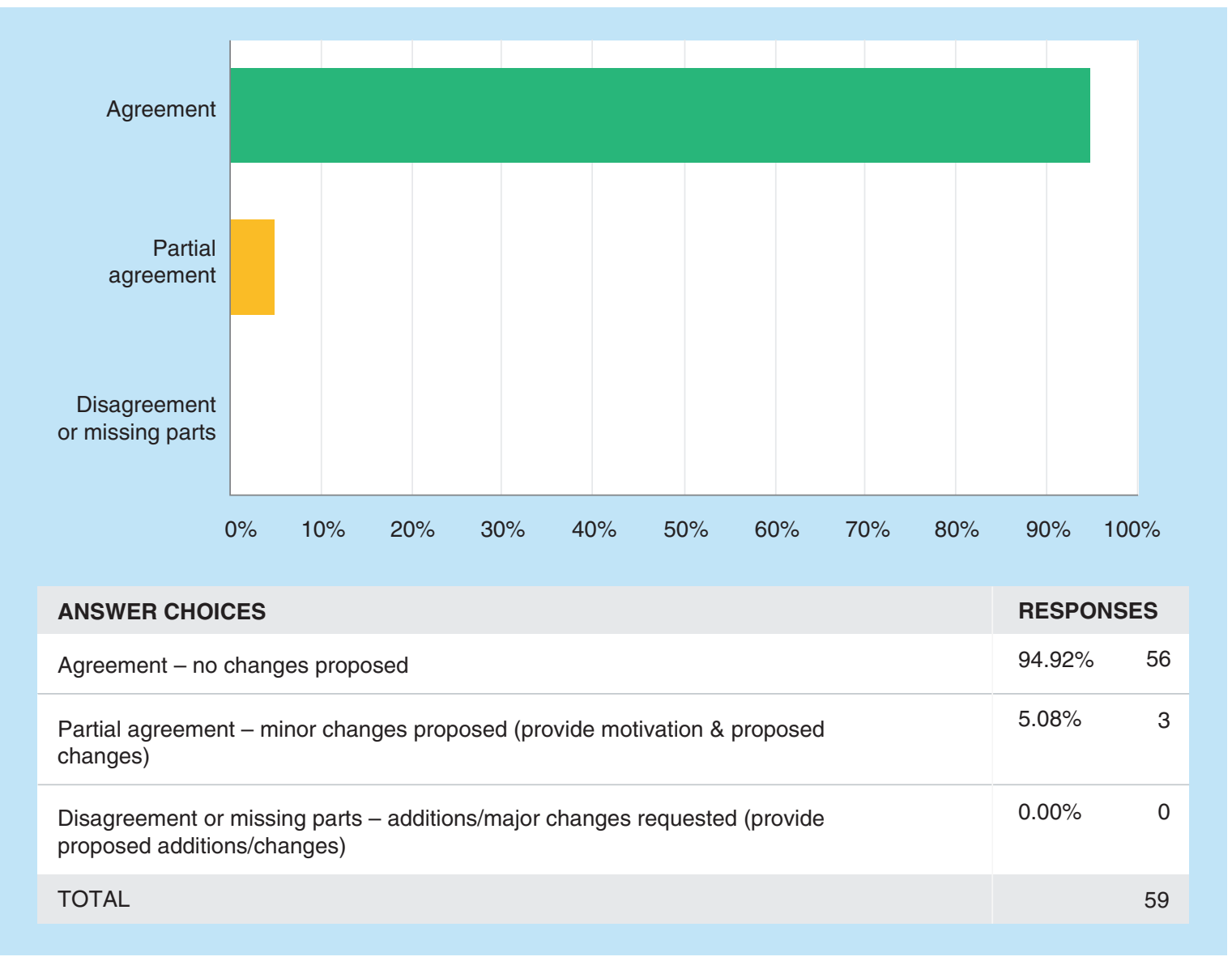

Partial agreement

No trend was found in these comments. 
T1Q20: Partial Validation - Stability; Lines 974-975: "If stability is established at one facility it does not necessarily need to be repeated at another facility."

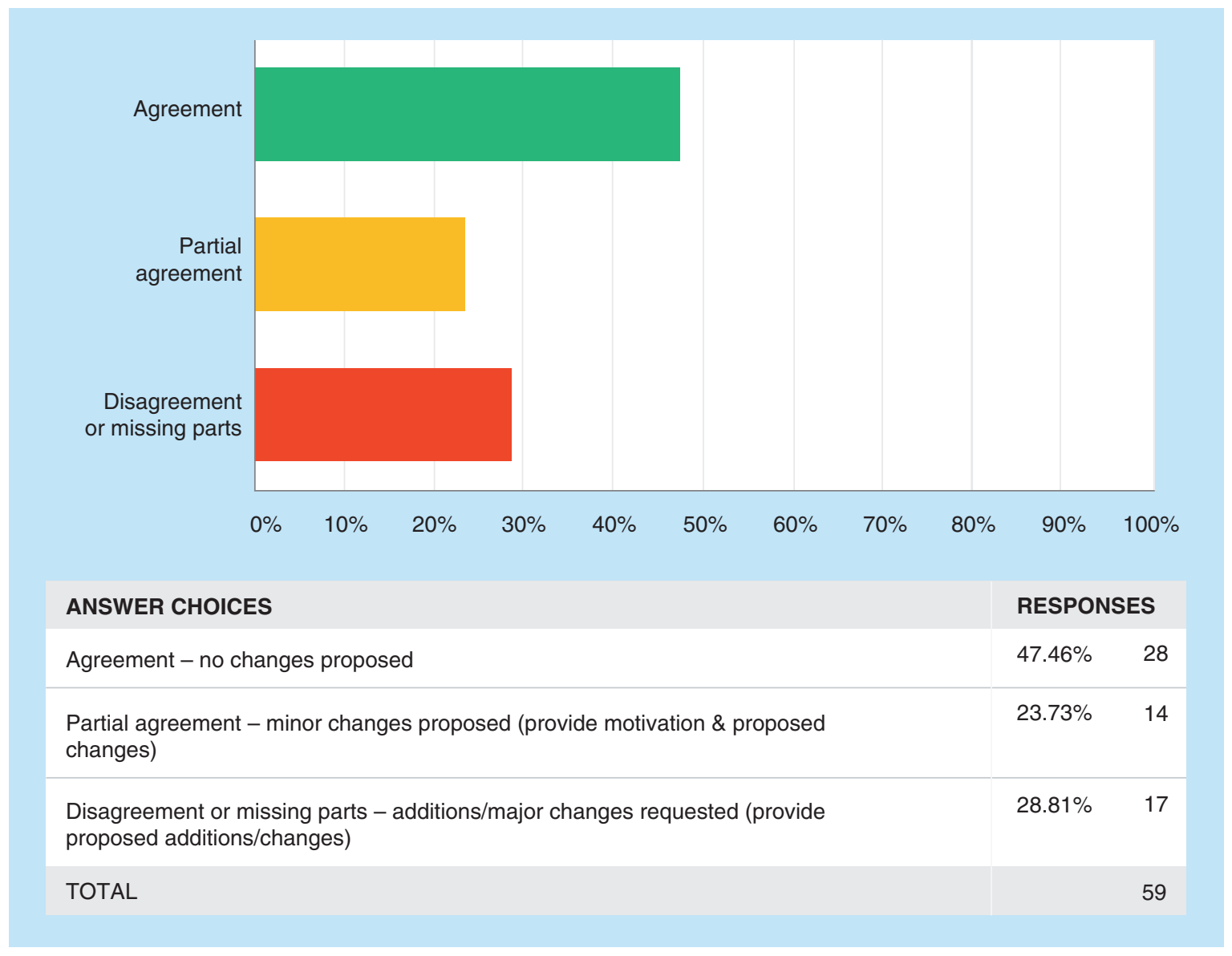

\section{Disagreement or missing parts}

All comments indicated strong disagreement with this statement and that it should be removed. Objections included clarification on what the background and scientific justification are in support for this statement, concerns on acceptability of this procedure by EMA and FDA, method bias to perform stability and previous experience in repeating stabilities at each facility and obtaining different results.

\section{Partial agreement}

No trend was found in these comments.

\section{Additional comments from the 13th GCC:}

The availability of documentation in order to be able to use stability results from a different CRO was identified as an important concern. Each CRO or sponsor must provide full access to all stability data (on short notice, in case of an inspection) and supporting information. 
T1Q21: Partial Validation - Chromatographic Methods; Lines 976-989: "For chromatographic methods, typical bioanalytical method modifications or changes that fall into this category include, but are not limited to, the following situations:

- Analytical site change using same method (i.e., bioanalytical method transfers between laboratories)

- A change in analytical methodology (e.g., change in detection systems, platform)

- A change in sample processing procedures

- A change in sample volume (e.g., the smaller volume of paediatric samples)

- Changes to the calibration concentration range

- A change in anticoagulant (but not changes in the counter-ion) in biological fluids (e.g., heparin to ethylenediaminetetraacetic acid (EDTA))

- Change from one matrix within a species to another (e.g., switching from human plasma to serum or cerebrospinal fluid) or changes to the species within the matrix (e.g., switching from rat plasma to mouse plasma)

- A change in storage conditions"

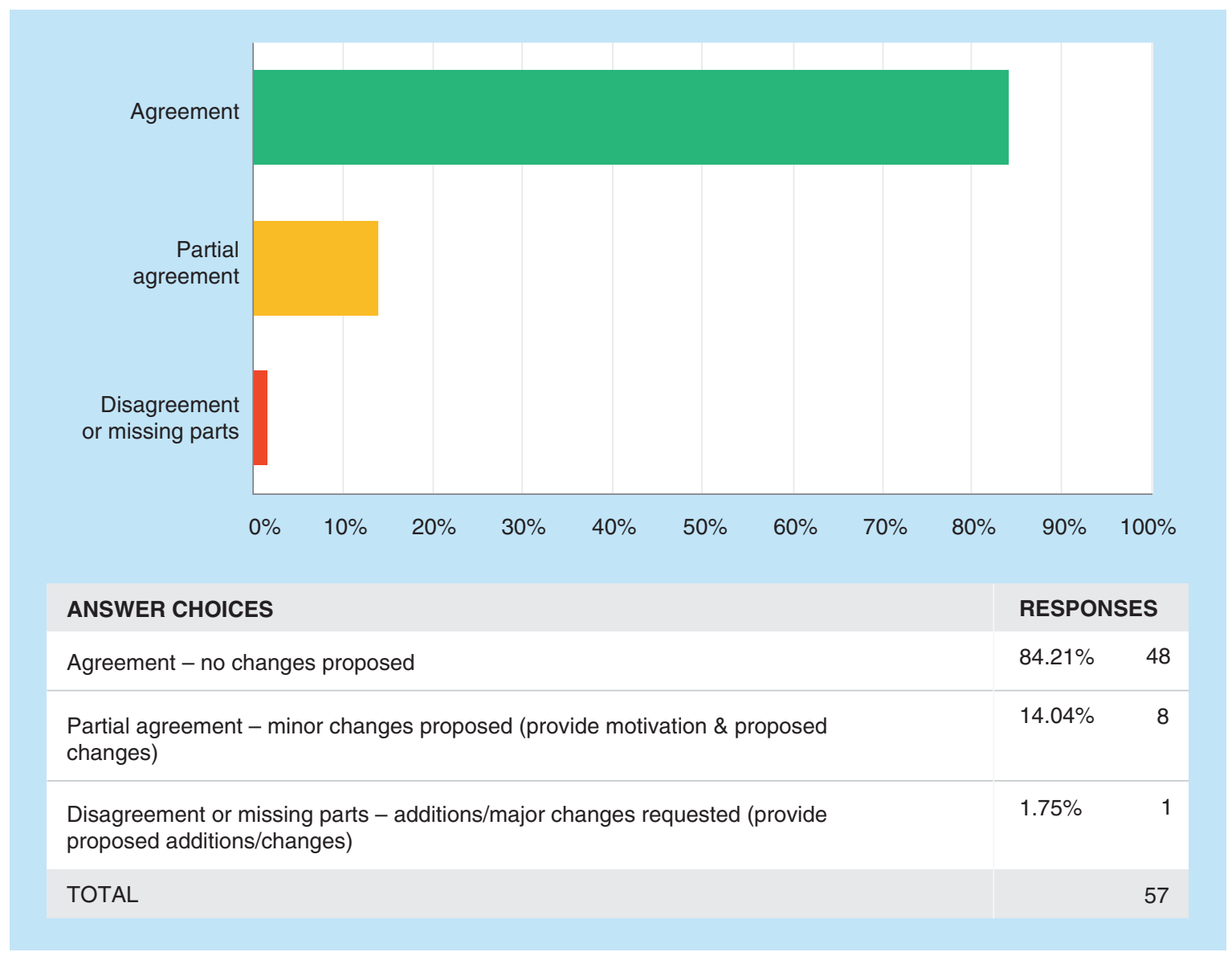

\section{Partial agreement}

Several comments mentioned a conflict with the EMA Guideline [19] and earlier language regarding a full validation for pivotal nonclinical studies and these lines which allow a change in species to be a partial validation. Clarity is needed for cases when there is a change of species for a pivotal study. 
T1Q22: Partial Validation - LBA; Lines 990-999: "For LBAs, typical bioanalytical method modifications or changes that fall into this category include, but are not limited to, the following situations:

- Changes in LBA critical reagents (e.g., lot-to-lot changes)

- Changes in MRD

- A change in storage conditions

- Changes to the calibration concentration range

- A change in analytical methodology (e.g., change in detection systems, platform)

- Analytical site change using same method (i.e., bioanalytical method transfers between laboratories)

- A change in sample preparation"

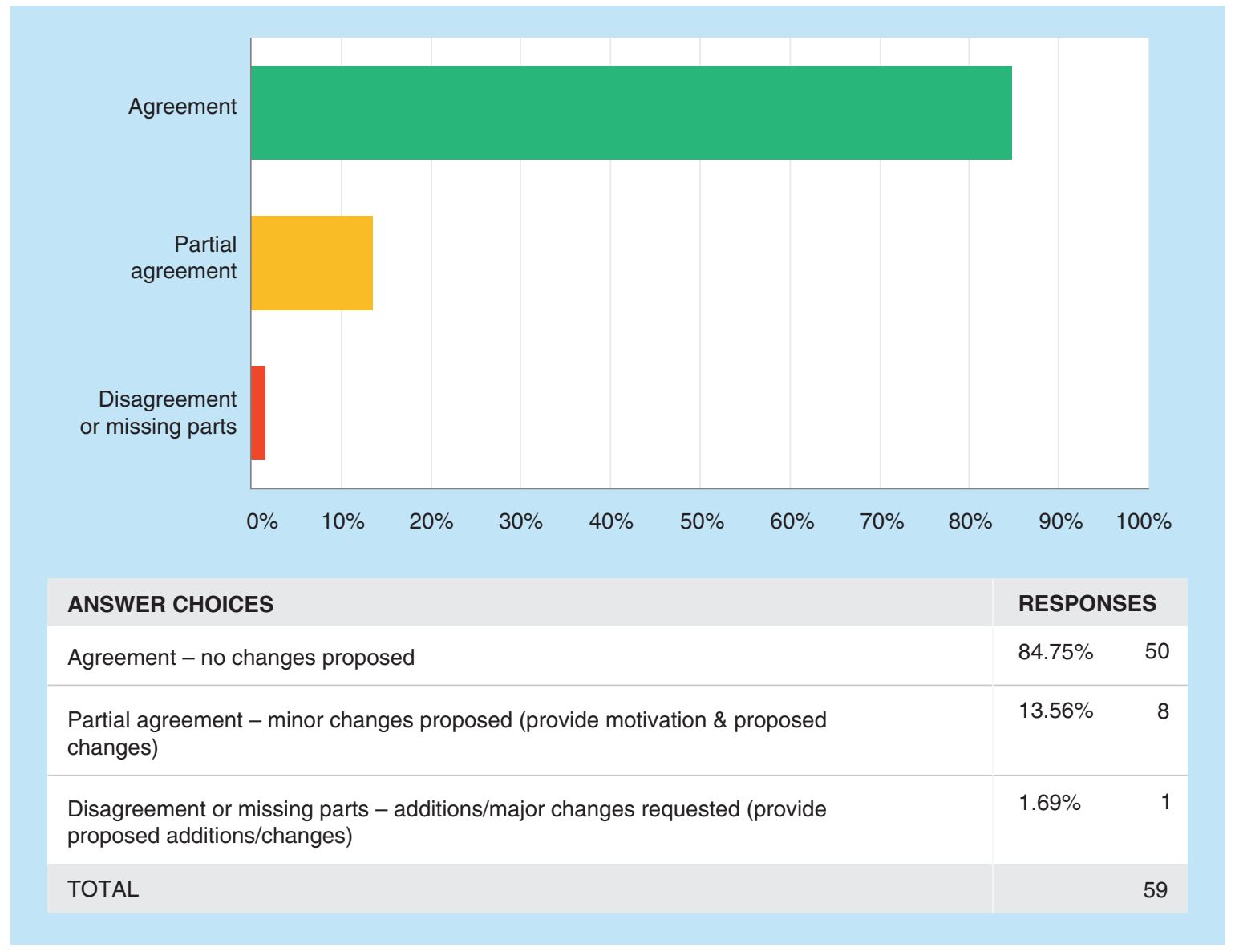

Partial agreement

No trend was found in these comments. 
T1Q23: Partial Validation - Acceptance; Lines 999-1001: "Partial validations are acceptable if the parameters tested meet the full validation criteria. If these criteria are not satisfied, additional investigation and validation is warranted."

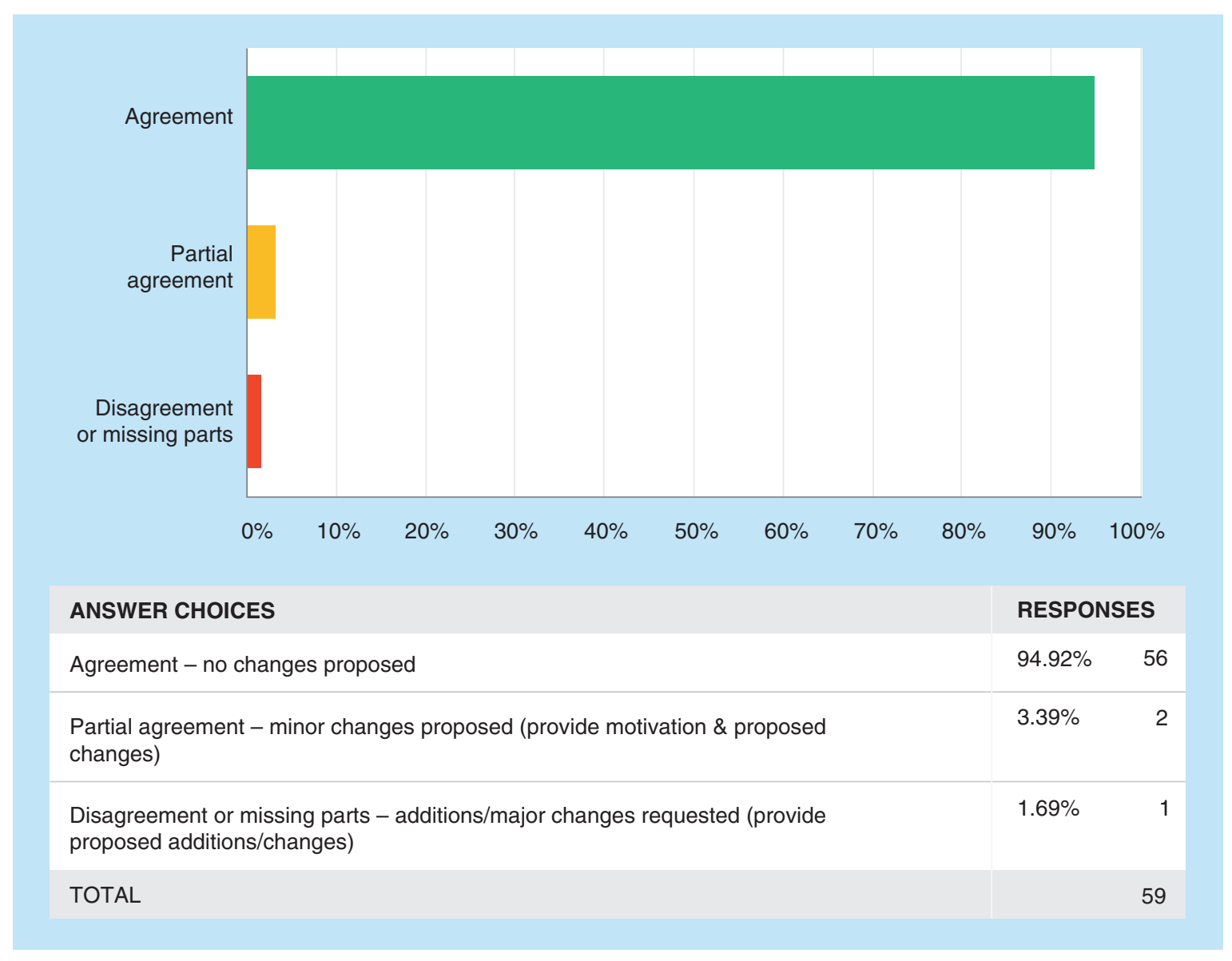

Partial agreement

No trend was found in these comments. 
T1Q24: Cross Validation - In Scope; Lines 1002-1009: "Cross validation is required to compare data under the following situations:

- Data are obtained from different fully validated methods within a study

- Data are obtained from different fully validated methods across studies that are going to be combined or compared to support special dosing regimens, or regulatory decisions regarding safety, efficacy and labelling.

- Data are obtained within a study from different laboratories with the same bioanalytical method."

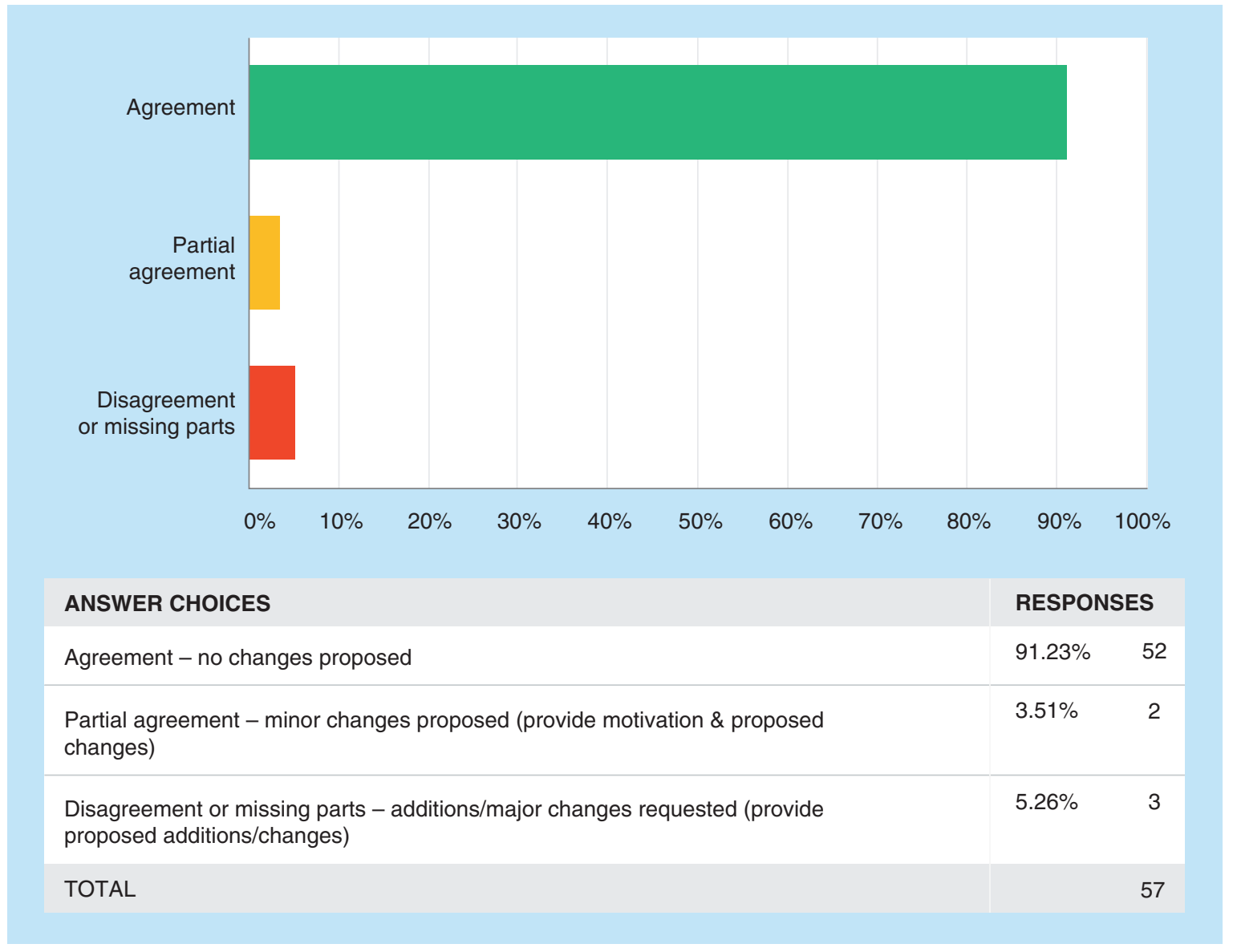

Disagreement or missing parts

No trend was found in these comments.

Partial agreement

No trend was found in these comments. 
T1Q25: Cross Validation - Out of Scope; Lines 1010-1011: "Cross validation is not generally required to compare data obtained across studies from different laboratories using the same validated method at each site."

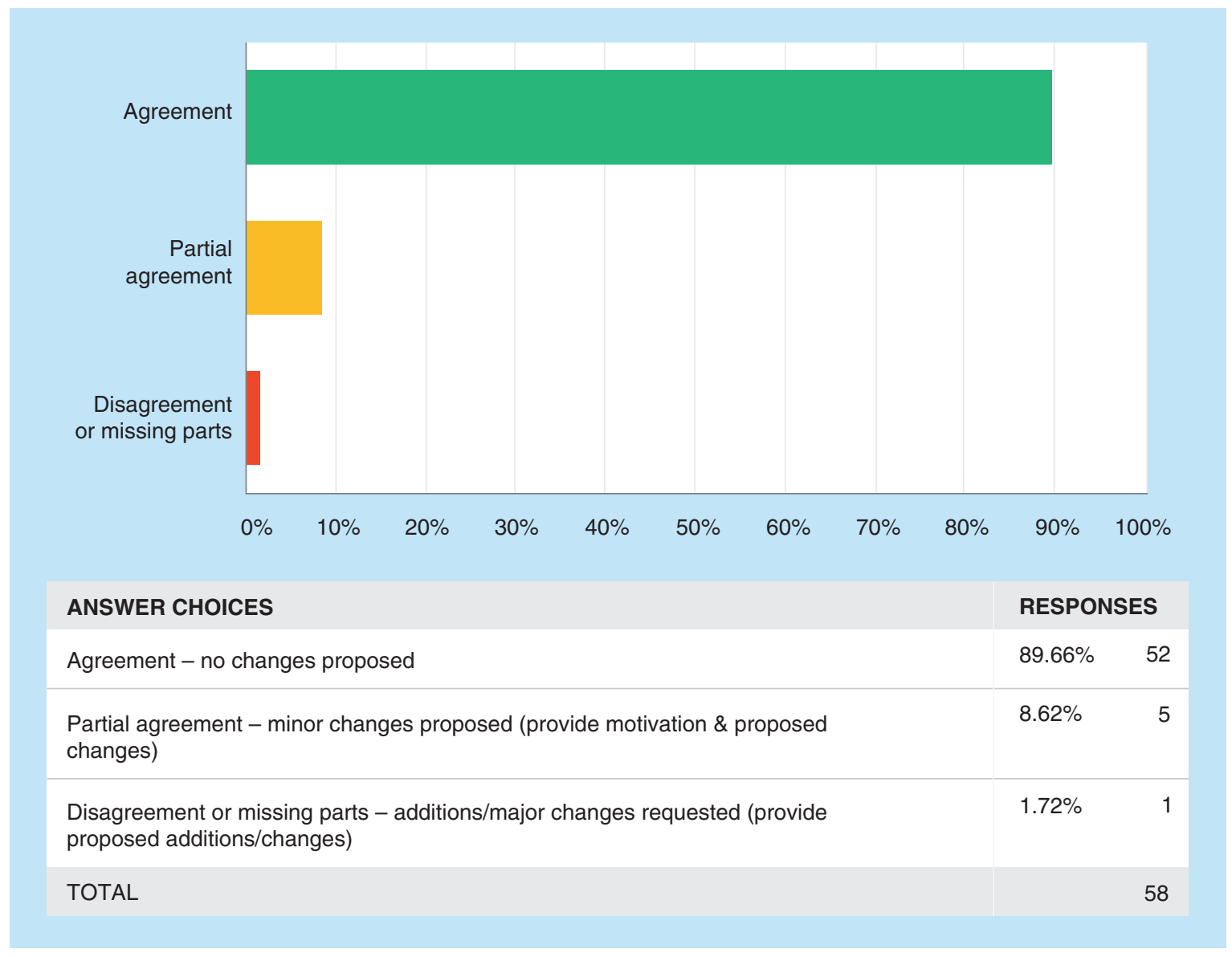

Partial agreement

No trend was found in these comments. 
T1Q26: Cross Validation - Timing Line 1012: "Cross validation should be performed in advance of study samples being analysed, if possible."

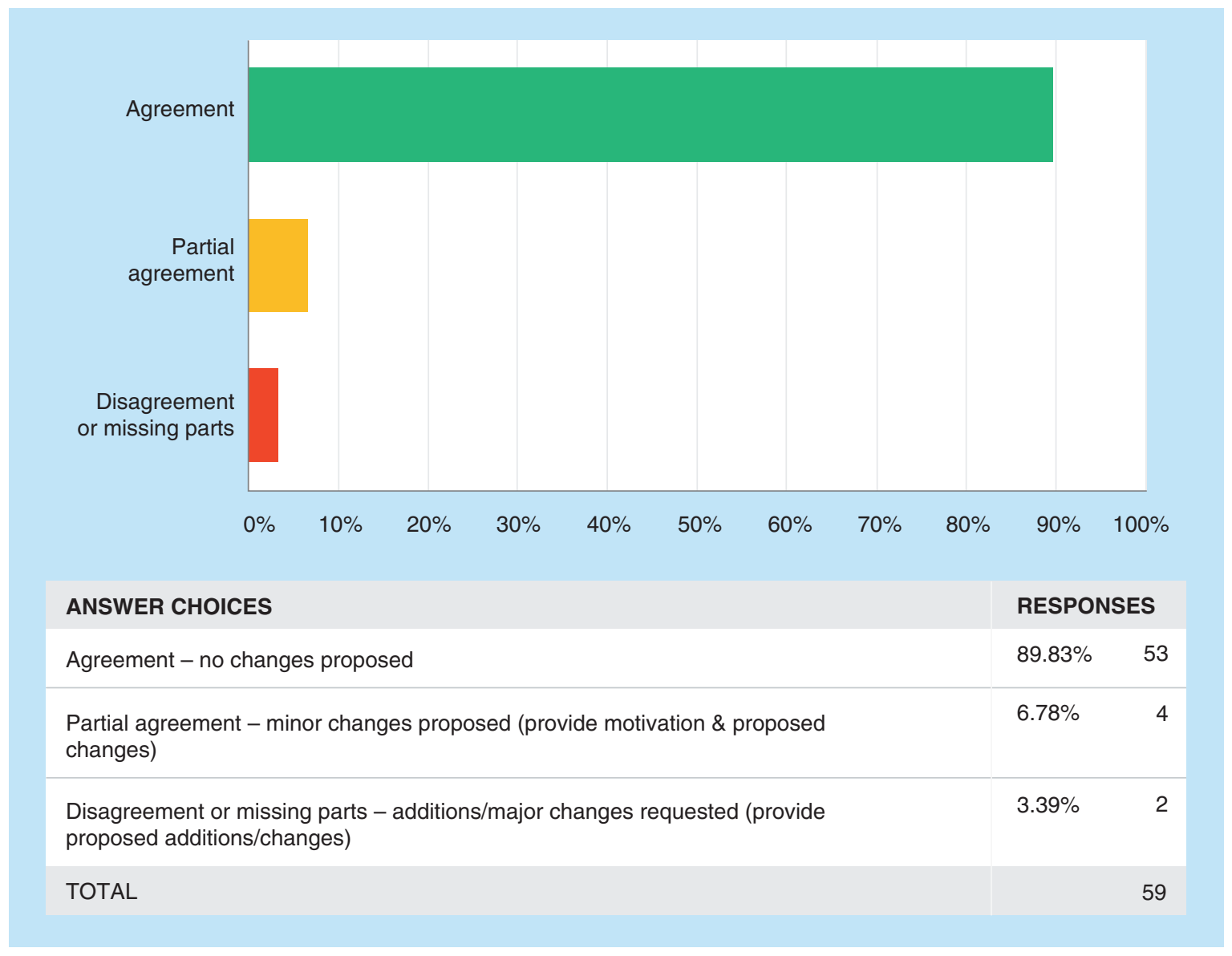

\section{Disagreement or missing parts}

No trend was found in these comments.

\section{Partial agreement}

These lines conflict with lines 1013-1015 (refer to T1Q27); if study samples are to be used for cross-validation, it cannot be performed in advance of sample analysis. 
T1Q27: Cross Validation - Method 1013-1015: "Cross validation should be assessed by measuring the same set of QCs (low, medium and high) in triplicate and study samples that span the study sample concentration range (if available $n \geq 30$ ) with both assays or in both laboratories."

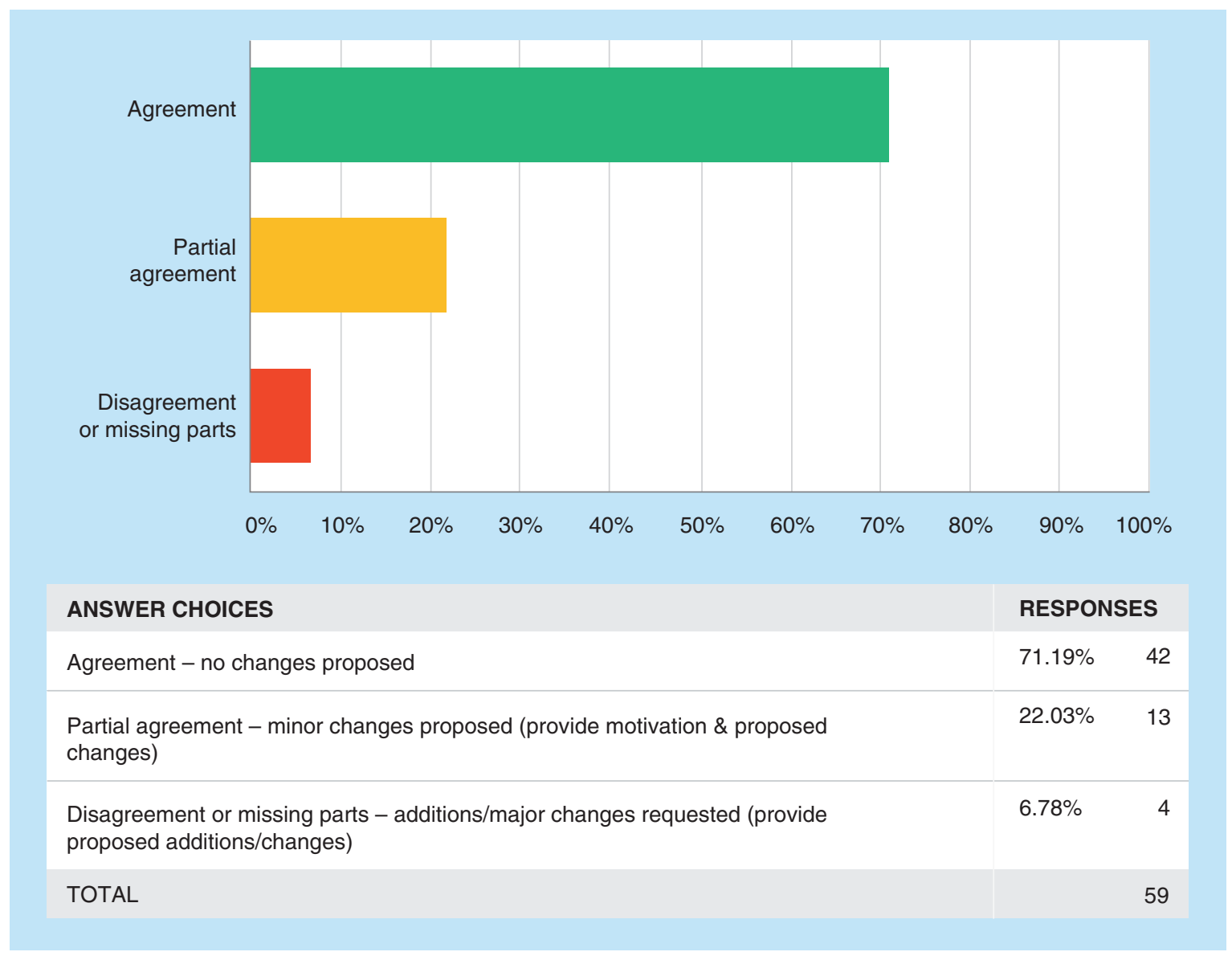

\section{Disagreement or missing parts}

- Acceptance criteria for the cross-validation should be provided. It is suggested to use similar criteria as for the ISR evaluation.

- These lines conflict with line 1012 (refer to T1Q26); if study samples are to be used for cross-validation, it cannot be performed in advance of sample analysis.

Partial agreement

The necessity of using incurred samples was queried, due to the generation of additional data. It should be clarified if pooled samples are permitted to alleviate this concern. 
T1Q28: Cross Validation - Bias/Alternative Approaches; Lines 1016-1020: "Bias can be assessed by BlandAltman plots or Deming regression. Other methods appropriate for assessing agreement between two assays (e.g., concordance correlation coefficient) may be used too. Alternatively, the concentration vs. time curves for incurred samples could be plotted for samples analysed by each method to assess bias. If disproportionate bias is observed between methods, the impact on the clinical data interpretation should be assessed."

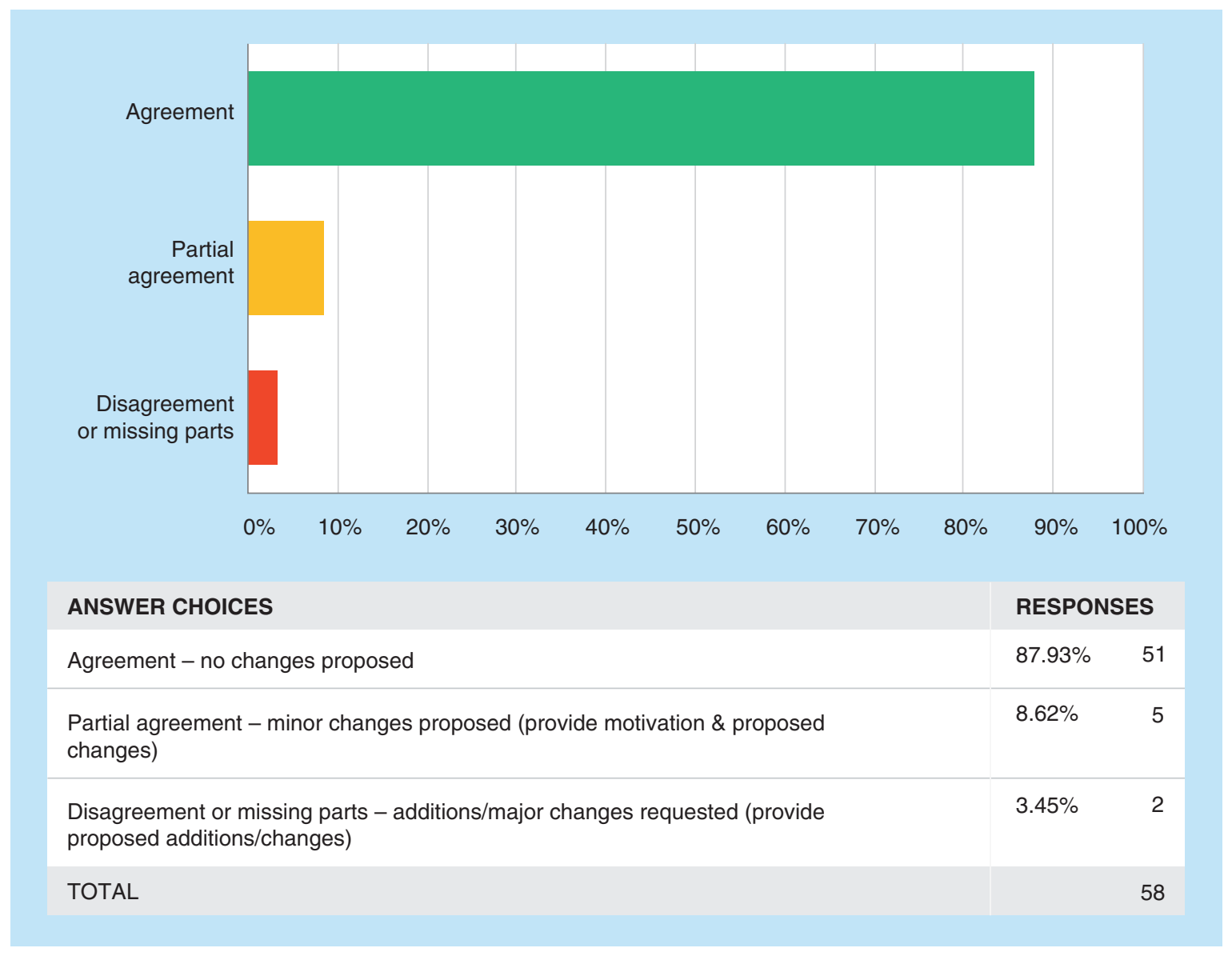

Disagreement or missing parts

As in T1Q7, it was recommended to use ISR criteria for incurred samples.

Partial agreement

No trend was found in these comments. 
Supplement Nehls, Buonarati, Cape et al.

T1Q29: Cross Validation - BA/BE; Lines 1021-1022: "The use of multiple bioanalytical methods in the conduct of one comparative BA/BE study is strongly discouraged."

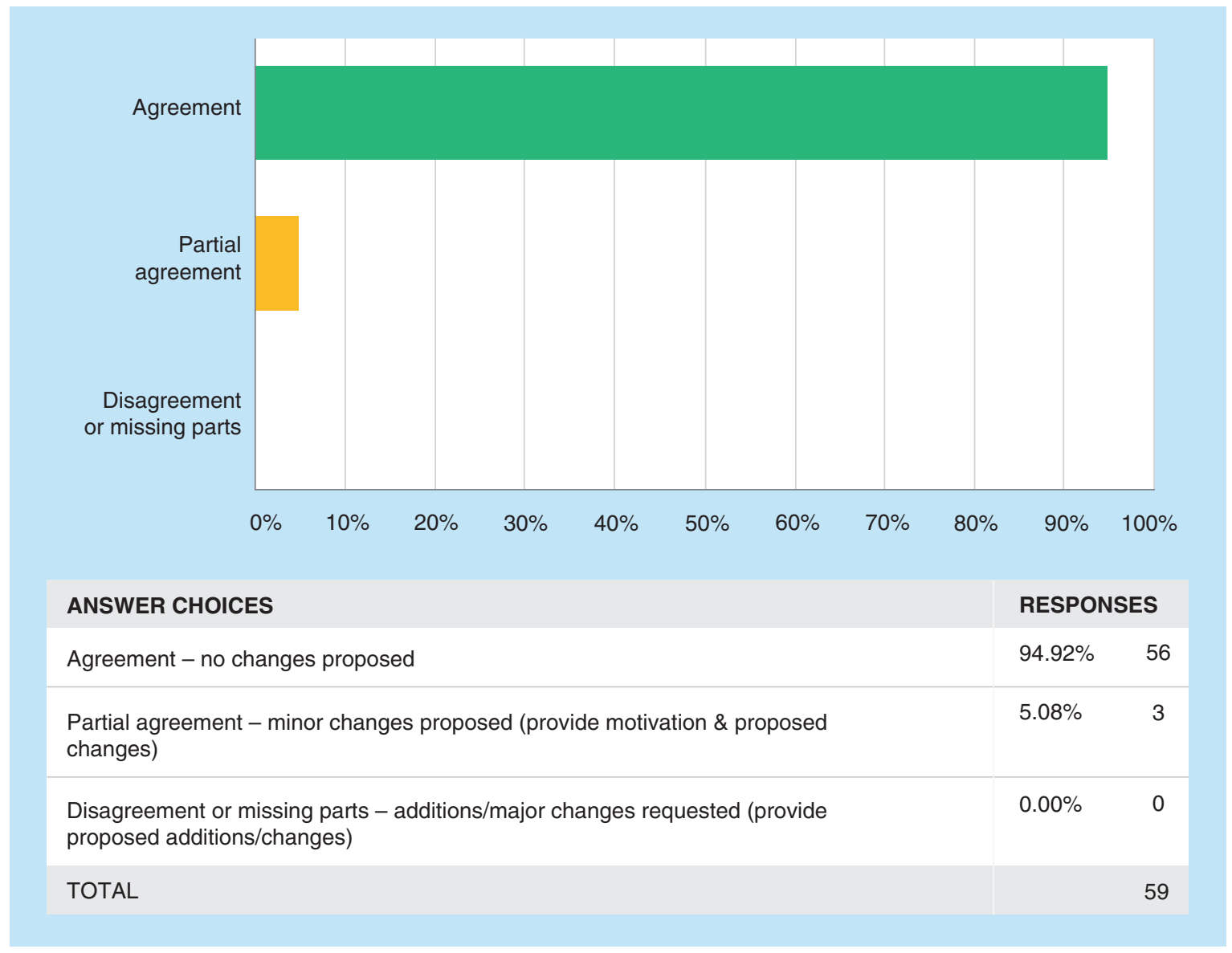

Partial agreement

No trend was found in these comments. 
T1Q30: Glossary - Accuracy; Lines 1273-1277: "The degree of closeness of the measured value to the nominal or known true value under prescribed conditions (or as measured by a particular method). In this document accuracy is expressed as percent relative error of the nominal value. Accuracy (\%) $=(($ Measured Value-Nominal Value $) /$ Nominal Value) $\times 100 "$

Glossary - Analysis; Lines 1279-1281: "A series of analytical procedures from sample processing/dilution to measurement on an analytical instrument."

Glossary - Analyte; Lines 1283-1285: "A specific chemical moiety being measured, including an intact drug, a biomolecule or its derivative or a metabolite in a biologic matrix."

Glossary - Analytical Procedure; Lines 1287-1289: "The analytical procedure refers to the way of performing the analysis. It should describe in detail the steps necessary to perform each analysis."

Glossary - Analytical Run; Lines 1291-1294: "A complete set of analytical and study samples with appropriate number of calibration standards and QCs for their validation. Several runs may be completed in one day or one run may take several days to complete."

Glossary - Anchor Calibrations Standards/Anchor Points; Lines 1295-1297: "Spiked samples set at concentrations below the LLOQ or above the ULOQ of the calibration curve and analysed to improve curve fitting in LBAs."

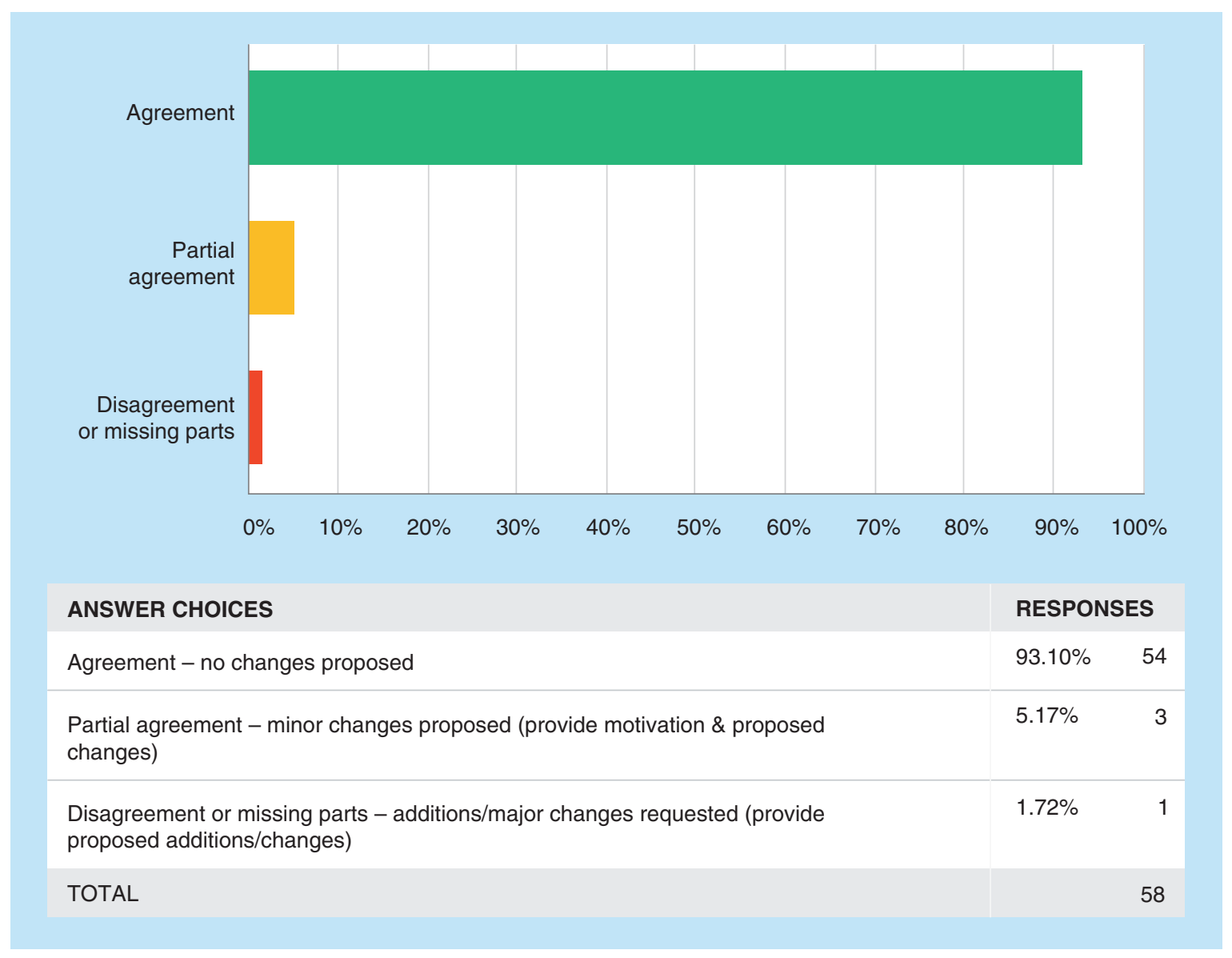

\section{Partial agreement}

It was suggested to provide examples to clarify the difference between an Analytical Run and a Batch (for Bioanalysis). 
T1Q31: Glossary - Batch (for Bioanalysis); Lines 1299-1301: "A batch is comprised of QCs and study samples which are handled during a fixed period of time and by the same group of analysts with the same reagents under homogenous conditions."

Glossary - Batch (for Reference Standards and Reagents); Lines 1303-1305: "A specific quantity of material produced in a process or series of processes so that it is expected to be homogeneous within specified limits. Also referred to as "Lot"."

Glossary - Biological Drugs; Lines 1307-1309: "Drugs manufactured by using biotechnology (e.g., therapeutic proteins). Also referred to as large molecule drugs."

Glossary - Biological Matrix; Lines 1311-1312: "A biological material including, but not limited to, blood, serum, plasma and urine."

Glossary - Binding Reagent; Lines 1314-1315: "A reagent that directly binds to the analyte in LBA-based bioanalytical methods."

Glossary - Blank Sample; Lines 1317-1318: "A sample of a biological matrix to which no analyte and no IS has been added."

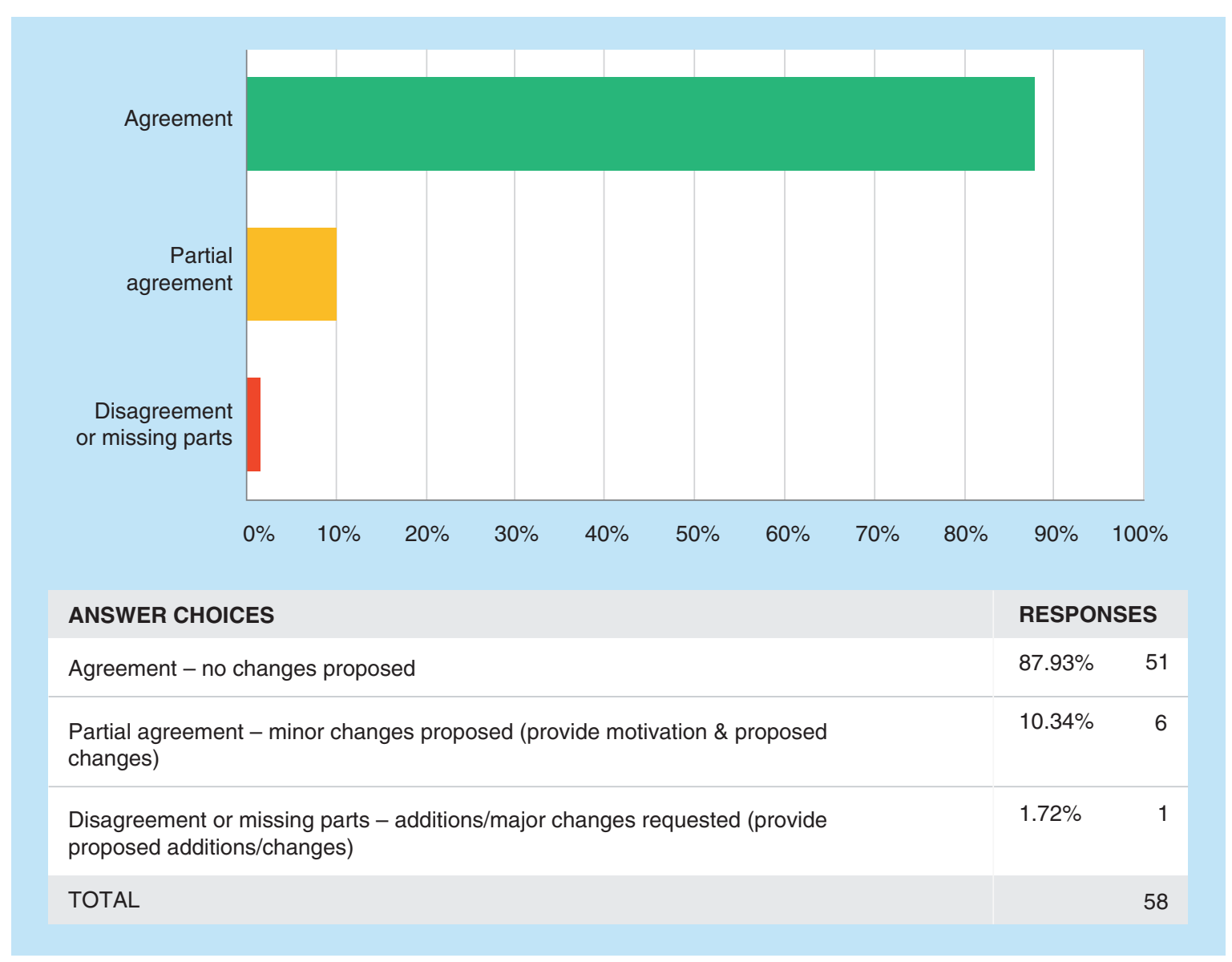

\section{Partial agreement}

As in T1Q30, it was suggested to provide examples to clarify the difference between an Analytical Run and a Batch (for Bioanalysis). 
T1Q32: Glossary - Calibration Curve; Lines 1319-1322: "The relationship between the instrument response (e.g., peak area, height or signal) and the concentration (amount) of analyte in the sample within a given range. Also referred to as Standard Curve."

Glossary - Calibration Range; Lines 1324-1328: "The calibration range of an analytical procedure is the interval between the upper and lower concentration (amounts) of analyte in the sample (including these concentrations) for which it has been demonstrated that the analytical procedure meets the requirements for precision, accuracy and response function." Glossary - Calibration Standards; Lines 1330-1332: "A matrix to which a known amount of analyte has been added or spiked. Calibration standards are used to construct calibration curves."

Glossary - Carry-over; Lines 1334-1335: "The appearance of an analyte signal in a sample from a preceding sample." Glossary - Chemical Drugs; Lines 1337-1338: "Chemically synthesised drugs. Also referred to as small molecule drugs."

Glossary - Critical Reagent; Lines 1340-1343: "Critical reagents for LBAs include binding reagents (e.g., antibodies, binding proteins, peptides) and those containing enzymatic moieties that have a direct impact on the results of the assay." Glossary - Cross Validation; Lines 1344-1346: "Comparison of two bioanalytical methods or the same bioanalytical method in different laboratories in order to demonstrate that the reported data are comparable."

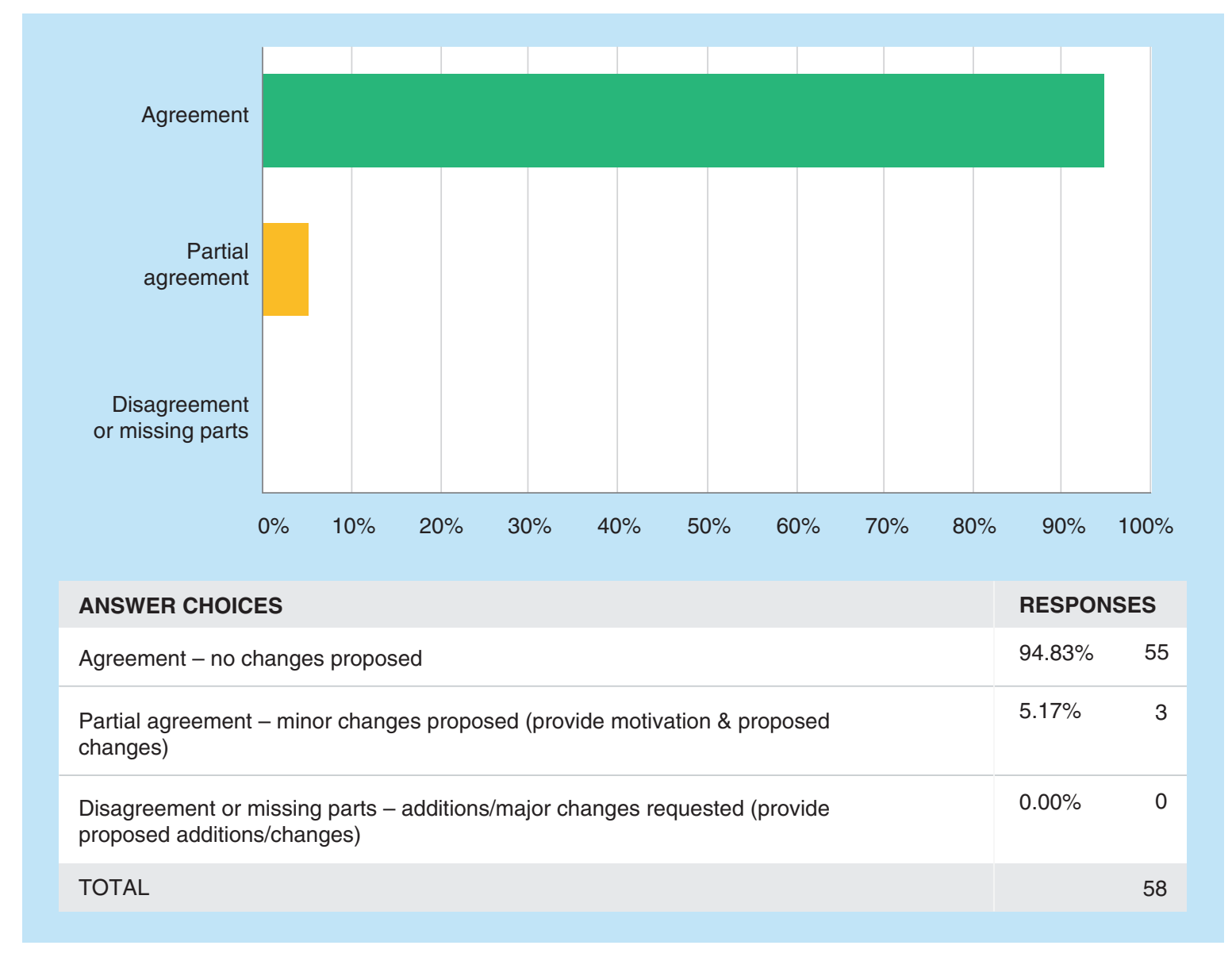

\section{Partial agreement}

No trend was found in these comments. 
T1Q33: Glossary - Dilution Integrity; Lines 1348-1350: "Assessment of the sample dilution procedure to confirm that the procedure does not impact the measured concentration of the analyte."

Glossary - Dilution Linearity; Lines 1352-1356: "A parameter demonstrating that the method can appropriately analyse samples at a concentration exceeding the ULOQ of the calibration curve without influence of hook effect or prozone effect and that the measured concentrations are not affected by dilution within the calibration range in LBAs."

Glossary - Full Validation; Lines 1358-1360: "Establishment of all validation parameters that ensure the integrity of the method when applied to sample analysis."

Glossary - Hook Effect; Lines 1362-1365: "Suppression of response due to very high concentrations of a particular analyte. A hook effect may occur in LBAs that use a liquid-phase reaction step for incubating the binding reagents with the analyte. Also referred to as prozone."

Glossary - Incurred Sample; Lines 1367-1368: "A sample obtained from study subjects or animals."

Glossary - Incurred Sample Reanalysis (ISR); Lines 1369-1371: "Reanalysis of a portion of the incurred samples in a separate analytical run on a different day to determine whether the original analytical results are reproducible."

Glossary - Interfering Substance; Lines 1373-1374: "A substance that is present in the matrix that may affect the analysis of an analyte."

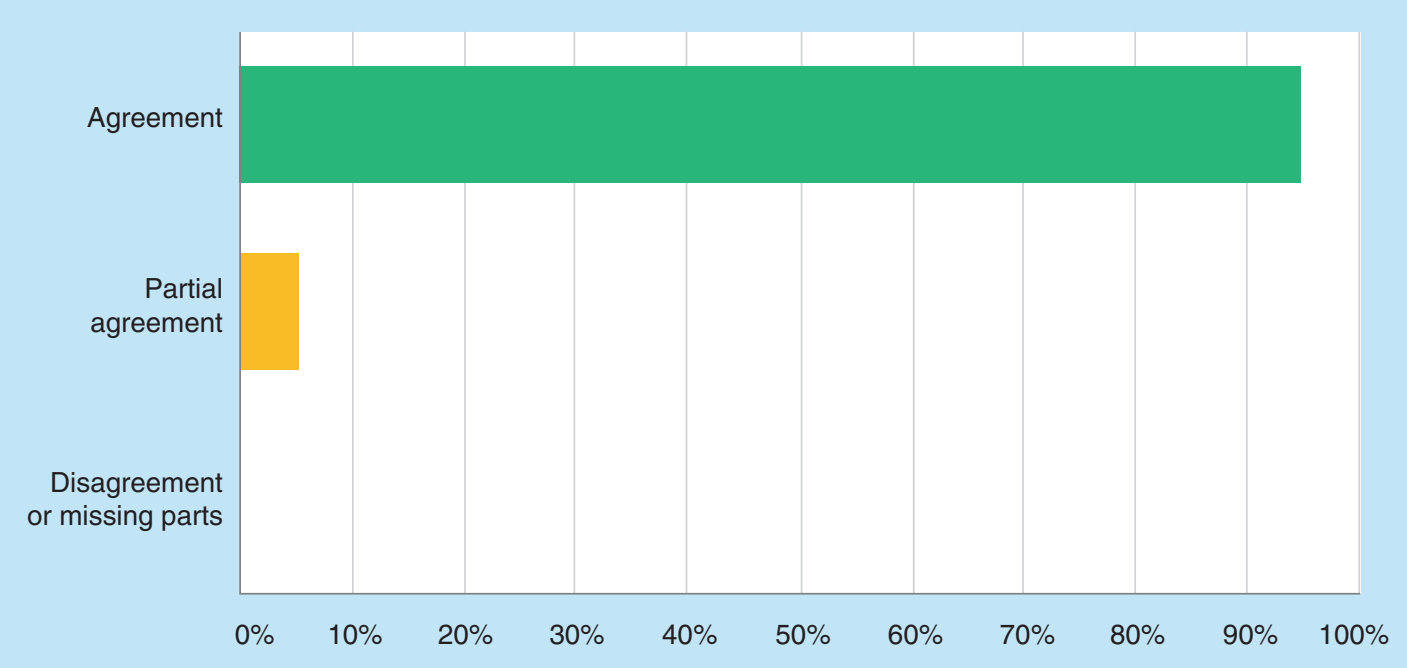

ANSWER CHOICES

RESPONSES

Agreement - no changes proposed

$94.83 \% \quad 55$

Partial agreement - minor changes proposed (provide motivation \& proposed

$5.17 \% \quad 3$ changes)

Disagreement or missing parts - additions/major changes requested (provide proposed additions/changes)

TOTAL

\section{Partial agreement}

No trend was found in these comments. 
T1Q34: Glossary - Internal Standard (IS); Lines 1376-1379: “A structurally similar analogue or stable isotope labelled compound added to calibration standards, QCs and study samples at a known and constant concentration to facilitate quantification of the target analyte."

Glossary - Ligand Binding Assay (LBA); Lines 1381-1384: "A method to analyse an analyte of interest using reagents that specifically bind to the analyte. The analyte is detected using reagents labelled with e.g. an enzyme, radioisotope, fluorophore or chromophore. Reactions are carried out in microtitre plates, test tubes, disks, etc."

Glossary - Lower Limit of Quantitation (LLOQ); Lines 1386-1388: "The lowest amount of an analyte in a sample that can be quantitatively determined with predefined precision and accuracy."

Glossary - Matrix Effect; Lines 1390-1392: "The direct or indirect alteration or interference in response due to the presence of unintended analytes or other interfering substances in the sample."

Glossary - Method; Lines 1393-1394: "A comprehensive description of all procedures used in sample analysis."

Glossary - Minimum Required Dilution (MRD); Lines 1396-1400: "The initial dilution factor by which biological samples are diluted with buffer solution for the analysis by LBAs. The MRD may not necessarily be the ultimate dilution but should be identical for all samples including calibration standards and QCS. However, samples may require further dilution."

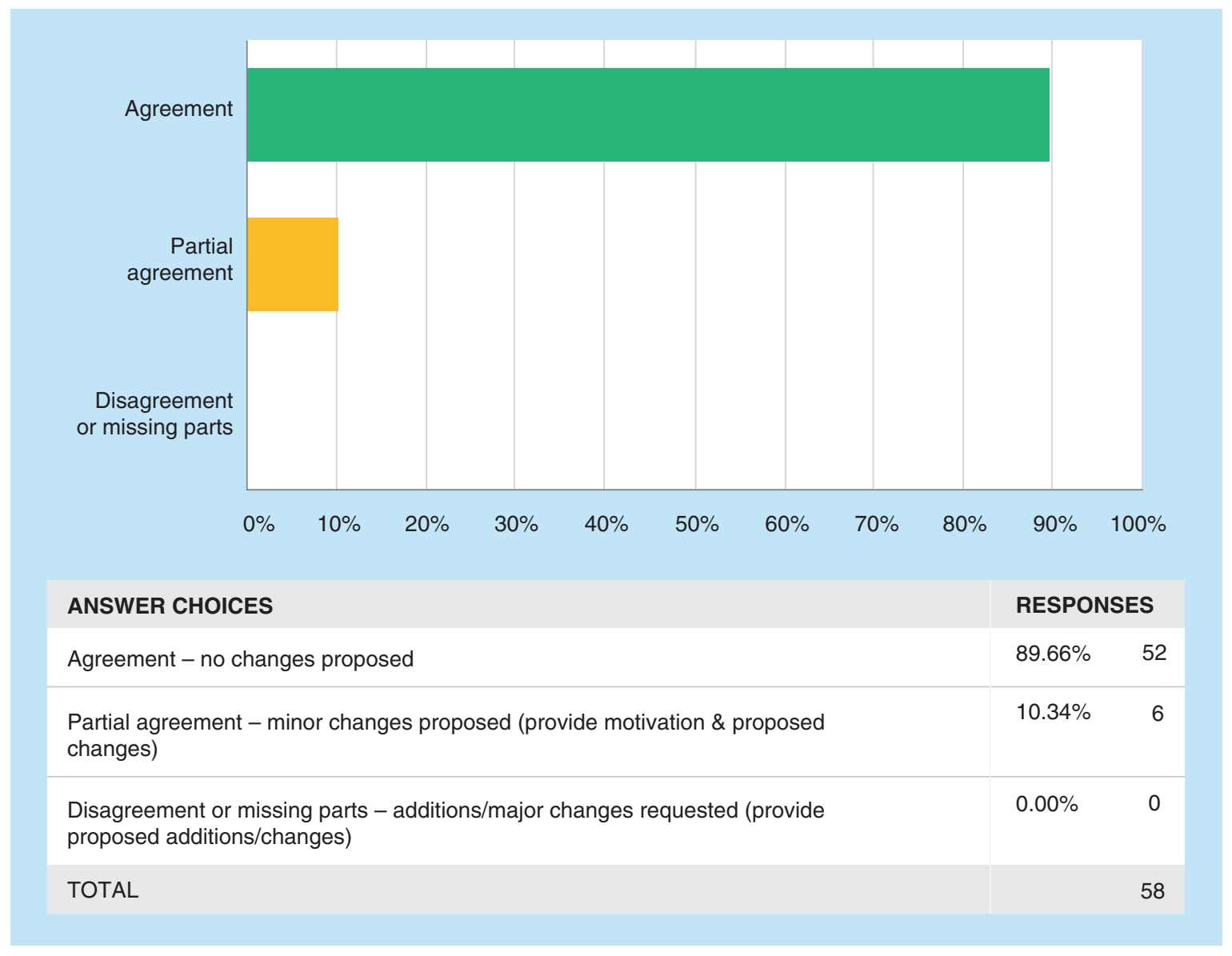

\section{Partial agreement}

It is suggested to clarify the definition of Internal Standard, since it may not always be a closely related analog or a stable isotope labelled compound. The following definition is proposed: "A compound added to calibration standards, QCs and study samples at a known and constant concentration with similar properties to the analyte to facilitate quantification of the target analyte based on response ratio." 
T1Q35: Glossary - Nominal Concentration; Lines 1402-1403: "Theoretical or expected concentration." Glossary - Parallelism; Lines 1405-1408: "Parallelism demonstrates that the serially diluted incurred sample response curve is parallel to the calibration curve. Parallelism is a performance characteristic that can detect potential matrix effects." Glossary - Partial Validation; Lines 1410-1411: "Evaluation of modifications to already fully validated analytical methods."

Glossary - Precision; Lines 1413-1417: "The closeness of agreement (i.e., degree of scatter) among a series of measurements. Precision is expressed as the coefficient of variation (CV) or the relative standard deviation (RSD) expressed as a percentage.

Precision (\%) $=($ Standard Deviation $/$ Mean $) \times 100 "$

Glossary - Processed Sample; Lines 1418-1420: "The final sample that has been subjected to various manipulations (e.g., extraction, dilution, concentration)."

Glossary - Quality Control Sample (QC); Lines 1422-1425: "A sample spiked with a known quantity of analyte that is used to monitor the performance of a bioanalytical method and assess the integrity and validity of the results of the unknown samples analysed in an individual batch or run."

Glossary - Recovery; Lines 1427-1429: "The extraction efficiency of an analytical process, reported as a percentage of the known amount of an analyte carried through the sample extraction and processing steps of the method."

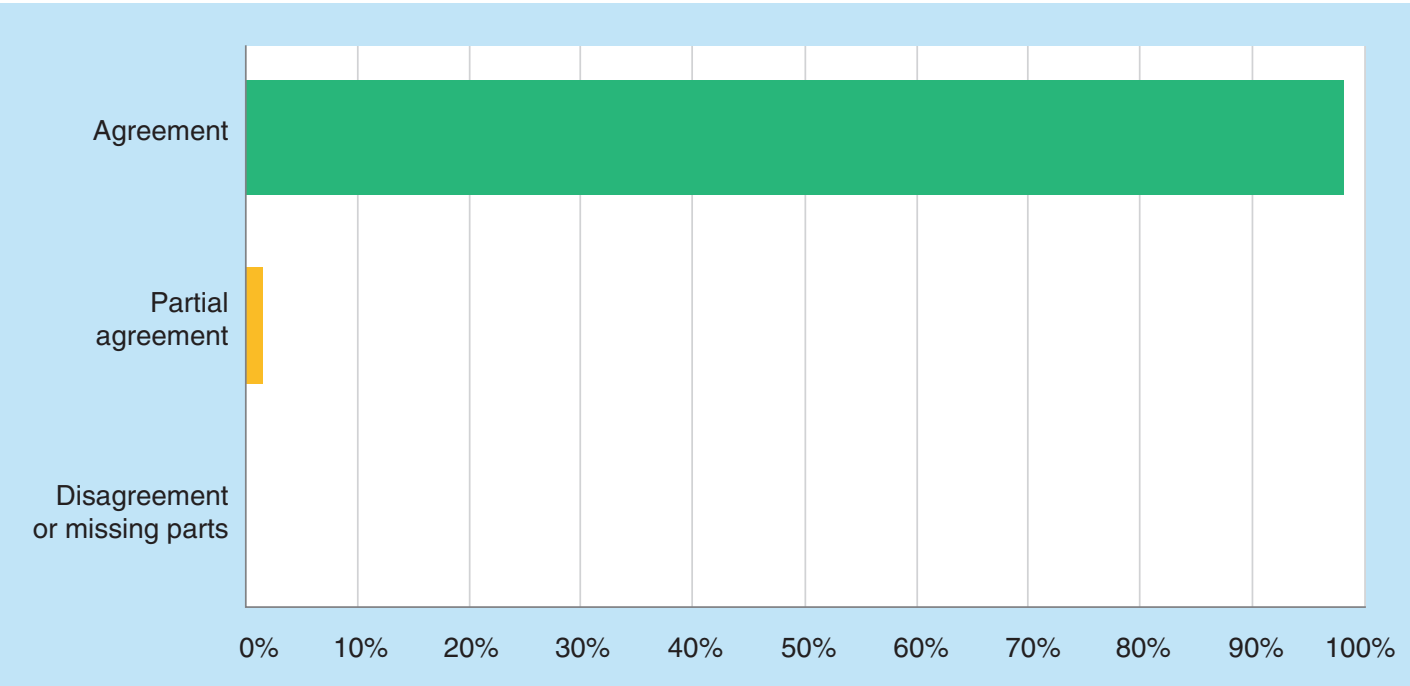

ANSWER CHOICES

RESPONSES

Agreement - no changes proposed

$98.28 \% \quad 57$

Partial agreement - minor changes proposed (provide motivation \& proposed

$1.72 \% \quad 1$ changes)

Disagreement or missing parts - additions/major changes requested (provide proposed additions/changes)

TOTAL 
T1Q36: Glossary - Reproducibility; Lines 1431-1432: "The extent to which consistent results are obtained when an experiment is repeated."

Glossary - Response Function; Lines 1434-1437: "A function which adequately describes the relationship between instrument response (e.g., peak area or height ratio or signal) and the concentration (amount) of analyte in the sample. Response function is defined within a given range. See also Calibration Curve."

Glossary - Selectivity; Lines 1439-1441: "Ability of an analytical method to differentiate and measure the analyte in the presence of interfering substances in the biological matrix (non-specific interference)."

Glossary - Sensitivity; Lines 1442-1444: "The lowest analyte concentration that can be measured with acceptable accuracy and precision (i.e., LLOQ)."

Glossary - Specificity; Lines 1446-1449: "Ability of an analytical method to detect and differentiate the analyte from other substances, including its related substances (e.g., substances that are structurally similar to the analyte, metabolites, isomers, impurities or concomitant medications)."

Glossary - Standard Curve; Lines 1451-1454: "The relationship between the instrument response (e.g., peak area, height or signal) and the concentration (amount) of analyte in the sample within a given range. Also referred to as calibration Curve."

Glossary - Standard Operating Procedure (SOP); Lines 1456-1457: "Detailed written instructions to achieve uniformity of the performance of a specific function."

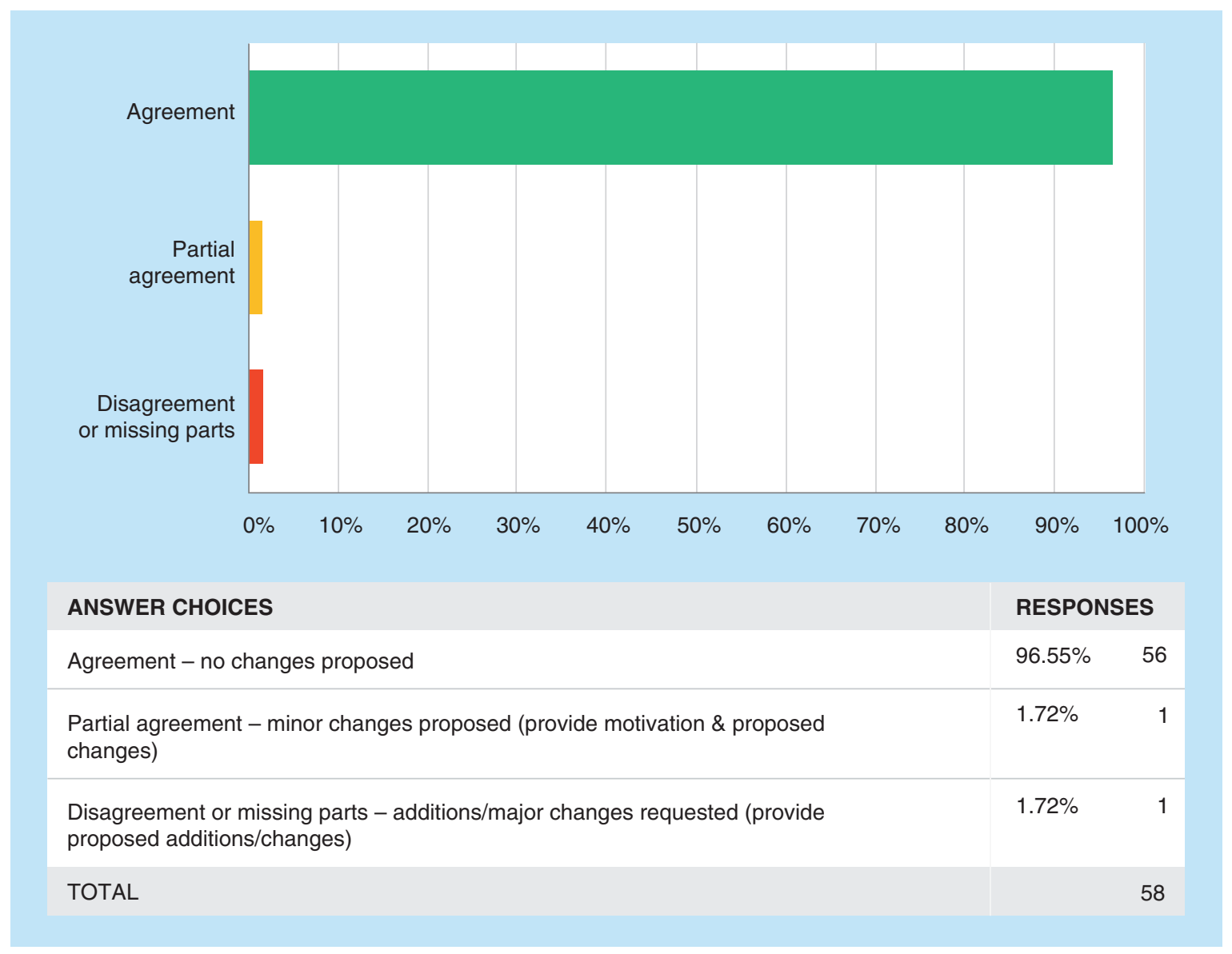


T1Q37: Glossary - Surrogate Matrix; Lines 1459-1461: "An alternative to a study matrix of limited availability (e.g., tissue, cerebrospinal fluid, bile) or where the study matrix contains an interfering endogenous counterpart."

Glossary - System Suitability; Lines 1463-1465: "Determination of instrument performance (e.g., sensitivity and chromatographic retention) by analysis of a set of reference standards conducted prior to the analytical run."

Glossary - Total Error; Lines 1466-1468: "The sum of the absolute value of the errors in accuracy (\%) and precision (\%). Total error is reported as percent (\%) error."

Glossary - Upper Limit of Quantitation (ULOQ); Lines 1470-1473: "The upper limit of quantification of an individual analytical procedure is the highest amount of analyte in a sample that can be quantitatively determined with pre-defined precision and accuracy."

Glossary - Validation; Lines 1475-1476: "Demonstration that a bioanalytical method is suitable for its intended purpose."

Glossary - Working Solution; Lines 1478-1480: "A non-matrix solution prepared by diluting the stock solution in an appropriate solvent. It is mainly added to matrix to prepare calibration standards and QCs."

Glossary - Zero Sample; Lines 1482-1483: "A blank sample spiked with an IS."

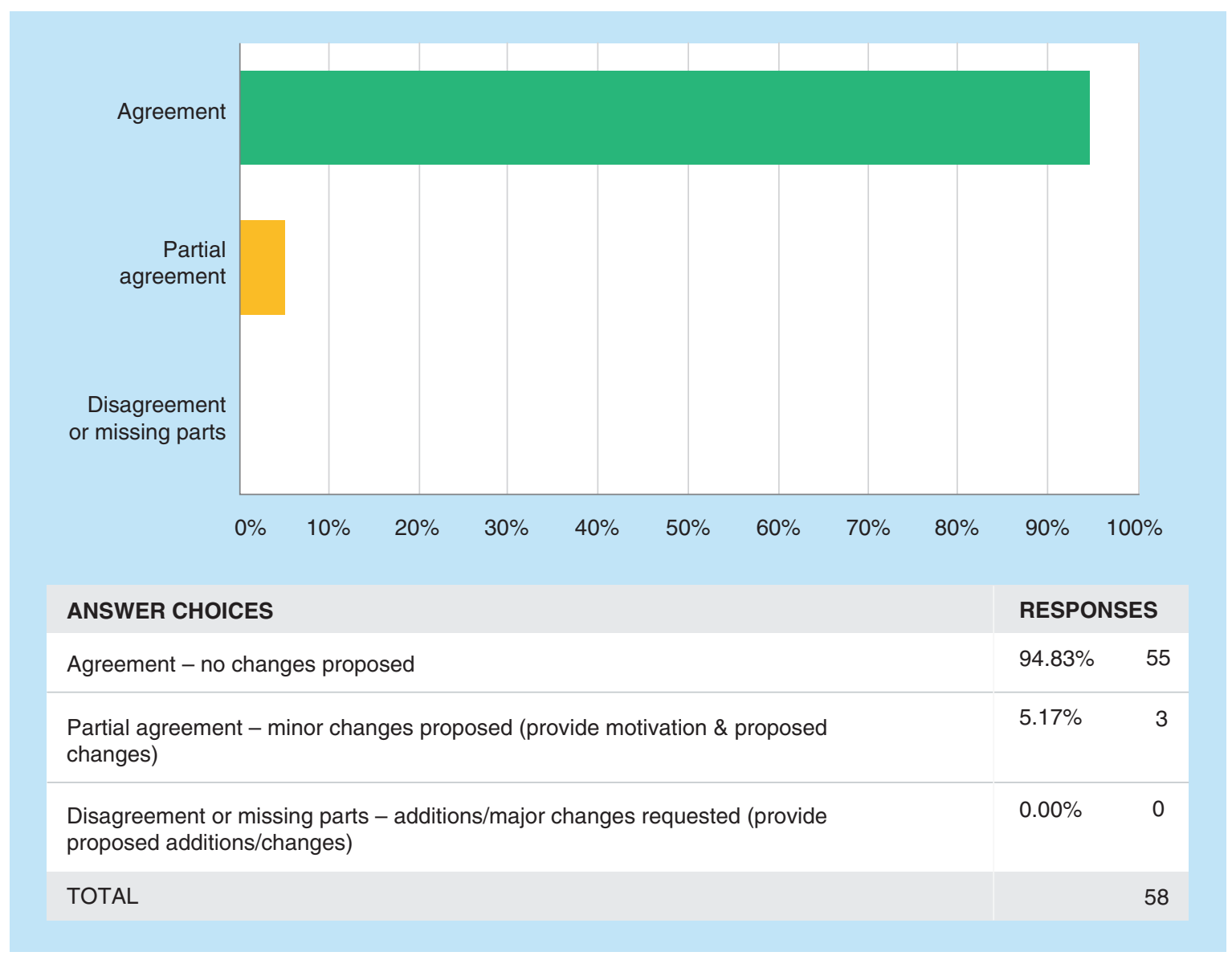

\section{Partial agreement}

Two comments suggested that System Suitability is not typically determined by a set of reference standards and to modify the definition as follows: "Determination of instrument performance (e.g., sensitivity and chromatographic retention) by analysis of a set of analyte samples (neat or in matrix) conducted prior to the analytical run."

\section{Additional comments from the 13th GCC:}

There were no further discussions during the GCC Closed Forum in support of the above proposal. 
Team 2 Chromatography I - Key Validation Parameters: Selectivity, Specificity, Matrix Effect, Calibration Curve, Accuracy, Precision, Quality Control, Carry-over, Stability, Dilution Integrity, Reinjections, Extraction Recovery

T2Q1: Selectivity Definition/Procedure; Lines 222-227: "Selectivity is the ability of an analytical method to differentiate and measure the analyte in the presence of potential interfering substances in the blank biological matrix. Selectivity is evaluated using blank samples (matrix samples processed without addition of an analyte or IS) obtained from at least 6 individual sources/lots (non-haemolysed and non-lipaemic). Use of fewer sources may be acceptable in the case of rare matrices. Selectivity for the IS should also be evaluated."

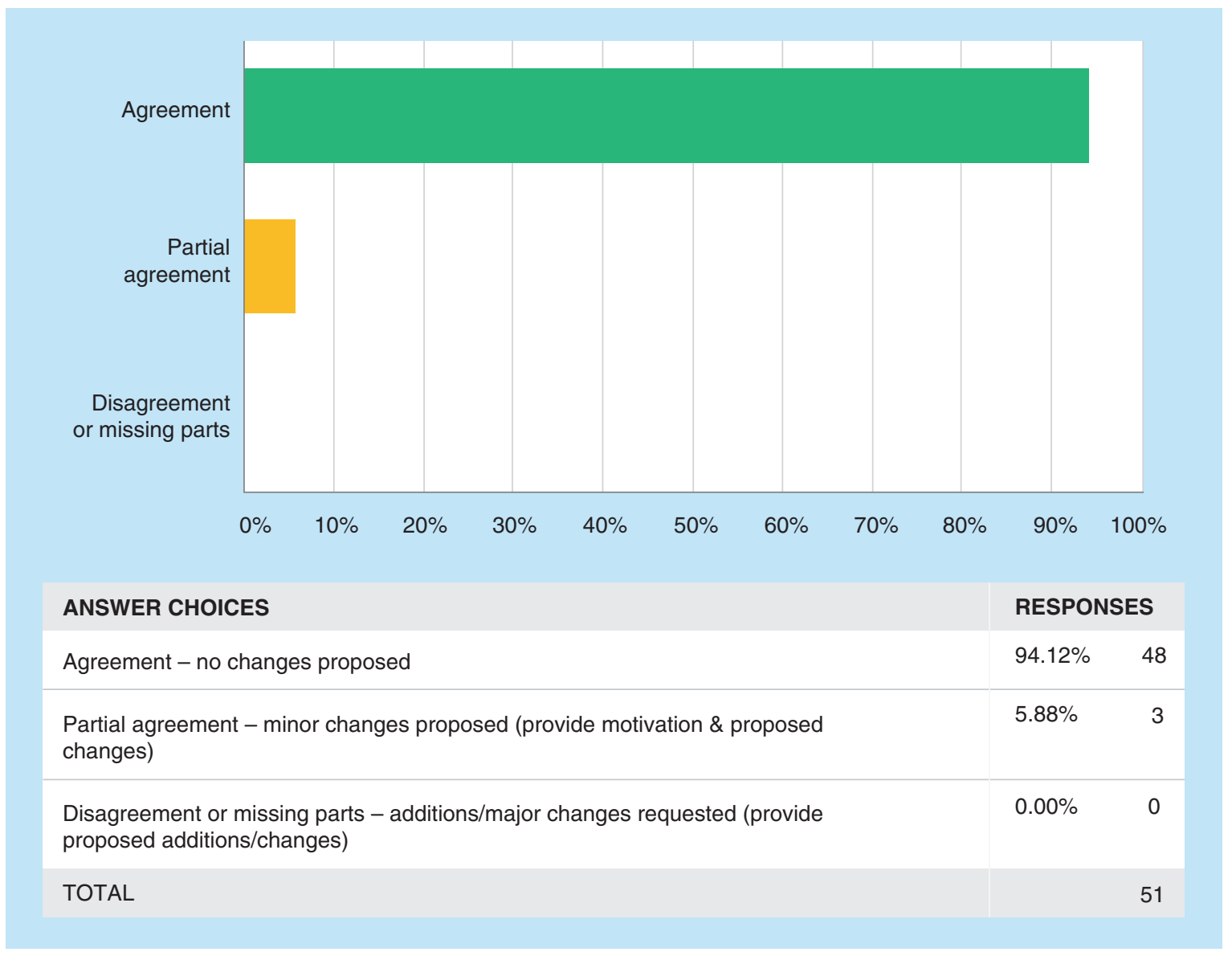

\section{Partial agreement}

No trend was found in these comments. 
T2Q2: Selectivity - Acceptance Criteria; Lines 228-232: "The evaluation of selectivity should demonstrate that no significant response attributable to interfering components is observed at the retention time(s) of the analyte or the IS in the blank samples. Responses detected and attributable to interfering components should not be more than 20\% of the analyte response at the LLOQ and not more than 5\% of the IS response in the LLOQ sample for each matrix."

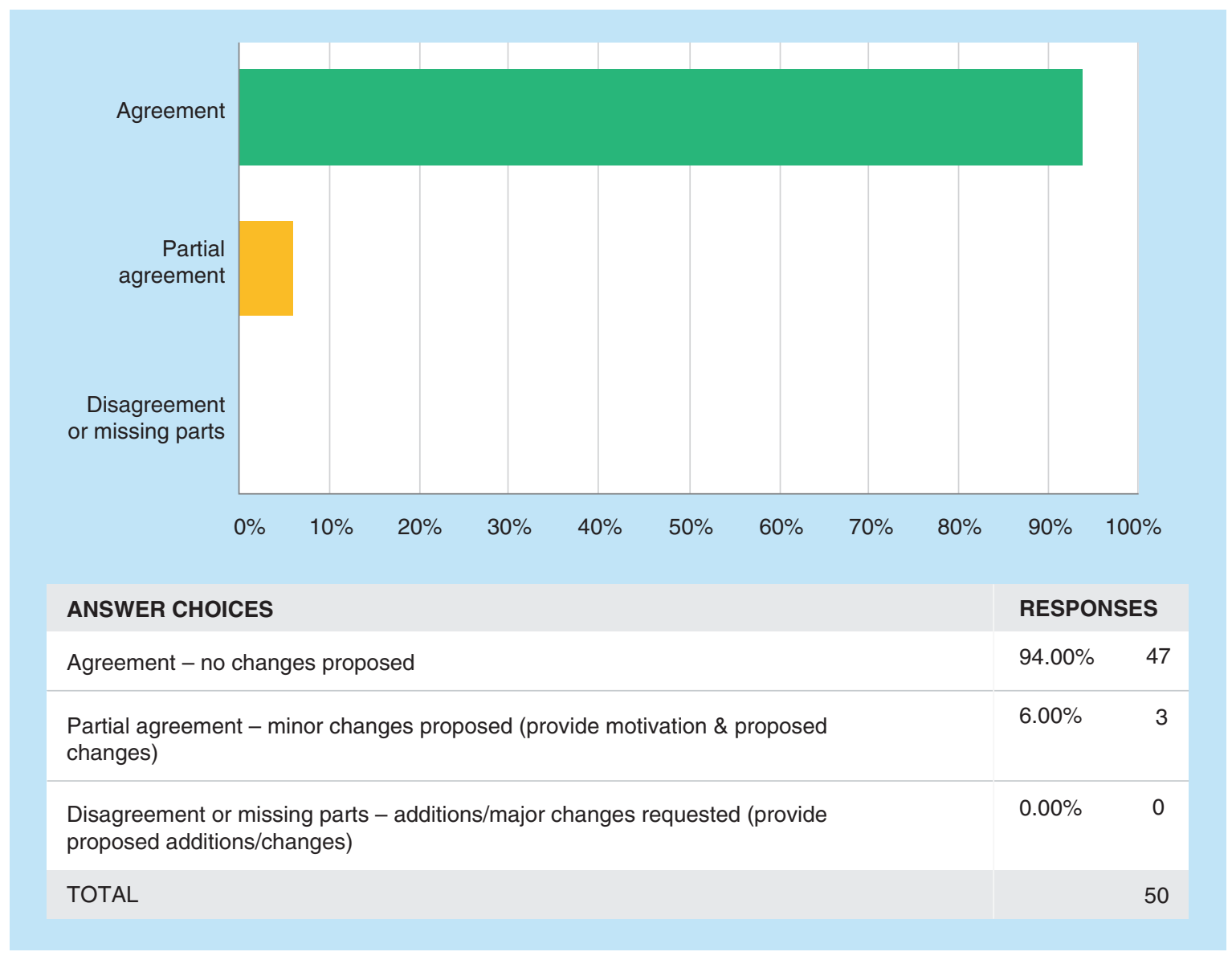

Partial agreement

No trend was found in these comments. 
T2Q3: Selectivity - Lipaemic Matrix; Lines 233-242: "For the investigation of selectivity in lipaemic matrices at least one source of matrix should be used. To be scientifically meaningful, the matrix used for these tests should be representative as much as possible of the expected study samples. A naturally lipaemic matrix with abnormally high levels of triglycerides should be obtained from donors. Although it is recommended to use lipaemic matrix from donors, if this is difficult to obtain, it is acceptable to spike matrix with triglycerides even though it may not be representative of study samples. However, if the drug impacts lipid metabolism or if the intended patient population is hyperlipidaemic, the use of spiked samples is discouraged. This evaluation is not necessary for preclinical studies unless the drug impacts lipid metabolism or is administered in a particular animal strain that is hyperlipidaemic."

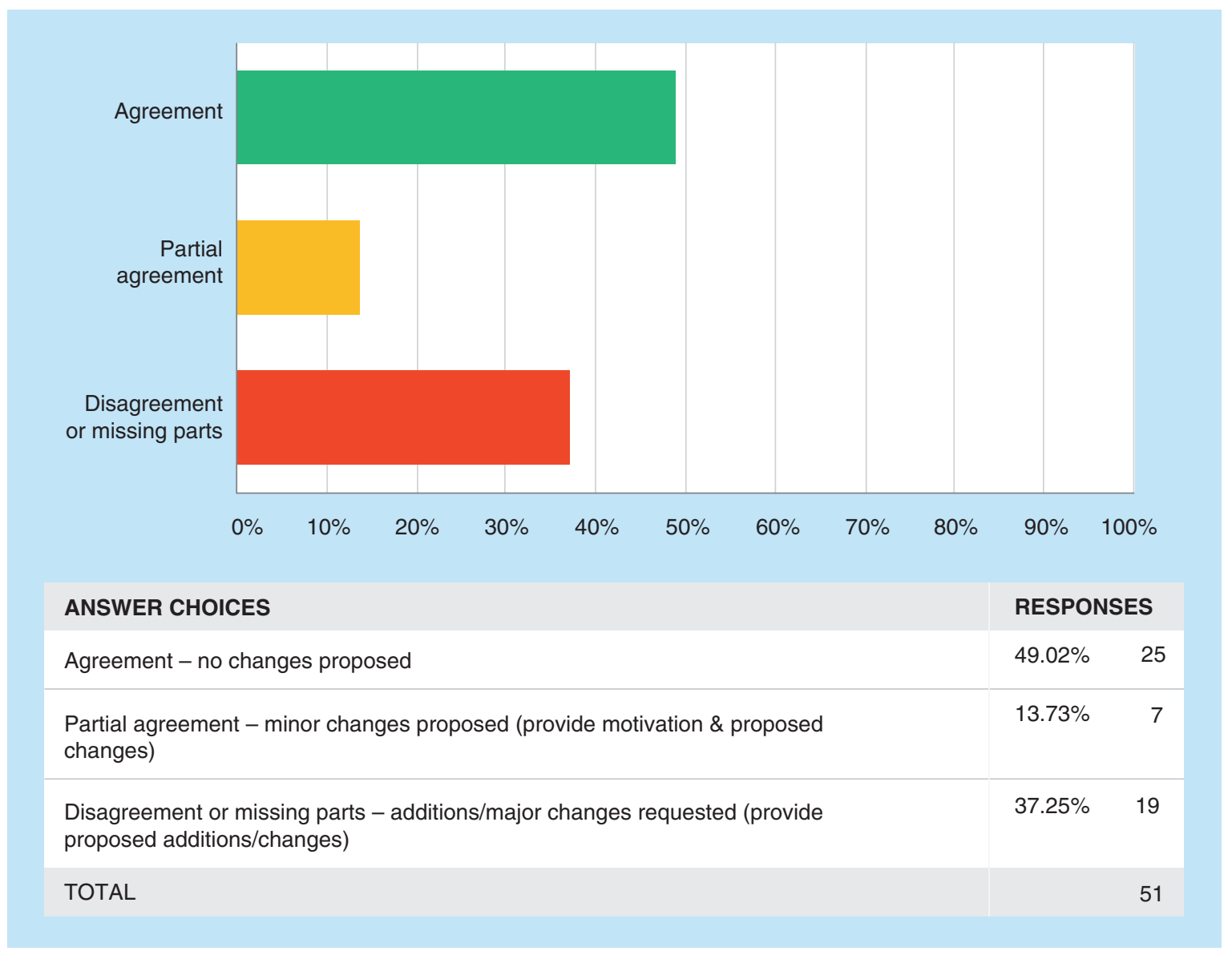

Disagreement or missing parts

The comments clearly indicated a strong disagreement with the statements:

- It is difficult to obtain naturally occurring lipaemic matrix.

- The possibility of spiking matrix with triglycerides.

Natural lipaemic plasma is readily available and spiking matrix with triglycerides is not acceptable. The sentence: "Although it is recommended to use lipaemic matrix from donors, if this is diffcult to obtain, it is acceptable to spike matrix with triglycerides even though it may not be representative of study samples." should be removed.

Moreover, there was overwhelming consensus that a level of triglycerides should be specified as at least more than $300 \mathrm{mg} / \mathrm{dL}$. Overall, the GCC white paper recommendations should be used [5].

Partial agreement

No additional trend was found in these comments. 
T2Q4: Selectivity - Haemolysed Matrix; Lines 243-245: "For the investigation of selectivity in haemolysed matrices at least one source of matrix should be used. Haemolysed matrices are obtained by spiking matrix with haemolysed whole blood (at least 2\% v/v) to generate a visibly detectable haemolysed sample."

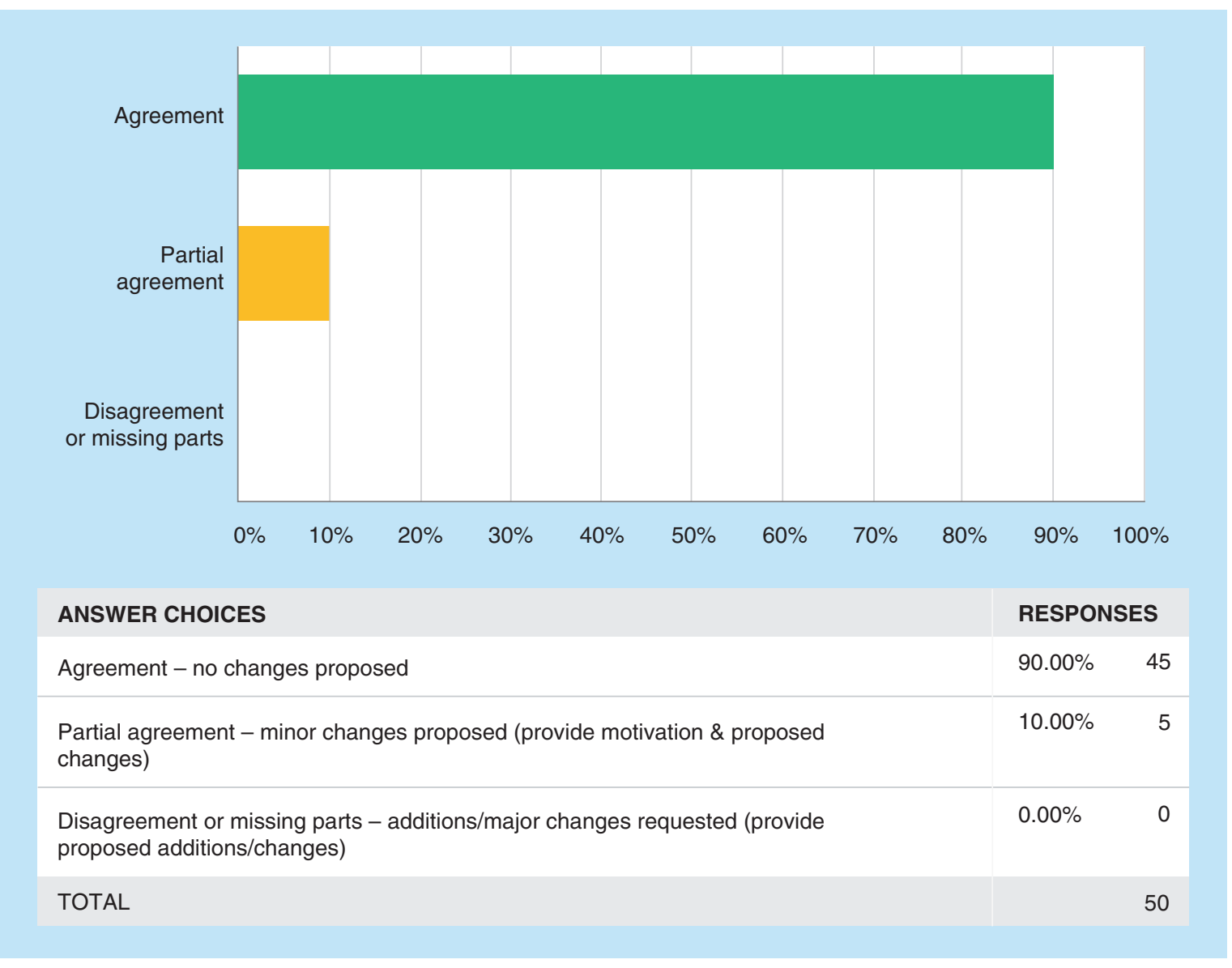

Partial agreement

No trend was found in these comments. 
T2Q5: Specificity - Definition; Lines 247-251: "Specificity is the ability of a bioanalytical method to detect and differentiate the analyte from other substances, including its related substances (e.g., substances that are structurally similar to the analyte, metabolites, isomer, impurities, degradation products formed during sample preparation, or concomitant medications that are expected to be used in the treatment of patients with the intended indication)."

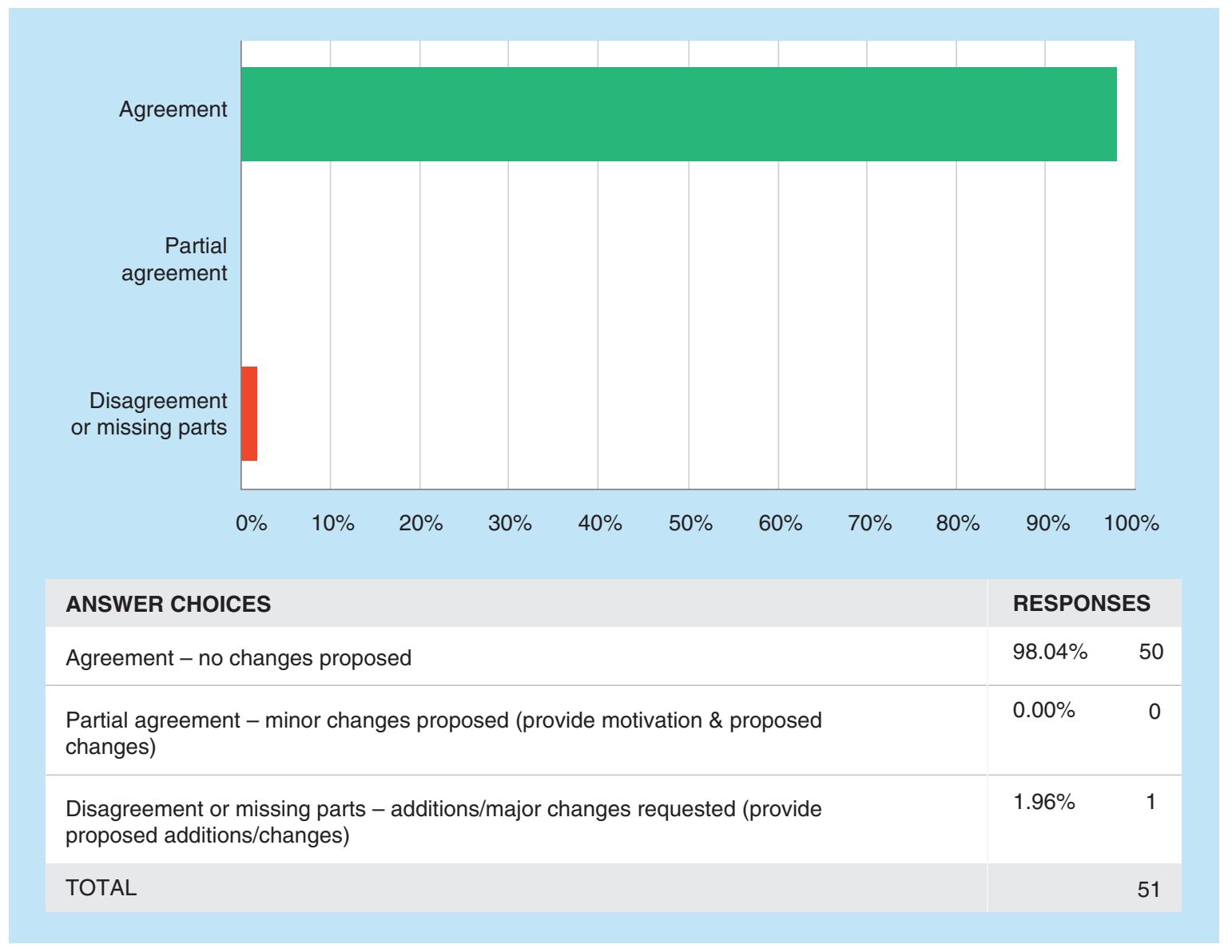


T2Q6: Specificity - Procedure; Lines 252-257: "If the presence of related substances is anticipated in the biological matrix of interest, the impact of such substances should be evaluated during method validation, or alternatively, in the pre-dose study samples. In the case of LC-MS based methods, to assess the impact of such substances, the evaluation may include comparing the molecular weight of a potential interfering related substance with the analyte and chromatographic separation of the related substance from the analyte."

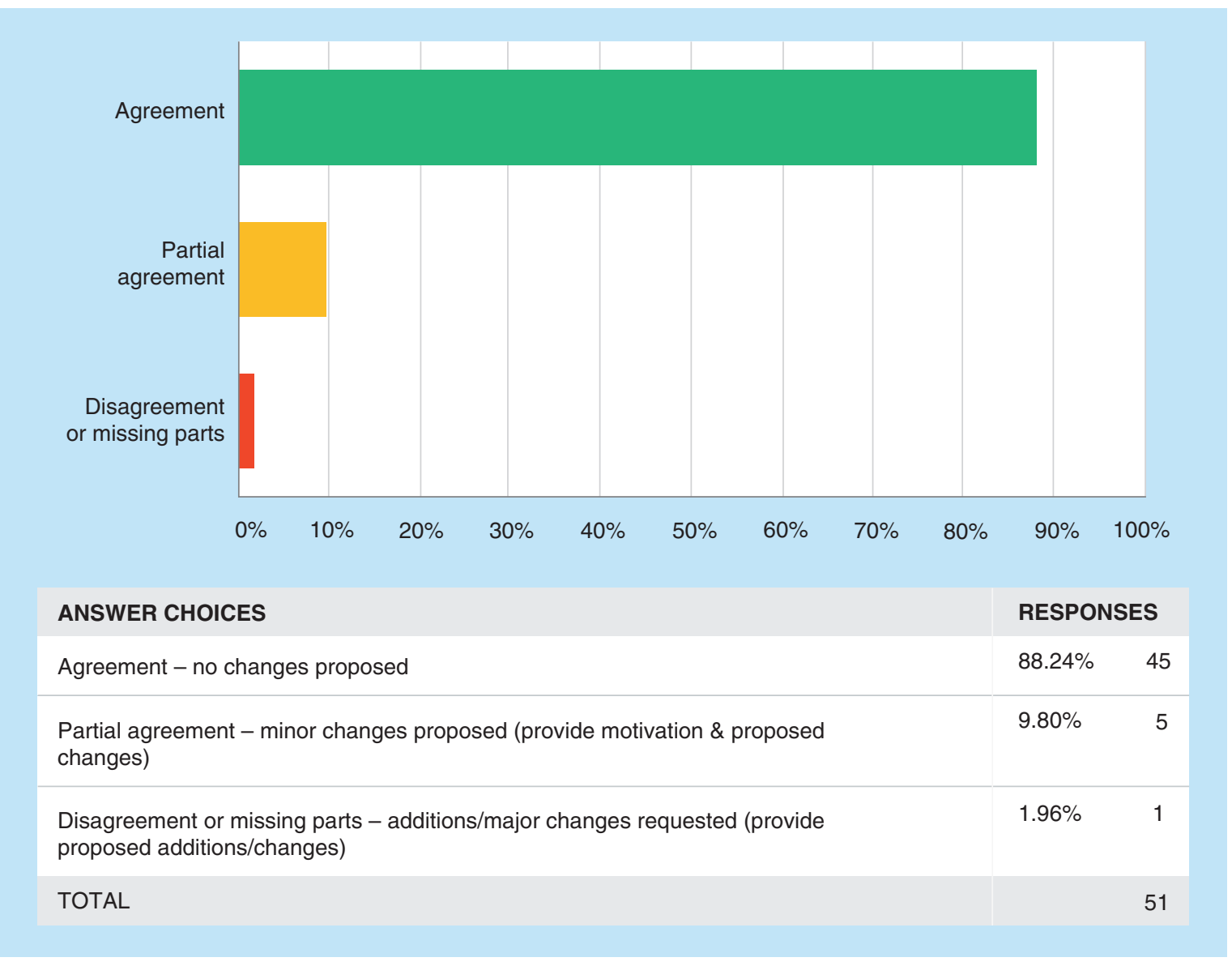

Partial agreement

No trend was found in these comments. 
T2Q7: Specificity - Acceptance Criteria; Lines 258-259: "Responses detected and attributable to interfering components should not be more than 20\% of the analyte response at the LLOQ and not more than 5\% of the IS response in the LLOQ sample."

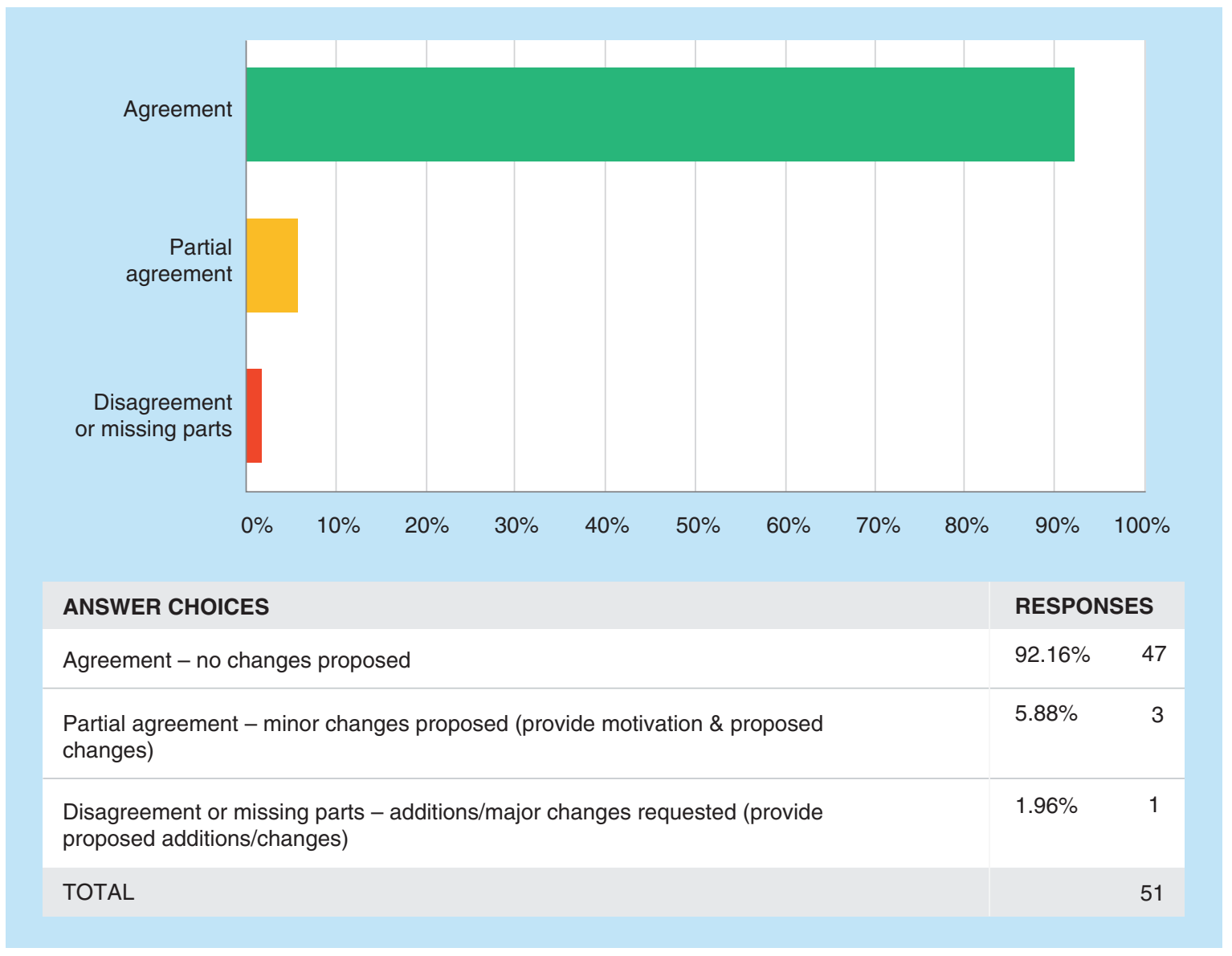

\section{Partial agreement}

No trend was found in these comments.

\section{Additional comments from the 13th GCC:}

GCC consensus was that no change to this section was required. 
T2Q8: Specificity - Back Conversion; Lines 260-268: "The possibility of back-conversion of a metabolite into the parent analyte during the successive steps of the analysis (including extraction procedures or in the MS source) should also be evaluated when relevant (i.e., potentially unstable metabolites such as ester analytes to ester/acidic metabolites, unstable $N$-oxides or glucuronide metabolites, lactone-ring structures). It is acknowledged that this evaluation will not be possible in the early stages of drug development of a new chemical entity when the metabolism is not yet evaluated. However, it is expected that this issue should be investigated and partial validation performed if needed. The extent of back-conversion, if any, should be established and the impact on the study results discussed in the Bioanalytical Report."

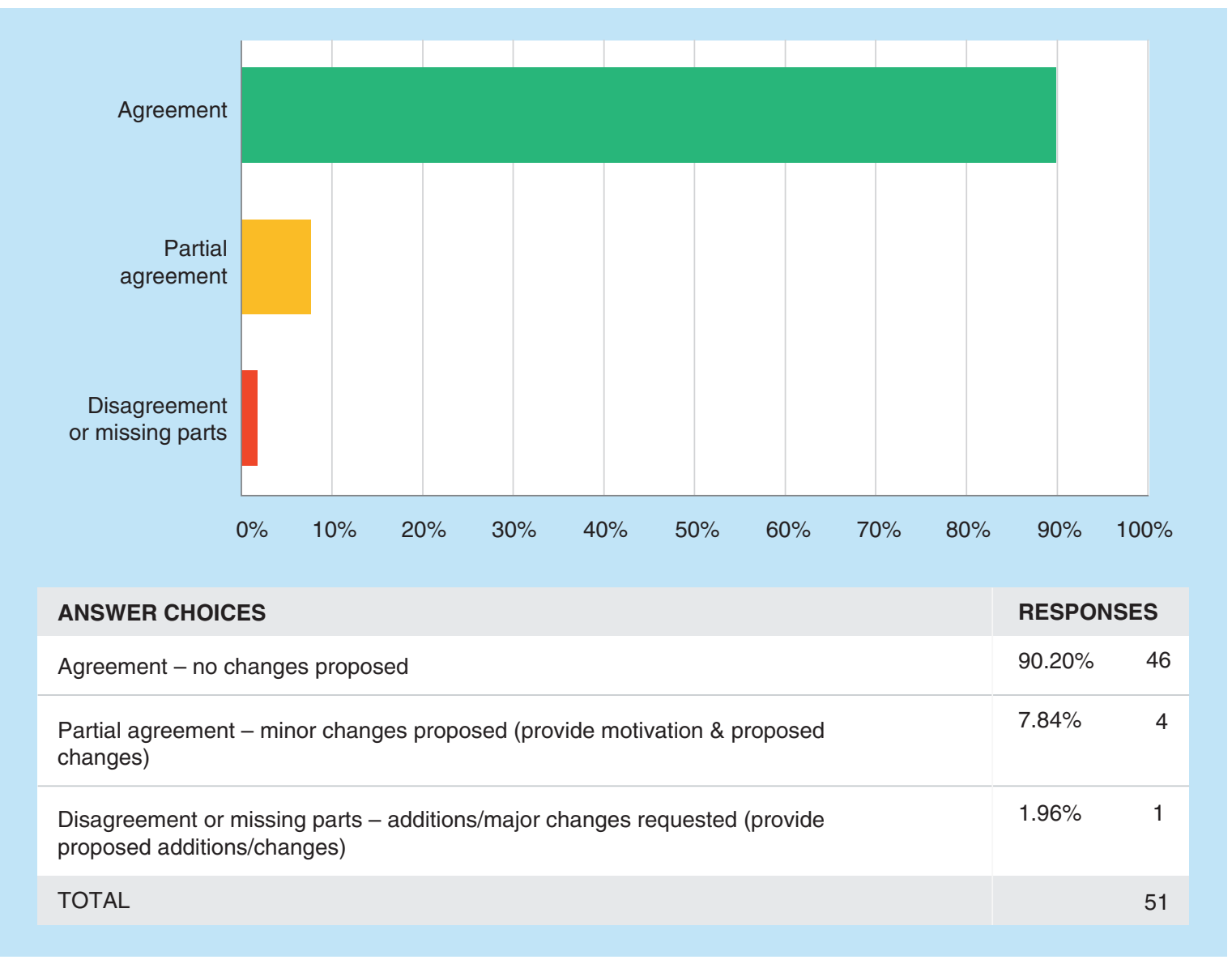

Partial agreement

No trend was found in these comments. 
T2Q9: Matrix Effect - Definition; Lines 270-271: "A matrix effect is defined as an alteration of the analyte response due to interfering and often unidentified component(s) in the sample matrix."

Matrix Effect - Procedure; Lines 271-274: "During method validation it is necessary to evaluate the matrix effect between different independent sources/lots. The matrix effect should be evaluated by analysing at least 3 replicates of low and high QCs, each prepared using matrix from at least 6 different sources/lots."

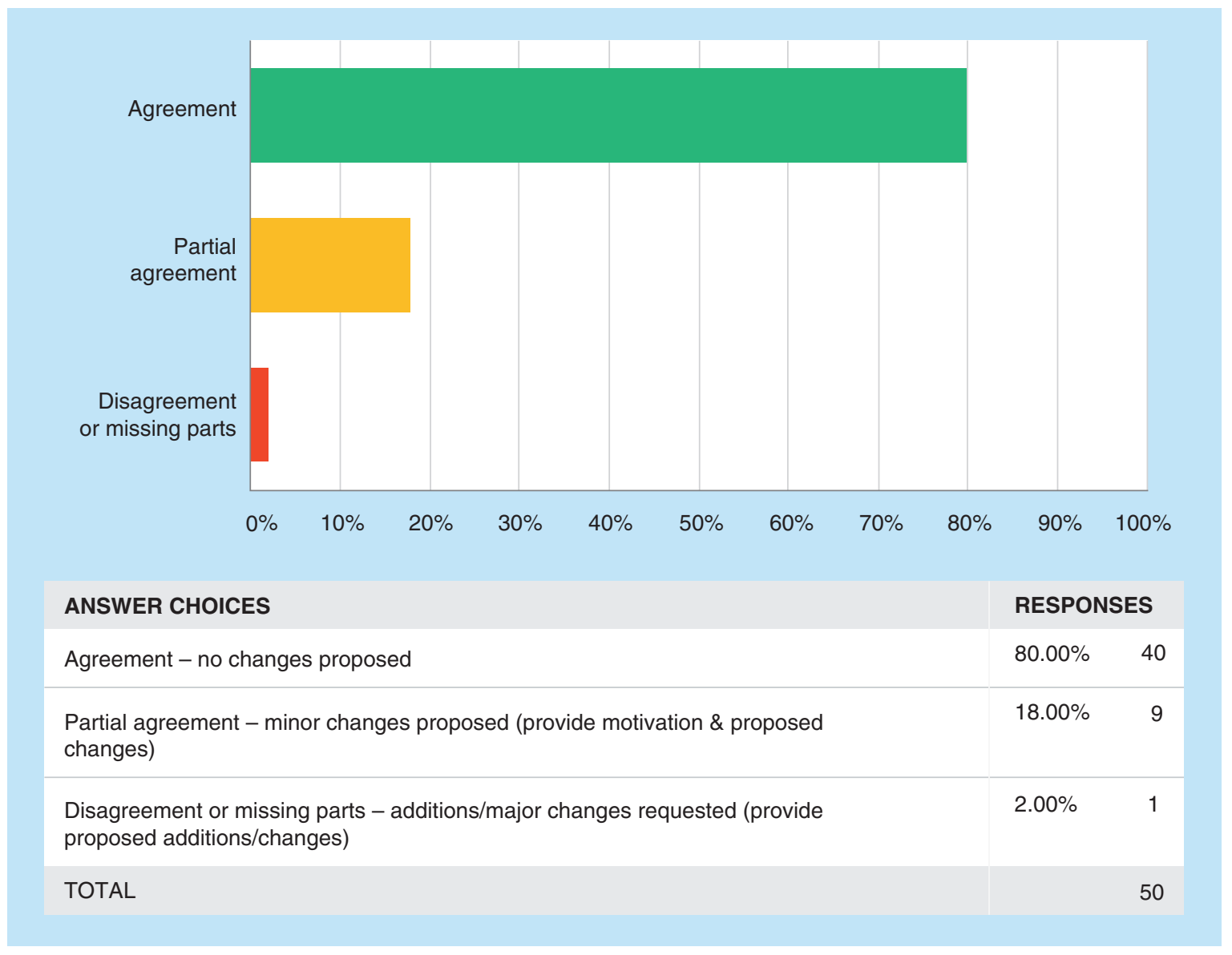

\section{Partial agreement}

- Several comments disagree with the requirement to perform the evaluation at the high QC level and suggested that this be removed since any matrix effect will be easier to detect at the low QC level.

- Some comments indicated that perfoming three replicates for each lot was not required, and that a singlet, as is done in current practice, is sufficient.

- Other comments suggested adding the matrix factor as an acceptable process for the matrix effect.

\section{Additional comments from the 13th GCC:}

It was further confirmed that this evaluation should not be performed at the high QC level since any matrix effect will be easier to detect at the low QC level. 
T2Q10: Matrix Effect - Acceptance Criteria; Lines 274-277: "The accuracy should be within $\pm 15 \%$ of the nominal concentration and the precision (per cent coefficient of variation (\%CV)) should not be greater than $15 \%$ in all individual matrix sources/lots. Use of fewer sources/lots may be acceptable in the case of rare matrices."

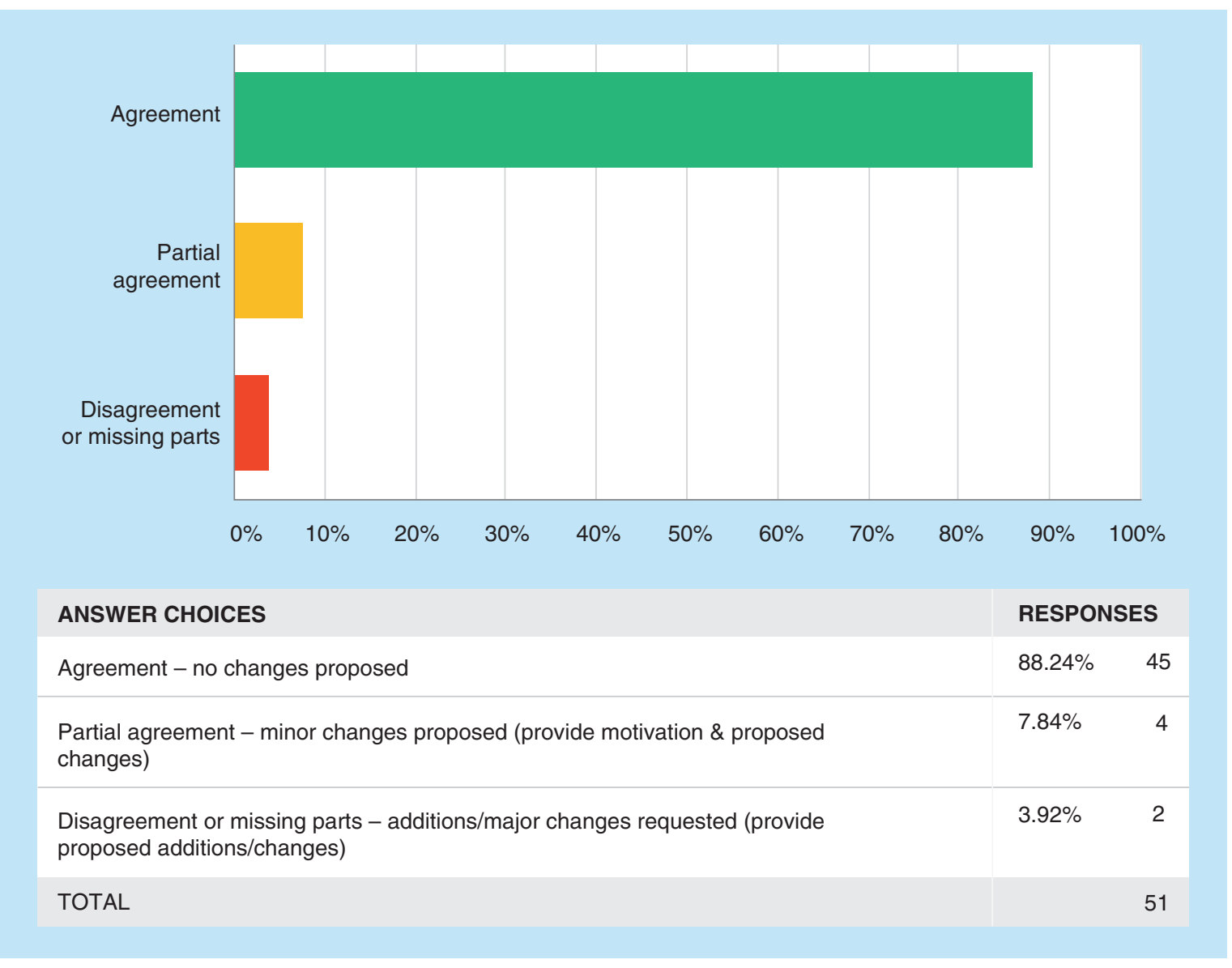

Disagreement or missing parts

No trend was found in these comments.

Partial agreement

It was suggested to clarify if the criteria are applicable to each individual lot or all the lots combined. 
T2Q11: Matrix Effect - Alternate Matrices; Lines 278-282: "The matrix effect should also be evaluated in relevant patient populations or special populations (e.g., hepatically impaired or renally impaired) when available. An additional evaluation of the matrix effect is recommended using haemolysed or lipaemic matrix samples during method validation on a case by case basis, especially when these conditions are expected to occur within the study.

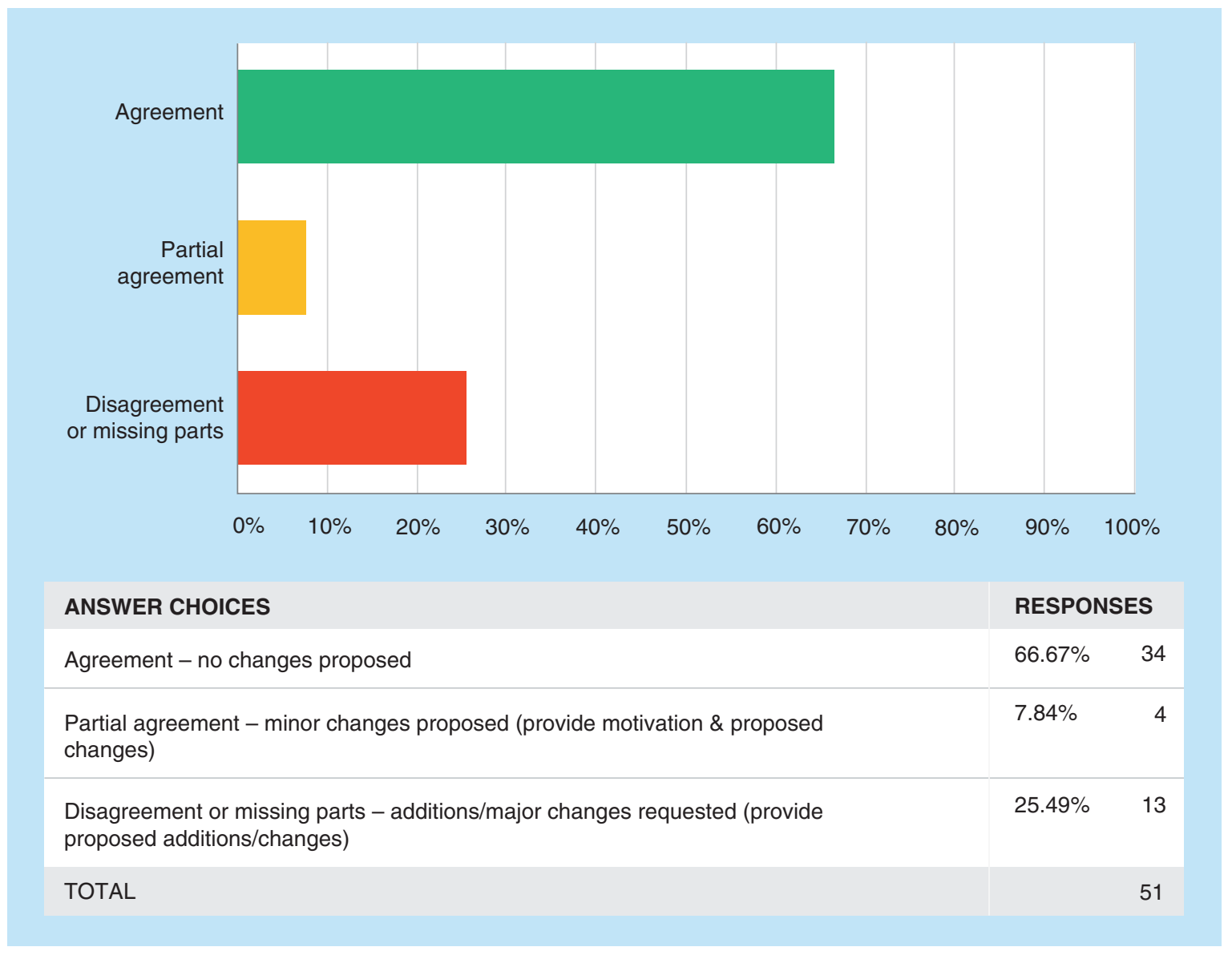

\section{Disagreement or missing parts}

The majority of the comments agreed that the evaluation of hemolyzed and lipaemic lots on a case by case basis was confusing, misleading and in disagreement with Regulatory Agencies' requests, since this could be interpreted differently by different agencies, and is normally performed in BMV.

This text: "a case by case basis, especially when these conditions are expected to occur within the study." should be removed.

\section{Partial agreement}

No additional trend was found in these comments. 
T2Q12: Calibration Curve - Purpose; Lines 284-285: "The calibration curve demonstrates the relationship between the nominal analyte concentration and the response of the analytical platform to the analyte."

Calibration Standards for Validation - Preparation; Lines 285-288: "Calibration standards, prepared by spiking matrix with a known quantity of analyte, span the calibration range and comprise the calibration curve. Calibration standards should be prepared in the same biological matrix as the study samples."

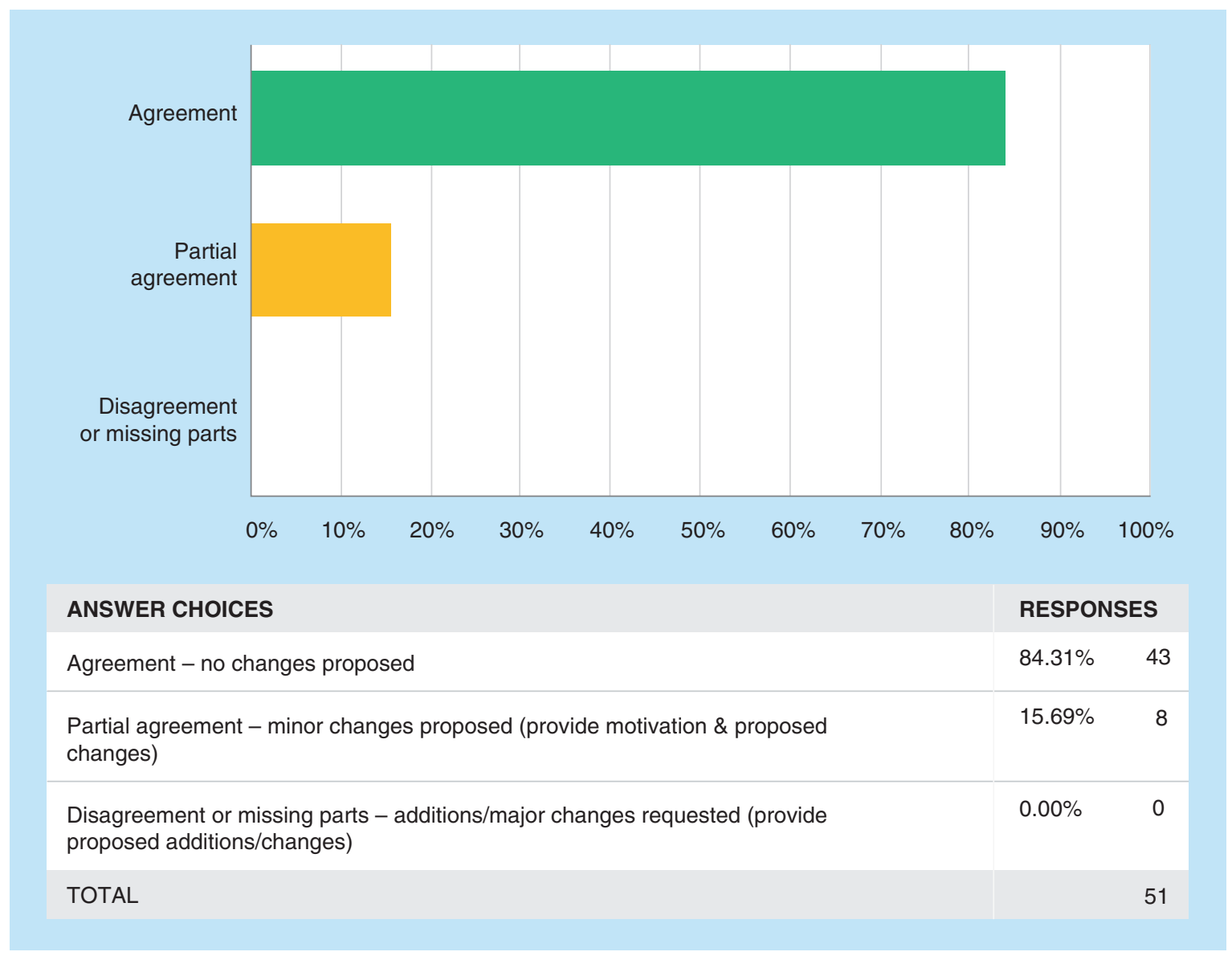

\section{Partial agreement}

All comments agreed that the last sentence should include language allowing the possibility of using surrogate matrix (e.g., for an endogenous compound). 
T2Q13: Calibration Curve - Range; Lines 288-290: "The calibration range is defined by the LLOQ, which is the lowest calibration standard, and the ULOQ, which is the highest calibration standard. There should be one calibration curve for each analyte studied during method validation and for each analytical run."

Calibration Curve - Contents; Lines 291-293: "A calibration curve should be generated with a blank sample, a zero sample (blank sample spiked with IS), and at least 6 concentration levels of calibration standards, including the LLOQ and the ULOQ."

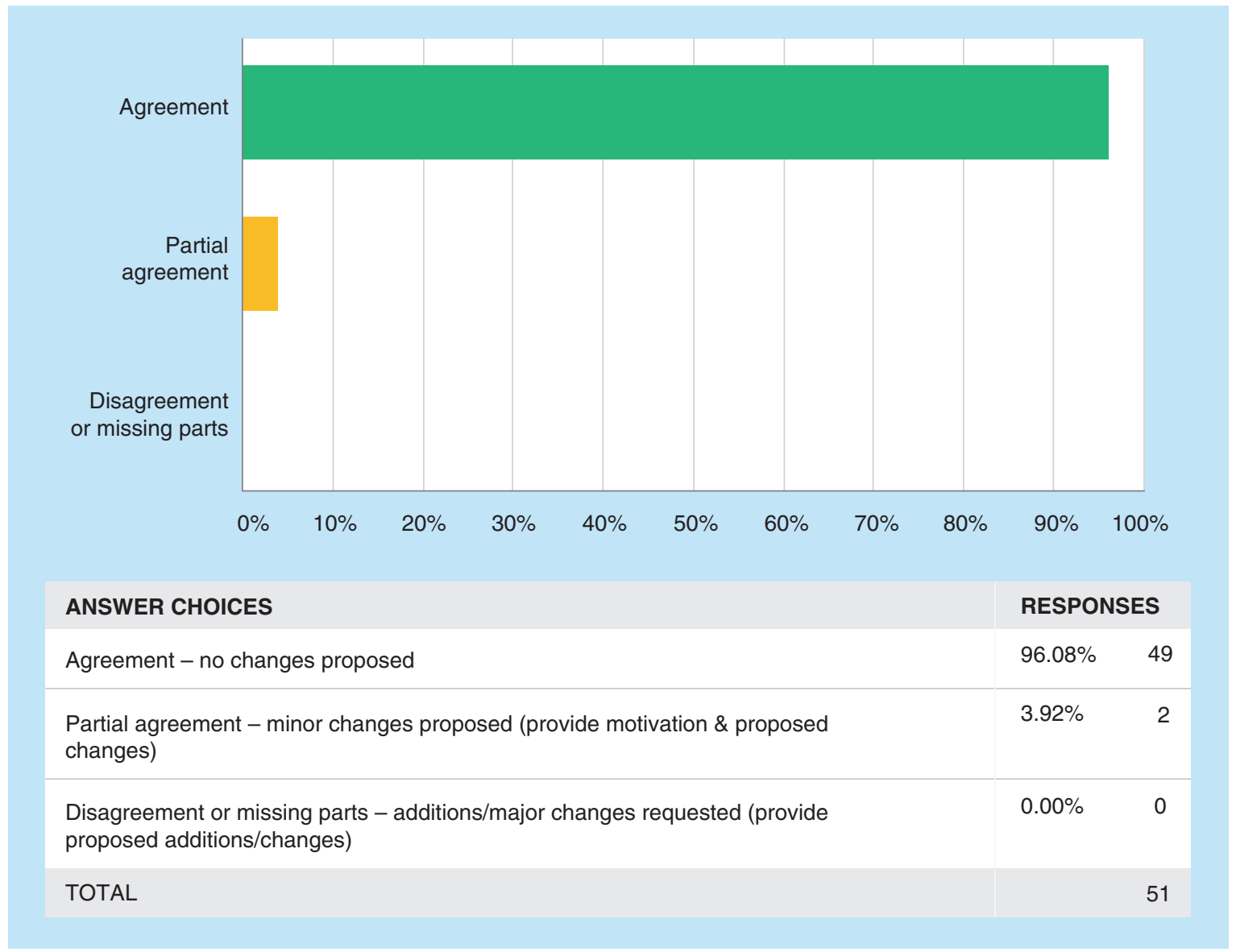

\section{Partial agreement}

No trend was found in these comments. 
T2Q14: Calibration Curve - Regression Model; Lines 294-298: "A simple regression model that adequately describes the concentration-response relationship should be used. The selection of the regression model should be directed by written procedures. The regression model, weighting scheme and transformation should be determined during the method validation. Blank and zero samples should not be included in the determination of the regression equation for the calibration curve."

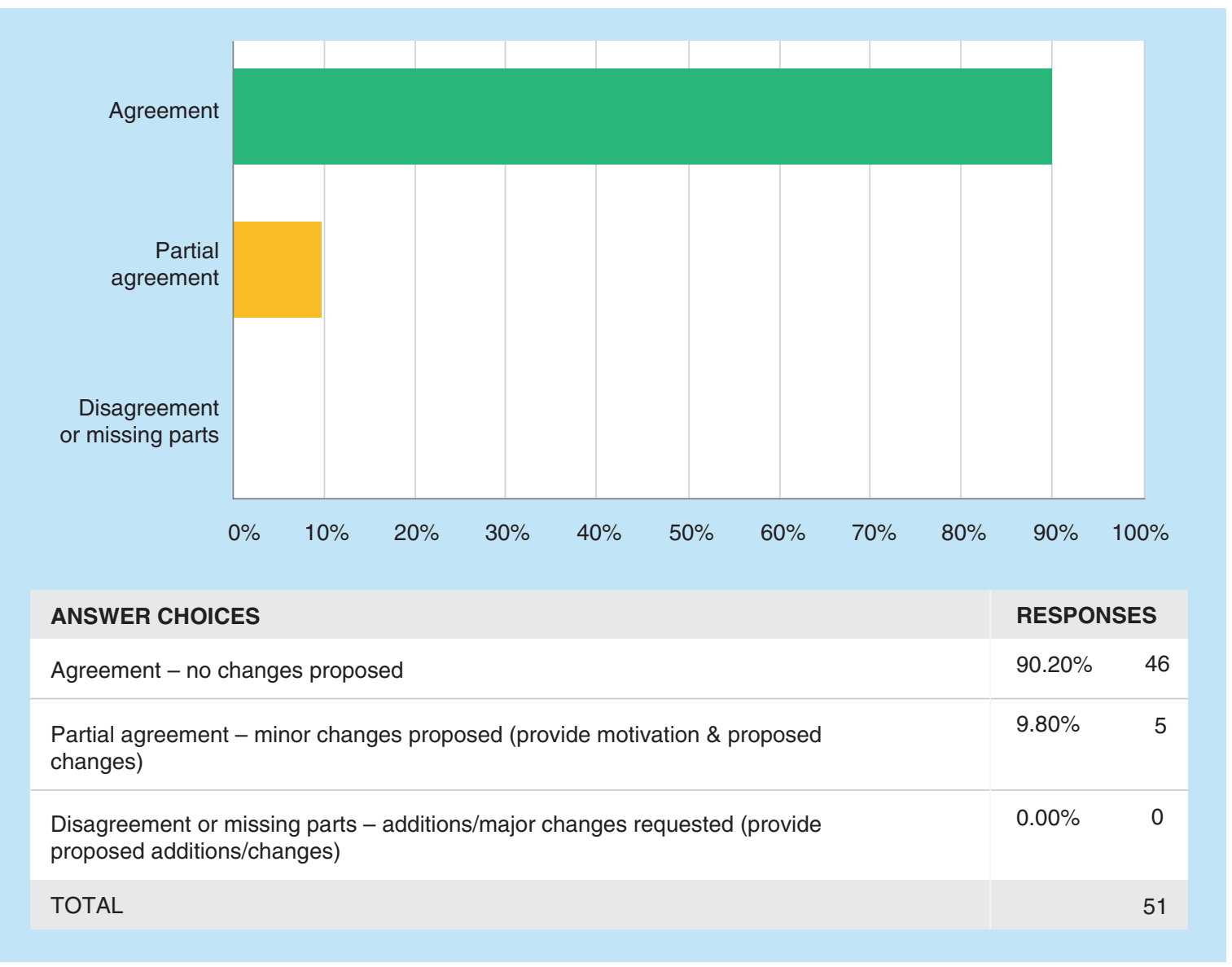

Partial agreement

A revision was suggested to state that the regression type should be determined in method development and confirmed in validation. 
T2Q15: Calibration Curve - Replicate Calibration Curves; Lines 298-300: "Each calibration standard may be analyzed in replicate, in which case data from all acceptable replicates should be used in the regression analysis."

Calibration Curve - Reporting; Lines 301-304: "The calibration curve parameters should be reported (slope and intercept in the case of a linear model). The back-calculated concentrations of the calibration standards should be presented together with the calculated mean accuracy values. All acceptable curves obtained during validation, based on a minimum of 3 independent runs over several days, should be reported."

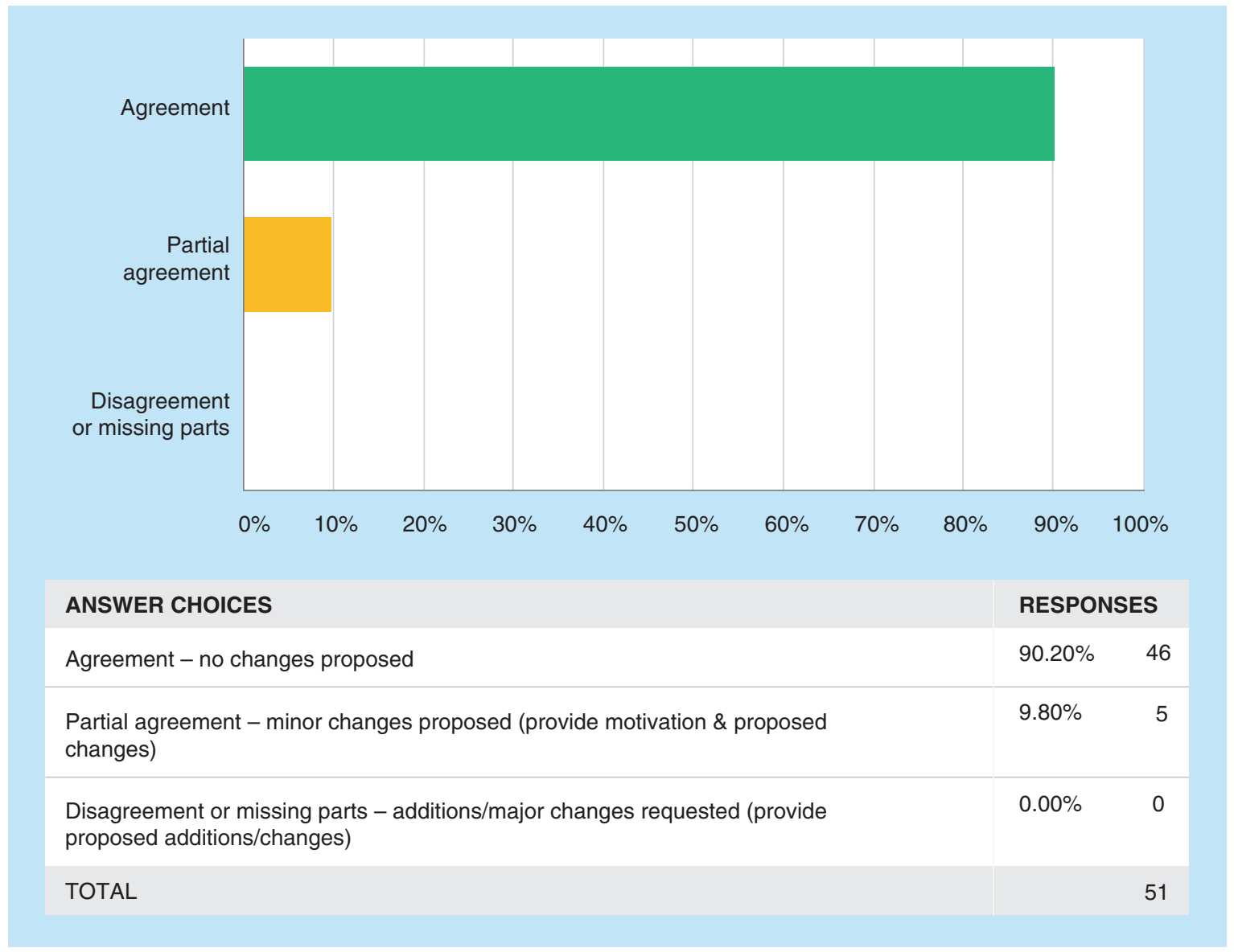

\section{Partial agreement}

It was suggested to change "over several days" to "at least 2 days" or "at least two seperate analytical occasions" (refer to T1Q13). 
T2Q16: Calibration Curve - Acceptance Criteria; Lines 305-310: "The accuracy of the back-calculated concentrations of each calibration standard should be within $\pm 20 \%$ of the nominal concentration at the LLOQ and within $\pm 15 \%$ at all the other levels. At least $75 \%$ of the calibration standards with a minimum of 6 calibration standard levels should meet the above criteria. In the case that replicates are used, the criteria (within $\pm 15 \%$ or $\pm 20 \%$ for LLOQ) should also be fulfilled for at least $50 \%$ of the calibration standards tested per concentration level."

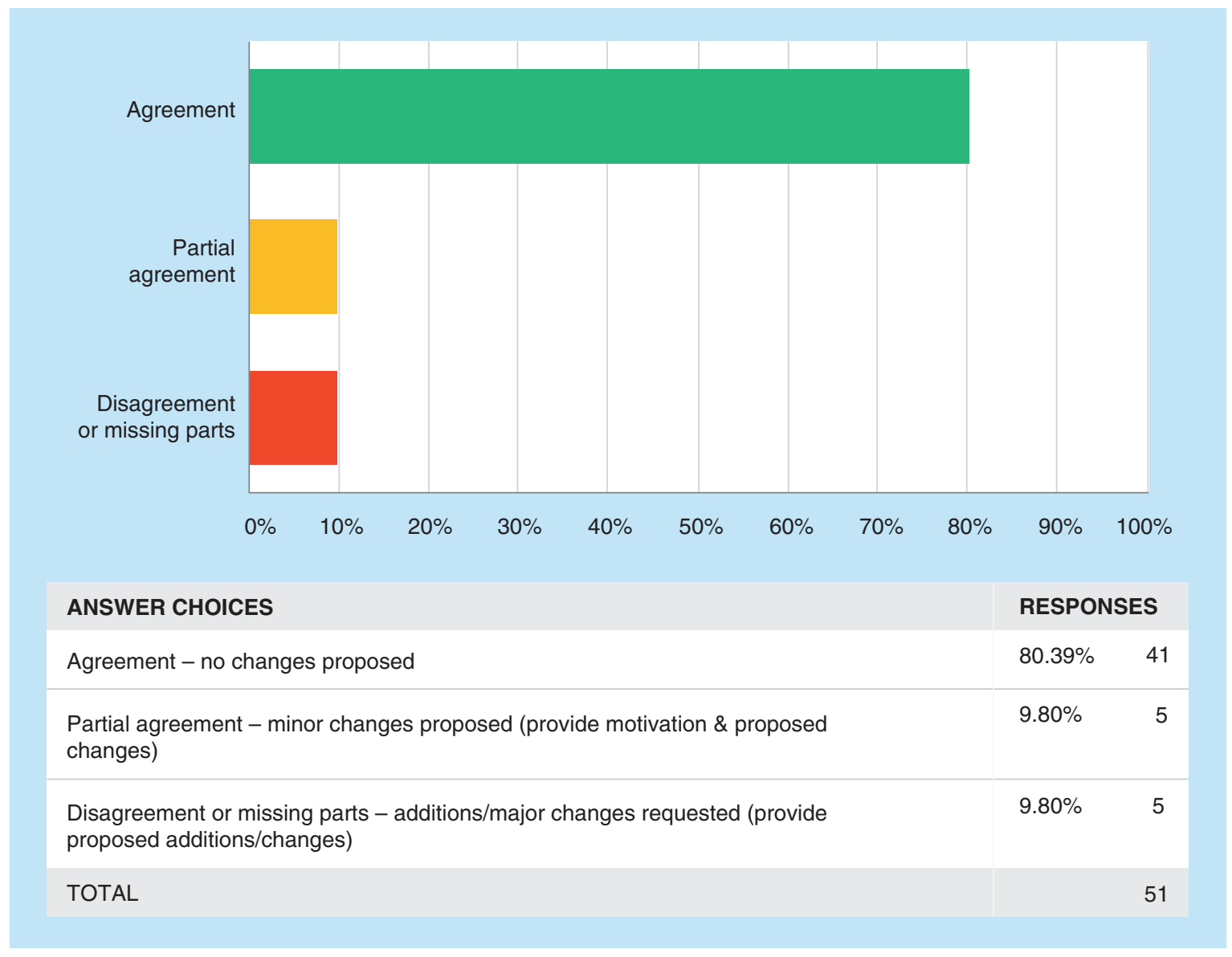

Disagreement or missing parts

All comments disagree with the sentence: "In the case that replicates are used, the criteria (within $\pm 15 \%$ or $\pm 20 \%$ for LLOQ) should also be fulfilled for at least 50\% of the calibration standards tested per concentration level." It is recommended that as long as $75 \%$ of the calibrant levels pass, the calibration curve should pass.

Partial agreement

No additional trend was found in these comments. 
T2Q17: Calibration Curve - Rejected Data; Lines 310-316: "In the case that a calibration standard does not comply with these criteria, this calibration standard sample should be rejected, and the calibration curve without this calibration standard should be re-evaluated, including regression analysis. For accuracy and precision runs, if all replicates of the LLOQ or the ULOQ calibration standard in a run are rejected then the run should be rejected, the possible source of the failure should be determined and the method revised if necessary. If the next validation run also fails, then the method should be revised before restarting validation."

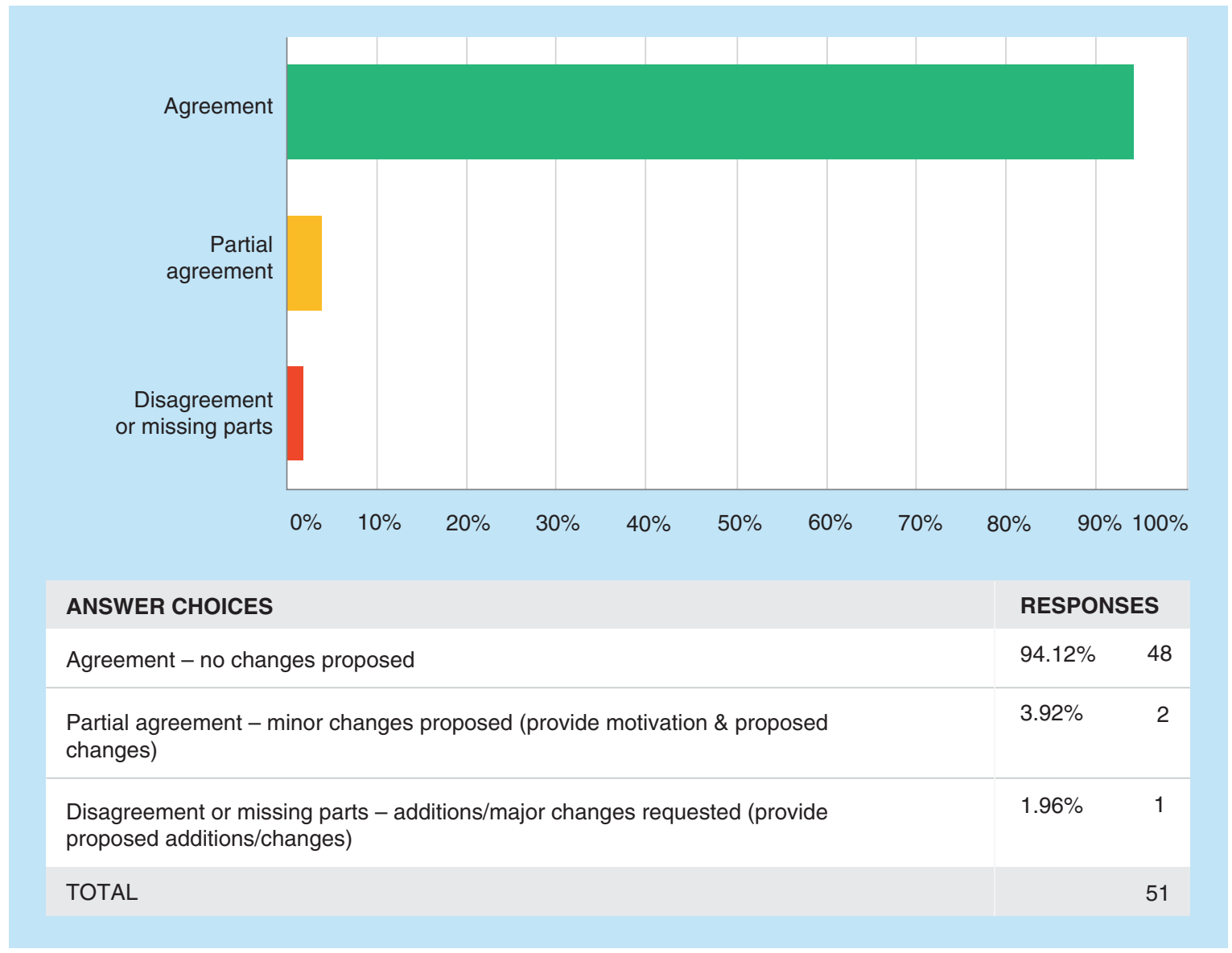

\section{Partial agreement}

It may be useful to mention that three comments suggested changing the last sentence as follows, since changing the method may not always be required: "If the next validation run also fails, analytical cause for the failure should be assessed and the method revised if needed before restarting the validation."

\section{Additional comments from the 13th GCC:}

There were no further discussions during the GCC Closed Forum in support of the above proposal. 
Supplement Nehls, Buonarati, Cape et al.

T2Q18: Calibration Curve - Fresh Spike; Lines 317-319: "The calibration curve should be prepared using freshly spiked calibration standards in at least one assessment. Subsequently, frozen calibration standards can be used within their defined period of stability."

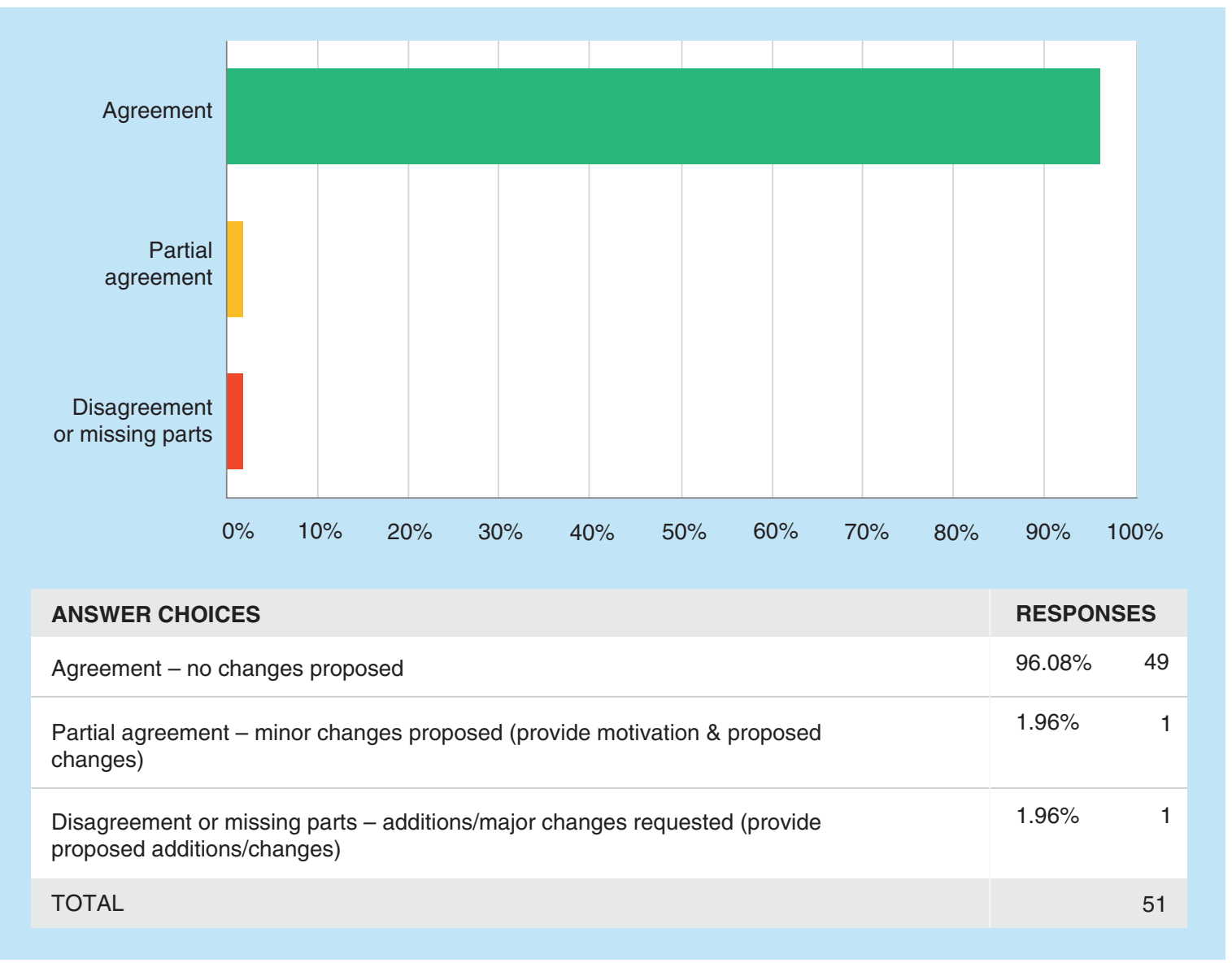


T2Q19: QCs for Validation - Purpose; Lines 322-324: "The QCs are intended to mimic study samples and should be prepared by spiking matrix with a known quantity of analyte, storing them under the conditions anticipated for study samples and analysing them to assess the validity of the analytical method."

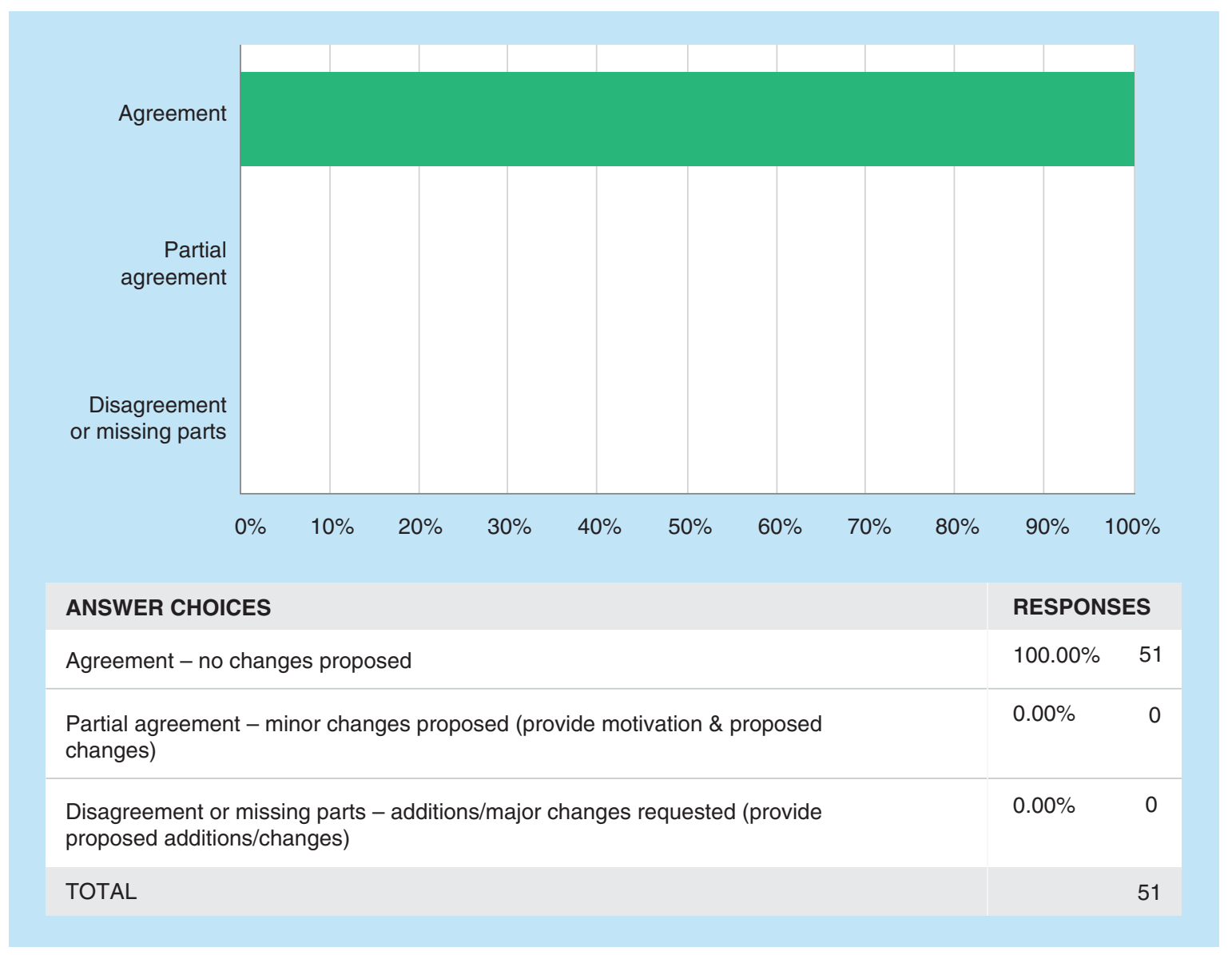


T2Q20: QCs for Validation - Preparation (Stocks); Lines 325-328: "Calibration standards and the QCs should be prepared from separate stock solutions in order to avoid biased estimations, which are not related to the analytical performance of the method. However, calibration standards and the QCs may be prepared from the same stock solution, provided the accuracy and stability of the stock solution have been verified."

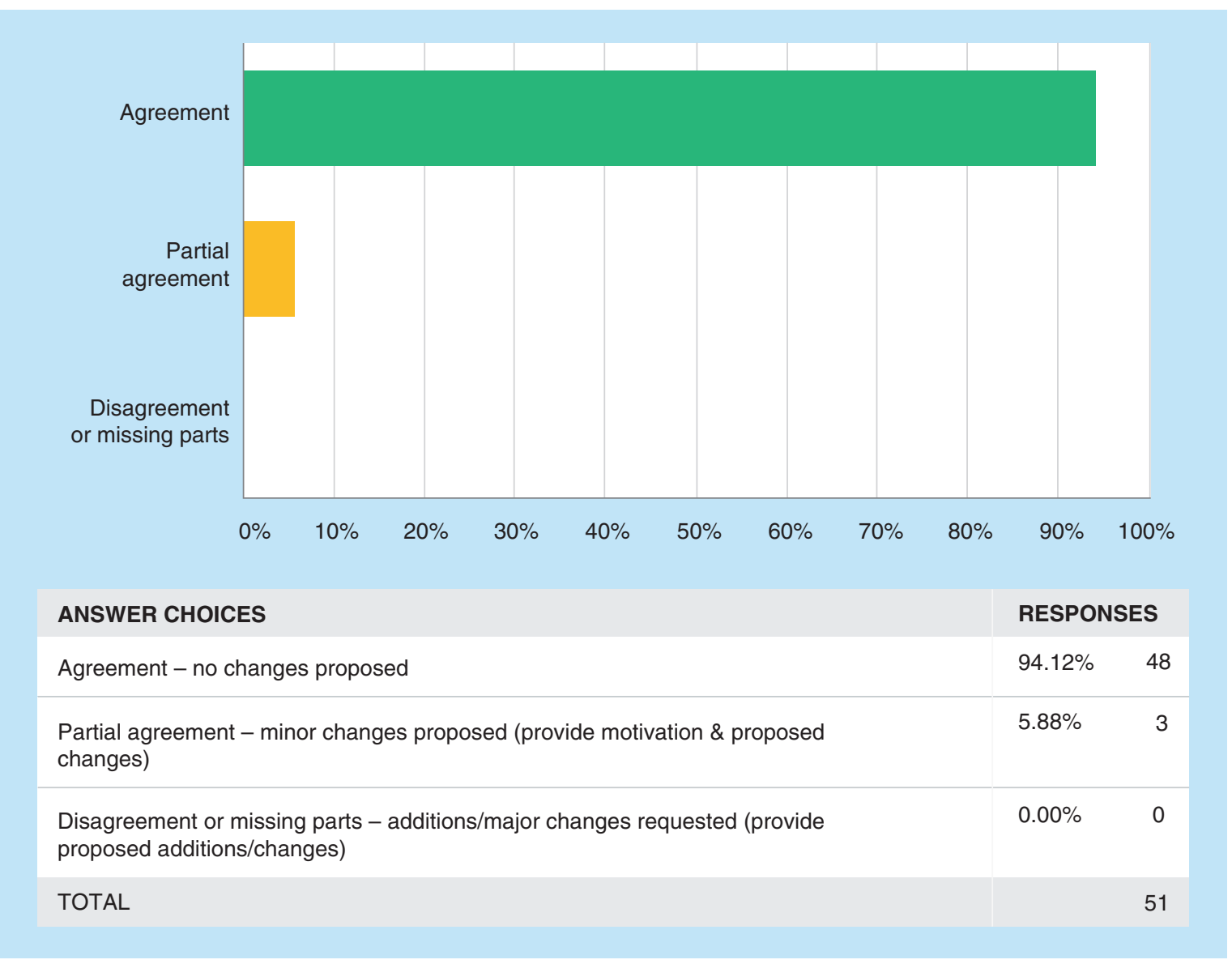

\section{Partial agreement}

Three comments suggested that this text should be reworded since both of these statements appear to contradict each other.

Additional comments from the 13th GCC:

There were no further discussions during the GCC Closed Forum in support of the above proposal. 
T2Q21: QCs for Validation - Preparation (Matrix); Lines 328-330: "A single source of blank matrix may be used, which should be free of interference or matrix effects, as described in Section 3.2.3."

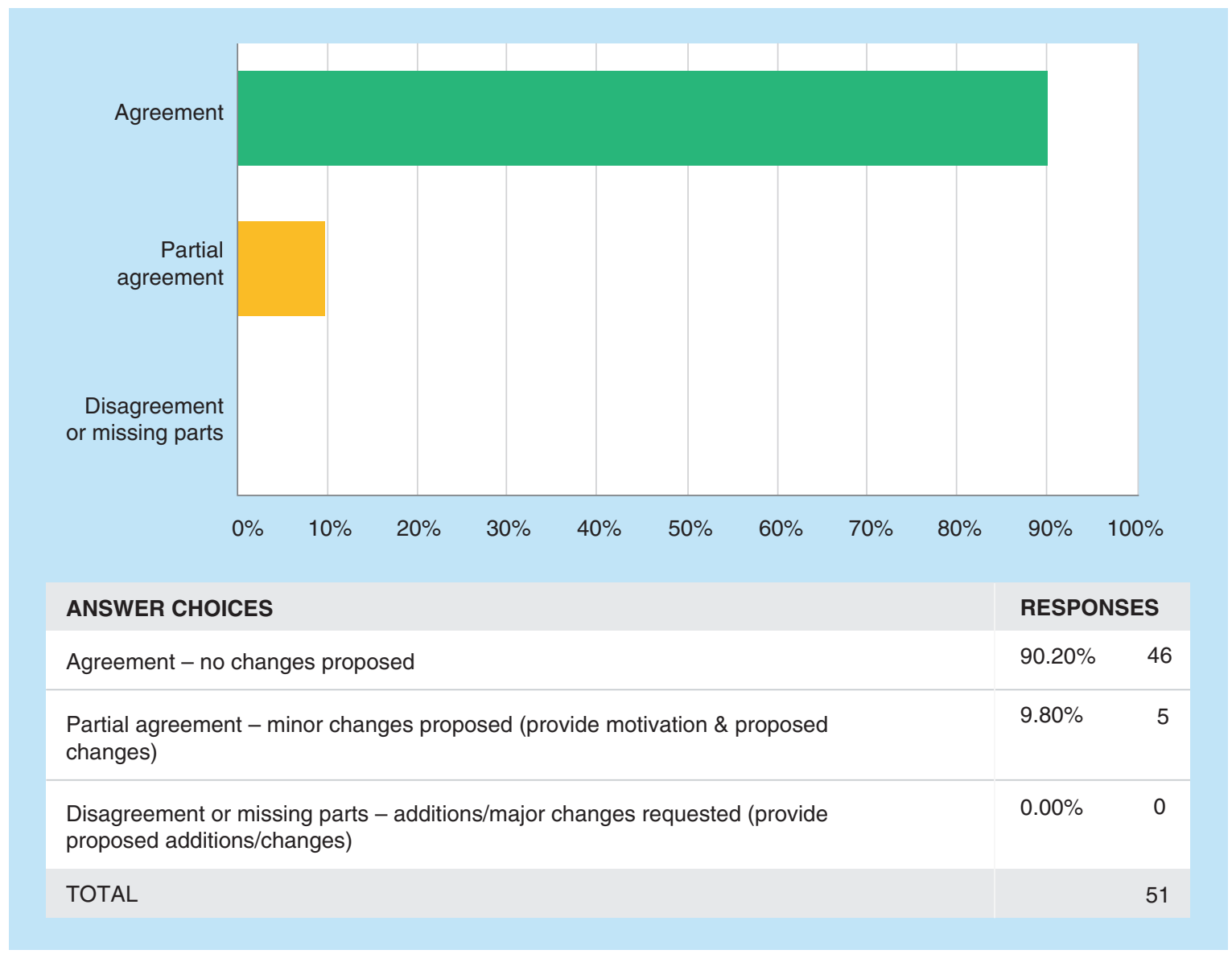

\section{Partial agreement}

It was suggested that this text be reworded since the presence of matrix effects have not been established prior to validation, when calibrants and QCs are being prepared. 
T2Q22: QCs for Validation - Concentrations; Lines 331-333: "During method validation the QCs should be prepared at a minimum of 4 concentration levels within the calibration curve range: the $L L O Q$, within three times of the LLOQ (low QC), around 30-50\% of the calibration curve range (medium QC) and at least $75 \%$ of the ULOQ (high QC)."

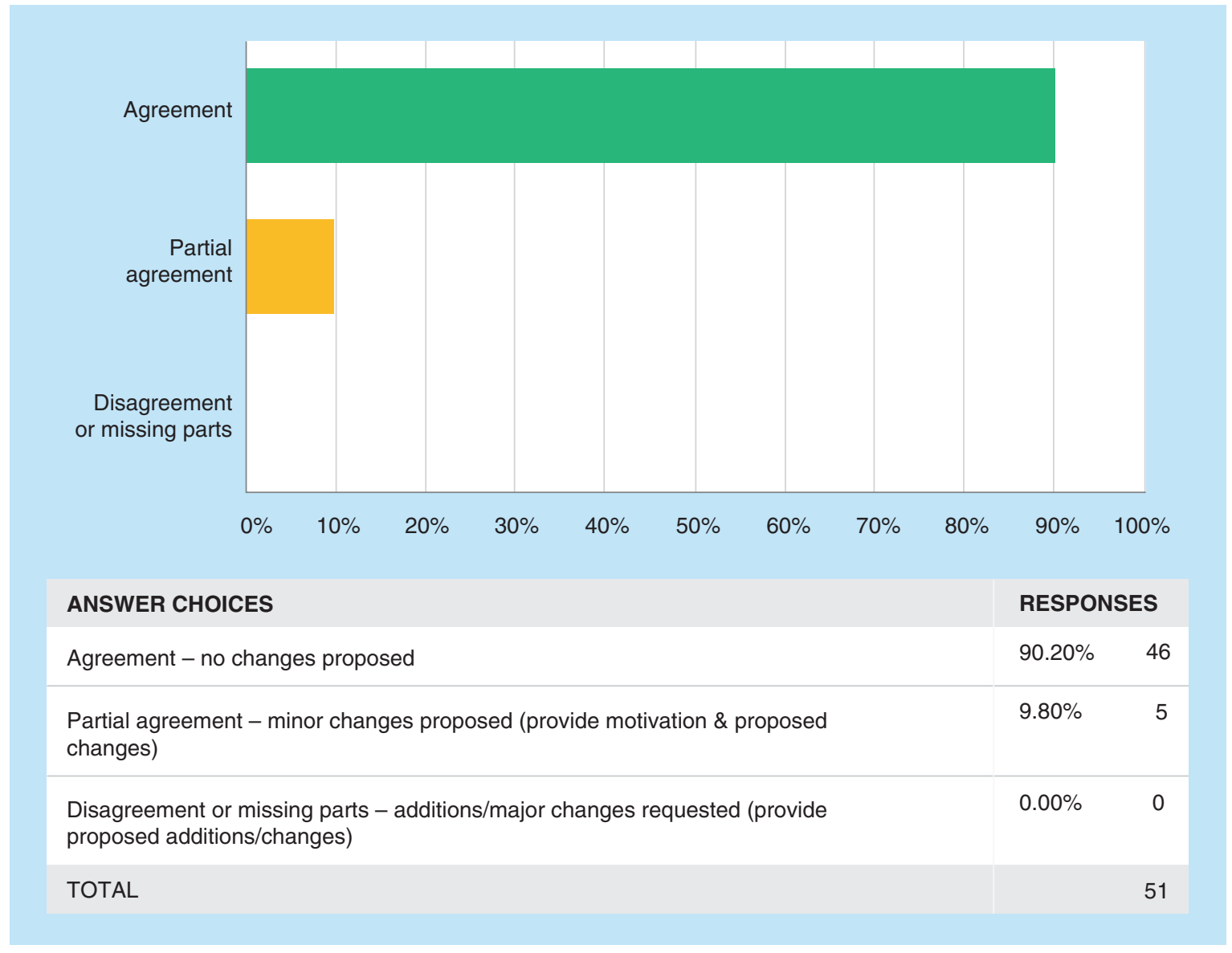

Partial agreement

It was requested to clarify the language describing if the medium QC referred to the geometric mean or the arithmetic mean. 
T2Q23: Accuracy and Precision - General; Lines 335-337: "Accuracy and precision should be determined by analyzing the QCs within each run (within-run) and in different runs (between-run). Accuracy and precision should be evaluated using the same runs and data."

Accuracy and Precision - Procedure; Lines 338-343: "Within-run accuracy and precision should be evaluated by analyzing at least five replicates at each QC concentration level in each analytical run. Between-run accuracy and precision should be evaluated by analyzing each QC concentration level in at least 3 analytical runs over at least two days. To enable the evaluation of any trends over time within one run, it is recommended to demonstrate accuracy and precision of the QCs over at least one of the runs in a size equivalent to a prospective analytical run of study samples."

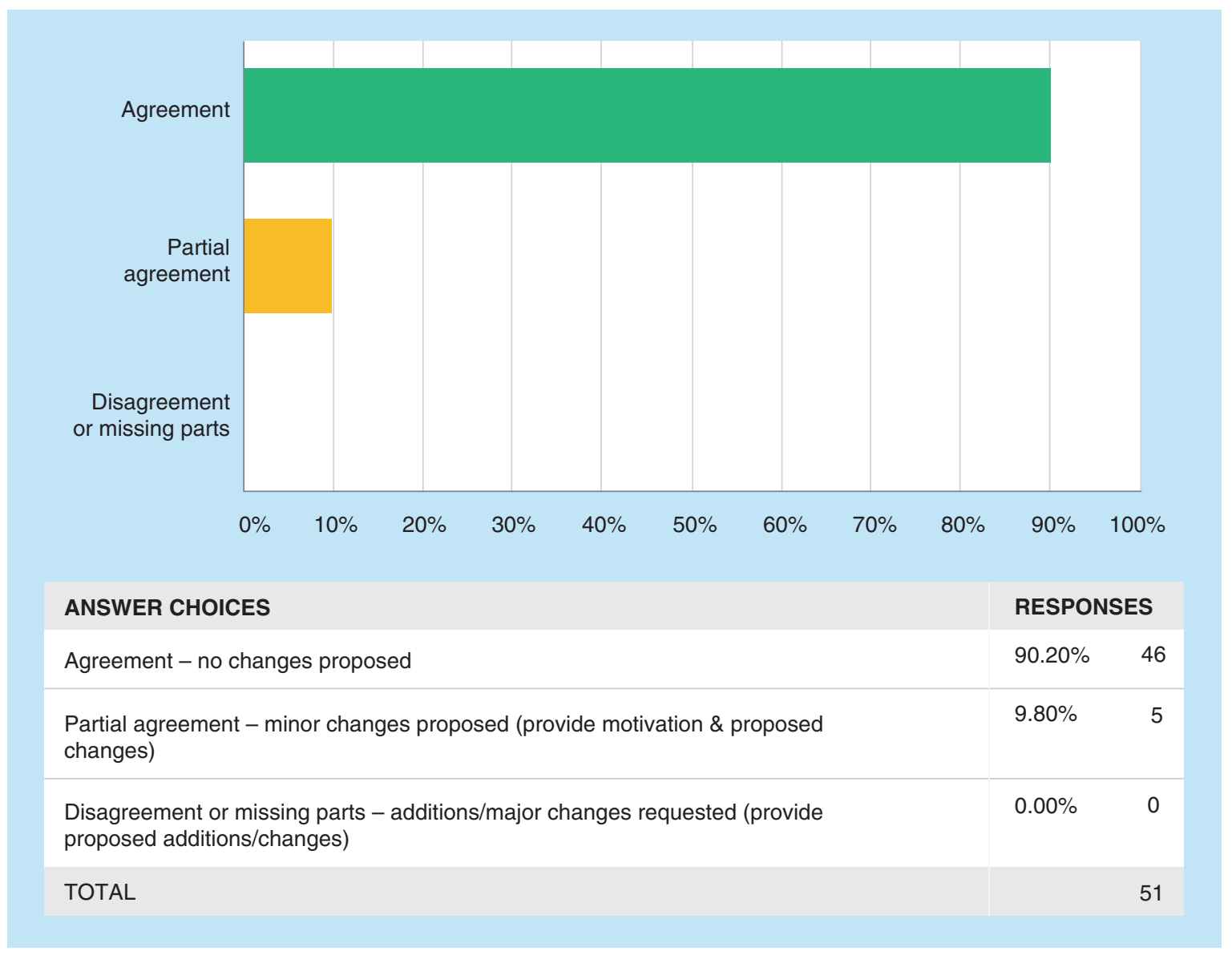

\section{Partial agreement}

No trend was found in these comments. 
T2Q24: Accuracy and Precision - Reporting; Lines 343-346: "Reported method validation data and the determination of accuracy and precision should include all results obtained, including individual QCs outside of the acceptance criteria, except those cases where errors are obvious and documented. Within-run accuracy and precision data should be reported for each run."

Accuracy and Precision - Rejected Data; Lines 346-348: "If the within-run accuracy or precision criteria are not met in all runs, an overall estimate of within-run accuracy and precision for each QC level should be calculated."

Accuracy and Precision; Lines 348-349: "Between-run (intermediate) precision and accuracy should be calculated by combining the data from all runs."

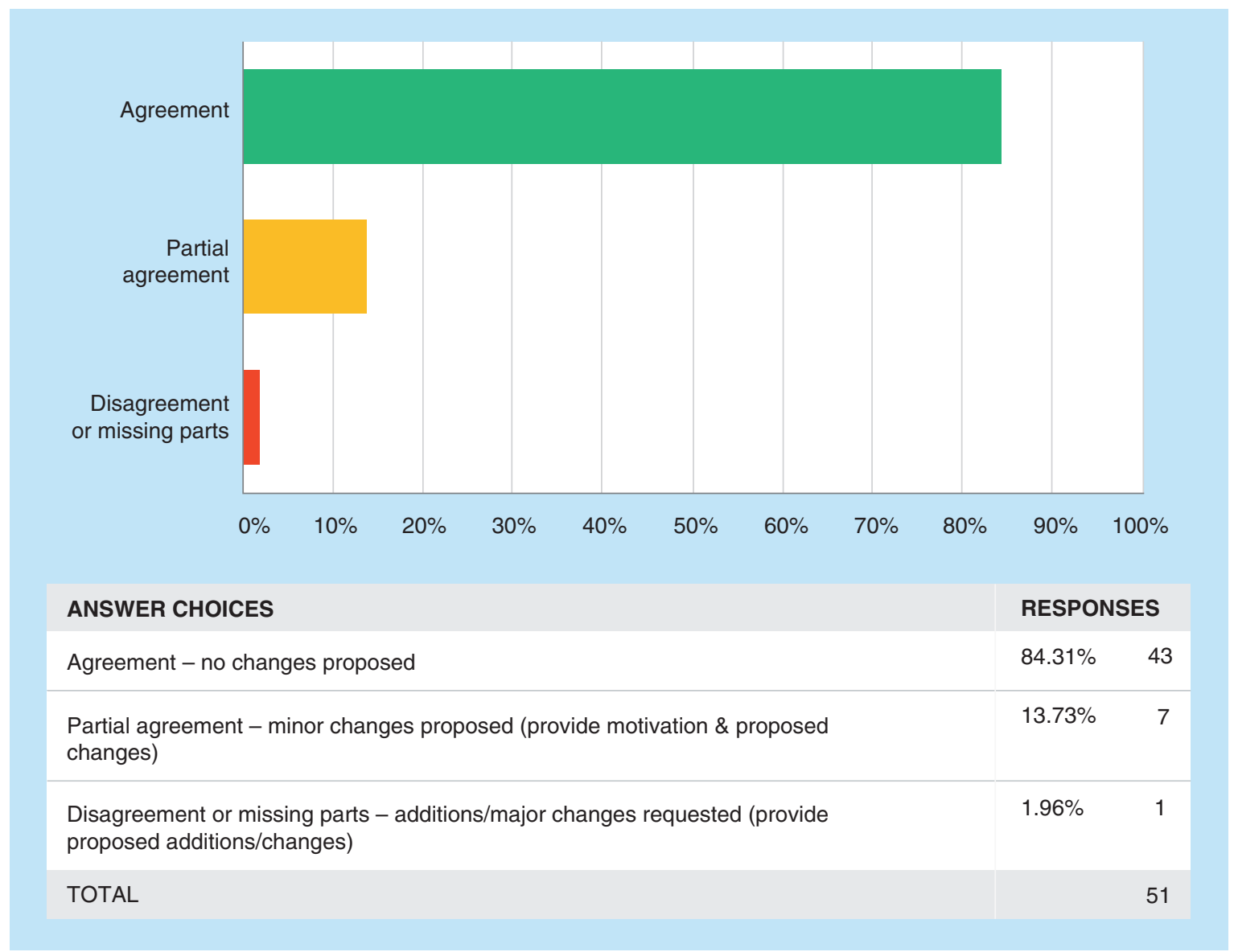

\section{Partial agreement}

Additional clarification is suggested to explain the recommendation in the following sentence: "If the within-run accuracy or precision criteria are not met in all runs, an overall estimate of within-run accuracy and precision for each QC level should be calculated' as it is unclear how this differs from the between-run accuracy and precision. 
T2Q25 Accuracy and Precision - Fresh Spike; Lines 350-352: "The calibration curves for these assessments should be prepared using freshly spiked calibration standards in at least one run. If freshly spiked calibration standards are not used in the other runs, stability of the frozen calibration standards should be demonstrated."

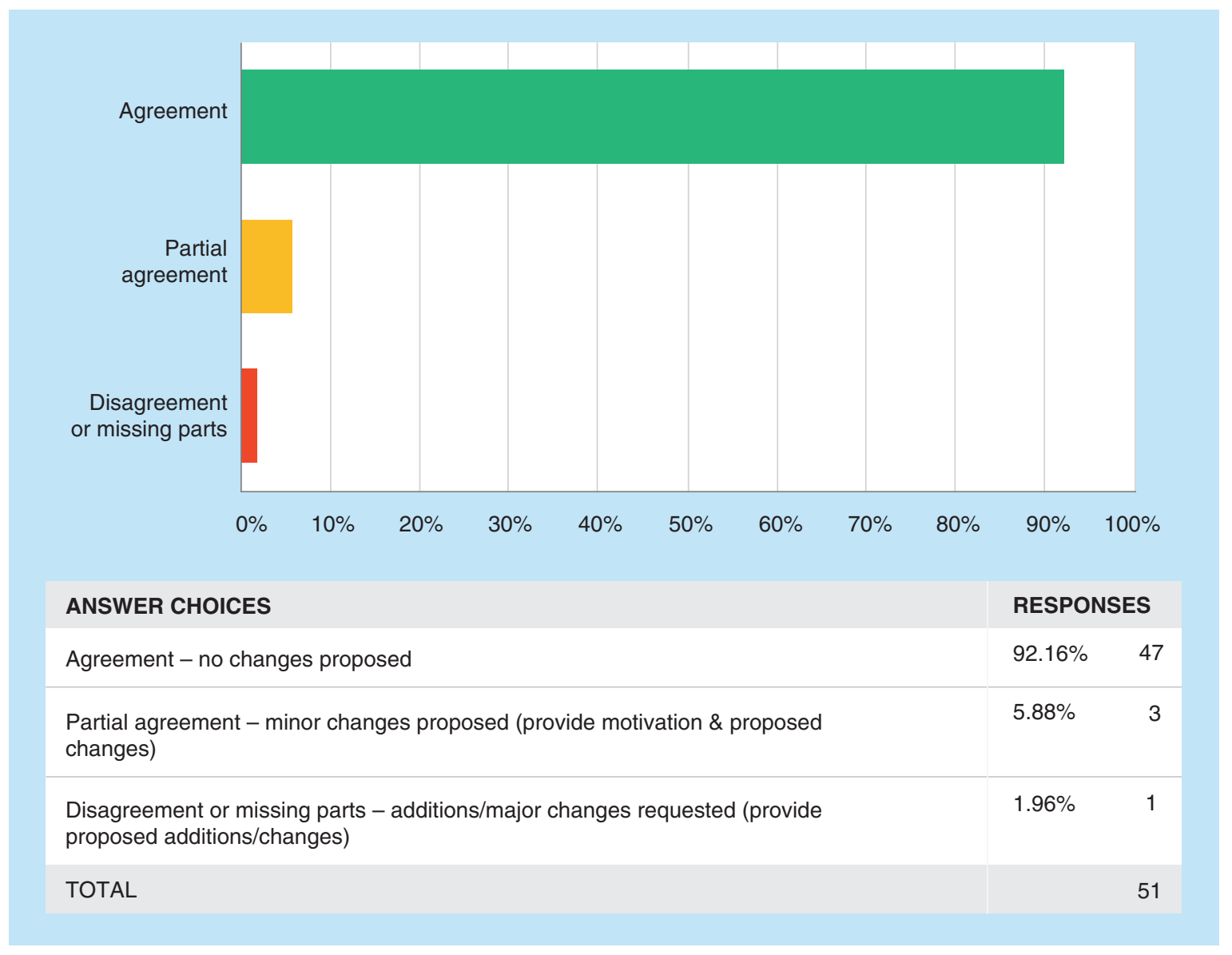

\section{Partial agreement}

No trend was found in these comments. 
T2Q26: Accuracy and Precision - Acceptance Criteria; Lines 353-356: "The overall accuracy at each concentration level should be within $\pm 15 \%$ of the nominal concentration, except at the $L L O Q$, where it should be within $\pm 20 \%$. The precision $(\% \mathrm{CV})$ of the concentrations determined at each level should not exceed $15 \%$, except at the LLOQ, where it should not exceed $20 \%$."

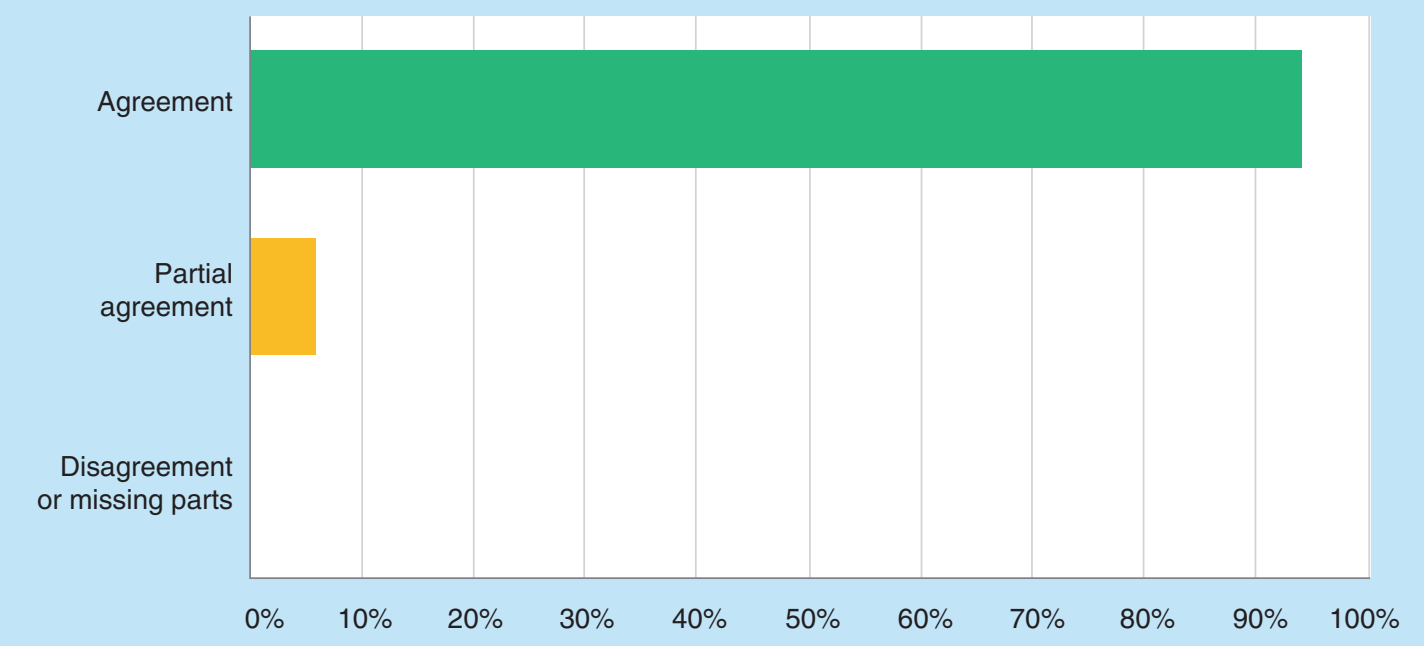

\section{ANSWER CHOICES}

RESPONSES

Agreement - no changes proposed

$94.12 \% \quad 48$

Partial agreement - minor changes proposed (provide motivation \& proposed

$5.88 \% \quad 3$ changes)

Disagreement or missing parts - additions/major changes requested (provide proposed additions/changes)

TOTAL

Partial agreement

No trend was found in these comments. 
T2Q27: Carryover - Definition; Lines 358-359: "Carryover is an alteration of a measured concentration due to residual analyte from a preceding sample that remains in the analytical instrument."

Carryover - Procedure; Lines 360-362: "Carryover should be assessed and minimised during method development. During validation carryover should be assessed by analysing blank samples after the calibration standard at the ULOQ."

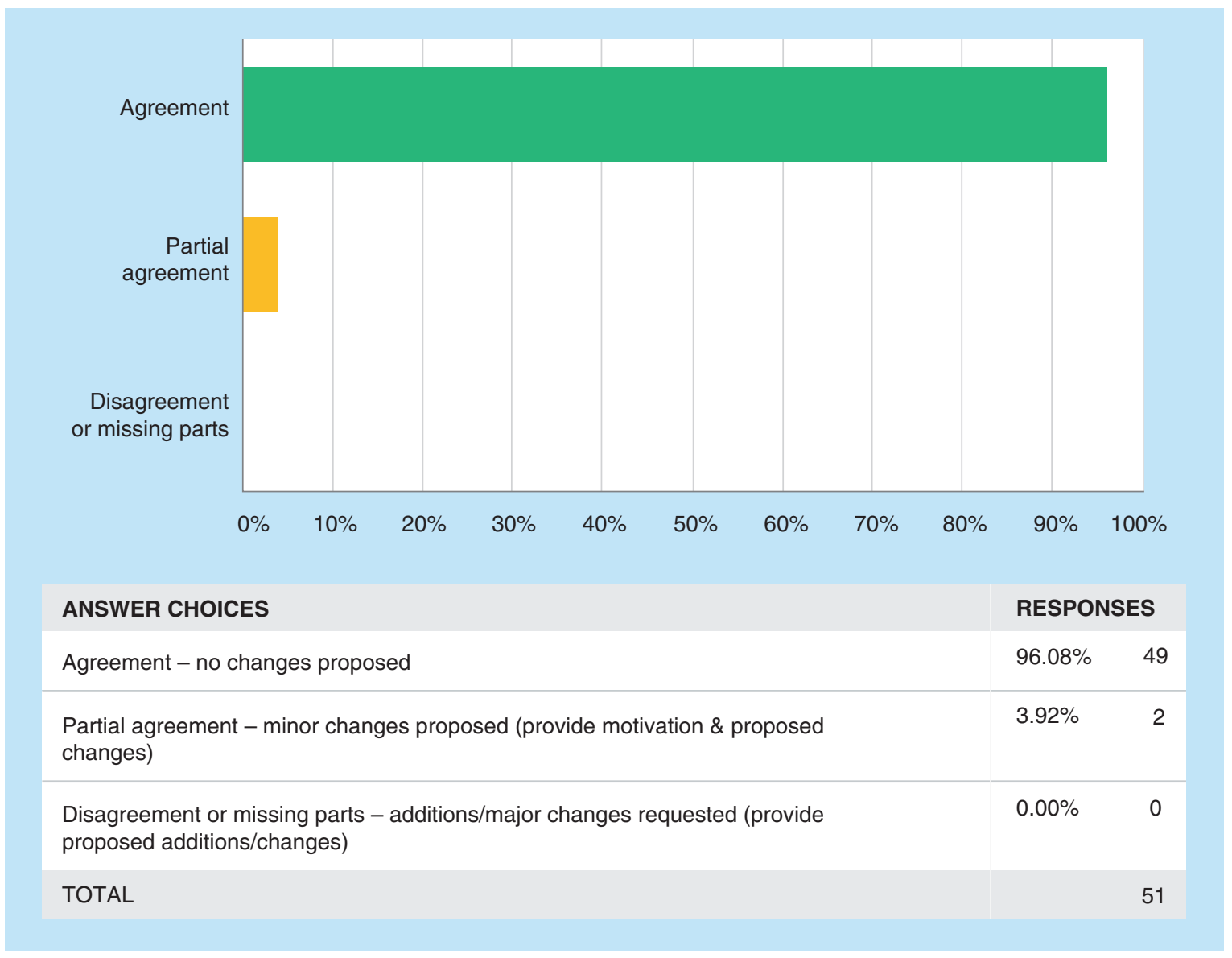

\section{Partial agreement}

No trend was found in these comments. 
T2Q28: Carryover - Acceptance Criteria; Lines 362-363: "Carryover in the blank samples following the highest calibration standard should not be greater than 20\% of the analyte response at the LLOQ and 5\% of the response for the IS."

Carryover - Mitigation; Lines 363-368: "If it appears that carryover is unavoidable, study samples should not be randomised. Specific measures should be considered, tested during the validation and applied during the analysis of the study samples, so that carryover does not affect accuracy and precision. This could include the injection of blank sample(s) after samples with an expected high concentration, before the next study sample."

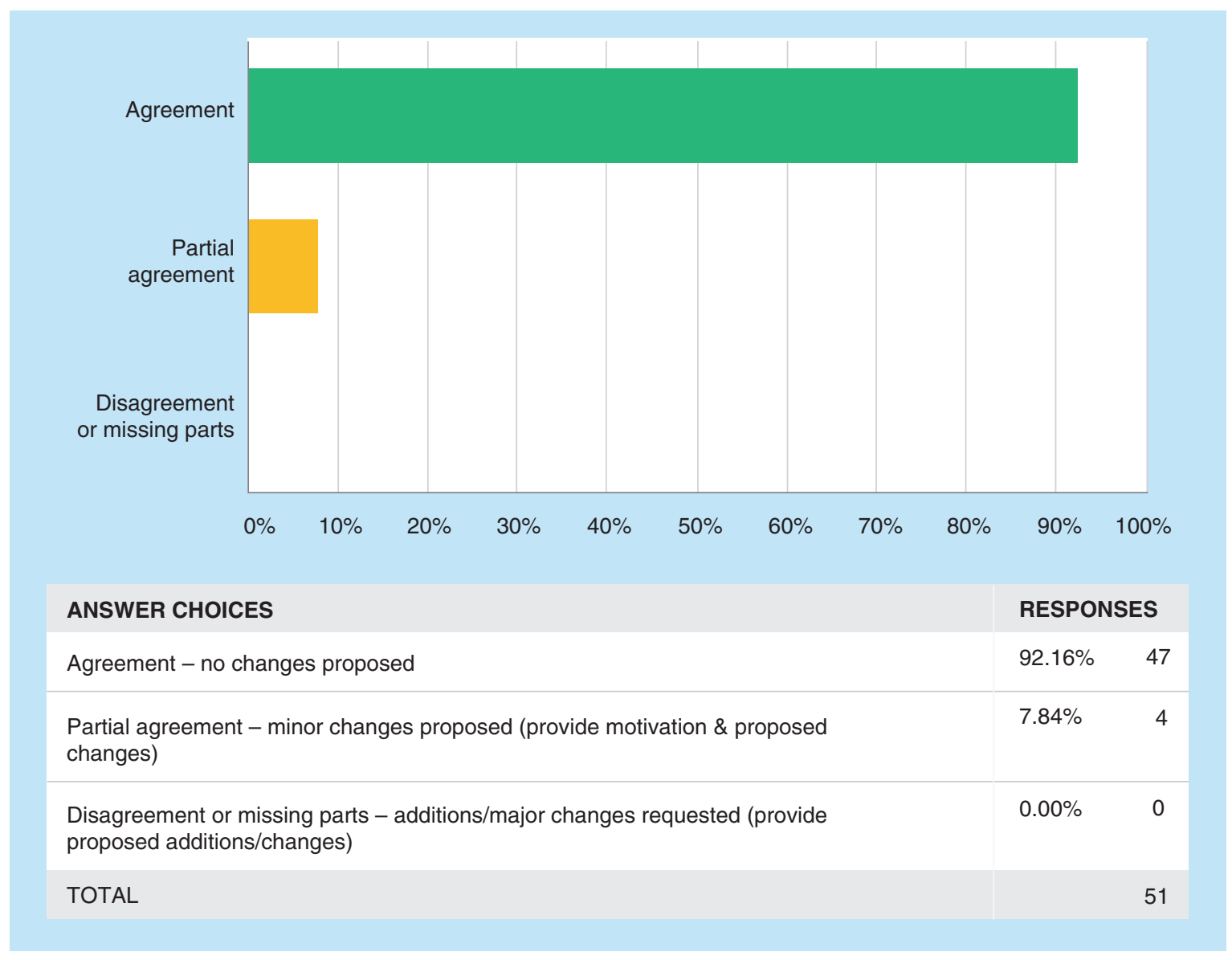

\section{Partial agreement}

No trend was found in these comments. 
T2Q29: Dilution Integrity - Definition; Lines 370-371: "Dilution integrity is the assessment of the sample dilution procedure, when required, to confirm that it does not impact the accuracy and precision of the measured concentration of the analyte."

Dilution Integrity - Procedure; Lines 372-378: "The same matrix from the same species used for preparation of the $Q C s$ should be used for dilution. Dilution QCs should be prepared with analyte concentrations in matrix that are greater than the ULOQ and then diluted with blank matrix. At least 5 replicates per dilution factor should be tested in one run to determine if concentrations are accurately and precisely measured within the calibration range. The dilution ratio(s) applied during study sample analysis should be within the range of the dilution ratios evaluated during validation."

Dilution Integrity - Acceptance Criteria; Lines 378-380: "The mean accuracy of the dilution QCs should be within $\pm 15 \%$ of the nominal concentration and the precision (\% CV) should not exceed $15 \%$.

Dilution Integrity - Surrogate Matrix; Lines 381-382: "In the cases of rare matrices use of a surrogate matrix for dilution may be acceptable, as long as it has been demonstrated that this does not affect precision and accuracy."

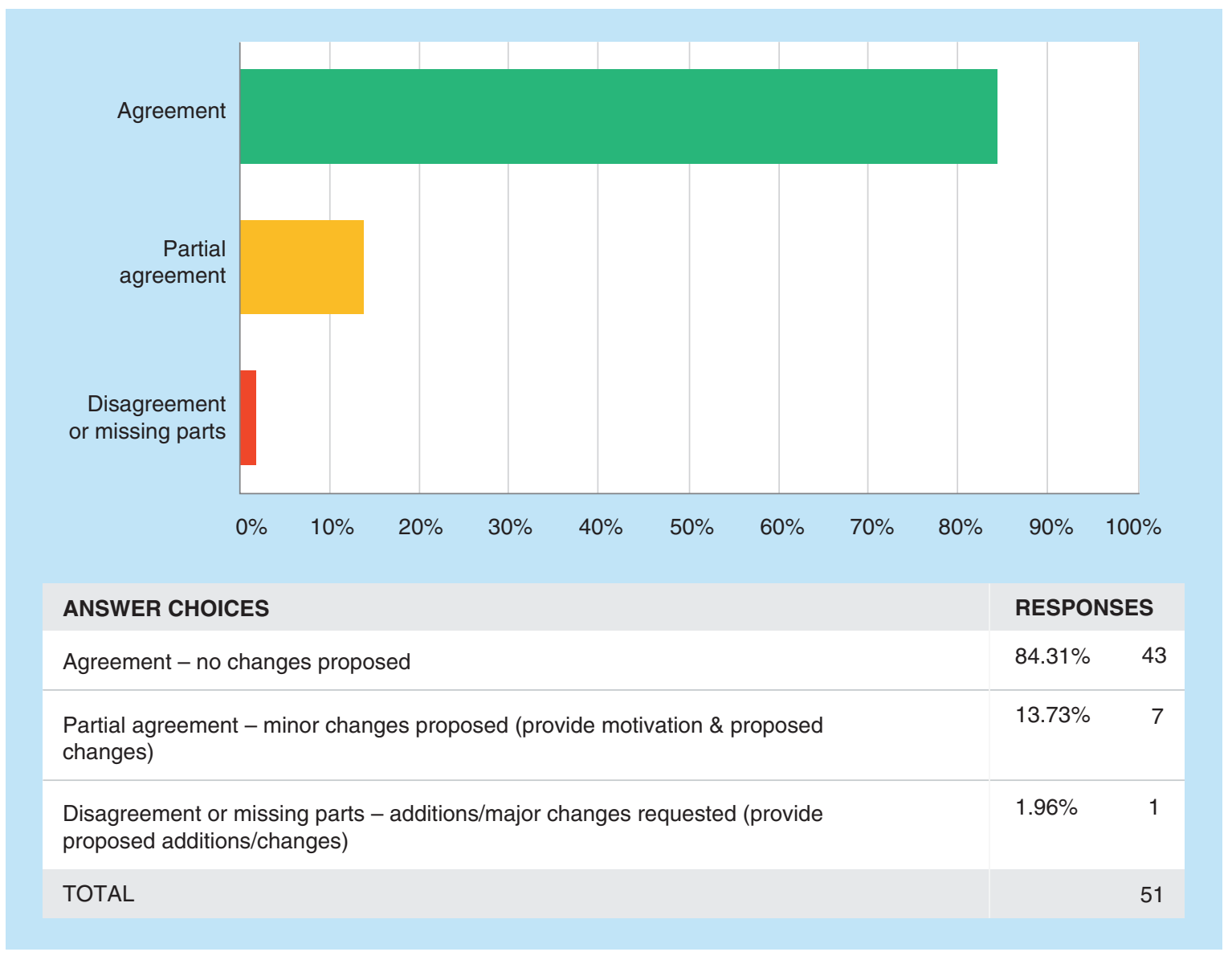

\section{Partial agreement}

- Several comments suggested adding that surrogate matrix for dilution may be used if sample matrix contains an endogenous analyte.

- Other comments suggested that only the highest dilution factor would be required for this evaluation.

\section{Additional comments from the 13th GCC:}

It was further recommended that language should be added to state that it is the dilution factor being monitored, not the maximum concentration capable of being diluted. 
T2Q30: Stability - General; Lines 384-389: "Stability evaluations should be carried out to ensure that every step taken during sample preparation, processing and analysis as well as the storage conditions used do not affect the concentration of the analyte. The storage and analytical conditions applied to the stability tests, such as the sample storage times and temperatures, sample matrix, anticoagulant and container materials, should reflect those used for the study samples."

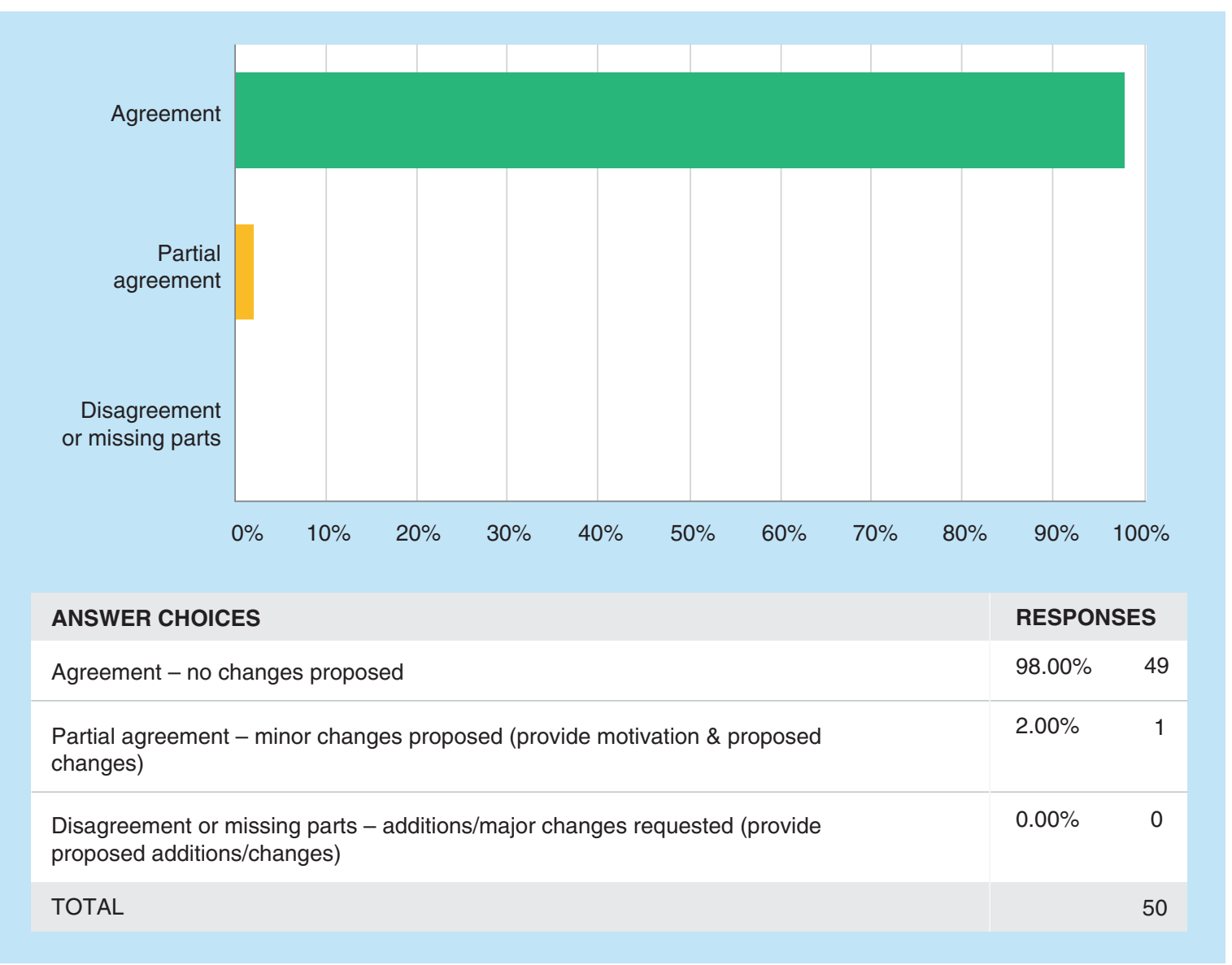


T2Q31: Stability - Use of Literature; Lines 389-390: "Reference to data published in the literature is not considered sufficient."

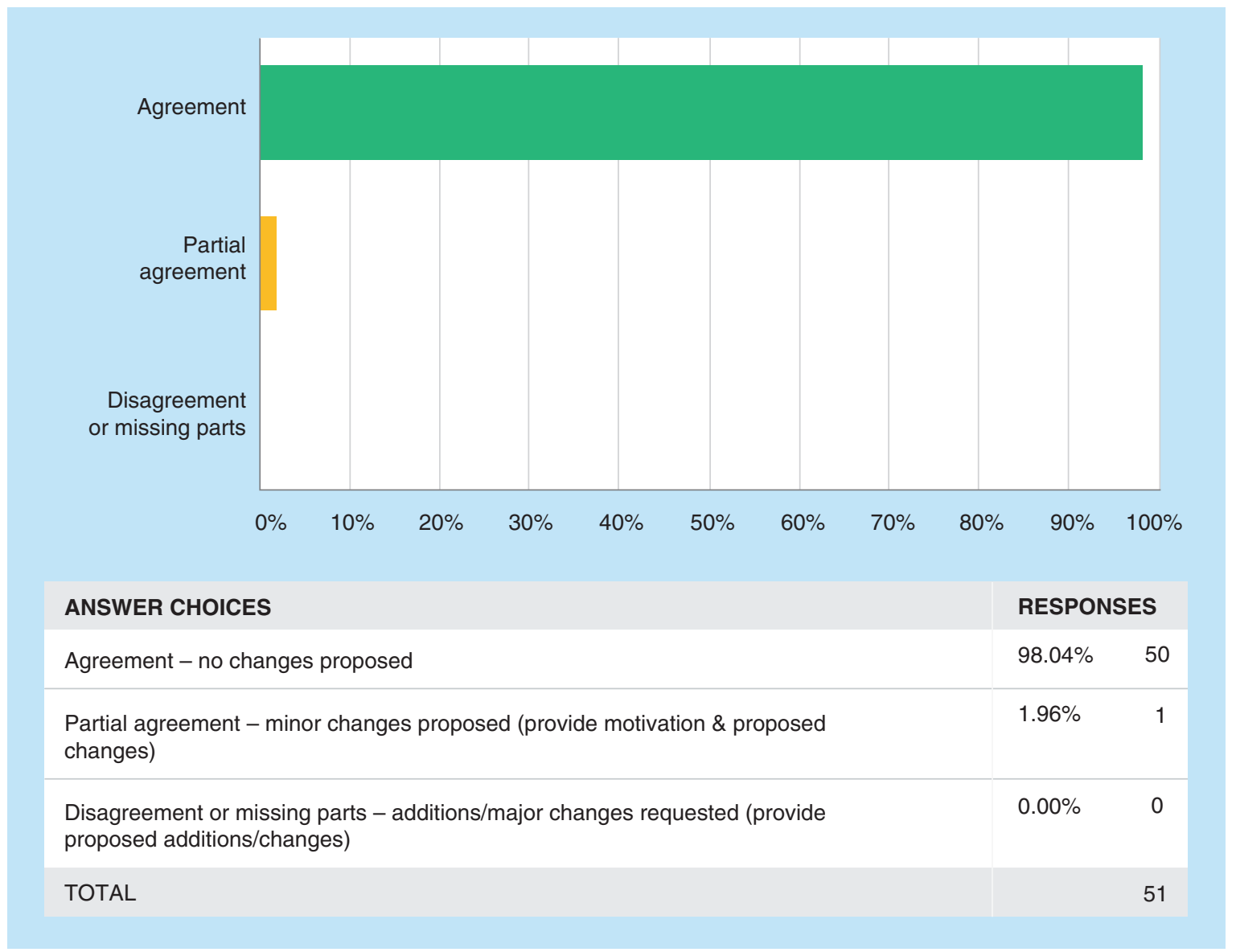


T2Q32: Stability - Procedure; Lines 390-395: "Validation of storage periods should be performed on stability QCs that have been stored for a time that is equal to or longer than the study sample storage periods. Stability of the analyte in the studied matrix is evaluated using low and high concentration stability QCs. Aliquots of the low and high stability QCs are analyzed at time zero and after the applied storage conditions that are to be evaluated. A minimum of three stability QCs should be prepared and analyzed per concentration level/storage condition/timepoint."

Stability - Fresh Curve; Lines 396-398: "The stability QCs are analyzed against a calibration curve, obtained from freshly spiked calibration standards in a run with its corresponding freshly prepared QCs or QCs for which stability has been proven."

Stability - Acceptance Criteria; Lines 398-399: "The mean concentration at each QC level should be within $\pm 15 \%$ of the nominal concentration."

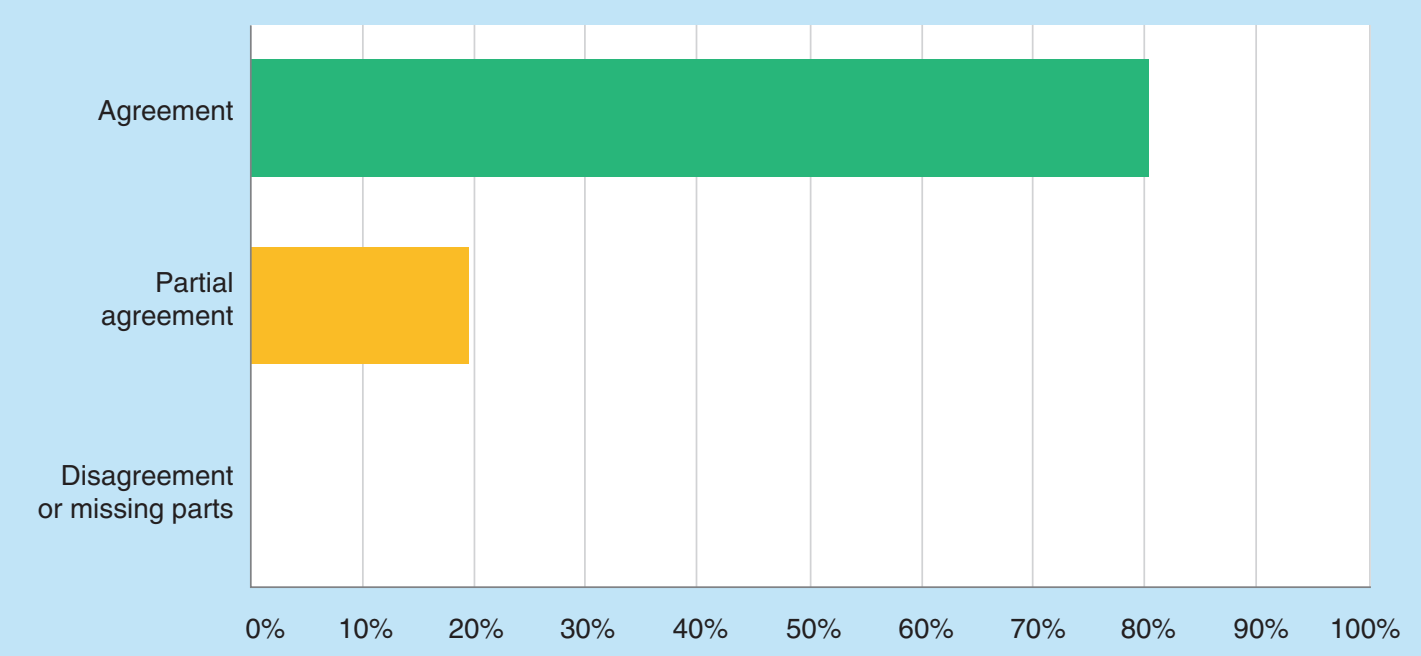

ANSWER CHOICES

RESPONSES

Agreement - no changes proposed

$80.39 \% \quad 41$

Partial agreement - minor changes proposed (provide motivation \& proposed

$19.61 \% \quad 10$ changes)

Disagreement or missing parts - additions/major changes requested (provide

$0.00 \%$ proposed additions/changes)

TOTAL

\section{Partial agreement}

Several comments suggested:

- to remove the requirement for assessment of QCs at time zero.

- to add criteria for precision (\%CV $\leq 15 \%)$.

- to clarify the text "three stability QCs" to regulatory expectations of tubes vs aliquots.

\section{Additional comments from the 13th GCC:}

The scientific rationale of potentially requiring three QC tubes for stability was questioned again, considering published literature demonstrates that no differences in stability have been seen when using three tubes vs three aliquots from one tube [8]. It was recommended that when clarifying the language, the requirement for three tubes be reconsidered by the participating Regulatory Agencies. 
T2Q33: Stability - Above ULQ Stability; Lines 399-402: "If the concentrations of the study samples are consistently higher than the ULOQ of the calibration range, the concentration of the high stability QC should be adjusted to reflect these higher concentrations. It is recognised that this may not be possible in nonclinical studies due to solubility limitations."

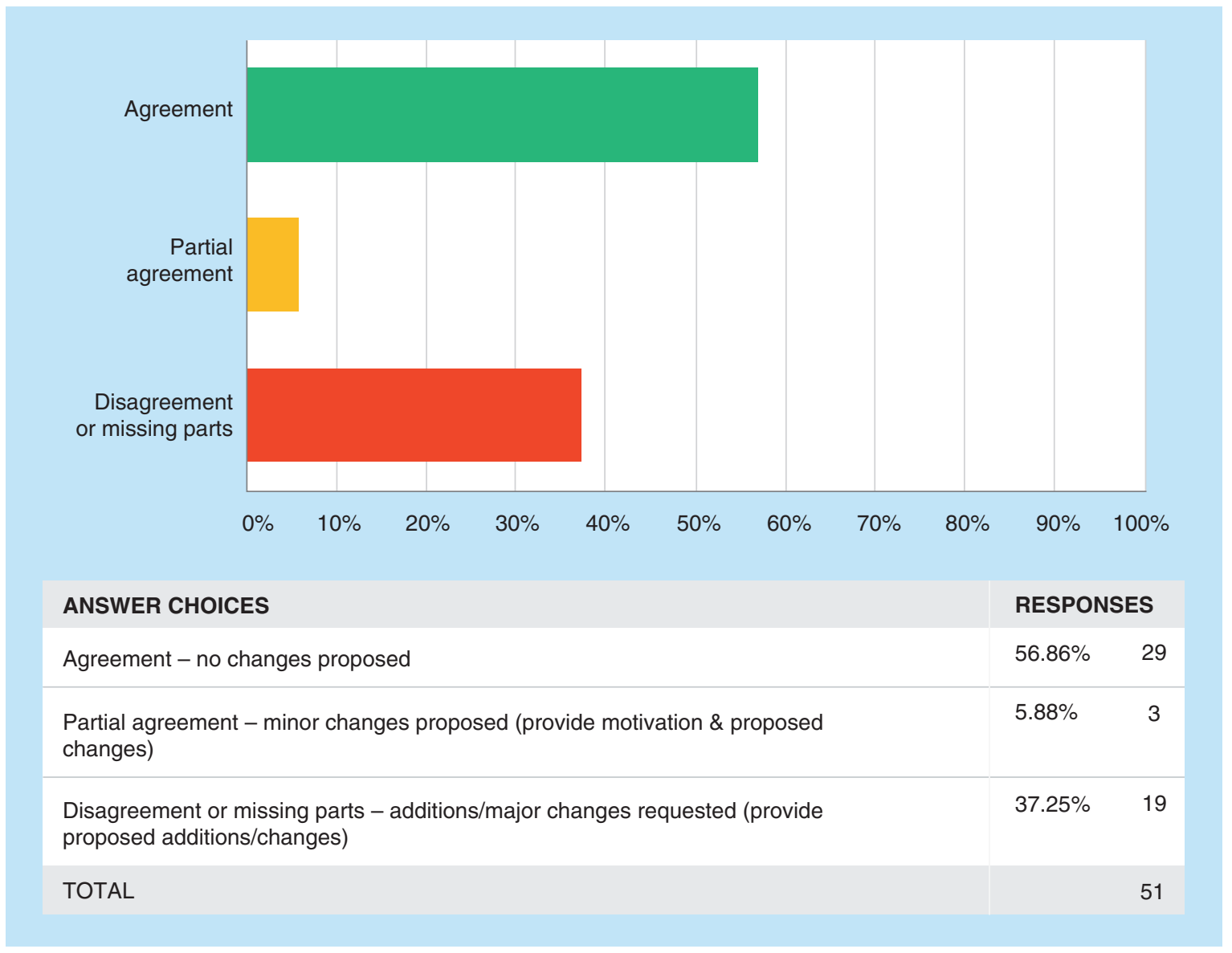

\section{Disagreement or missing parts}

- Almost all the comments strongly disagreed with the sentence: "It is recognised that this may not be possible in nonclinical studies due to solubility limitations" and recommend that it be removed in the final version. The disagreement is mainly based on CROs experience and expertise to overcome solubility issues with different approaches and to then successfully perform this test.

- It is worthy to report that a few comments made the point that if samples are consistently higher than the ULOQ, the range (and so the high QC) would be adjusted, making this evaluation obsolete. It was recommended to remove the requirement to test stability of QC samples above the ULOQ.

\section{Partial agreement}

No additional trend was found in these comments.

\section{Additional comments from the 13th GCC:}

There was agreement during the GCC Closed Forum to fully support all of the above comments. 
T2Q34: Stability - Multi-Analyte; Lines 403-405: "If multiple analytes are present in the study samples (e.g., studies with a fixed combination, or due to a specific drug regimen) the stability test of an analyte in matrix should be conducted with the matrix containing all of the analytes."

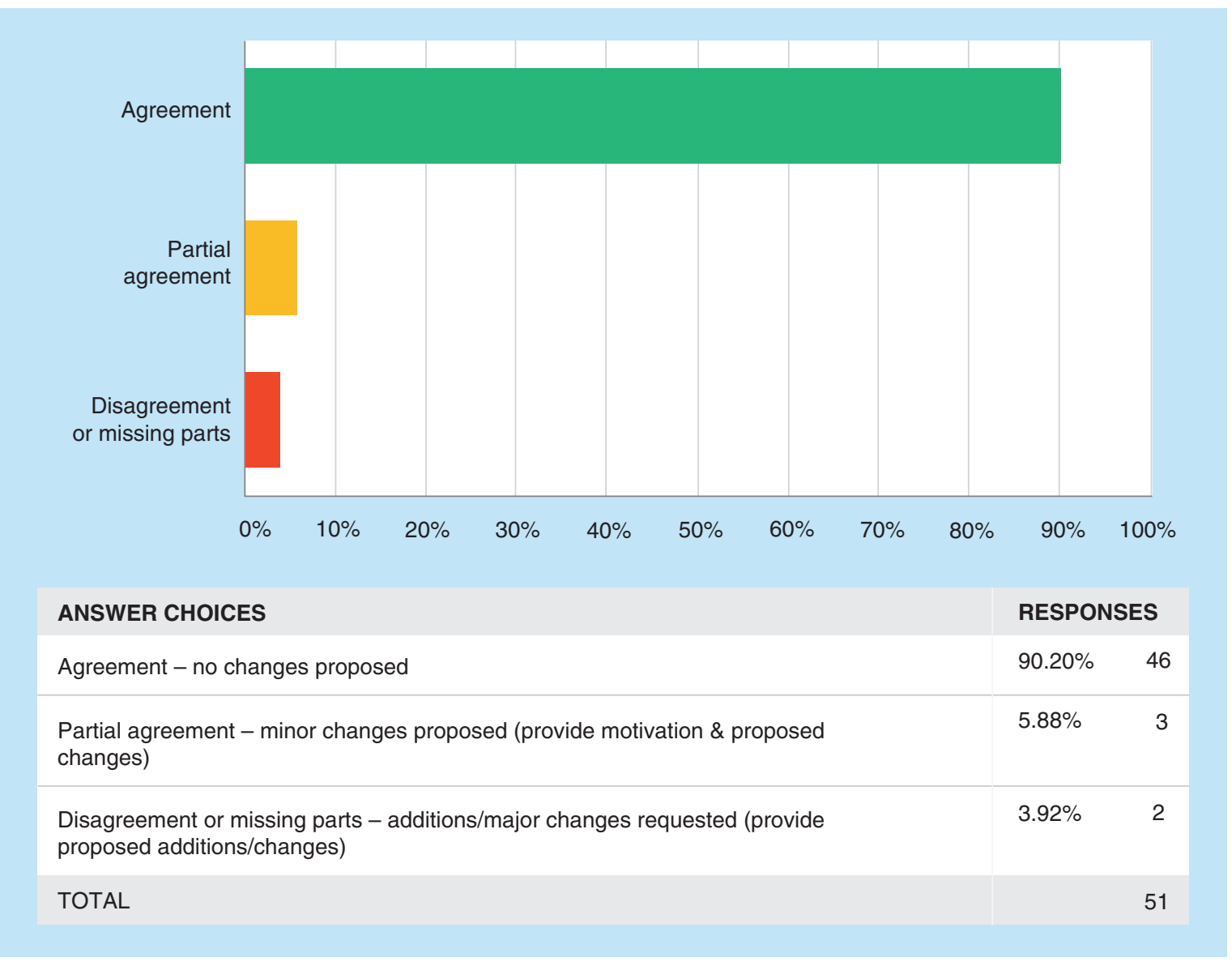

Disagreement or missing parts

Two comments asked to remove the recommendation to test co-administered compound stability, since there are no data in the literature to demonstrate that this is an issue.

Partial agreement

The three comments of this section made the same point as above.

Additional comments from the 13th GCC:

GCC asked regulators to consider the data published in a White Paper [13] while reviewing this paragraph. 
T2Q35: Stability - Stabilities Required; Lines 406-407, 422, 431, 440, 447, 457-458: "The following stability tests should be evaluated:

1) Stability of stock and working solutions

2) Freeze-thaw matrix stability

3) Bench-top (short-term) matrix stability

4) Processed sample stability

5) Long-term matrix stability

In addition, the following test should be performed if applicable:

6) Whole blood stability"

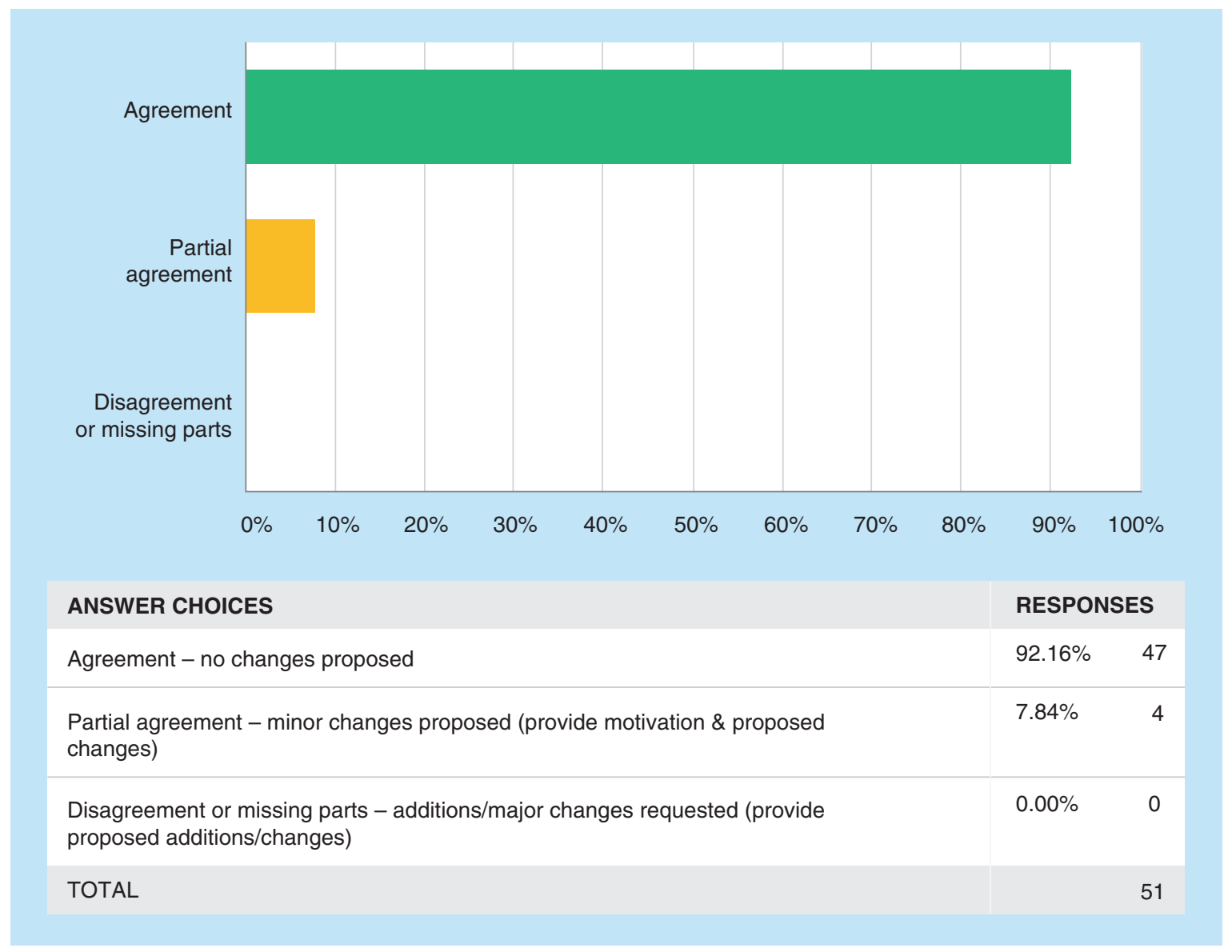

\section{Partiall agreement}

No trend was found in these comments.

\section{Additional comments from the 13th GCC:}

It was recommended to replace the assessment of processed sample stability with the reinjection reproducibility evaluation since the latter is a better representation of what might occur during sample analysis. 
T2Q36: Stock and Working Solution Stability - Procedure; Lines 408-414: "The stability of the stock and working solutions of the analyte and IS should be determined under the storage conditions used during the analysis of study samples by using the lowest and the highest concentrations of these solutions. They are assessed using the response of the detector. Stability of the stock and working solutions should be tested with an appropriate dilution, taking into consideration the linearity and measuring range of the detector. If the stability varies with concentration, then the stability of all concentrations of the stock and working solutions needs to be assessed."

Stock and Working Solution Stability - SIL IS; Lines 414-416: "If no isotopic exchange occurs for the stable isotope-labeled IS under the same storage conditions as the analyte for which the stability is demonstrated, then no additional stability determinations for the IS are necessary."

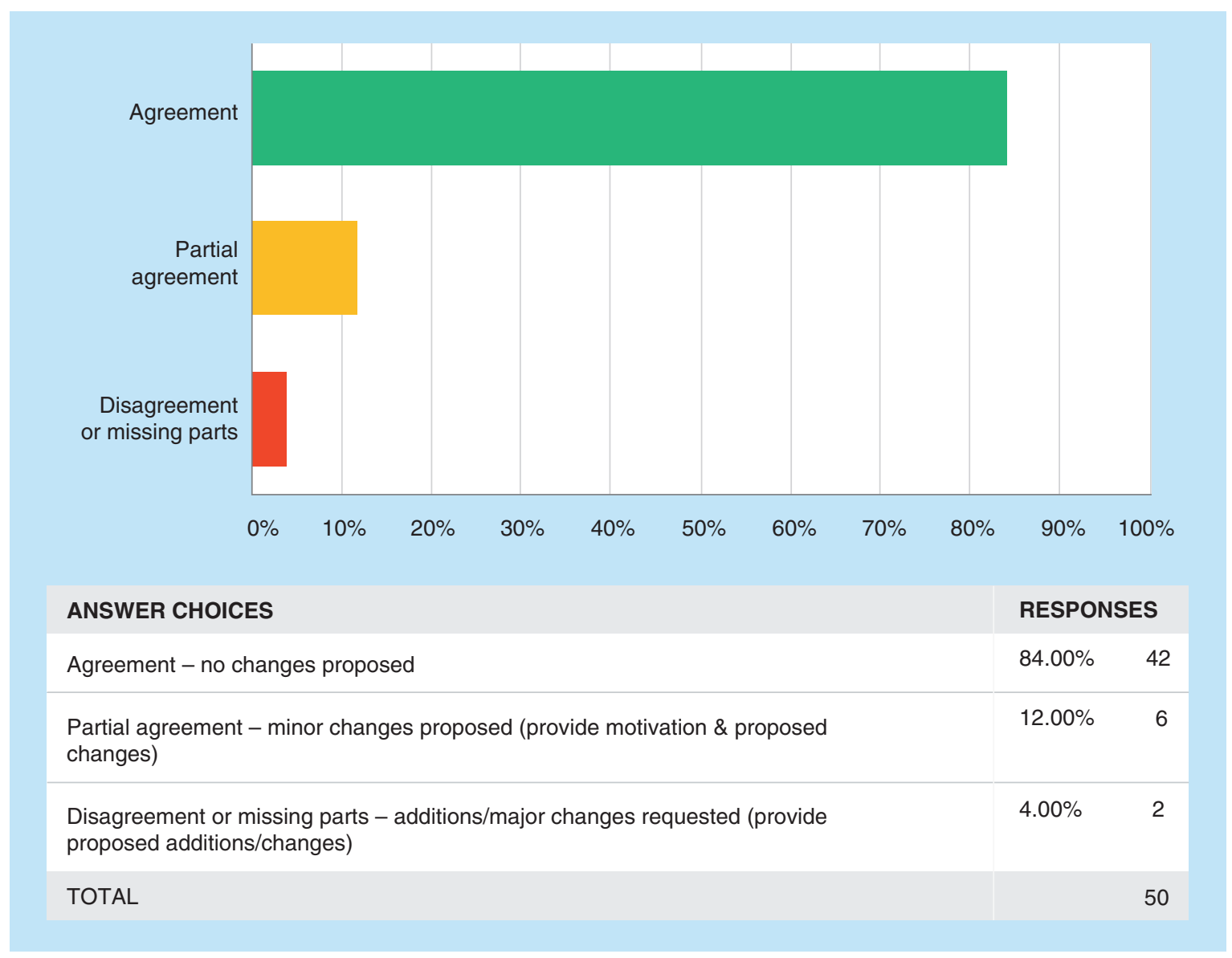

Disagreement or missing parts

It is recommended to add criteria for stock stability since the $15 \%$ criteria mentioned in earlier lines pertaining to stability does not apply.

Partial agreement

It is suggested to provide some clarification of testing all solution concentrations if stability varies with concentration. 
T2Q37: Stock and Working Solution Stability - Expired Reference Standard; Lines 417-421: "If the reference standard expires, or it is past the retest date, the stability of the stock solutions made previously with this lot of reference standard are defined by the expiration or retest date established for the stock solution. The routine practice of making stock and working solutions from reference standards solely for extending the expiry date for the use of the reference standard is not acceptable."

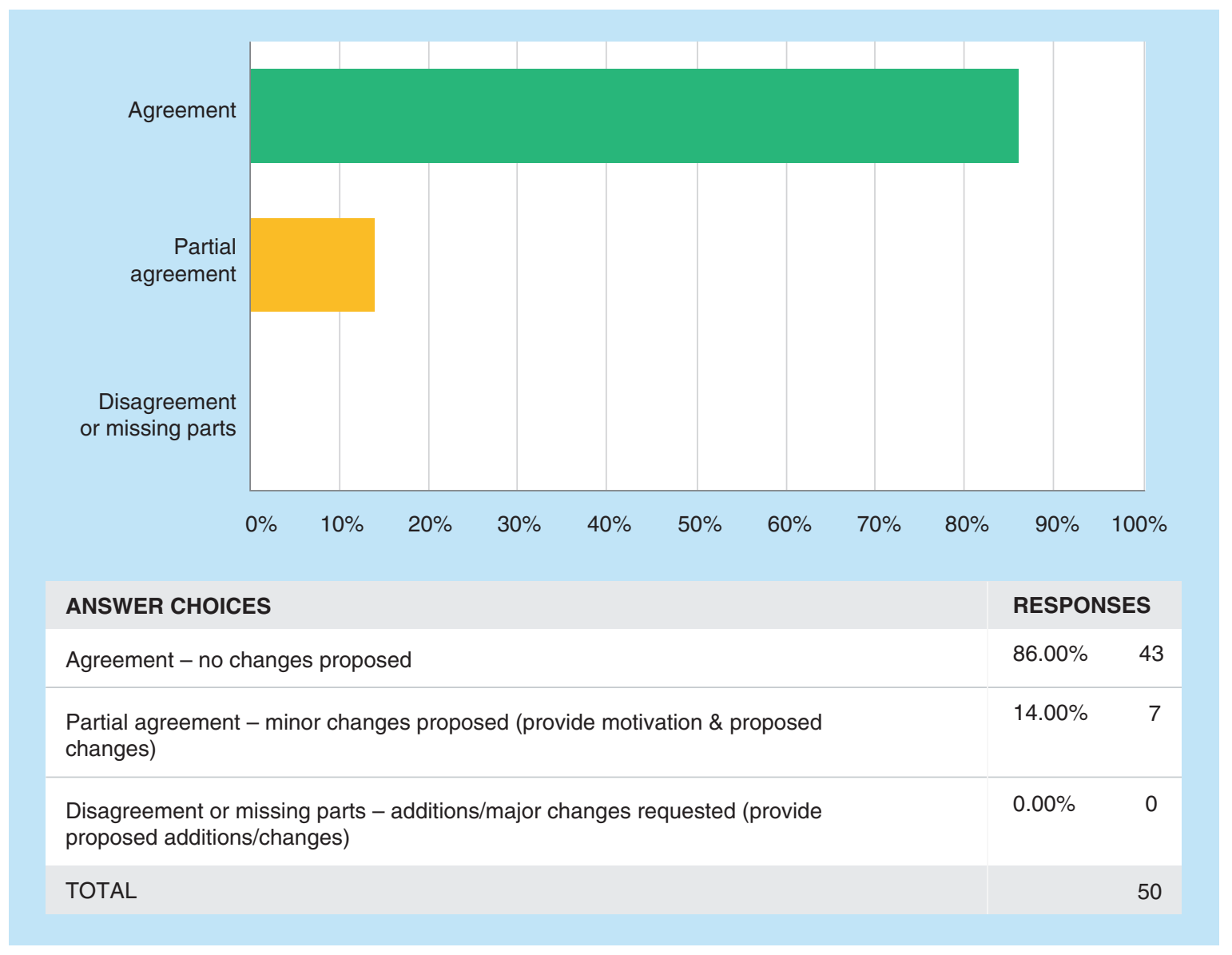

\section{Partial agreement}

No trend was found in these comments. 
T2Q38: Freeze-Thaw Stability - Purpose; Lines 423-424: "To assess the impact of repeatedly removing samples from frozen storage, the stability of the analyte should be assessed after multiple cycles of freezing and thawing."

Freeze-Thaw Stability - Procedure; Lines 424-428: "Low and high stability QCs should be thawed and analyzed according to the same procedures as the study samples. Stability QCs should be kept frozen for at least 12 hours between the thawing cycles. Stability QCs for freeze-thaw stability should be assessed using freshly prepared calibration standards and QCs or QCs for which stability has been proven."

Freeze-Thaw Stability - Cycles; Lines 428-430: "Low and high stability QCs should be thawed and analyzed according to the same procedures as the study samples. Stability QCs should be kept frozen for at least 12 hours between the thawing cycles. Stability QCs for freeze-thaw stability should be assessed using freshly prepared calibration standards and QCs or QCs for which stability has been proven. The number of freeze-thaw cycles validated should equal or exceed that of the freeze-thaw cycles undergone by the study samples, but a minimum of three cycles should be conducted."

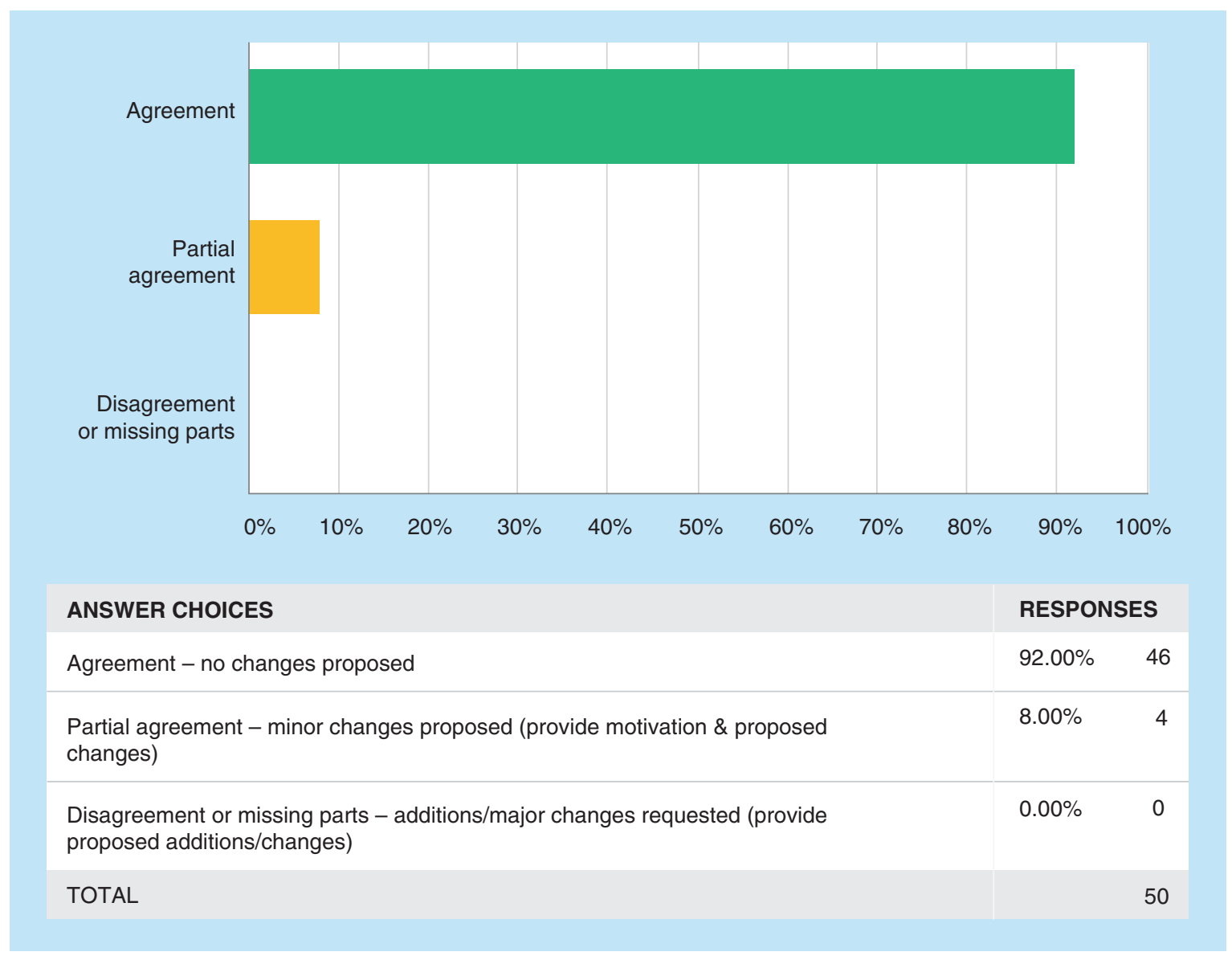

\section{Partial agreement}

A few comments suggested that the requirement to perform three cycles be removed. It is only important to cover the freeze-thaw cycles used for the analysis of study samples.

\section{Additional comments from the 13th GCC:}

There were no further discussions during the GCC Closed Forum in support of the above proposal. 
T2Q39: Bench-Top (Short-Term) Stability - Purpose; Lines 432-433: "Bench-top matrix stability experiments should be designed and conducted to cover the laboratory handling conditions for the study samples."

Bench-Top (Short-Term) Stability - Procedure; Lines 434-436: "Low and high stability QCs should be thawed in the same manner as the study samples and kept on the bench-top at the same temperature and for at least the same duration as the study samples."

Bench-Top (Short-Term) Stability - Duration; Lines 437-439: "The total time on the bench-top should be concurrent; it is not acceptable to use additive exposure to bench-top conditions (i.e., adding up time from each freezethaw evaluation is not acceptable)."

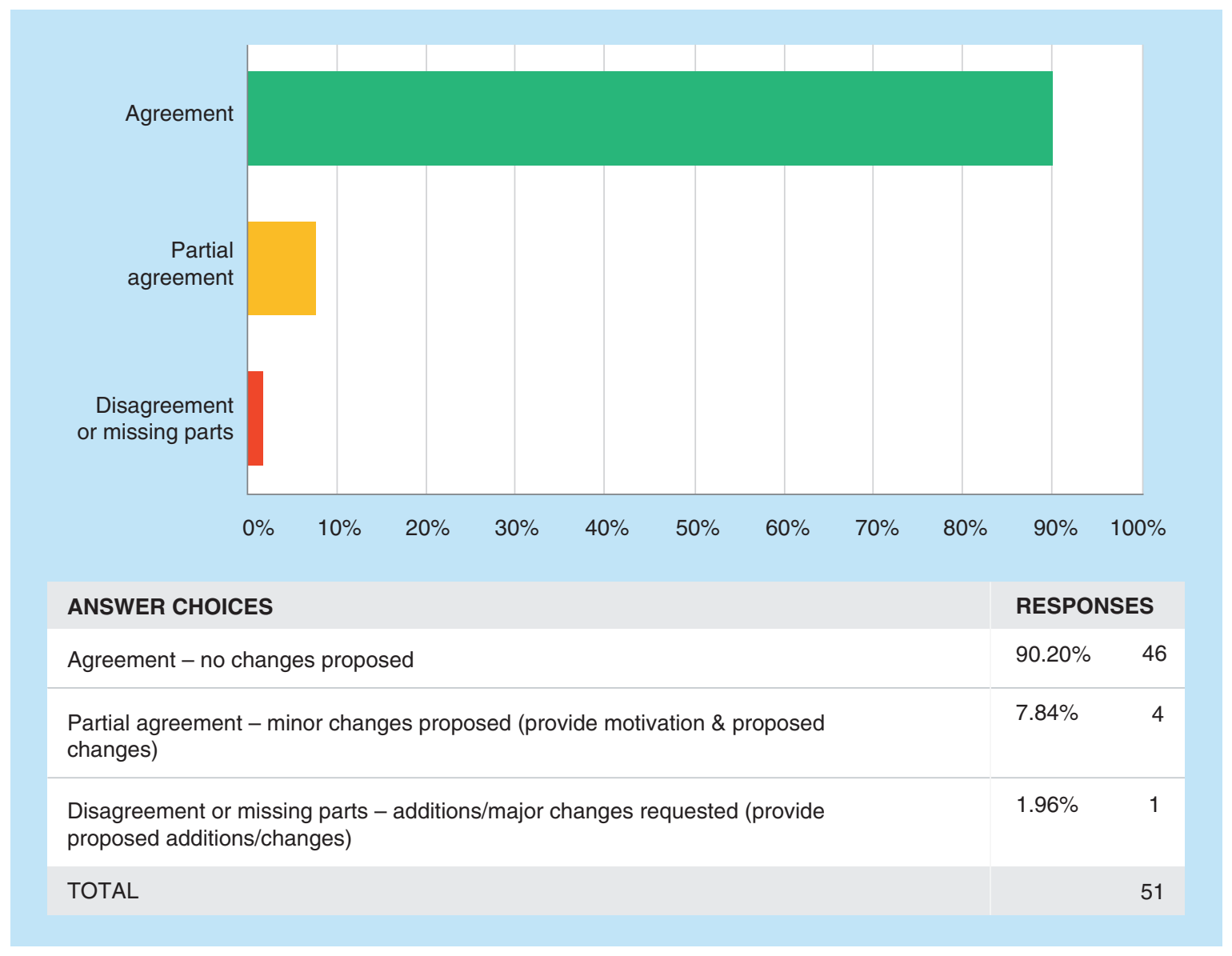

\section{Partial agreement}

All five comments disagree with or ask for clarification regarding the statement that additive exposure is not acceptable, since this best represents how study samples are handled. The scientific rationale is unclear for not permitting additive exposure and leads the reader to assume that when determining the short-term exposure during sample analysis, adding each duration that the sample is thawed should not be used.

\section{Additional comments from the 13th GCC:}

It was recommended that the sentence: "The total time on the bench-top should be concurrent; it is not acceptable to use additive exposure to bench-top conditions (i.e., adding up time from each freeze-thaw evaluation is not acceptable)" be removed. 
T2Q40: Processed Sample Stability; Lines 441-446: "The stability of processed samples, including the time until completion of analysis (in the autosampler/instrument), should be determined. For example:

- Stability of the processed sample at the storage conditions to be used during the analysis of study samples (dry extract or in the injection phase)

- On-instrument/ autosampler stability of the processed sample at injector or autosampler temperature."

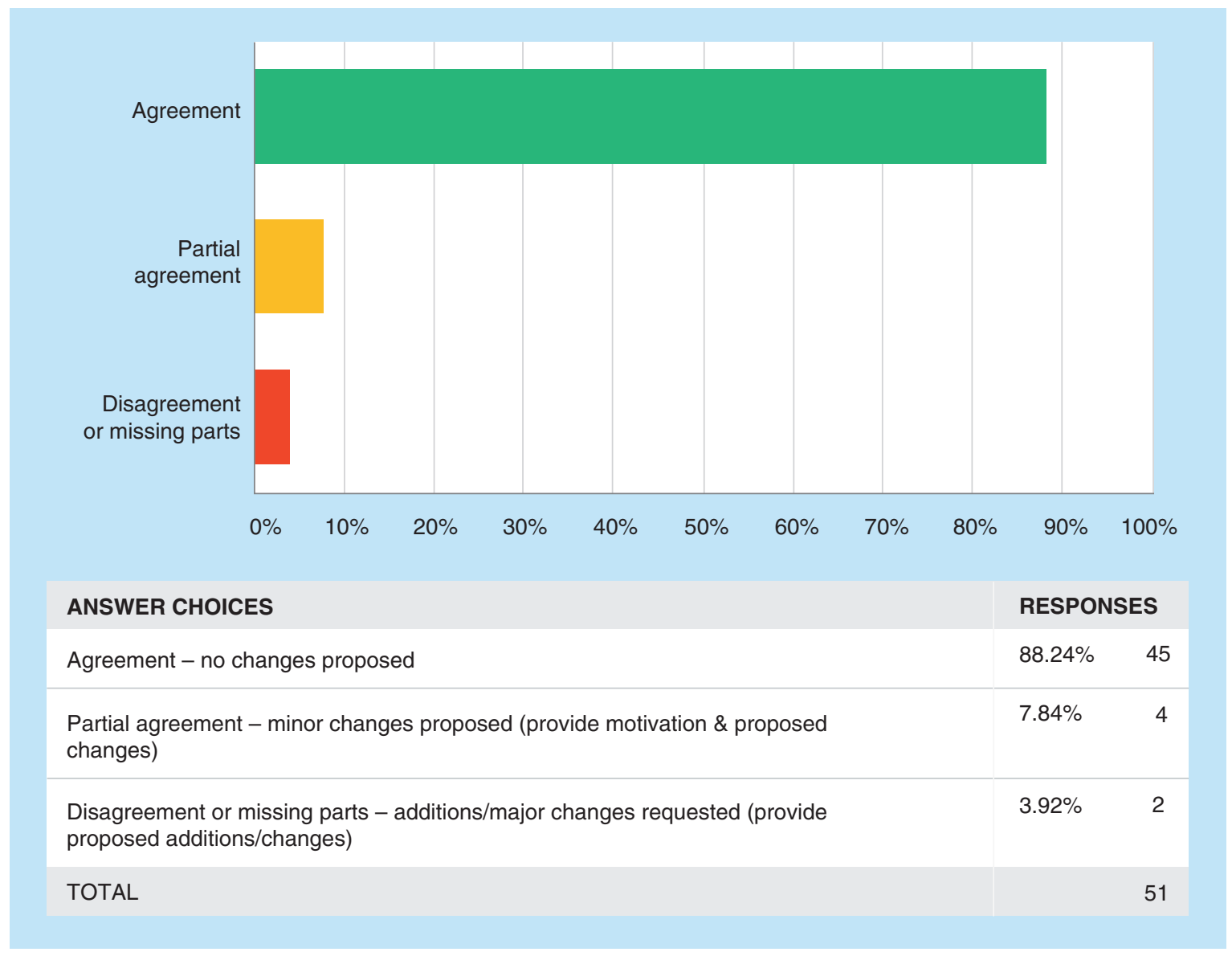

Disagreement or missing parts

No trend was found in these comments.

Partial agreement

No trend was found in these comments. 
T2Q41: Long-Term Stability - Purpose; Line 448: "The long-term stability of the analyte in matrix stored in the freezer should be established."

Long-Term Stability - Procedure; Lines 449-450: "Low and high stability QCs should be stored in the freezer under the same storage conditions and at least for the same duration as the study samples."

Long-Term Stability - Chemical Drugs; Lines 451-452: "For chemical drugs, it is considered acceptable to extrapolate the stability at one temperature (e.g., $\left.-20^{\circ} \mathrm{C}\right)$ to lower temperatures $\left(\right.$ e.g., $\left.-70^{\circ} \mathrm{C}\right)$."

Long-Term Stability - Biological Drugs; Lines 453-456: "For biological drugs, it is acceptable to apply a bracketing approach, e.g., in the case that the stability has been demonstrated at $-70^{\circ} \mathrm{C}$ and at $-20^{\circ} \mathrm{C}$, then it is not necessary to investigate the stability at temperatures in between those two points at which study samples will be stored."

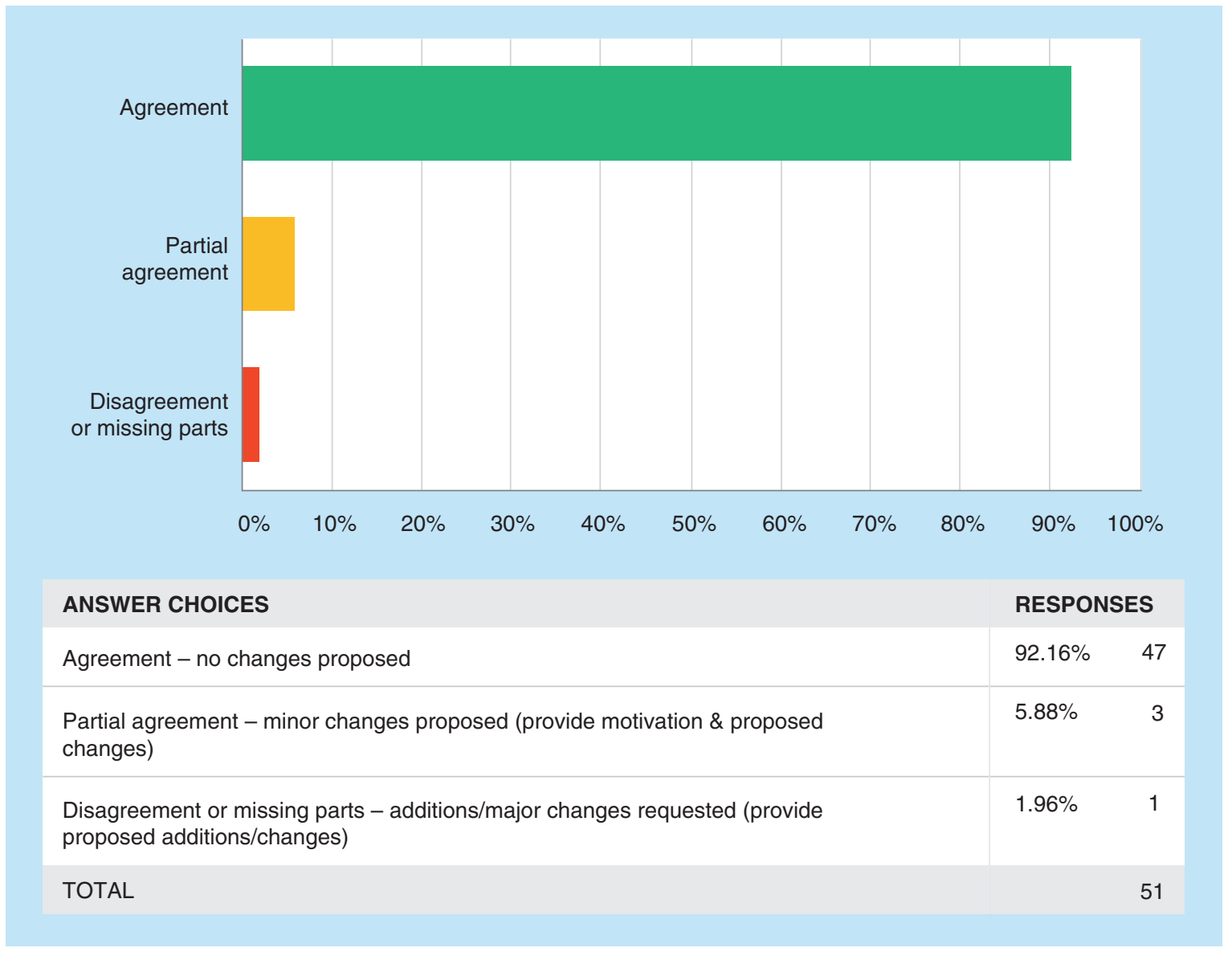

\section{Partial agreement}

No trend was found in these comments. 
Supplement Nehls, Buonarati, Cape et al.

T2Q42: Whole Blood Stability - Purpose; Lines 459-462: "Sufficient attention should be paid to the stability of the analyte in the sampled matrix (blood) directly after collection from subjects and prior to preparation for storage to ensure that the concentrations obtained by the analytical method reflect the concentrations of the analyte in the subject's blood at the time of sample collection."

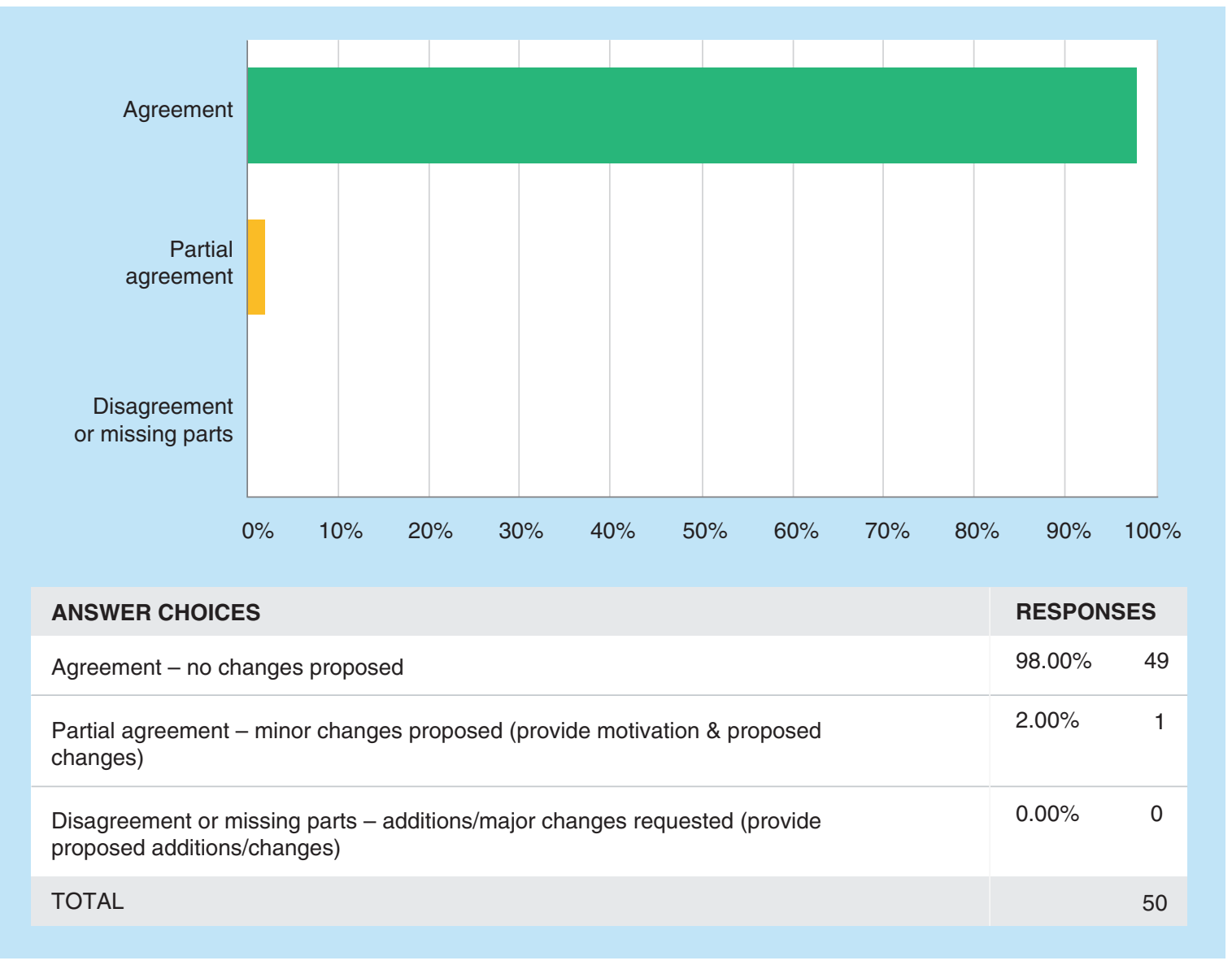


T2Q43: Whole Blood Stability - MD vs Validation; Lines 463-465: "If the matrix used is plasma or serum, the stability of the analyte in blood should be evaluated during method development (e.g., using an exploratory method in blood) or during method validation. The results should be provided in the Validation Report."

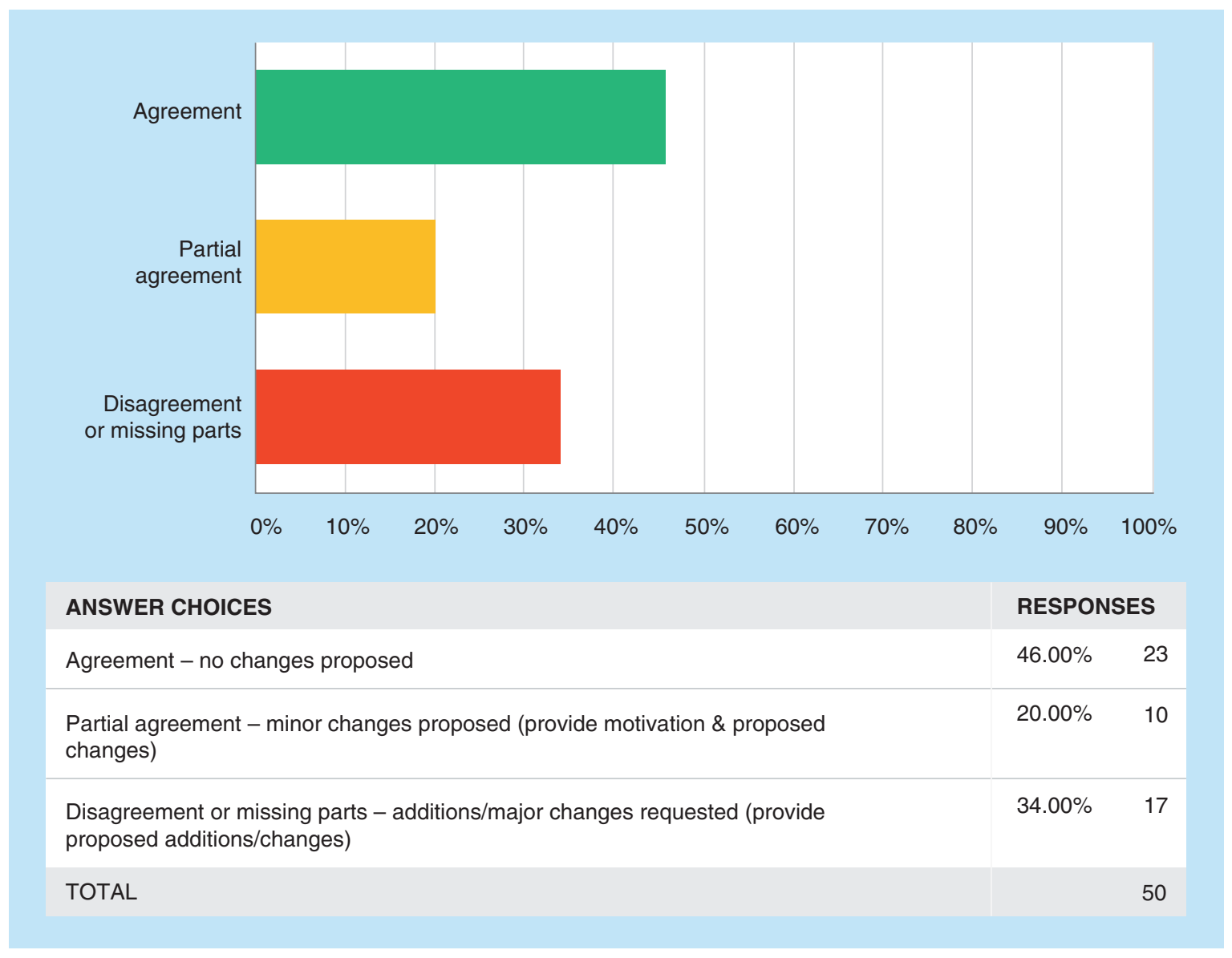

\section{Disagreement or missing parts}

- The majority of the comments strongly disagree with the inclusion of using an exploratory method in blood for whole blood stability. The following text should be removed: "(e.g., using an exploratory method in blood)." The main concerns were the following:

- An exploratory method in blood does not reflect what is happening in plasma;

- An exploratory method is not acceptable in validation and/or in the validation report;

- If an exploratory method in blood should be developed before or after the plasma method;

- If the blood method should be validated like the plasma method;

- Nobody uses an exploratory blood method for plasma samples;

- It was suggested at EBF as a possibility in discovery/non-regulated but it should not be in a guidance.

- Moreover, the majority of comments also request clarification for how the whole blood stability in serum can be performed.

Partial agreement

No additional trend was found in these comments. 
T2Q44: Reinjection Reproducibility; Lines 467-471: "Reproducibility of the method is assessed by replicate measurements of the QCs and is usually included in the assessment of precision and accuracy. However, if samples could be reinjected (e.g., in the case of instrument interruptions or other reasons such as equipment failure), reinjection reproducibility should be evaluated and included in the Validation Report or provided in the Bioanalytical Report of the study where it was conducted."

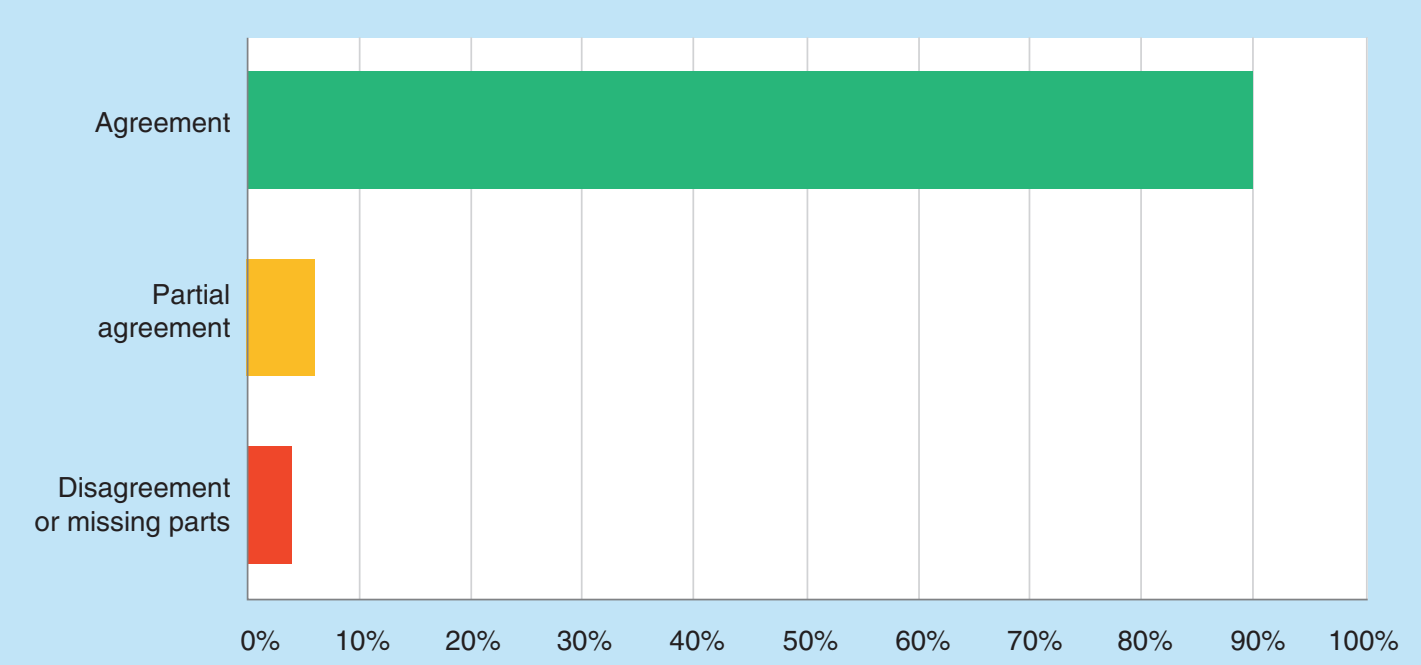

\section{ANSWER CHOICES}

RESPONSES

Agreement - no changes proposed

Partial agreement - minor changes proposed (provide motivation \& proposed changes)

Disagreement or missing parts - additions/major changes requested (provide proposed additions/changes)

TOTAL

Disagreement or missing parts

No trend was found in these comments.

Partial agreement

No trend was found in these comments. 
T2Q45: Recovery - General; Lines 1146-1148: "For methods that employ sample extraction, the recovery (extraction efficiency) should be evaluated. Recovery is reported as a percentage of the known amount of an analyte carried through the sample extraction and processing steps of the method."

Recovery - Procedure; Lines 1148-1154: "Recovery is determined by comparing the analyte response in a biological sample that is spiked with the analyte and processed, with the response in a biological blank sample that is processed and then spiked with the analyte. Recovery of the analyte does not need to be 100\%, but the extent of recovery of an analyte and of the IS (if used) should be consistent. Recovery experiments are recommended to be performed by comparing the analytical results for extracted samples at multiple concentrations, typically three concentrations (low, medium and high)."

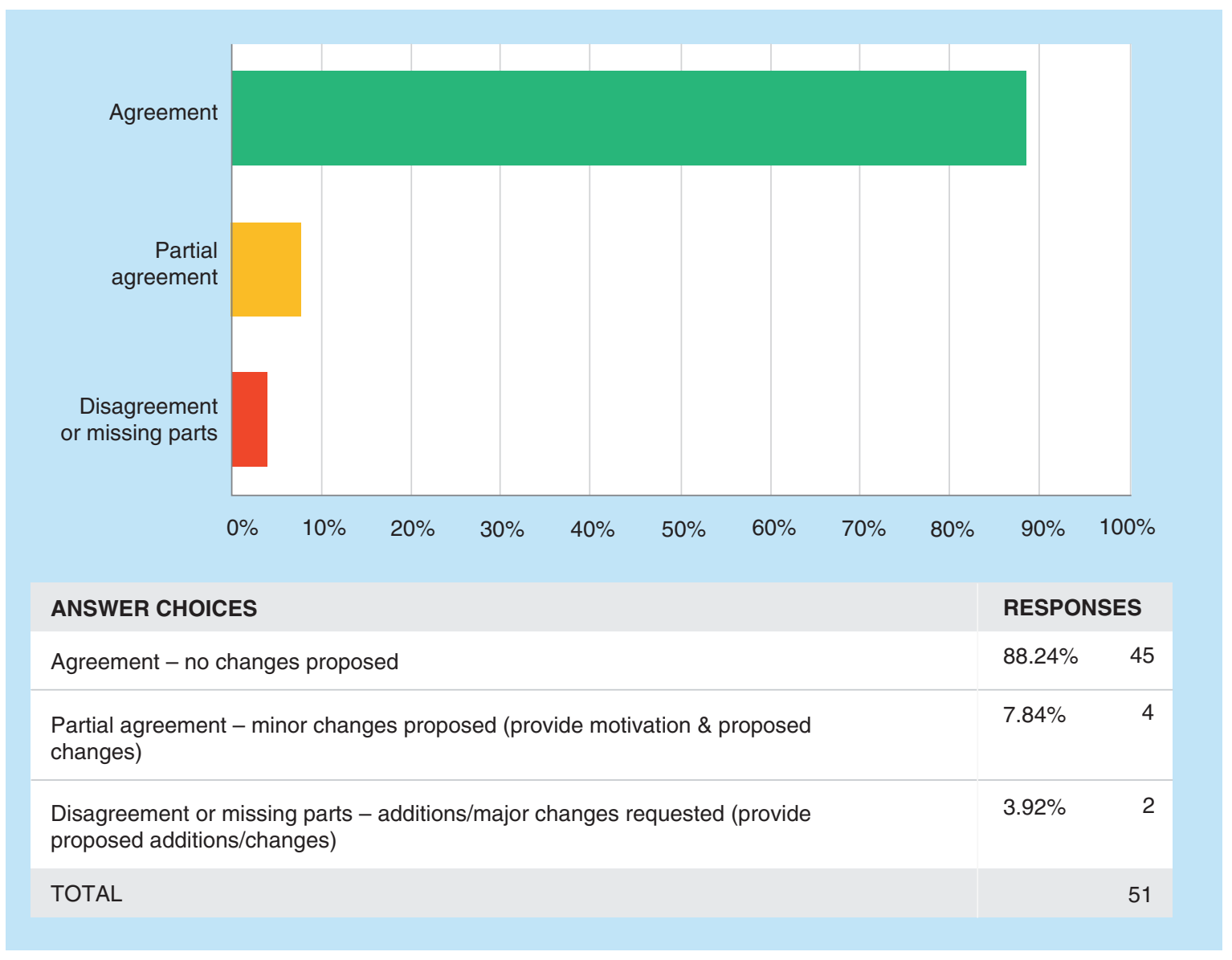

Disagreement or missing parts

No trend was found in these comments.

Partial agreement

No trend was found in these comments.

\section{Additional comments from the 13th GCC:}

It was recommended that the recovery evaluation could be performed in either method development or validation. 
Team 3 Chromatography II - Study Sample Analysis, Acceptance Criteria for an Analytical Run, Calibration Range Reanalysis, Reinjection of Study Samples, Reintegration of Chromatograms

T3Q1: General; Lines 473-477: "The analysis of study samples can be carried out after validation has been completed, however, it is understood that some parameters may be completed at a later stage (e.g., long-term stability). By the time the data are submitted to a regulatory authority, the bioanalytical method validation should have been completed. The study samples, QCs and calibration standards should be processed in accordance with the validated analytical method."

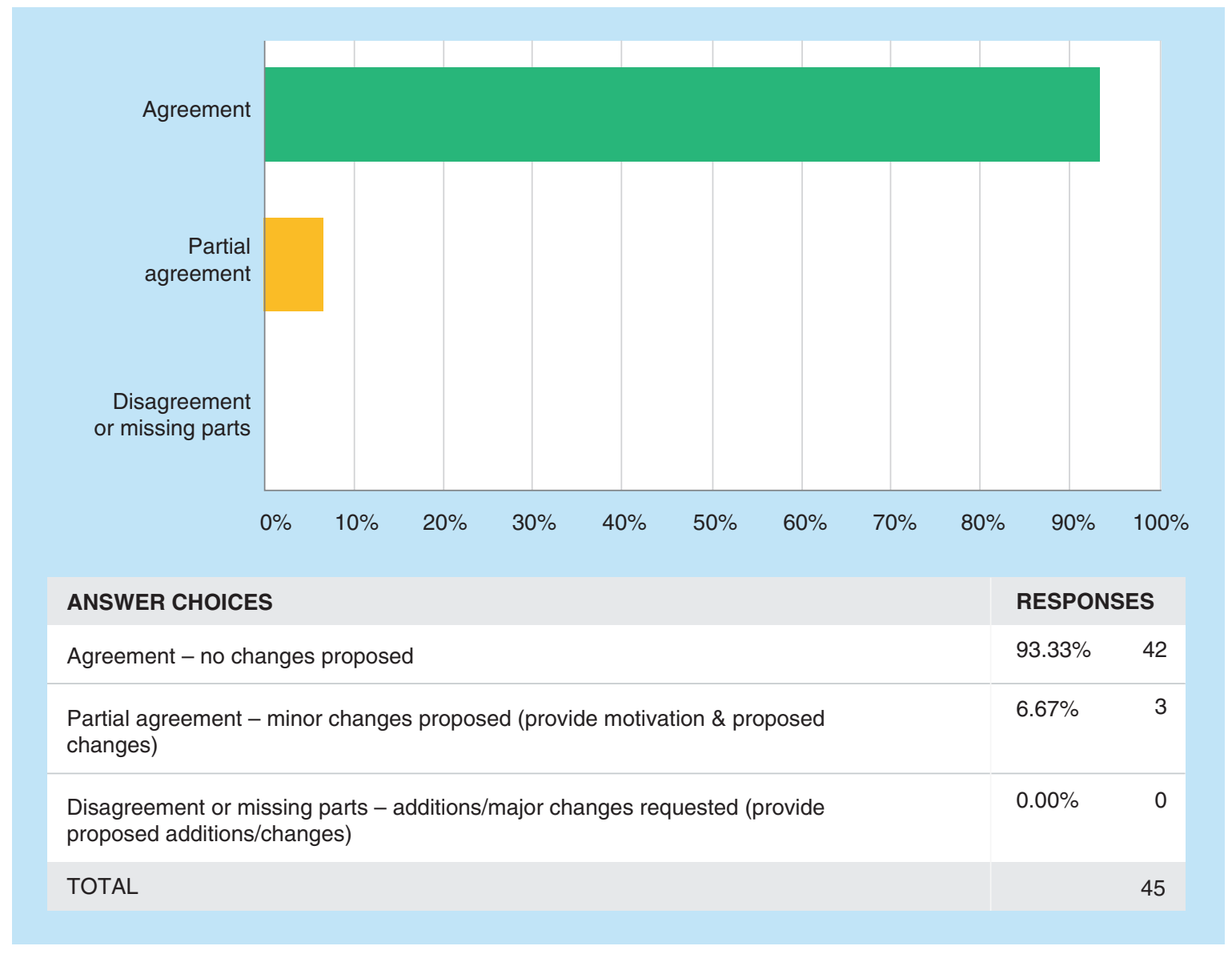

Partial agreement

No trend was found in these comments. 
T3Q2: System Suitability; Lines 477-481: "If system suitability is assessed, a predefined specific study plan, protocol or SOP should be used. System suitability, including apparatus conditioning and instrument performance, should be determined using samples that are independent of the calibration standards and QCs for the run. Subject samples should not be used for system suitability."

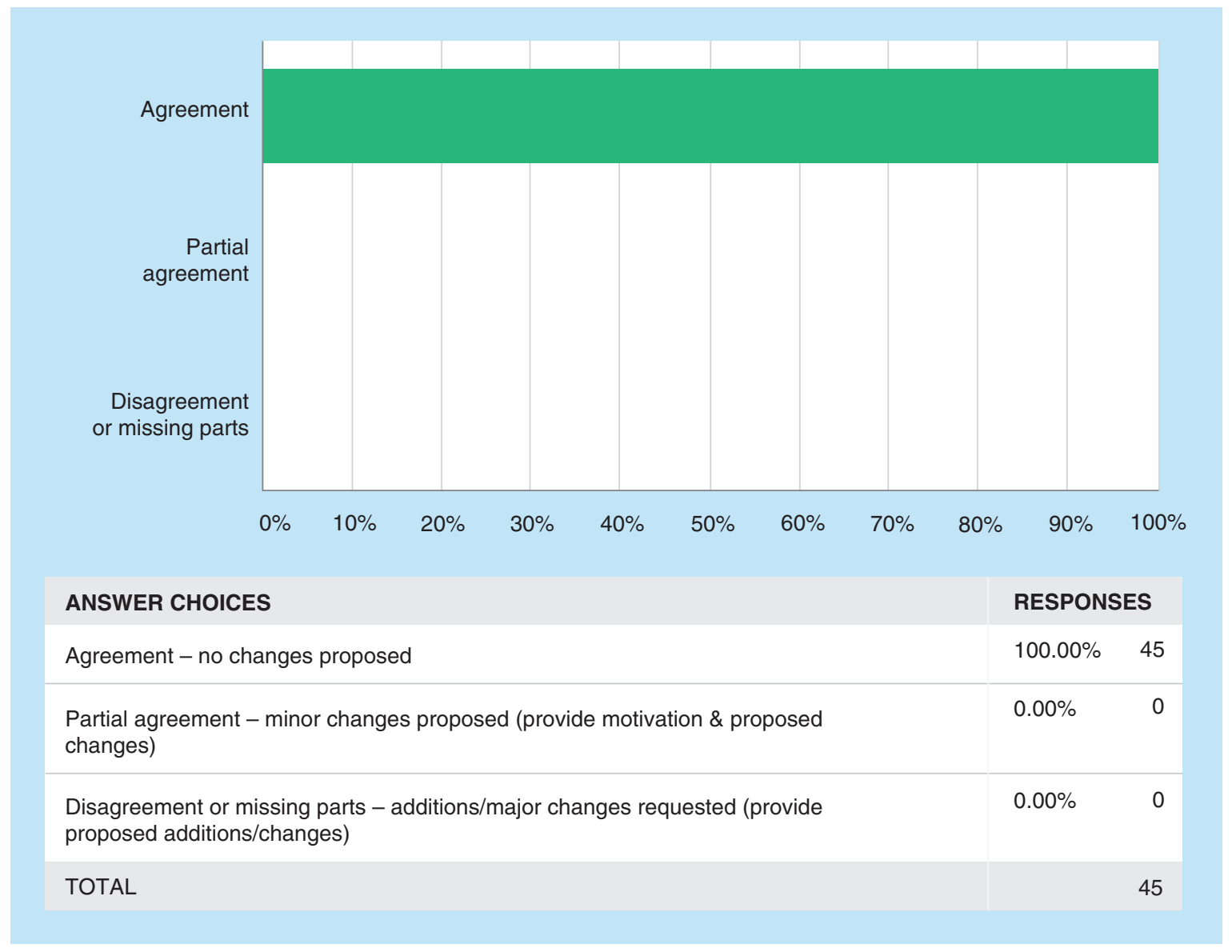


T3Q3: IS Variability; Lines 481-482: "The IS responses of the study samples should be monitored to determine whether there is systemic IS variability."

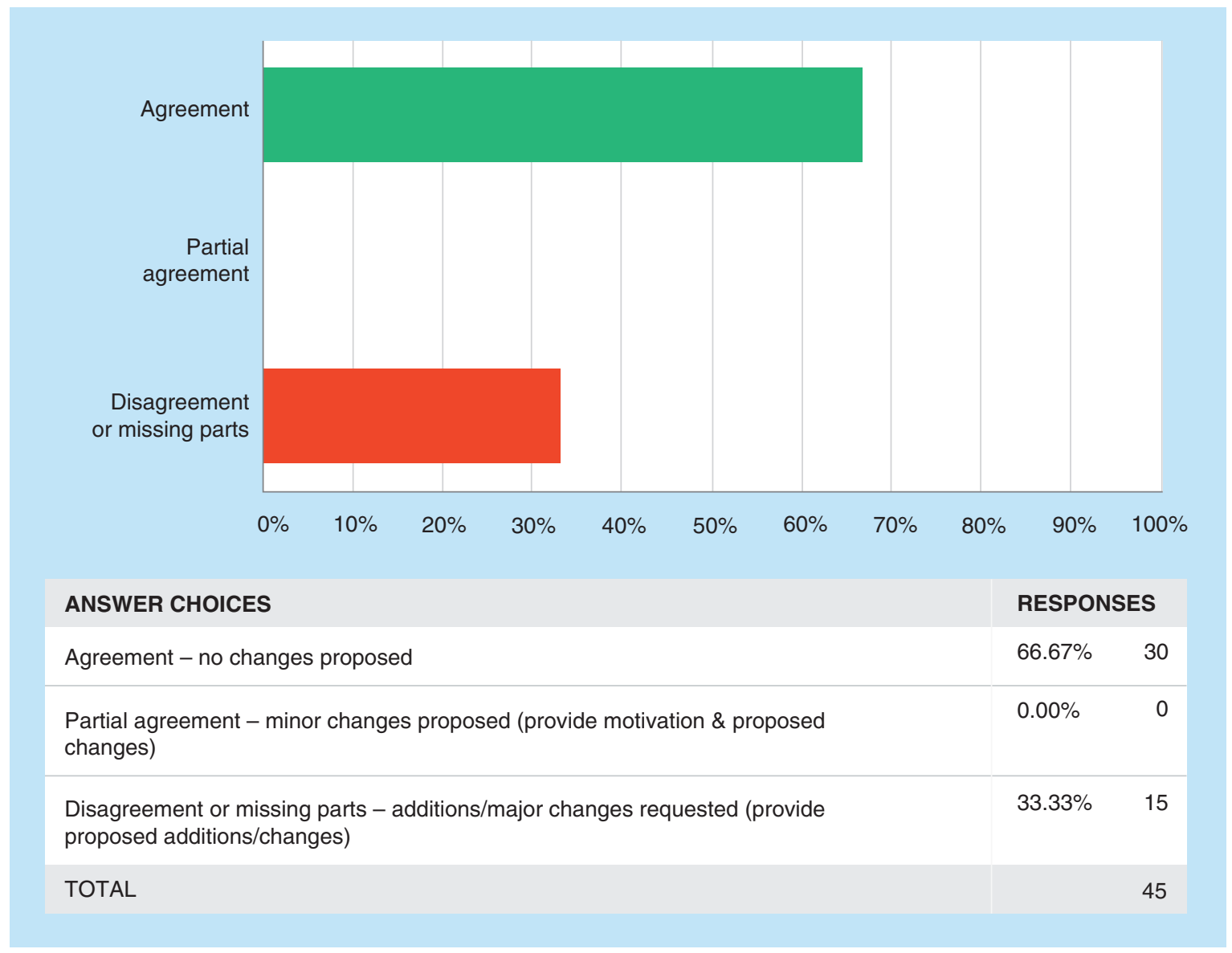

\section{Disagreement or missing parts}

The respondents who commented strongly requested that more clarity be provided to help guide implementation of criteria and regulatory expectations for when investigation is necessary. It was suggested to follow GCC White Paper recommendations [10]. Concerns were around the recent FDA interest in the impact of IS variability on data reliability. 
T3Q4: Analytical Run - Contents; Lines 485-488: "An analytical run consists of a blank sample (processed matrix sample without analyte and without IS), a zero sample (processed matrix with IS), calibration standards at a minimum of six concentration levels, at least three levels of QCs (low, medium and high) in duplicate (or at least $5 \%$ of the number of study samples, whichever is higher) and the study samples to be analyzed."

Analytical Run - QC Samples; Lines 489-490: "The QCs should be divided over the run in such a way that the accuracy and precision of the whole run is ensured. Study samples should always be bracketed by QCs."

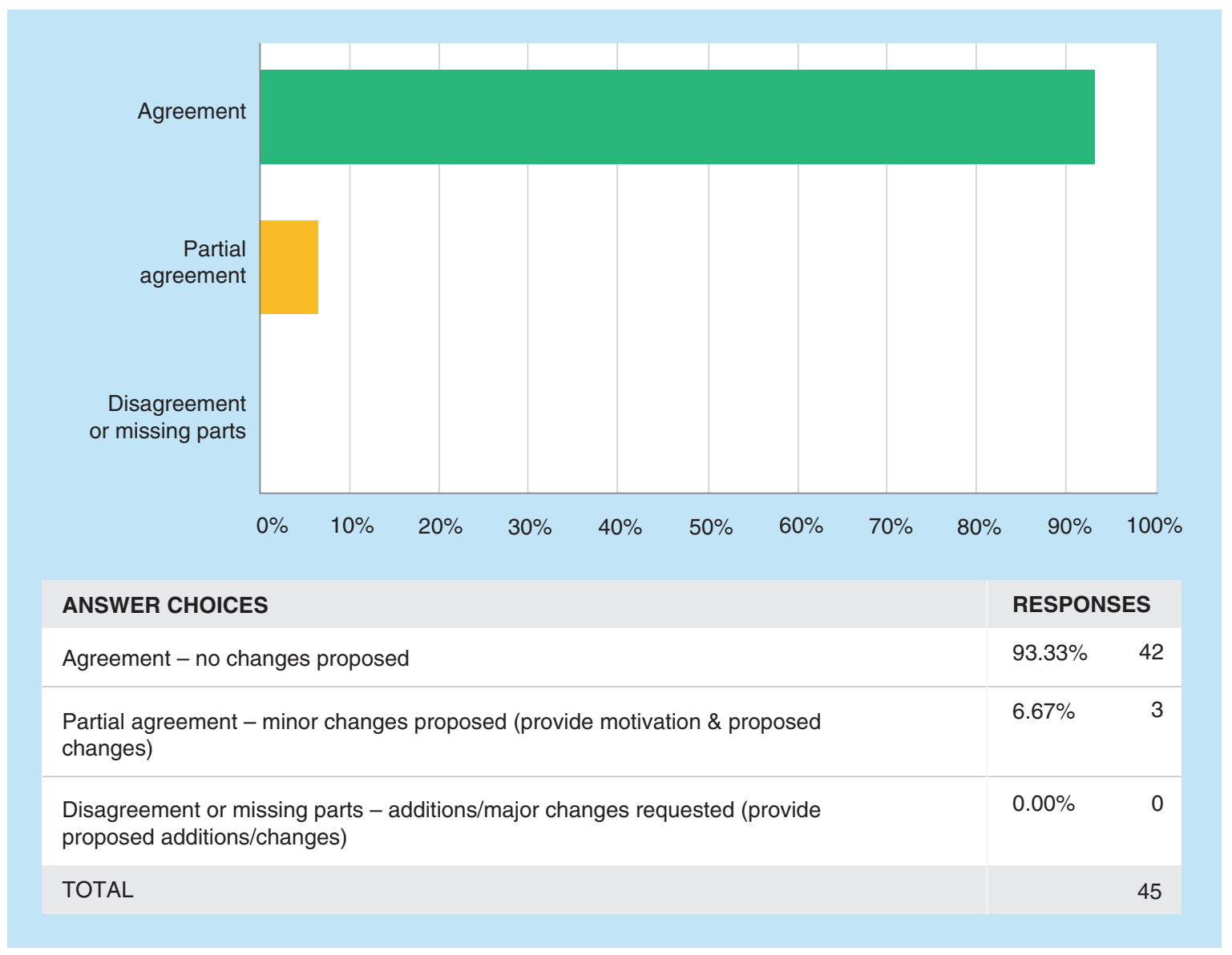

\section{Partial agreement}

Three comments suggested adding that study samples can also be bracketed by calibration standards.

\section{Additional comments from the 13th GCC:}

There were no further discussions during the GCC Closed Forum in support of the above proposal. 
T3Q5: Analytical Run - Curve Preparation; Lines 491-492: "The calibration standards and QCs should be spiked independently using separately prepared stock solutions, unless the accuracy and stability of the stock solutions have been verified."

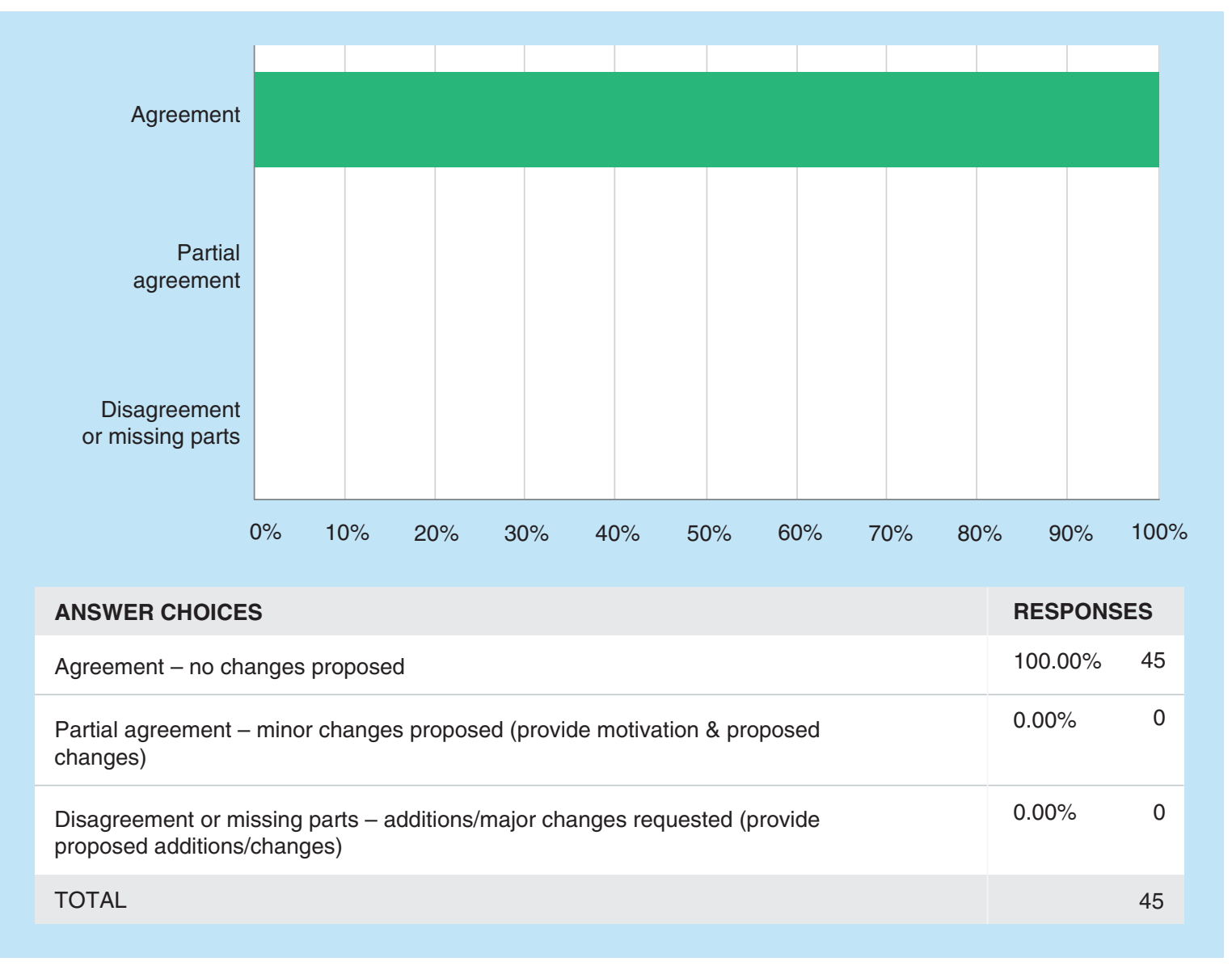


T3Q6: Analytical Run - Definition of a Batch; Lines 492-496: "All samples (calibration standards, QCs and study samples) should be processed and extracted as one single batch of samples in the order in which they are intended to be analyzed. A single batch is comprised of study samples and QCs, which are handled during a fixed period of time and by the same group of analysts with the same reagents under homogeneous conditions."

Analytical Run - Multiple Batches in a Run; Lines 497-499: "Analyzing samples that were processed as several separate batches in a single analytical run is discouraged. If such an approach cannot be avoided, for instance due to bench-top stability limitations, each batch of samples should include low, medium and high QCs."

Analytical Run - Defining Acceptance Criteria; Lines 500-501: "Acceptance criteria should be pre-established in an SOP or in the study plan and should be defined for the whole analytical run and the separate batches in the run, if applicable."

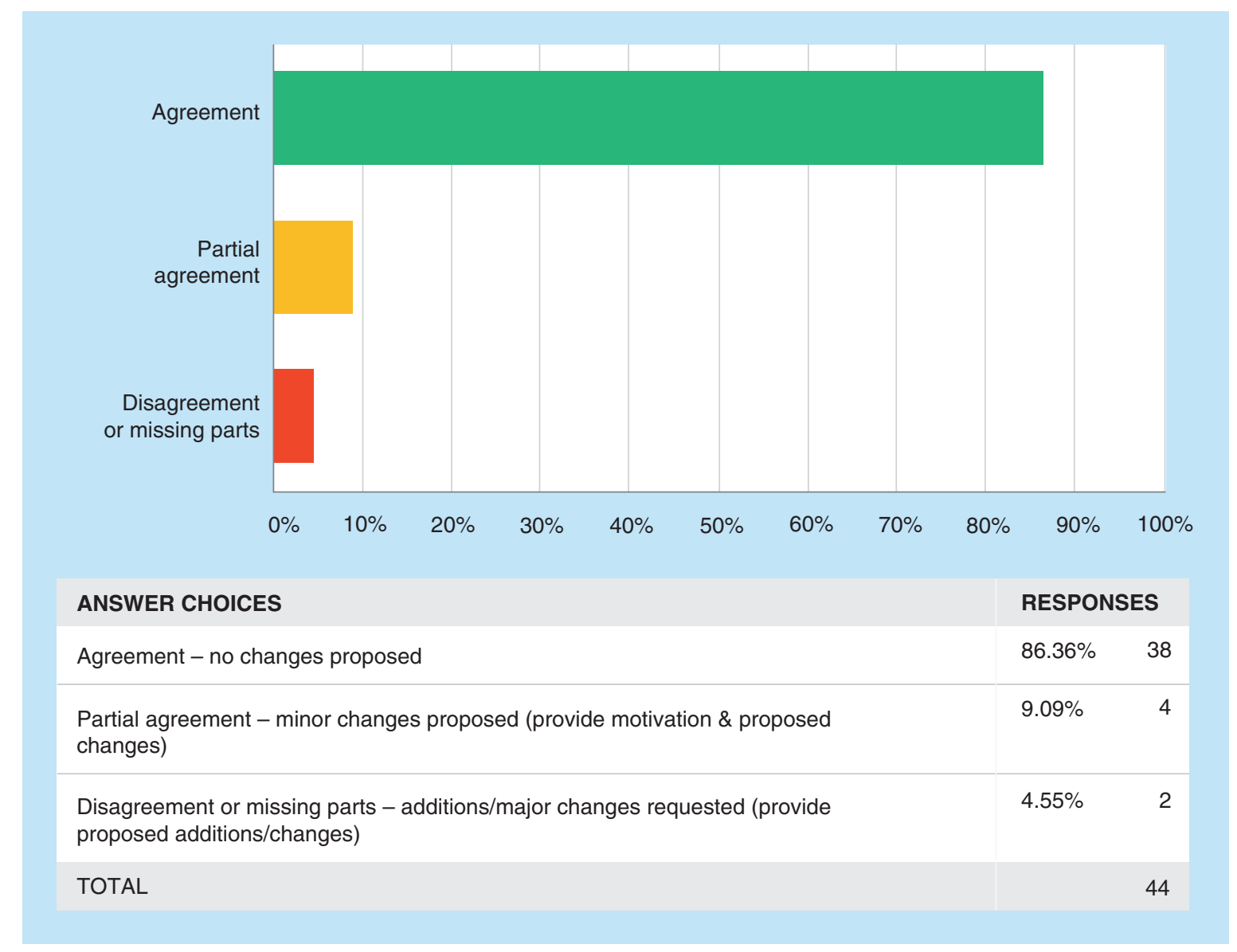

Disagreement or missing parts

It may be worthy to mention that both comments requested clarifying the definition of a batch, perhaps by including examples.

\section{Partiall agreement}

No trend was found in these comments.

\section{Additional comments from the 13th GCC:}

There were no further discussions during the GCC Closed Forum regarding the above proposal. 
Supplement Nehls, Buonarati, Cape et al.

T3Q7: Analytical Run - BA/BE; Lines 501-503: "For comparative BA/BE studies it is advisable to analyze all samples of one subject together in one analytical run to reduce variability."

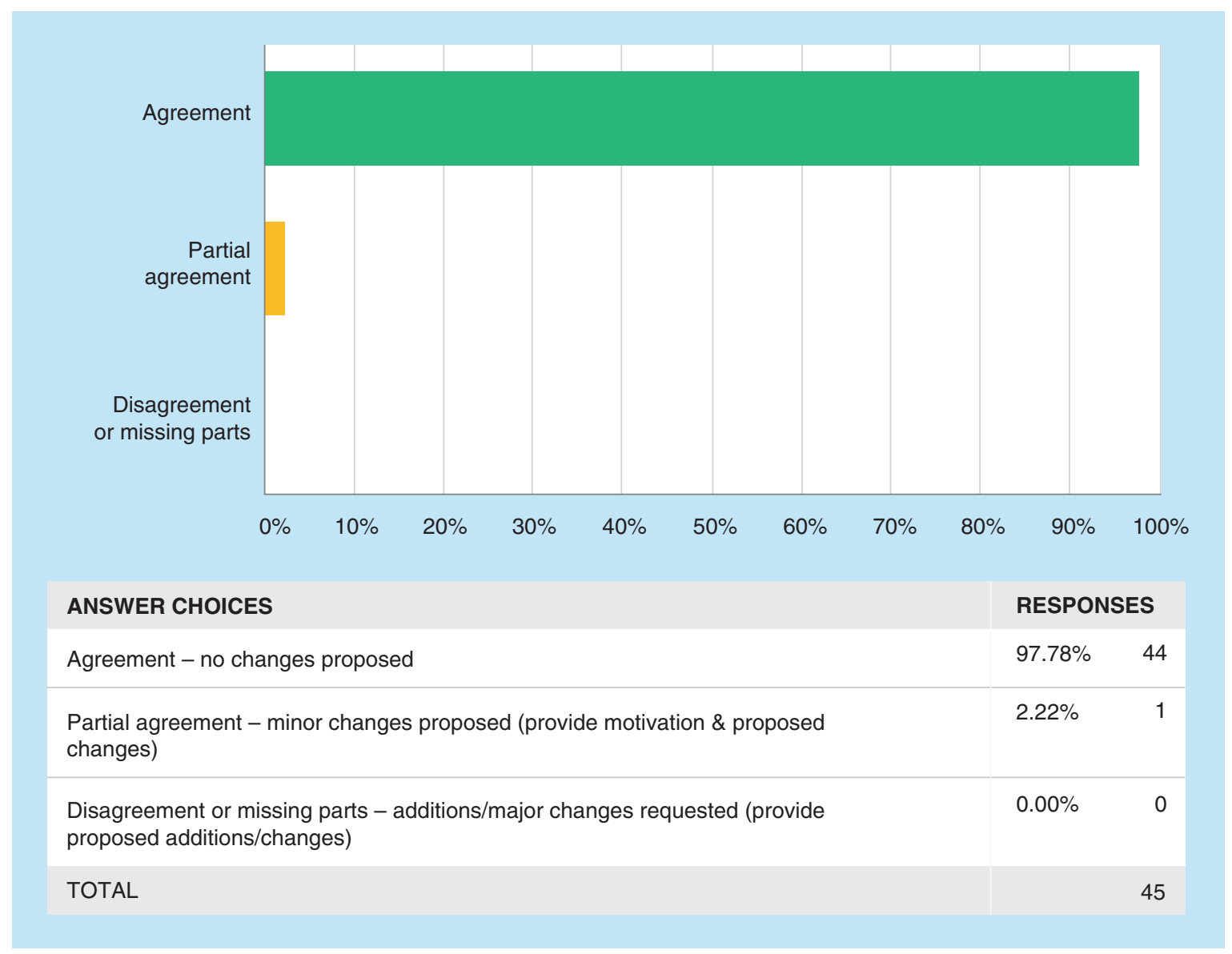


T3Q8: Carryover; Lines 504-508: "The impact of any carryover that occurs during study sample analysis should be assessed and reported (Refer to Section 3.2.6). If carryover is detected its impact on the measured concentrations should be mitigated (e.g., non-randomisation of study samples, injection of blank samples after samples with an expected high concentration) or the validity of the reported concentrations should be justified in the Bioanalytical Report."

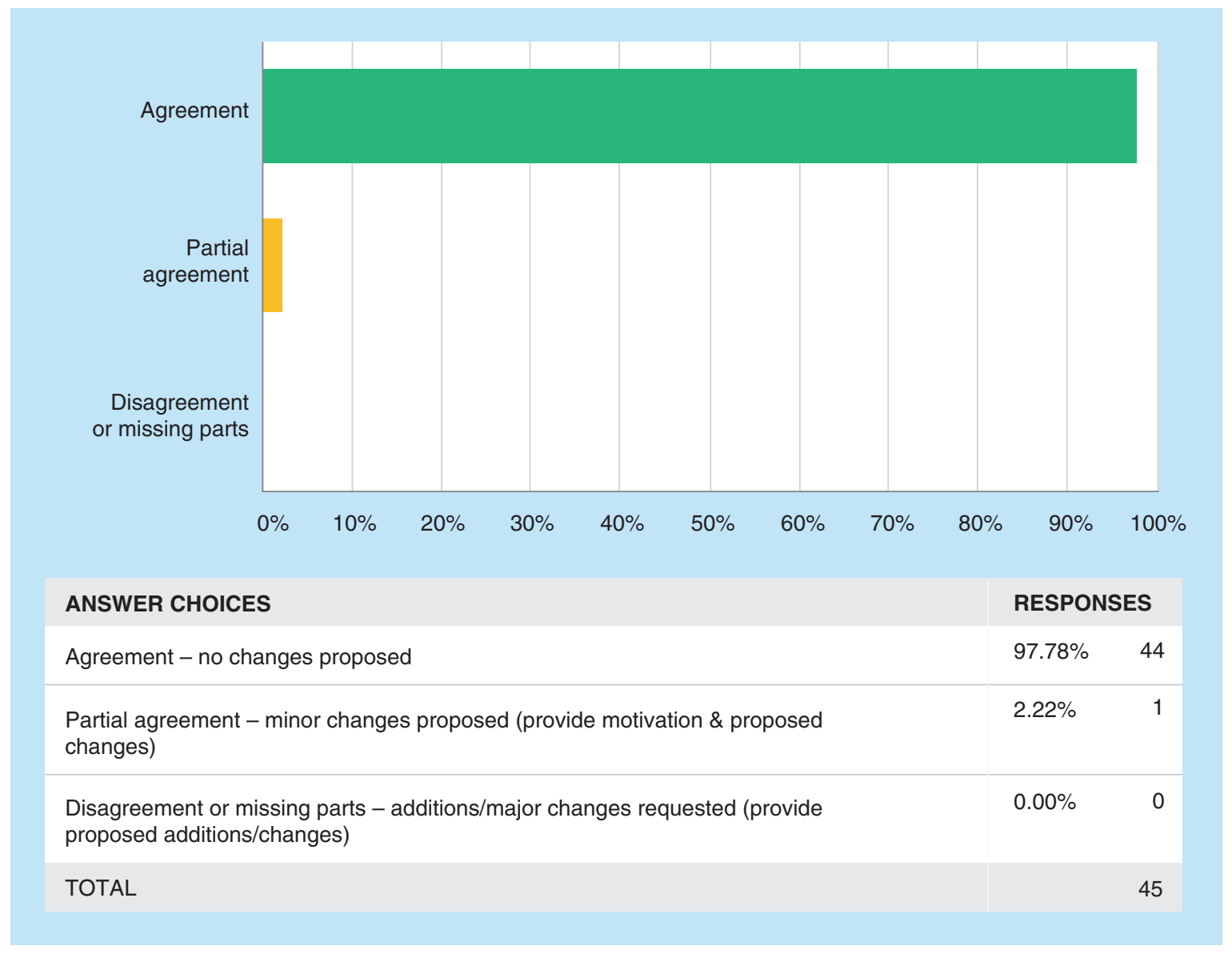


T3Q9: Analytical Run - Acceptance Criteria Batch vs Run; Lines 510-514: "Criteria for the acceptance or rejection of an analytical run should be defined in the protocol, in the study plan or in an SOP. In the case that a run contains multiple batches, acceptance criteria should be applied to the whole run and to the individual batches. It is possible for the run to meet acceptance criteria, even if a batch within that run is rejected for failing to meet the batch acceptance criteria."

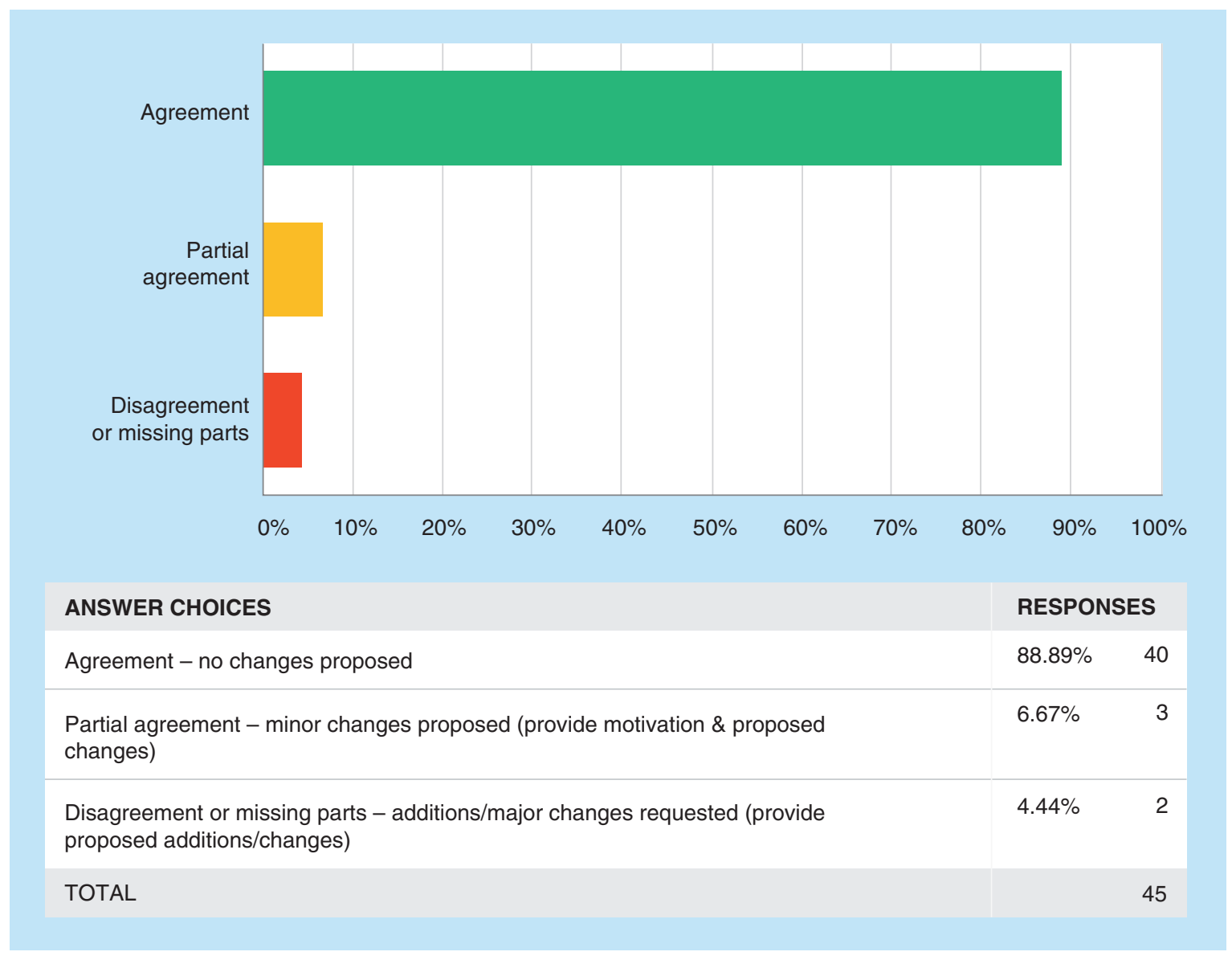

Disagreement or missing parts

No trend was found in these comments.

Partial agreement

No trend was found in these comments. 
T3Q10: Analytical Run - Acceptance Criteria of Calibration Standards; Lines 515-521: “The back-calculated concentrations of the calibration standards should be within $\pm 15 \%$ of the nominal value, except for the LLOQ for which it should be within $\pm 20 \%$. At least $75 \%$ of the calibration standard concentrations, with a minimum of six concentration levels, should fulfil these criteria. If more than six calibration standard levels are used and one of the calibration standards does not meet the criteria, this calibration standard should be rejected and the calibration curve without this calibration standard should be re-evaluated and a new regression analysis performed."

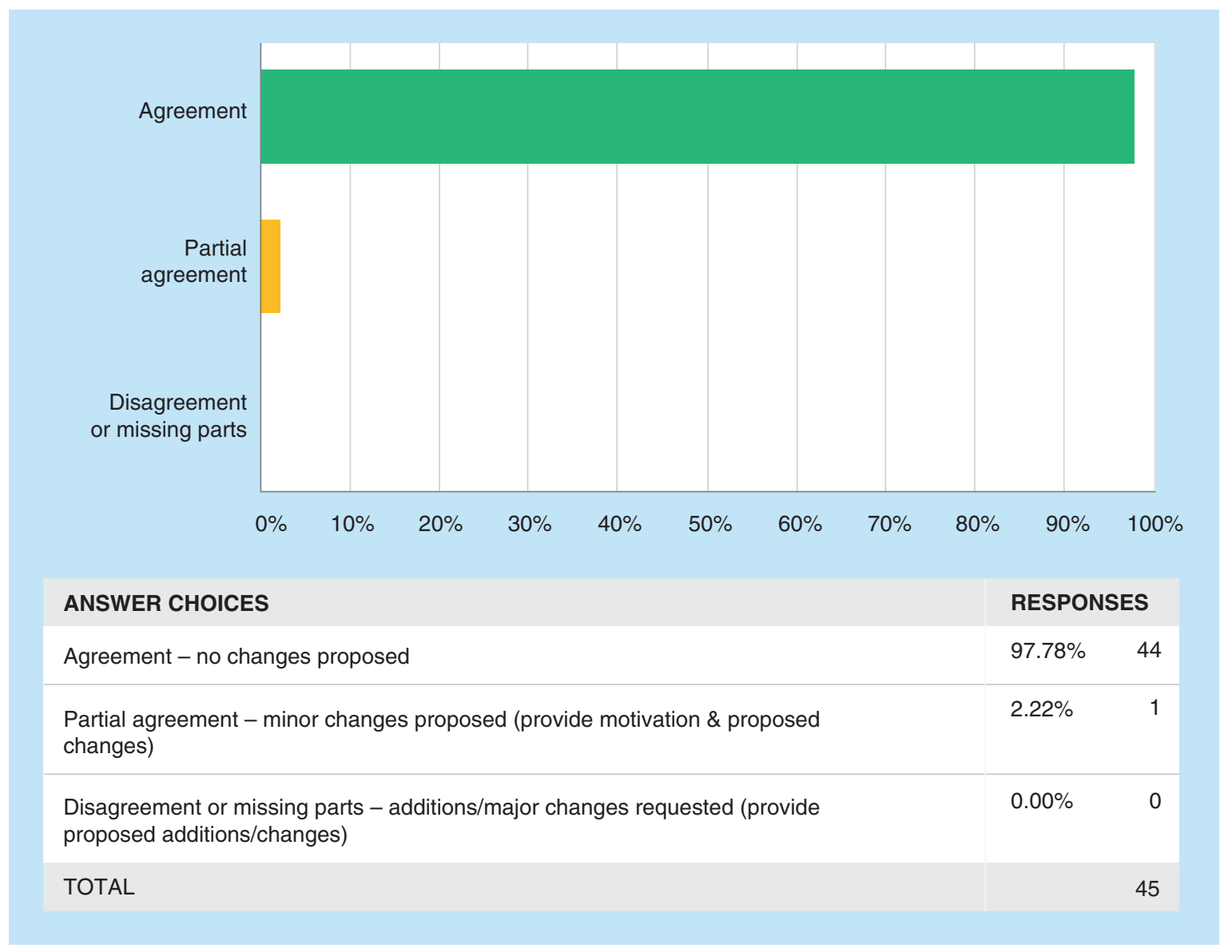


T3Q11: Analytical Run - Acceptance Criteria Rejected LLOQ or ULQ; Lines 522-529: "If the rejected calibration standard is the $L L O Q$, the new lower limit for this analytical run is the next lowest acceptable calibration standard of the calibration curve. This new lower limit calibration standard will retain its original acceptance criteria (i.e., $\pm 15 \%)$. If the highest calibration standard is rejected, the ULOQ for this analytical run is the next acceptable highest calibration standard of the calibration curve. The revised calibration range should cover at least three $Q C$ concentration levels (low, medium and high). Study samples outside of the revised range should be reanalyzed. If replicate calibration standards are used and only one of the LLOQ or ULOQ standards fails, the calibration range is unchanged."

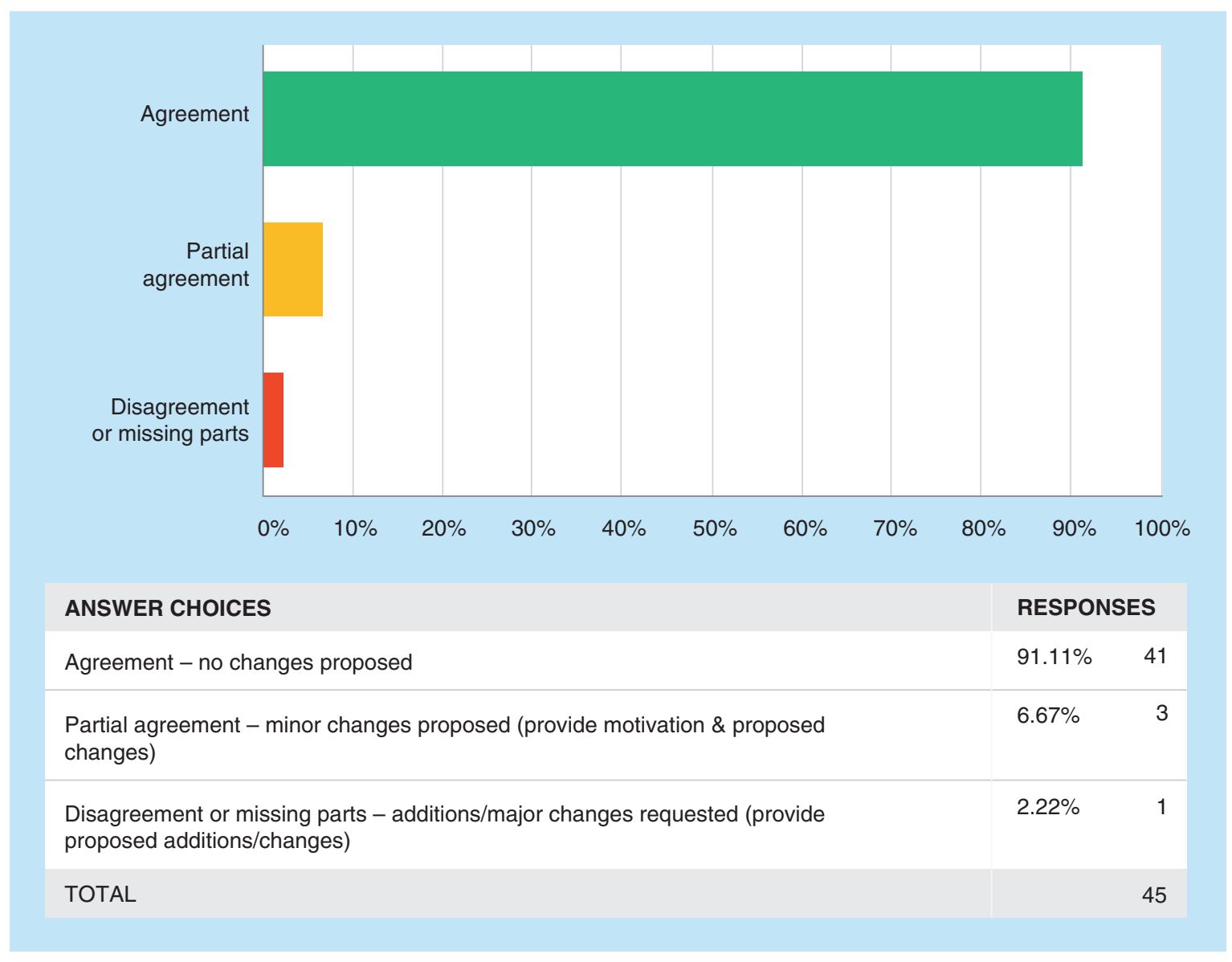

\section{Partial agreement}

No trend was found in these comments. 
T3Q12: Analytical Run - Acceptance Criteria QC Samples; Lines 530-531: "At least 2/3 of the total QCs and at least $50 \%$ at each concentration level should be within $\pm 15 \%$ of the nominal values."

Analytical Run - Rejected Runs; Lines 531-534: "If these criteria are not fulfilled the analytical run should be rejected. A new analytical batch needs to be prepared for all study samples within the failed analytical run for subsequent analysis. In the cases where the failure is due to an assignable technical cause, samples may be reinjected."

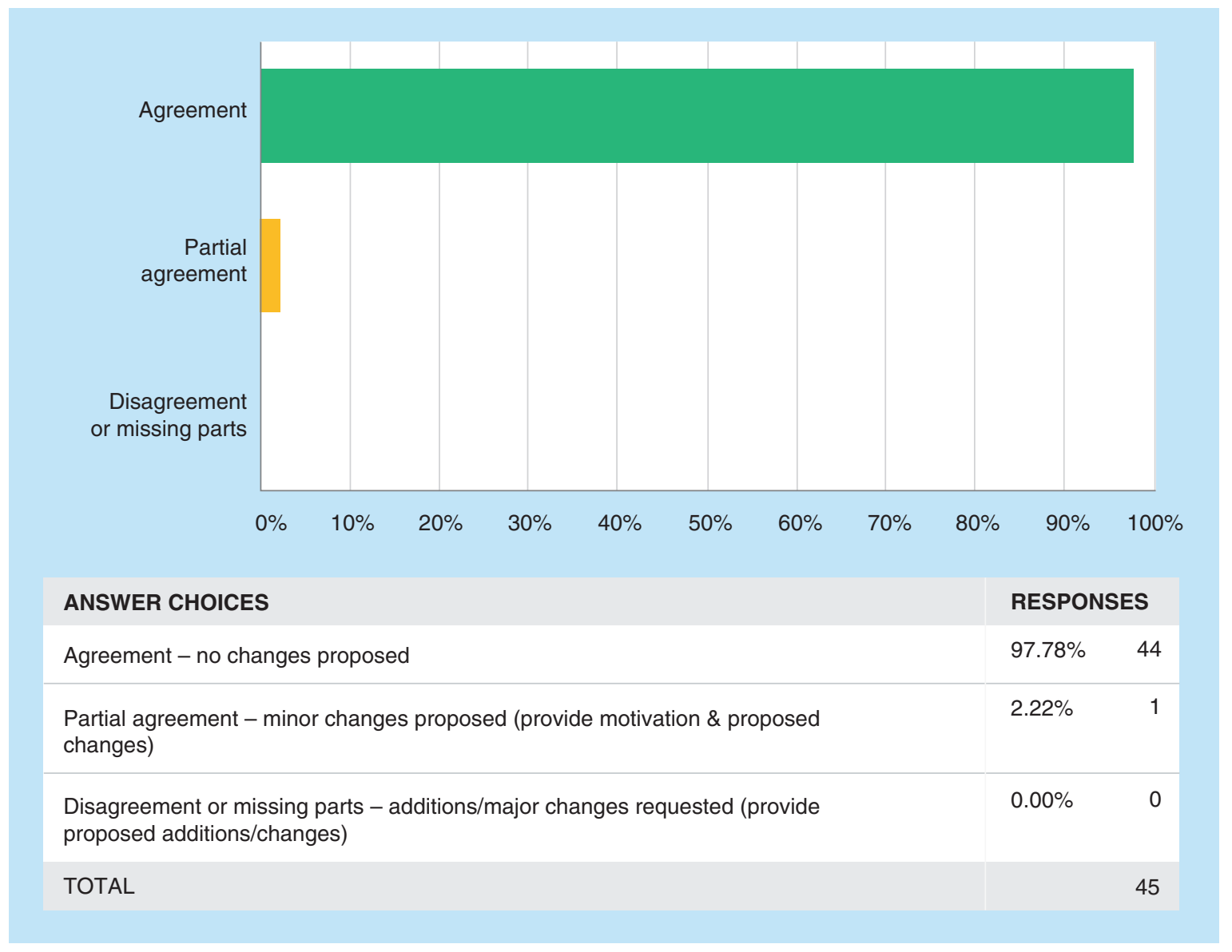


T3Q13: Analytical Run - Sample Dilutions; Lines 535-536: "Analytical runs containing samples that are diluted and reanalysed should include dilution QCs to verify the accuracy and precision of the dilution method during study sample analysis."

Analytical Run - Dilution QCs; Lines 536-540: "The concentration of the dilution QCs should exceed that of the study samples being diluted (or of the ULOQ) and they should be diluted using the same dilution factor. The within-run acceptance criteria of the dilution $Q C(s)$ will only affect the acceptance of the diluted study samples and not the outcome of the analytical run."

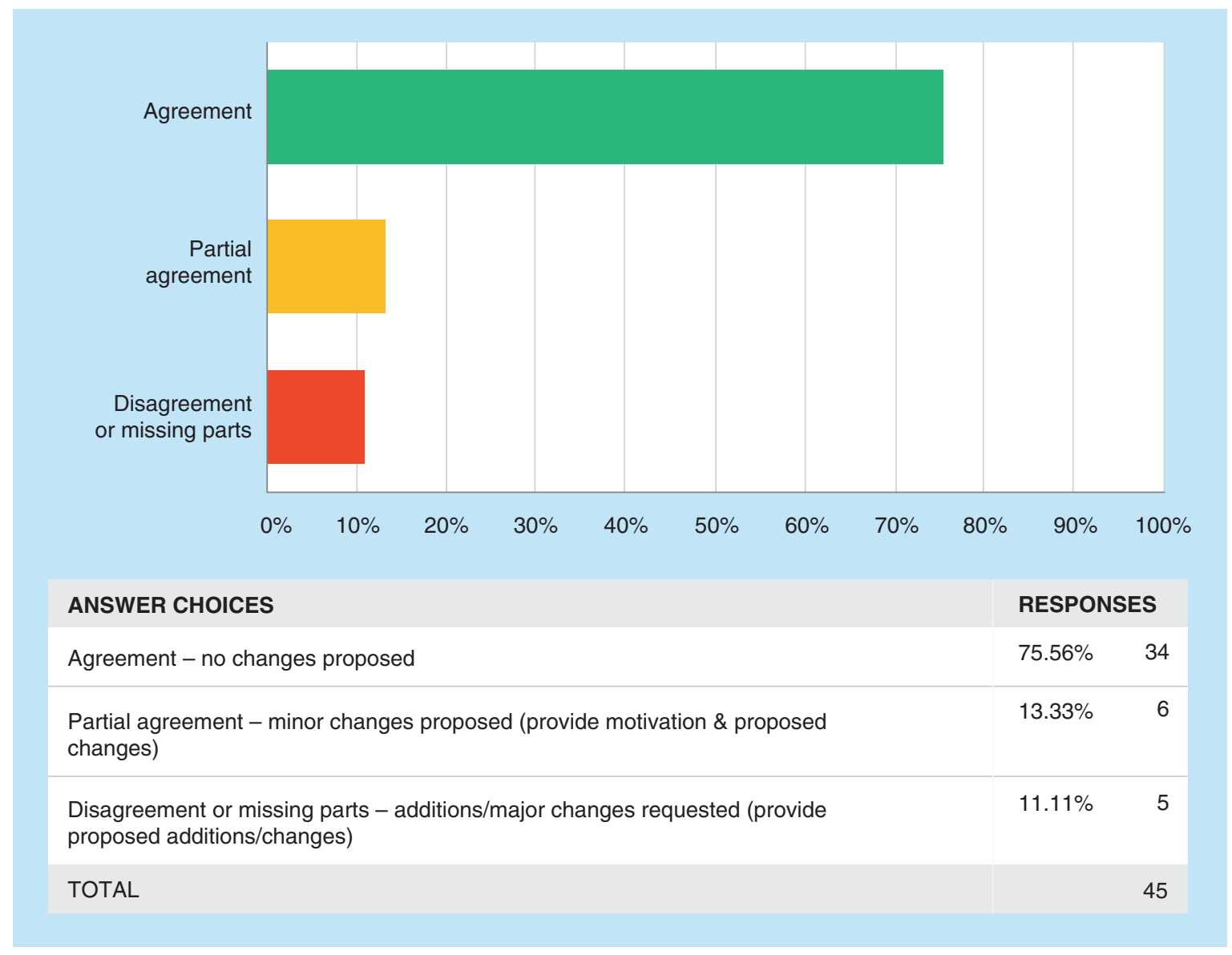

Disagreement or missing parts

All five comments disagreed with the requirement to include dilution QCs during sample analysis, since dilutions are already validated as part of the dilution integrity assessment.

\section{Partial agreement}

Since the maximum study sample concentration is typically unknown prior to analysis, it was suggested to adjust the wording to accommodate this practicality when defining the requirements of the concentration of the dilution QC.

\section{Additional comments from the 13th GCC:}

The discussion within the GCC Closed Forum confirmed the recommendation that dilution QCs should not be required during sample analysis as long as it is validated appropriately. Additionally, there is no added value in testing all dilution factors. 
T3Q14: Multi - Analytes; Lines 541-544: "When several analytes are assayed simultaneously, there should be one calibration curve for each analyte studied. If an analytical run is acceptable for one analyte but has to be rejected for another analyte, the data for the accepted analyte should be used. The determination of the rejected analyte requires a re-extracted analytical batch and analysis."

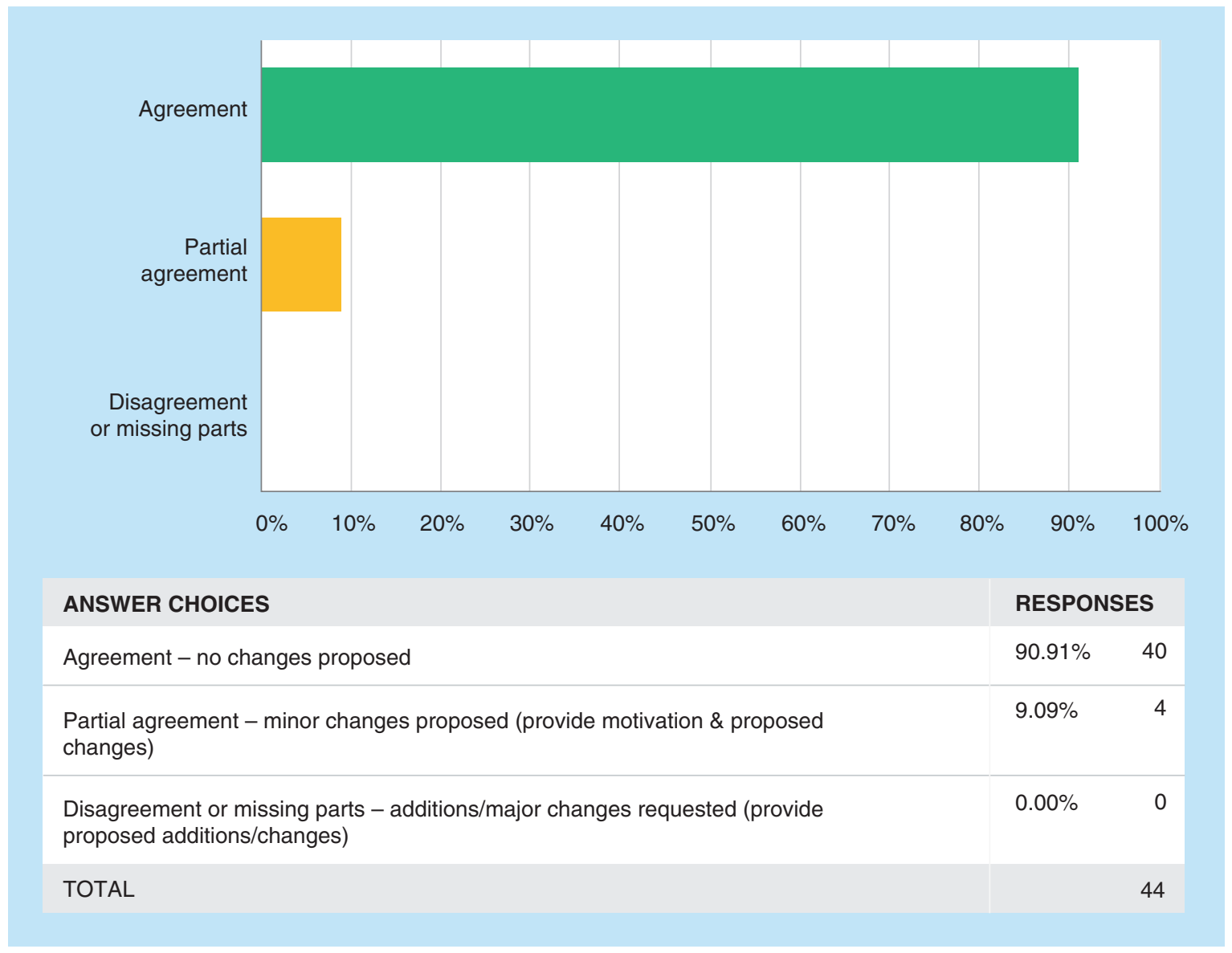

\section{Partiall agreement}

All four comments suggested allowing for reinjection of the failed analyte if the cause of failure is system or chromatography related and can be subsequently resolved.

\section{Additional comments from the 13th GCC:}

There were no further discussions during the GCC Closed Forum regarding the above proposal. 
T3Q15: Analytical Run - Reporting; Lines 545-546: "The back-calculated concentrations of the calibration standards and QCs of passed and accepted runs should be reported."

Analytical Run - Precsision and Accuracy Criteria; Lines 546-551: "The back-calculated concentrations of the calibration standards and QCs of passed and accepted runs should be reported. The overall (between-run) accuracy and precision of the QCs of all accepted runs should be calculated at each concentration level and reported in the analytical report (Refer to Section 8 Documentation and Table 1). If the overall mean accuracy or precision fails the $15 \%$ criterion, an investigation to determine the cause of the deviation should be conducted. In the case of comparative BA/BE studies it may result in the rejection of the data."

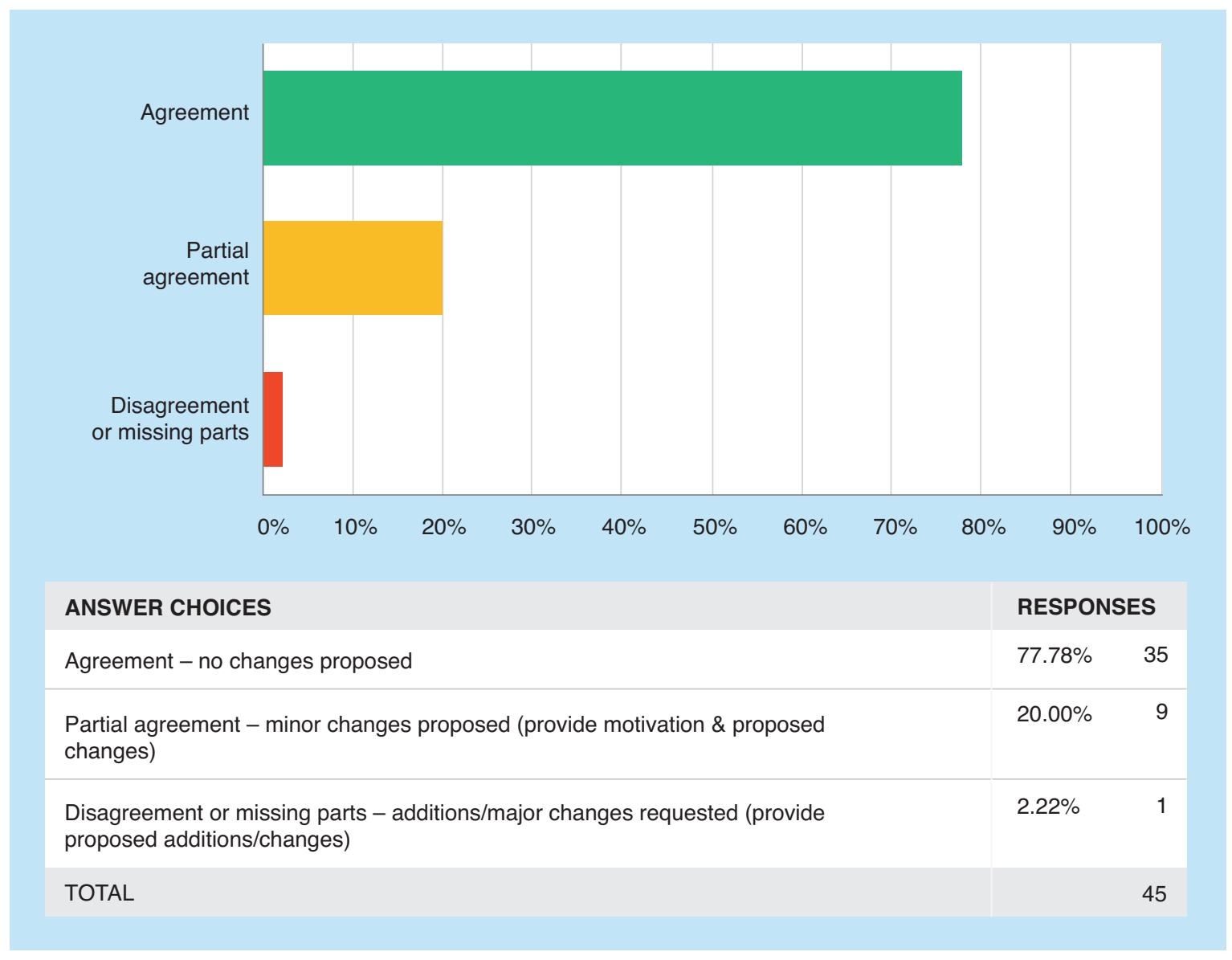

\section{Partial agreement}

It was suggested that the use of an outlier test be permitted in cases where a single out of specification QC result negatively affects inter- or intra-run precision and accuracy data. 
T3Q16: Calibration Range - Incorrect Range; Lines 553-556: "If a narrow range of analyte concentrations of the study samples is known or anticipated before the start of study sample analysis, it is recommended to either narrow the calibration curve range, adapt the concentrations of the QCs, or add new QCs at different concentration levels as appropriate, to adequately reflect the concentrations of the study samples."

Calibration Range - Sample Clustering; Lines 557-562: "At the intended therapeutic dose(s), if an unanticipated clustering of study samples at one end of the calibration curve is encountered after the start of sample analysis, the analysis should be stopped and either the standard calibration range narrowed (i.e., partial validation), existing QC concentrations revised, or QCs at additional concentrations added to the original curve within the observed range before continuing with study sample analysis. It is not necessary to reanalyse samples analysed before optimising the calibration curve range or QC concentrations.”

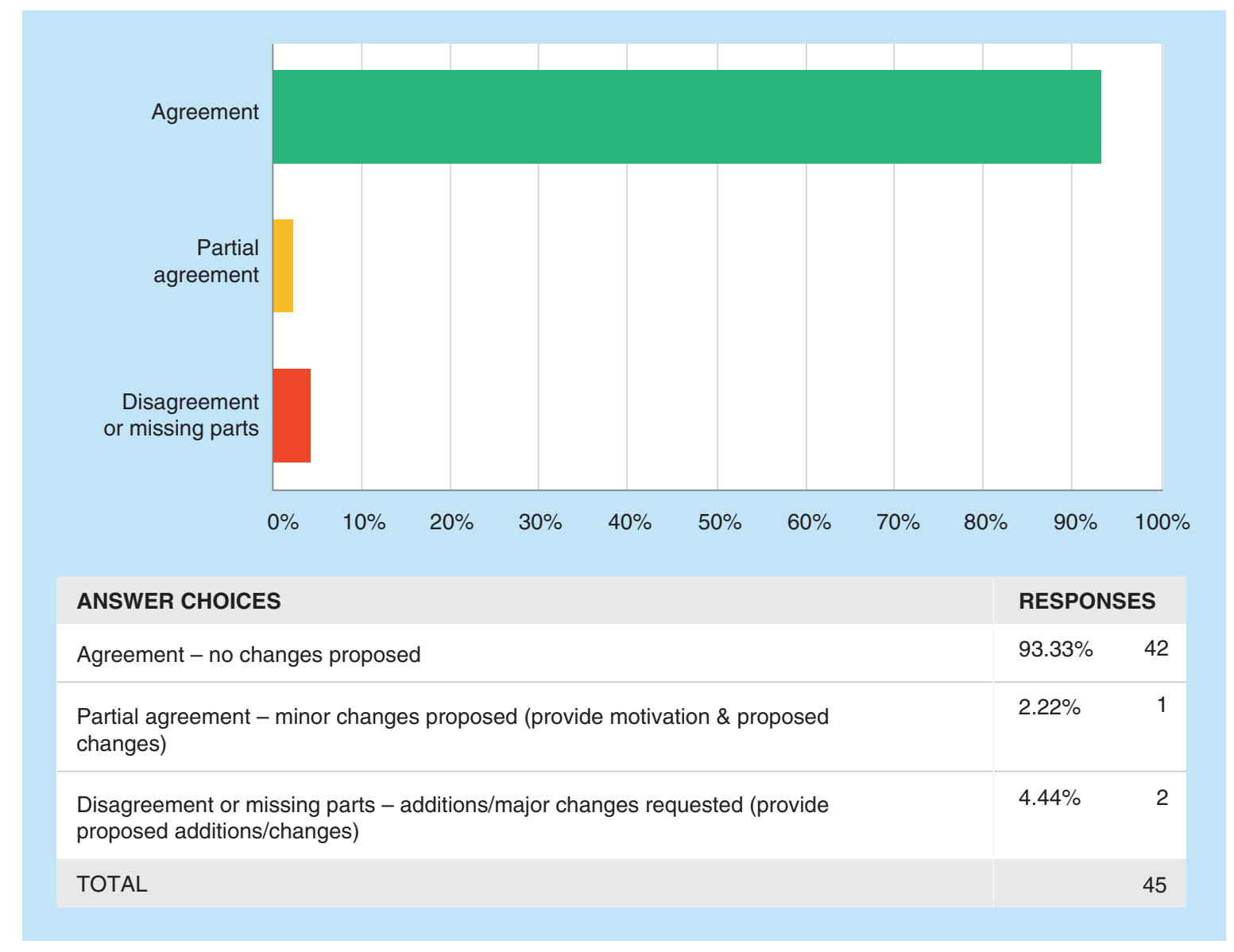

\section{Partial agreement}

It may be worthy to mention that in two comments it was noted that the entire range is validated for an assay, demonstrating the reliability of study sample concentrations across all sections of the range. Therefore, it is not necessary to add QCs when sample concentrations cluster in one area of the curve.

\section{Additional comments from the 13th GCC:}

There were no further discussions during the GCC Closed Forum in support of the above proposal. 
T3Q17: Calibration Range - Samples > ULOQ; Lines 563-567: "The same applies if a large number of the analyte concentrations of the study samples are above the ULOQ. The calibration curve range should be changed, if possible, and $Q C(s)$ added or their concentrations modified. If it is not possible to change the calibration curve range or the number of samples with a concentration above the ULOQ is not large, samples should be diluted according to the validated dilution method."

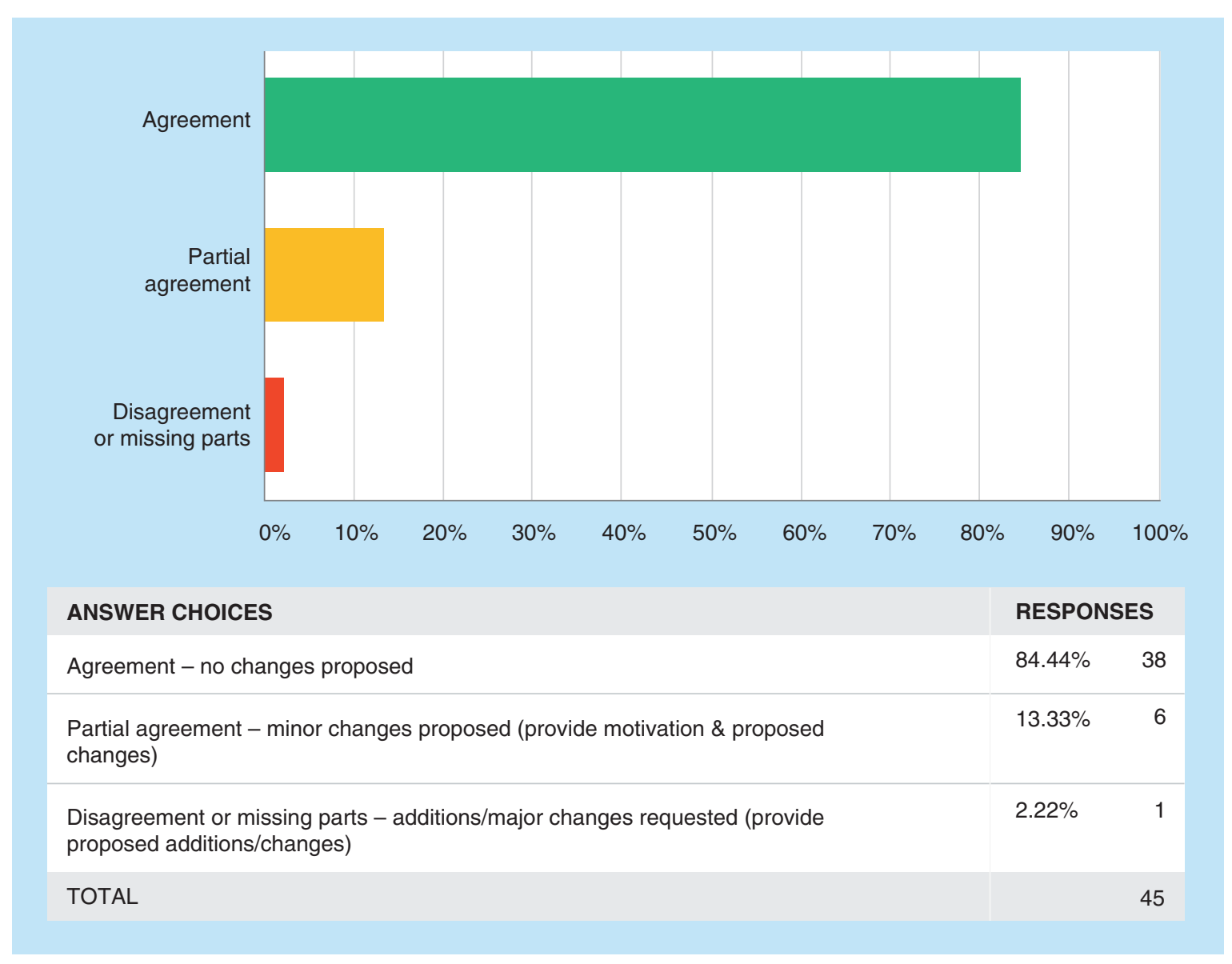

Partial agreement

Clarity was requested for the meaning of "large number of samples."

\section{Additional comments from the 13th GCC:}

It was suggested that the last word be changed from "method" to "factor." 
T3Q18: Calibration Range - QC Samples; Lines 568-570: "At least 2 QC levels should fall within the range of concentrations measured in study samples. If the calibration curve range is changed, the bioanalytical method should be revalidated (partial validation) to verify the response function and to ensure accuracy and precision."

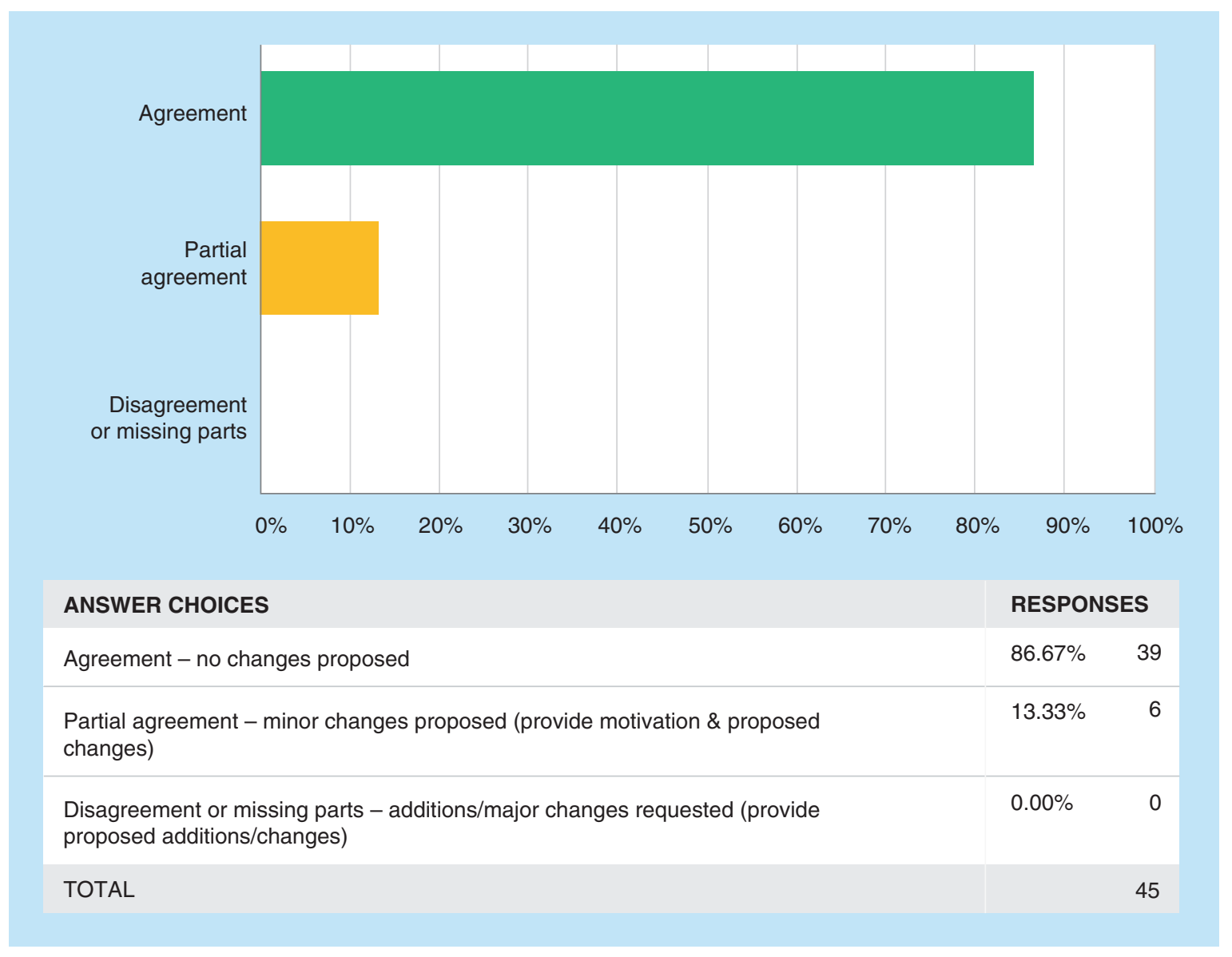

\section{Partial agreement}

No trend was found in these comments. 
T3Q19: Sample Reanalysis - Predefined Criteria; Lines 572-574: "Possible reasons for reanalysis of study samples, the number of replicates and the decision criteria to select the value to be reported should be predefined in the protocol, study plan or SOP, before the actual start of the analysis of the study samples."

Sample Reanalysis - Number of Repeats; Lines 575-576: "The number of samples (and percentage of total number of samples) that have been reanalyzed should be reported and discussed in the Bioanalytical Report."

Sample Reanalysis - Examples; Lines 577-589: "Some examples of reasons for study sample reanalysis are:

- Rejection of an analytical run because the run failed the acceptance criteria with regard to accuracy of the calibration standards and/or the precision and accuracy of the QCs

- IS response significantly different from the response for the calibration standards and QCs (as pre-defined in an SOP)

- The concentration obtained is above the ULOQ

- The concentration observed is below the revised LLOQ in runs where the lowest calibration standard has been rejected from a calibration curve, resulting in a higher LLOQ compared with other runs

- Improper sample injection or malfunction of equipment

- The diluted study sample is below the LLOQ

- Identification of quantifiable analyte levels in predose samples, control or placebo samples

- Poor chromatography (as pre-defined in an SOP)."

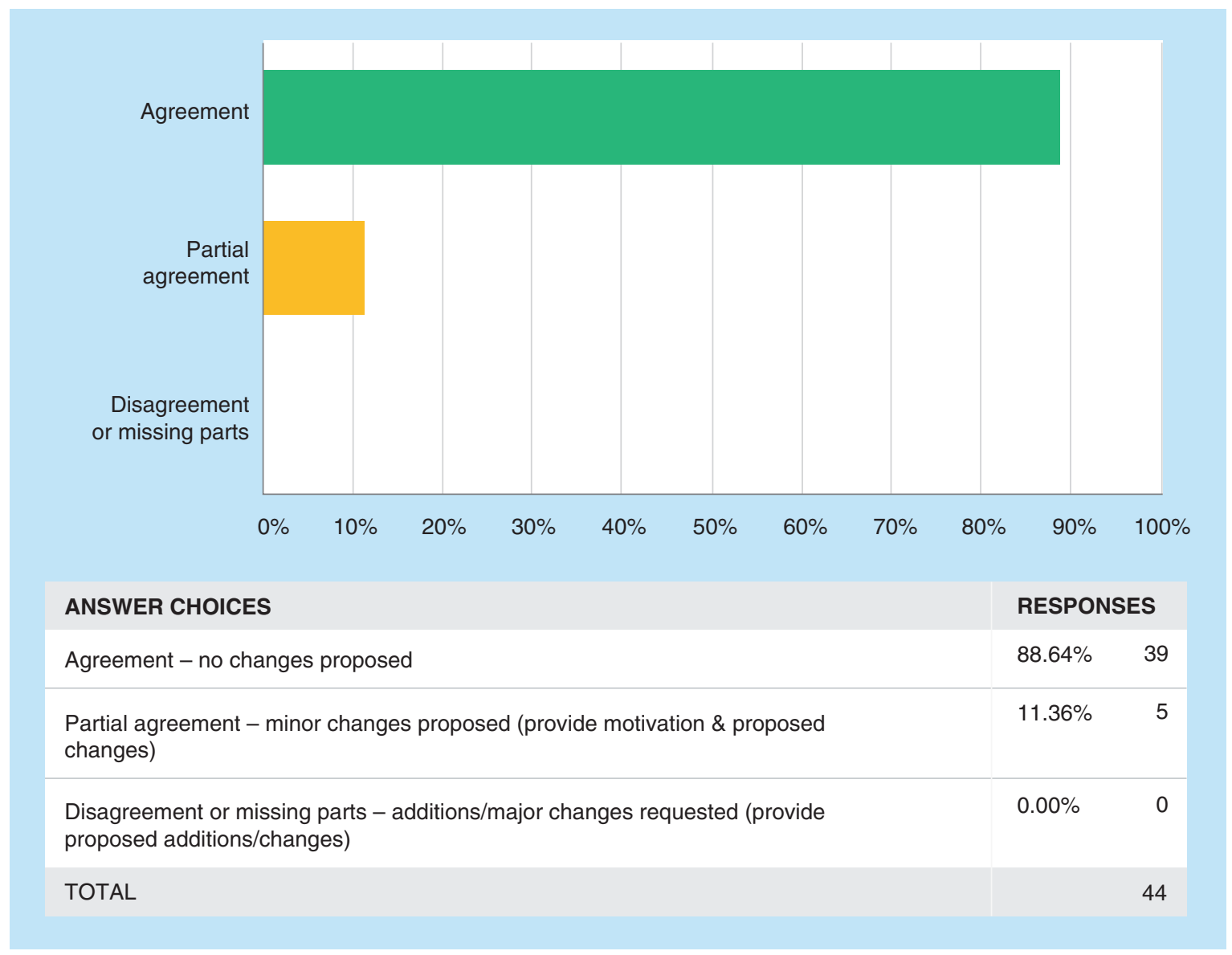

\section{Partial agreement}

It was suggested that samples repeated due to batch rejection are not considered as reanalyzed samples because the original concentration data is not valid.

Additional comments from the 13th GCC:

It was recommended to add definitions to differentiate reinjection from reanalysis to the glossary. 
T3Q20: Sample Reanalysis - PK Repeats; Lines 590-592: "For comparative BA/BE studies, reanalysis of study samples for a PK reason (e.g., a sample concentration does not fit with the expected profile) is not acceptable, as it may bias the study result."

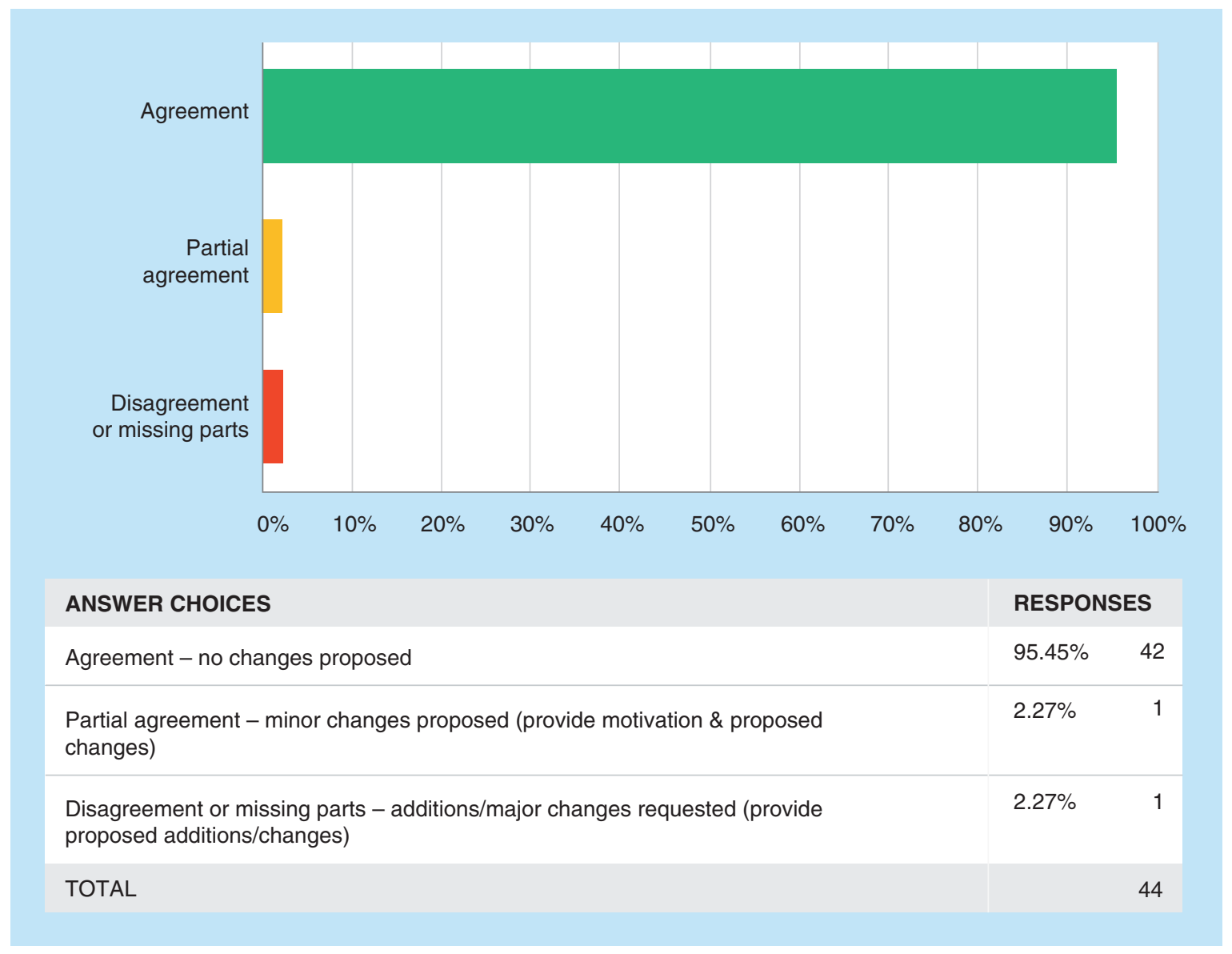


T3Q21: Sample Reanalysis - Reporting; Lines 593-598: "Any reanalysed samples should be identified in the Bioanalytical Report and the initial value, the reason for reanalysis, the values obtained in the reanalyses, the final accepted value and a justification for the acceptance should be provided. Further, a summary table of the total number of samples that have been reanalysed for each reason should be provided. In cases where the first analysis yields a non-reportable result, a single reanalysis is considered sufficient (e.g., concentration above the ULOQ or equipment malfunction)."

Sample Reanalysis - Confirmatory Reassays; Lines 598-600: "In cases where the value needs to be confirmed (e.g., predose sample with measurable concentrations) replicate determinations are required if sample volume allows."

Sample Reanalysis - Investigations; Lines 601-603: "The safety of trial subjects should take precedence over any other aspect of the trial. Consequently, there may be other circumstances when it is necessary to reanalyse specific study samples for the purpose of an investigation."

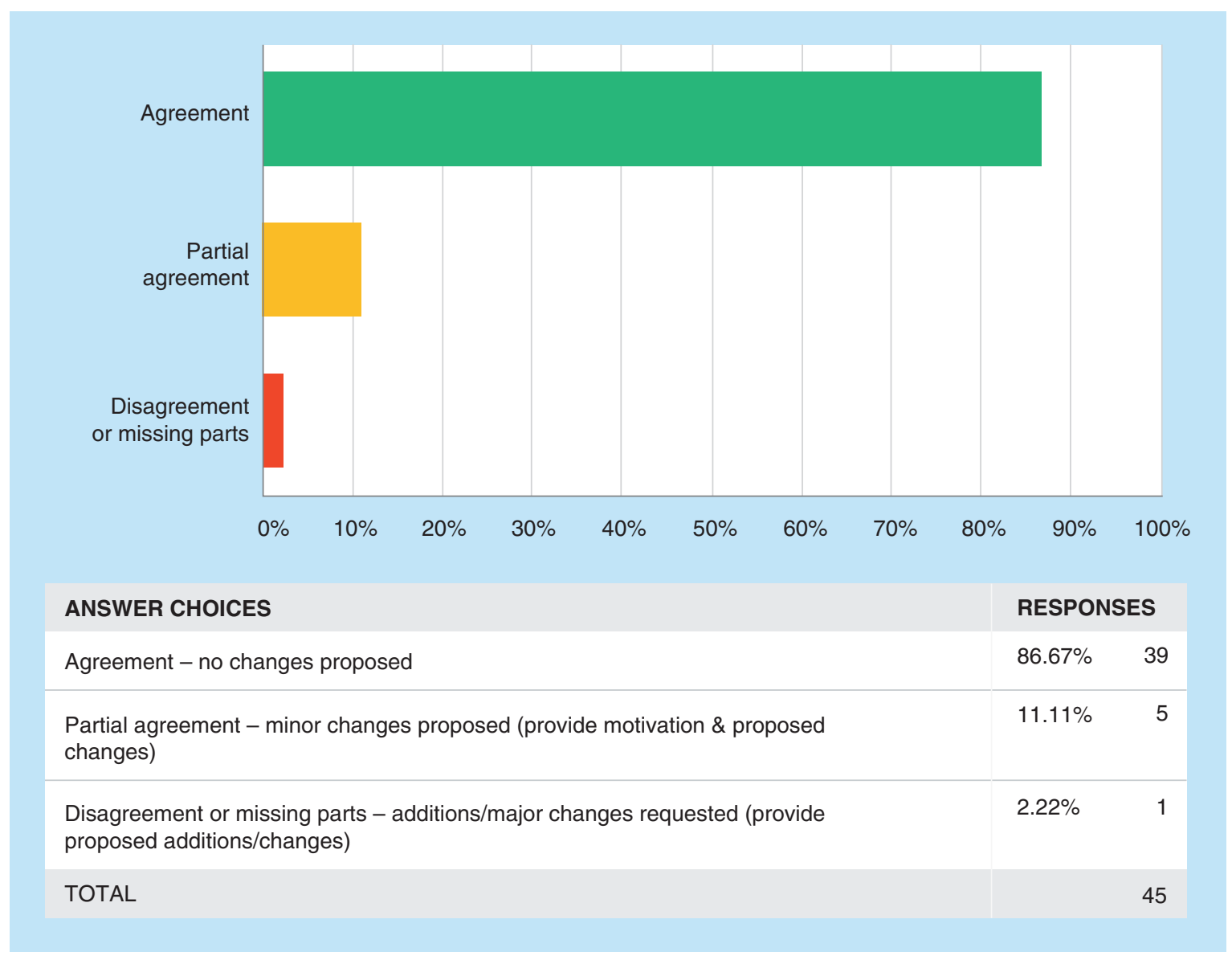

\section{Partial agreement}

It is worthy to note that a few comments indicated that invalid results should not be reported. 
T3Q22: Reinjection; Lines 605-609: "Reinjection of processed samples can be made in the case of equipment failure if reinjection reproducibility has been demonstrated during validation or provided in the Bioanalytical Report where it was conducted. Reinjection of a full analytical run or of individual calibration standards or QCs simply because the calibration standards or QCs failed, without any identified analytical cause, is not acceptable."

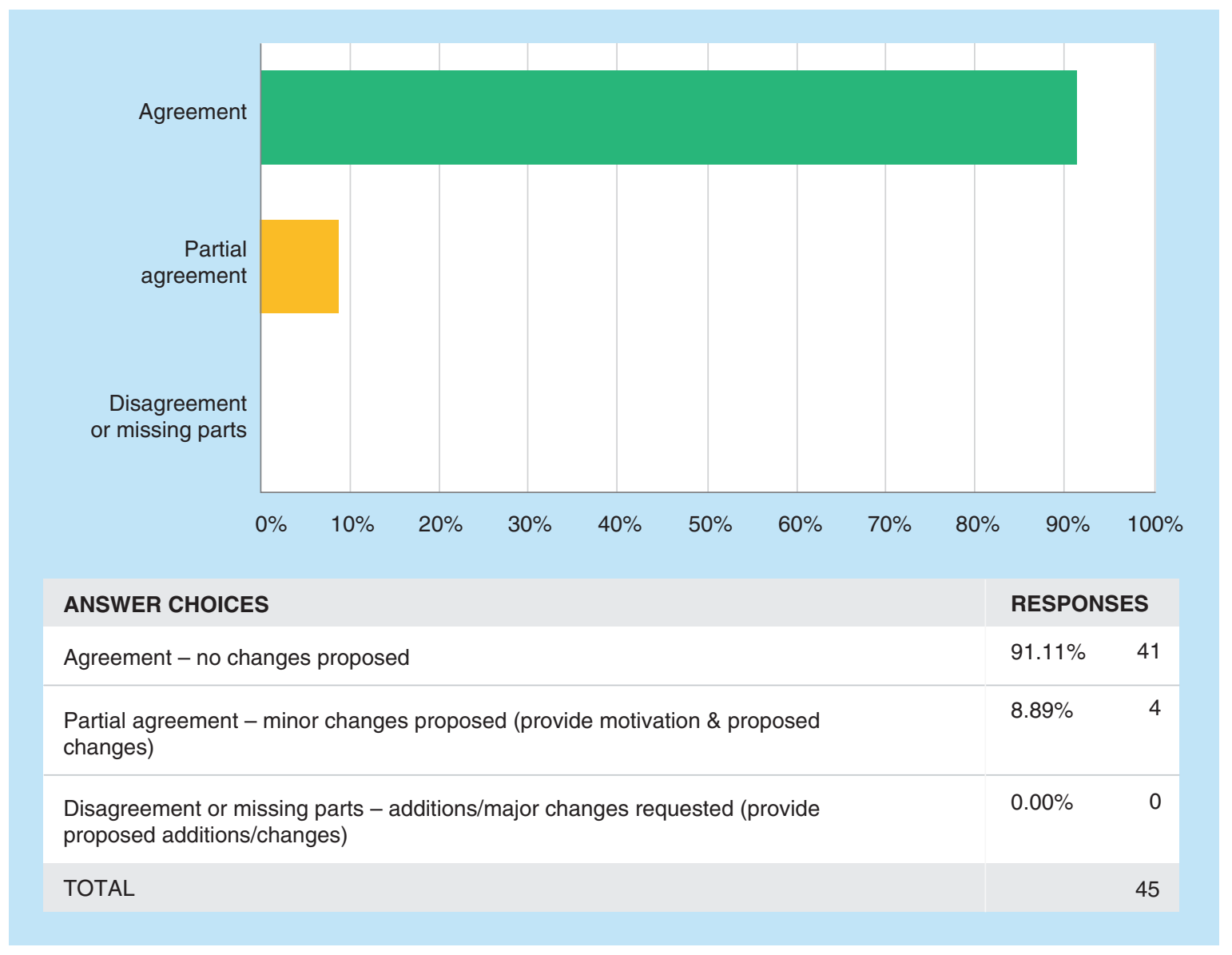

\section{Partial agreement}

No trend was found in these comments. 
T3Q23: Chromatogram Integration - Predefined Process; Lines 611-612: "Chromatogram integration and reintegration should be described in a study plan, protocol or SOP."

Chromatogram Integration - Deviations to Predefined Process; Lines 612-613: "Any deviation from the procedures described a priori should be discussed in the Bioanalytical Report."

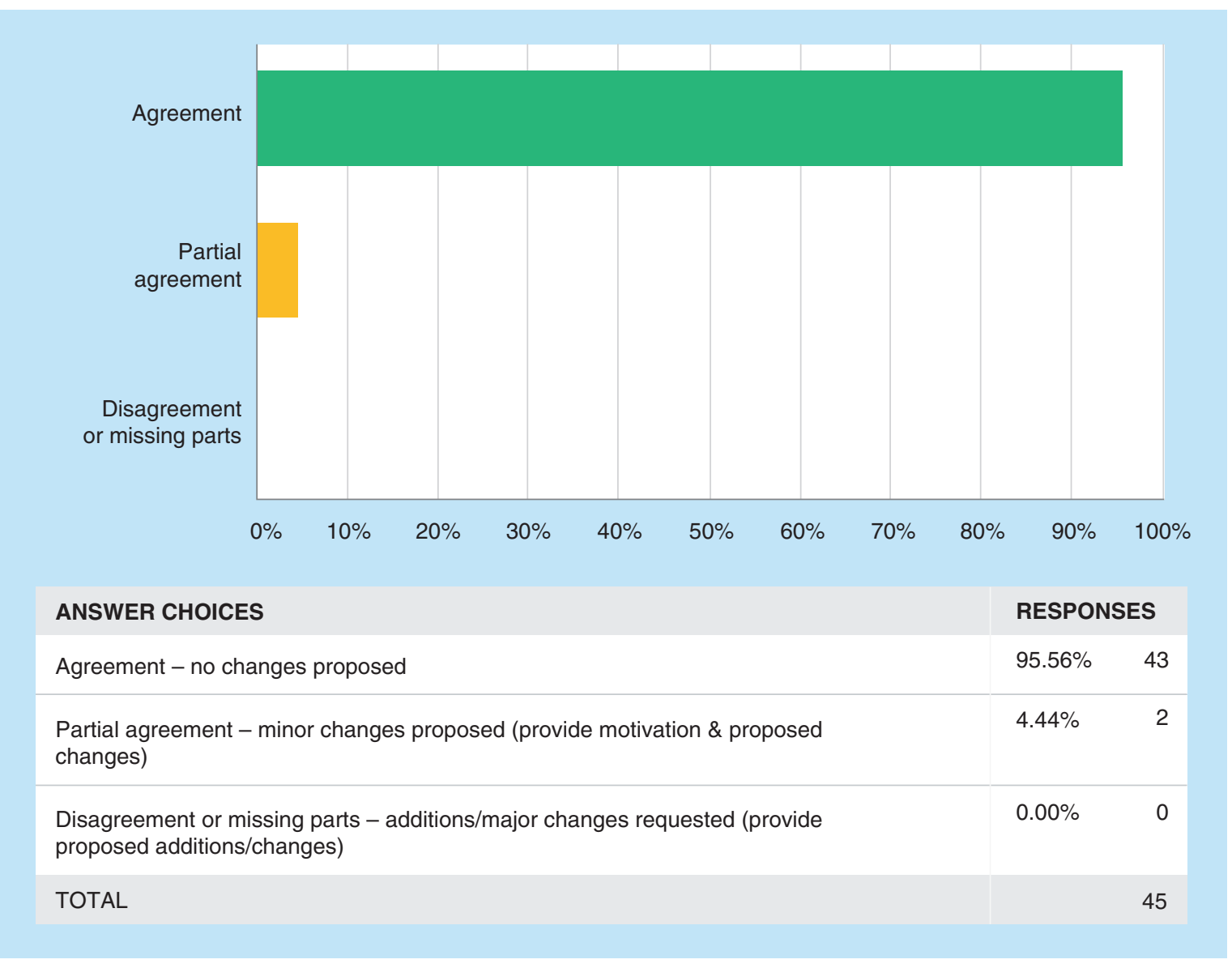

\section{Partial agreement}

No trend was found in these comments. 
T3Q24: Chromatogram Integration - Reintegration; Lines 613-615: "The list of chromatograms that required reintegration, including any manual integrations, and the reasons for reintegration should be included in the Bioanalytical Report."

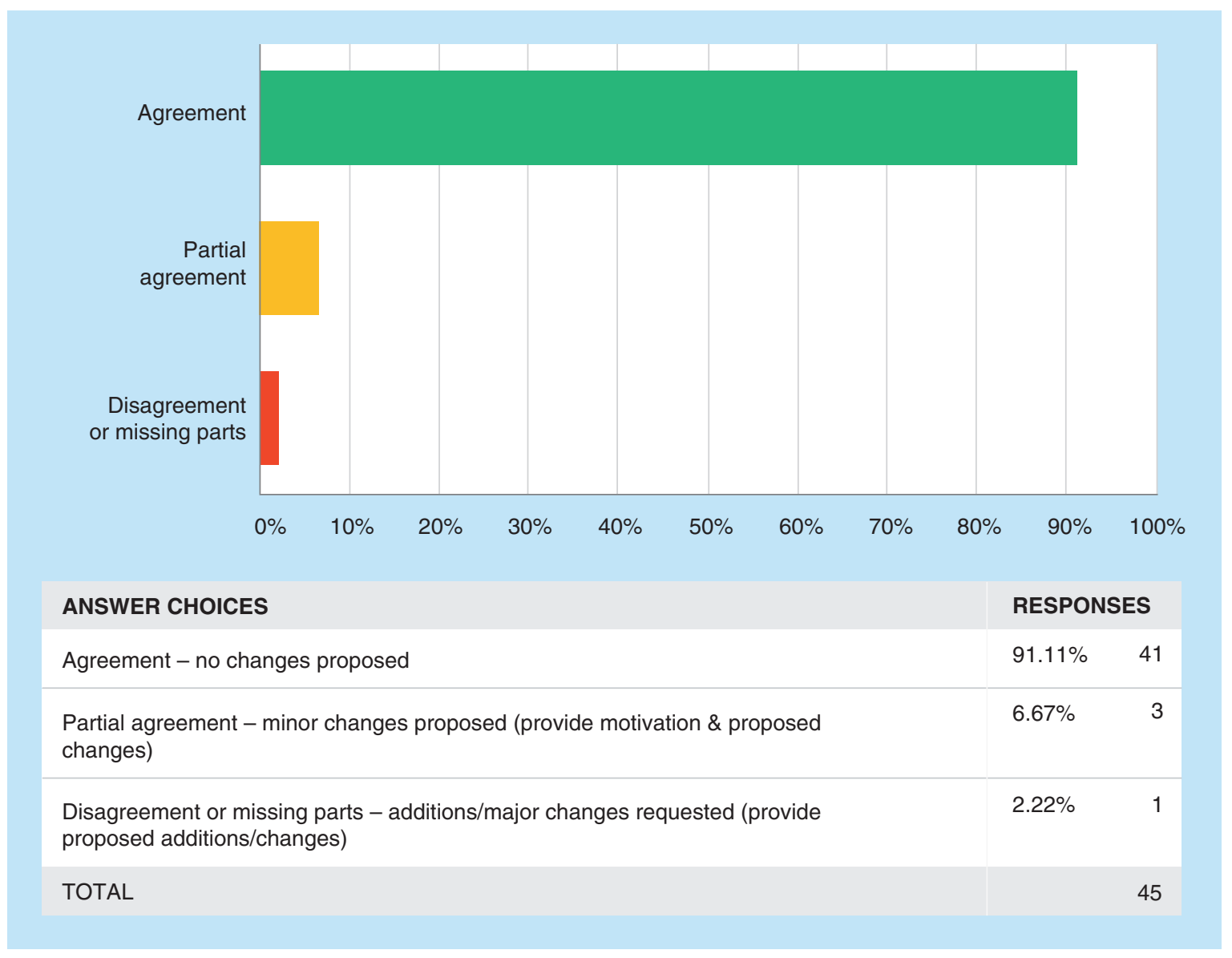

\section{Partial agreement}

A few comments suggested clarifying that this refers to reintegration of single samples. If the entire batch is integrated with new parameters, this should not require additional reporting.

\section{Additional comments from the 13th GCC:}

There were no further discussions during the GCC Closed Forum regarding the above proposal. 
T3Q25: Chromatogram Integration - Reintegrated Chromatograms; Lines 615-617: "Original and reintegrated chromatograms and initial and repeat integration results should be kept for future reference and submitted in the Bioanalytical Report for comparative BA/BE studies."

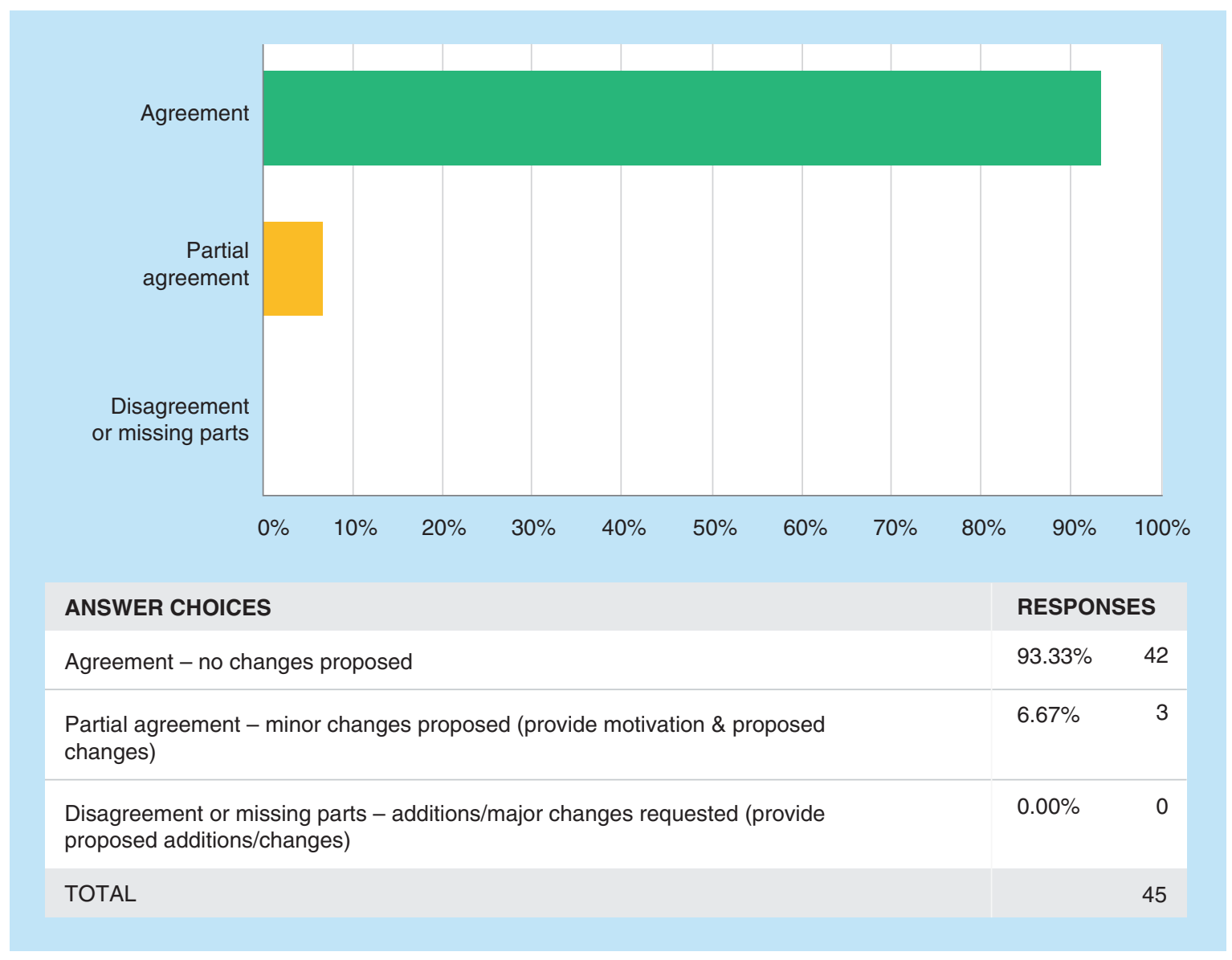

Partial agreement

No trend was found in these comments. 
Team 4 LBA I - Key Validation parameters: Selectivity, Specificity, Calibration Curve, Accuracy, Precision, Quality Controls, Carryover, Stability, Dilution Linearity, Hook Effect, including Parallelism, Minimum Required Dilution

T4Q1: Replicate Analysis - Assay Format; Lines 659-662: "When using LBA, study samples can be analysed using an assay format of 1 or more well(s) per sample. The assay format should be specified in the protocol, study plan or SOP. If method development and assay validation are performed using 1 or more well(s) per sample, then study sample analysis should also be performed using 1 or more well(s) per sample, respectively."

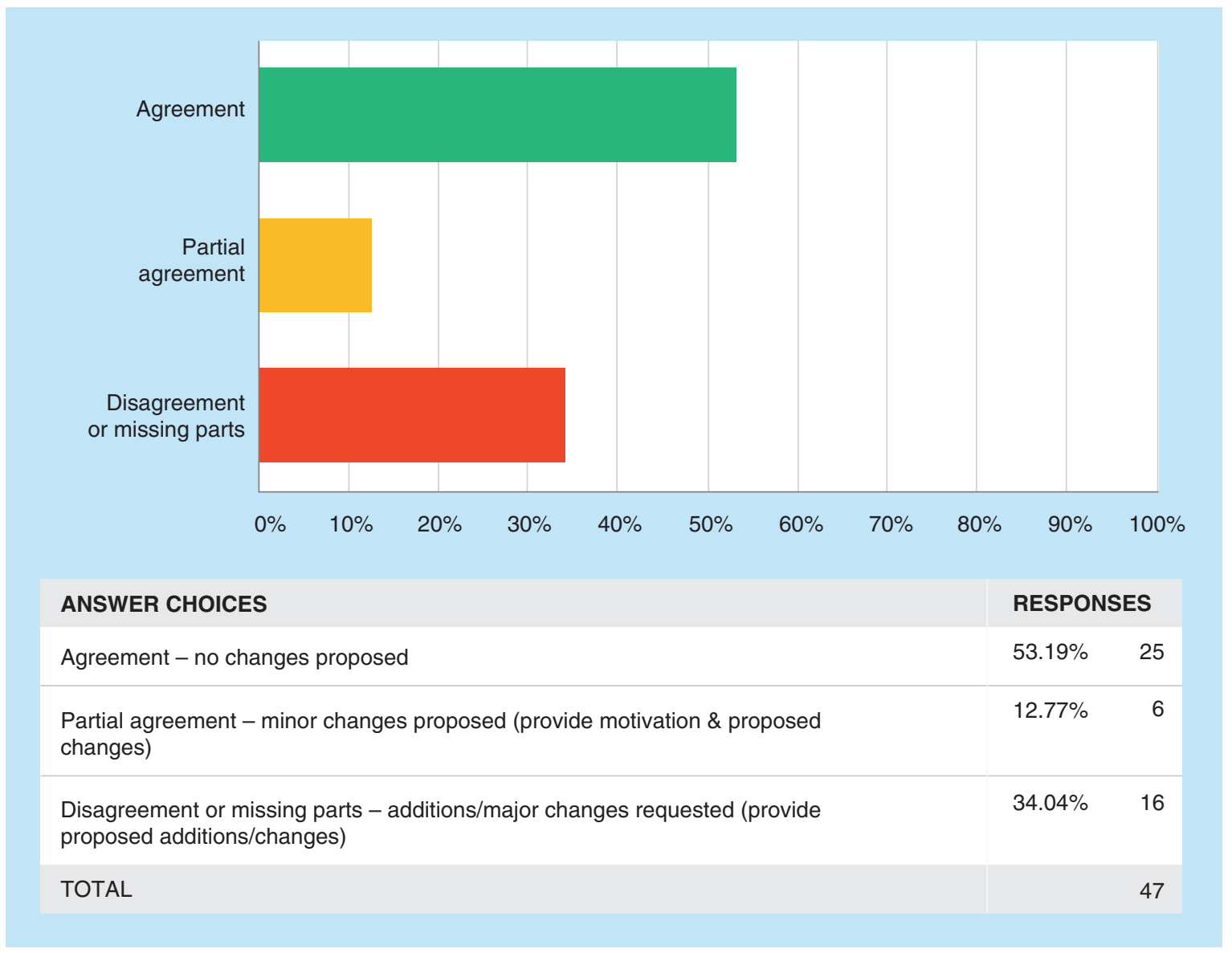

Disagreement or missing parts

- Numerous comments requested additional guidance on LBA singlicate analysis (one-well) specifically when using the Gyros platform. Concerns were perceived lack of regulatory support for singlicate analysis, missing acceptance criteria and sponsor requests not to use singlicate analysis due to lack of precedent or guidance.

- Additional confirmation is requested that specifically states that singlicate analysis is permitted for LBAs, based on published literature [23].

Partial agreement

No trend was found in these comments. 
T4Q2: Replicate Analysis - Concentrations; Lines 662-666: "If multiple wells per sample are used, the reportable sample concentration value should be determined either by calculating the mean of the responses from the replicate wells or by averaging the concentrations calculated from each response. Data evaluation should be performed on reportable concentration values."

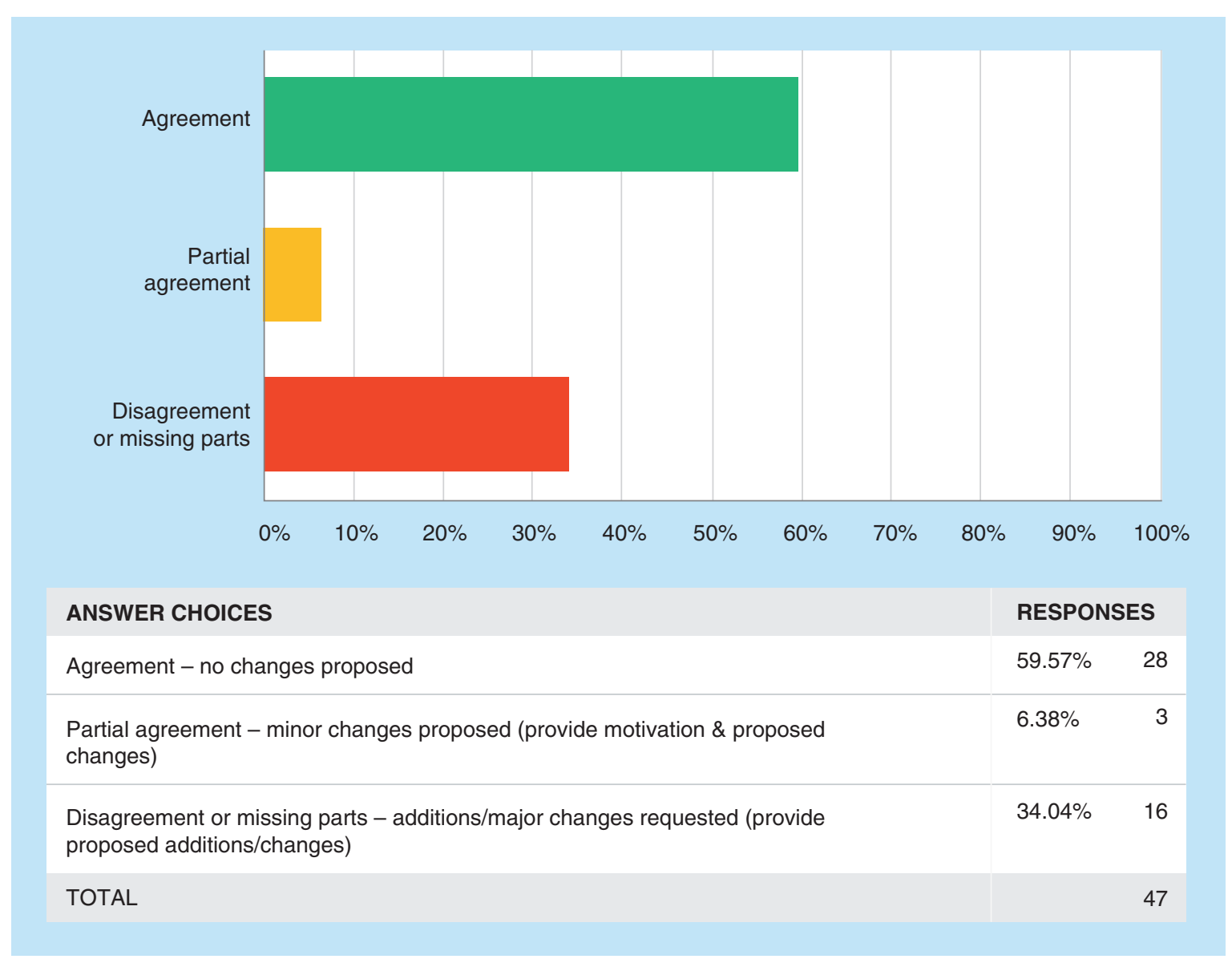

Disagreement or missing parts

- The majority of comments strongly requested adding criteria for the precision $(\% \mathrm{CV})$ between replicate wells since this is missing in both the FDA and EMA guidance/guideline [18,19] and to refer to current recommendations on this topic [23]. The main concern seems to be the lack of guidance on how to determine sample concentration from replicate wells (either by using the mean of the responses from the replicate wells or by averaging the concentration calculated from each response), or if either is acceptable if a consistent approach is applied.

- Other comments were in support of adding guidance for LBA singlicate analysis (see T4Q1).

Partial agreement

No additional trend was found in these comments. 
T4Q3: Specificity - Procedure; Lines 668-671: "Specificity is evaluated by spiking blank matrix samples with related molecules at the maximal concentration(s) of the structurally related molecule anticipated in study samples. The accuracy of the target analyte at the LLOQ and at the ULOQ should be investigated in the presence of related molecules at the maximal concentration(s) anticipated in study samples."

Specificity - Acceptance criteria; Lines 671-674: "The response of blank samples spiked with related molecules should be below the LLOQ. The accuracy of the target analyte in presence of related molecules should be within $\pm 25 \%$ of the nominal values."

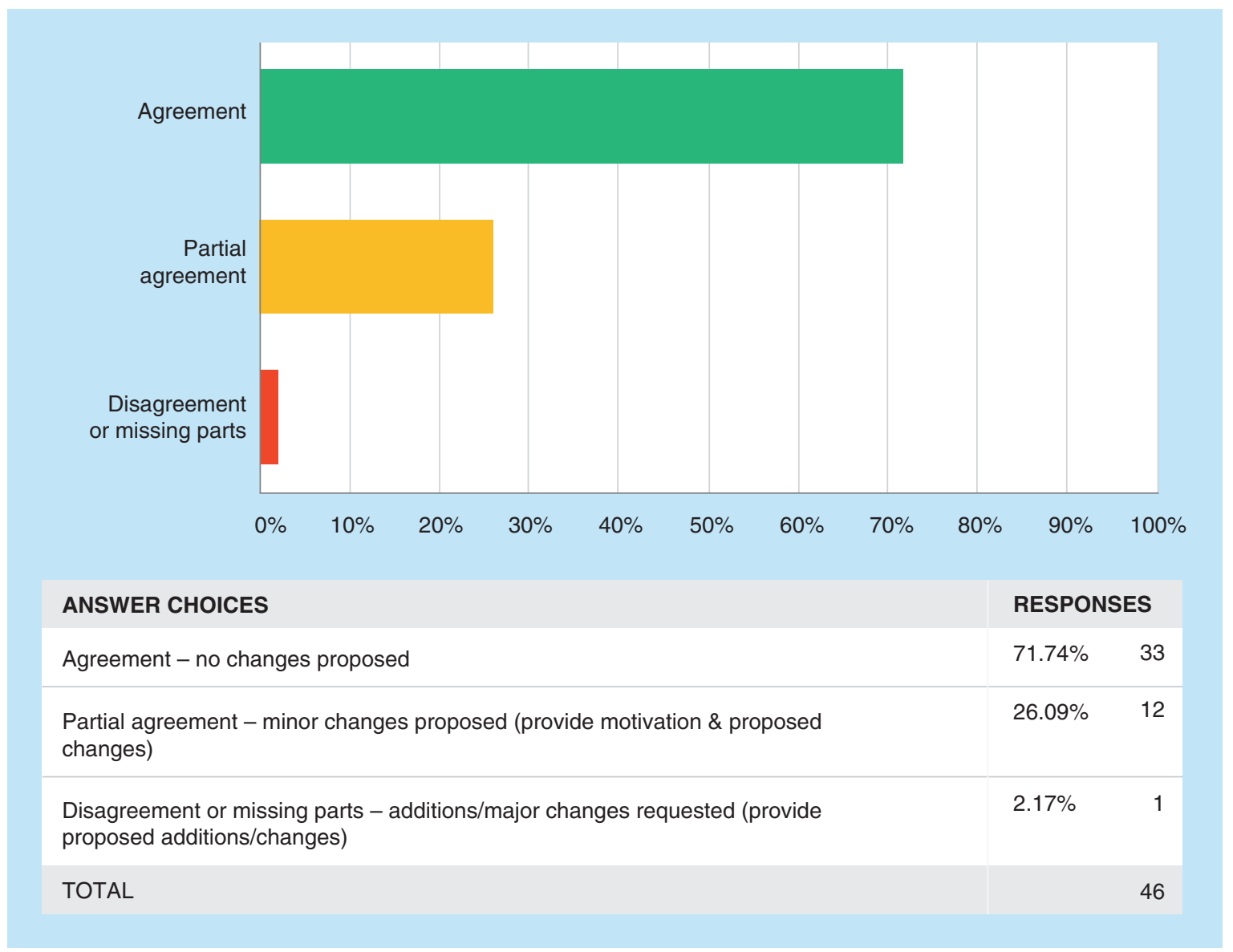

\section{Partiall agreement}

- Several comments suggested assessing specificity at HQC instead of the ULOQ and disagreement with the FDA Guidance.

- A few comments suggested clarifying or providing examples of “related molecules”. 
T4Q4: Specificity - Nonspecificity; Lines 675-680: "In the event of non-specificity, the impact on the method should be evaluated by spiking increasing concentrations of interfering molecules in blank matrix and measuring the accuracy of the target analyte at the LLOQ and ULOQ. It is essential to determine the minimum concentration of the related molecule where interference occurs. Appropriate mitigation during sample analysis should be employed, e.g., it may be necessary to adjust the LLOQ/ULOQ accordingly or consider a new method."

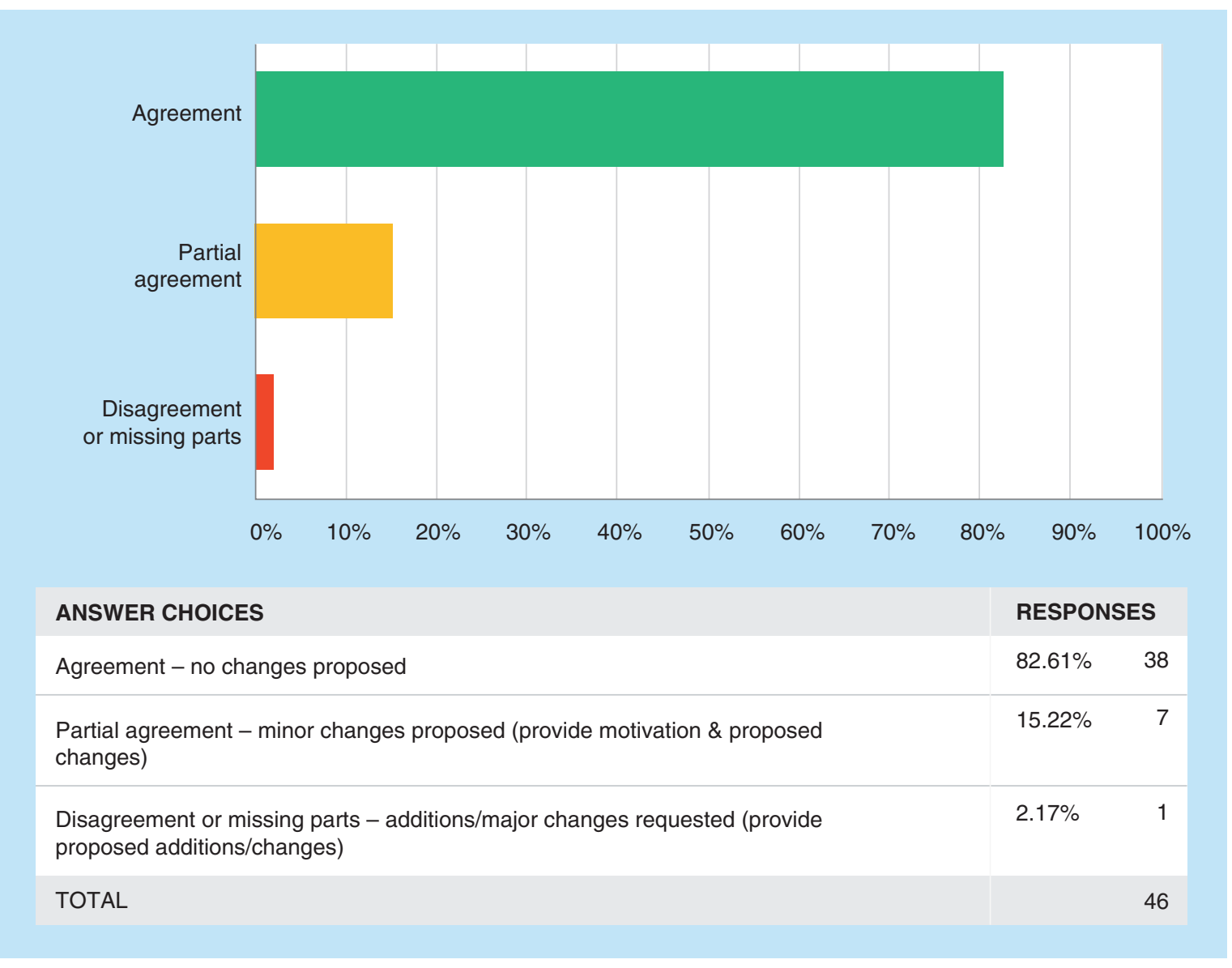

\section{Partial agreement}

As in T4Q3, several comments requested that assessing this evaluation at ULOQ be replaced with the HQC. 
T4Q5: Specificity - Additional Testing; Lines 681-683: "During method development and early assay validation, these "related molecules" are frequently not available. Additional evaluation of specificity may be conducted after the original validation is completed."

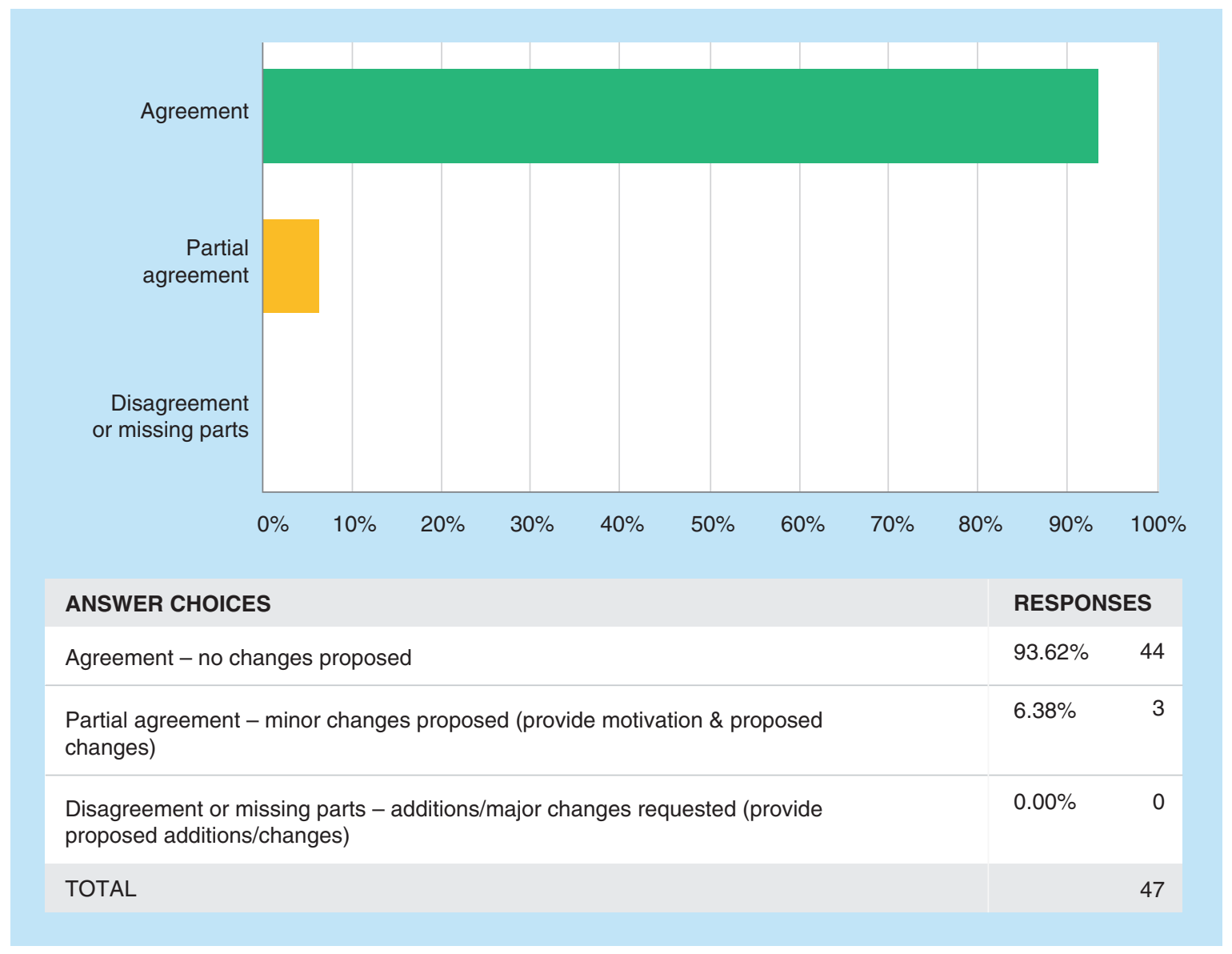

\section{Partial agreement}

No trend was found in these comments. 
T4Q6: Selectivity - Definition; Lines 685-688: "Selectivity is the ability of the method to detect and differentiate the analyte of interest in the presence of other "unrelated compounds" (non-specific interference) in the sample matrix. The matrix can contain non-specific matrix component such as degrading enzymes, heterophilic antibodies or rheumatoid factor which may interfere with the analyte of interest."

Selectivity - Procedure; Lines 689-692: "Selectivity should be evaluated at the low end of an assay where problems occur in most cases, but it is recommended that selectivity is also evaluated at higher analyte concentrations. Therefore, selectivity is evaluated using blank samples obtained from at least 10 individual sources and by spiking the individual blank matrices at the LLOQ and at the high QC level."

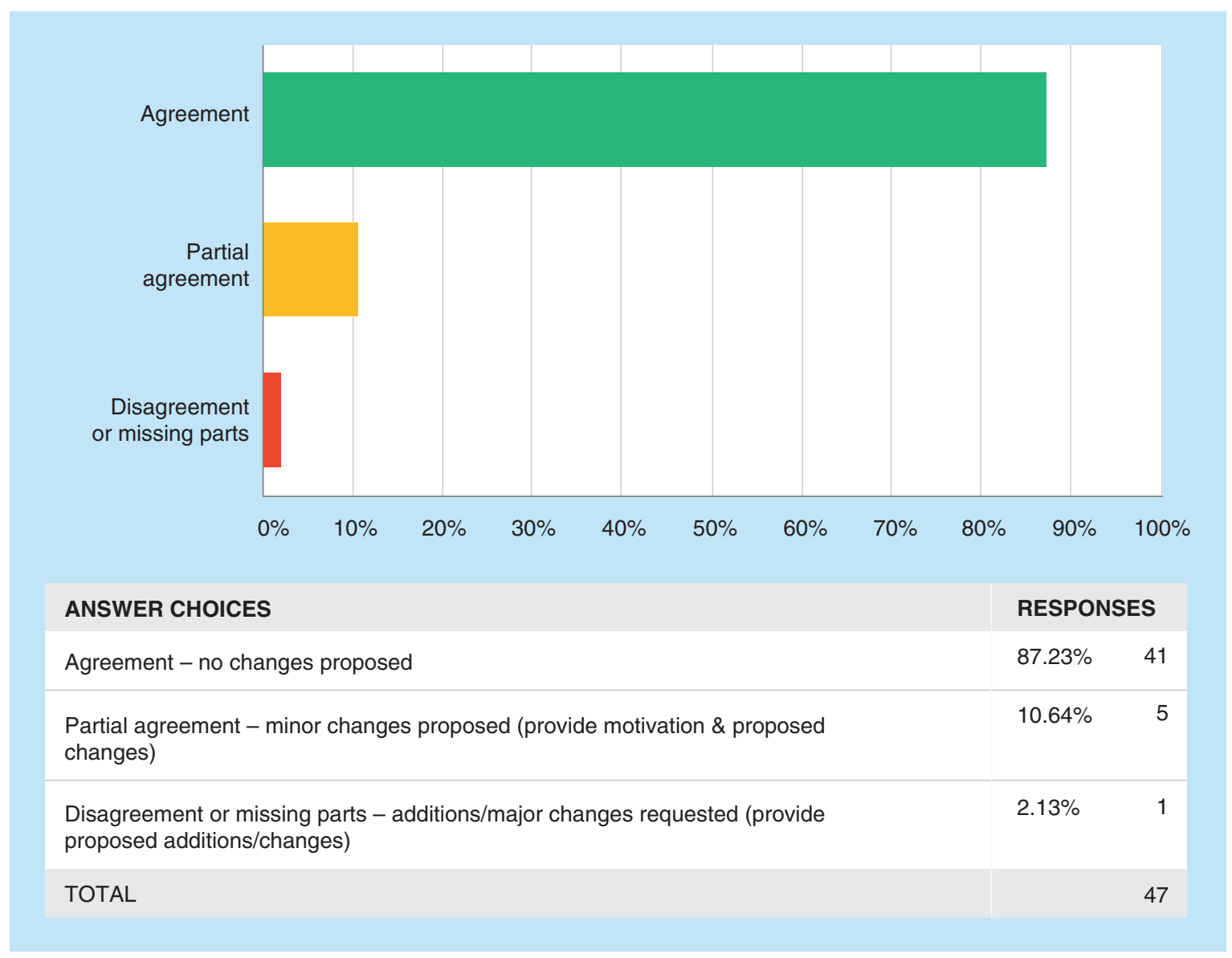

\section{Partial agreement}

It is worthy to mention that a few comments suggested using ULOQ rather than the high QC level.

\section{Additional comments from the 13th GCC:}

There were no further discussions during the GCC Closed Forum in support of the above proposal. 
T4Q7: Selectivity - Acceptance Criteria; Lines 693-696: "The response of the blank samples should be below the LLOQ in at least $80 \%$ of the individual sources. The accuracy should be within $\pm 25 \%$ at the LLOQ and within $\pm 20 \%$ at the high QC level of the nominal concentration in at least $80 \%$ of the individual sources evaluated."

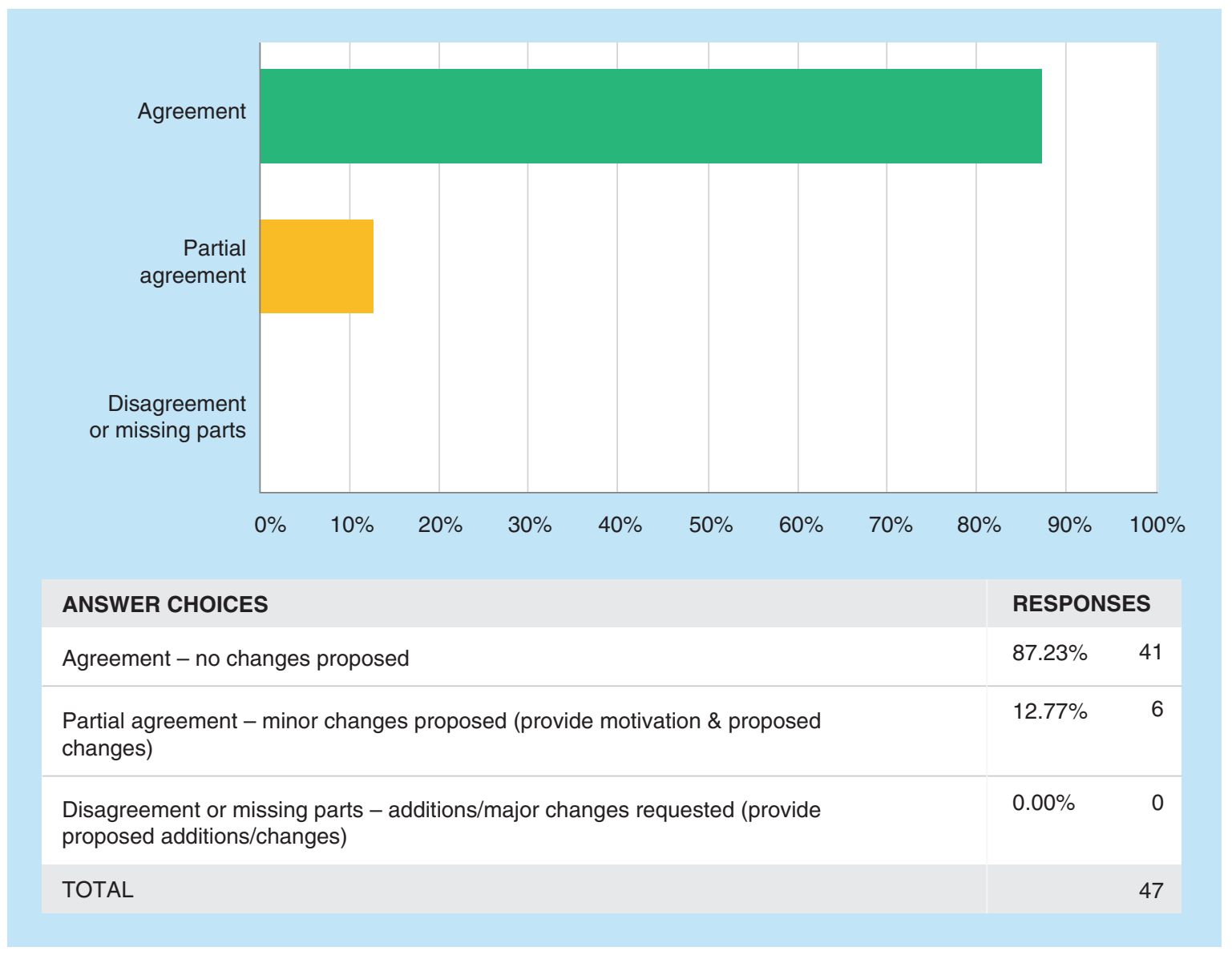

\section{Partial agreement}

It is worthy to mention that a few comments suggested using $25 \%$ at LLOQ and ULOQ.

\section{Additional comments from the 13th GCC:}

There were no further discussions during the GCC Closed Forum in support of the above proposal. 
T4Q8: Selectivity - Hemolysis and Lipaemic; Lines 697-699: "Selectivity should be evaluated in lipaemic samples and haemolysed samples (Refer to Section 697 3.2.1). For lipaemic and haemolysed samples, tests can be evaluated once using a single source of matrix."

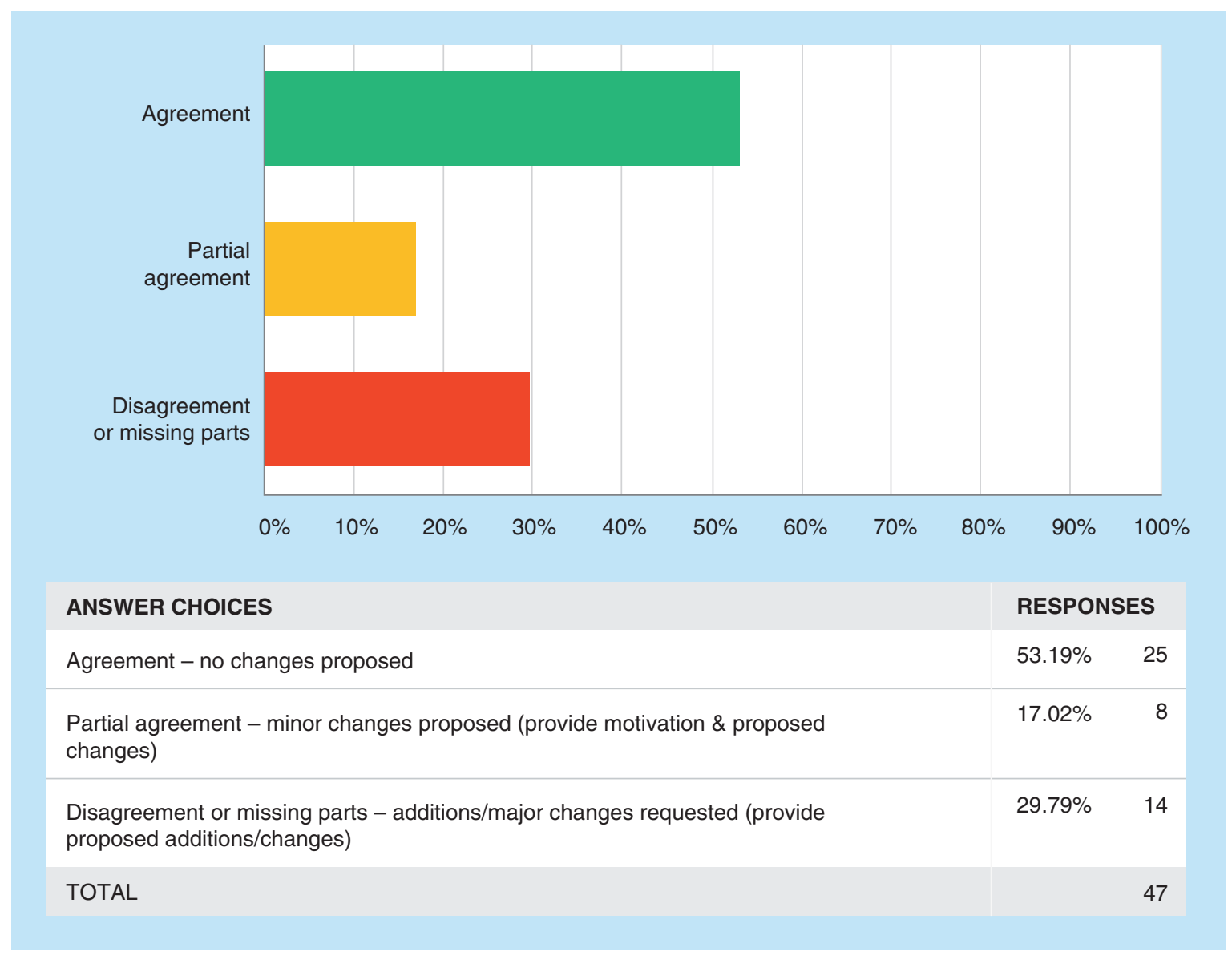

Disagreement or missing parts

- Several comments suggested that full text be provided in this section directed specifically for LBA as opposed to referring to the chromatography section.

- Specific limits for both lipaemic (>300 mg/mL) and haemolysed ( $2 \% \mathrm{v} / \mathrm{v})$ samples should be added.

Partial agreement

No additional trend was found in these comments. 
T4Q9: Selectivity - Patient Populations; Lines 699-700: "Selectivity should be assessed in samples from relevant patient populations. In the case of relevant patient populations there should be at least five individual patients."

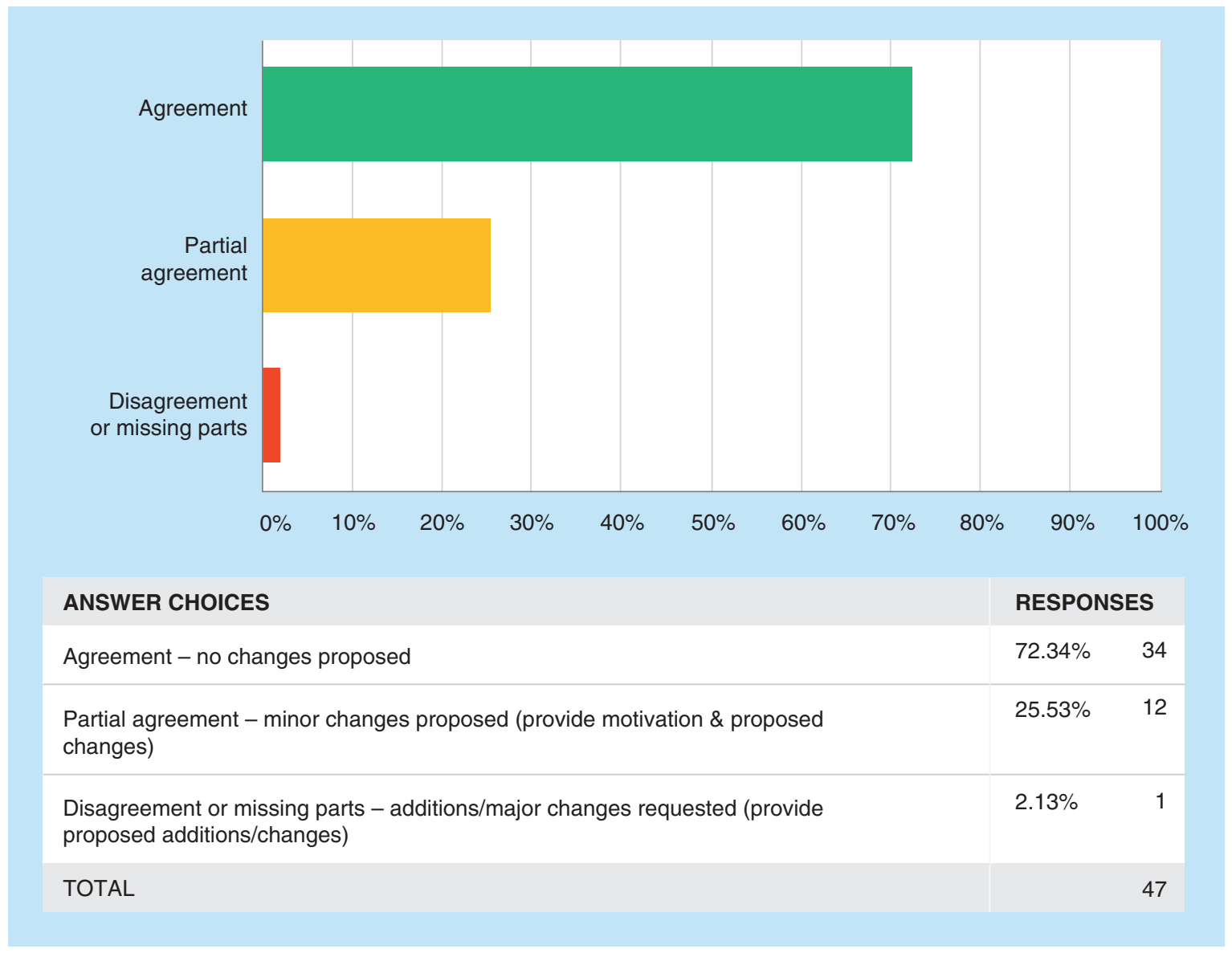

\section{Partial agreement}

Several comments suggested that the wording be revised to at least ten individual patients, and that "if possible" be added to the end of the sentence to account for rare matrices. 
T4Q10: Calibration Curve - Definition; Lines 702-705: "The calibration curve demonstrates the relationship between the nominal analyte concentration and the response of the analytical platform to the analyte. Calibration standards, prepared by spiking matrix with a known quantity of analyte, span the calibration range and comprise the calibration curve."

Calibration Curve - Calibration Standards; Lines 706-708: "Calibration standards should be prepared in the same biological matrix as the study samples. The calibration range is defined by the LLOQ, which is the lowest calibration standard, and the ULOQ, which is the highest calibration standard. There should be one calibration curve for each analyte studied during method validation and for each analytical run."

Calibration Curve - Contents; Lines 709-713: "A calibration curve should be generated with at least 6 concentration levels of calibration standards, including LLOQ and ULOQ standards, plus a blank sample. The blank sample should not be included in the calculation of calibration curve parameters. Anchor point samples at concentrations below the LLOQ and above the ULOQ of the calibration curve may also be used to improve curve fitting."

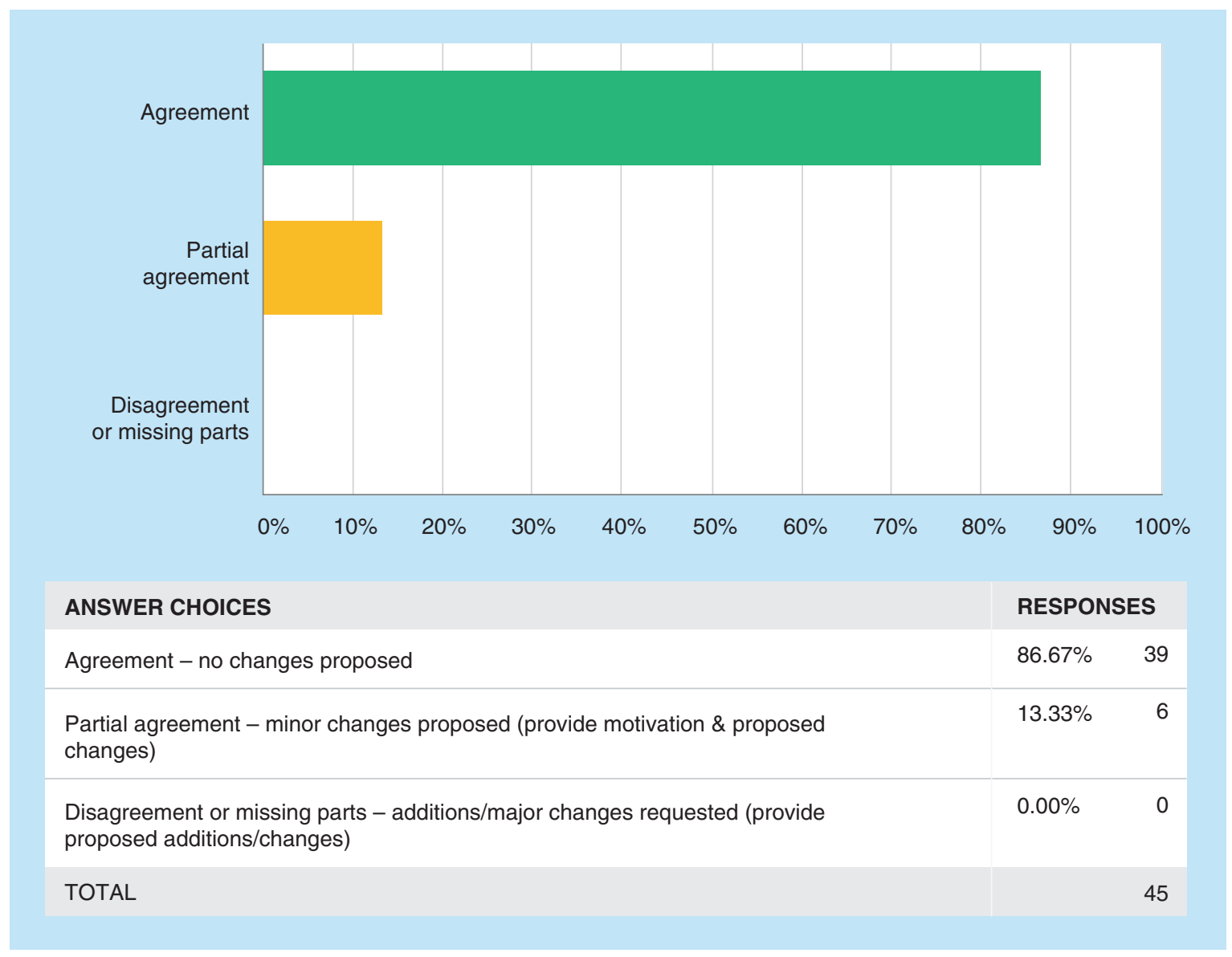

\section{Partial agreement}

- In order to clarify that anchor points are not included, it was recommended to add the bold text to the sentence "The calibration range is defined by the $L L O Q$, which is the lowest calibration standard demonstrating acceptable accuracy and precision, and the ULOQ, which is the highest calibration standard, demonstrating acceptable accuracy and precision."

- It was suggested to add language specifying when anchor points can be masked.

- It should be also mentioned that a few comments strongly recommended adding language to allow use of a surrogate matrix for calibration standards in the case of, for example, endogenous compounds or rare matrices. 
T4Q11: Calibration Curve - Linearity; Lines 713-716: "The relationship between response and concentration for a calibration curve is most often fitted by a 4-or 5-parameter logistic model if there are data points near the lower and upper asymptotes, although other models may be used with suitable justification."

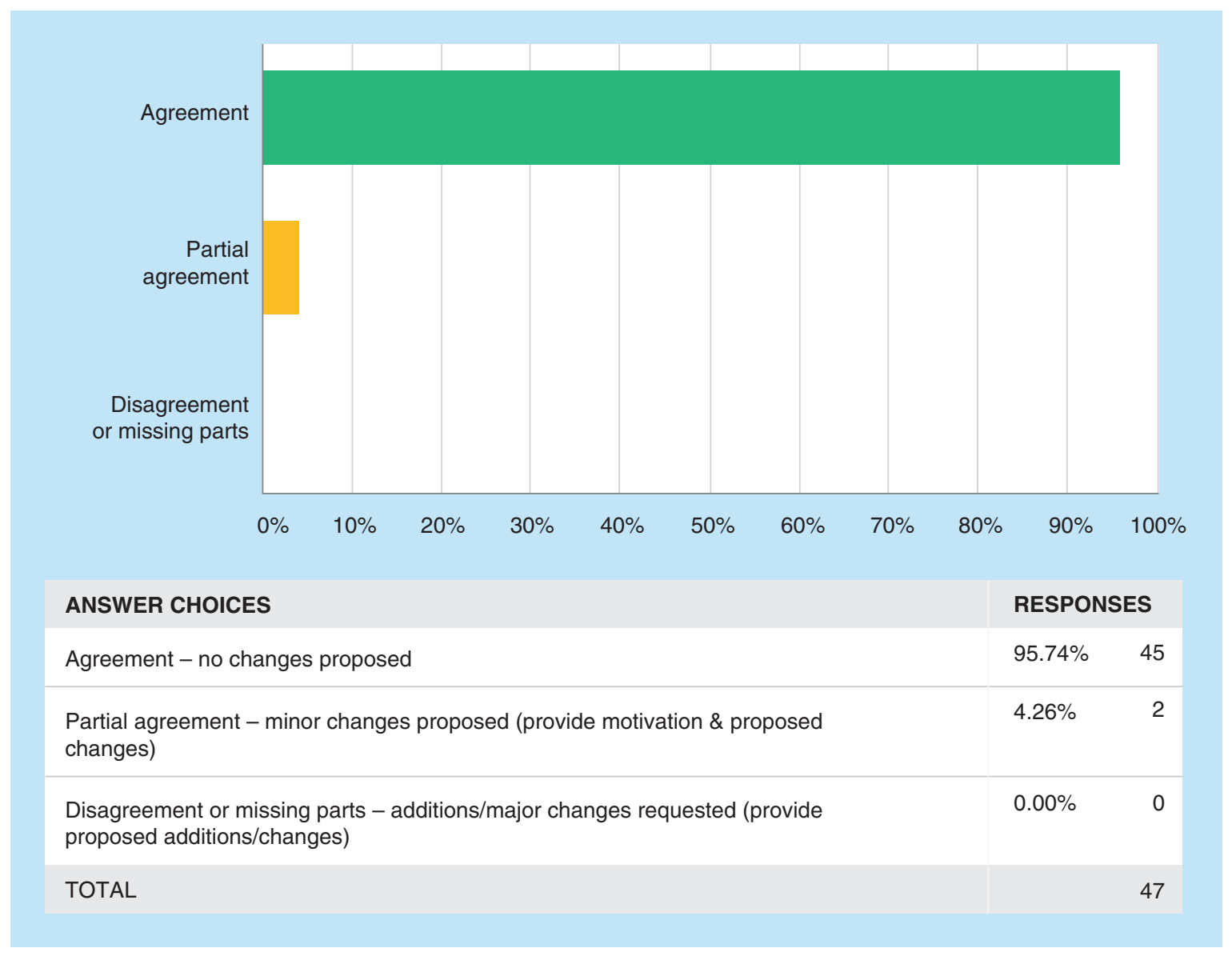

\section{Partial agreement}

It may be worth noting that both comments suggested adding language around the use of appropriate weighting. 
T4Q12: Calibration Curve - Acceptance Criteria; Lines 717-724: "A minimum of 6 independent runs should be evaluated over several days considering the factors that may contribute to between-run variability. The accuracy and precision of back-calculated concentrations of each calibration standard should be within $\pm 25 \%$ of the nominal concentration at the $L L O Q$ and $U L O Q$, and within $\pm 20 \%$ at all other levels. At least $75 \%$ of the calibration standards excluding anchor points, and a minimum of 6 concentration levels of calibration standards, including the LLOQ and ULOQ, should meet the above criteria. The anchor points do not require acceptance criteria since they are beyond the quantifiable range of the curve."

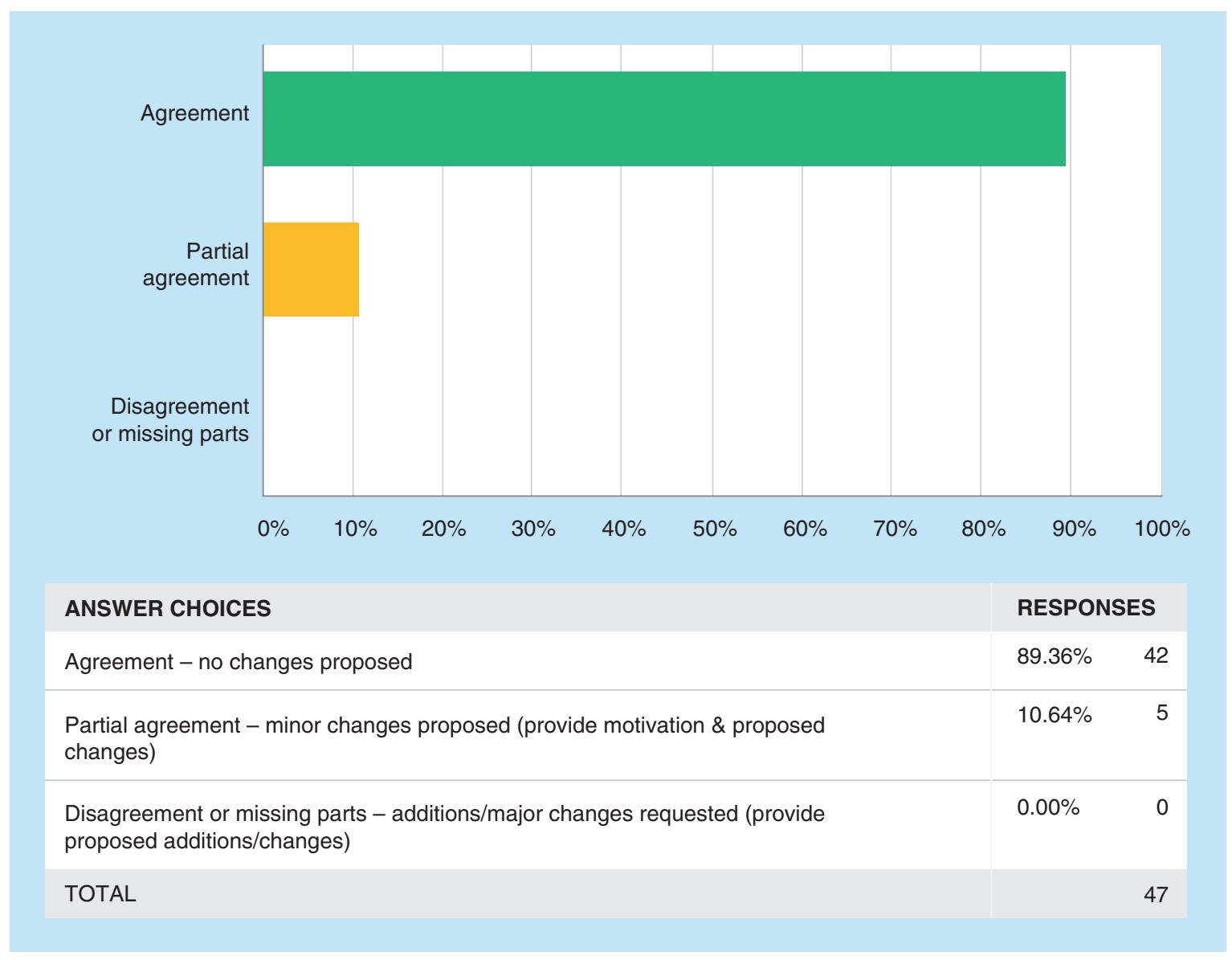

\section{Partial agreement}

It is worthy to mention that a few comments suggest the addition that consecutive calibrations standards should not be masked and to state that anchor points can be removed to improve curve fit.

\section{Additional comments from the 13th GCC:}

There was a general agreement that anchor points may be removed to improve curve fit. 
T4Q13. Calibration Curve-fresh spike; Lines 725-727: "The calibration curve should preferably be prepared using freshly spiked calibration standards. If freshly spiked calibration standards are not used, the frozen calibration standards can be used within their defined period of stability."

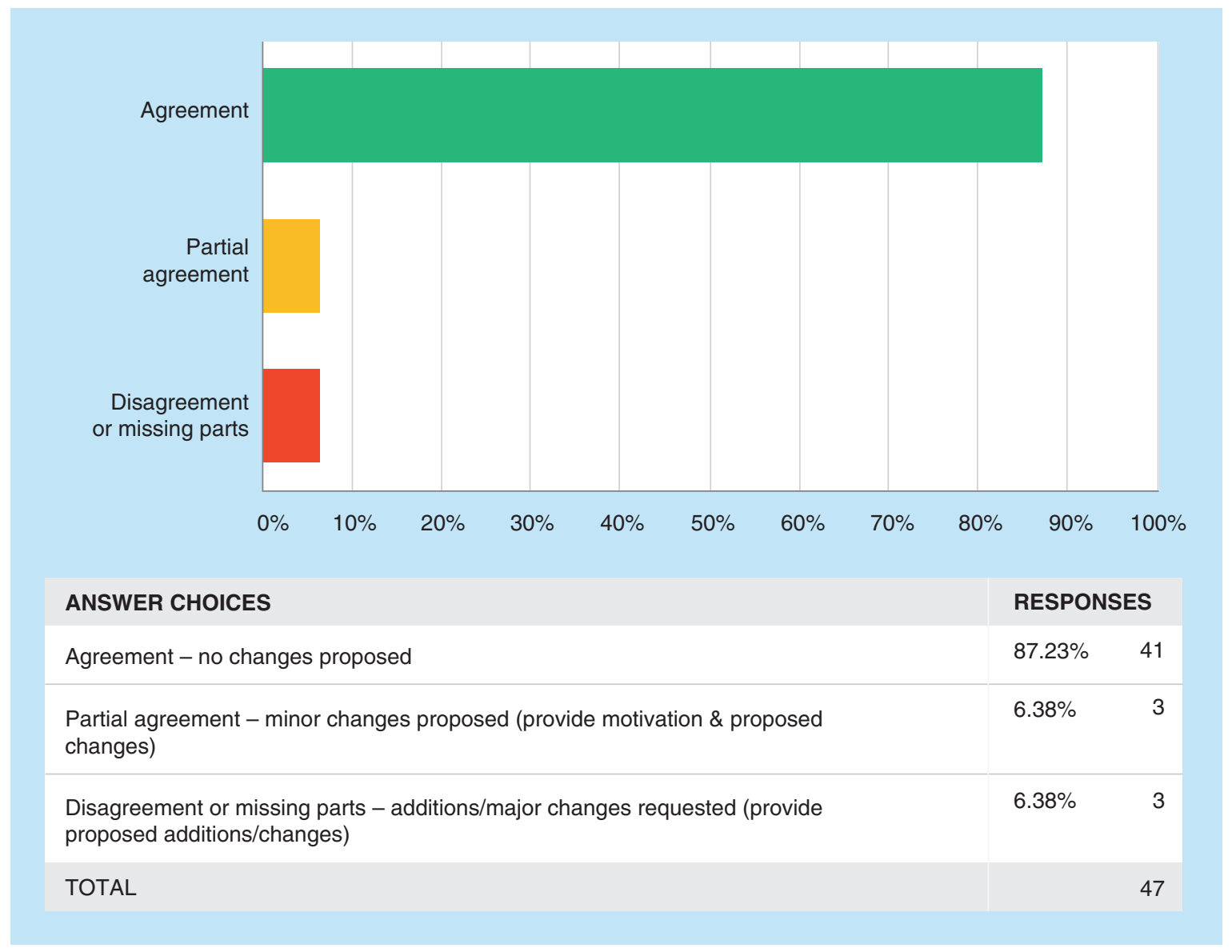

\section{Partial agreement}

There was overall disagreement demonstrated in the comments with the requirement to "preferably" use freshly spiked calibration standards. It was suggested to clarify that this recommendation is "for validation experiments" and remove "preferably" to be aligned with the chromatographic assay section. 
T4Q14: QCs for Validation - Purpose; Lines 730-732: "The QCs are intended to mimic study samples and should be prepared by spiking matrix with a known quantity of analyte, stored under the conditions anticipated for study samples and analysed to assess the validity of the analytical method."

QCs for Validation - Preparation; Lines 733-735: "The dilution series for the preparation of the QCs should be completely independent from the dilution series for the preparation of calibration standard samples. They may be prepared from a single stock provided that its accuracy has been verified or is known."

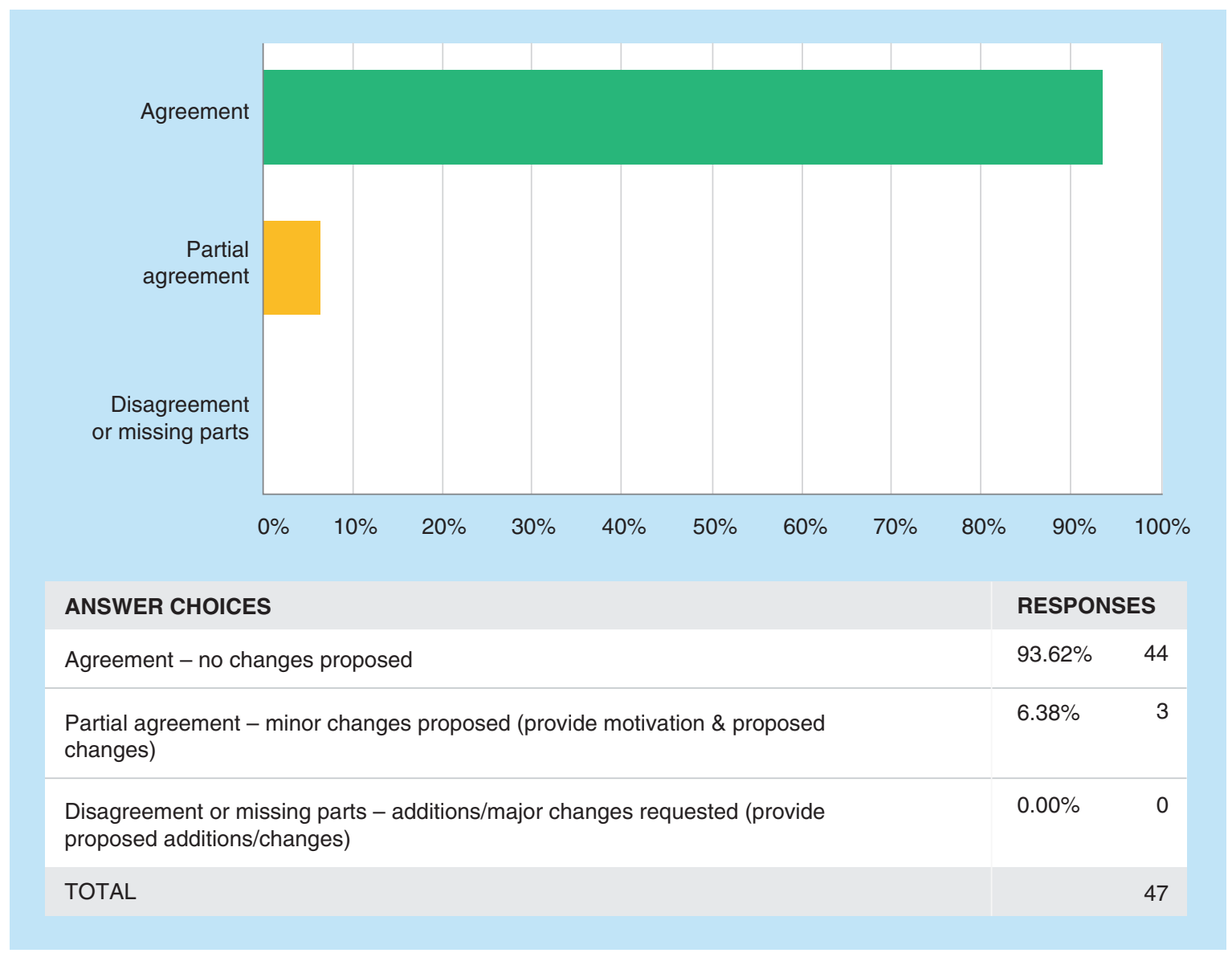

Partial agreement

No trend was found in these comments. 
T4Q15: QCs for Validation - Concentration Levels; Lines 735-739: "The QCs should be prepared at a minimum of 5 concentration levels within the calibration curve range: The analyte should be spiked at the LLOQ, within three times of the LLOQ (low QC), around the geometric mean of the calibration curve range (medium QC), and at least at 75\% of the ULOQ (high QC) and at the ULOQ."

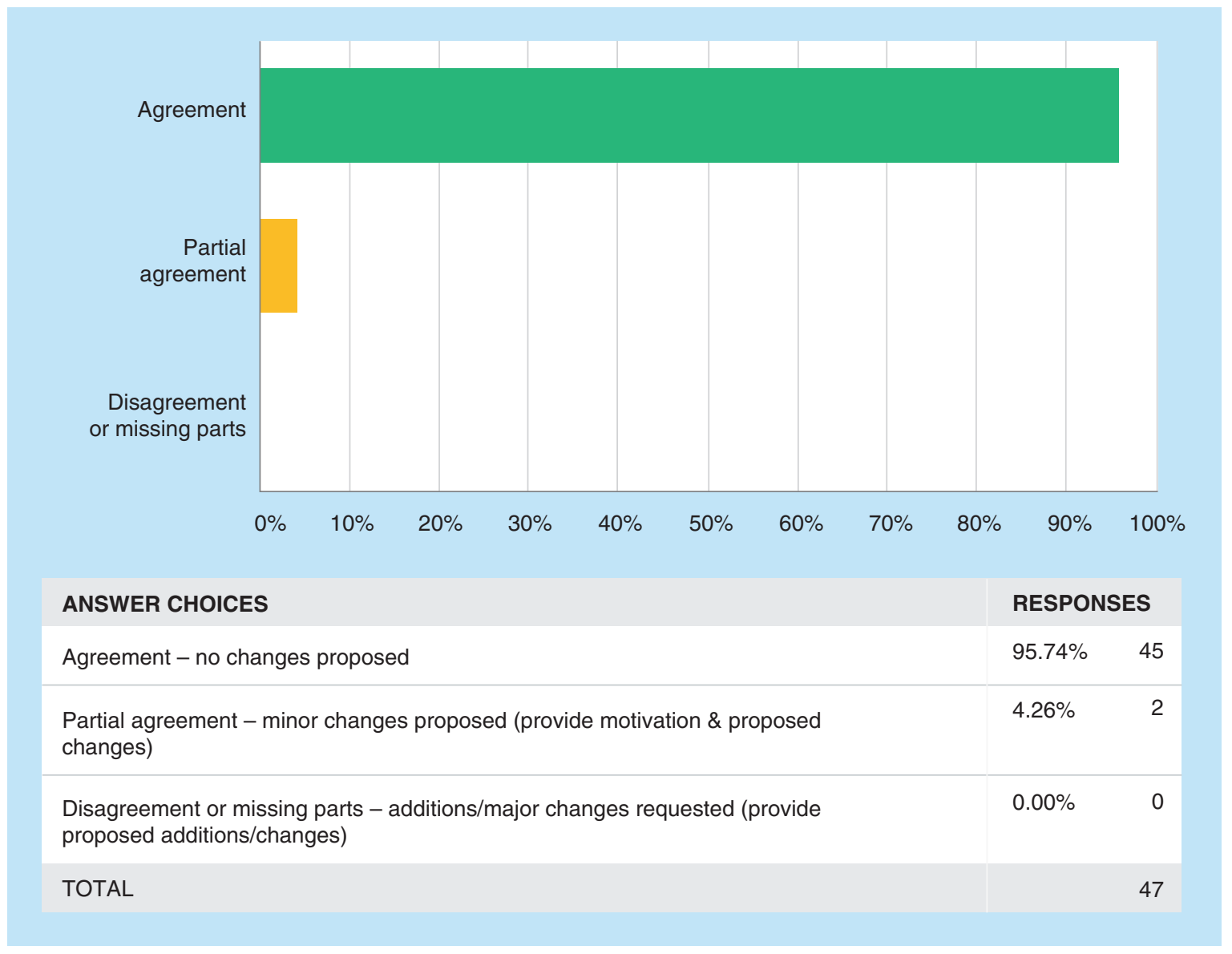

\section{Partial agreement}

No trend was found in these comments. 
T4Q16: Accuracy and Precision - General; Lines 741-743: "Accuracy and precision should be determined by analysing the QCs within each run (within-run) and in different runs (between-run). Accuracy and precision should be evaluated using the same runs and data."

Accuracy and Precision - Procedure; Lines 744-746: "Accuracy and precision should be determined by analysing at least 3 replicates per run at each QC concentration level (LLOQ, low, medium, high, ULOQ) in at least 6 runs over 2 or more days."

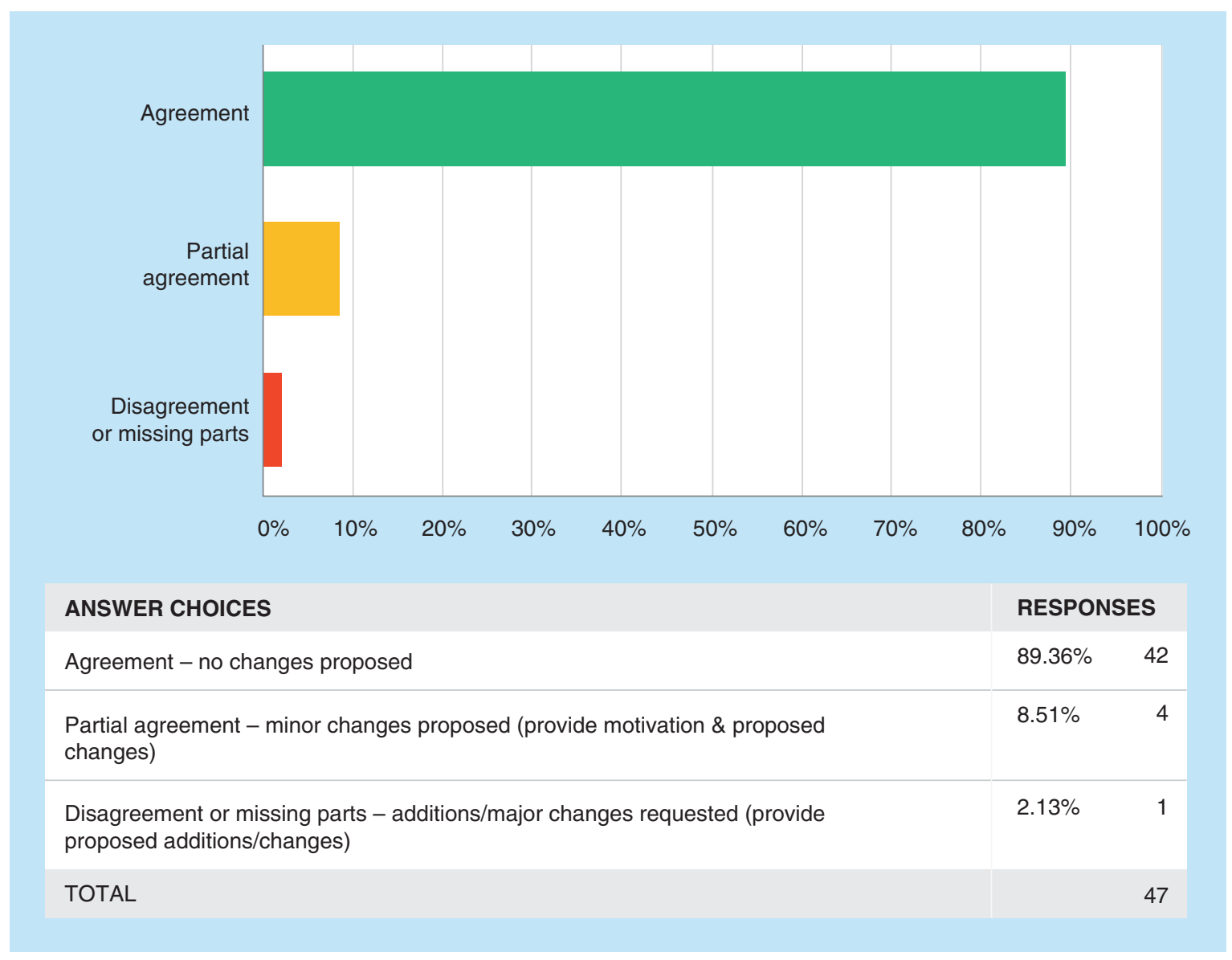

Partial agreement

No trend was found in these comments. 
T4Q17: Accuracy and Precision - Reporting; Lines 746-751: "Reported method validation data and the determination of accuracy and precision should include all results obtained, except those cases where errors are obvious and documented. Within-run accuracy and precision data should be reported for each run. If the within-run accuracy or precision criteria are not met in all runs, an overall estimate of within-run accuracy and precision for each QC level should be calculated. Between-run (intermediate) precision and accuracy should be calculated by combining the data from all runs."

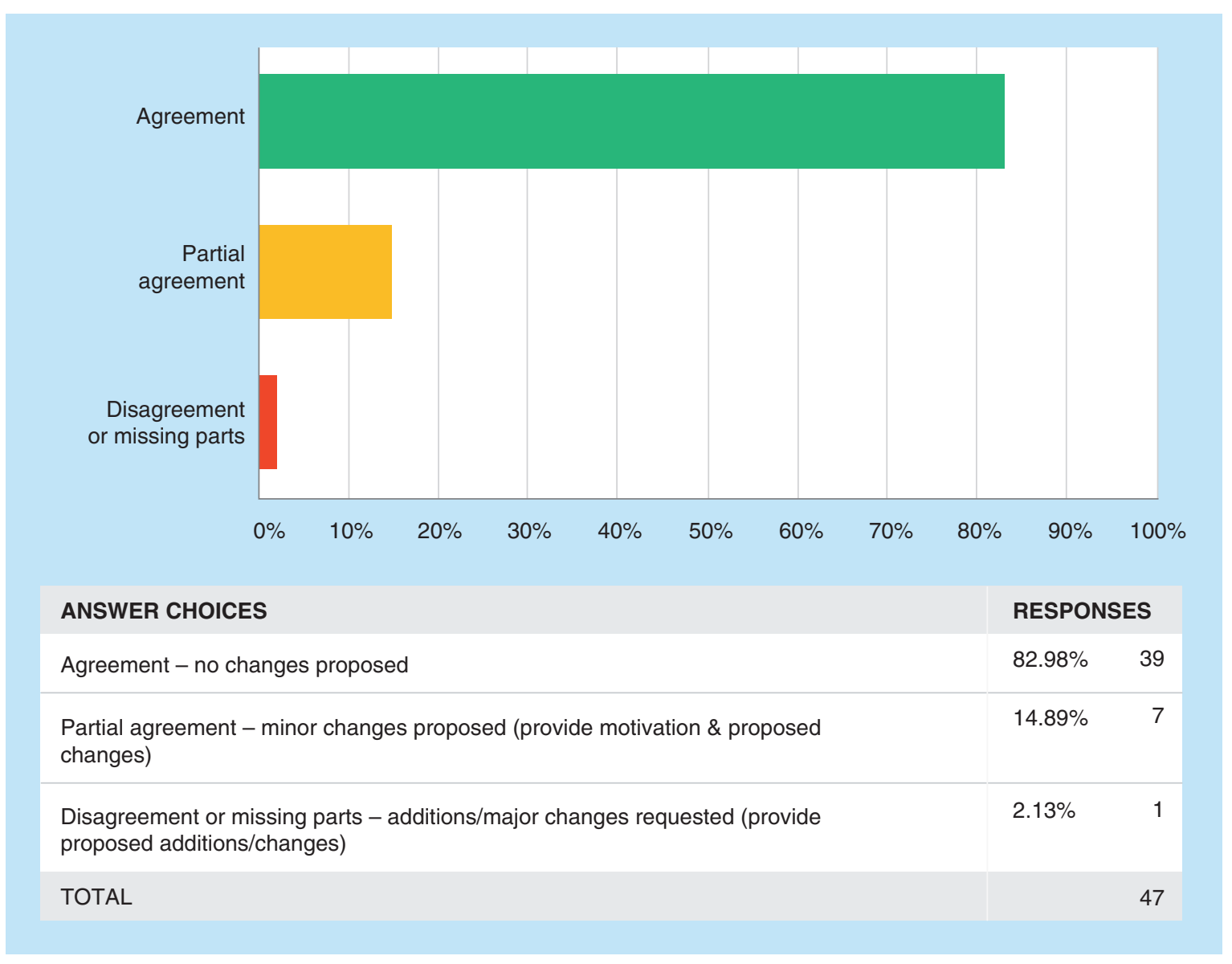

\section{Partial agreement}

Clarity was requested regarding how to proceed if the within-run accuracy and precision are not met in all runs. More details were recommended to outline how the required "overall estimate" would be performed. 
T4Q18: Accuracy and Precision - Acceptance Criteria; Lines 752-759: "The overall within-run and between-run accuracy at each concentration level should be within $\pm 20 \%$ of the nominal values, except for the LLOQ and ULOQ, which should be within $\pm 25 \%$ of the nominal value. Within-run and between-run precision of the QC concentrations determined at each level should not exceed 20\%, except at the LLOQ and ULOQ, where it should not exceed 25\%. Furthermore, the total error (i.e., sum of absolute value of the errors in accuracy (\%) and precision (\%)) should be evaluated. The total error should not exceed 30\% (40\% at LLOQ and ULOQ)."

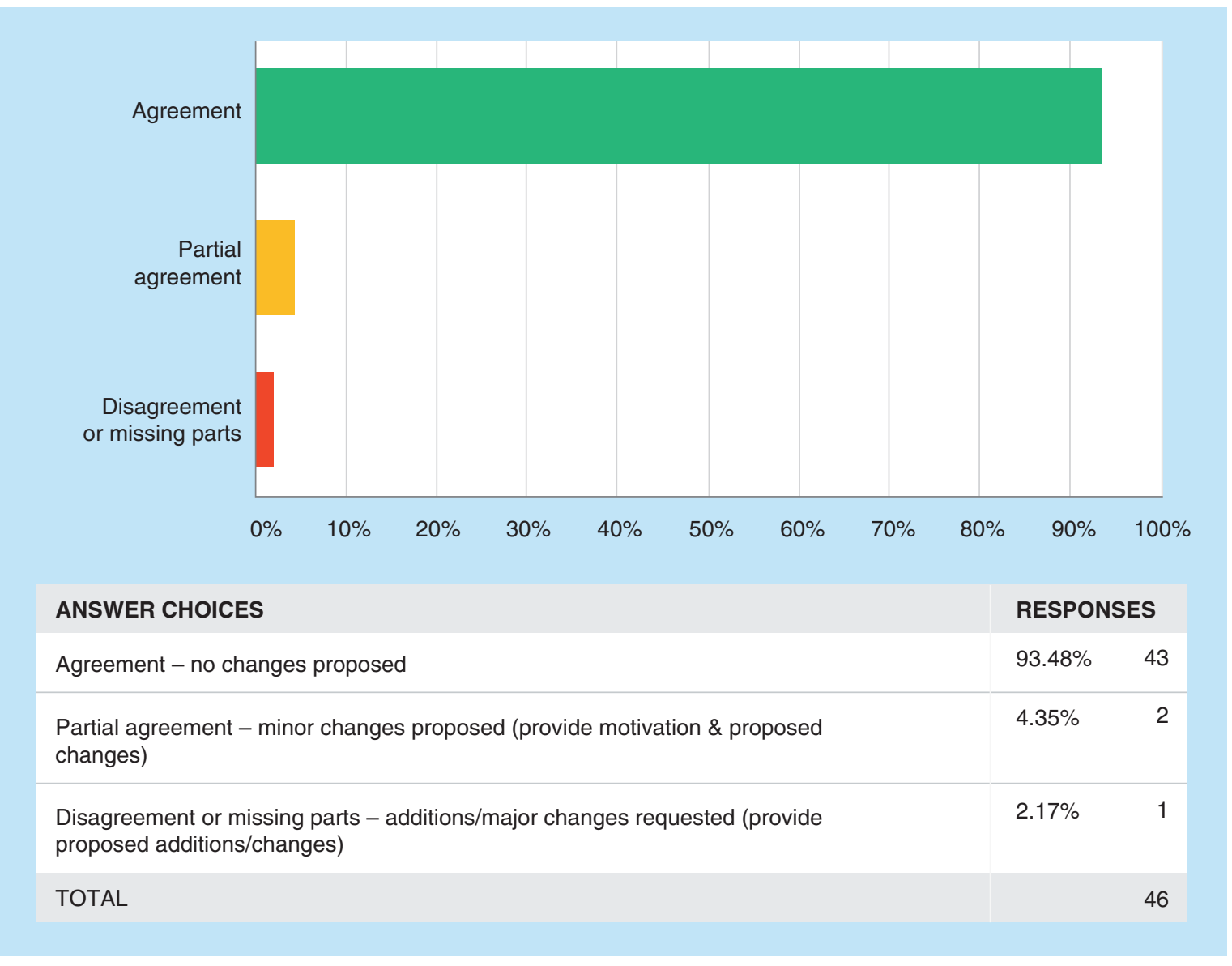

Partial agreement

It was suggested to clarify what "overall" refers to. 
T4Q19: Carryover; Lines 761-764: "Carry-over is generally not an issue for LBA analyses. However, if the assay platform is prone to carry-over, the potential of carry-over should be investigated by placing blank samples after the calibration standard at the ULOQ. The response of blank samples should be below the LLOQ."

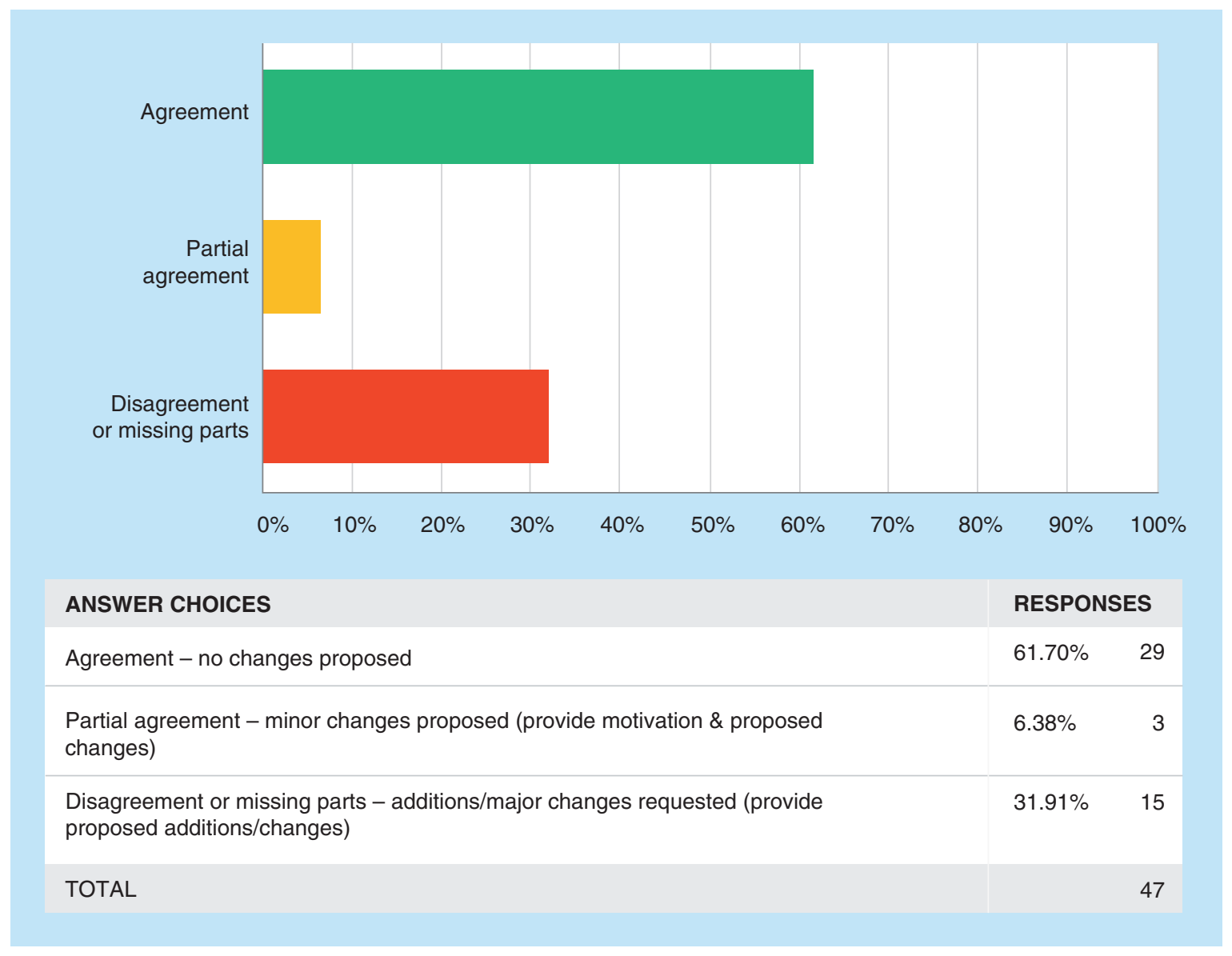

\section{Disagreement or missing parts}

There was strong disagreement with this section. LBA methods have not previously required carryover assessments. Therefore, it was highly recommended to rewrite the section with focus on LBA rather than chromatographic assays, with more details and criteria around regulatory expectations for methods using Gyros or automated LBAs.

\section{Partial agreement}

No additional trend was found in these comments.

\section{Additional comments from the 13th GCC:}

The survey comments were confirmed during the GCC Closed Forum. 
T4Q20: Dilution Linearity and Hook Effect - Purpose; Lines 766-771: "Due to the narrow assay range in many $L B A s$, study samples may require dilution in order to achieve analyte concentrations within the range of the assay. Dilution linearity is assessed to confirm: (i) that measured concentrations are not affected by dilution within the calibration range and (ii) that sample concentrations above the ULOQ of a calibration curve are not impacted by hook effect (i.e., a signal suppression caused by high concentrations of the analyte), whereby yielding an erroneous result."

Dilution Linearity and Hook Effect - Procedure; Lines 772-778: "The same matrix as that of the study sample should be used for preparation of the QCs for dilution. Dilution linearity should be demonstrated by generating a dilution $Q C$, i.e., spiking the matrix with an analyte concentration above the ULOQ, analysed undiluted (for hook effect) and diluting this sample (to at least 3 different dilution factors) with blank matrix to a concentration within the calibration range. For each dilution factor tested, at least 3 runs should be performed using the number of replicates that will be used in sample analysis."

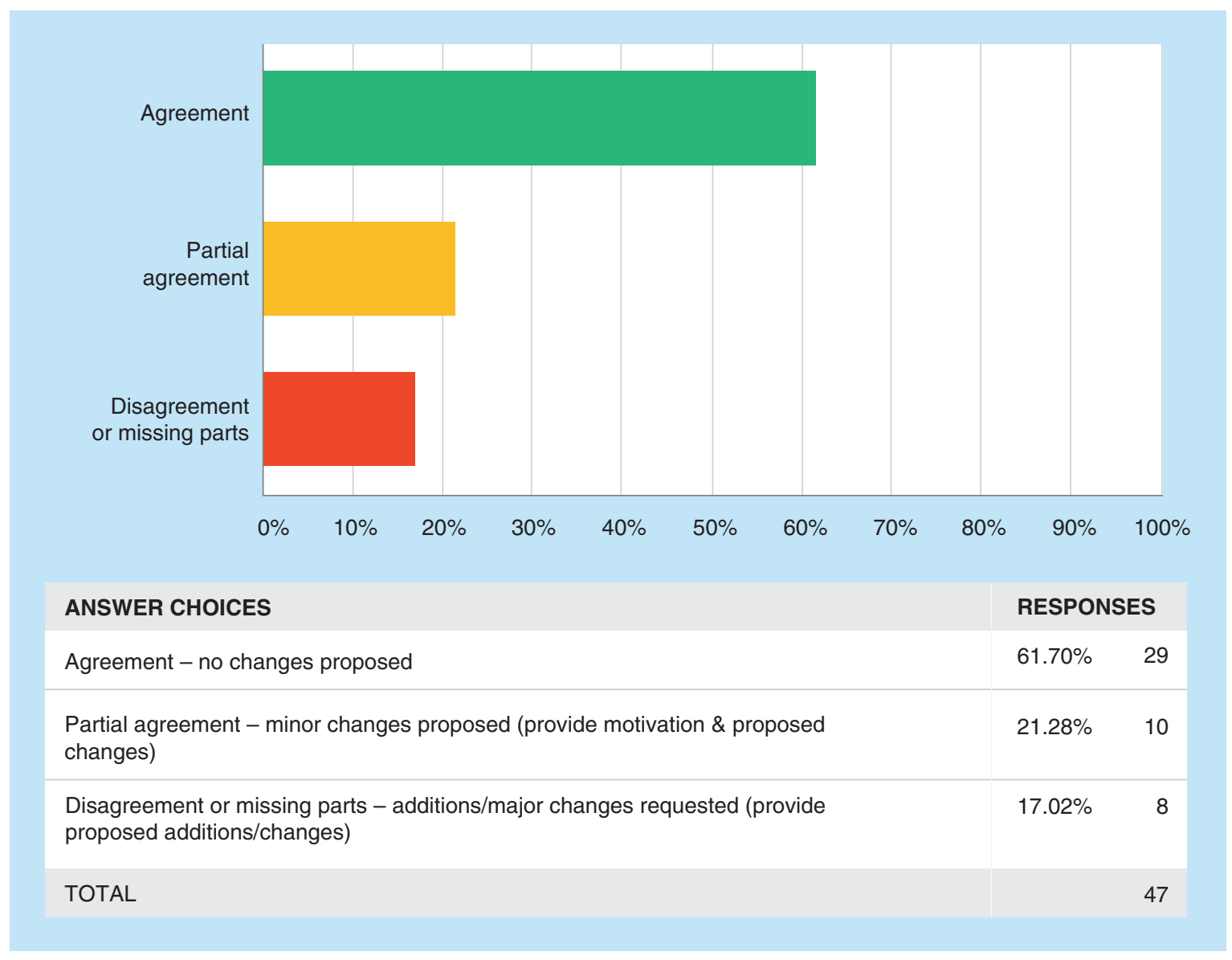

Disagreement or missing parts

The majority of comments disagreed with the requirement to perform three runs for Dilution Linearity. It is recommended that one run with three independently prepared replicates per dilution factor is sufficient.

Partial agreement

No additional trend was found in these comments.

Additional comments from the 13th GCC:

The survey comments were confirmed during the GCC Closed Forum. 
T4Q21: Dilution Linearity and Hook Effect - Acceptance Criteria Hook Effect; Lines 778-780: "The absence or presence of response reduction (hook effect) is checked in the dilution QCs and, if observed, measures should be taken to eliminate response reduction during the analysis of study samples."

Dilution Linearity and Hook Effect - Acceptance Criteria Dilution Linearity; Lines 781-783: "The calculated concentration for each dilution should be within $\pm 20 \%$ of the nominal concentration after correction for dilution and the precision of the final concentrations across all the dilutions should not exceed 20\%."

Dilution Linearity and Hook Effect - Dilution Factors; Lines 784-785: "The dilution factor(s) applied during study sample analysis should be within the range of dilution factors evaluated during validation."

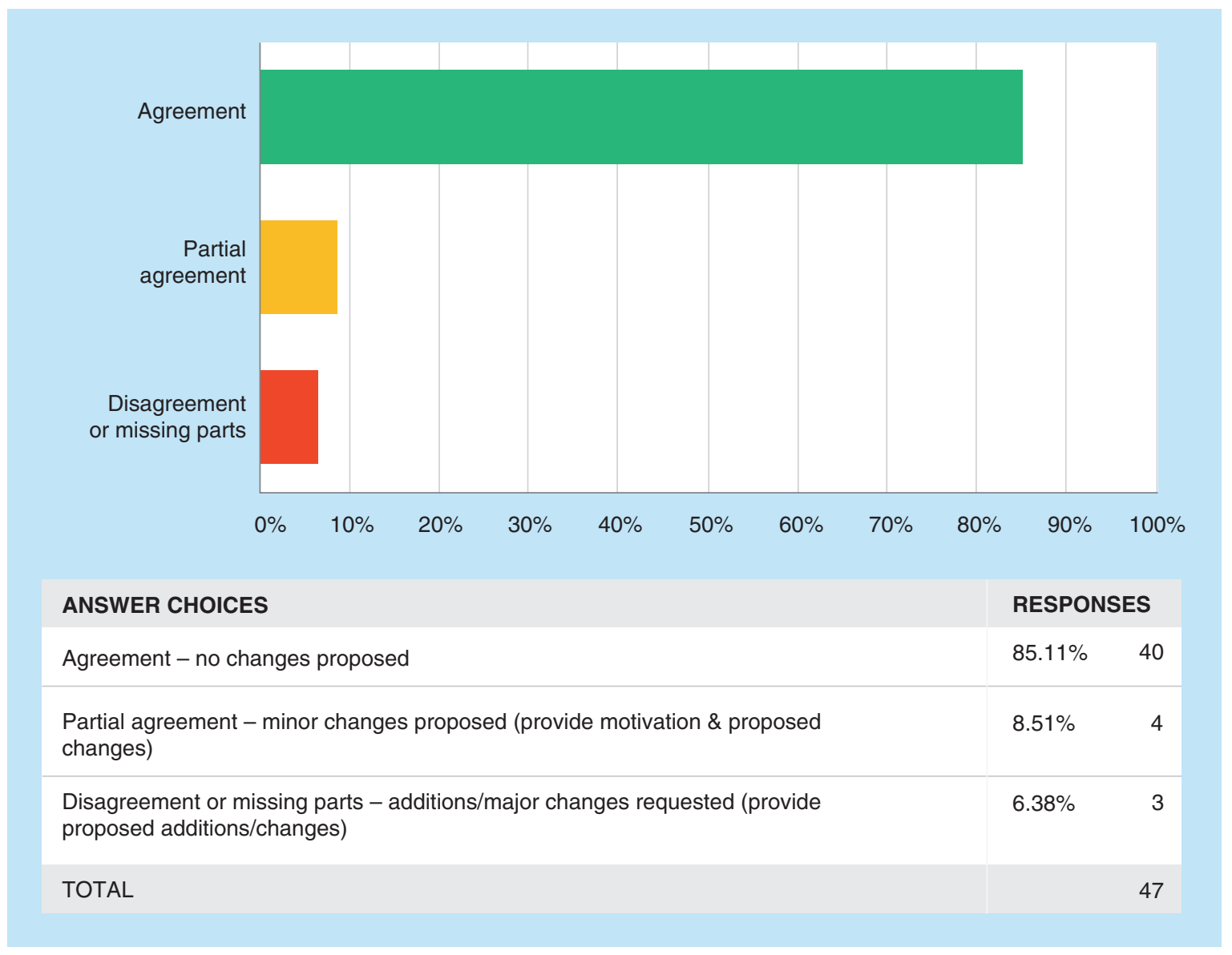

Disagreement or missing parts

No trend was found in these comments.

\section{Partial agreement}

No trend was found in these comments.

\section{Additional comments from the 13th GCC:}

It was recommended to add the statement to the end of this section: "When not possible, an alternate approach may be used." 
T4Q22: Stability - General; Lines 787-792: "Stability evaluations should be carried out to ensure that every step taken during sample preparation, processing and analysis as well as the storage conditions used do not affect the concentration of the analyte. The storage and analytical conditions applied to the stability tests, such as the sample storage times and temperatures, sample matrix, anticoagulant, and container materials should reflect those used for the study samples."

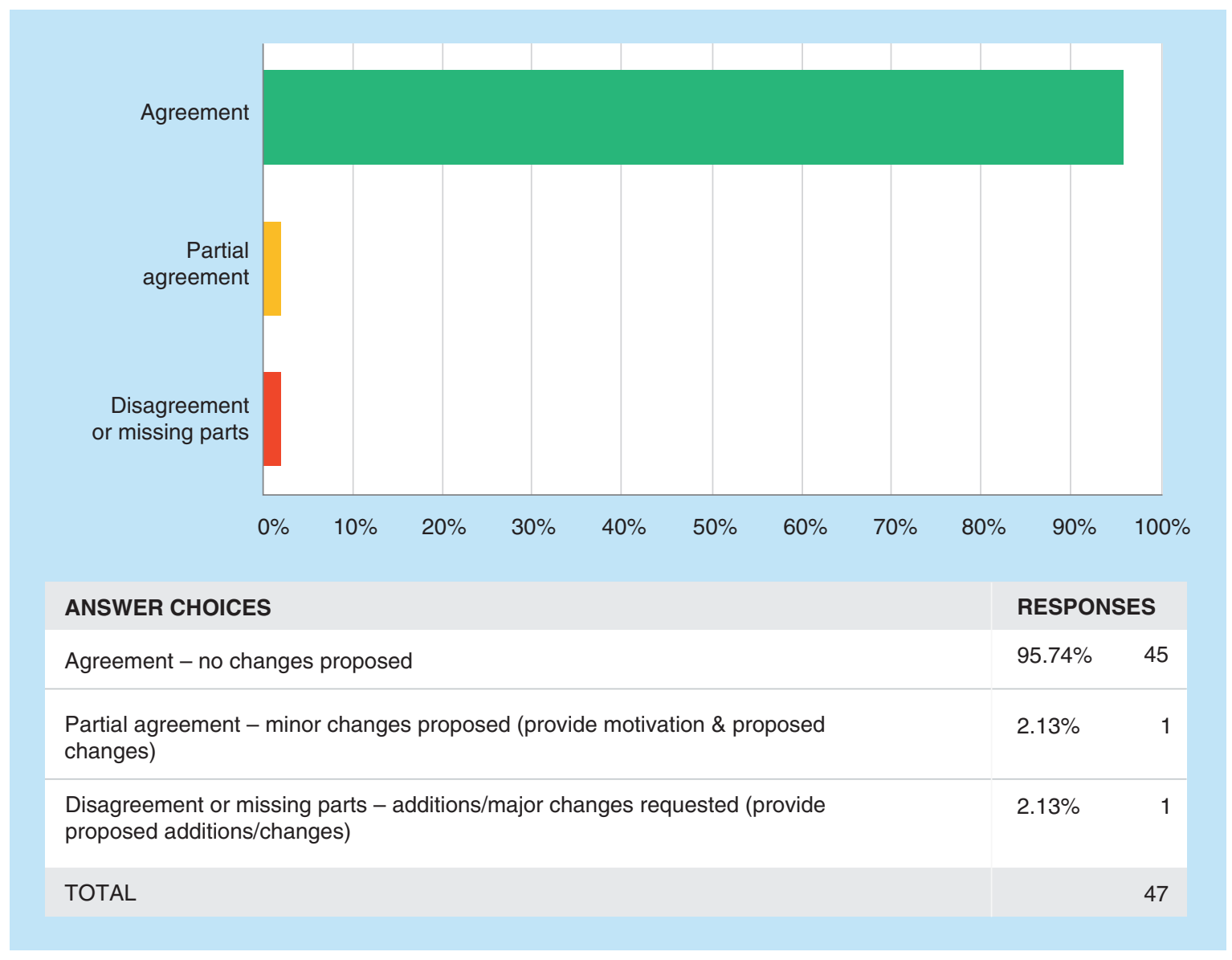

\section{Additional comments from the 13th GCC:}

lt was suggested that full text be provided in this section directed specifically for LBA as opposed to referring to the chromatography section. 
T4Q23: Stability - Reference to Literature; Lines 792-793: "Reference to data published in the literature is not considered sufficient."

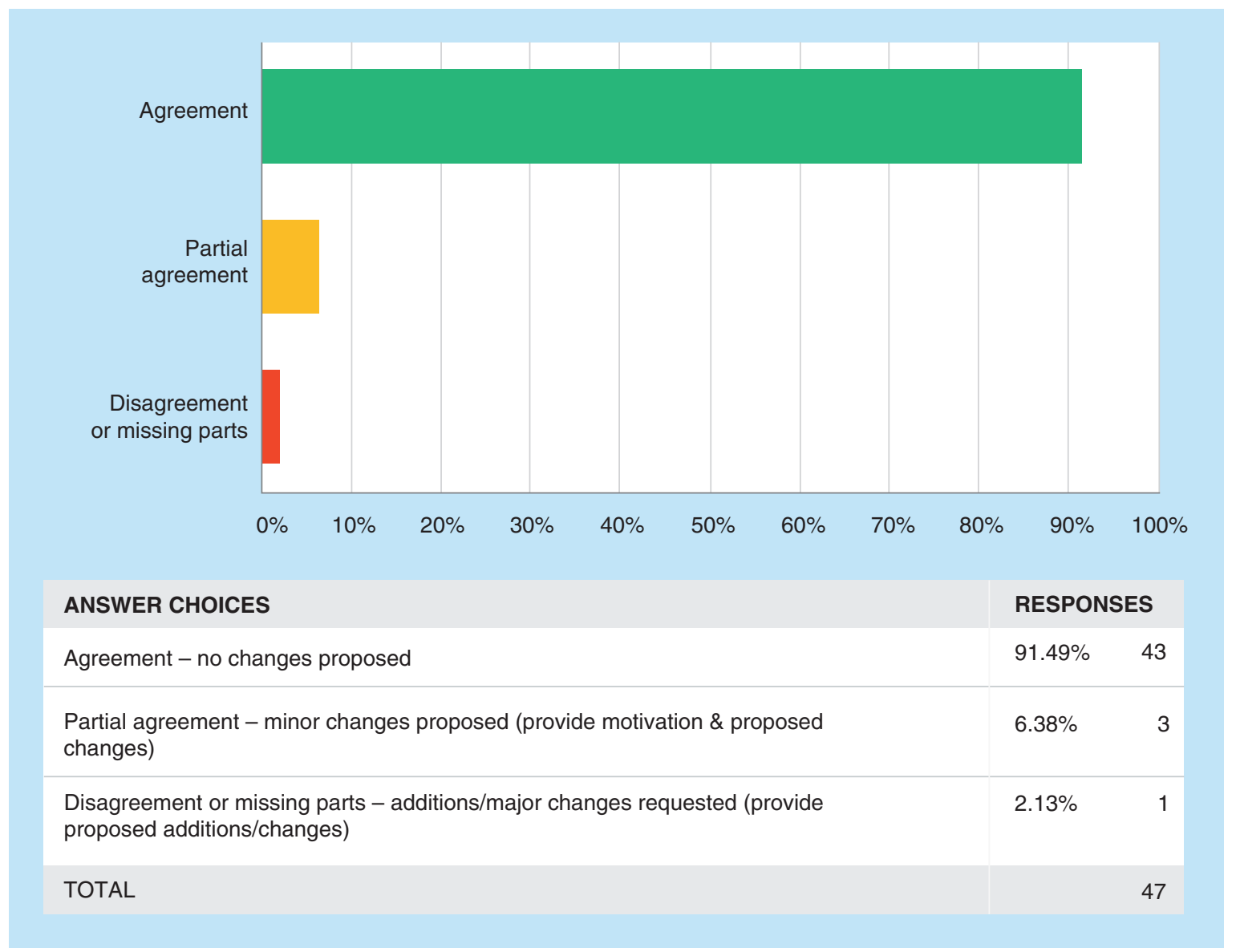

Disagreement or missing parts

No trend was found in these comments.

Partial agreement

No trend was found in these comments. 
T4Q24: Stability - Tested Conditions; Lines 793-794: "Validation of storage periods should be performed on stability QCs that have been stored for a time that is equal to or longer than the study sample storage periods."

Stability - Procedure; Lines 795-798: "Stability of the analyte in the studied matrix is evaluated using low and high concentration stability QCs. Aliquots of the low and high stability QCs are analysed at time zero and after the applied storage conditions that are to be evaluated. A minimum of three stability QCs should be prepared and analysed per concentration level/storage condition/timepoint."

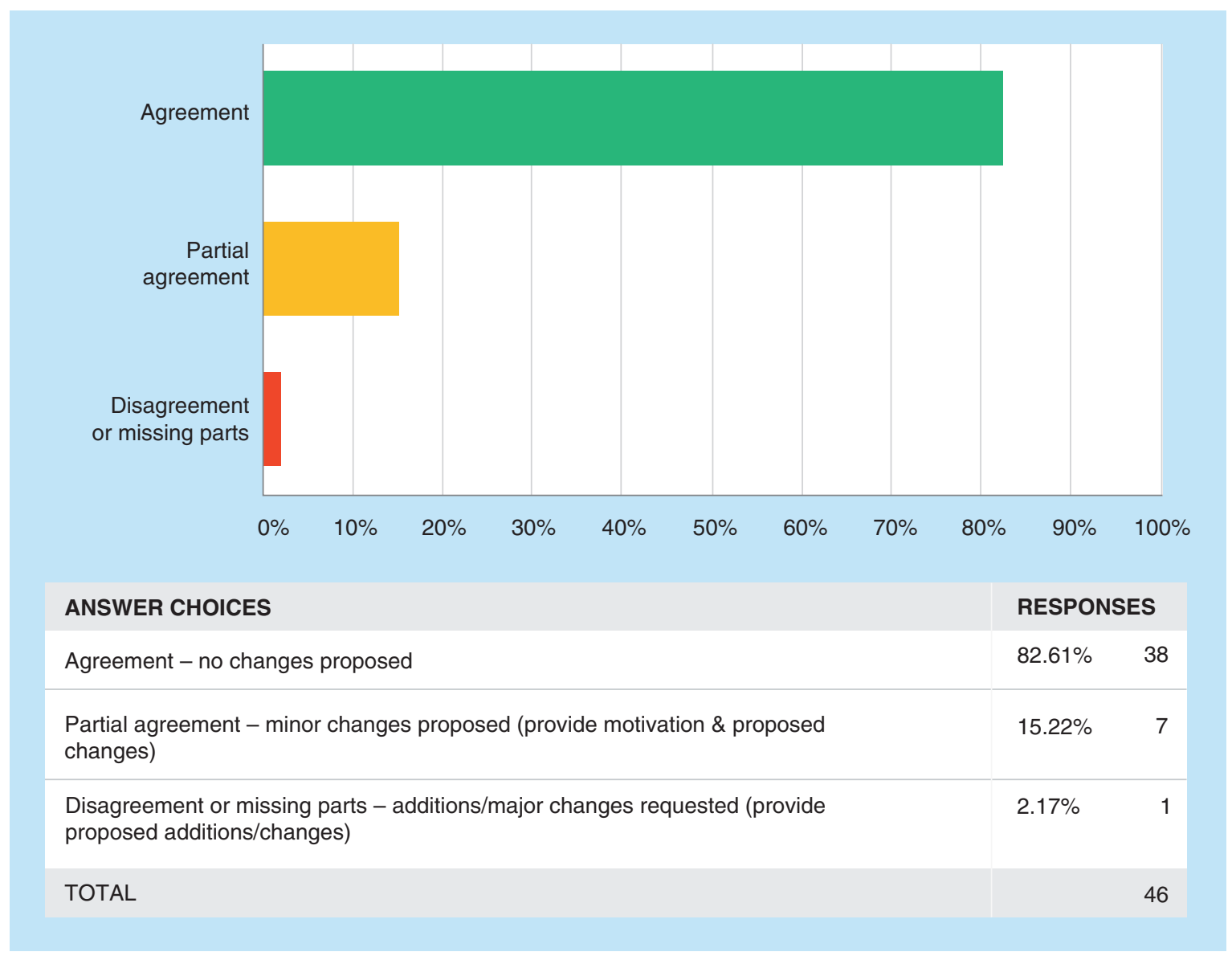

\section{Partial agreement}

Several comments suggested:

- to remove the requirement for assessment of QCs at time zero.

- to clarify the text "three stability QCs" to regulatory expectations of tubes vs aliquots.

\section{Additional comments from the 13th GCC:}

The scientific rationale of potentially requiring three QC tubes for stability was questioned again, considering published literature demonstrates that no differences in stability have been seen when using three tubes vs three aliquots from one tube [8]. It was recommended that when clarifying the language, the requirement for three tubes were reconsidered. 
T4Q25: Stability - Comparison Curve; Lines 799-804: "The stability QCs are analysed against a calibration curve, obtained from freshly spiked calibration standards in a run with its corresponding freshly prepared QCs or QCs for which stability has been proven. While the use of freshly prepared calibration standards and QCs is the preferred approach, it is recognised that in some cases, for macromolecules, it may be necessary to freeze them overnight. In such cases, valid justification should be provided and freeze-thaw stability demonstrated."

Stability - Acceptance Criteria; Lines 804-805: "The mean concentration at each level should be within $\pm 20 \%$ of the nominal concentration."

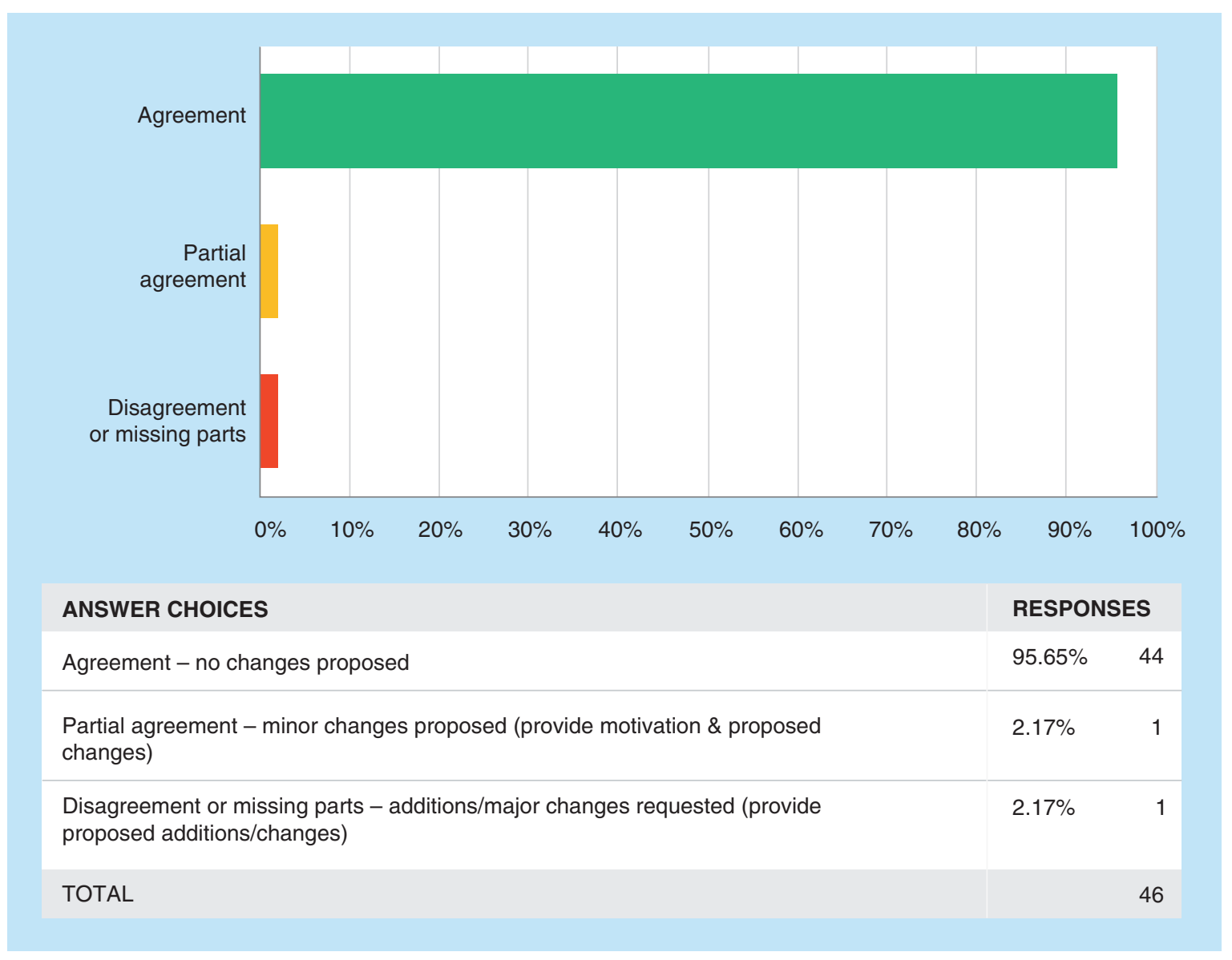

\section{Partiall agreement}

Both comments recommended adding criteria for precision.

\section{Additional comments from the 13th GCC:}

There were no further discussions during the GCC Closed Forum regarding the above proposal. 
T4Q26: Stability - Stability of >ULOQ; Lines 806-809: "Since sample dilution may be required for many LBA assays due to a narrow calibration range, the concentrations of the study samples may be consistently higher than the ULOQ of the calibration curve. If this is the case, the concentration of the stability QCs should be adjusted, considering the applied sample dilution, to represent the actual sample concentration range."

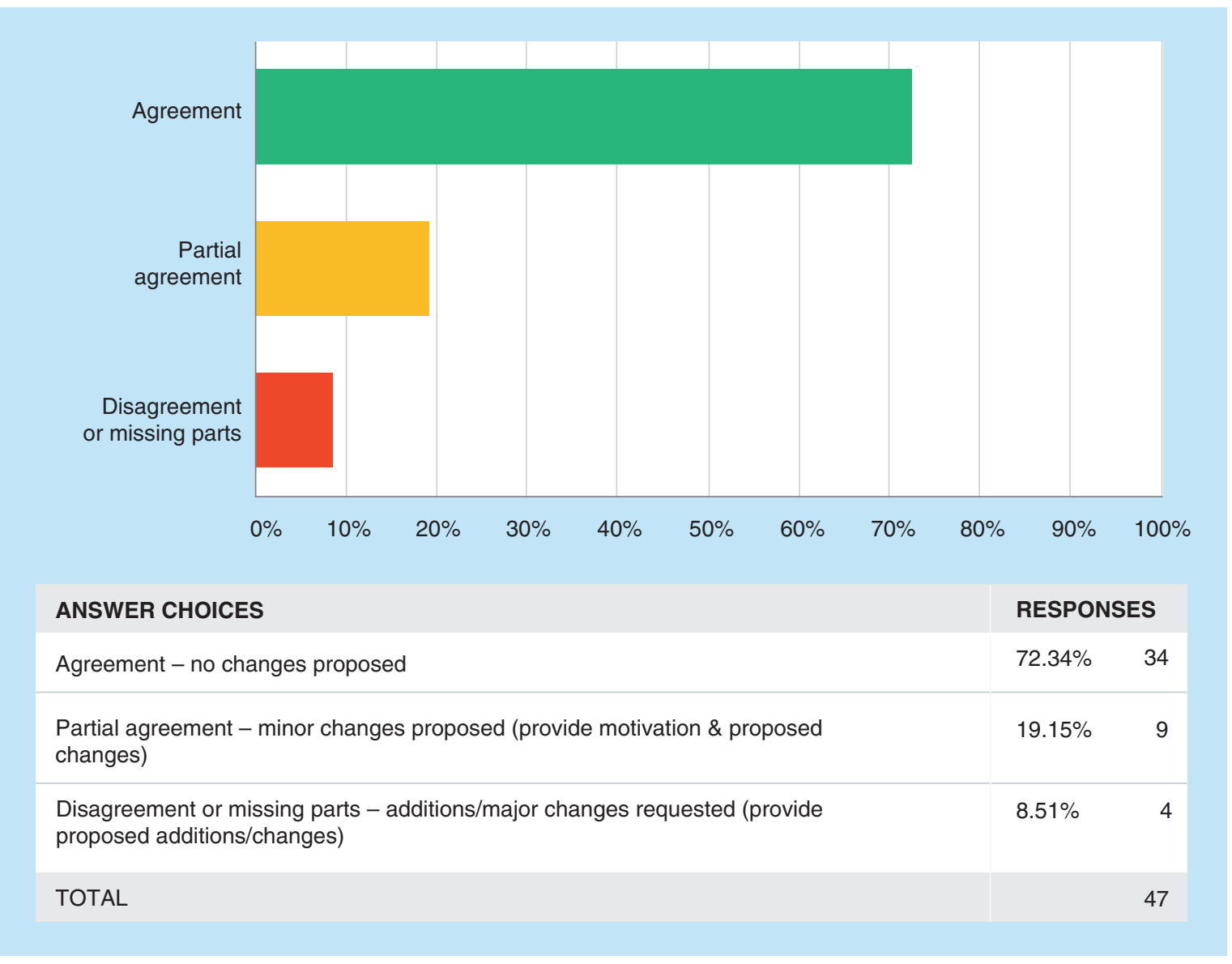

Disagreement or missing parts

No trend was found in these comments.

Partial agreement

Additional details on this evaluation were suggested regarding:

- If both a HQC and an ultra-high QC are required.

- How to proceed when study sample concentrations cannot be predicted in validation. 
T4Q27: Stability - Required Stabilities; Lines 810-812: "As mentioned in Section 3.2.8, the investigation of stability should cover bench-top (short-term) stability at room temperature or sample preparation temperature and freeze-thaw stability. In addition, long-term stability should be studied."

Stability - Chemical Drugs; Lines 813-814: "For chemical drugs, it is considered acceptable to extrapolate the stability at one temperature (e.g., $\left.-20^{\circ} \mathrm{C}\right)$ to lower temperatures (e.g., $\left.-70^{\circ} \mathrm{C}\right)$."

Stability - Biological Drugs; Lines 815-817: "For biological drugs, it is acceptable to apply a bracketing approach, e.g., in the case that the stability has been demonstrated at $-70^{\circ} \mathrm{C}$ and at $-20^{\circ} \mathrm{C}$, then it is not necessary to investigate the stability at temperatures in between those two points at which study samples will be stored."

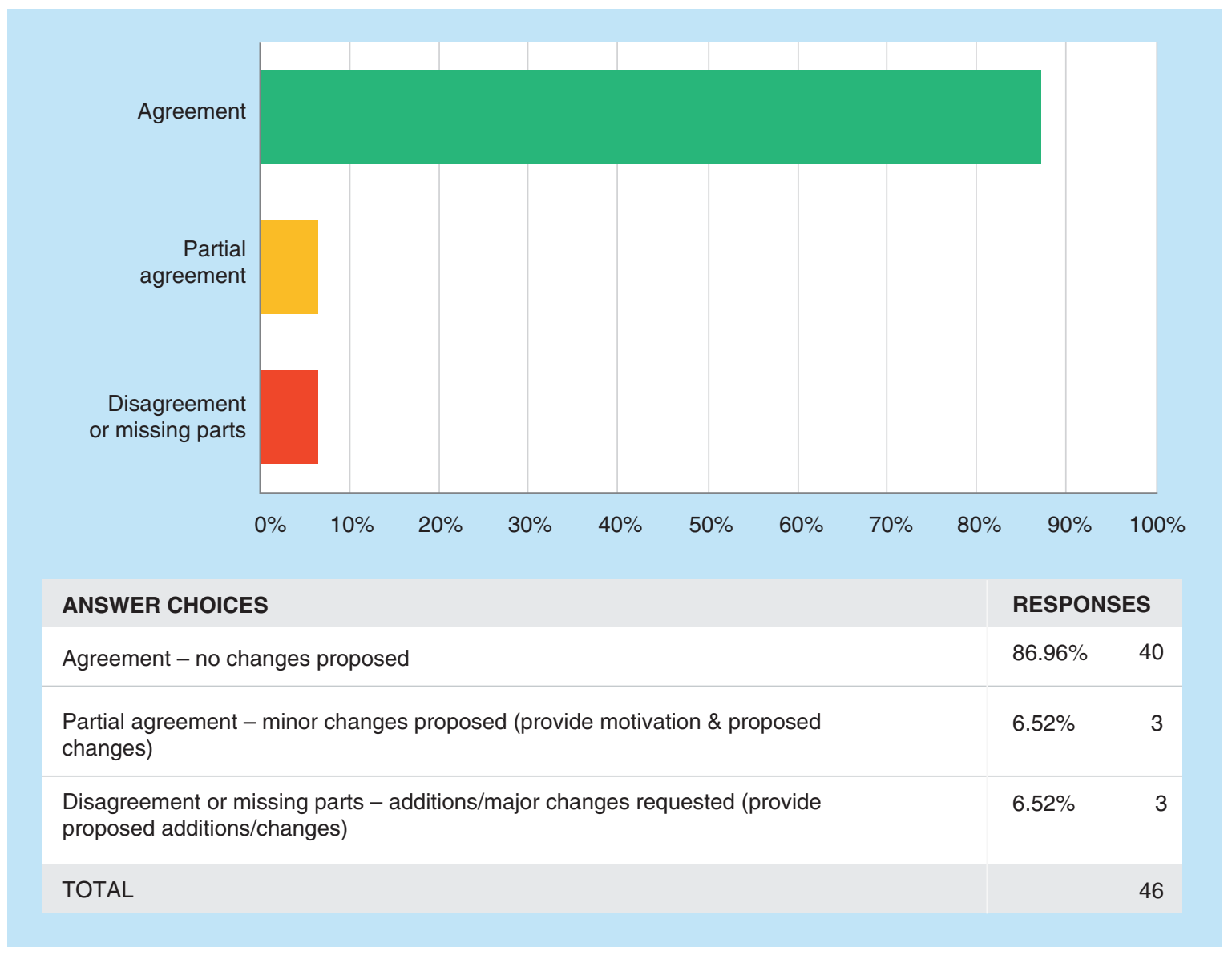

Disagreement or missing parts

Three comments recommend that it should be acceptable to extrapolate stability at $-20^{\circ} \mathrm{C}$ for all drug types.

\section{Additional comments from the 13th GCC:}

The survey comments were confirmed during the GCC Closed Forum and consensus was reached to support extrapolation of stability at $-20^{\circ} \mathrm{C}$ for all drug types. This is another section that references chromatography sections for criteria, and preferable for full text to be provided in this section directed specifically for LBA. 
T4Q28: Parallelism - Definition; Lines 1129-1130: "Parallelism is defined as a parallel relationship between the calibration curve and serially diluted study samples to detect any influence of dilution on analyte measurement."

Parallelism - Requirement; Lines 1130-1134: "Although lack of parallelism is a rare occurrence for PK assays, parallelism of LBA should be evaluated on a case-by-case basis, e.g., where interference caused by a matrix component (e.g., presence of endogenous binding protein) is suspected during study sample analysis. Parallelism investigation or the justification for its absence should be included in the Bioanalytical Report."

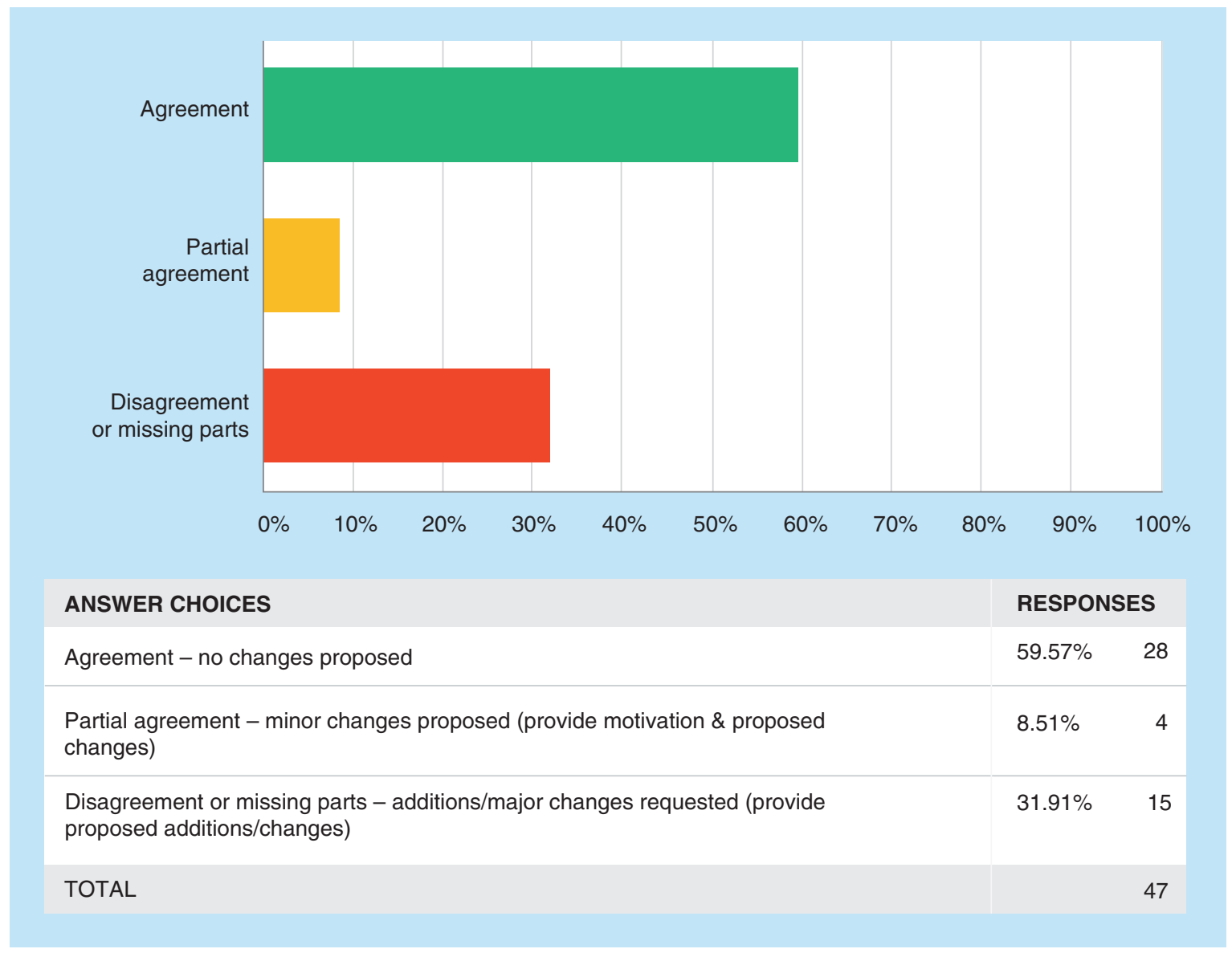

Disagreement or missing parts

There was strong disagreement with what to include in the justification/investigation for the absence of parallelism. Additional details are required to clarify regulatory expectations. Moreover, recommendations/guidelines to design a specific template for this justification were requested.

Partial agreement

No additional trend was found in these comments.

Additional comments from the 13th GCC:

The survey comments were confirmed during the GCC Closed Forum. 
T4Q29: Parallelism - Timing; Lines 1135-1139: "As parallelism assessments are rarely possible during method development and method validation due to the unavailability of study samples and parallelism is strictly linked to the study samples (i.e., an assay may have perfectly suitable parallelism for a certain population of samples, yet lack it for another population), these experiments should be conducted during the analysis of the study samples."

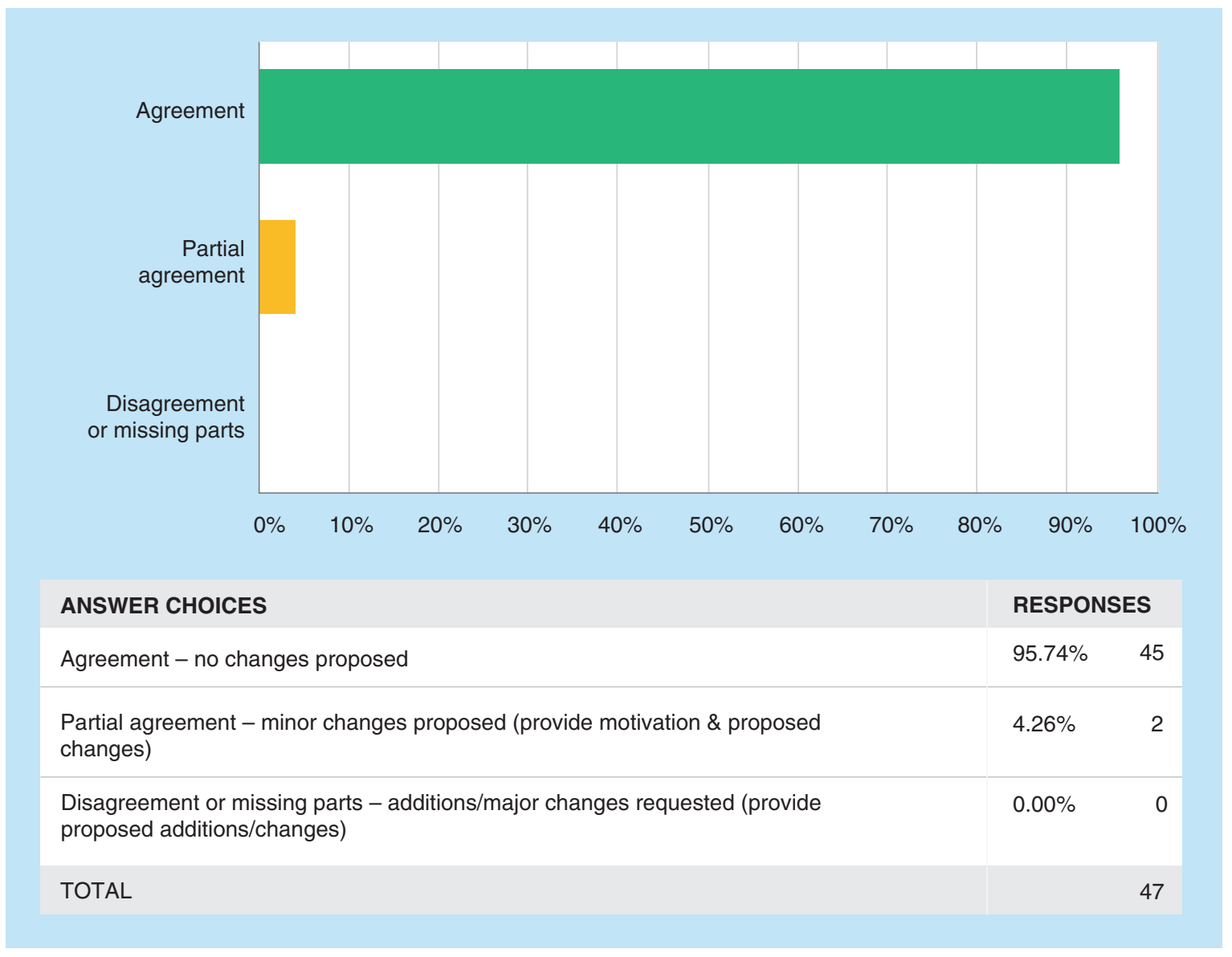

\section{Partial agreement}

No trend was found in these comments. 
T4Q30: Parallelism - Procedure; Lines 1139-1140: "A high concentration study sample (preferably close to Cmax) should be diluted to at least three concentrations with blank matrix."

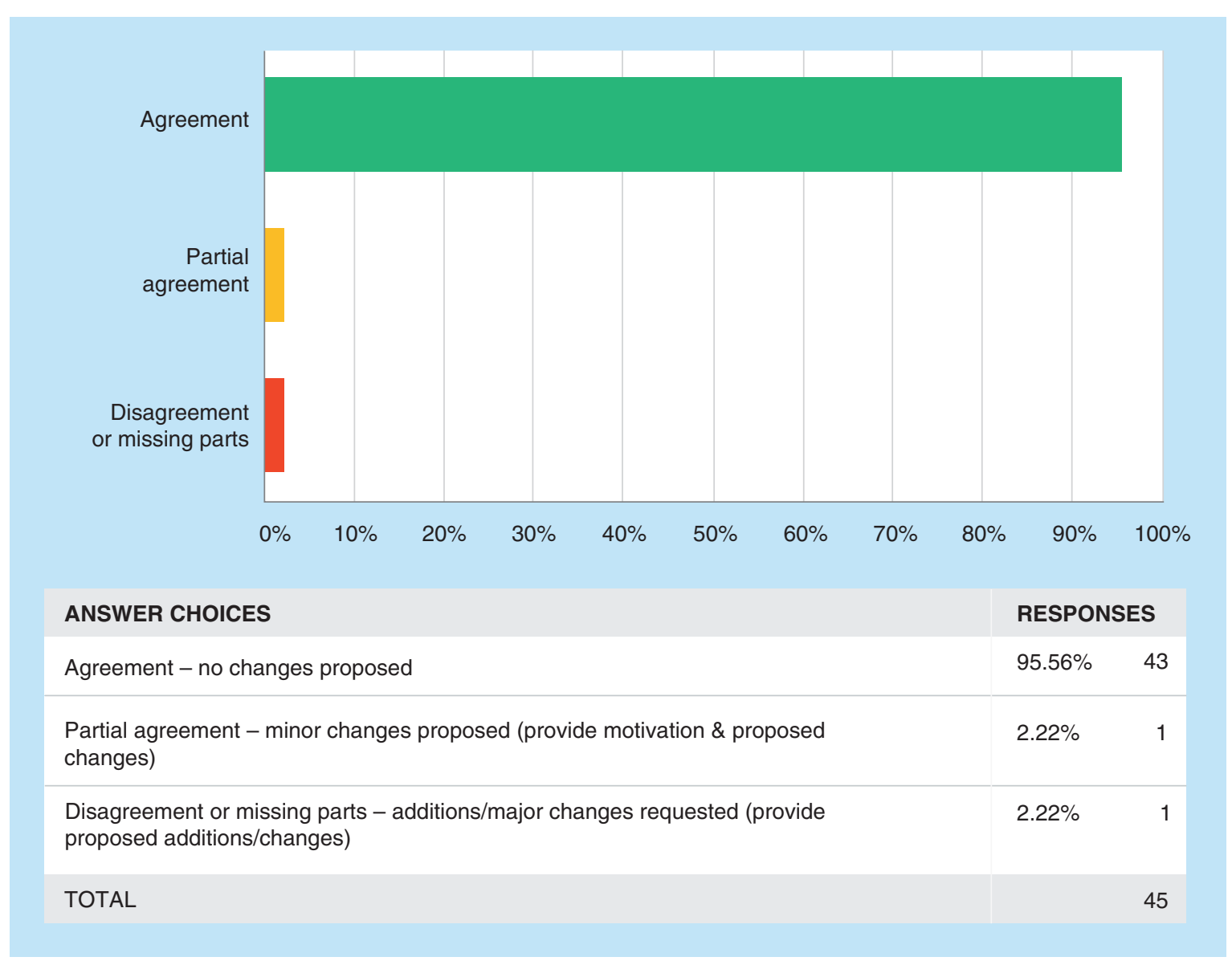

Partial agreement

No trend was found in these comments. 
T4Q31: Parallelism - Criteria; Lines 1140-1144: "The precision between samples in a dilution series should not exceed 30\%. However, when applying the 30\% criterion, data should be carefully monitored as results that pass this criterion may still reveal trends of non-parallelism. In the case that the sample does not dilute linearly (i.e., in a non-parallel manner), a procedure for reporting a result should be defined a priori."

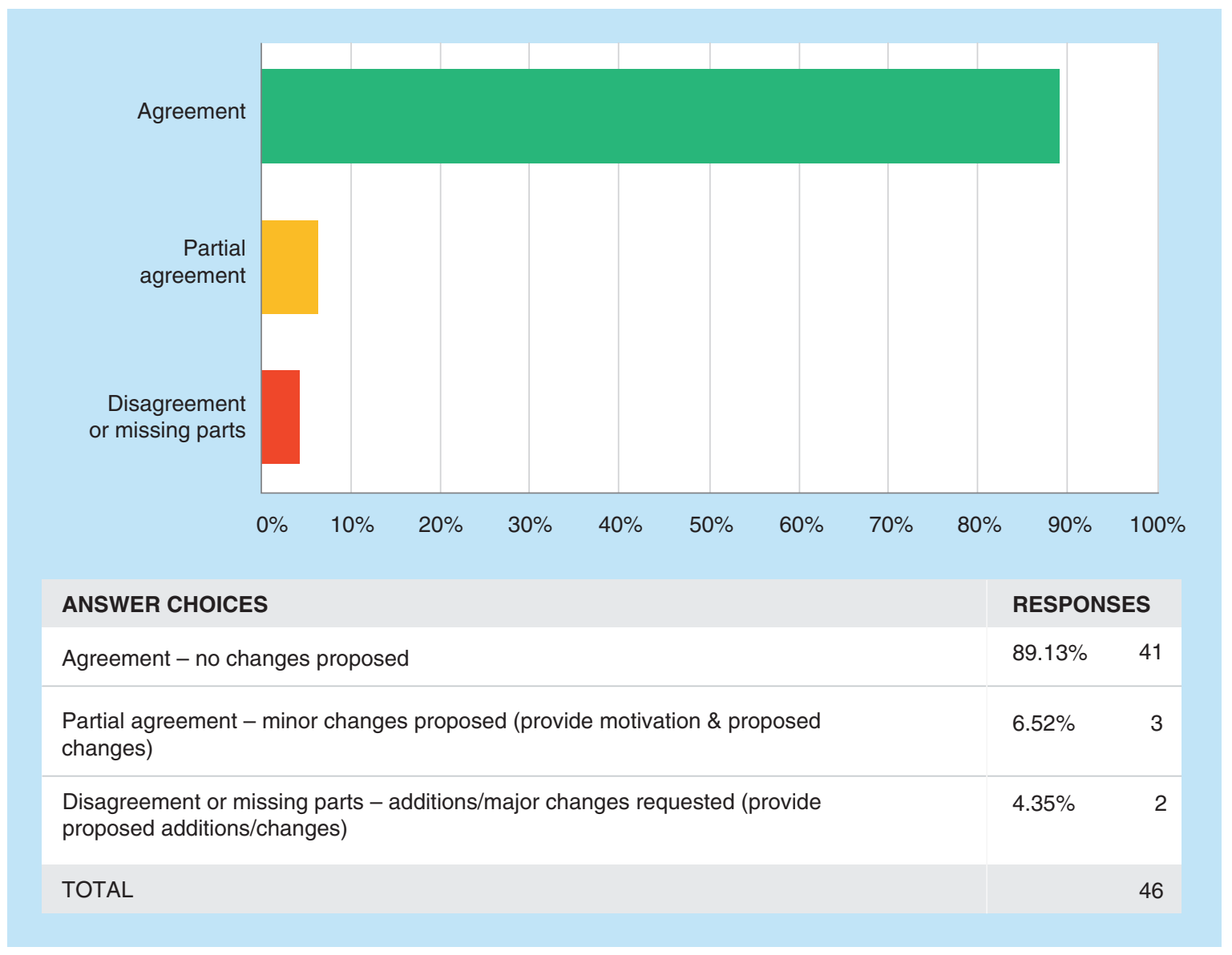

Disagreement or missing parts

No trend was found in these comments.

Partial agreement

No trend was found in these comments. 
T4Q32: MRD; Lines 1156-1161: "MRD is a dilution factor employed in samples that are diluted with buffer solution to reduce the background signal or matrix interference on the analysis using LBA. The MRD should be identical for all samples including calibration standards and the $Q C s$ and it should be determined during method development. If MRD is changed after establishment of the method, partial validation is necessary. MRD should be defined in the Validation Report of the analytical method."

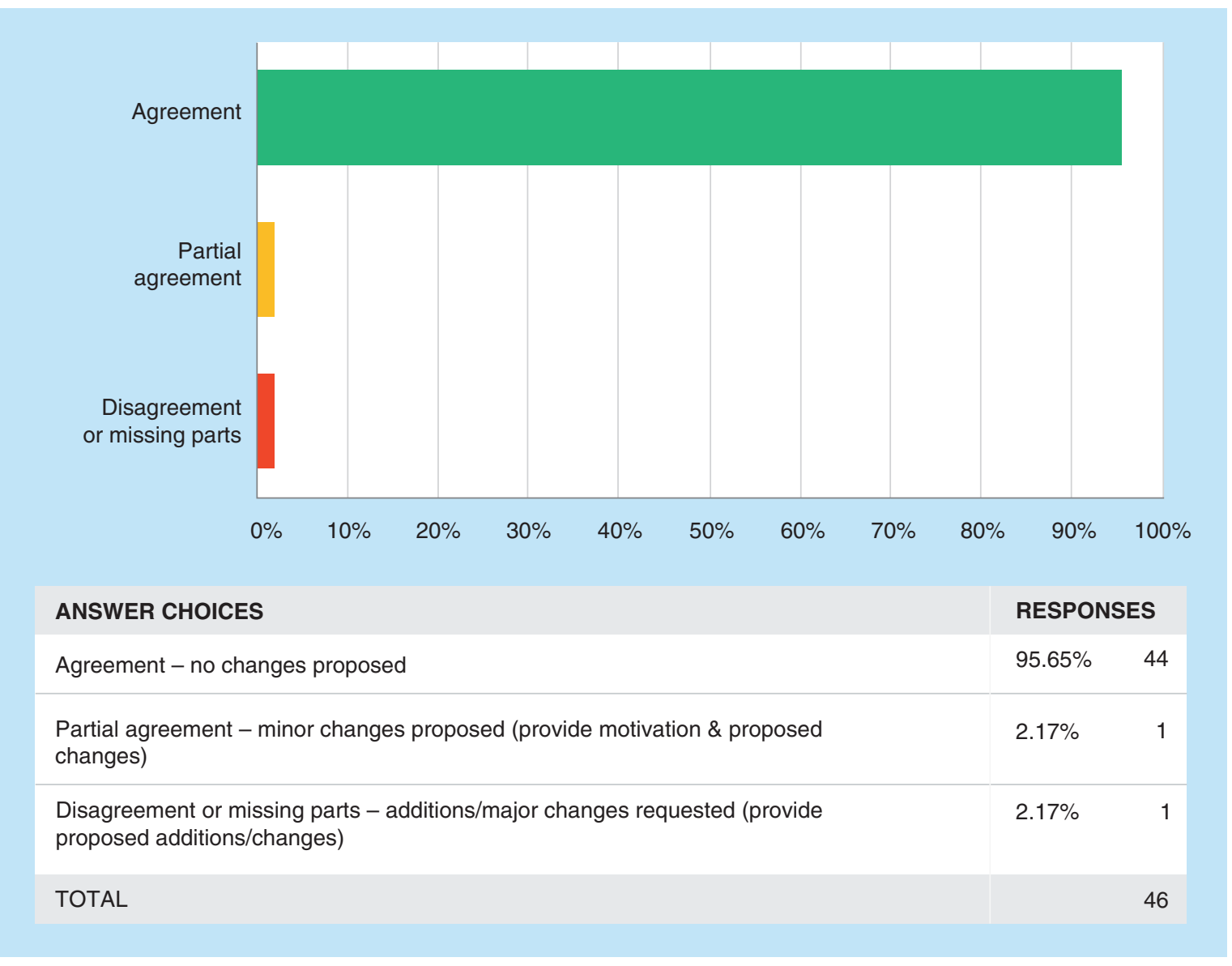


Team 5 LBA II - Study Sample Analysis, Acceptance Criteria for an Analytical Run, Calibration Range, Reanalysis of Study Samples including Critical Reagents

T5Q1: Critical Reagents - Background; Lines 631-635: "Critical reagents, including binding reagents (e.g., binding proteins, aptamers, antibodies or conjugated antibodies) and those containing enzymatic moieties, have direct impact on the results of the assay and, therefore, their quality should be assured. Critical reagents bind the analyte and, upon interaction, lead to an instrument signal corresponding to the analyte concentration. The critical reagents should be identified and defined in the assay method."

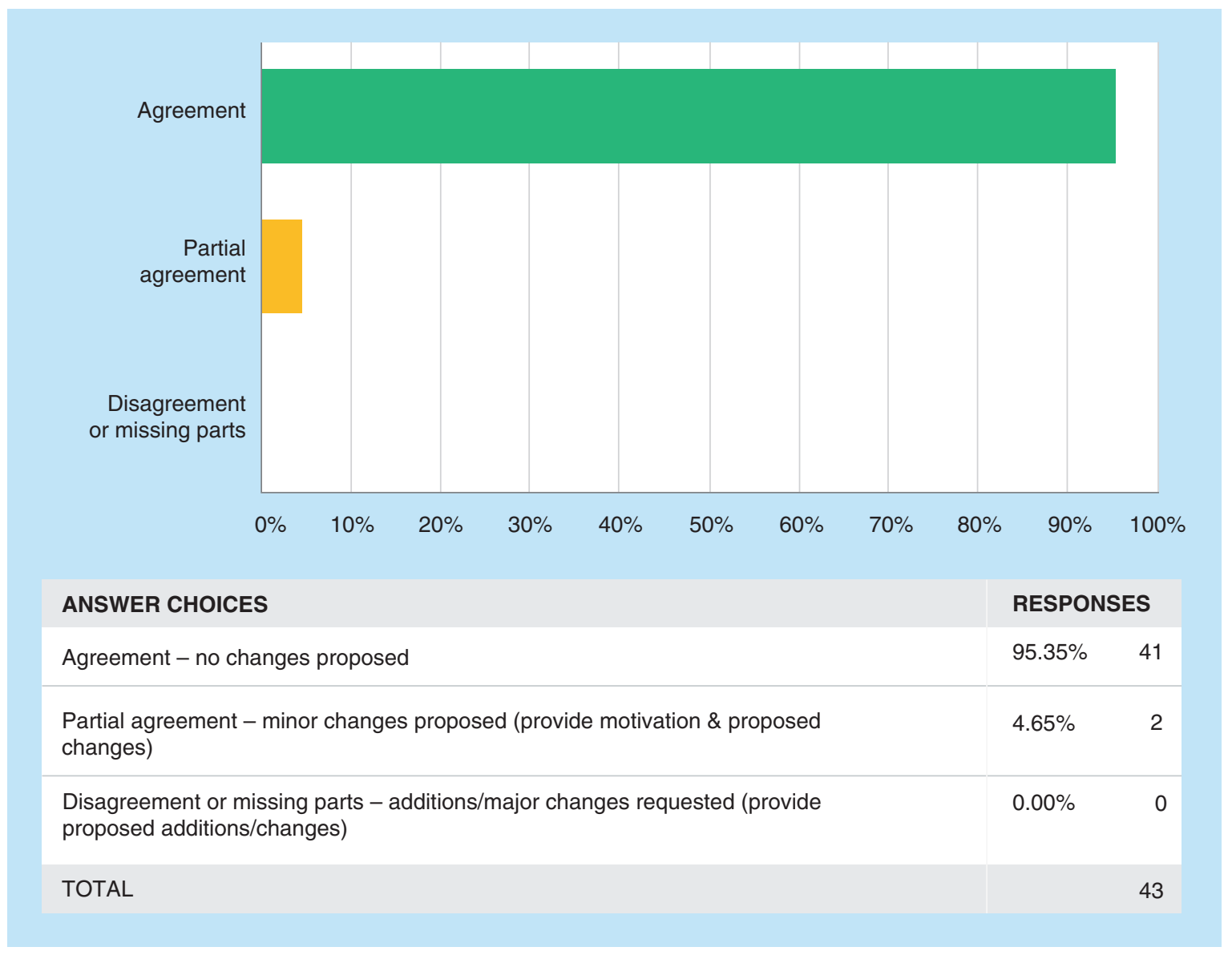

\section{Partial agreement}

No trend was found in these comments.

\section{Additional comments from the 13th GCC:}

It was suggested to use a risk-based approach when identifying and defining critical reagents. Some reagents may be critical for one assay but not for another all due to assay characteristics. 
T5Q2: Critical Reagents - Procurement; Lines 636-637: "Reliable procurement of critical reagents, whether manufactured in-house or purchased commercially, should be considered early in method development."

Critical Reagents - Data Sheet; Lines 637-640: "The data sheet for the critical reagent should include at a minimum identity, source, batch/lot number, purity (if applicable), concentration (if applicable) and stability/storage conditions (Refer to Table 1). Additional characteristics may be warranted."

Critical Reagents - Lifecycle Management; Lines 641-643: "A critical reagent lifecycle management procedure is necessary to ensure consistency between the original and new batches of critical reagents. Reagent performance should be evaluated using the bioanalytical assay."

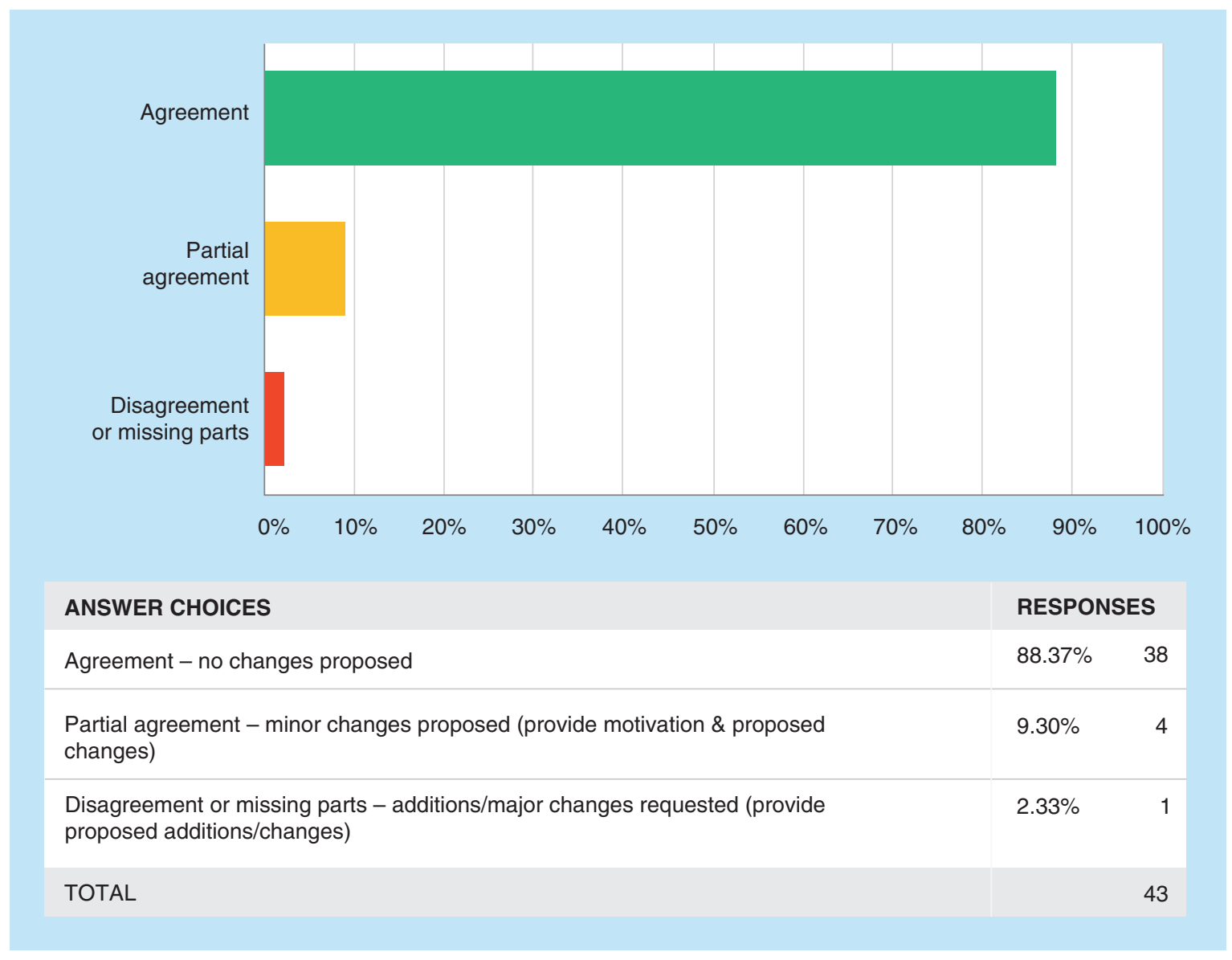

\section{Partial agreement}

No trend was found in these comments.

\section{Additional comments from the 13th GCC:}

- It was suggested to clarify the meaning of "source" in this section (e.g., vendor or animal).

- It was suggested to define "data sheet" and "life cycle management". 
T5Q3: Critical Reagents - Impact of Changes; Lines 643-645: "Minor changes to critical reagents would not be expected to influence the assay performance, whereas major changes may significantly impact the performance."

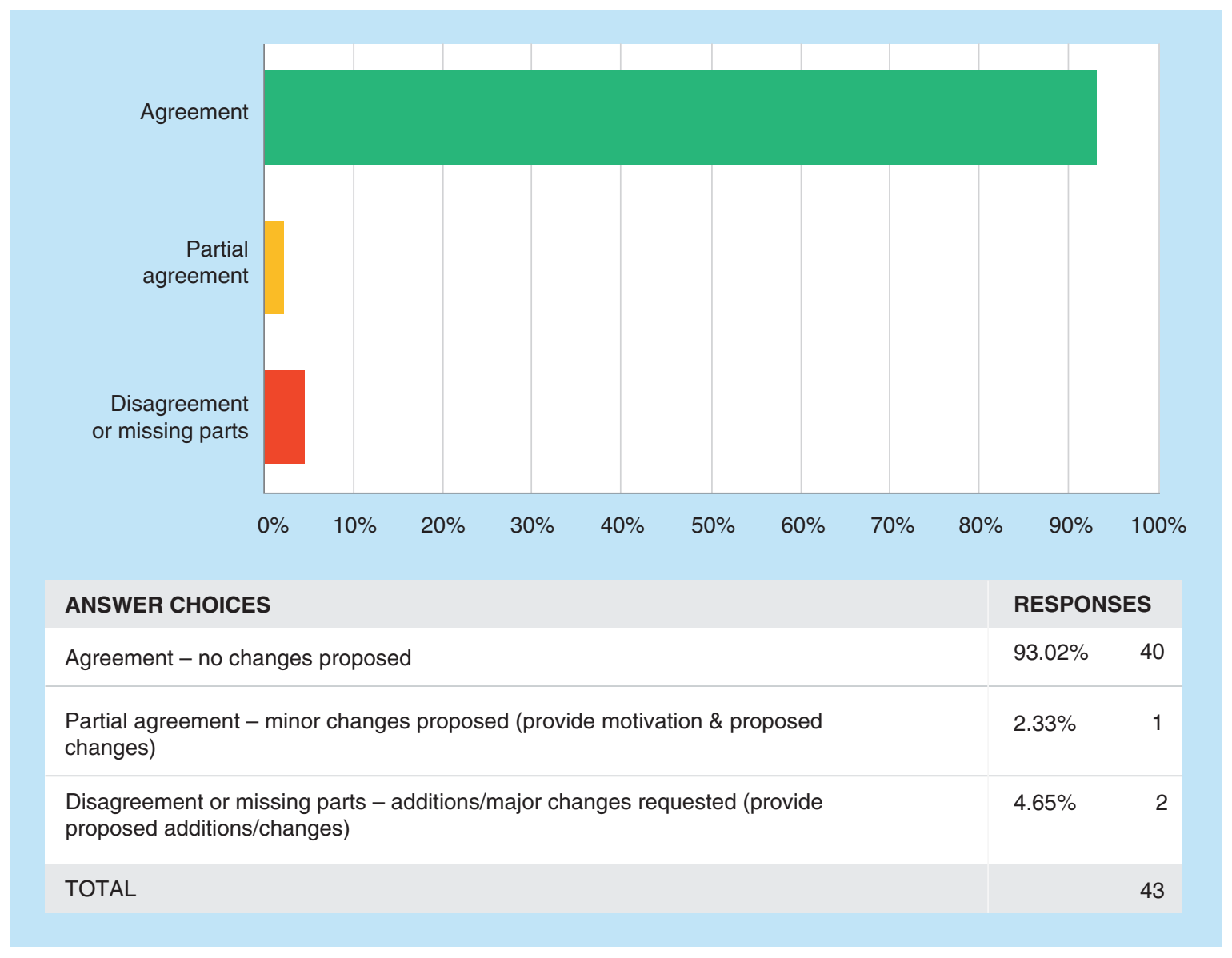

Disagreement or missing parts

Overall the comments indicated concerns with the meaning of minor vs major changes. 
T5Q4: Critical Reagents - Minor changes; Lines 645-646: "If the change is minor (e.g., the source of one reagent is changed), a single comparative accuracy and precision assessment is sufficient for characterisation."

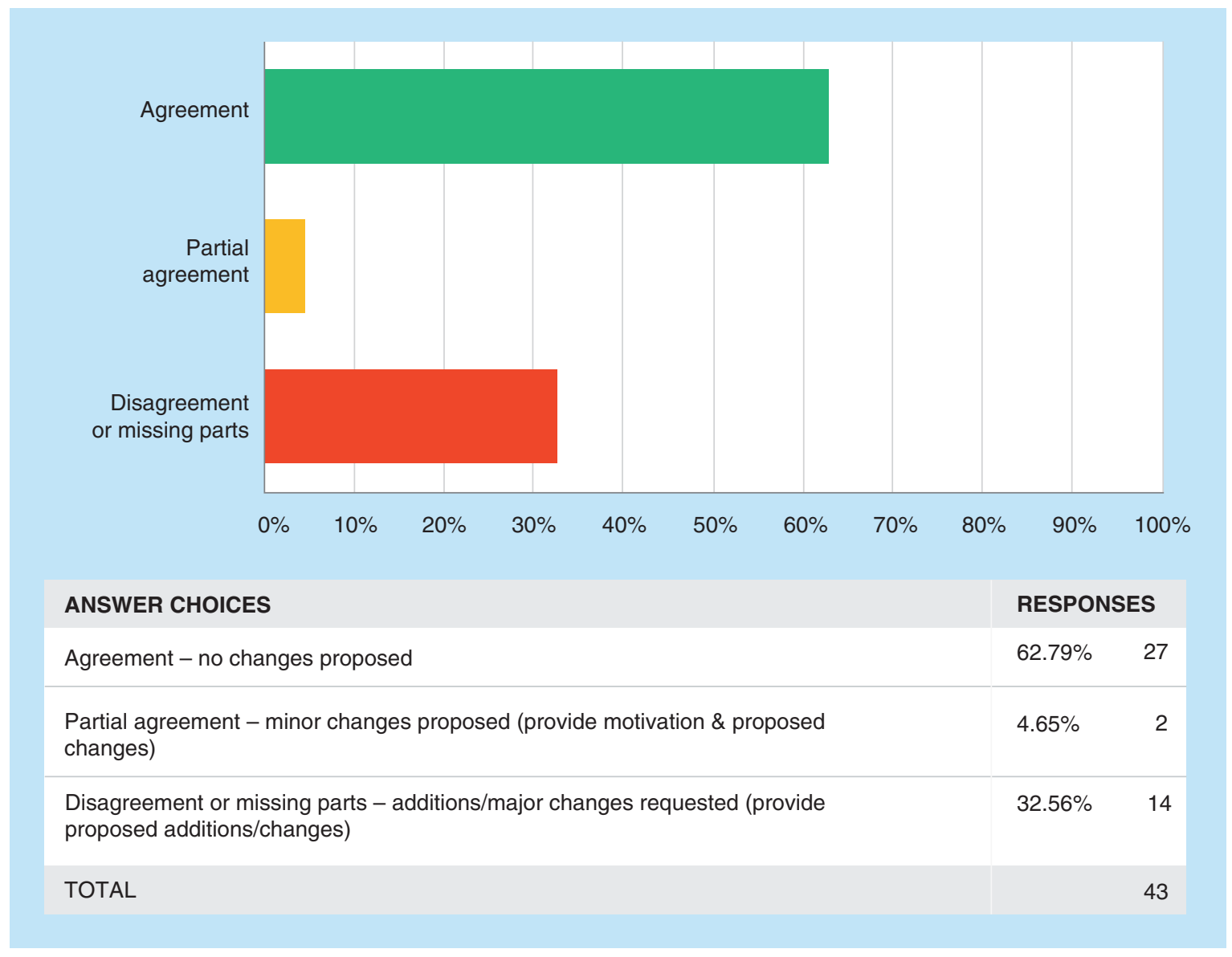

\section{Disagreement or missing parts}

There was strong disagreement in all the comments regarding the language around major and minor changes (see also T5Q3), especially that changing the source of a critical reagent is a minor change. In many cases, this can cause significant impact on an assay. The major concern was the fact that ICH M10 may have used EBF recommendations for critical reagents. It seems that $\mathrm{CRO}$ s are in disagreement with $\mathrm{EBF}$ recommendations which are considered too complicated to be applied to actual situations. It was highly recommended to keep this section simple and to follow current EMA or FDA guideline/guidance instead of EBF recommendations. It was also suggested to generate a White Paper from GCC on critical reagents.

Partial agreement

No additional trend was found in these comments. 
T5Q5: Critical Reagents - Major Changes; Lines 646-651: "If the change is major, then additional validation experiments are necessary. Ideally, assessment of changes will compare the assay with the new reagents to the assay with the old reagents directly. Major changes include, but are not limited to, change in production method of antibodies, additional blood collection from animals for polyclonal antibodies and new clones or new supplier for monoclonal antibody production."

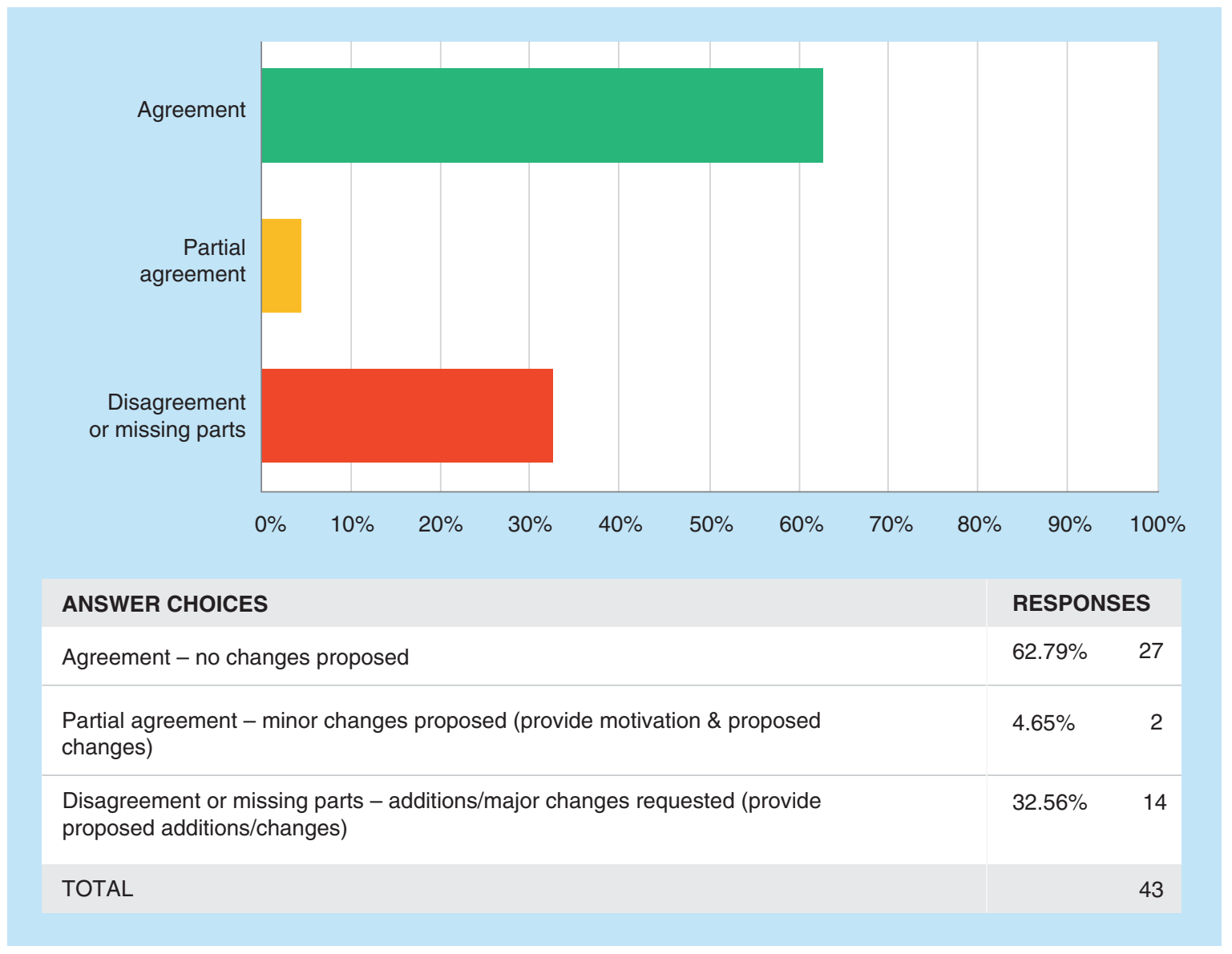

Disagreement or missing parts

Comments are similar as in T5Q4 with the same recommendations:

There was strong disagreement in all the comments regarding the language around major and minor changes (see also T5Q3). The major concern was the fact that ICH M10 may have used EBF recommendations for critical reagents. It seems that CROs are in disagreement with $\mathrm{EBF}$ recommendations which are considered too complicated to be applied to actual situations. It was highly recommended to keep this section simple and to follow current EMA or FDA guideline/guidance instead of EBF. It was also suggested to generate a White Paper from GCC on critical reagents.

Partial agreement

No additional trend was found in these comments. 
T5Q6: Critical Reagents - Performance Parameters; Lines 652-657: "Retest dates and validation parameters should be documented in order to support the extension or replacement of the critical reagent. Stability testing of the reagents should be based upon the performance in the bioanalytical assay and be based upon general guidance for reagent storage conditions and can be extended beyond the expiry date from the supplier. The performance parameters should be documented in order to support the extension or replacement of the critical reagent."

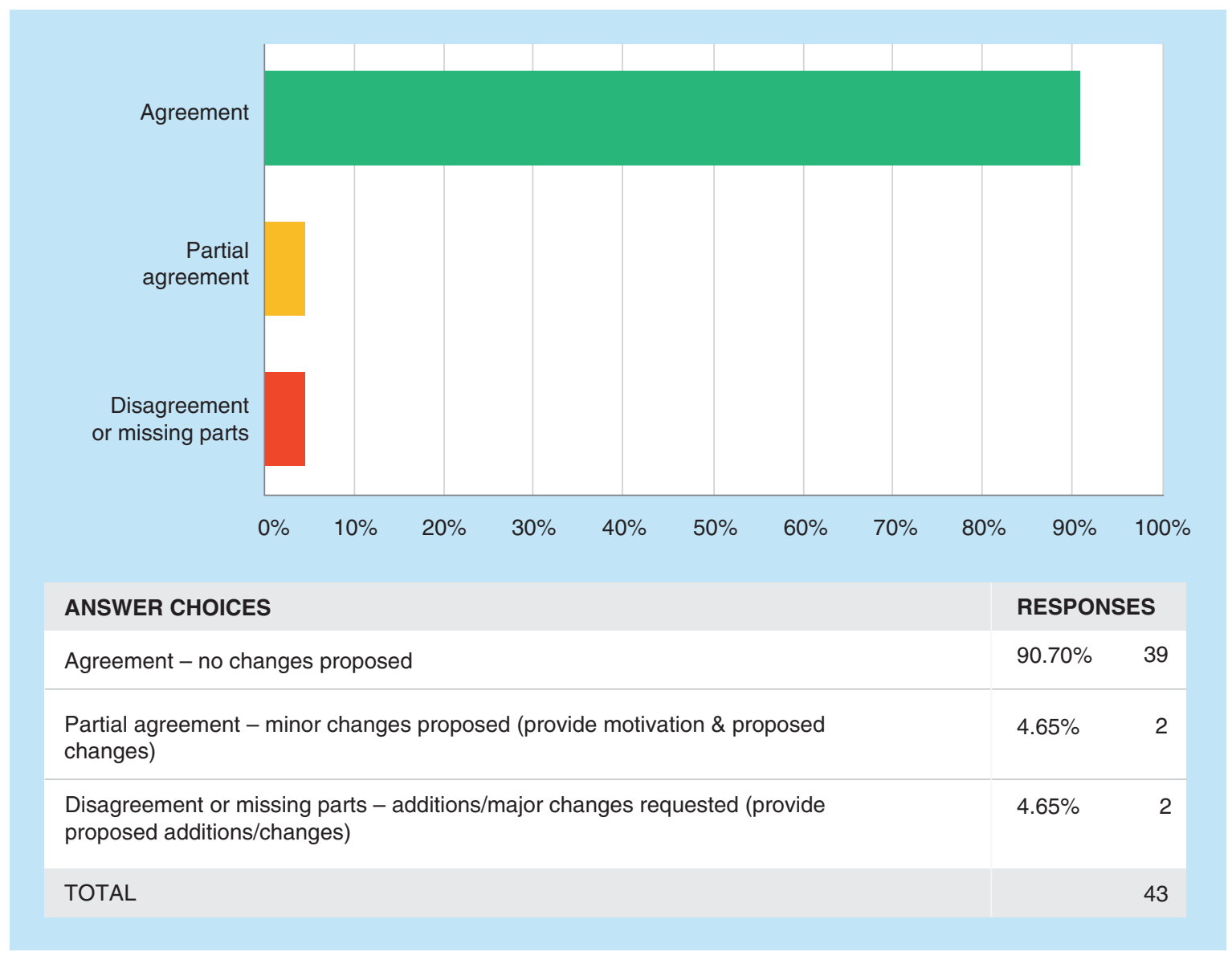

Disagreement or missing parts

No additional trend was found in these comments.

Partial agreement

It was suggested that clarification is needed to specify if the extension of stability can be performed also by bioanalytical laboratories (e.g., based on assay performance) or only by the vendor.

\section{Additional comments from the 13th GCC:}

There was consensus that $\mathrm{CRO}$ s are using retest dates and not expiration dates for their critical reagents. 
T5Q7: General; Lines 819-824: "The analysis of study samples can be carried out after validation has been completed however it is understood that some parameters may be completed at a later stage (e.g., long-term stability). By the time the data are submitted to a regulatory authority, the bioanalytical method validation should have been completed. The study samples, QCs and calibration standards should be processed in accordance with the validated analytical method. Refer to Table 1 for expectations regarding documentation."

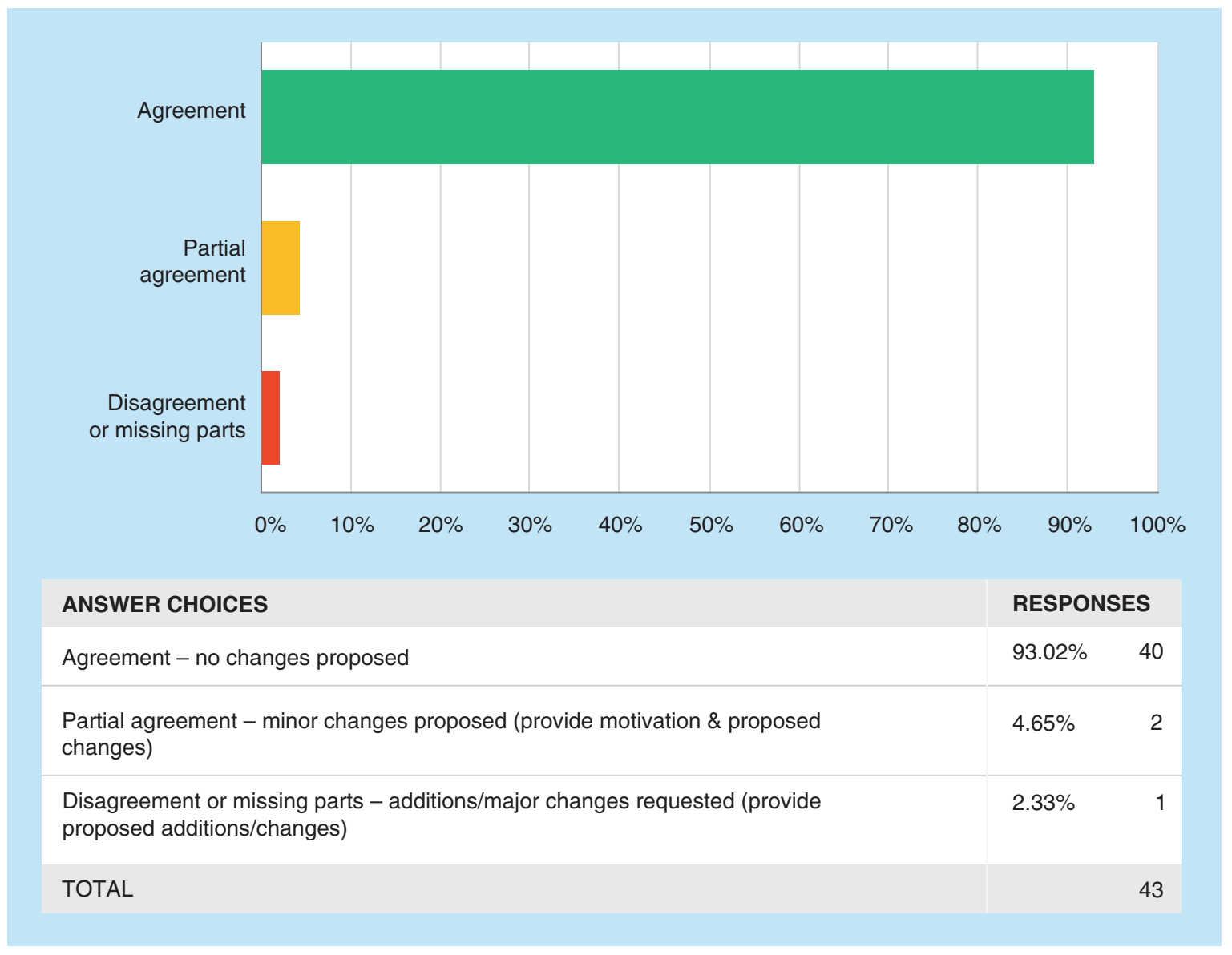

\section{Partial agreement}

No trend was found in these comments. 
T5Q8: Analytical Run - Contents; Lines 826-830: "An analytical run consists of a blank sample, calibration standards at a minimum of 6 concentration levels, at least 3 levels of QCs (low, medium and high) applied as two sets (or at least 5\% of the number of study samples, whichever is higher) and the study samples to be analysed. The blank sample should not be included in the calculation of calibration curve parameters."

Analytical Run - QC Placement; Lines 830-832: "The QCs should be placed in the run in such a way that the accuracy and precision of the whole run is ensured taking into account that study samples should always be bracketed by QCs."

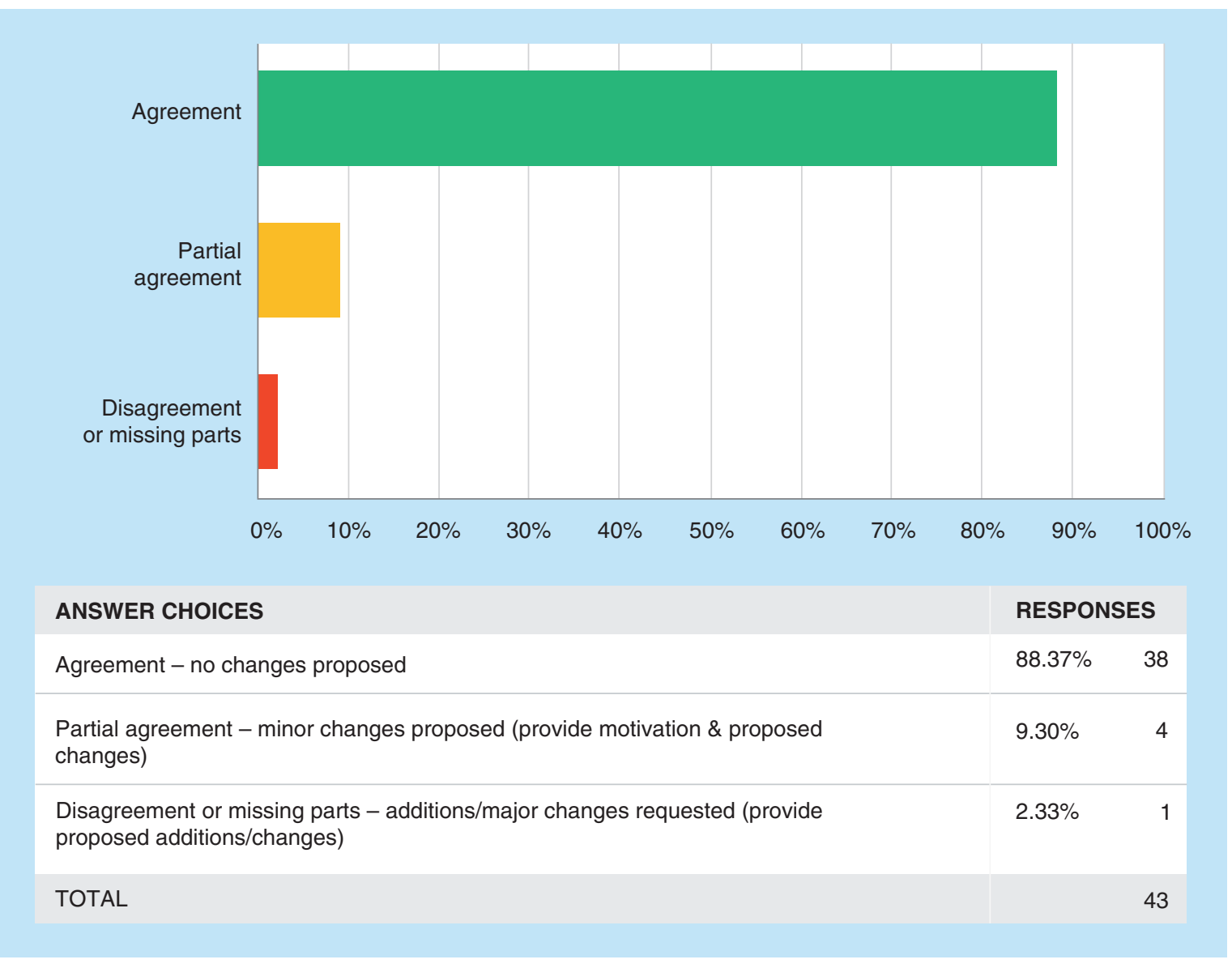

Partial agreement

No trend was found in these comments. 
T5Q9: Analytical Run - Plate Contents; Lines 833-836: "Most often microtitre plates are used for LBAs. An analytical run may comprise of one or more plate(s). Typically, each plate contains an individual set of calibration standards and QCs. If each plate contains its own calibration standards and QCs then each plate should be assessed on its own."

Analytical Run - Plate Space Constraints; Lines 836-842: "However, for some platforms the sample capacity may be limited. In this case, sets of calibration standards may be placed on the first and the last plate, but QCs should be placed on every single plate. QCs should be placed at least at the beginning (before) and at the end (after) of the study samples of each plate. The QCs on each plate and each calibration curve should fulfil the acceptance criteria (Refer to Section 4.3.2). For the calculation of concentrations, the calibration standards should be combined to conduct one regression analysis. If the combined calibration curve does not pass the acceptance criteria the whole run fails."

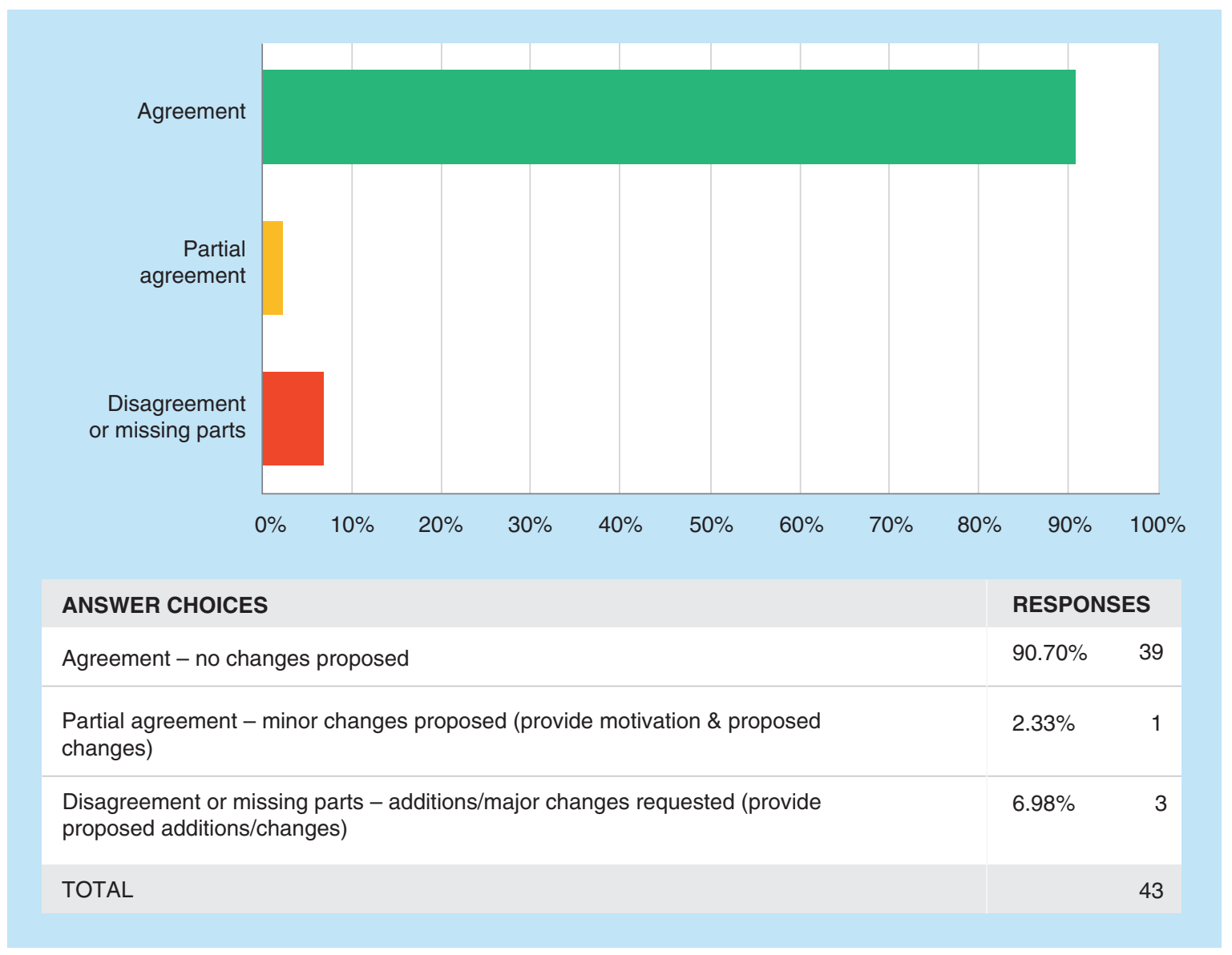

Disagreement or missing parts

No trend was found in these comments. 
T5Q10: Analytical Run - Predefining Acceptance Criteria; Lines 844-845: "Criteria for the acceptance or rejection of an analytical run should be defined in the protocol, in the study plan or in an SOP."

Analytical Run - Batch vs Run Acceptance Criteria; Lines 845-848: "In the case that a run contains multiple batches, acceptance criteria should be applied to the whole run and to the individual batches. It is possible for the run to meet acceptance criteria, even if a batch within that run is rejected for failing to meet the batch acceptance criteria."

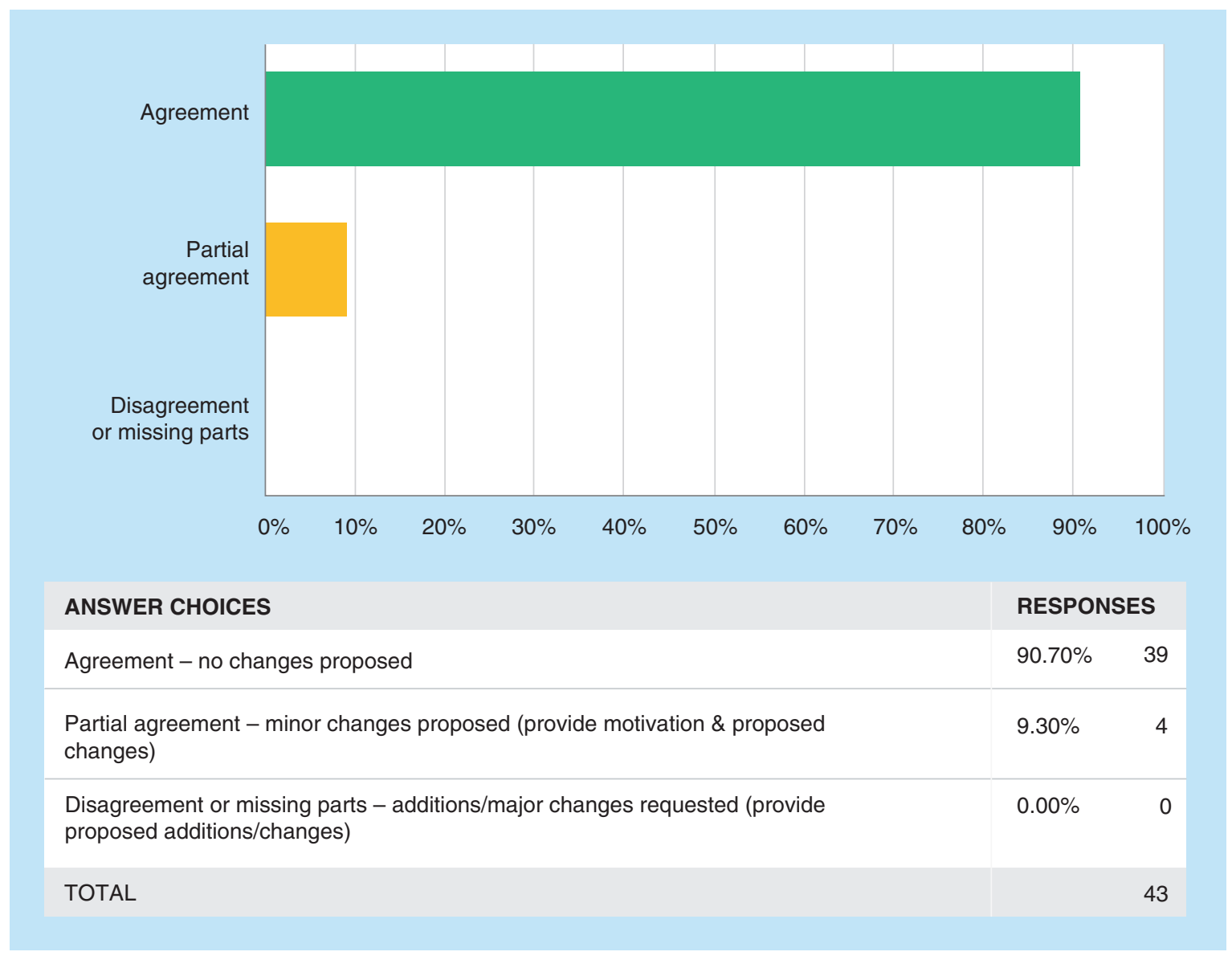

\section{Partial agreement}

All four comments request clarification on the difference between a run and a batch. This section is unclear. Similar comments were also found in the chromatographic assays section (lines 492-499).

\section{Additional comments from the 13th GCC:}

The comments of the survey were confirmed by consensus that batch and run in this context should be more clearly defined since the definition of batch seems to be different than how it is used in this section. In practice, most considered an analytical run to be as defined in the glossary (lines 1291-1294), "A complete set of analytical and study samples with appropriate number of calibration standards and QCs for their validation"; however, a batch (for bioanalysis) is usually considered as set of runs performed at the same period of time. Under these definitions, a batch can contain multiple runs, but a run cannot contain multiple batches. 
T5Q11: Analytical Run - Acceptance Criteria; Lines 849-856: "The back-calculated concentrations of the calibration standards should be within $\pm 20 \%$ of the nominal value at each concentration level, except for the LLOQ and the ULOQ, for which it should be within $\pm 25 \%$. At least $75 \%$ of the calibration standards, with a minimum of 6 concentration levels, should fulfil this criterion. This requirement does not apply to anchor calibration standards. If more than 6 calibration standards are used and one of the calibration standards does not meet these criteria, this calibration standard should be rejected and the calibration curve without this calibration standard should be re-evaluated and a new regression analysis performed."

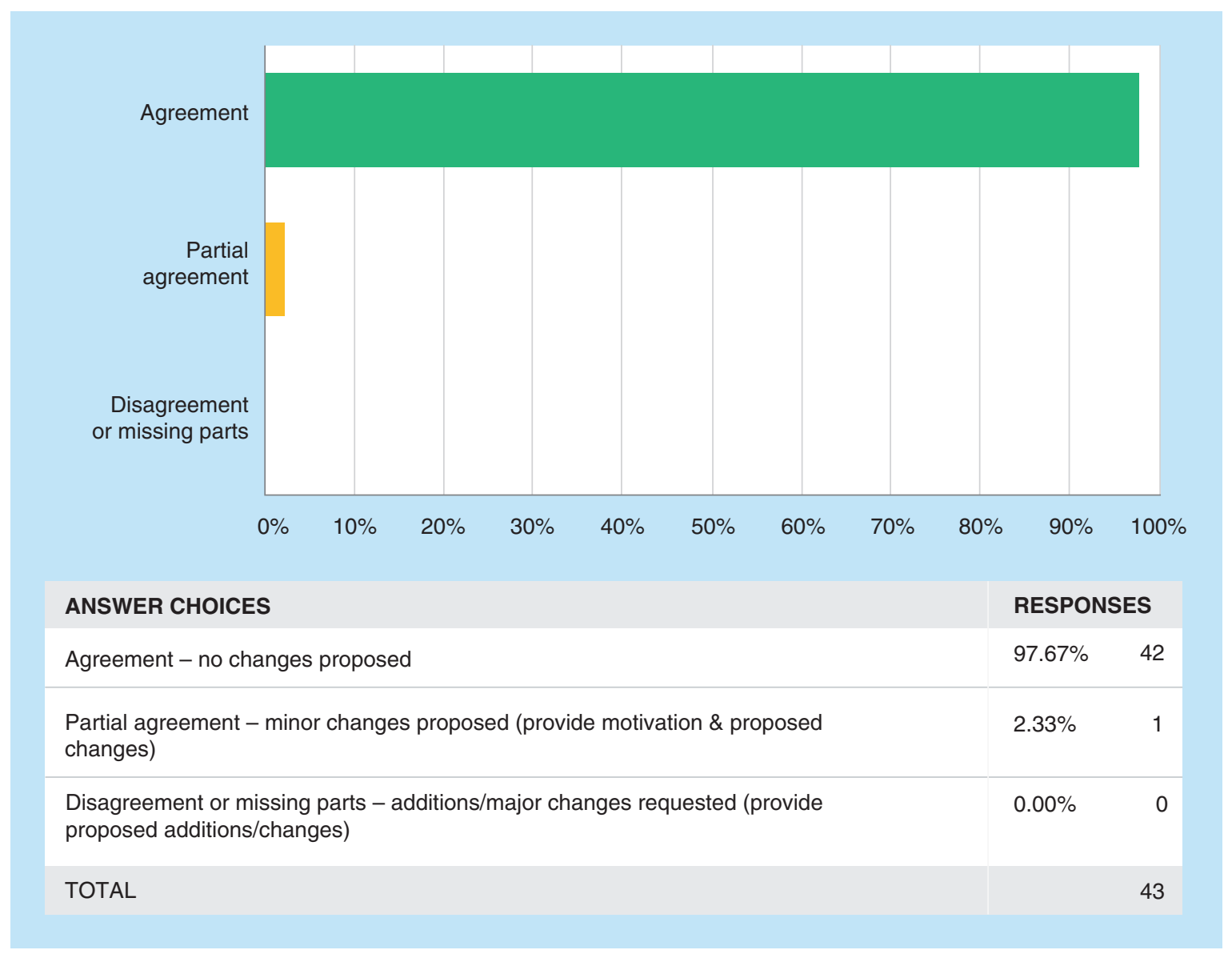


T5Q12: Analytical Run - Rejected LOQ or ULQ; Lines 857-863: "If the rejected calibration standard is the $L L O Q$, the new lower limit for this analytical run is the next lowest acceptable calibration standard of the calibration curve. If the highest calibration standard is rejected, the new upper limit for this analytical run is the next acceptable highest calibration standard of the calibration curve. The new lower and upper limit calibration standard will retain their original acceptance criteria (i.e., $\pm 20 \%$ ). The revised calibration range should cover all QCs (low, medium and high). The study samples outside of the revised assay range should be reanalysed."

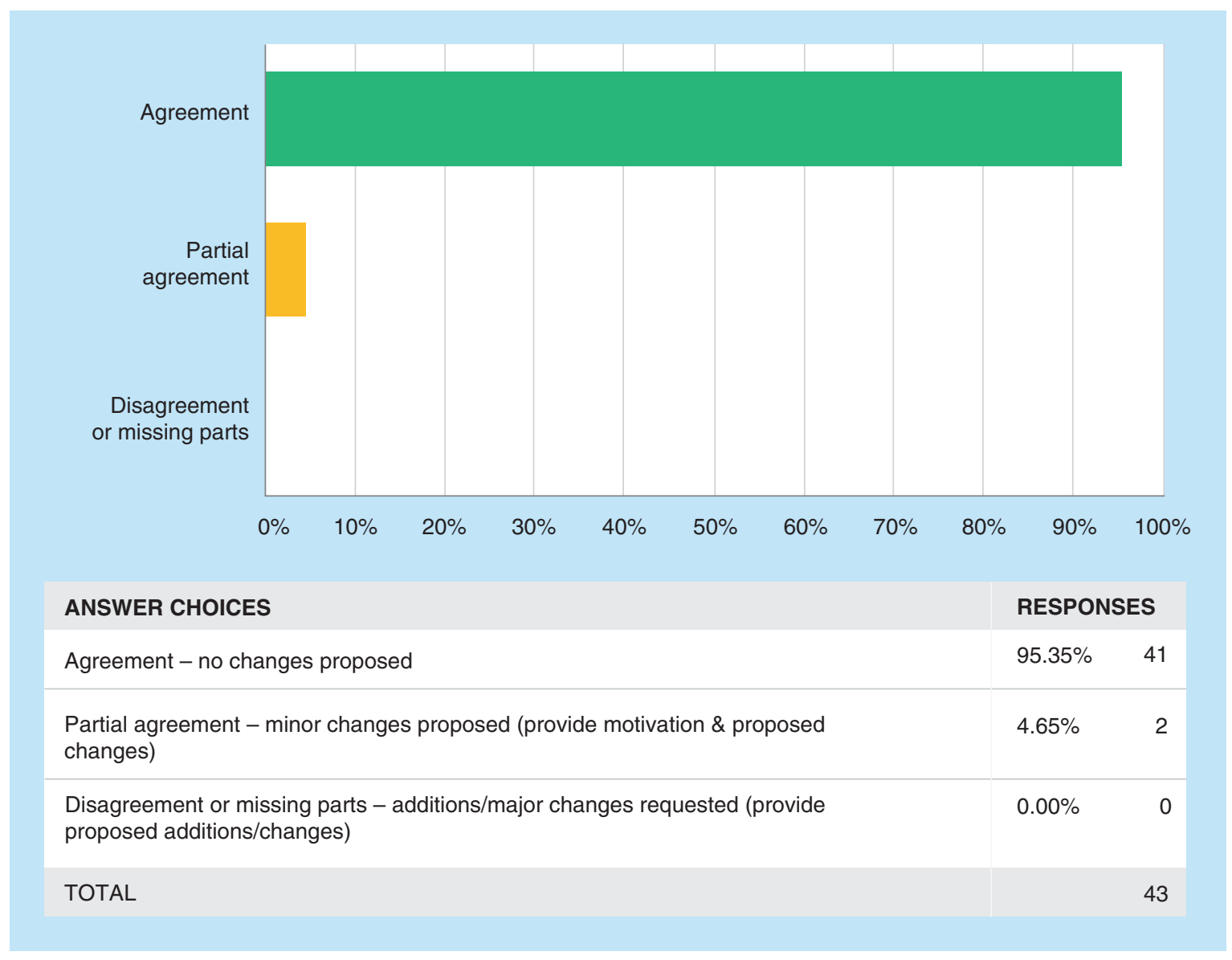

Partial agreement

No trend was found in these comments. 
T5Q13: Analytical Run - QC levels; Lines 864-868: "Each run should contain at least 3 levels of QCs (low, medium and high). During study sample analysis, the calibration standards and QCs should mimic the analysis of the study sample with regard to the number of wells used per study sample. At least 2/3 of the QCs and 50\% at each concentration level should be within $\pm 20 \%$ of the nominal value at each concentration level. Exceptions to these criteria should be justified and predefined in the SOP or protocol."

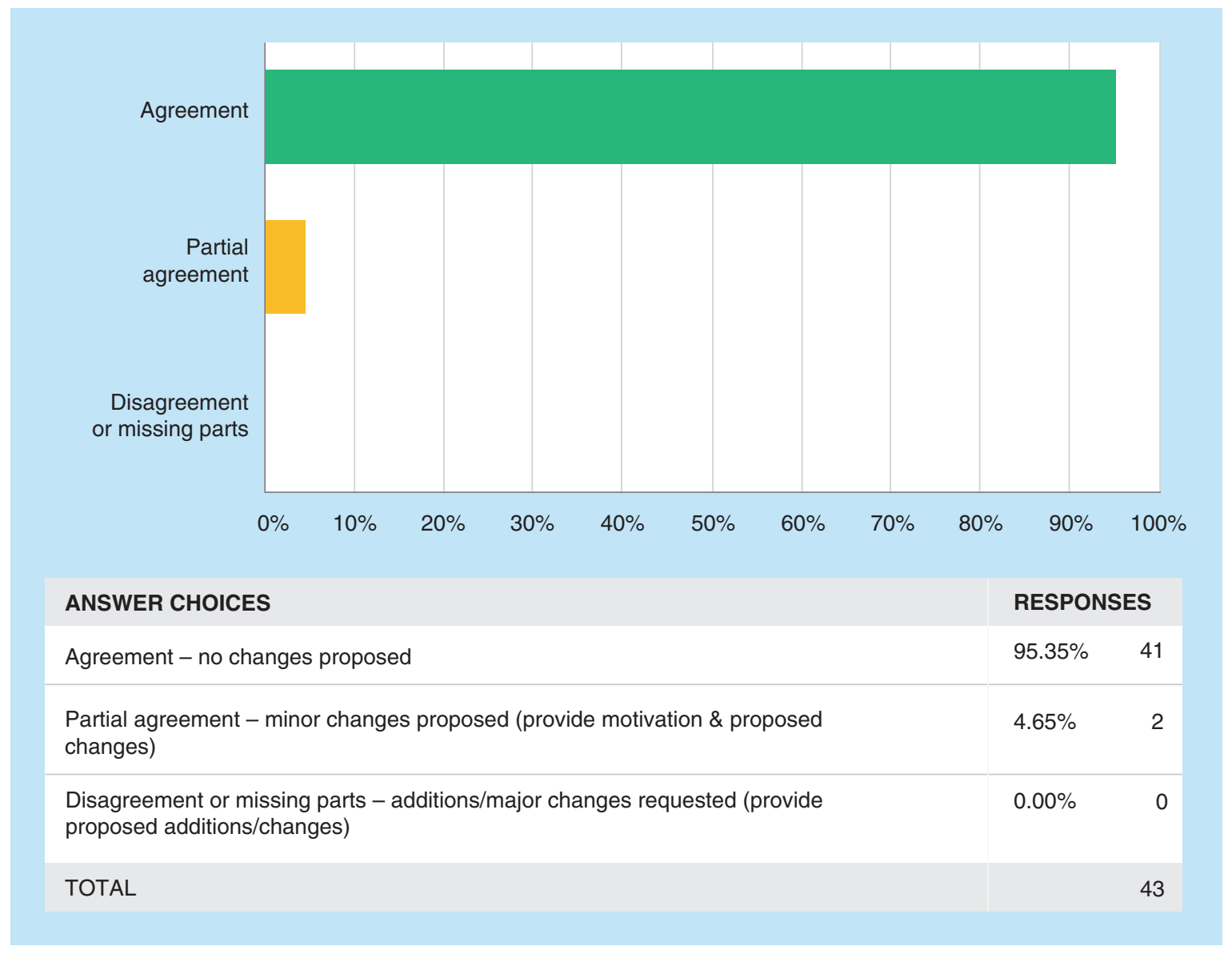

\section{Partial agreement}

No trend was found in these comments. 
T5Q14: Analytical Run - Precision and Accuracy Criteria; Lines 869-873: "The overall mean accuracy and precision of the QCs of all accepted runs should be calculated at each concentration level and reported in the analytical report. In the case that the overall mean accuracy and/or precision exceeds 20\%, additional investigations should be conducted to determine the cause(s) of this deviation. In the case of comparative BA/BE studies it may result in the rejection of the data."

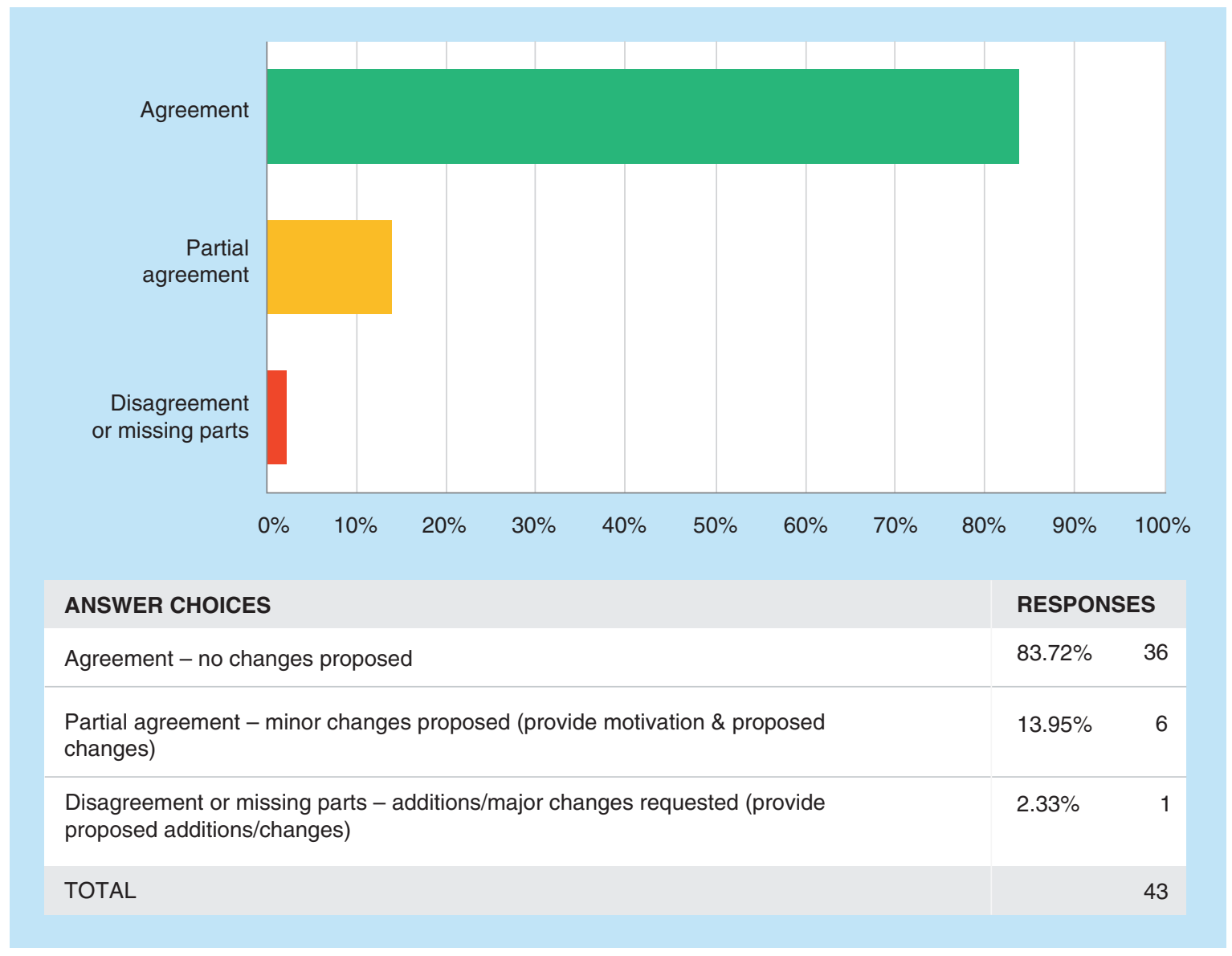

Partial agreement

No trend was found in these comments. 
T5Q15: Calibration Range; Lines 875-882: "At least 2 QC sample levels should fall within the range of concentrations measured in study samples. At the intended therapeutic dose(s), if an unanticipated clustering of study samples at one end of the calibration curve is encountered after the start of sample analysis, the analysis should be stopped and either the standard calibration range narrowed (i.e., partial validation), existing QC concentrations revised, or QCs at additional concentrations added to the original curve within the observed range before continuing with study sample analysis. It is not necessary to reanalyse samples analysed before optimising the calibration curve range or QC concentrations."

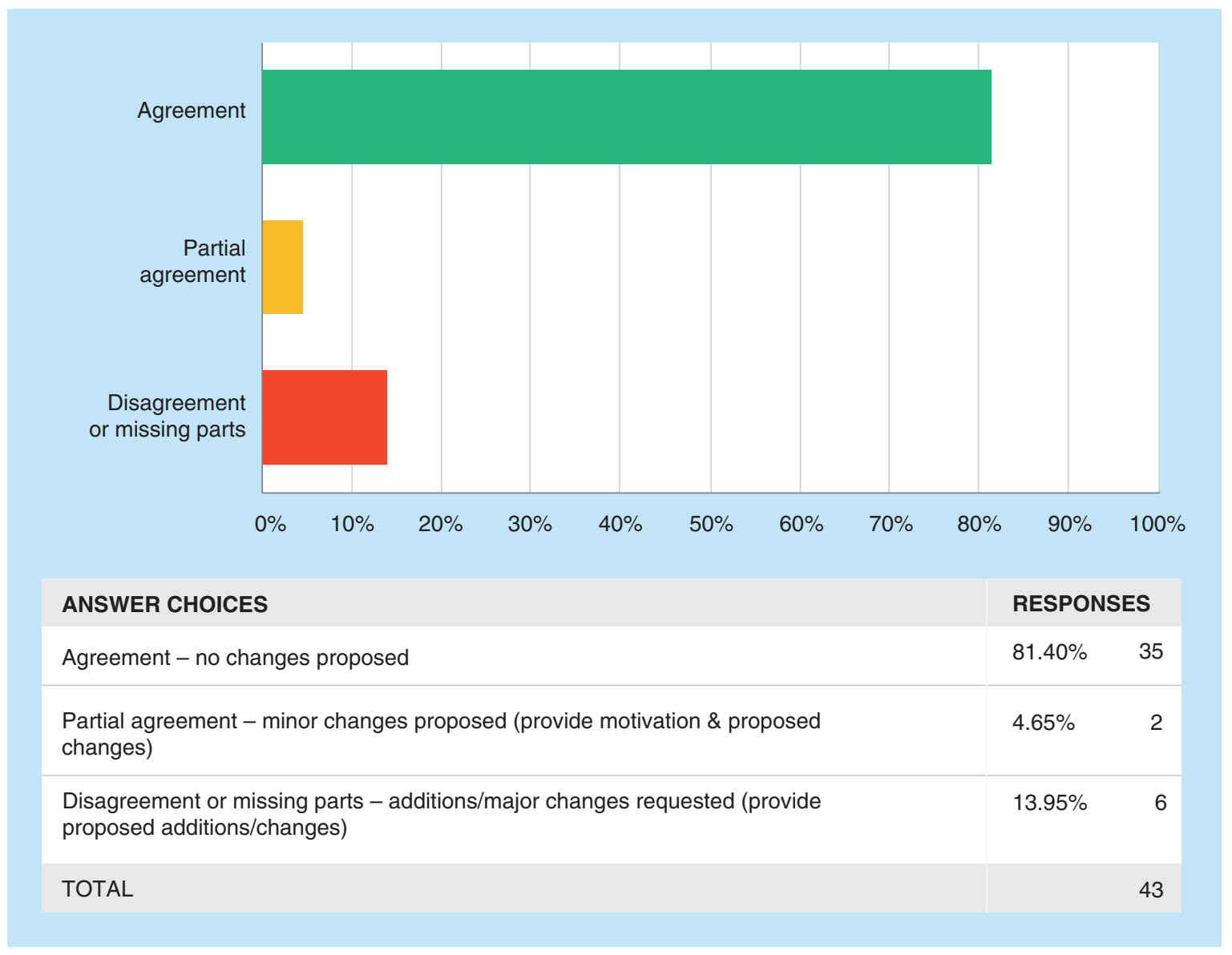

Disagreement or missing parts

All comments disagreed with the need to narrow the range if clustering occurs since the range is typically narrow for LBAs; dilution linearity precision and accuracy across the entire range are all assessed.

\section{Additional comments from the 13th GCC:}

There was consensus that the text regarding narrowing the range should be removed. 
T5Q16: Sample Reanalysis - Predefined Criteria; Lines 884-886: "Possible reasons for reanalysis of study samples, the number of reanalyses and the decision criteria to select the value to be reported should be predefined in the protocol, study plan or SOP, before the actual start of the analysis of the study samples."

Sample Reanalysis - Number of Repeats; Lines 887-888: "The number of samples (and percentage of total number of samples) that have been reanalysed should be reported and discussed in the Bioanalytical Report."

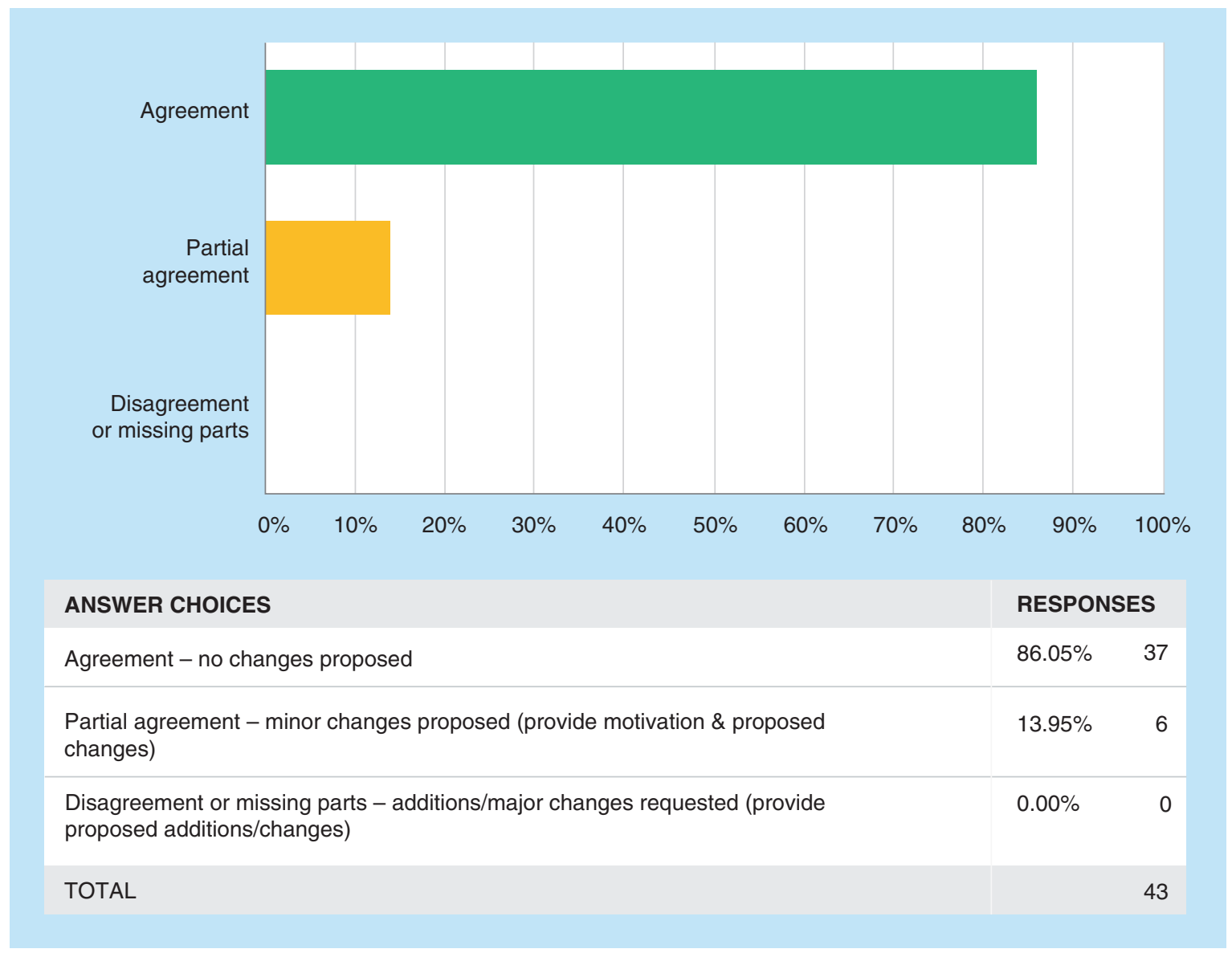

\section{Partial agreement}

All the comments recommend that the number and the percentage of samples repeated are not required since all the repeated samples are reported in various tables in the bioanalytical report.

\section{Additional comments from the 13th GCC:}

It was recommended to align the language with the chromatography section since it is difficult to project reasons for reanalysis in the protocol. The scope of this section should be that every method should have a discussion of sample acceptance as well as the reporting criteria. 
T5Q17: Sample Reanalysis - Examples; Lines 889-901: "Some examples of reasons for study sample reanalysis are:

- Rejection of an analytical run because the run failed the acceptance criteria with regard to accuracy of the calibration standards and/or the precision and accuracy of the QCs,

- The concentration obtained is above the ULOQ,

- The concentration obtained is below the LLOQ in runs where the lowest calibration standard has been rejected from a calibration curve, resulting in a higher LLOQ compared with other runs,

- Malfunction of equipment

- The diluted sample is below the LLOQ

- Identification of quantifiable analyte levels in pre-dose samples, control or placebo samples.

- When samples are analysed in more than one well and non-reportable values are obtained due to one replicate failing the pre-defined acceptance criteria (e.g., excessive variability between wells, one replicate being above the ULOQ or below the LLOQ)."

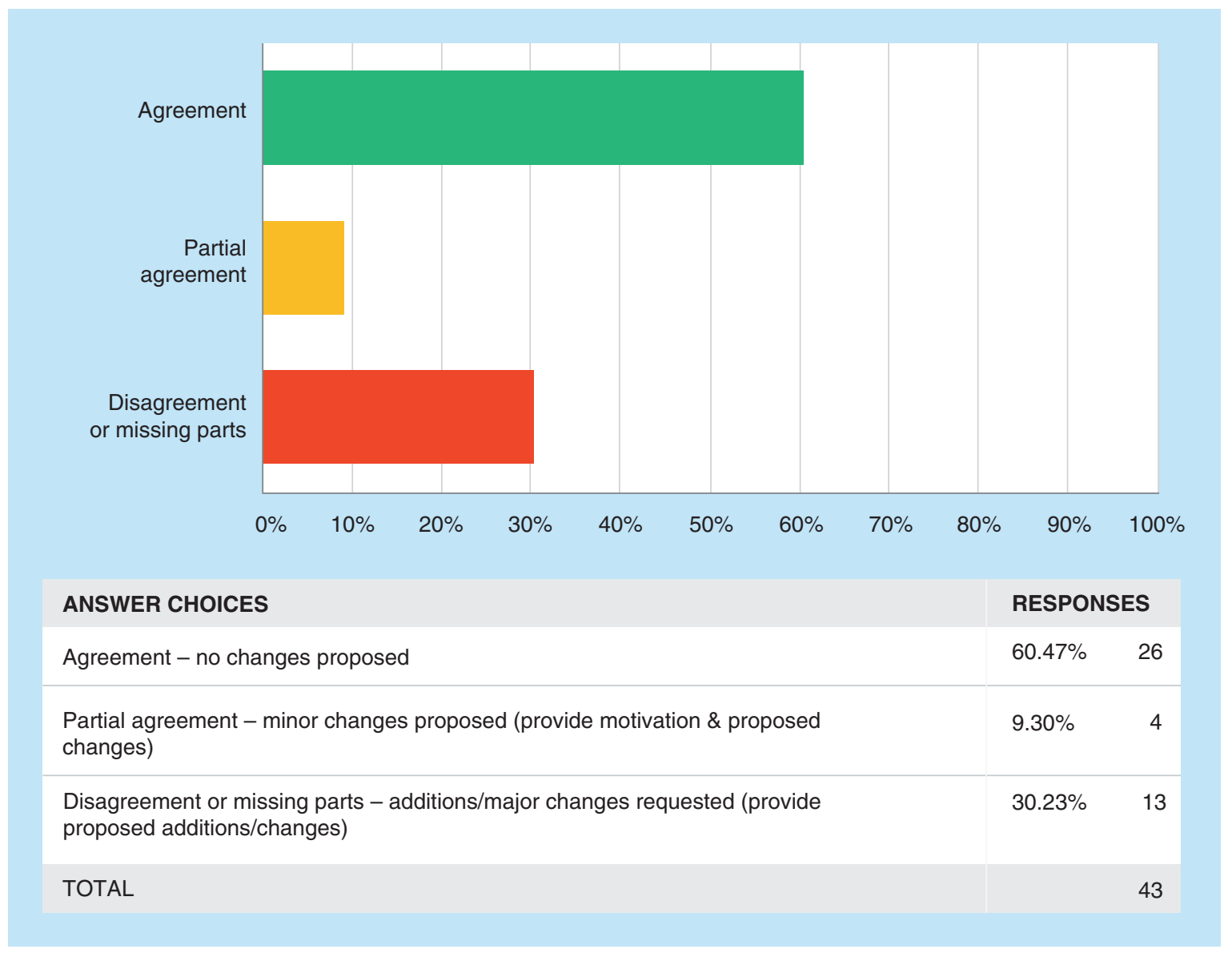

Disagreement or missing parts

It was recommended to add criteria to define excessive variability between wells. Also see comments in T4Q2: "The majority of comments strongly requested to add criteria for the precision $(\% \mathrm{CV})$ between replicate wells since they are missing in both FDA \& EMA guidance/guideline and to refer to current recommendations on this topic [23]. The main concern seems to be the lack of guidance on how to determine sample concentrations from replicate wells (either by using the mean of the responses from the replicate wells or by averaging the concentration calculated from each responses), or if either is acceptable if a consistent approach is applied."

\section{Partial agreement}

It was recommended that samples originally assayed as part of a rejected batch should not be considered as a repeated sample. 
T5Q18: Sample Reanalysis - PK Repeats; Lines 902-904: "For comparative BA/BE studies, reanalysis of study samples for a PK reason (e.g., a sample concentration does not fit with the expected profile) is not acceptable, as it may bias the study result."

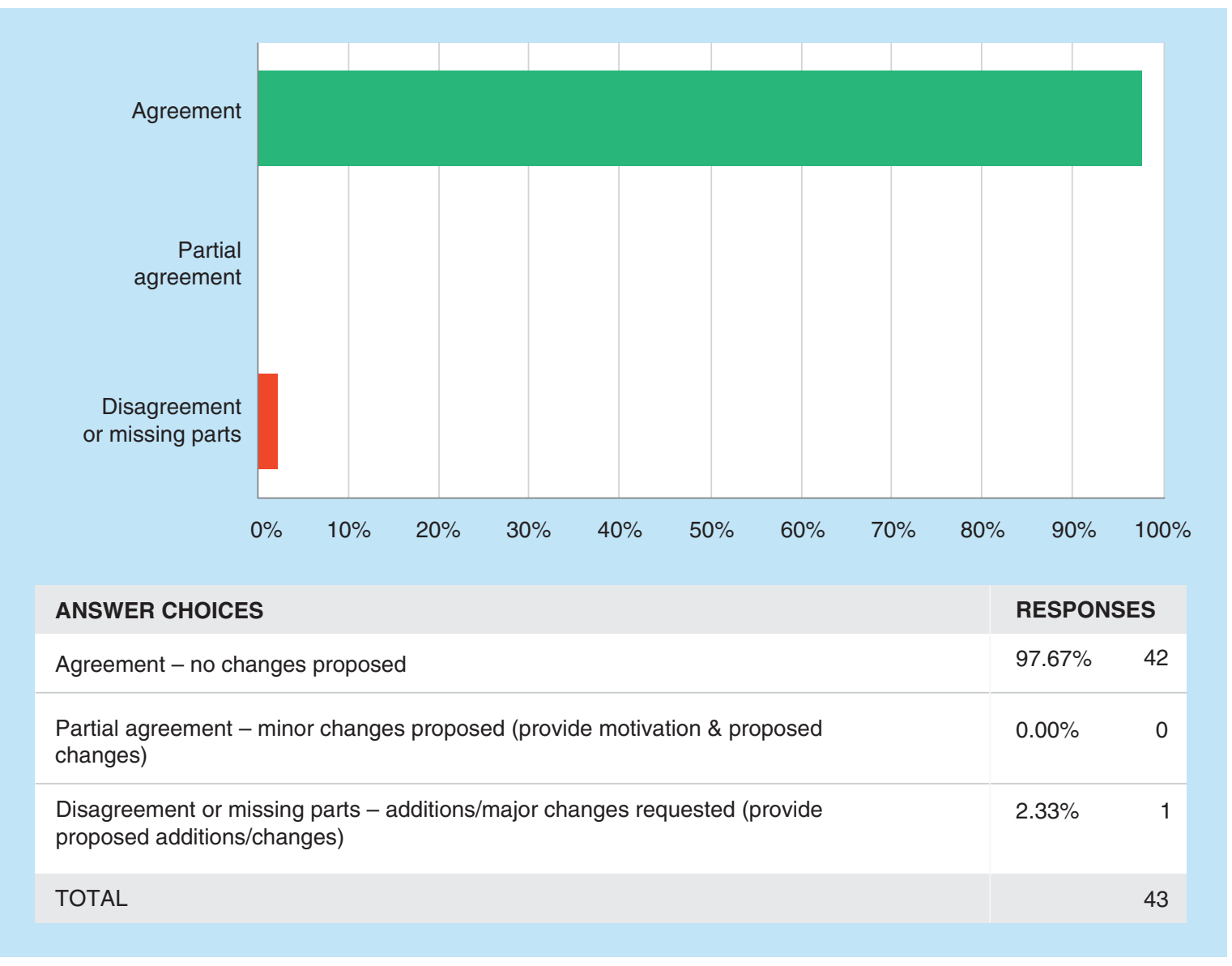


T5Q19: Sample Reanalysis - Reporting; Lines 905-908: "The reanalysed samples should be identified in the Bioanalytical Report and the initial value, the reason for reanalysis, the values obtained in the reanalyses, the final accepted value and a justification for the acceptance should be provided. Further, a summary table of the total number of samples that have been reanalysed due to each reason should be provided."

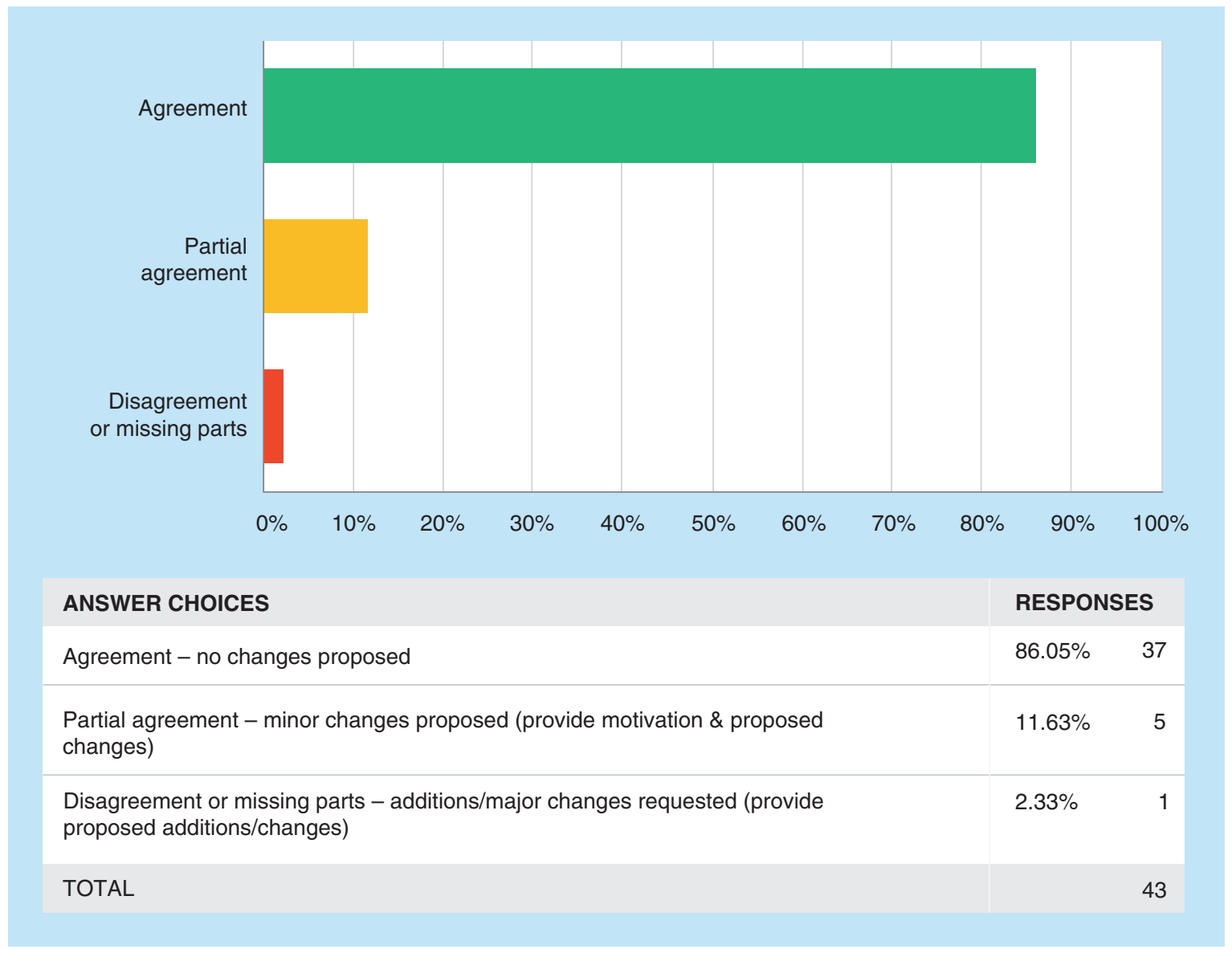

\section{Partial agreement}

It was suggested that the sentence: "Further, a summary table of the total number of samples that have been reanalysed due to each reason should be provided" is redundant.

\section{Additional comments from the 13th GCC:}

- It was recommended that the following text should be removed: "the final accepted value and a justification for the acceptance should be provided."

- It was recommended that the "summary table" in the last sentence should be renamed as a "reassay history table". 
T5Q20: Sample Reanalysis - Confirmatory Reassays; Lines 908-913: "In cases where the first analysis yields a non-reportable result, a single reanalysis is considered sufficient (e.g., concentration above the ULOQ or excessive variability between wells). The analysis of the samples should be based on the same number of wells per study sample as in the initial analysis. In cases where the value needs to be confirmed, (e.g., pre-dose sample with measurable concentrations) multiple determinations are required where sample volume allows."

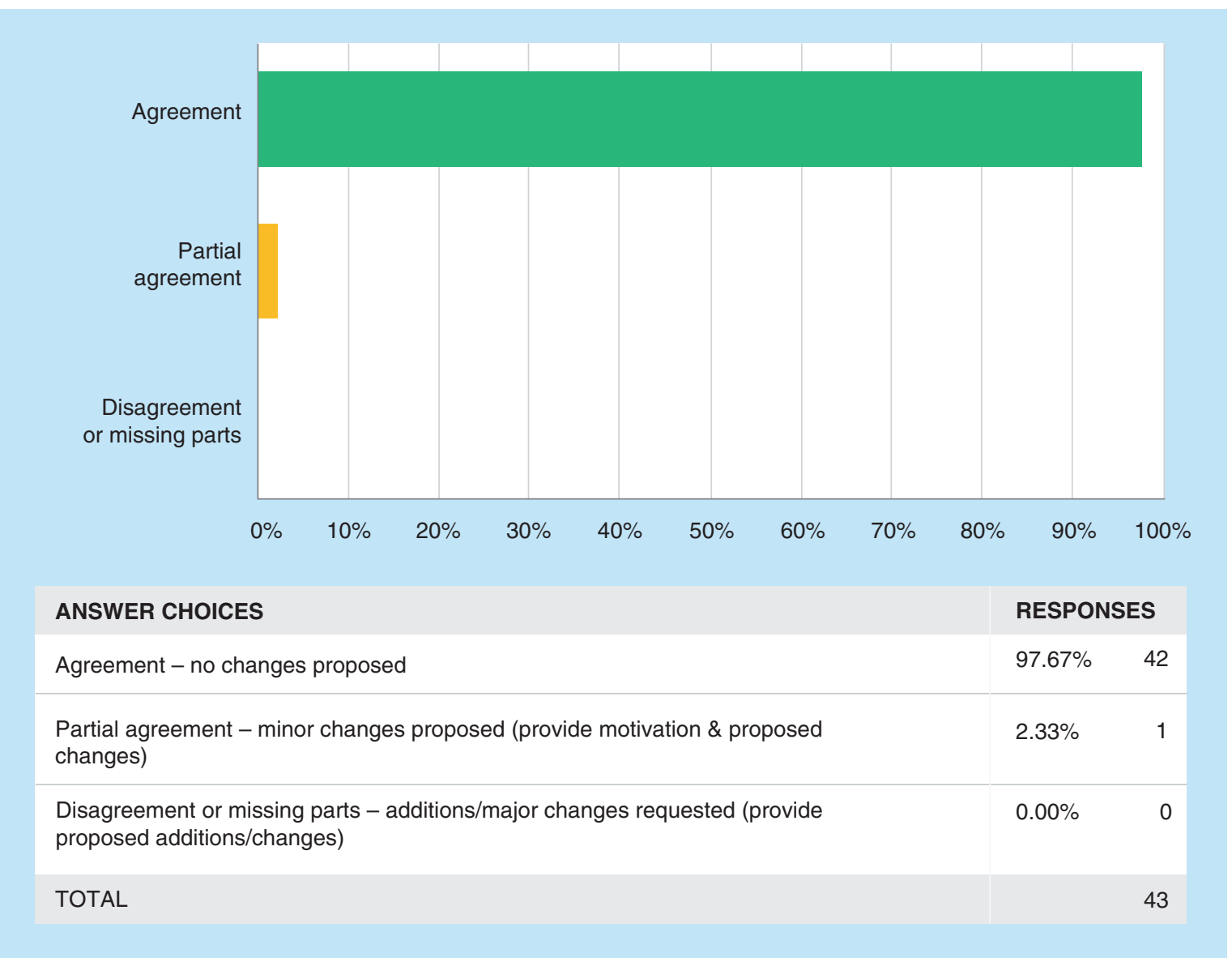


T5Q21: Sample Reanalysis - Investigations; Lines 914-916: “The safety of trial subjects should take precedence over any other aspect of the trial. Consequently, there may be other circumstances when it is necessary to reanalyse specific study samples for the purpose of an investigation."

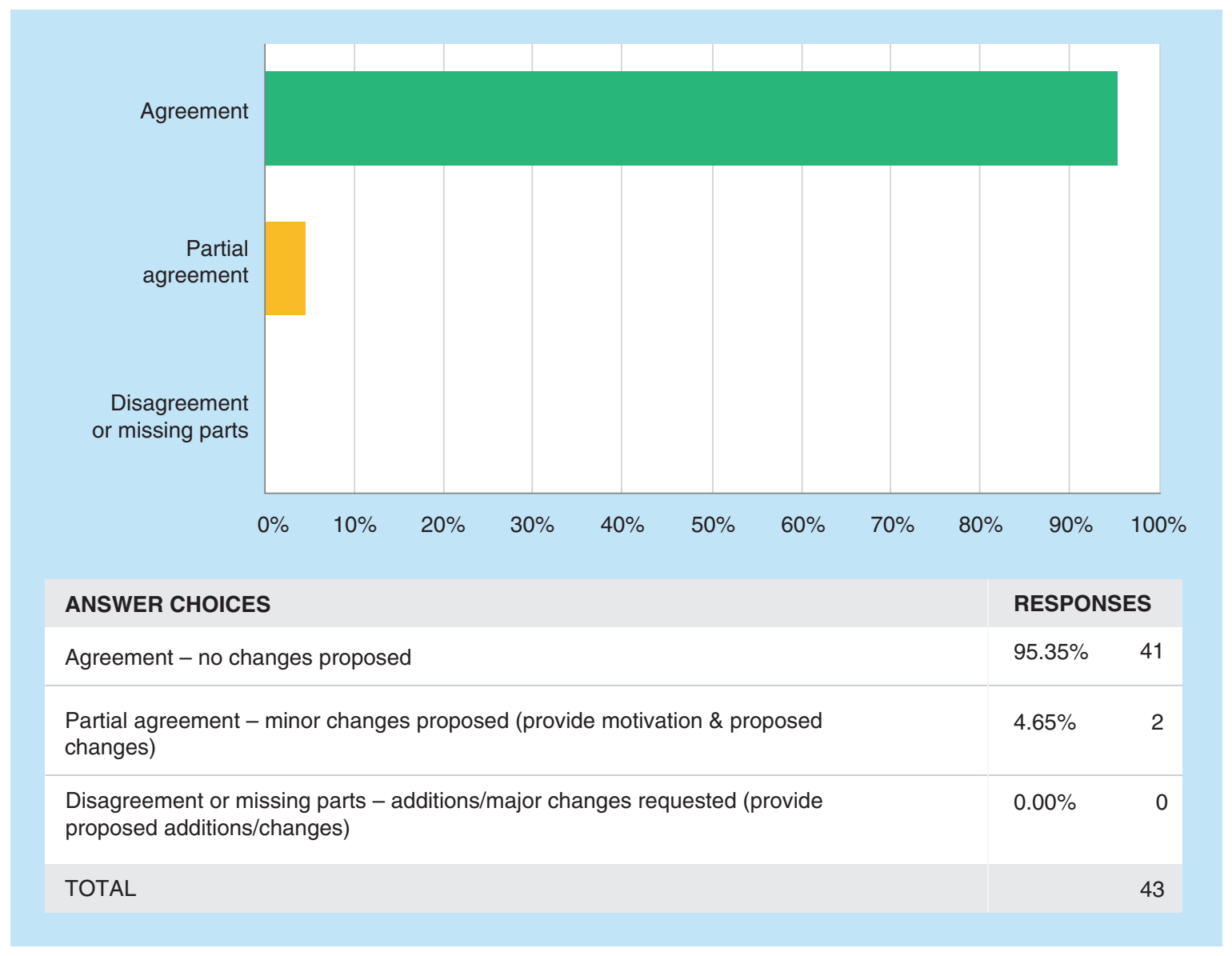

\section{Partial agreement}

Two comments suggested that guidance should be provided for documentation and reporting requirements for this case.

\section{Additional comments from the 13th GCC:}

There were no further discussions during the GCC Closed Forum in support of the above proposal. 
Team 6 Reference Standards, Dried Matrix Methods, New or Alternative Technologies, Commercial and Diagnostic Kits, Analytes that are also Endogenous Compounds

T6Q1: Reference Standards (CC) - Use in Stds and QCs; Lines 192-196: "During method validation and the analysis of study samples, a blank biological matrix is spiked with the analyte(s) of interest using solutions of reference standard(s) to prepare calibration standards, QCs and stability QCs. Calibration standards and QCs should be prepared from separate stock solutions. However, calibration standards and QCs may be prepared from the same stock solution provided the accuracy and stability of the stock solution have been verified."

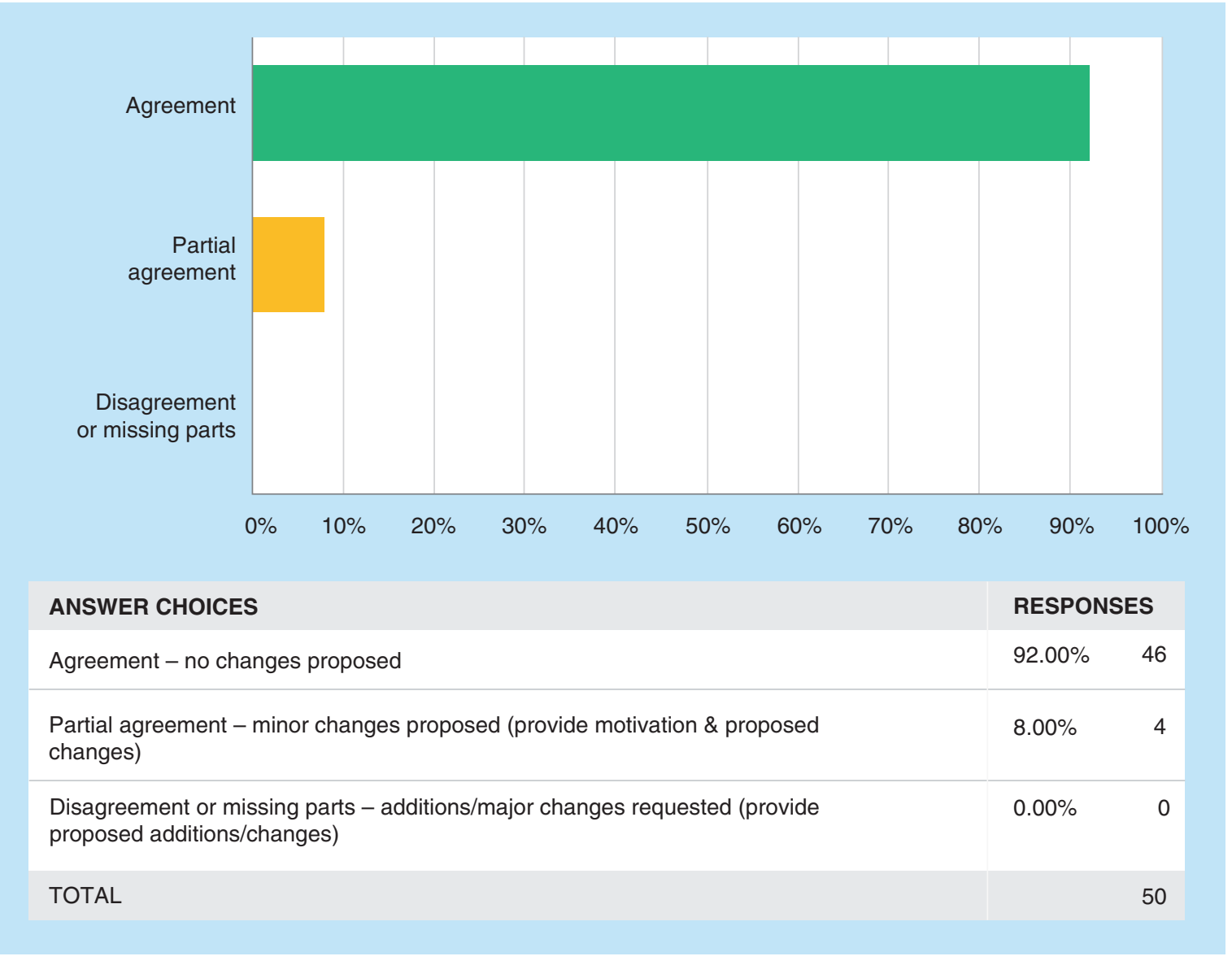

\section{Partial agreement}

Three comments suggested that guidance be provided regarding sufficient verification of accuracy between stocks.

Additional comments from the 13th GCC:

There were no further discussions during the GCC Closed Forum regarding the above proposal. 
T6Q2: Reference Standards (CC) - Use as IS; Lines 197-198: "A suitable internal standard (IS) should be added to all calibration standards, QCs and study samples during sample processing. The absence of an IS should be technically justified."

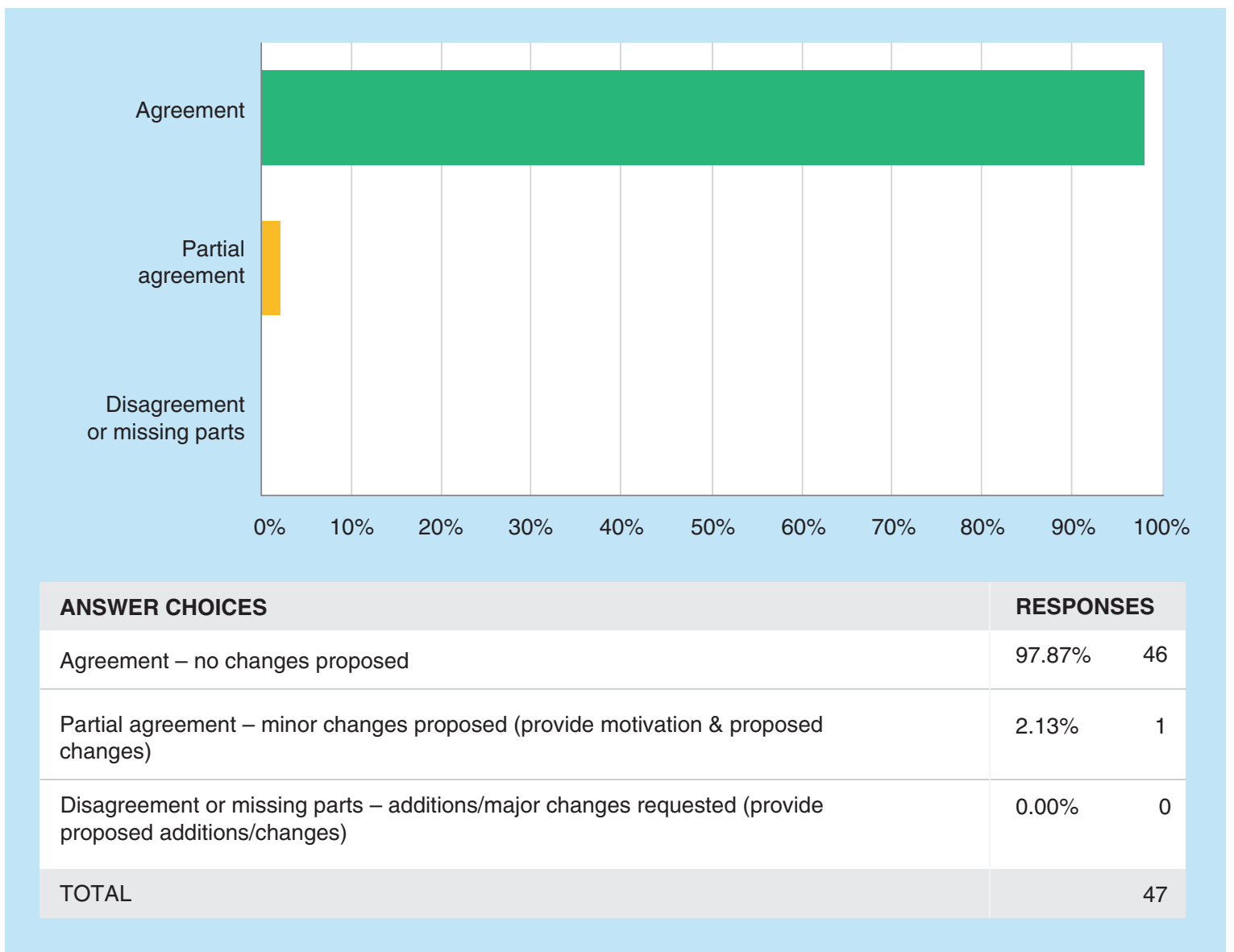


T6Q3: Reference Standards (CC) - Quality; Lines 199-204: "It is important that the reference standard is well characterised and the quality (purity, strength, identity) of the reference standard and the suitability of the IS is ensured, as the quality will affect the outcome of the analysis and, therefore, the study data. The reference standard used during validation and study sample analysis should be obtained from an authentic and traceable source. The reference standard should be identical to the analyte. If this is not possible, an established form (e.g., salt or hydrate) of known quality may be used."

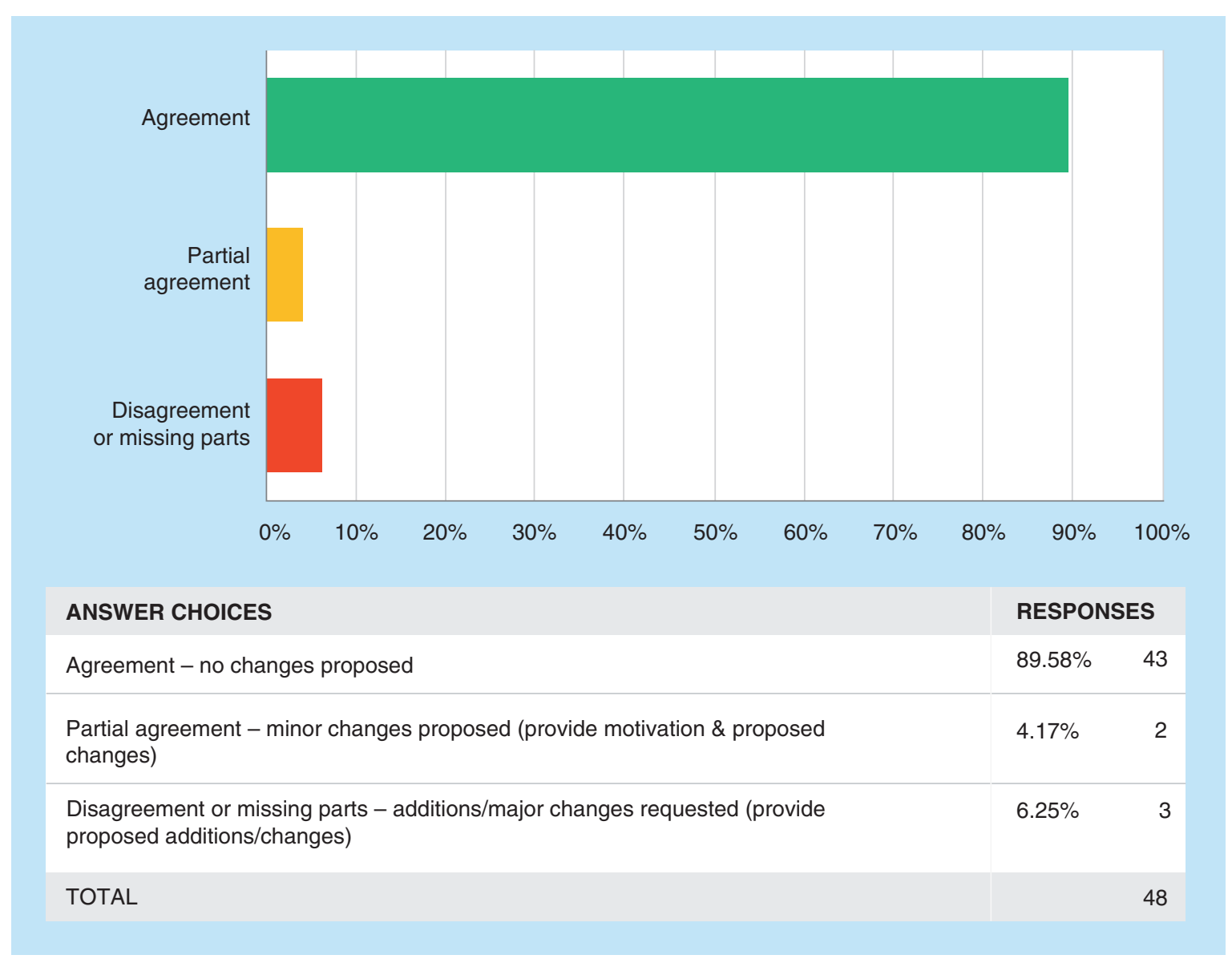

Disagreement or missing parts

It may be worthy to mention that three comments requested more detail in the Guideline for reference standards since it is important to have them well-charactherized. Moreover, harmonization of the contents of a CoA, as per 2018 GCC Closed Forum discussion [9], is key to the industry.

\section{Partial agreement}

No trend was found in these comments.

\section{Additional comments from the 13th GCC:}

Since "strength" is typically used in the CMC space, it was recommended to remove this word. The importance of reference standard characterization and CoA harmonization were discussed in depth in 2018 [9]. Hence, there were no further discussions during the 2019 GCC Closed Forum. 
T6Q4: Reference Standards (CC) - Suppliers; Lines 205-207: "Suitable reference standards include compendial standards, commercially available standards or sufficiently characterised standards prepared in-house or by an external non-commercial organisation."

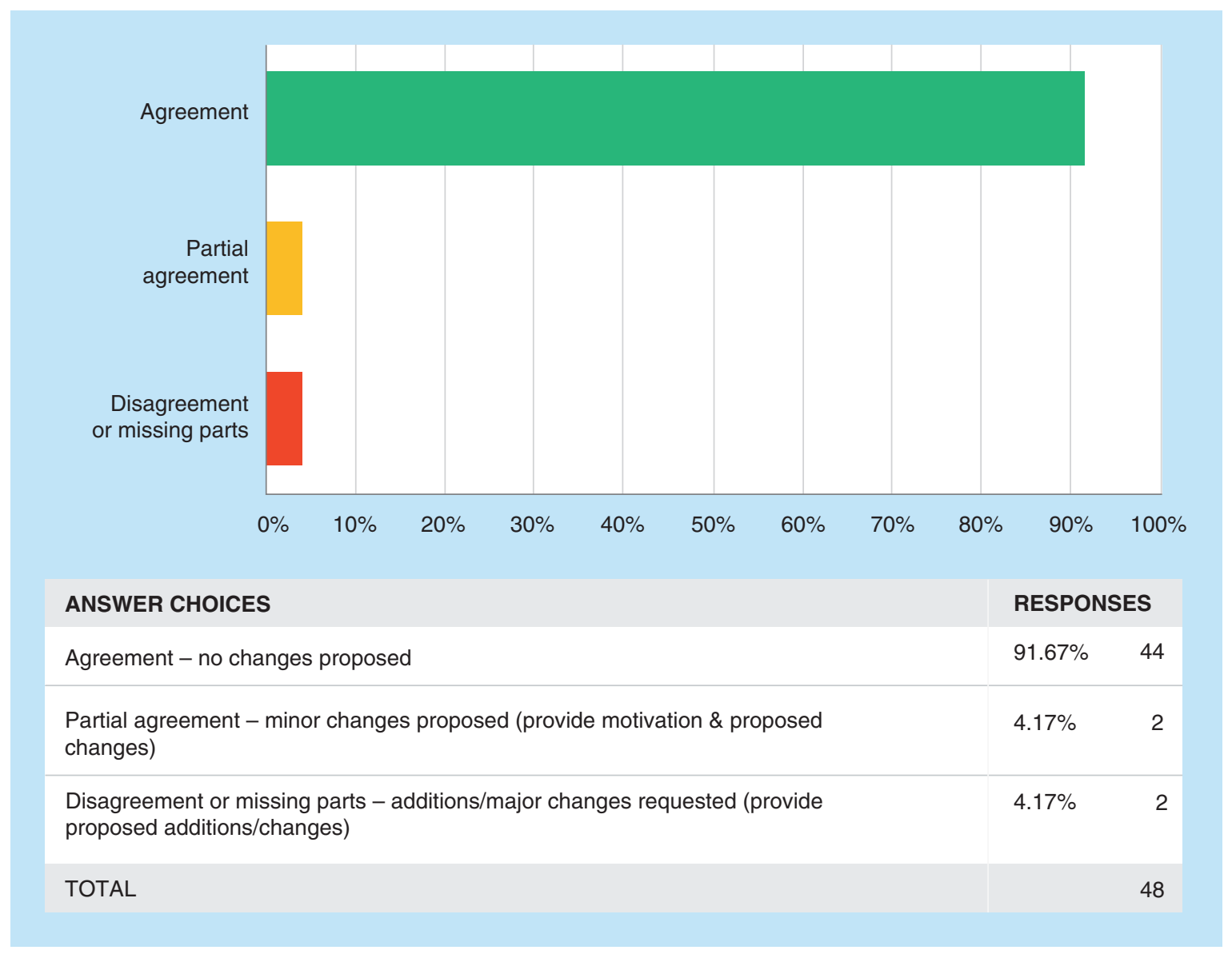

Disagreement or missing parts

No trend was found in these comments.

Partial agreement

No trend was found in these comments. 
T6Q5: Reference Standards (CC) - Certificate of Analysis Analyte; Lines 207-209: "A certificate of analysis (CoA) or an equivalent alternative is required to ensure quality and to provide information on the purity, storage conditions, retest/expiration date and batch number of the reference standard."

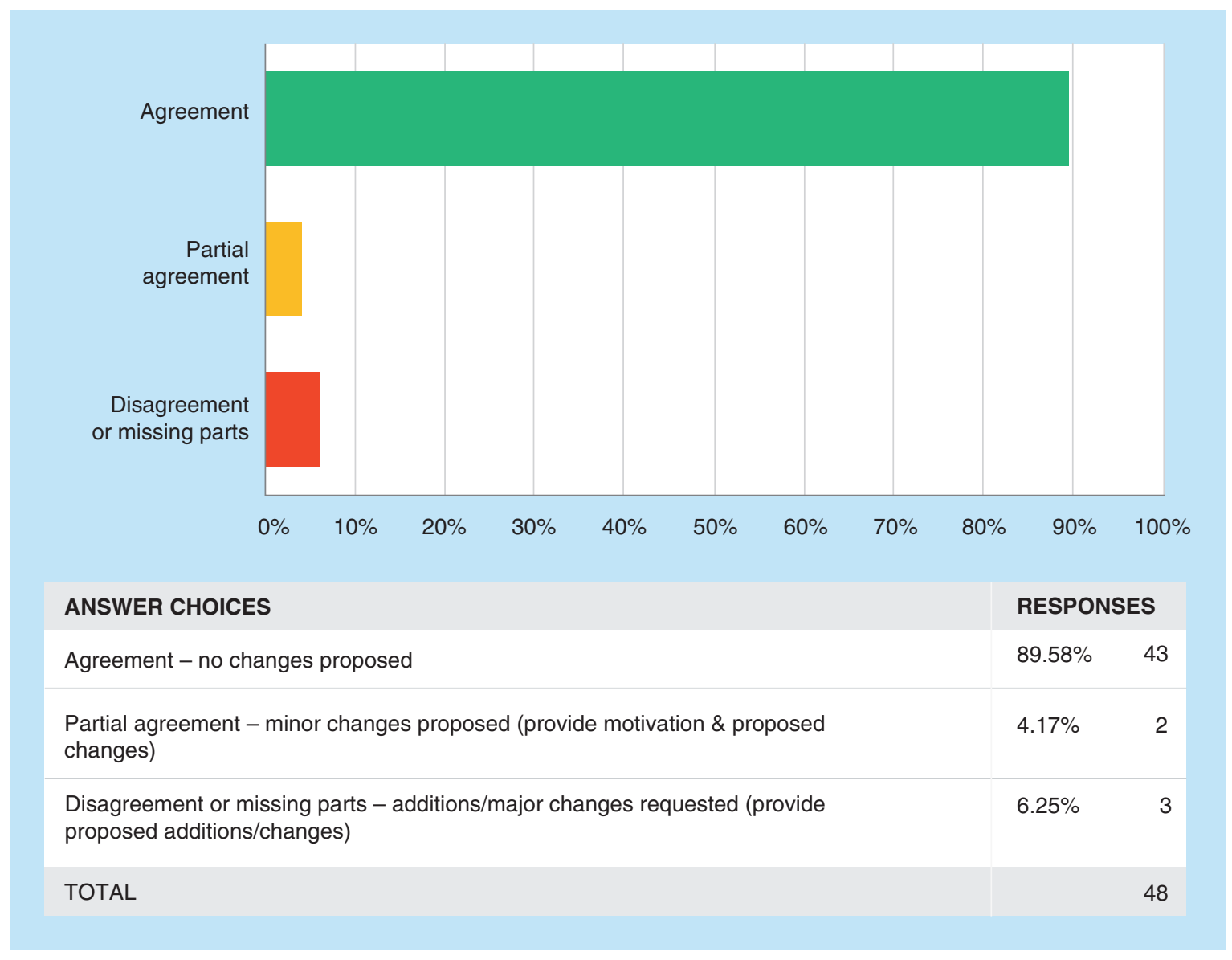

Disagreement or missing parts

No trend was found in these comments.

Partial agreement

No trend was found in these comments. 
T6Q6: Reference Standards (CC) - Certificate of Analysis IS; Lines 210-211: "A CoA is not required for the IS as long as the suitability for use is demonstrated, e.g., a lack of analytical interference is shown for the substance itself or any impurities thereof."

Reference Standards (CC) - Stable Isotope Labelled IS; Lines 212-216: "When MS detection is used, the use of the stable isotope-labelled analyte as the IS is recommended whenever possible. However, it is essential that the labelled standard is of high isotope purity and that no isotope exchange reaction occurs. The presence of unlabelled analyte should be checked and if unlabelled analyte is detected, the potential infuence should be evaluated during method validation."

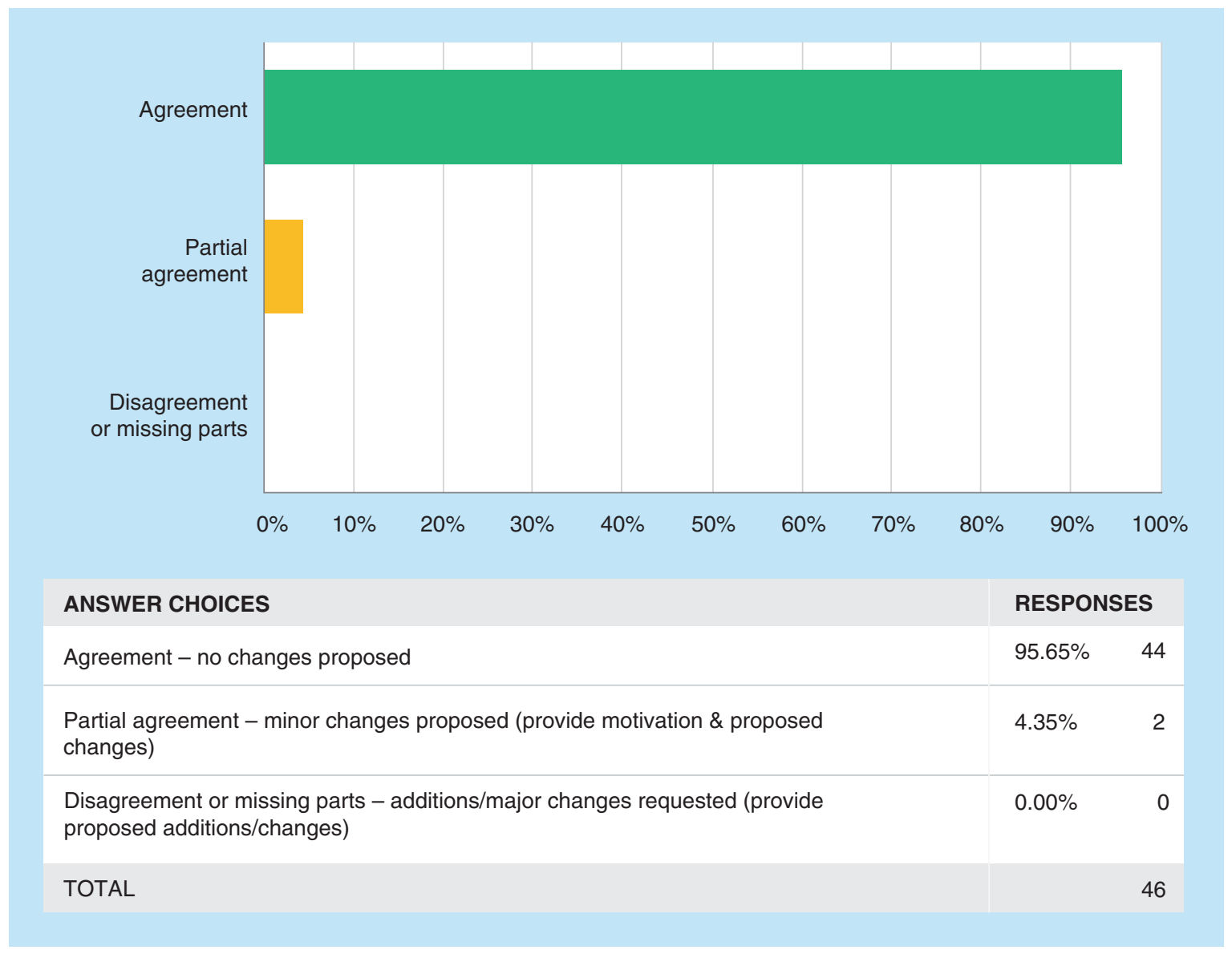

\section{Partial agreement}

No trend was found in these comments. 
T6Q7: Reference Standards (CC) - Stock and Working Solutions; Lines 217-219: "Stock and working solutions can only be prepared from reference standards that are within the stability period as documented in the CoA (either expiration date or the retest date in early development phase)."

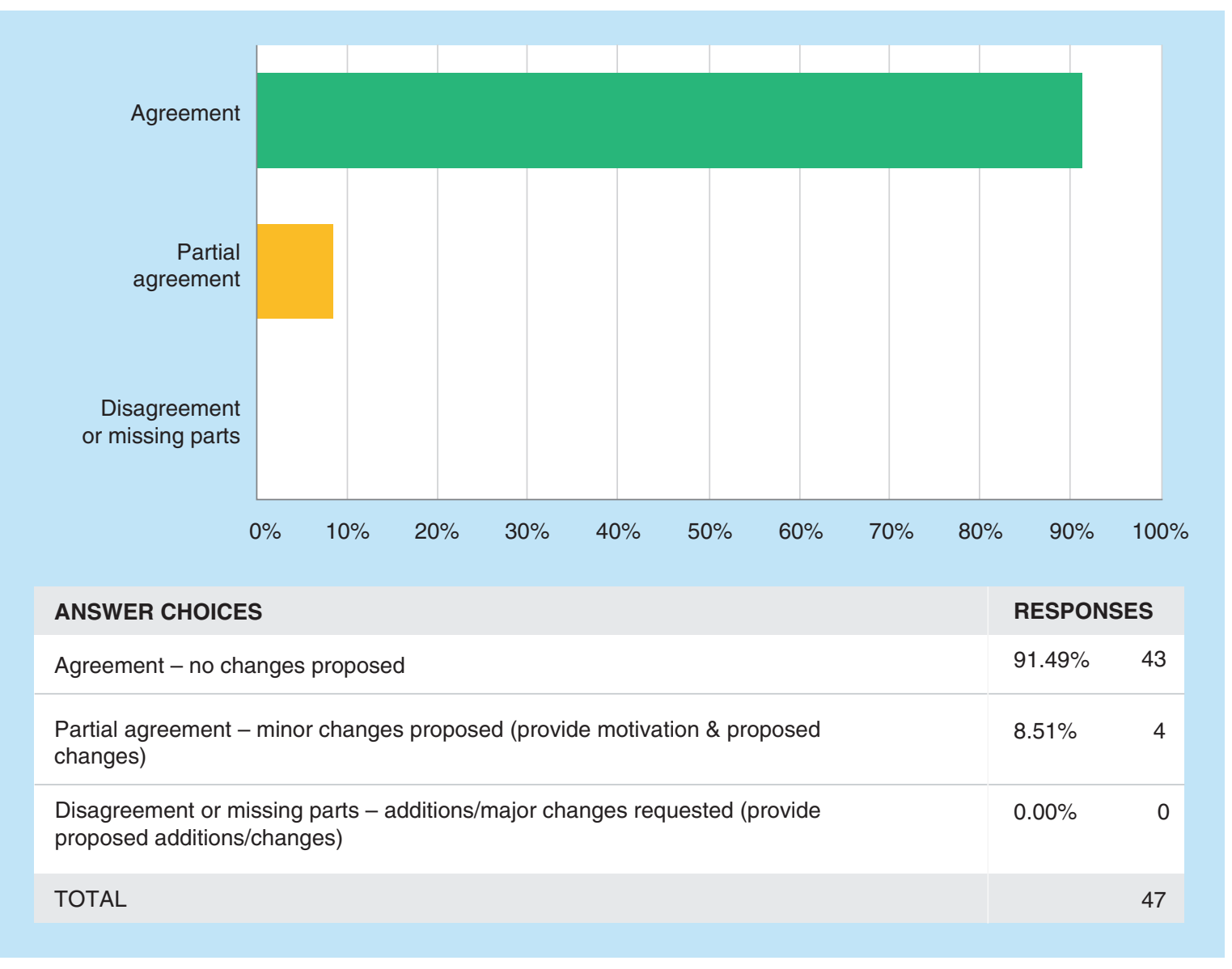

\section{Partial agreement}

It was suggested to remove "working solutions".

Additional comments from the 13th GCC:

The comments of the survey were confirmed by consensus on the removal of "working solutions". 
T6Q8: Reference Standards - Requirement; Line 621: "The reference standard should be well characterised and documented (e.g., CoA and origin)."

Reference Standards - Background; Lines 621-623: "A biological drug has a highly complex structure and its reactivity with binding reagents for bioanalysis may be influenced by a change in the manufacturing process of the drug substance."

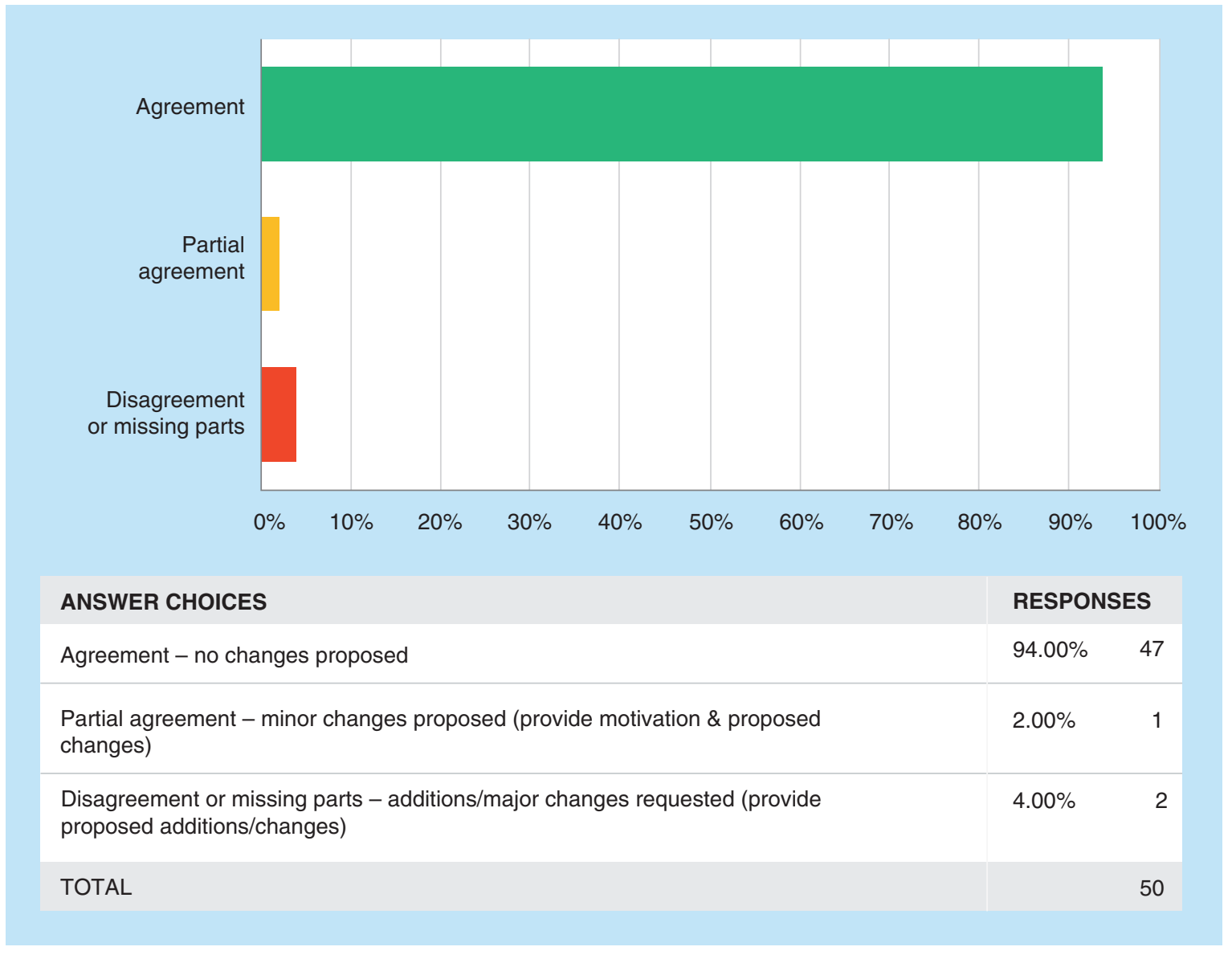

Disagreement or missing parts

No trend was found in these comments. 
T6Q9: Reference Standards (CC) -Batch Used; Lines 624-626: "It is recommended that the manufacturing batch of the reference standard used for the preparation of calibration standards and QCs is derived from the same batch of drug substance as that used for dosing in the nonclinical and clinical studies whenever possible."

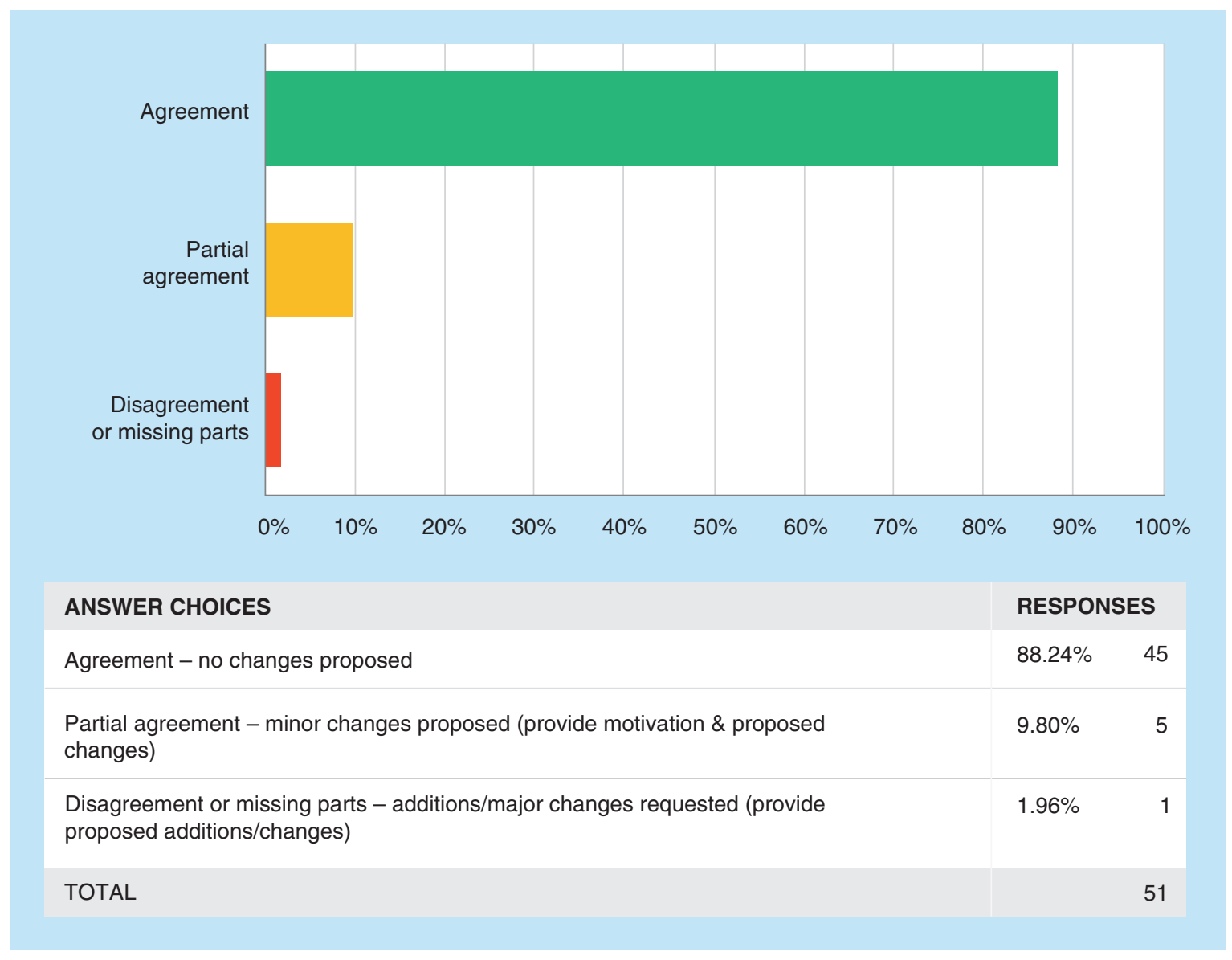

\section{Partial agreement}

Multiple clarifications were requested in the comments. It was also suggested that this is necessary for biological drugs only, not for chemical entities.

\section{Additional comments from the 13th GCC:}

- Comments from the survey were further discussed and expanded during the GCC Closed Forum by confirming that the language and criteria should be harmonized for independent reference standards and those used as part of kits, since it appears that there is more flexibility when using the latter. The expectations for reference standards from commercial kits should also be added.

- It was also confirmed that the requirement to use the same batch is almost never possible because it is not typically available when the method is being validated. 
T6Q10: Reference Standards - Batch Changed; Lines 626-629: "If the reference standard batch used for bioanalysis is changed, bioanalytical evaluation should be carried out prior to use to ensure that the performance characteristics of the method are within the acceptance criteria."

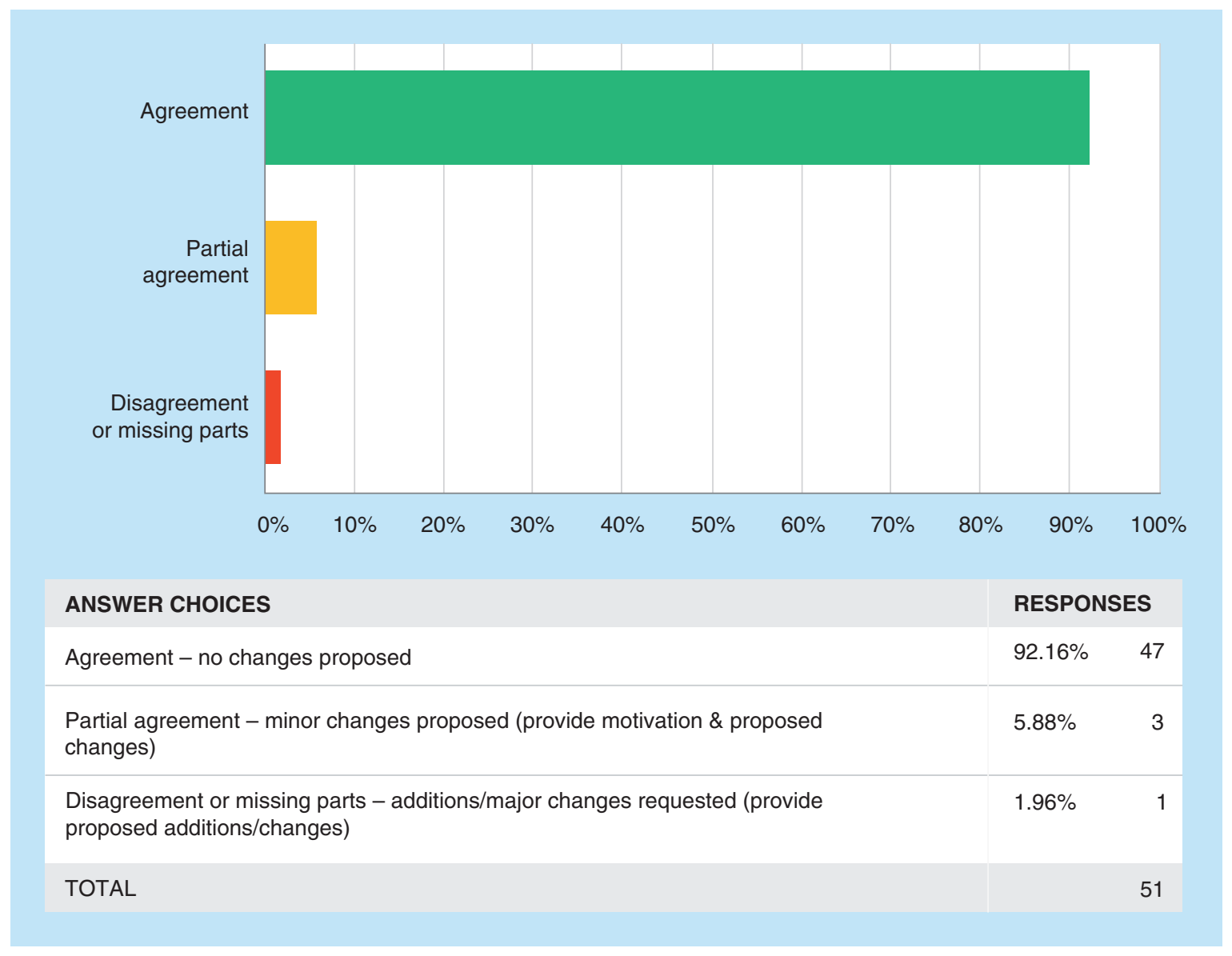

Partial agreement

As in T6Q9, it was suggested that this is necessary for biological drugs only, not for chemical entities. 
T6Q11: Endogenous Compounds - Background; Lines 1025-1029: "For analytes that are also endogenous compounds, the accuracy of the measurement of the analytes poses a challenge when the assay cannot distinguish between the therapeutic agent and the endogenous counterpart. The endogenous levels may vary because of age, gender, diurnal variations, illness or as a side effect of drug treatment."

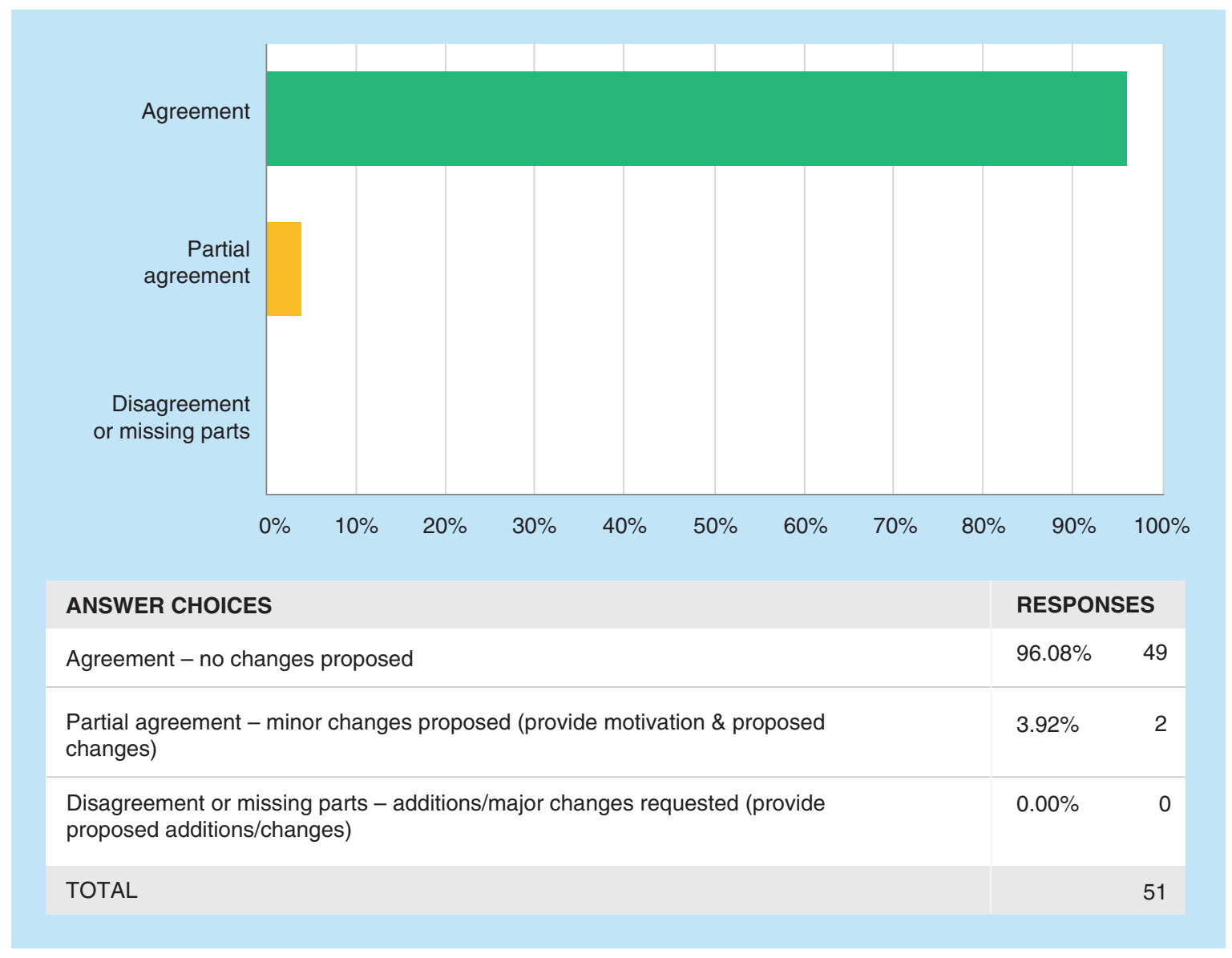

Partial agreement

No trend was found in these comments. 
T6Q12: Endogenous Compounds - Matrix Lot Selection; Lines 1029-1034: "If available, biological matrix with an adequate signal-to-noise ratio (i.e., endogenous level sufficiently low for the desired LLOQ, e.g., <20\% of the LLOQ) should be used as blank matrix to prepare calibration standards and QCs since the biological matrix used to prepare calibration standards and QCs should be the same as the study samples (i.e., authentic biological matrix) and should be free of matrix effect and endogenous analyte at the level that causes interference."

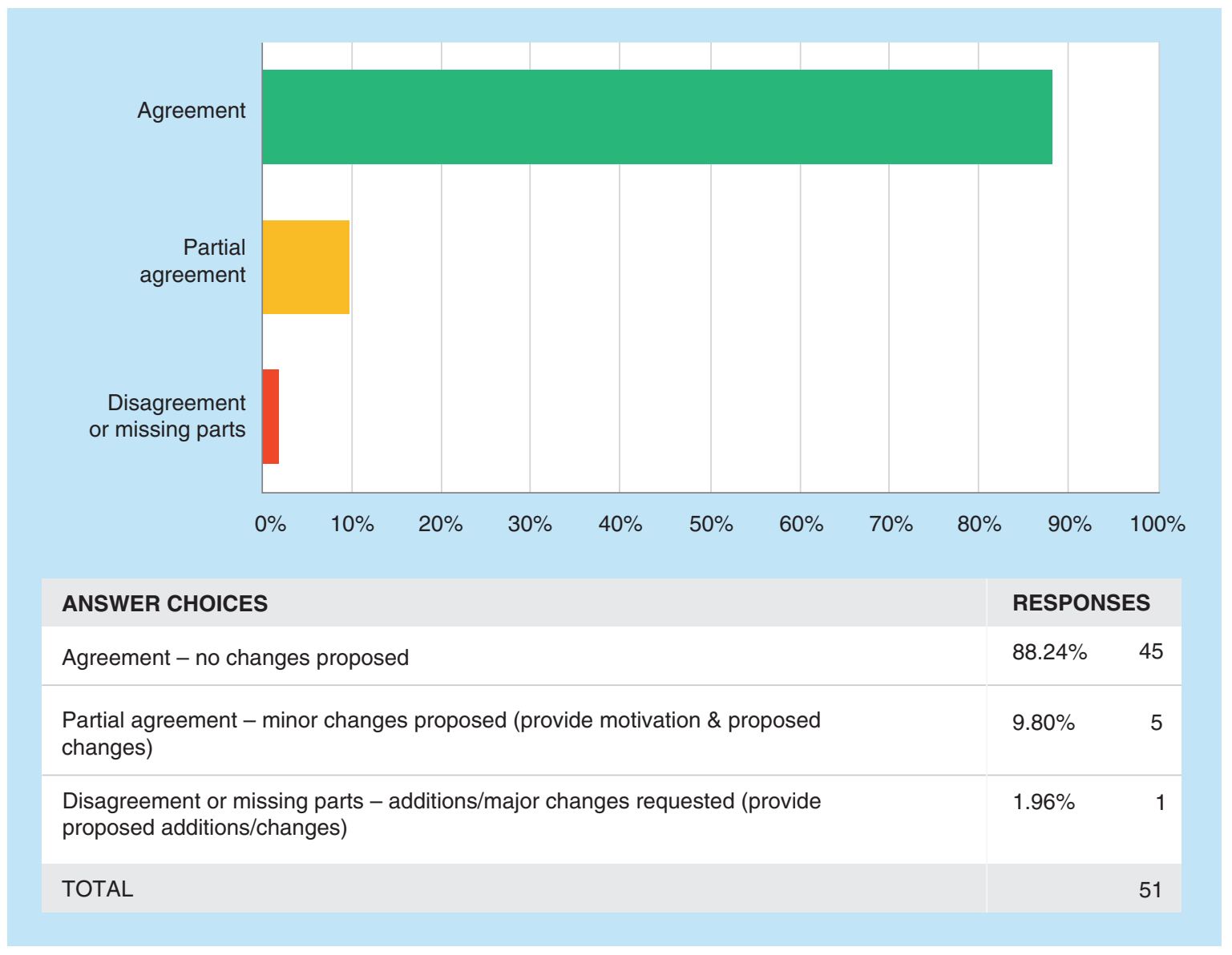

\section{Partiall agreement}

A few comments suggested that the text stating the matrix used for preparing QCs "should be free of matrix effect" should be rewritten since it is often not possible.

\section{Additional comments from the 13th GCC:}

There were no further discussions during the GCC Closed Forum regarding the above proposal. 
T6Q13: Calculating Endogenous Level; Lines 1035-1039: "In those cases where matrices without interference are not available, there are four possible approaches to calculate the concentration of the endogenous analyte in calibration standards, QCs and, consequently, study samples: 1) the standard addition approach, 2) the background subtraction approach, 3) the surrogate matrix (neat, artificial or stripped matrices) approach and 4) the surrogate analyte approach."

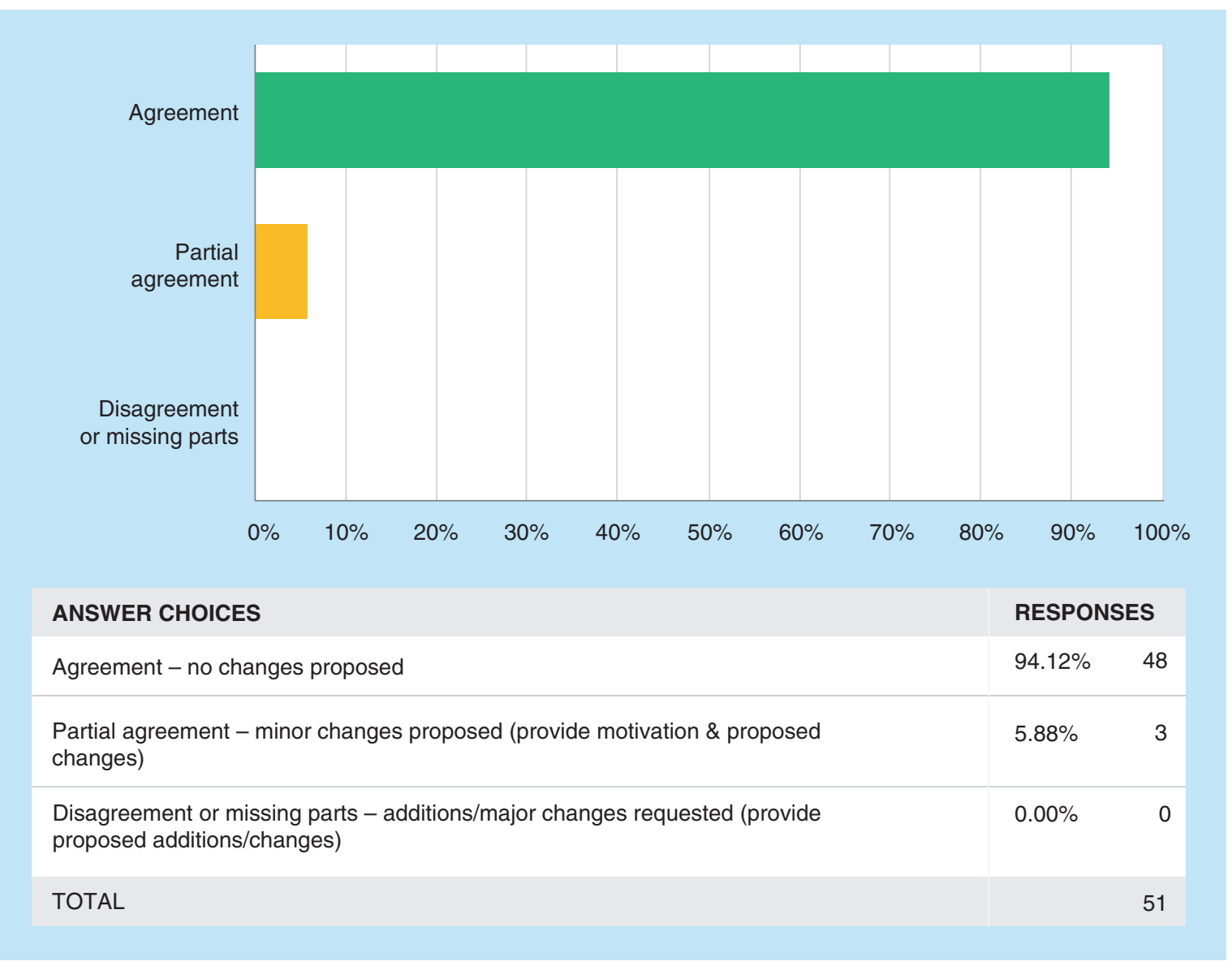

\section{Partial agreement}

Three comments suggested that the standard addition approach be removed for LBA assays. This opinion was also expressed in several responses to other questions.

Additional comments from the 13th GCC:

These comments were discussed and it was agreed that this approach should be removed for LBA. 
T6Q14: Standard Addition Approach; Lines 1040-1045: "Every study sample is divided into aliquots of equal volume. All aliquots, but one, are separately spiked with known and varying amounts of the analyte standards to construct a calibration curve for every study sample. The study sample concentration is then determined as the negative $x$-intercept of the standard calibration curve prepared in that particular study sample."

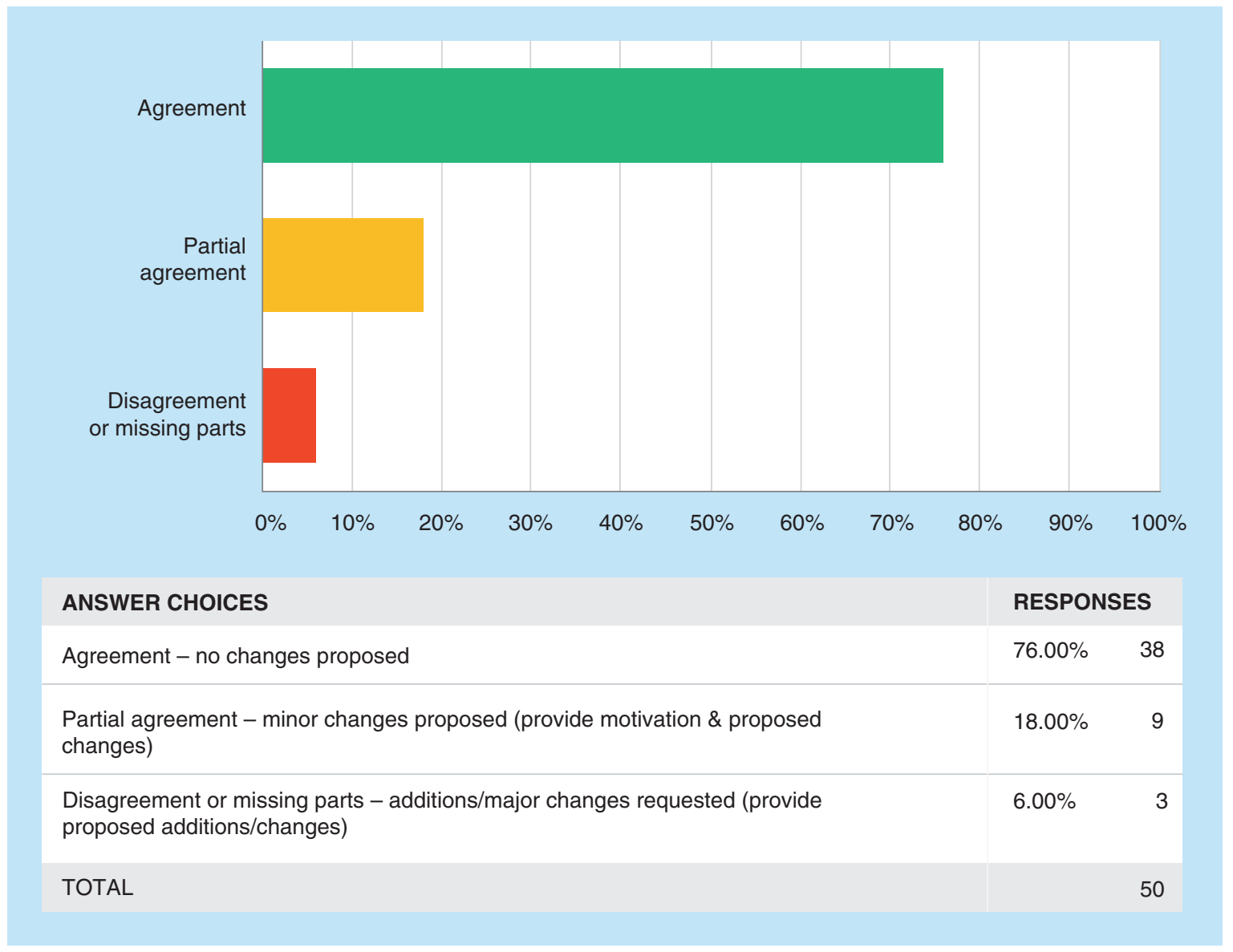

Disagreement or missing parts

All comments stated that this approach is only relevant with a linear regression and chromatography assays (see also T6Q13).

\section{Partiall agreement}

Some comments mentioned this approach is rarely used for incurred samples due to limited sample volume and the fact that it is time-consuming. 
T6Q15: Background Subtraction Approach; Lines 1046-1049: "The endogenous background concentrations of analytes in a pooled/representative matrix are subtracted from the concentrations of the added standards, subsequently the subtracted concentrations are used to construct the calibration curve."

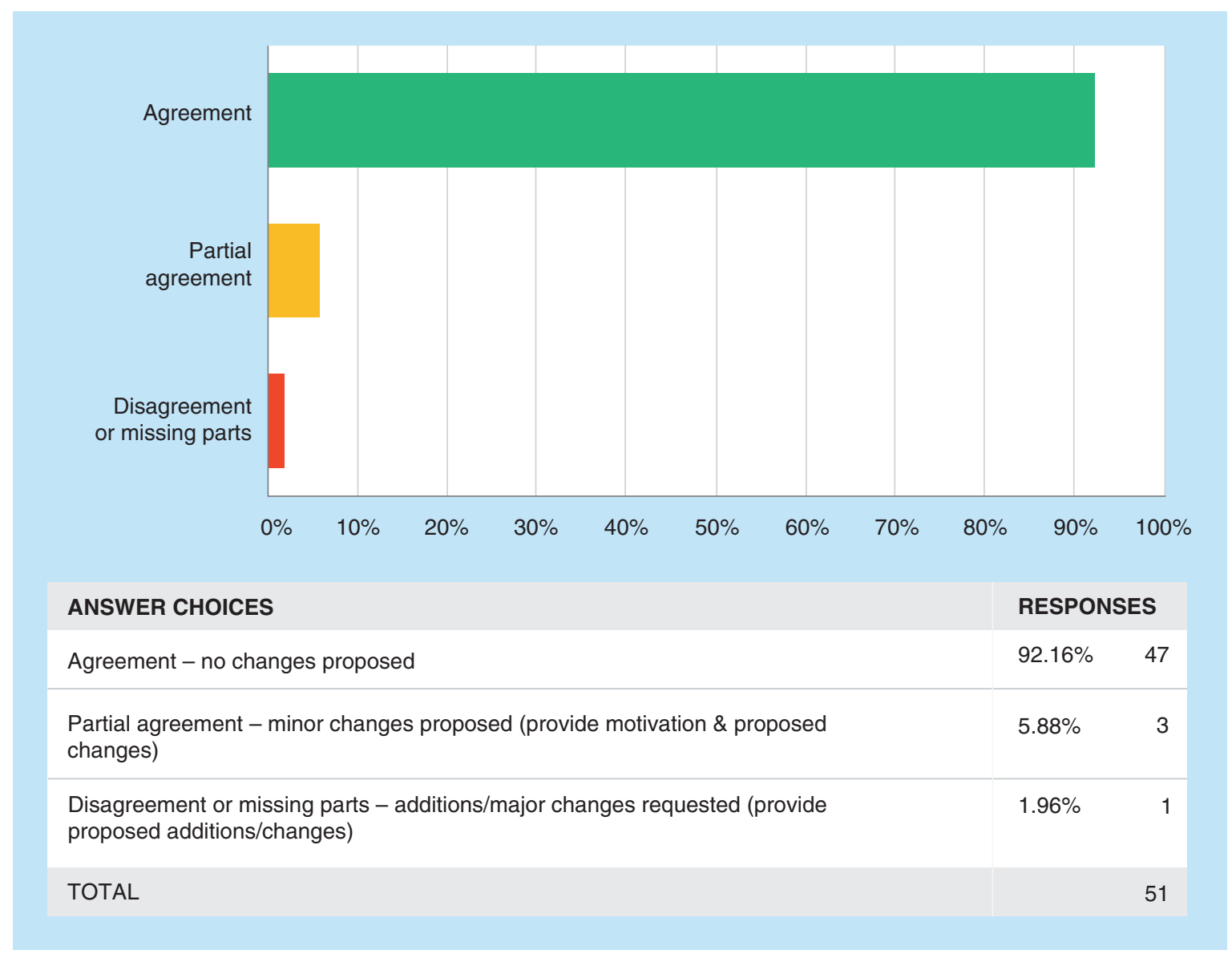

Partial agreement

No trend was found in these comments. 
T6Q16: Surrogate Matrix Approach; Lines 1050-1053: "The matrix of the study samples is substituted by a surrogate matrix. Surrogate matrices can vary widely in complexity from simple buffers or artificial matrices that try to mimic the authentic one, to stripped matrices."

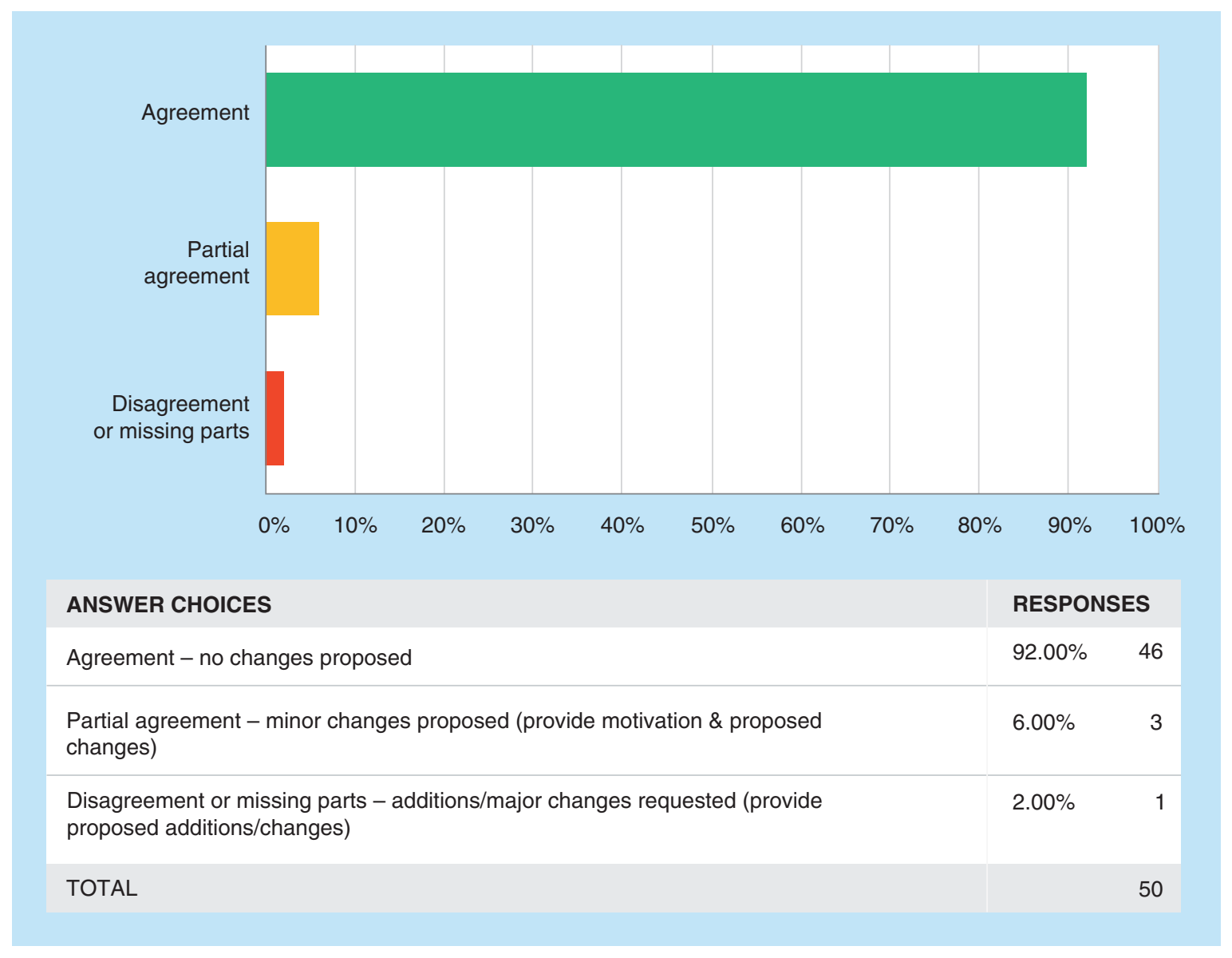

\section{Partial agreement}

No trend was found in these comments. 
T6Q17: Surrogate Analyte Approach - Background; Lines 1054-1058: "Stable-isotope labelled analytes are used as surrogate standards to construct the calibration curves for the quantification of endogenous analytes. In this method it is assumed that the physicochemical properties of the authentic and surrogates analytes are the same with the exception of molecular weight."

Surrogate Analyte Approach; Lines 1058-1063: "However, isotope standards may differ in retention time and MS sensitivity, therefore, before application of this approach, the ratio of the labelled to unlabelled analyte MS responses (i.e., the response factor) should be close to unity and constant over the entire calibration range. If the response factor does not comply with these requirements, it should be incorporated into the regression equation of the calibration curve."

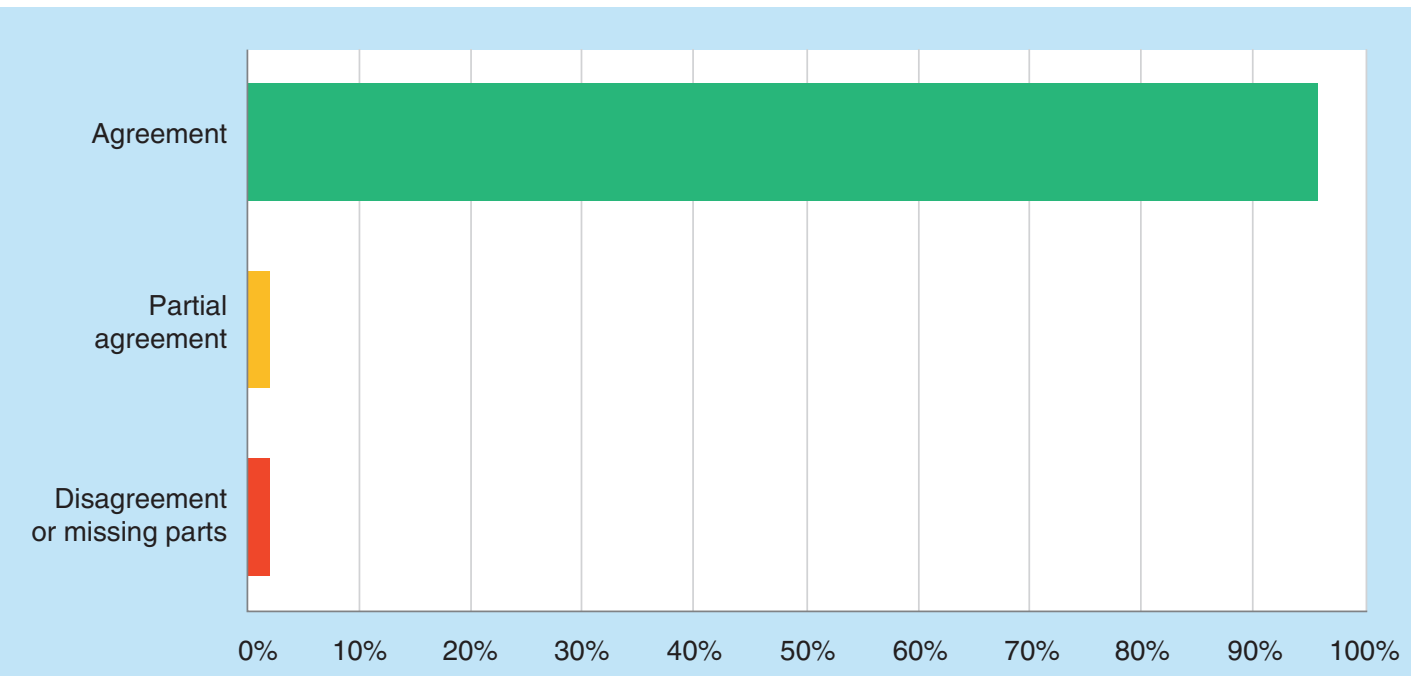

ANSWER CHOICES

RESPONSES

Agreement - no changes proposed

Partial agreement - minor changes proposed (provide motivation \& proposed

$2.13 \%$

1 changes)

Disagreement or missing parts - additions/major changes requested (provide

$2.13 \%$ proposed additions/changes)

TOTAL 
T6Q18: Endogenous Compounds - General; Lines 1064-1065: "Validation of an analytical method for an analyte that is also an endogenous compound will require the following considerations."

QC Samples - Concentrations; Lines 1067-1071: "The endogenous concentrations of the analyte in the biological matrix should be evaluated prior to QC preparation (e.g., by replicate analysis). The blank matrices with the minimum level of the endogenous analyte should be used. The concentrations of the QCs should account for the endogenous concentrations in the biological matrix (i.e., additive) and be representative of the expected study concentrations."

QC Samples - Preparation; Lines 1072-1075: "The QCs used for validation should be aliquots of the authentic biological matrix unspiked and spiked with known amounts of the authentic analyte. In spiked samples, the added amount should be enough to provide concentrations that are statistically different from the endogenous concentration."

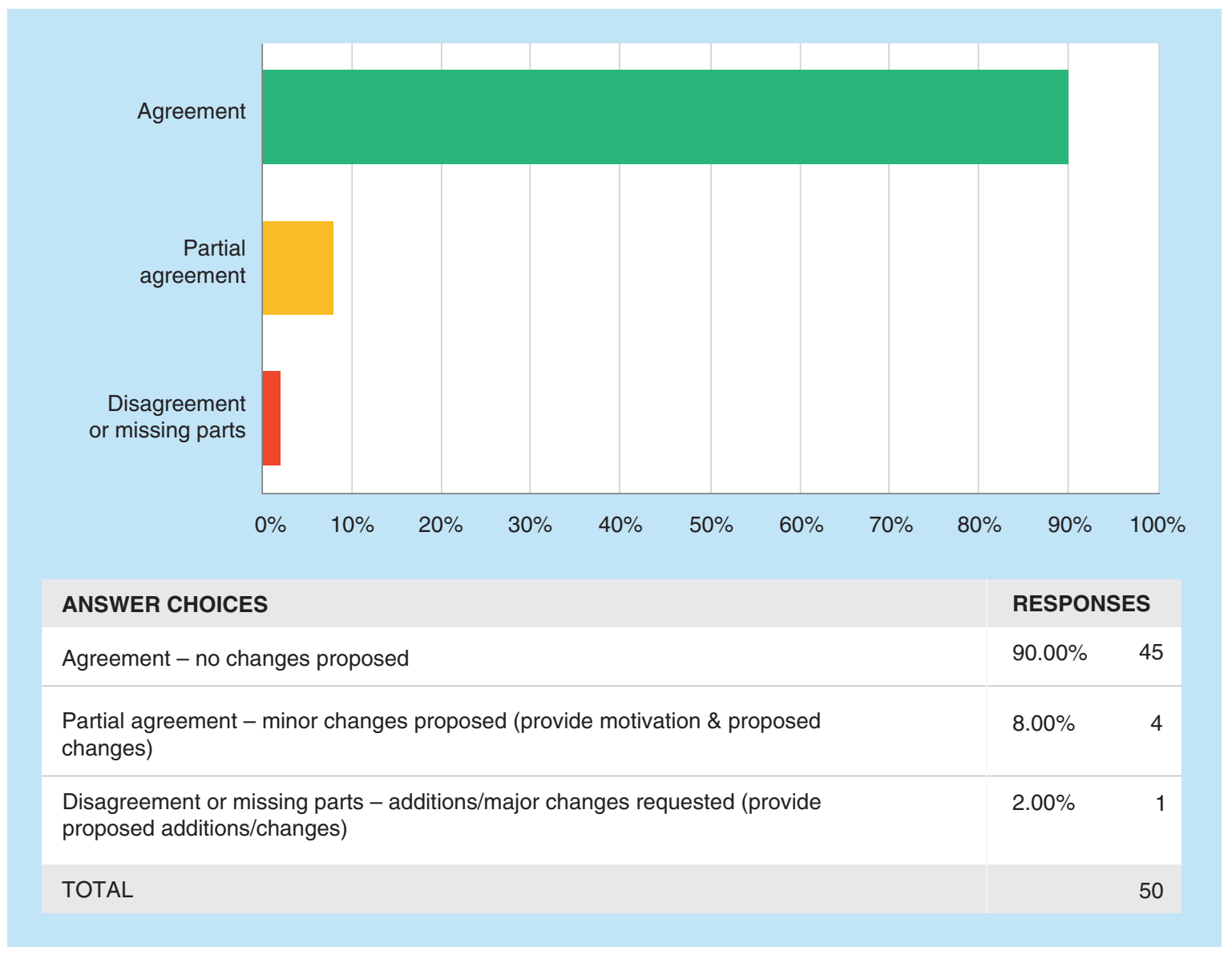

\section{Partial agreement}

It was recommended to use a mixture of authentic biological matrix and surrogate matrix QCs when the endogenous level of the actual matrix is higher than the lower QC levels. 
T6Q19: Calibration Standards - Surrogate Matrix; Lines 1077-1078: "In the Surrogate Matrix and Surrogate Analyte Approaches, these surrogates should be used only for the preparation of the calibration standards."

Calibration Standards - Standard Matrix; Lines 1079-1084: "In the Standard Addition and Background Subtraction Approaches the same biological matrix and analyte as the study samples is used to prepare the calibration standards. However, when the background concentrations are lowered by dilution of the blank matrices before spiking with the standards (e.g., if a lower LLOQ is required in the Background Subtraction Approach) the composition of the matrices in the study samples and the calibration standards is different, which may cause different recoveries and matrix effects."

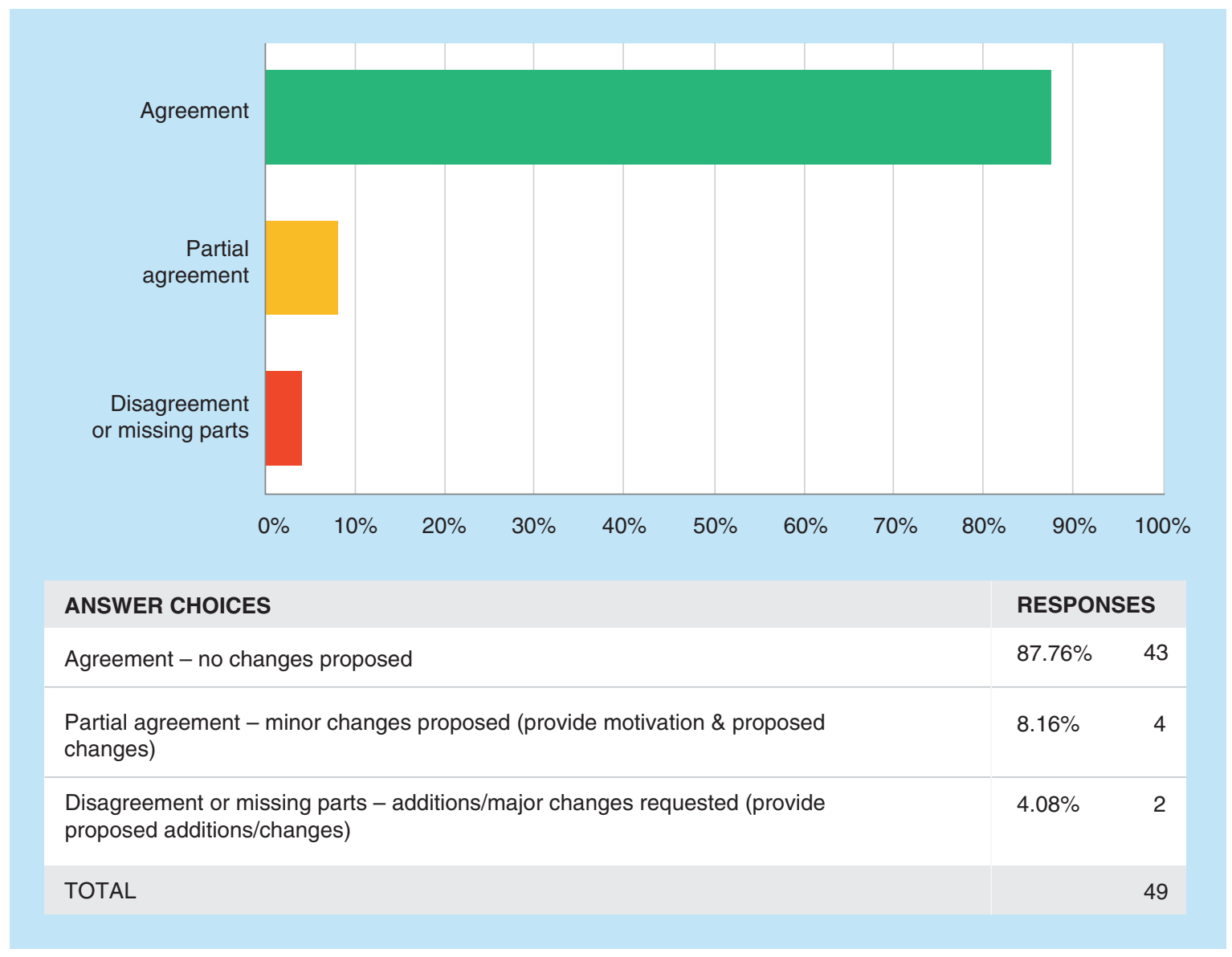

\section{Partial agreement}

Similar to comments in T6Q18, it was recommended to use a mixture of authentic biological matrix and surrogate matrix QCs when the endogenous level of the actual matrix is higher than the lower QC levels. 
T6Q20: Selectivity; Lines 1086-1090: "The assessment of selectivity is complicated by the absence of interference-free matrix. For chromatography, peak purity should be investigated as part of method validation by analysing matrices obtained from several donors using a discriminative detection system (e.g., tandem mass spectrometry (MS/MS)). Other approaches, if justified by scientific principles, may also be considered."

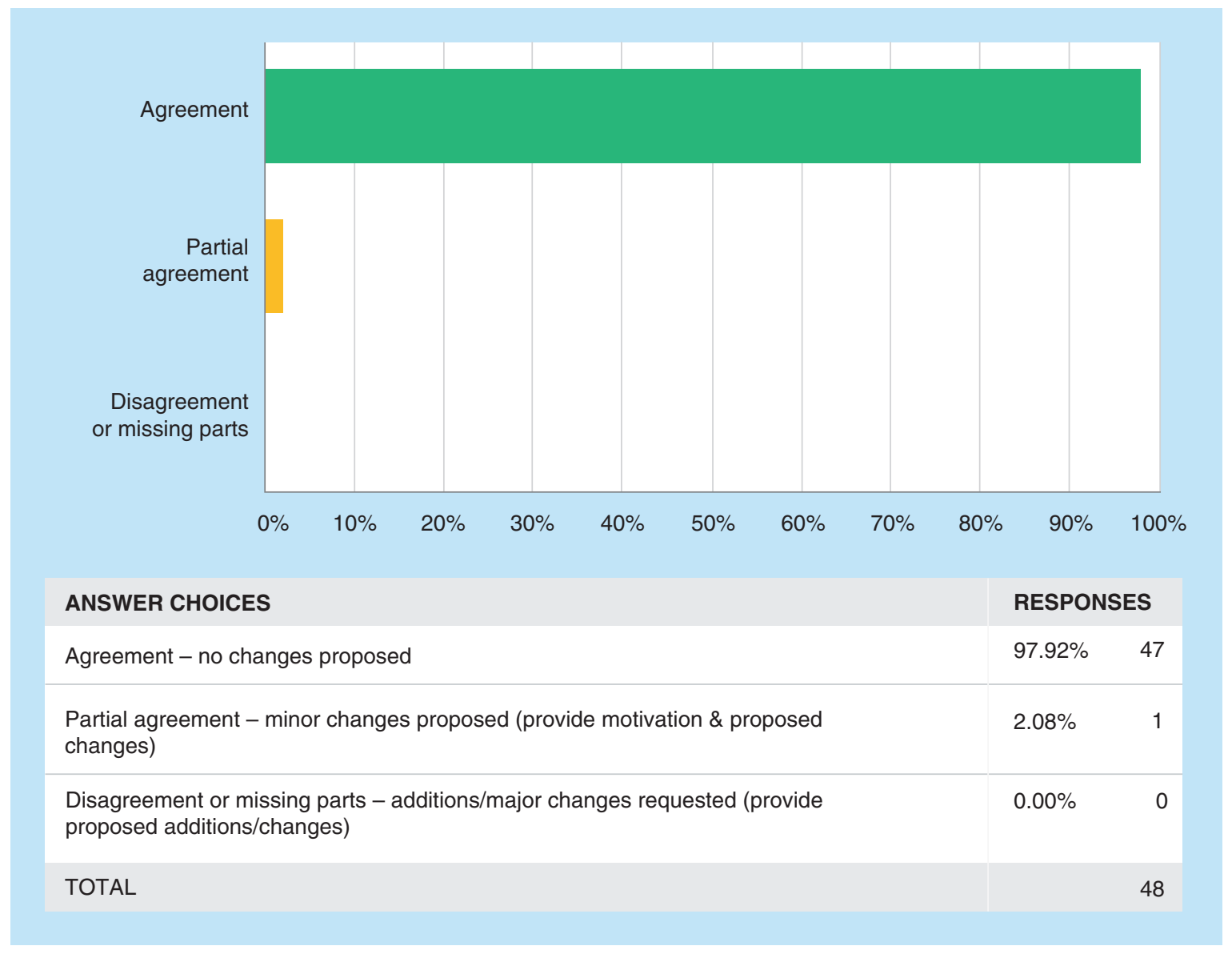


T6Q21: Recovery and Matrix Effects - General; Lines 1091-1095: "For the Standard Addition and Background Subtraction Approaches, as the same biological matrix and analyte are used for study samples and calibration standards, the same recovery and matrix effect occurs in the study samples and the calibration standards. For the Surrogate Matrix and Surrogate Analyte Approaches, the matrix effect and the extraction recovery may differ between calibration standards and study samples."

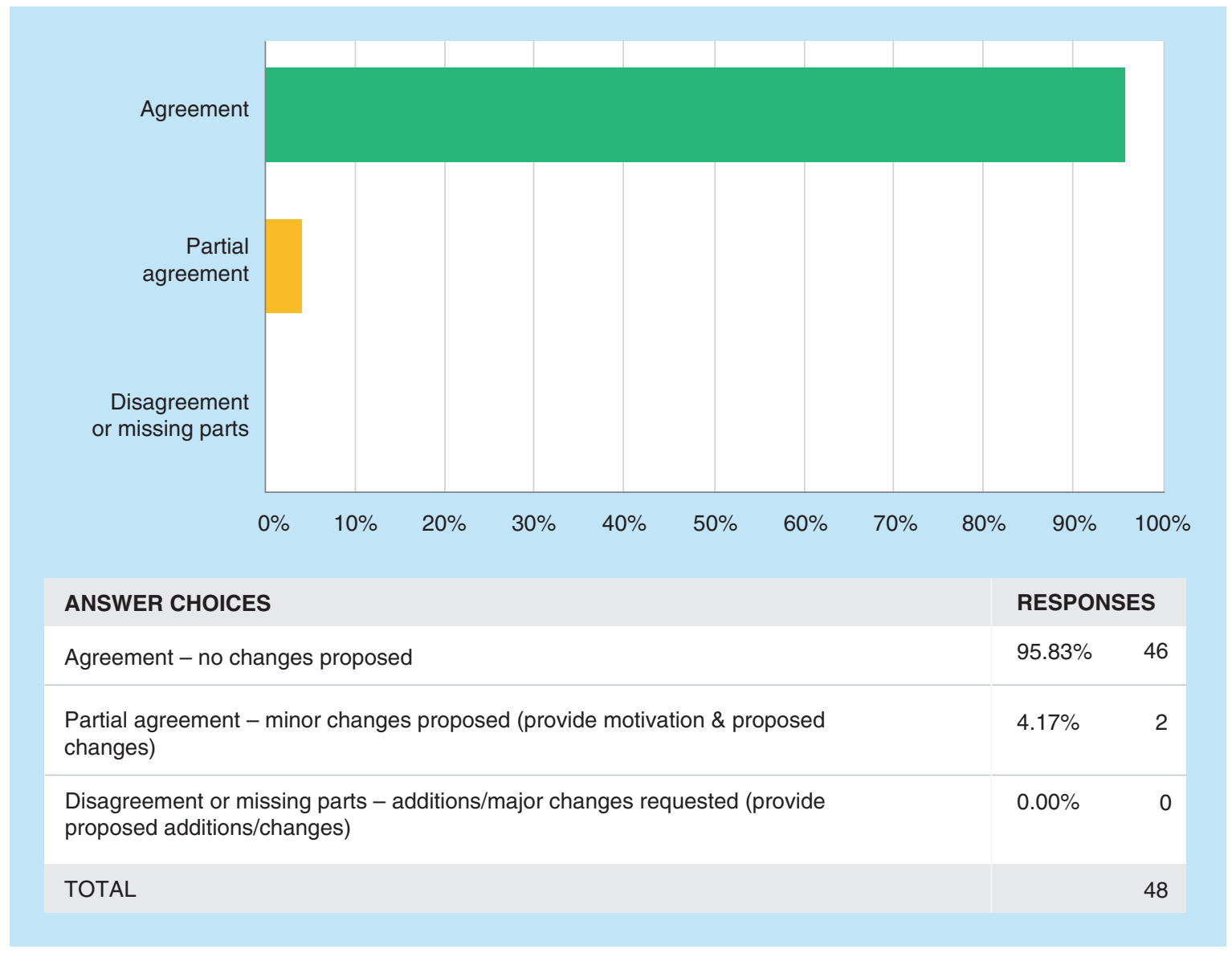

Partial agreement

No trend was found in these comments. 
T6Q22: Recovery and Matrix Effects - Surrogate Matrix Approach; Lines 1096-1100: "If the Surrogate Matrix Approach is used, demonstration of similar matrix effect and extraction recovery in both the surrogate and original matrix is required. This should be investigated in an experiment using QCs spiked with analyte in the matrix against the surrogate calibration curve and should be within $\pm 15 \%$ for chromatographic assays and within $\pm 20 \%$ for LBA assays."

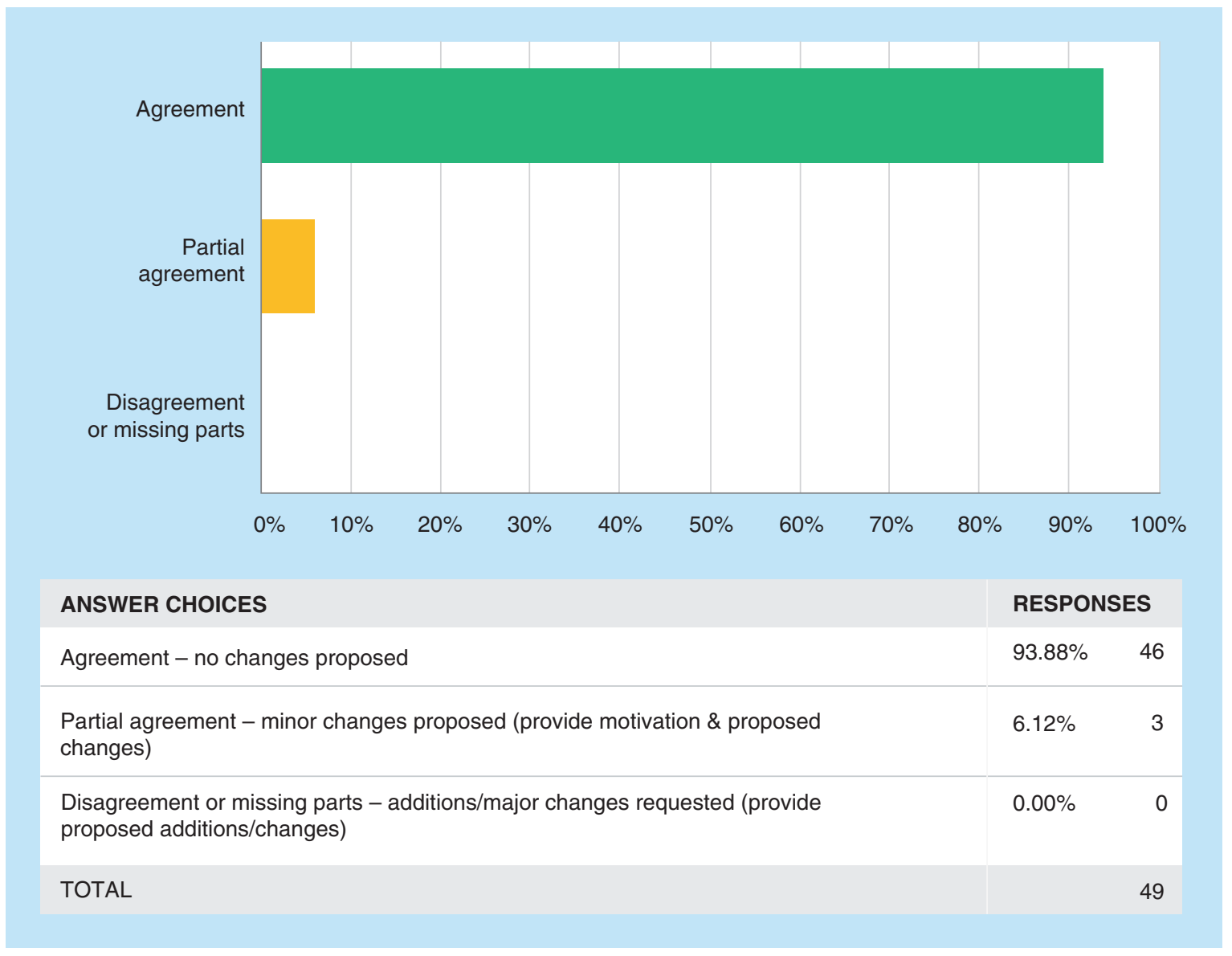

\section{Partial agreement}

No trend was found in these comments. 
T6Q23: Recovery and Matrix Effects - Surrogate Analyte Approach; Lines 1101-1104: "•If the Surrogate Analyte Approach is used, demonstration of similarity in matrix effect and recovery between surrogate and authentic endogenous analytes is required. This should be investigated in an experiment within $\pm 15 \%$ for chromatographic assays and within $\pm 20 \%$ for LBA assays."

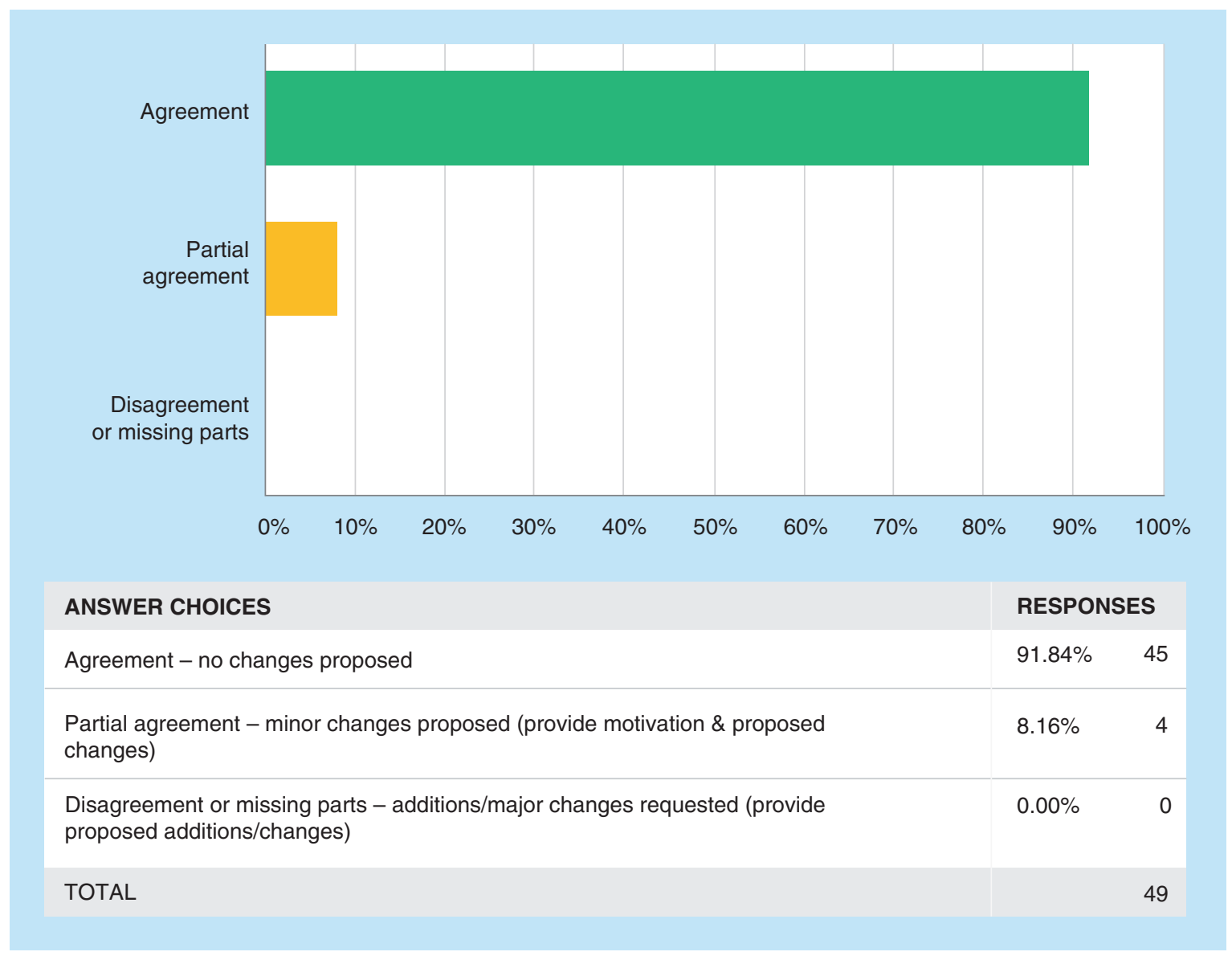

Partial agreement

No trend was found in these comments. 
T6Q24: Selectivity, Recovery and Matrix Effects - Number of Donors; Lines 1105-1107: "Since the composition of the biological matrix might affect method performance, it is necessary to investigate matrices from different donors, except in the Standard Addition Approach, where each sample is analysed with its own calibration curve."

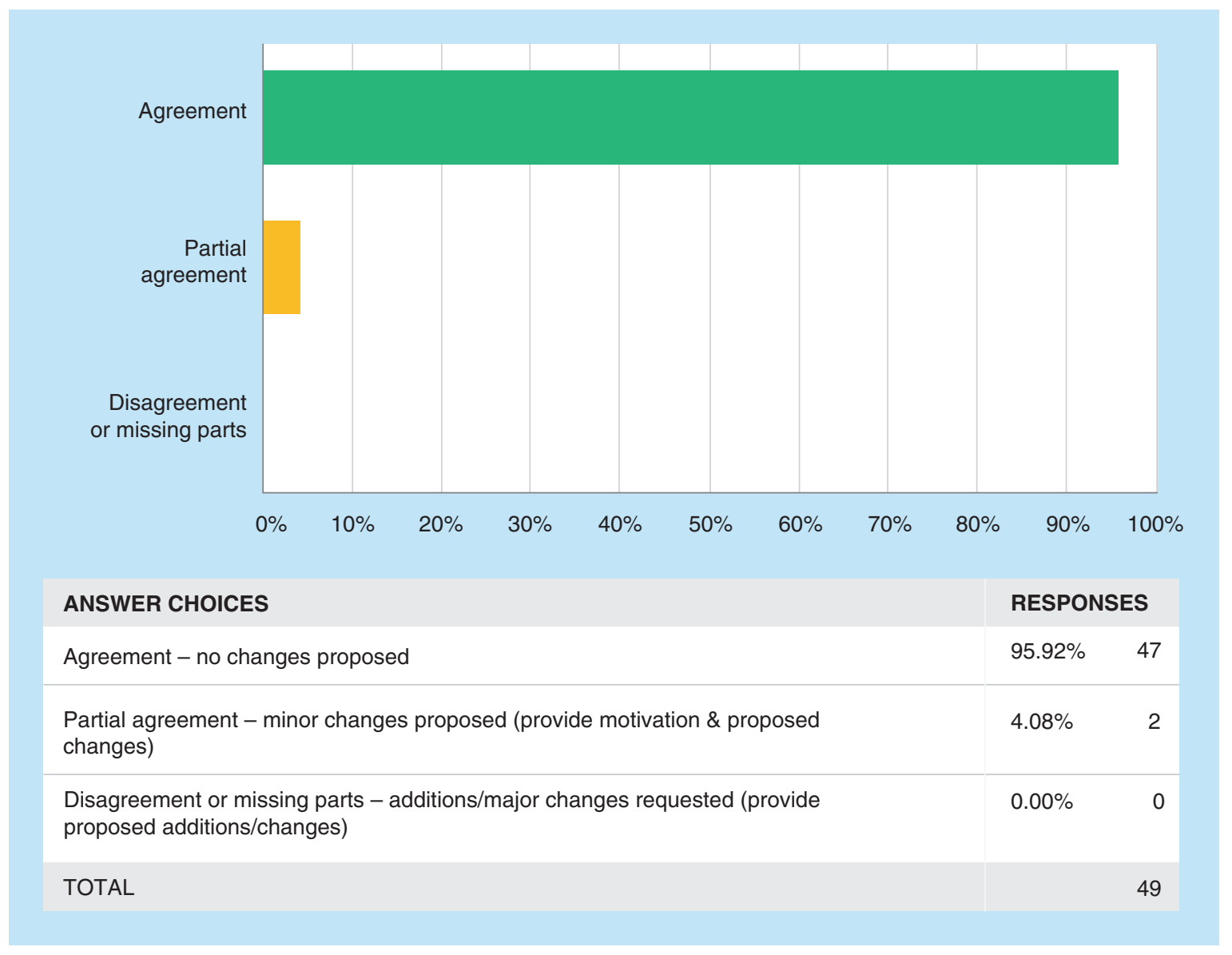

\section{Partial agreement}

No trend was found in these comments. 
T6Q25: Parallelism; Lines 1108-1110: "Parallelism should be evaluated in the Surrogate Matrix and Surrogate Analyte Approaches by means of the Standard Addition approach, spike recovery or dilutional linearity."

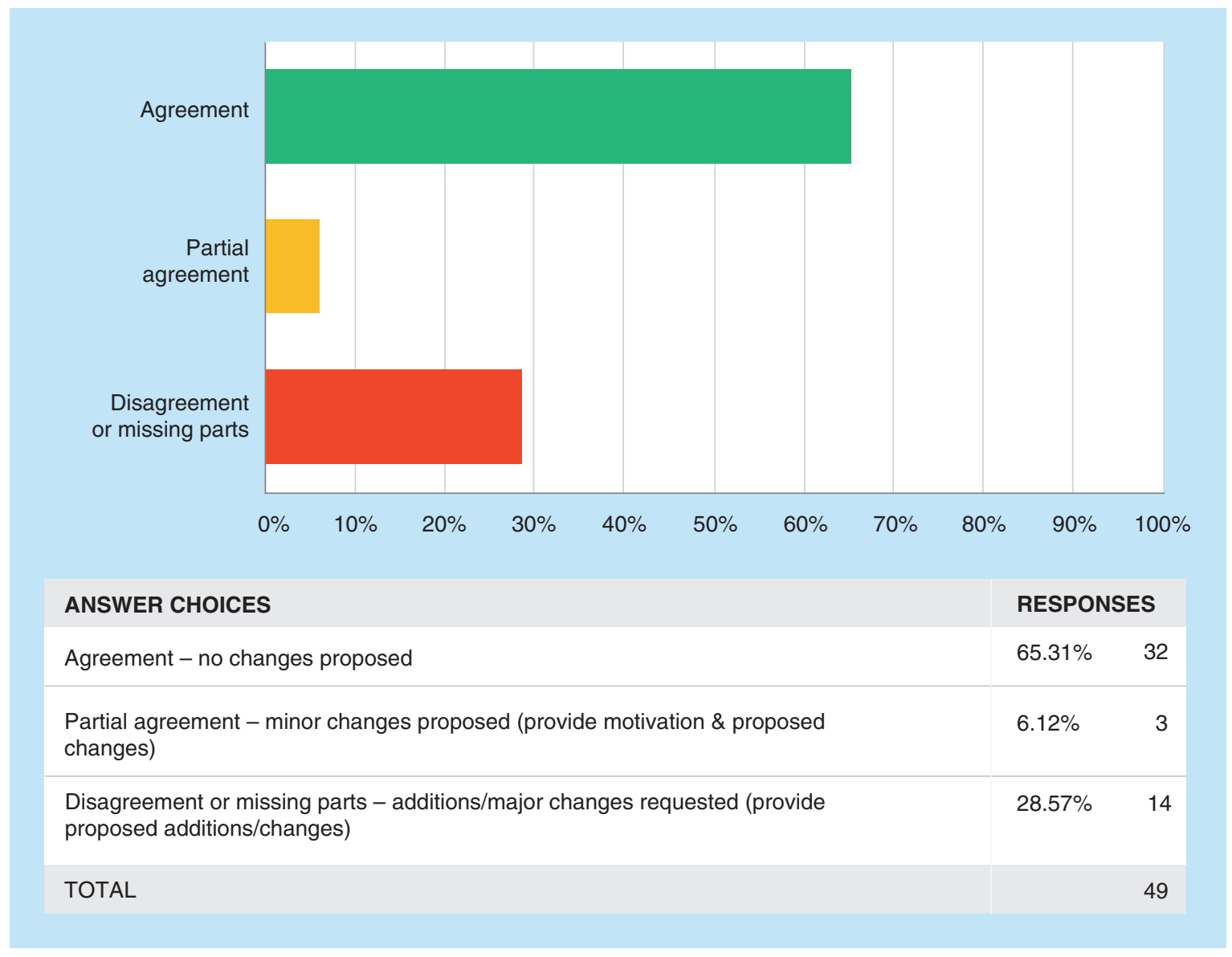

\section{Disagreement or missing parts}

All comments showed a strong disagreement with this section. It was recommended to move this text to section 7.2 and add more details on the procedure and criteria for parallelism. Parallelism tesing for LBA may be different than for chromatographic methods. Specifically, for hybrid assays (hybrid LBA/LC-MS) and LC-MS/MS parallelism evaluations, it was recommended to follow the recent recommendations from literature for biomarkers/endogenous analytes [24-27].

\section{Additional comments from the 13th GCC:}

It was recommended that additional emphasis should be added on the importance and rationale of parallelism in the endogenous space. For small and large molecules, the approaches are different and should be expanded. 
T6Q26: Accuracy and Precision - Procedure; Lines 1112-1117: "In case of using a surrogate matrix or analyte, the assessment of accuracy and precision should be performed by analysing the QCs against the surrogate calibration curve. In certain cases, dilution of the QCs with surrogate matrix may be necessary. These experiments should be repeated with authentic biological matrices from different donors to address variability due to the matrix. Analysis of the unspiked QCs will give the mean endogenous background concentration and only precision and no accuracy can be determined for this QCs."

Accuracy and Precision - Concentration Determination; Lines 1118-1119: "The concentration of the endogenous substance in the blank sample may be determined and subtracted from the total concentrations observed in the spiked samples."

Accuracy and Precision - Accuracy Formula; Lines 1119-1121: "Accuracy is recommended to be calculated using the following formula:

$$
\operatorname{Accuracy}(\%)=100 \times \frac{(\text { Measured concentration of spiked sample }- \text { endogenous concentration })}{\text { Nominal concentration }}
$$

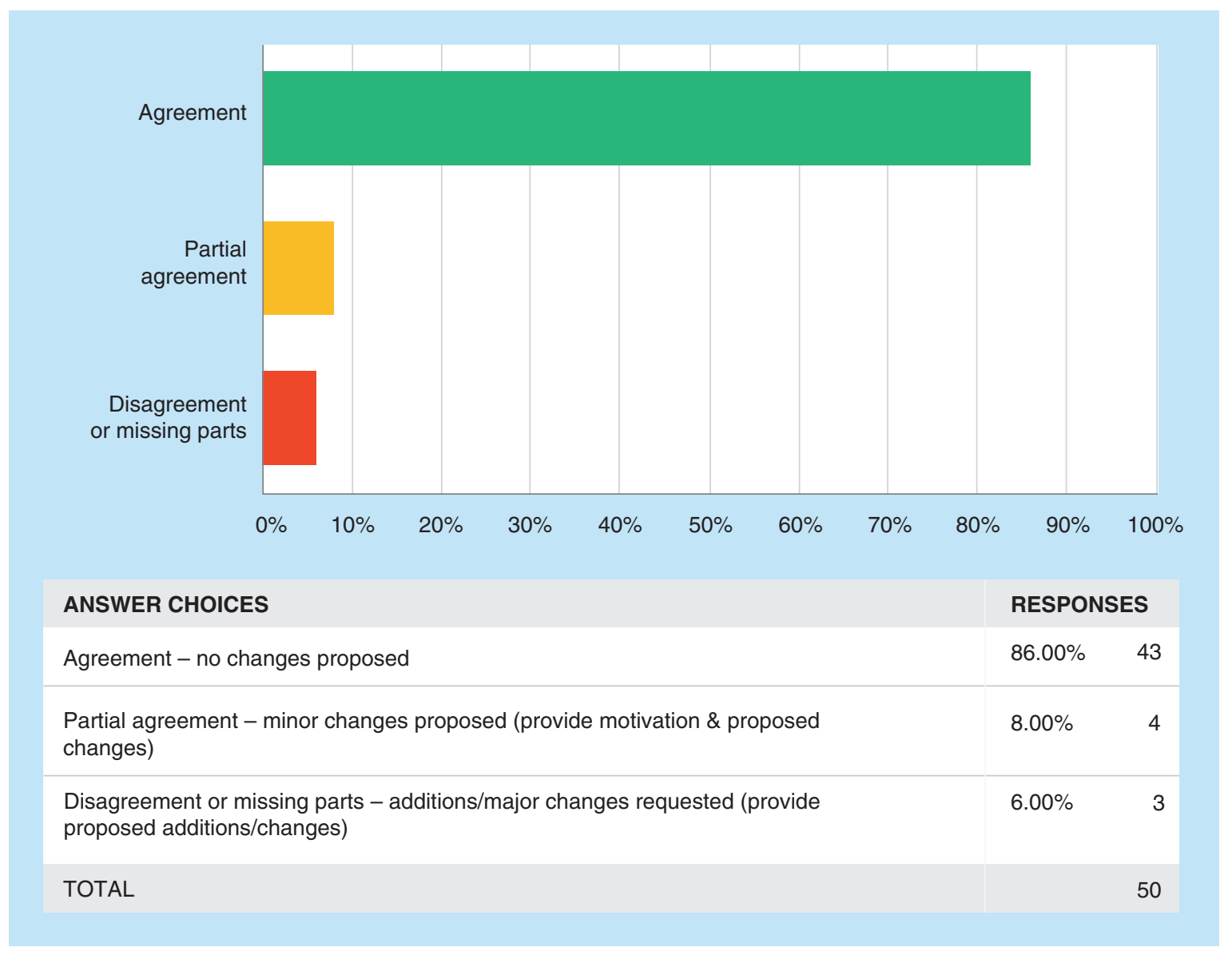

Disagreement or missing parts

It was recommended that the denominator of the formula should be the spiked concentration. 
T6Q27: Stability - General; Lines 1123-1125: "In order to mimic study samples as much as possible, stability experiments should be investigated with the authentic analyte in the authentic biological matrix and with unspiked and spiked samples."

Stability - Surrogate; Lines 1125-1127: "However, if a surrogate matrix is used for calibration standards, stability should also be demonstrated for the analyte in the surrogate matrix, as this could differ from stability in the authentic biological matrix."

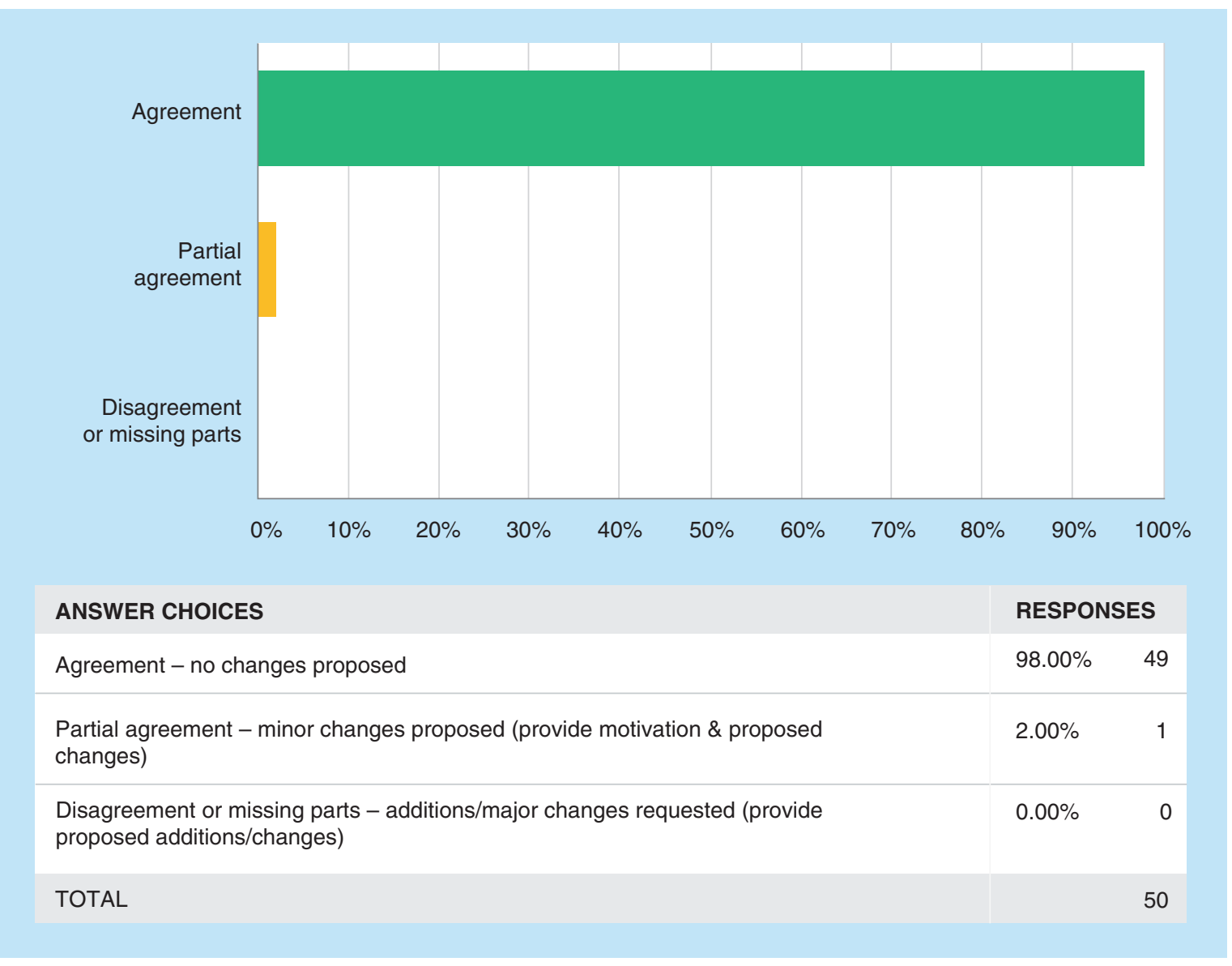


T6Q28: Commercial and Diagnostic Kits - Background; Lines 1163-1168: "Commercial or diagnostic kits (referred to as kits) are sometimes co-developed with new drugs or therapeutic biological products for point-of-care patient diagnosis. The recommendations in this section of the guideline do not apply to the development of kits that are intended for point-of-care patient diagnosis (e.g., companion or complimentary diagnostic kits). Refer to the appropriate guideline documents regarding regulatory expectations for the development of these kits."

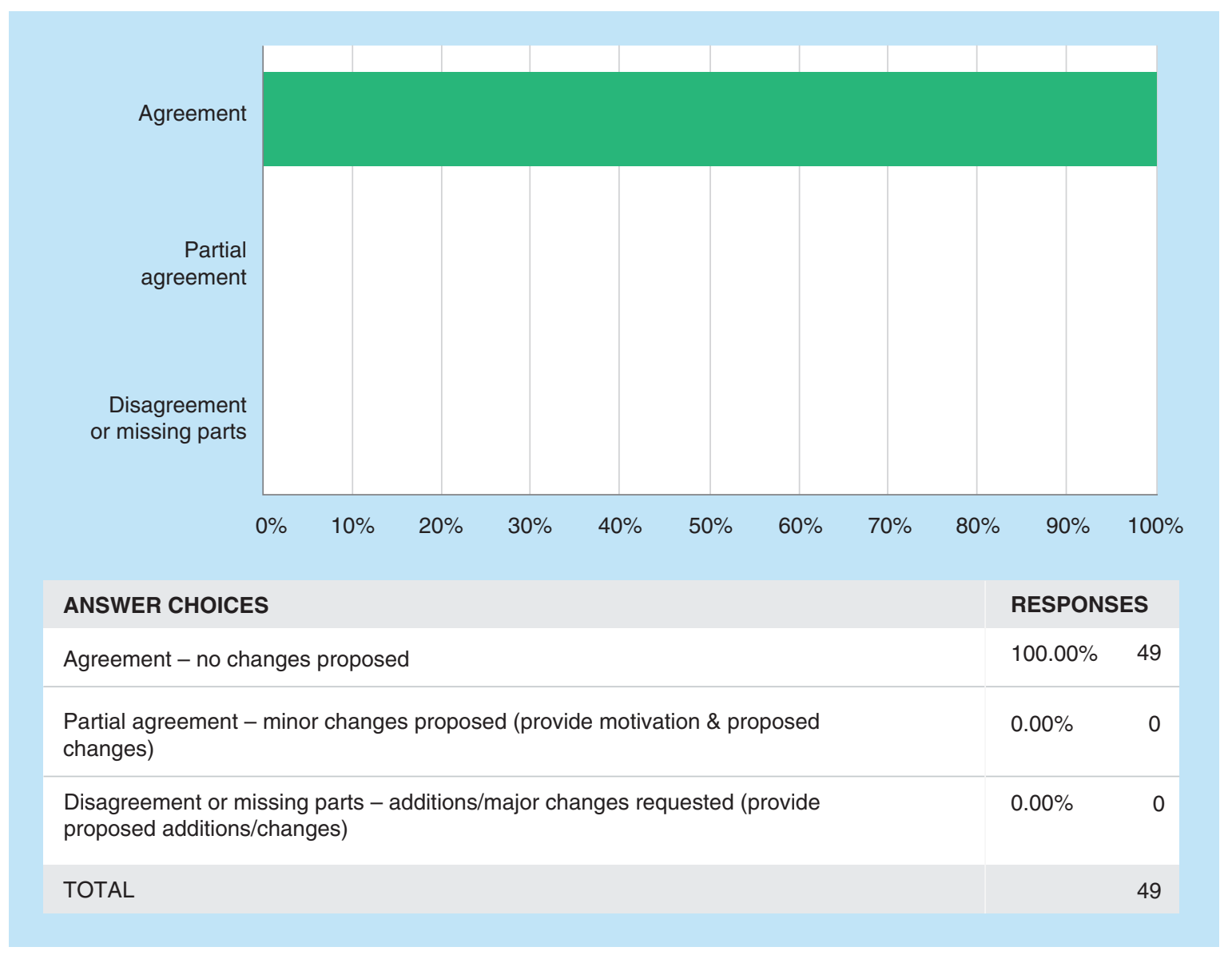


T6Q29: Commercial and Diagnostic Kits - Validation; Lines 1169-1172: "If an applicant repurposes a kit (instead of developing a new assay) or utilises "research use only" kits to measure chemical or biological drug concentrations during the development of a novel drug, the applicant should assess the kit validation to ensure that it conforms to the drug development standards described in this guideline."

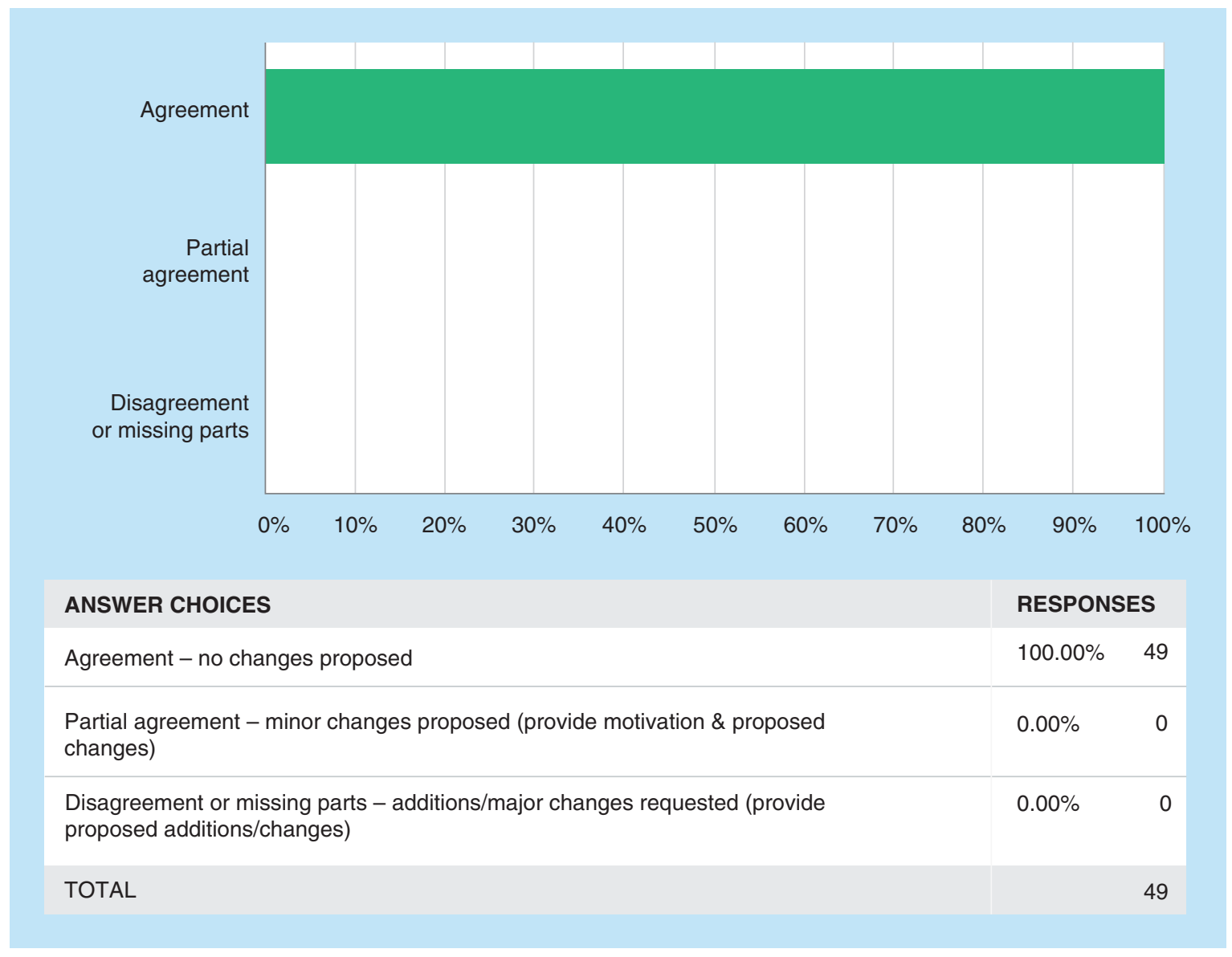


T6Q30: Commercial and Diagnostic Kits - Validation Requirements; Lines 1173-1192: "Validation considerations for kit assays include, but are not limited to, the following:

- If the reference standard in the kit differs from that of the study samples, testing should evaluate differences in assay performance of the kit reagents. The specificity, accuracy, precision and stability of the assay should be demonstrated under actual conditions of use in the facility conducting the sample analysis. Modifications from kit processing instructions should be completely validated.

- Kits that use sparse calibration standards (e.g., one-or two-point calibration curves) should include in-house validation experiments to establish the calibration curve with a sufficient number of standards across the calibration range.

- Actual QC concentrations should be known. Concentrations of QCs expressed as ranges are not sufficient for quantitative applications. In such cases QCs with known concentrations should be prepared and used, independent of the kit-supplied QCs.

- Calibration standards and QCs should be prepared in the same matrix as the study samples. Kits with calibration standards and QCs prepared in a matrix different from the study samples should be justified and appropriate experiments should be performed.

- If multiple kit lots are used within a study, lot-to-lot variability and comparability should be addressed for any critical reagents included in the kits.

- If a kit using multiple assay plates is employed, sufficient replicate QCs should be used on each plate to monitor the accuracy of the assay. Acceptance criteria should be established for the individual plates and for the overall analytical run."

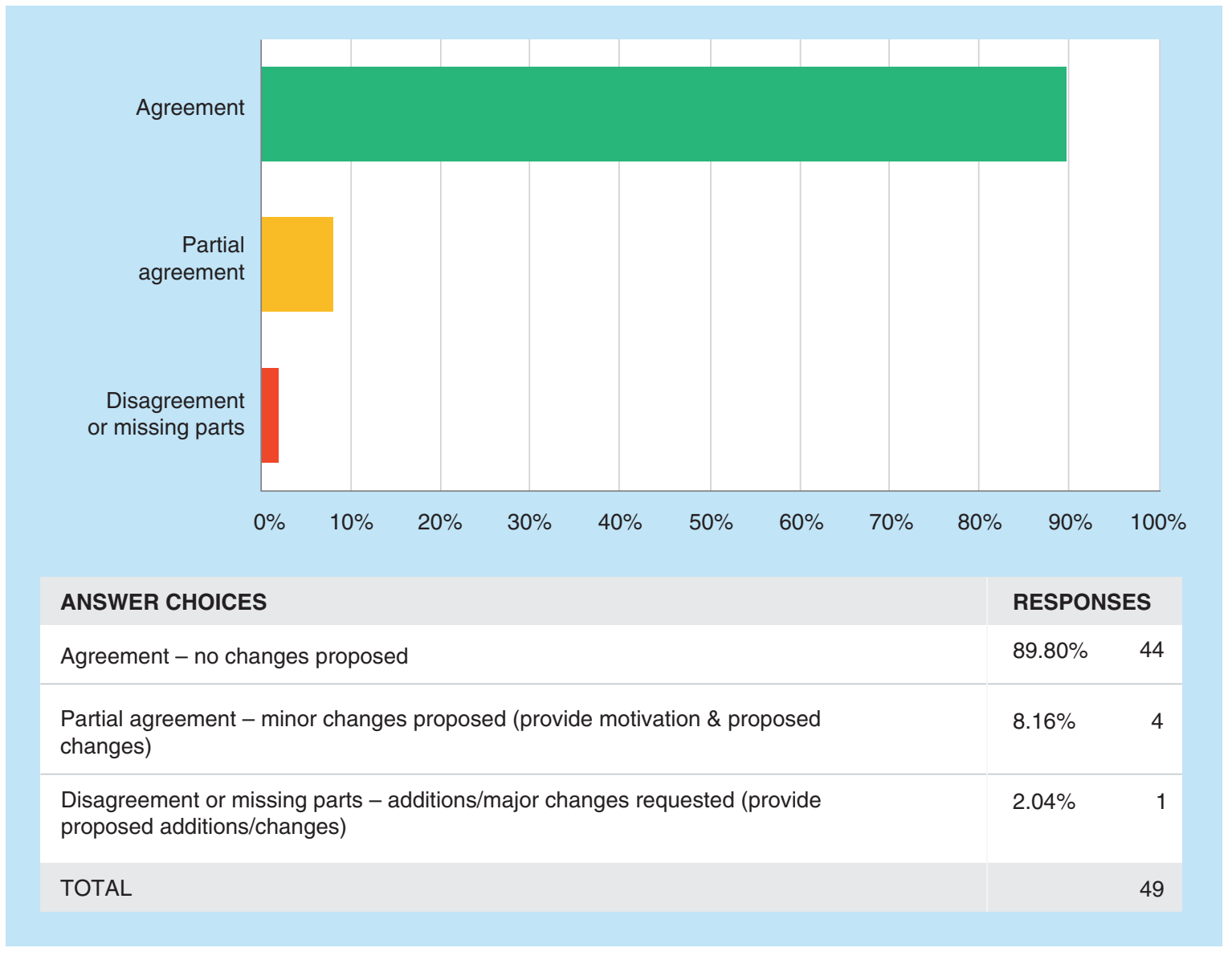

Partial agreement

No trend was found in these comments. 
T6Q31: New or Alternative Technologies - Cross Validation; Lines 1194-1195: "When a new or alternative technology is used as the sole bioanalytical technology from the onset of drug development, cross validation with an existing technology is not required."

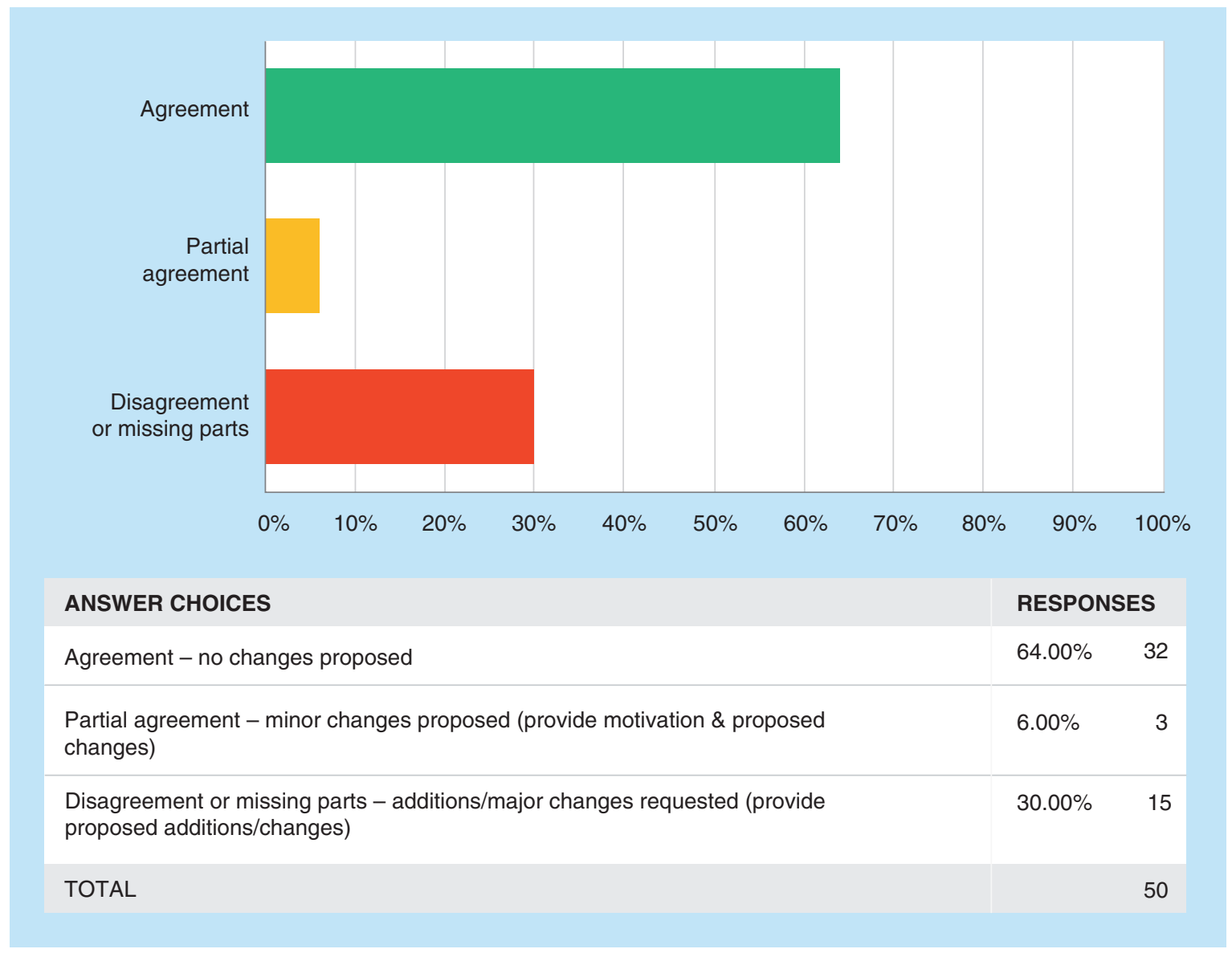

Disagreement or missing parts

It was strongly recommended again to provide guidance and add hybrid assays (hybrid LBA/LCMS) into this guideline. Similar comments and requests were also clearly stated in T1Q6 and T1Q9.

T1Q6 excerpt:

- The addition of hybrid LBA/LCMS assays for large molecules, as an established technology in bioanalysis, is strongly recommended.

- Additional clarification when using new technologies is recommended.

T1Q9 excerpt:

- The majority of the comments requested a guideline for hybrid LBA/LCMS for biotherapeutics. Hence, it is strongly recommended to add the bold wording to the first sentence: "The information in this guideline applies to the quantitative analysis by ligand binding assays (LBAs) and chromatographic methods such as liquid chromatography $(L C)$, or gas chromatography $(G C)$, which are typically used in combination with mass spectrometry (MS) detection and occasionally with other detectors. The information in this guideline also applies to hybrid assays (hybrid LBA/LCMS)."

\section{Additional comments from the 13th GCC:}

- It was recommended to adopt the LBA criteria for hybrid LBA/LCMS methods.

- It was suggested to modify the section title to "Technologies Used at the Onset of Discovery". 
T6Q32: New or Alternative Technologies - Changing Platforms; Lines 1196-1204: "The use of two different bioanalytical technologies for the development of a drug may generate data for the same product that could be difficult to interpret. This outcome can occur when one platform generates drug concentrations that differ from those obtained with another platform. Therefore, when a new or alternative analytical platform is replacing a previous platform used in the development of a drug it is important that the potential differences are well understood. The data generated from the previous platform/technology should be cross validated to that of the new or alternative platform/technology. Seeking feedback from the regulatory authorities is encouraged early in drug development. The use of two methods or technologies within a comparative $B A / B E$ study is strongly discouraged."

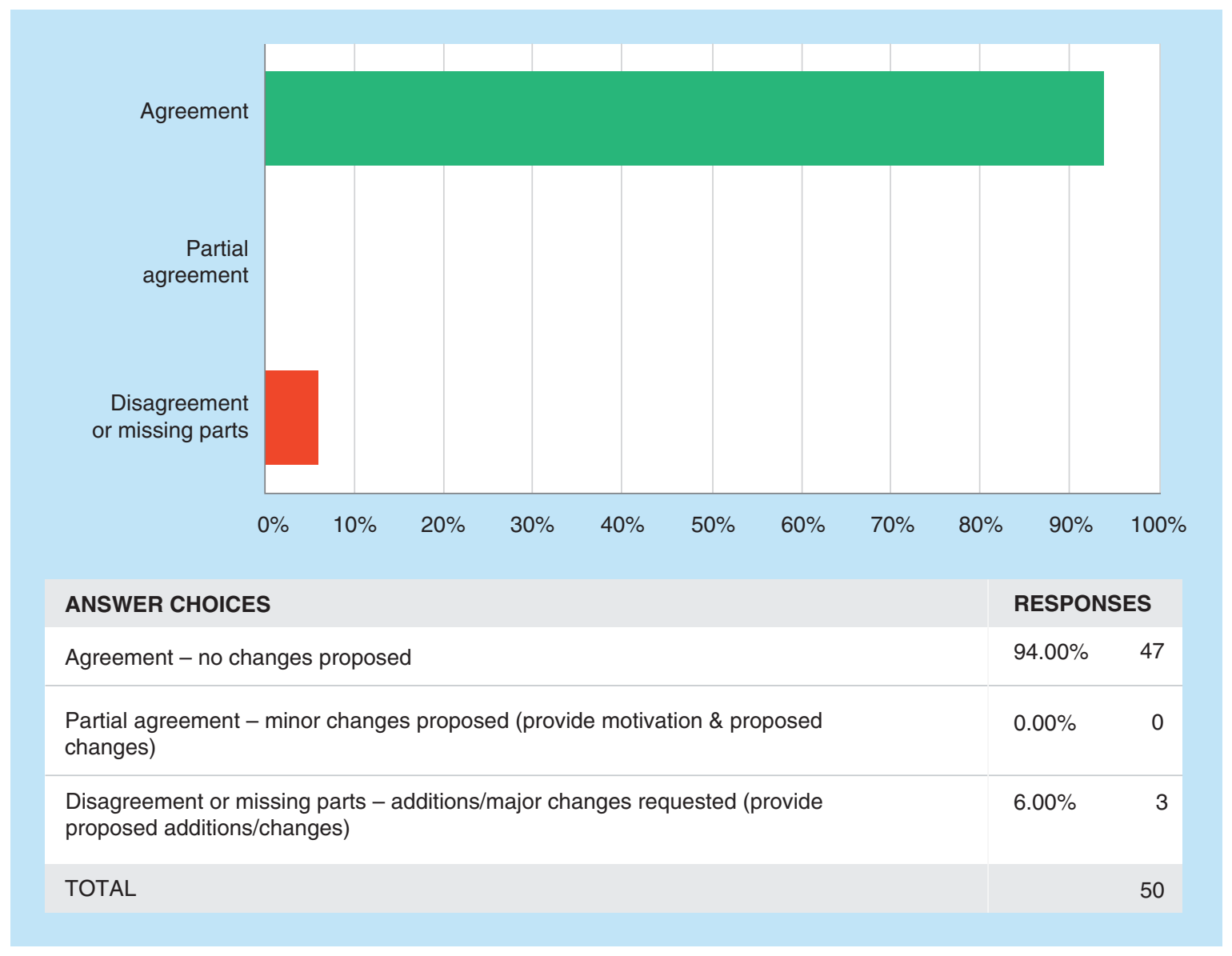

Disagreement or missing parts

No trend was found in these comments. 
T6Q33: New or Alternative Technologies - Acceptance Criteria; Lines 1205-1206: "The use of new technology in regulated bioanalysis should be supported by acceptance criteria established a priori based on method development and verified in validation."

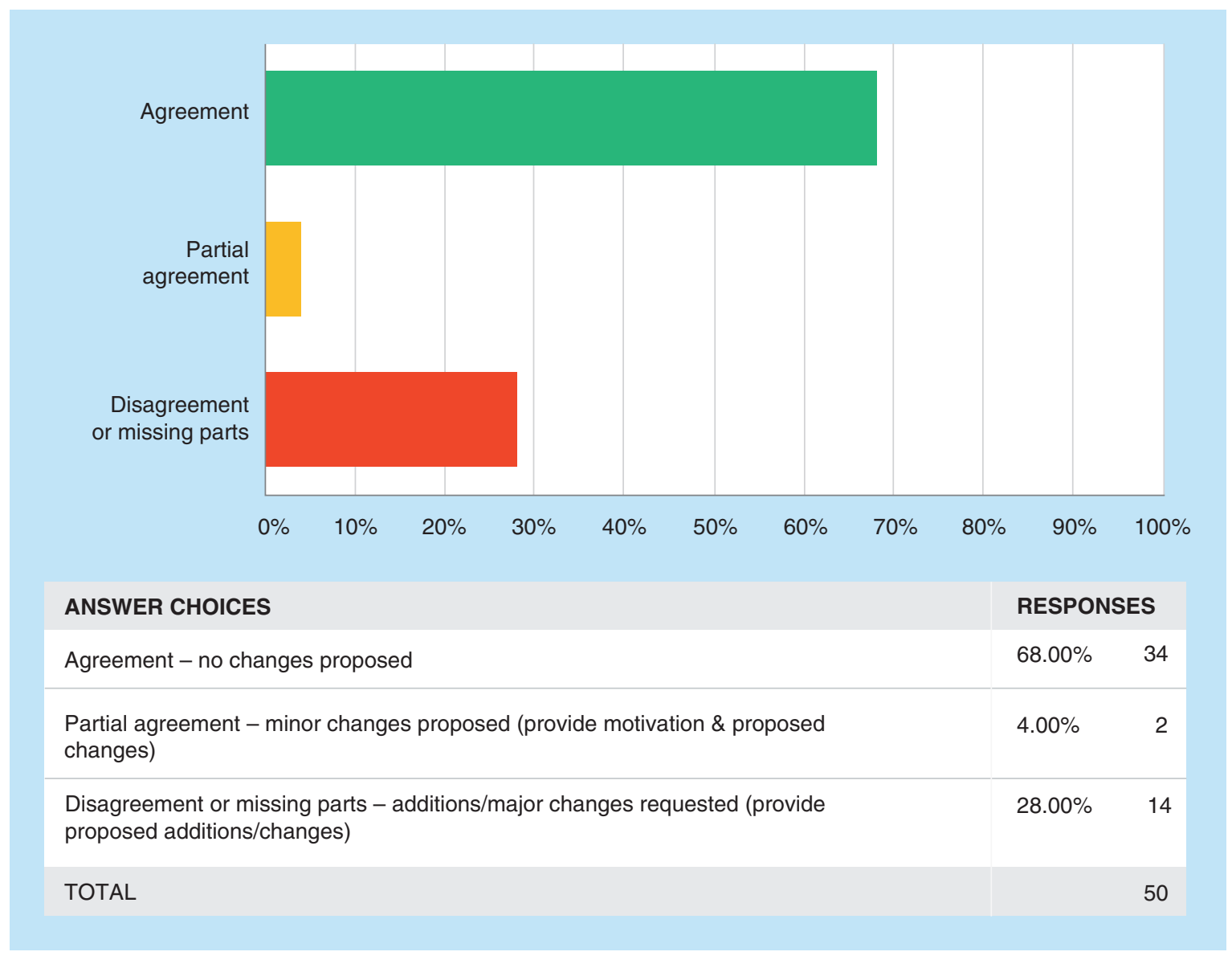

Disagreement or missing parts

Same comments as in T6Q31, T1Q6 and T1Q9. It was strongly recommended to specify criteria for hybrid assays (hybrid LBA/LCMS).

\section{Partial agreement}

It was recommended that the acceptance criteria be based on the validation results.

\section{Additional comments from the 13th GCC:}

- It was recommended to adopt the LBA criteria for hybrid LBA/LCMS methods.

- It was suggested to modify the text in this section to say the guidance can be used to define the space when using technologies not already explicitly covered by the guidance. 
T6Q34: Dried Matrix Methods - Definition; Lines 1208-1210: "Dried matrix methods (DMM) is a sampling methodology that offers benefits such as collection of reduced blood sample volumes as a microsampling technique for drug analysis and ease of collection, storage and transportation."

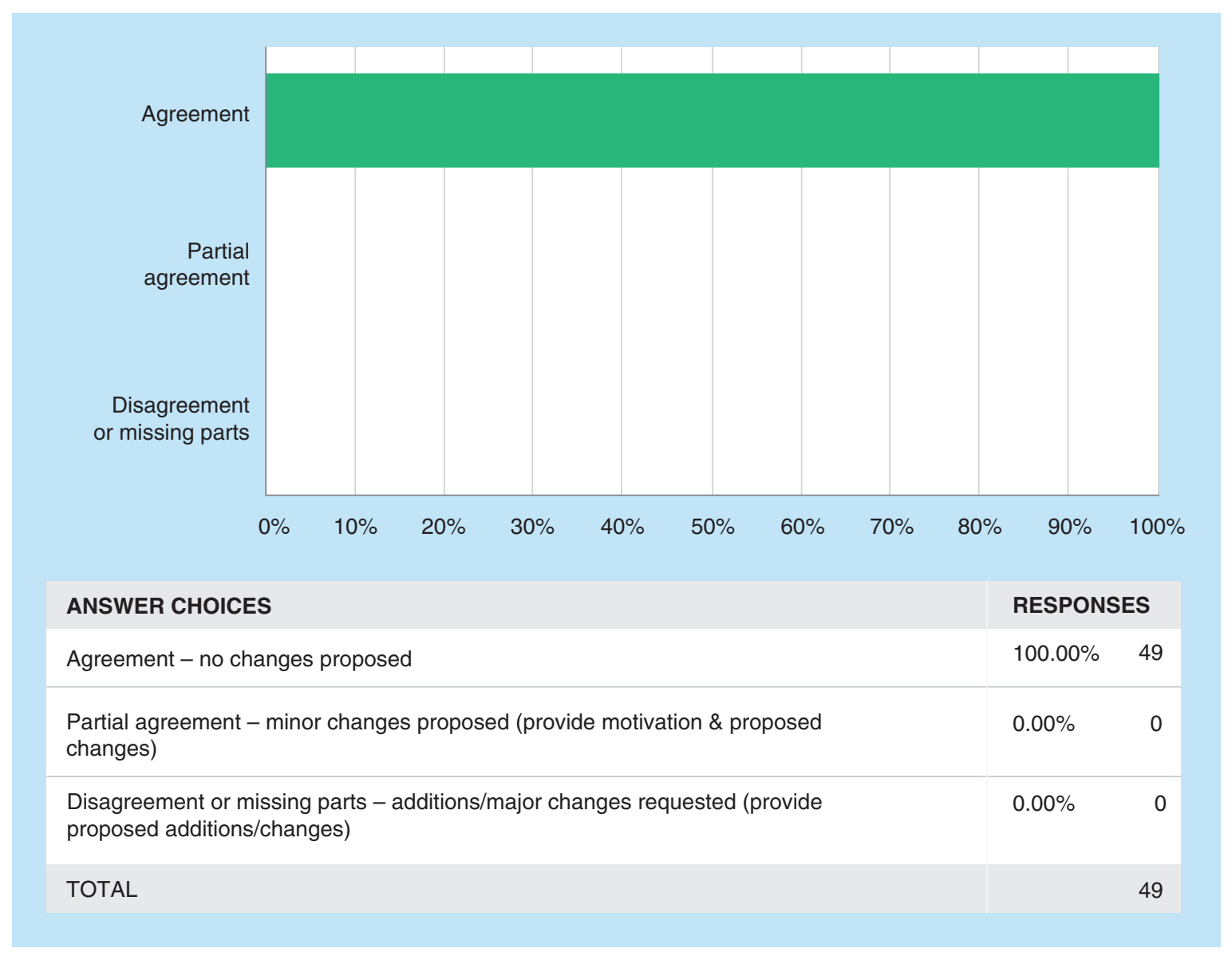


T6Q35: Dried Matrix Methods - Extra Evaluations; Lines 1210-1220: "In addition to the typical methodological validation for $L C-M S$ or $L B A$, use of DMM necessitates further validation of this sampling approach before using DMM in studies that support a regulatory application, such as:

- Haematocrit (especially for spotting of whole blood into cards)

- Sample homogeneity (especially for sub-punch of the sample on the card/device)

- Reconstitution of the sample

- DMM sample collection for ISR

- Care should be taken to ensure sufficient sample volumes or numbers of replicates are retained for ISR

- Should be assessed by multiple punches of the sample or samples should be taken in duplicate"

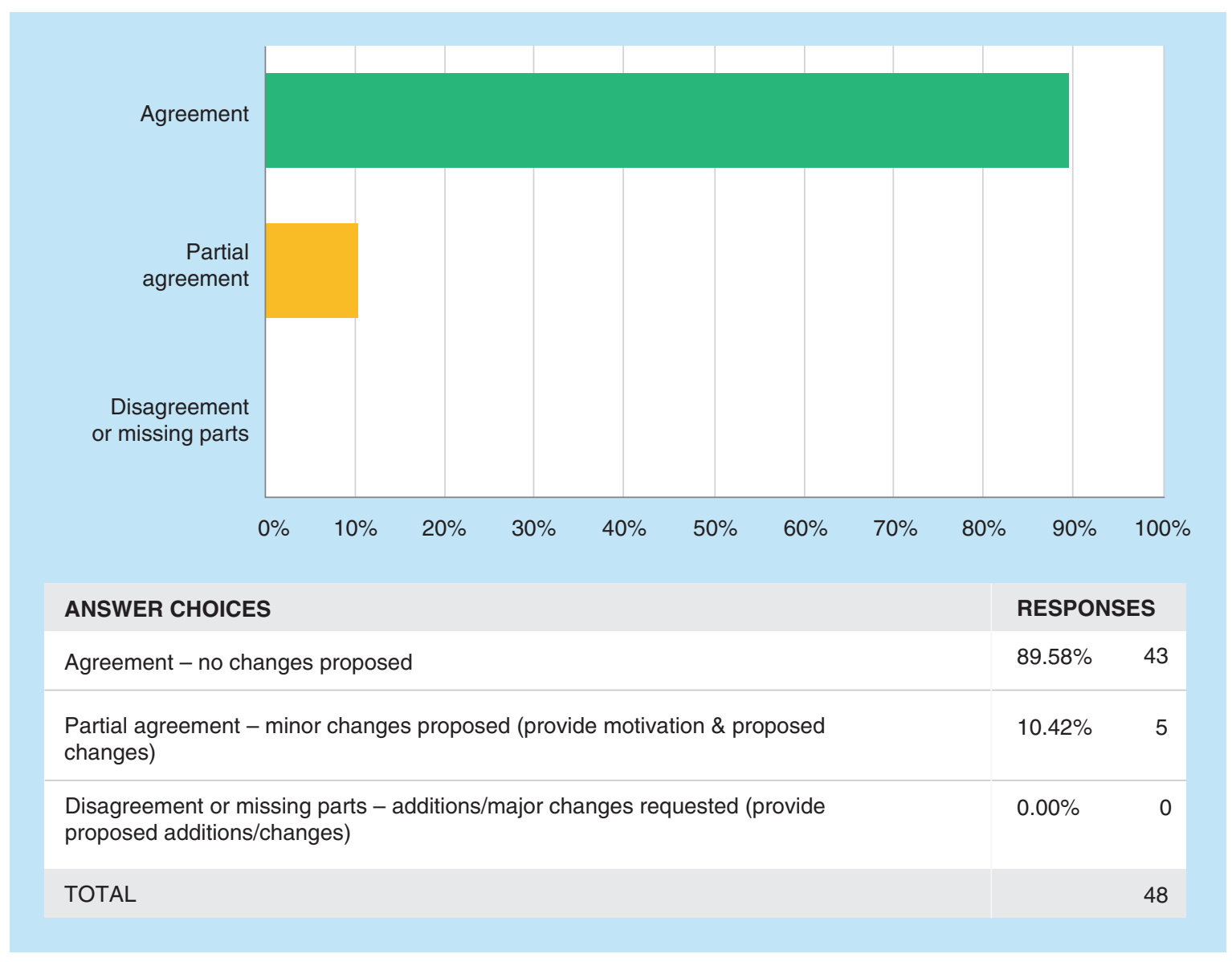

Partial agreement

No trend was found in these comments. 
T6Q36: Dried Matrix Methods - Cross Validation; Lines 1221-1225: "When DMM is used for clinical or nonclinical studies in addition to typical liquid approaches (e.g., liquid plasma samples) in the same studies, these two methods should be cross validated as described (Refer to Section 6.2). For nonclinical TK studies, refer to Section 4.1 of ICH S3A Q\&A. Feedback from the appropriate regulatory authorities is encouraged in early drug development."

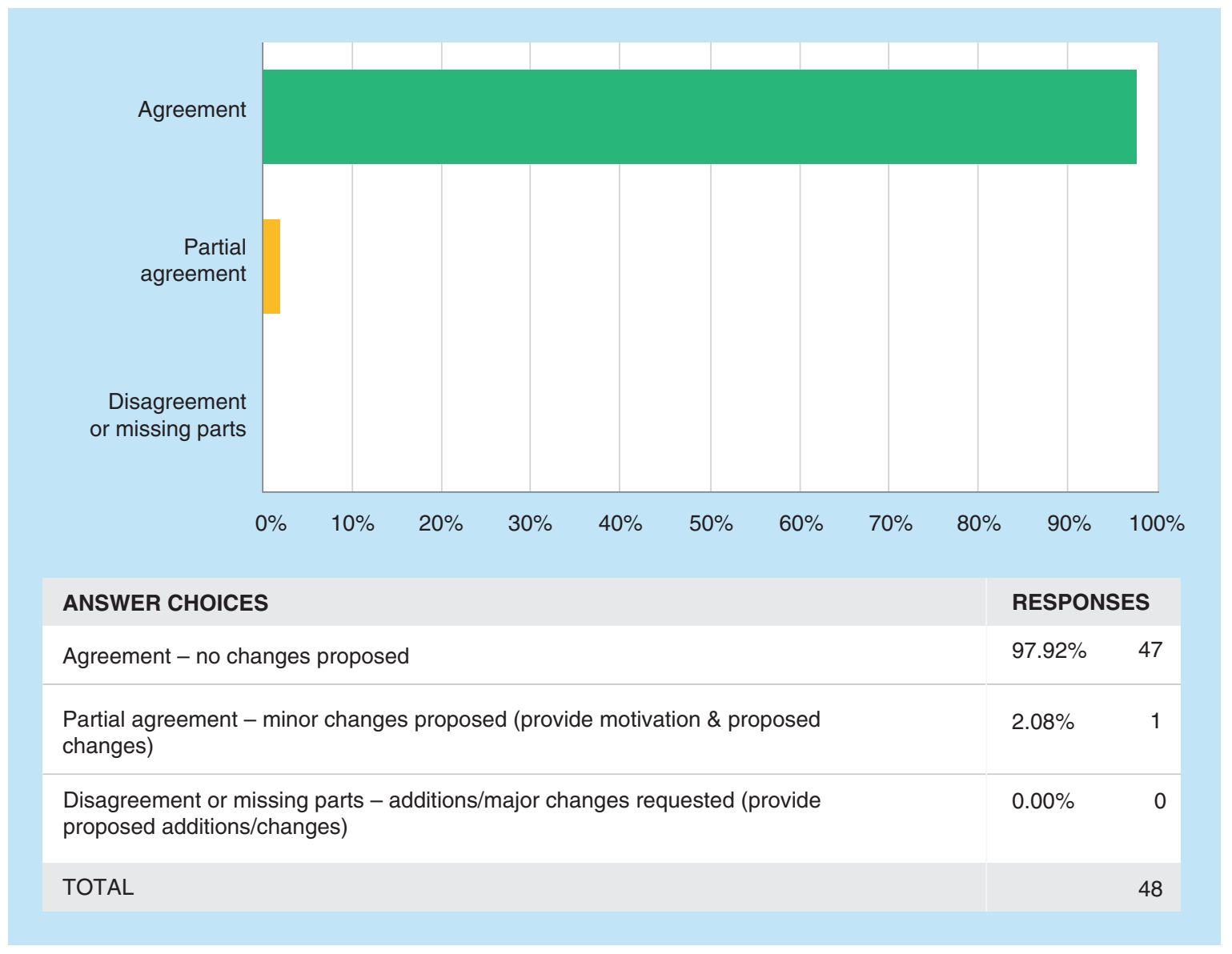


Team 7 General Principles of Method Development/Validation, Documentation for Validation and Bioanalytical Reports

T7Q1: Method Development - Purpose; Lines 125-127: "The purpose of bioanalytical method development is to define the design, operating conditions, limitations and suitability of the method for its intended purpose and to ensure that the method is optimised for validation."

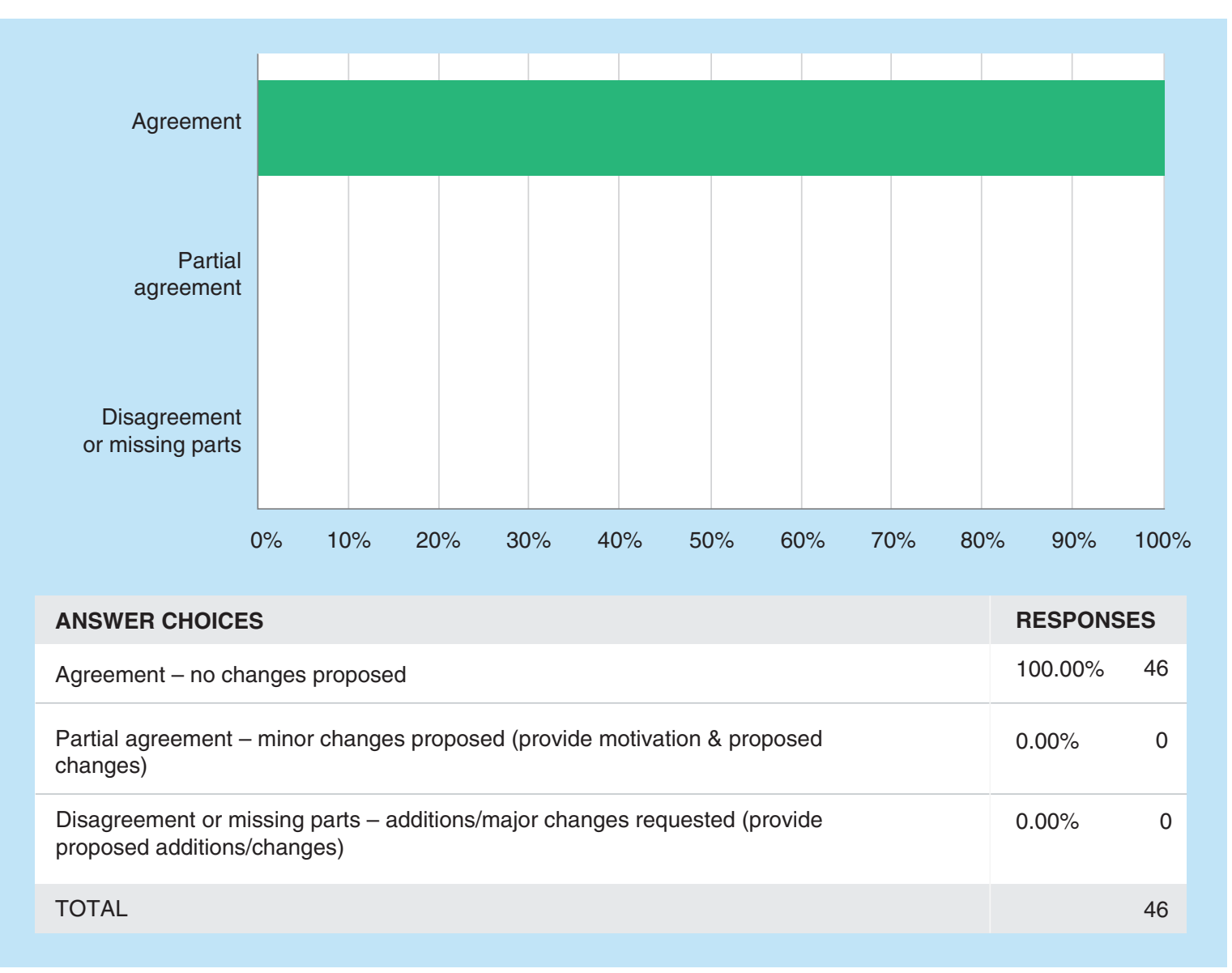


T7Q2: Method Development - Context of Use; Lines 128-130: "Before the development of a bioanalytical method, the applicant should understand the analyte of interest (e.g., the physicochemical properties of the drug, in vitro and in vivo metabolism and protein binding) and consider aspects of any prior analytical methods that may be applicable."

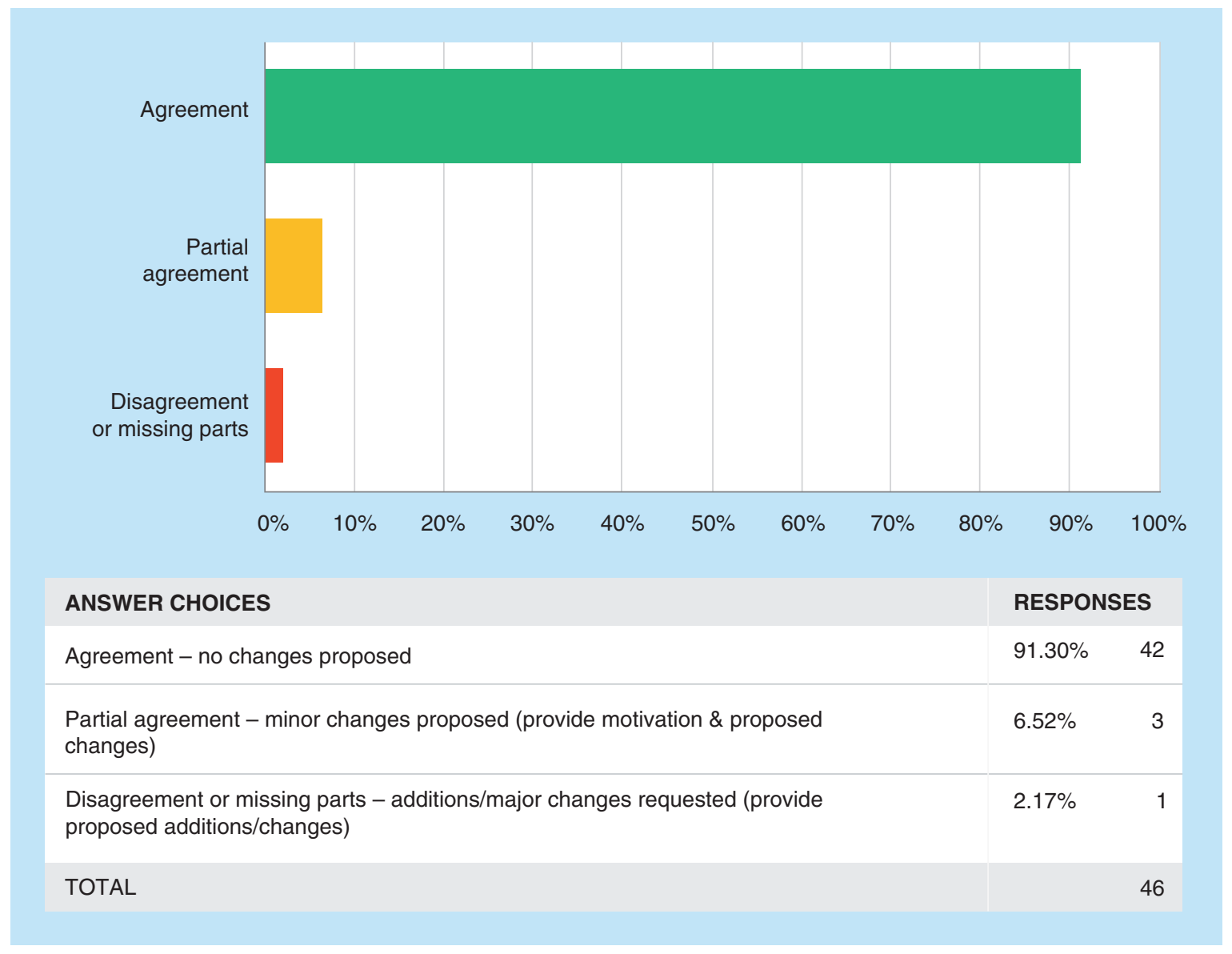

\section{Partial agreement}

It was suggested in three comments to add "if available" to the examples since this information is often not available at the time of method development.

\section{Additional comments from the 13th GCC:}

It was suggested to add "structure" to the list of examples. 
T7Q3: Method Development - Inclusions; Lines 131-144: "Method development involves optimising the procedures and conditions involved with extracting and detecting the analyte. Method development can include the optimisation of the following bioanalytical parameters to ensure that the method is suitable for validation:

- Reference standards

- Critical reagents

- Calibration curve

- Quality control samples (QCs)

- Selectivity and specificity

- Sensitivity

- Accuracy

- Precision

- Recovery

- Stability of the analyte in the matrix

- Minimum Required Dilution (MRD)"

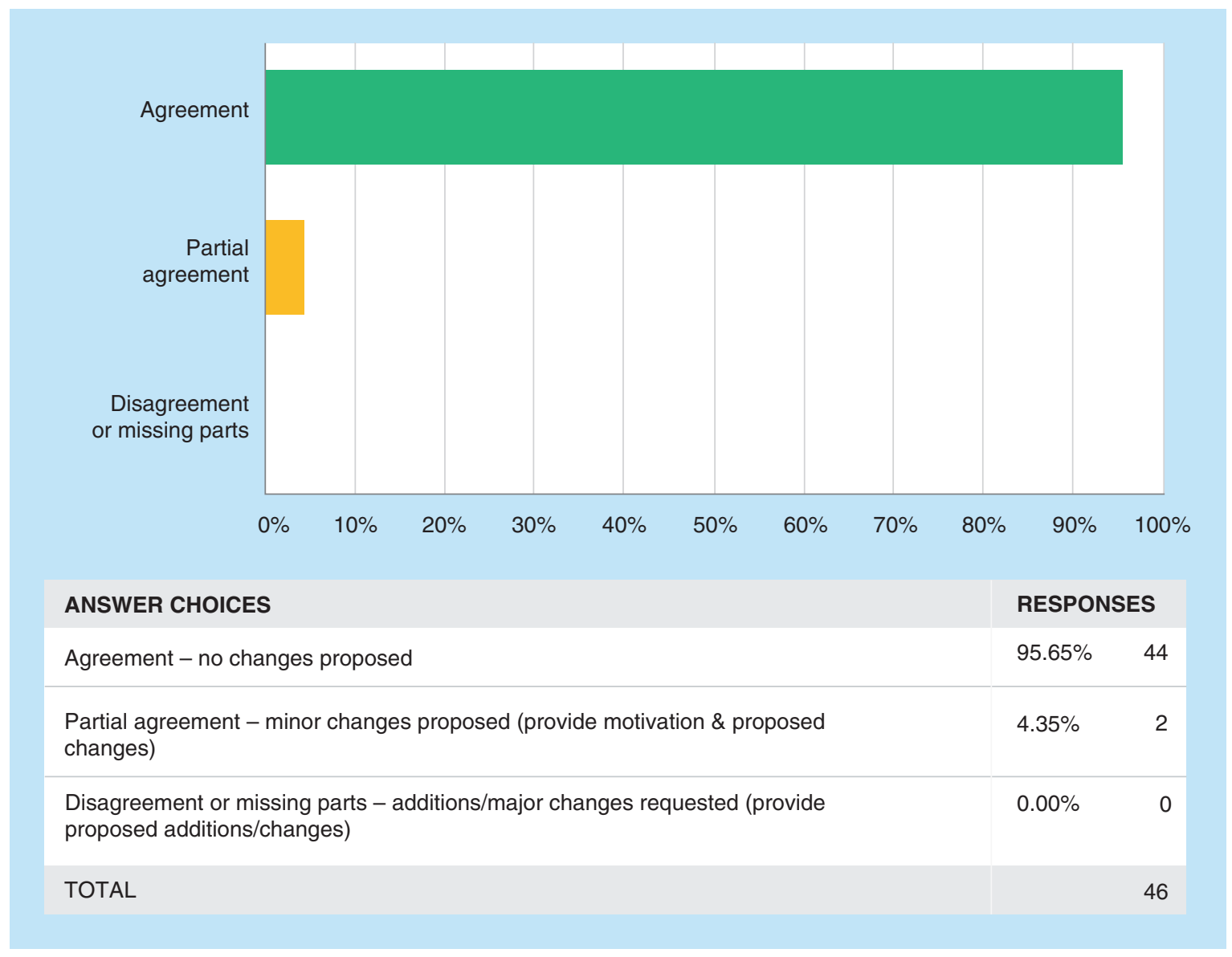

\section{Partial agreement}

No trend was found in these comments.

Additional comments from the 13th GCC:

It was suggested to add the bold text to the following sentence: "Method development can include but is not limited to the optimisation of the following bioanalytical parameters to ensure that the method is suitable for validation" 
T7Q4: Method Development - Documentation; Lines 145-148: "Bioanalytical method development does not require extensive record keeping or notation. However, the applicant should record the changes to procedures as well as any issues and their resolutions to provide a rationale for any changes made to validated methods immediately prior to or in the course of analysing study samples for pivotal studies."

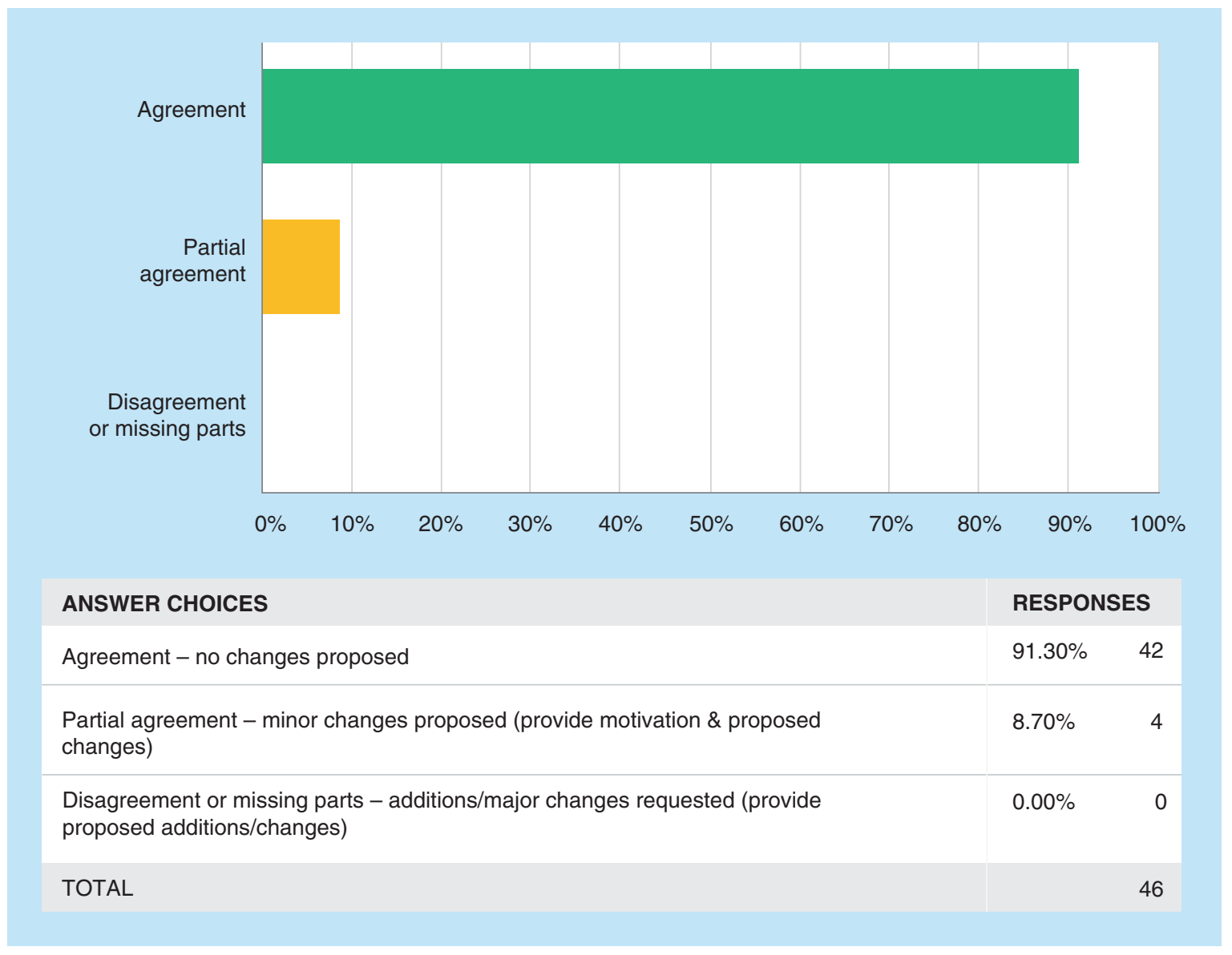

\section{Partial agreement}

All comments request clarification for the documentation of method development (e.g., only if method is sent back to development, during initial development, changes to validated method, synopsis, other).

\section{Additional comments from the 13th GCC:}

There were no further discussions during the GCC Closed Forum regarding the above proposal. 
T7Q5: Method Development - Purpose of Validation; Lines 149-150: "Once the method has been developed, bioanalytical method validation proves that the optimised method is suited to the analysis of the study samples."

Full Validation - Definition of Method; Lines 153-155: "Bioanalytical method validation is essential to ensure the acceptability of assay performance and the reliability of analytical results. A bioanalytical method is defined as a set of procedures used for measuring analyte concentrations in biological samples."

Full Validation; Lines 155-159: "A full validation of a bioanalytical method should be performed when establishing a bioanalytical method for the quantification of an analyte in clinical and in pivotal nonclinical studies. Full validation should also be performed when implementing an analytical method that is reported in the literature and when a commercial kit is repurposed for bioanalytical use in drug development."

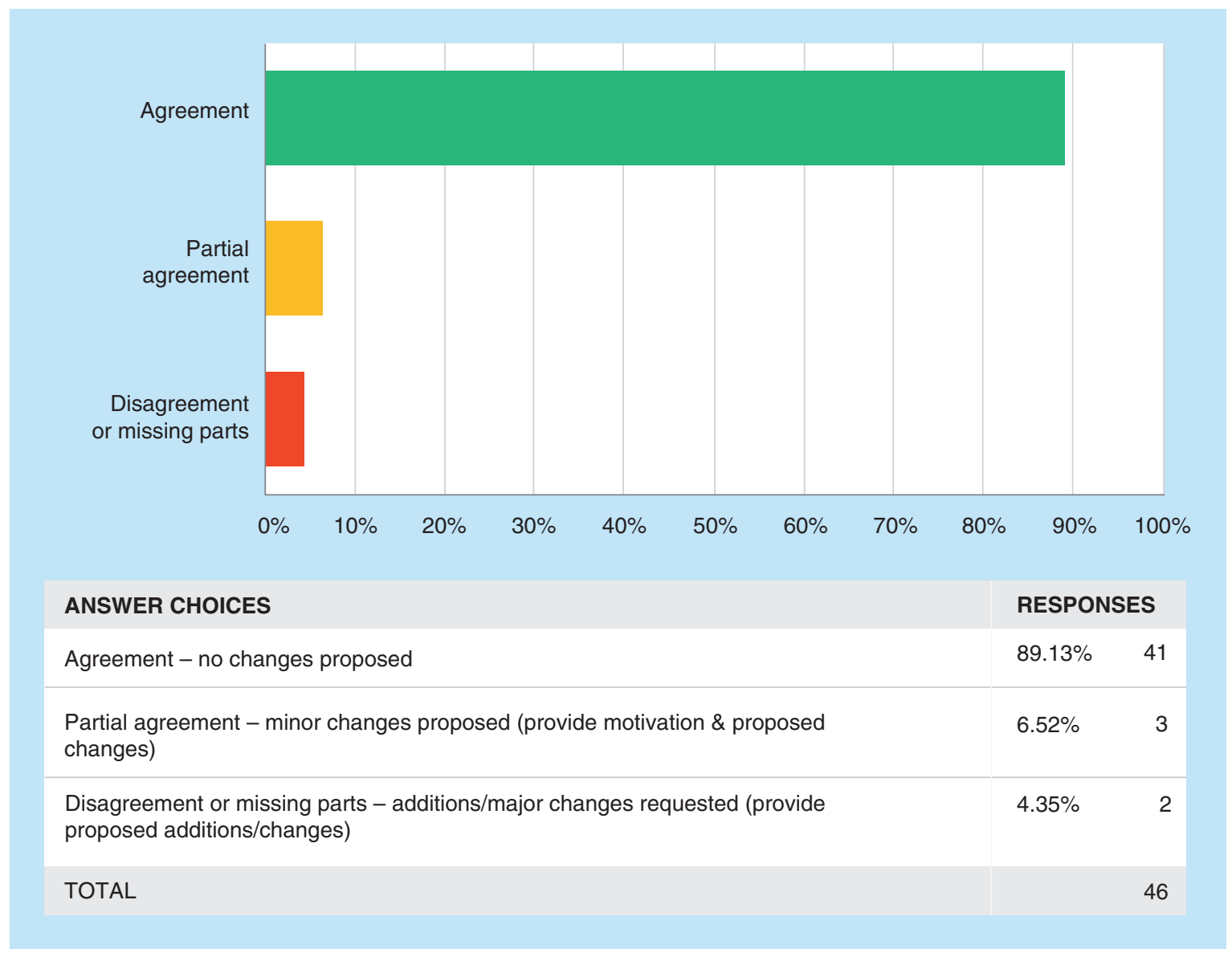

\section{Partial agreement}

It was suggested that the language should be adjusted to imply that a full validation should be based on the intent of the study data. Several examples could be given where a full validation is not required under the statements of lines $155-157$.

Additional comments from the 13th GCC:

It was recommended to add "unless scientifically justified" to the end of the last sentence. 
T7Q6: Full Validation - Multianalyte; Lines 159-163: "Usually one analyte has to be determined, but on occasion it may be appropriate to measure more than one analyte. This may involve two different drugs, a parent drug with its metabolites or the enantiomers or isomers of a drug. In these cases, the principles of validation and analysis apply to all analytes of interest."

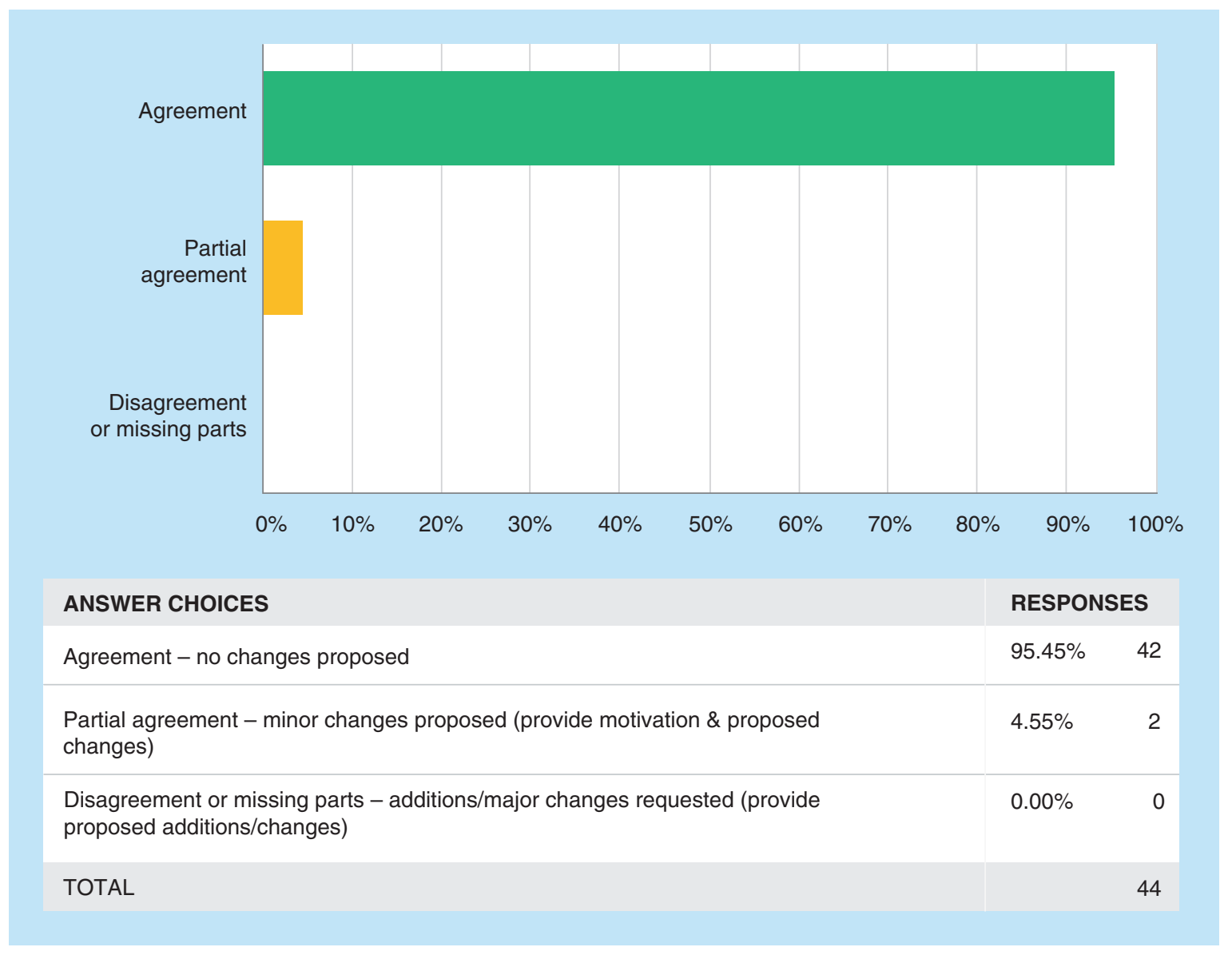

\section{Partial agreement}

No trend was found in these comments. 
T7Q7: Full Validation - Chromatographic Method Requirements; Lines 164-167: "For chromatographic methods a full validation should include the following elements: selectivity, specificity (if necessary), matrix effect, calibration curve (response function), range (lower limit of quantification [LLOQ] to upper limit of quantification [ULOQ]), accuracy, precision, carry-over, dilution integrity, stability and reinjection reproducibility."

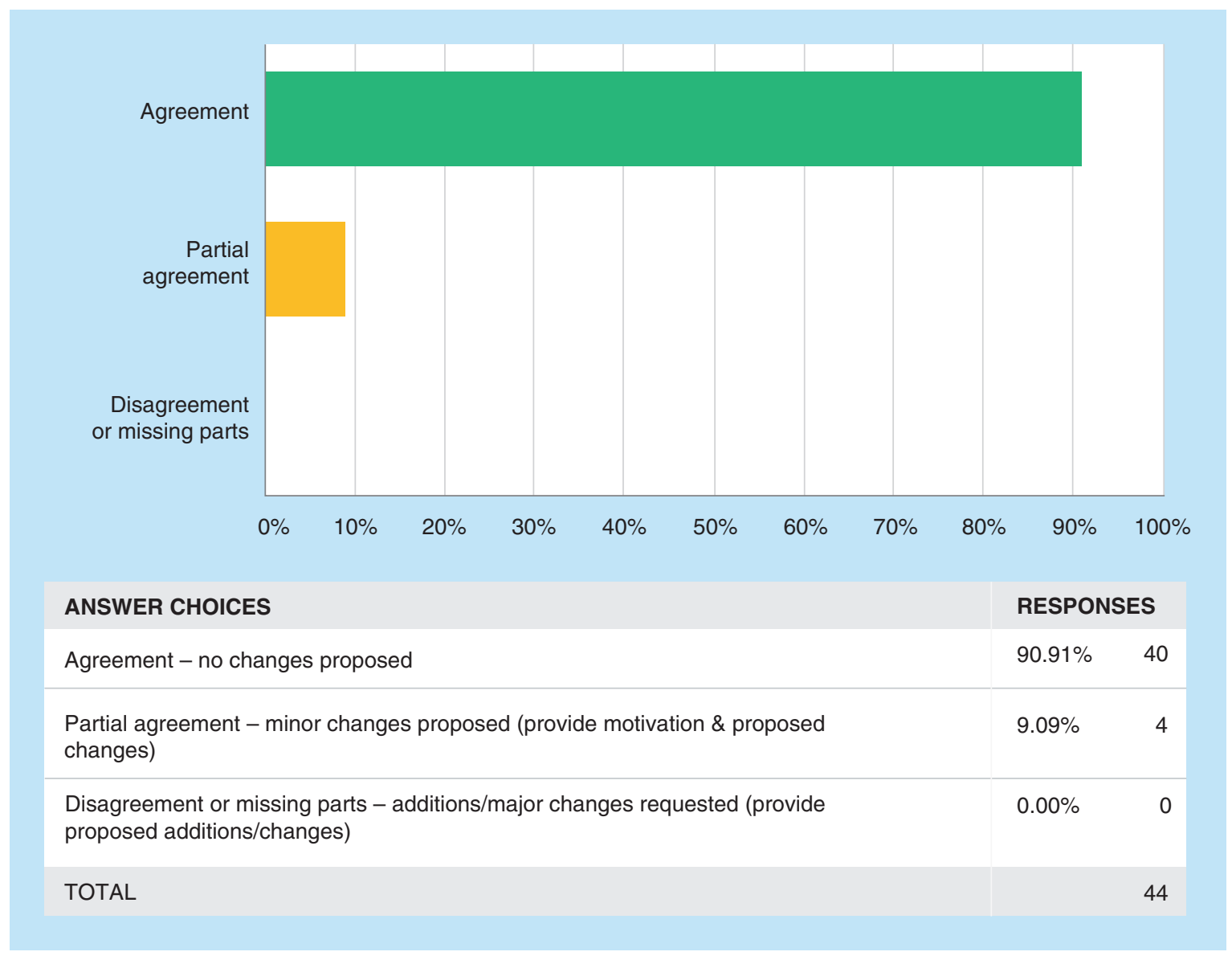

\section{Partial agreement}

No trend was found in these comments.

\section{Additional comments from the 13th GCC:}

Minor additions were suggested in bold text to this section: "For chromatographic methods a full validation should minimally include the following elements (see Table 1): selectivity, specificity (if necessary), matrix effect, calibration curve (response function), range (lower limit of quantification (LLOQ) to upper limit of quantification (ULOQ)), accuracy, precision, recovery, carry-over, dilution integrity, stability and reinjection reproducibility." 
T7Q8: Full Validation - LBA Requirements; Lines 168-170: "For LBAs the following elements should be evaluated: specificity, selectivity, calibration curve (response function), range (LLOQ to ULOQ), accuracy, precision, carry-over (if necessary), dilution linearity, parallelism (if necessary, conducted during sample analysis) and stability."

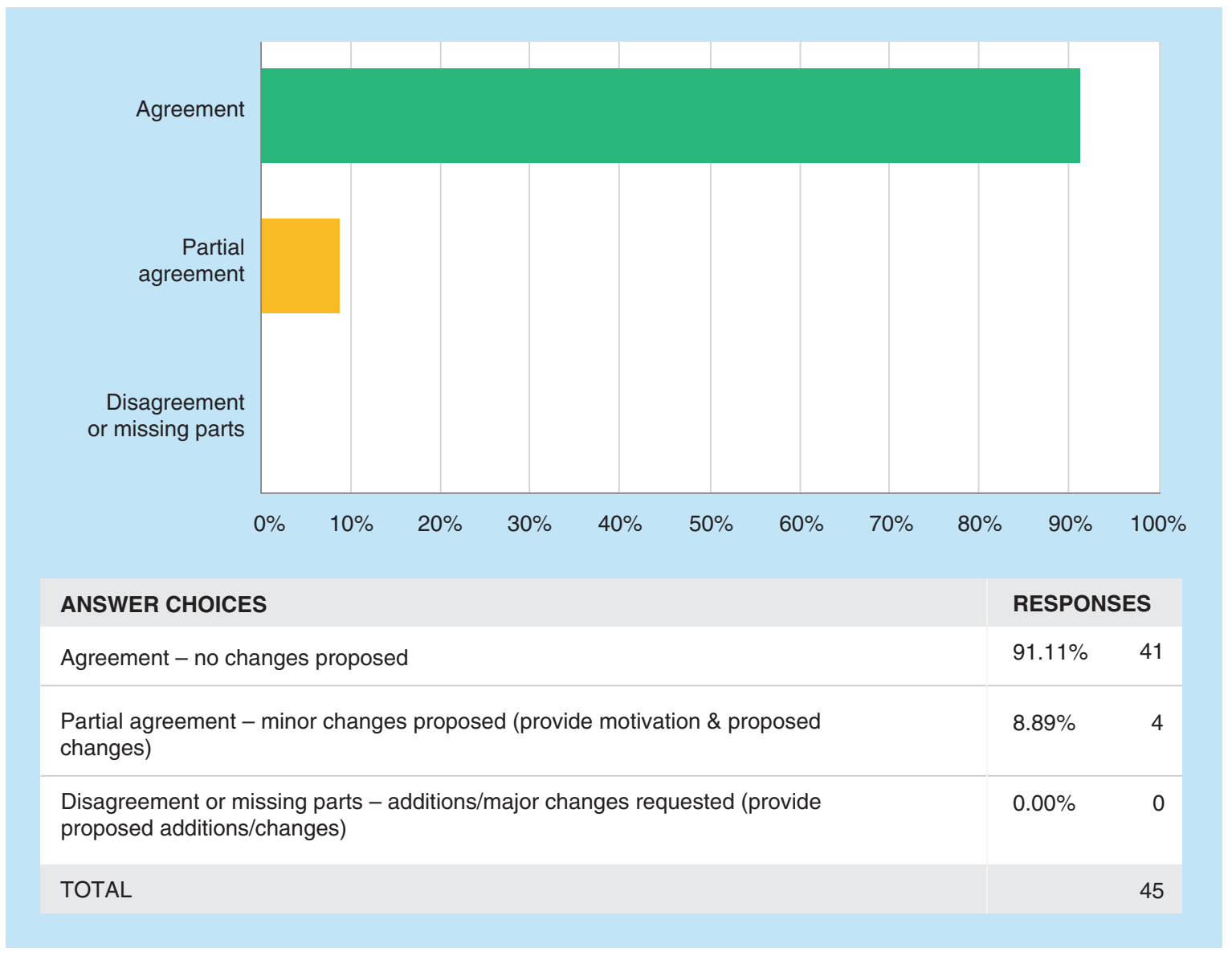

\section{Partial agreement}

No trend was found in these comments.

\section{Additional comments from the 13th GCC:}

Minor additions were suggested:

- As for T7Q7, it was recommended to add "minimally".

- Hook effect should be added.

- Parallelism should be removed. 
T7Q9: Full Validation - Matrix Selection; Lines 171-172: "The matrix used for analytical method validation should be the same as the matrix of the study samples, including anticoagulants and additives."

Full Validation - Surrogate Matrix; Lines 172-176: "In some cases, it may be difficult to obtain an identical matrix to that of the study samples (e.g., rare matrices such as tissue, cerebrospinal fluid, bile). In such cases surrogate matrices may be acceptable for analytical method validation. The surrogate matrix should be selected and justified scientifically for use in the analytical method."

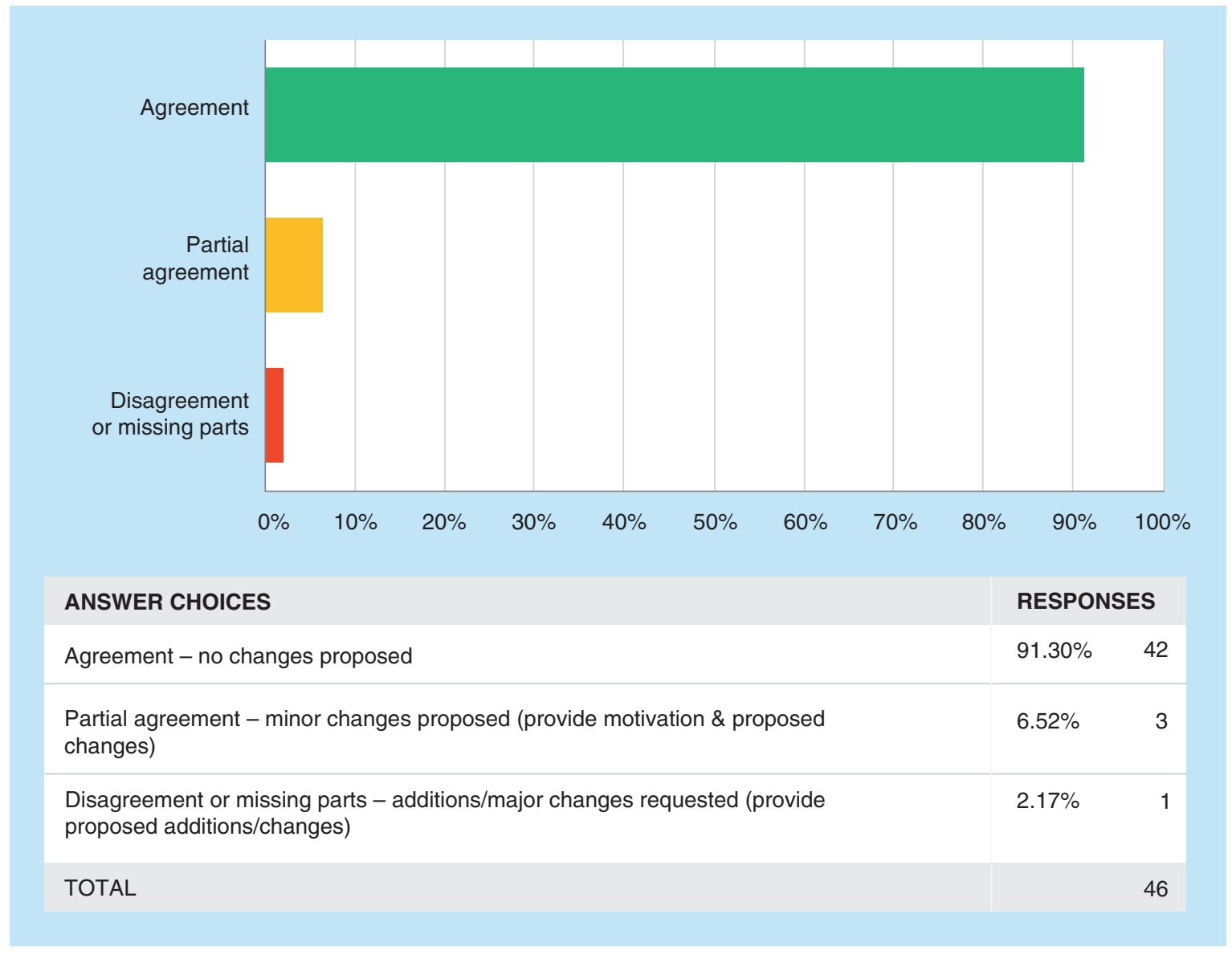

\section{Partial agreement}

Two comments suggested adding regulatory expectations for anticoagulant counter-ions.

\section{Additional comments from the 13th GCC:}

Comments from the survey were discussed during the GCC Closed Forum and the need to clarify and include that changes to anticoagulant counter-ions does not require additional testing was confirmed. 
T7Q10: Full Validation - Method SOP; Lines 177-179: "A specific, detailed, written description of the bioanalytical method should be established a priori. This description may be in the form of a protocol, study plan, report, or Standard Operating Procedure (SOP)."

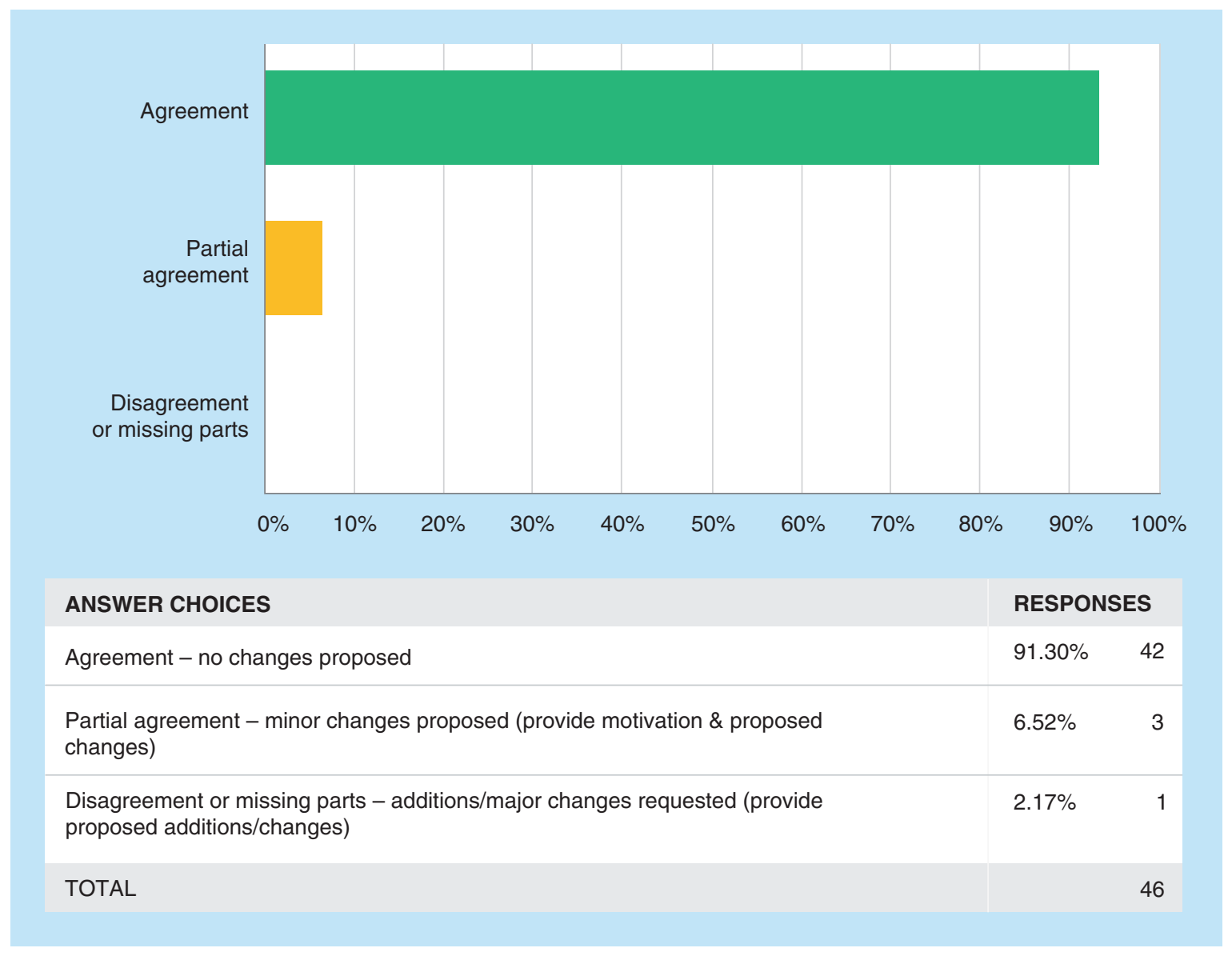

\section{Partial agreement}

No trend was found in these comments.

\section{Additional comments from the 13th GCC:}

A minor addition was suggested to remove "report" and add "analytical procedure." 
Supplement Nehls, Buonarati, Cape et al.

T7Q11: Partial Validation - General; Lines 181-184: "Modifications to a fully validated analytical method may be evaluated by partial validation. Partial validation can range from as little as one accuracy and precision determination to a nearly full validation (Refer to Section 6.1). The items in a partial validation are determined according to the extent and nature of the changes made to the method."

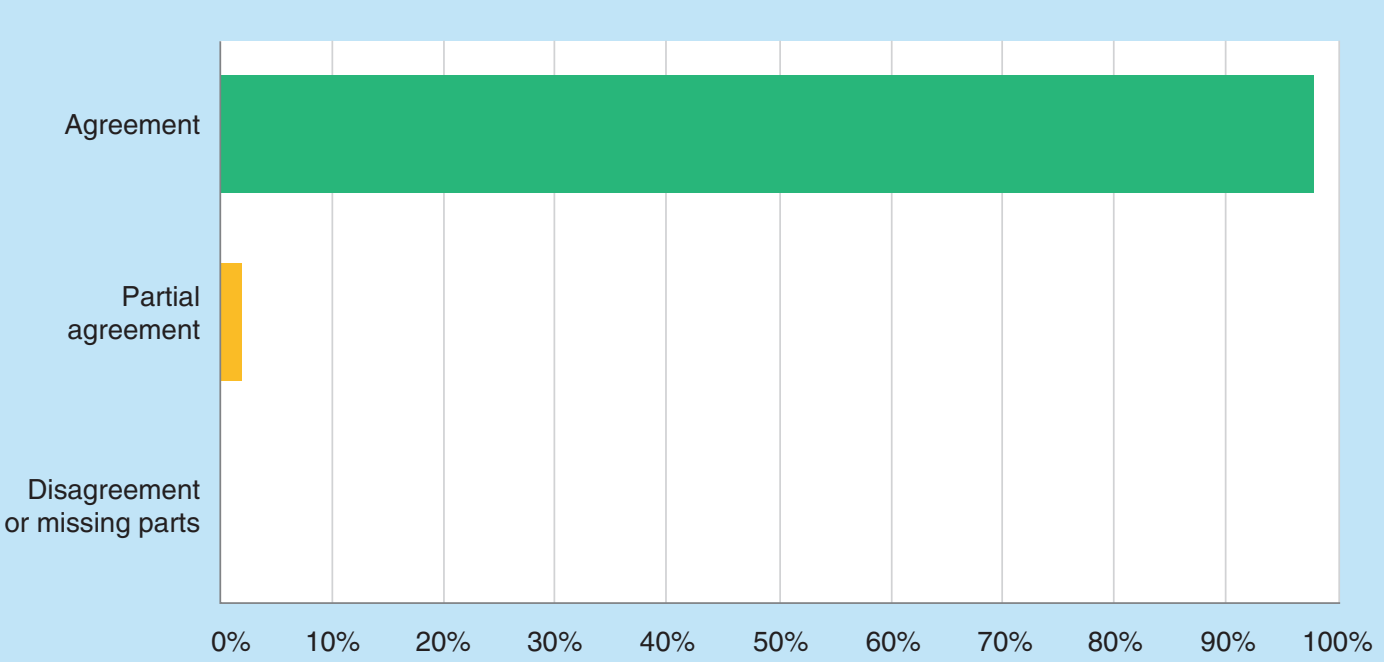

ANSWER CHOICES

RESPONSES

Agreement - no changes proposed

$97.83 \% \quad 45$

Partial agreement - minor changes proposed (provide motivation \& proposed changes)

$2.17 \% \quad 1$

Disagreement or missing parts - additions/major changes requested (provide

$0.00 \%$

0 proposed additions/changes)

TOTAL 
T7Q12: Cross Validation; Lines 186-189: "Where data are obtained from different methods within or across studies, or when data are obtained within a study from different laboratories applying the same method, comparison of those data is needed and a cross validation of the applied analytical methods should be carried out (Refer to Section 6.2)."

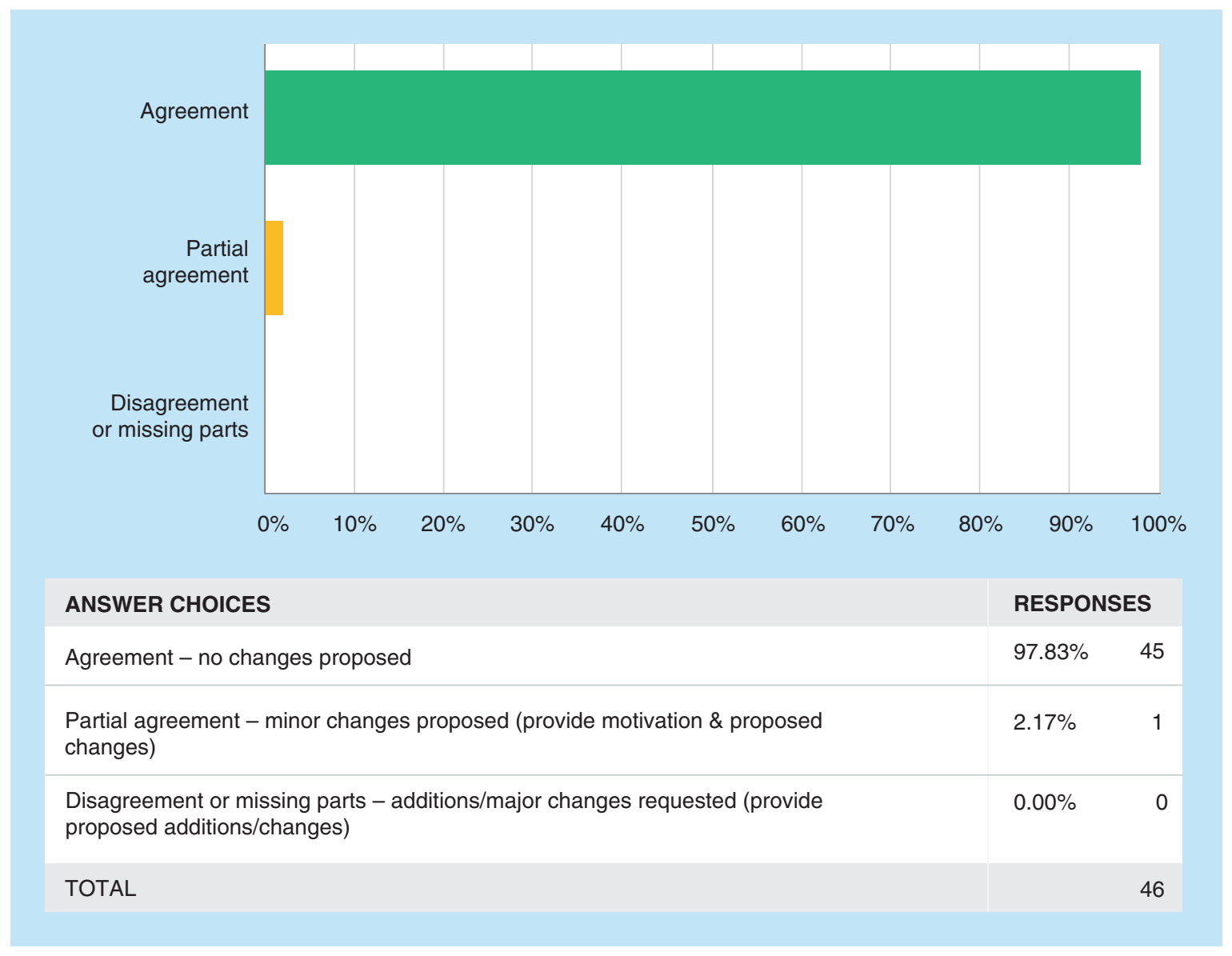

\section{Additional comments from the 13th GCC:}

It was suggested that cross validation is needed when different methods are used within or across pivotal studies. 
Supplement Nehls, Buonarati, Cape et al.

T7Q13: Documentation - Background; Lines 1227-1229: "General and specific SOPs and good record keeping are essential to a properly validated analytical method. The data generated for bioanalytical method validation should be documented and available for data audit and inspection."

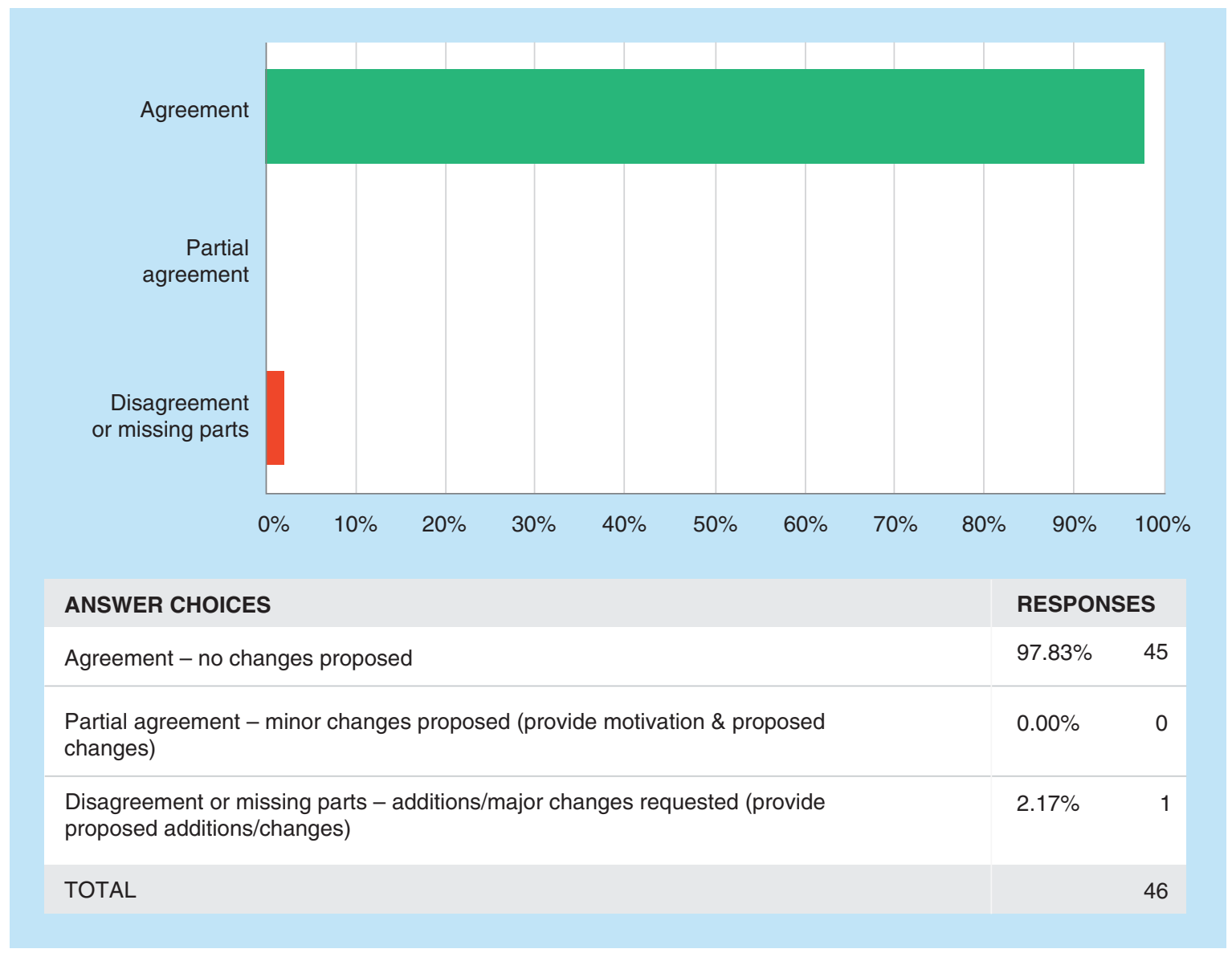


T7Q14: Data Availability; Lines 1229-1233: "Table 1 describes the recommended documentation for submission to the regulatory authorities and documentation that should be available at the analytical site at times of inspection. This documentation may be stored at the analytical site or at another secure location. In this case the documentation should be readily available when requested."

Data Retention; Lines 1234-1237: "All relevant documentation necessary for reconstructing the study as it was conducted and reported should be maintained in a secure environment. Relevant documentation includes, but is not limited to, source data, protocols and reports, records supporting procedural, operational, and environmental concerns and correspondence records between all involved parties."

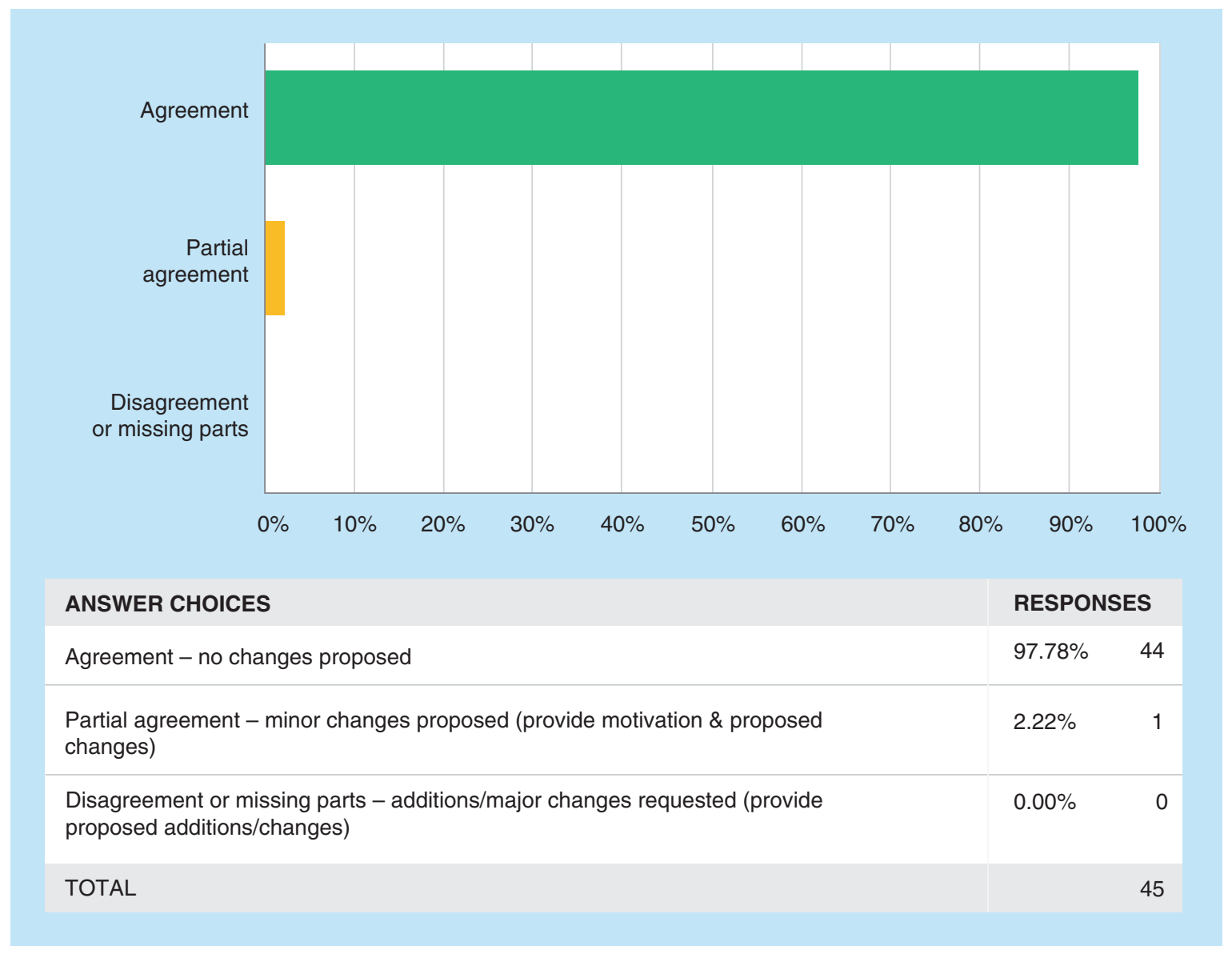


T7Q15: Good Documentation Practices; Lines 1238-1242: "Regardless of the documentation format (i.e., paper or electronic), records should be contemporaneous with the event and subsequent alterations should not obscure the original data. The basis for changing or reprocessing data should be documented with sufficient detail, and the original record should be maintained. Transcripts/copies of data derived from analyses in biohazardous areas should be maintained if applicable."

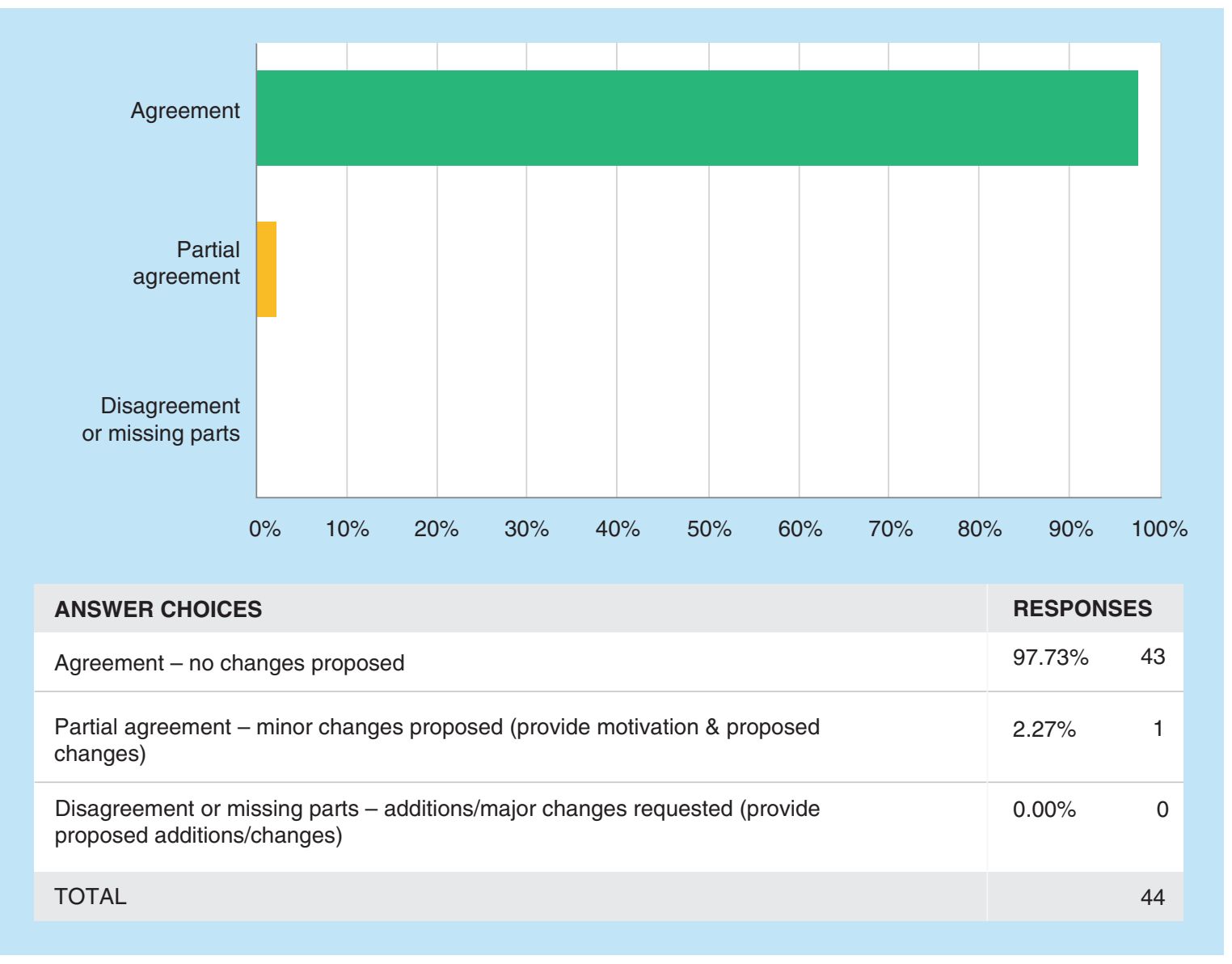


T7Q16: Summary; Lines 1244-1245: "Summary information should include the following items in Section 2.6.4/2.7.1 of the Common Technical Document (CTD) or reports:"

Summary of Assay Methods; Lines 1246-1249: "- A summary of assay methods used for each study should be included. Each summary should provide the protocol number, the assay type, the assay method identification code, the Bioanalytical Report code, effective date of the method, and the associated Validation Report codes."

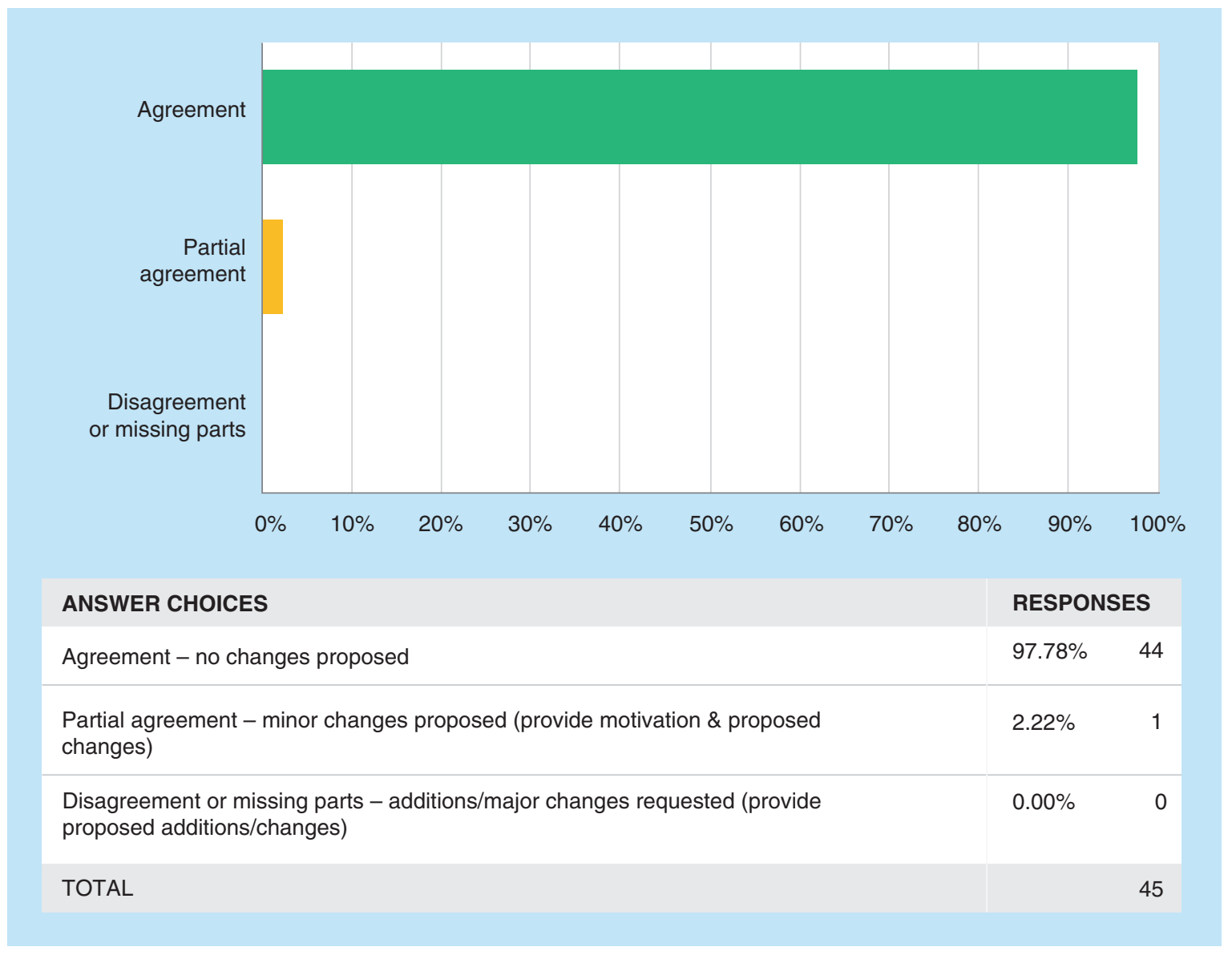


T7Q17: Summary of Reports; Lines 1250 - 1254: “• A summary table of all the relevant Validation Reports should be provided for each analyte, including Partial Validation and Cross Validation Reports. The table should include the assay method identification code, the type of assay, the reason for the new method or additional validation (e.g., to lower the limit of quantification). Changes made to the method should be clearly identified."

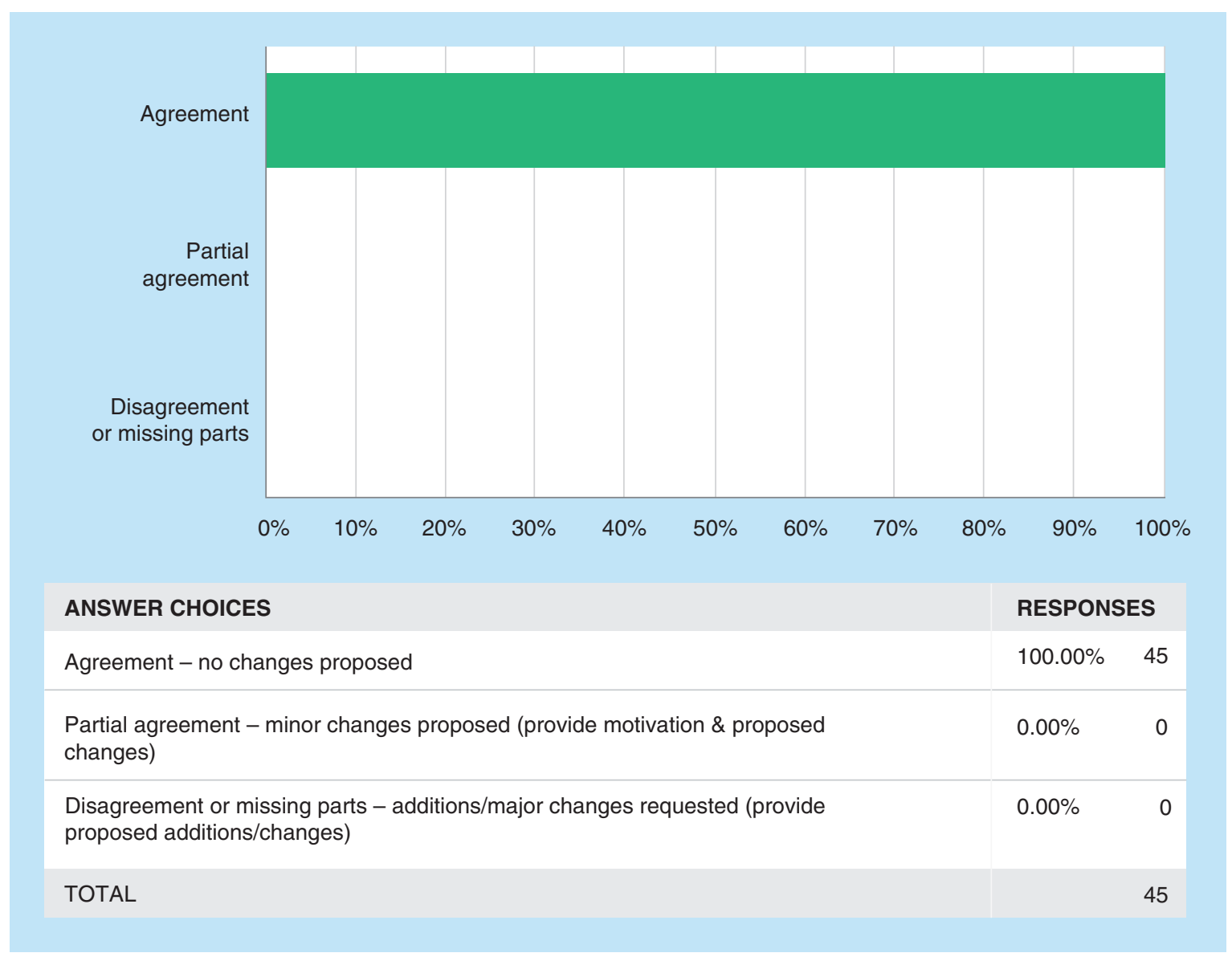


T7Q18: Summary Cross - References; Lines 1255-1257: “• A summary table cross-referencing multiple identifcation codes should be provided when an assay has different codes for the assay method, the Validation Reports and the Bioanalytical Reports."

Summary - Method Changes; Lines 1258-1259: "• Discussion of method changes in the protocol (e.g., evolution of methods, reason(s) for revisions, unique aspects."

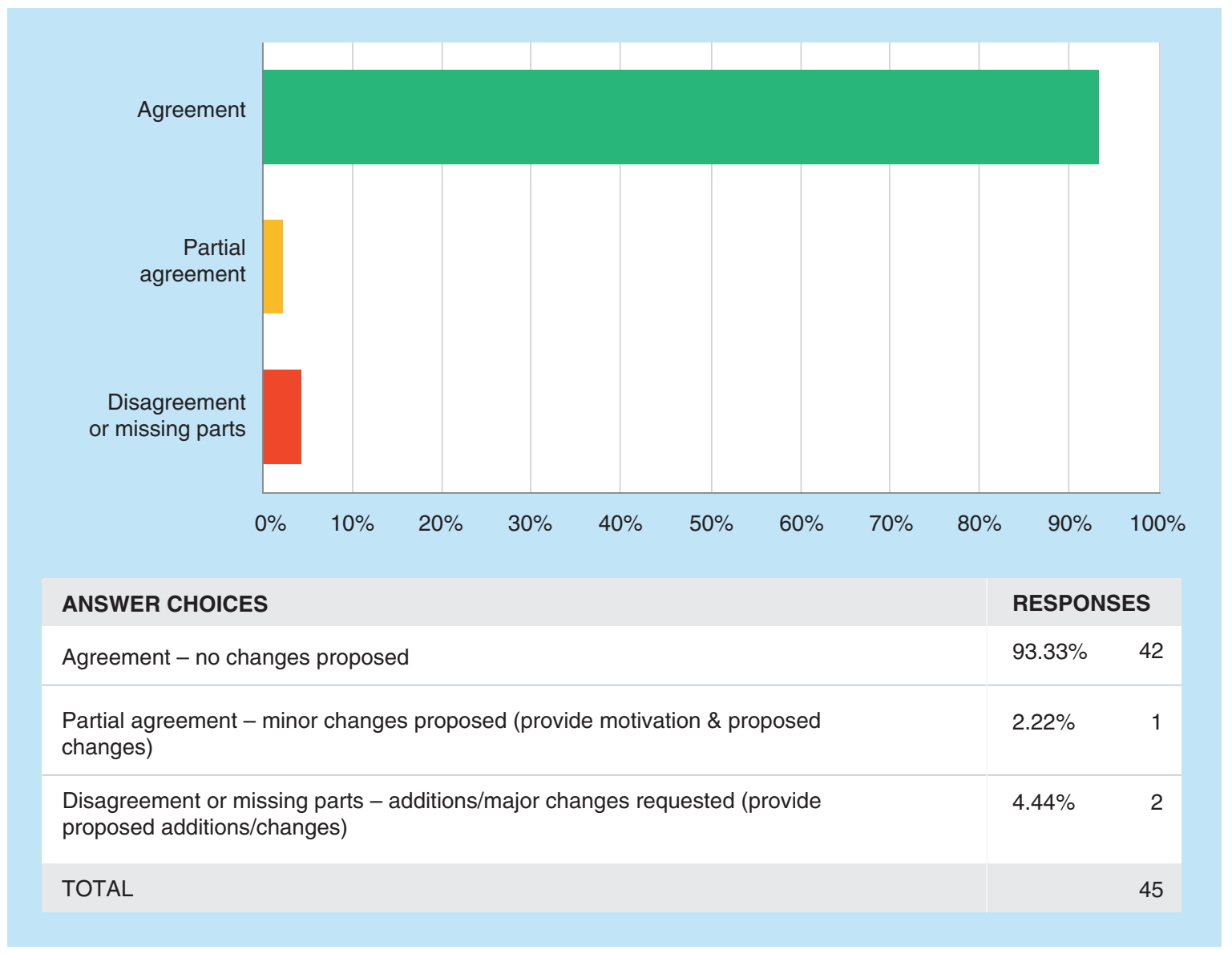

Disagreement or missing parts

No trend was found in these comments. 
T7Q19: Summary - Inspection History; Lines 1260-1261: “• For comparative BA/BE studies a list of regulatory site inspections including dates and outcomes for each analytical site if available."

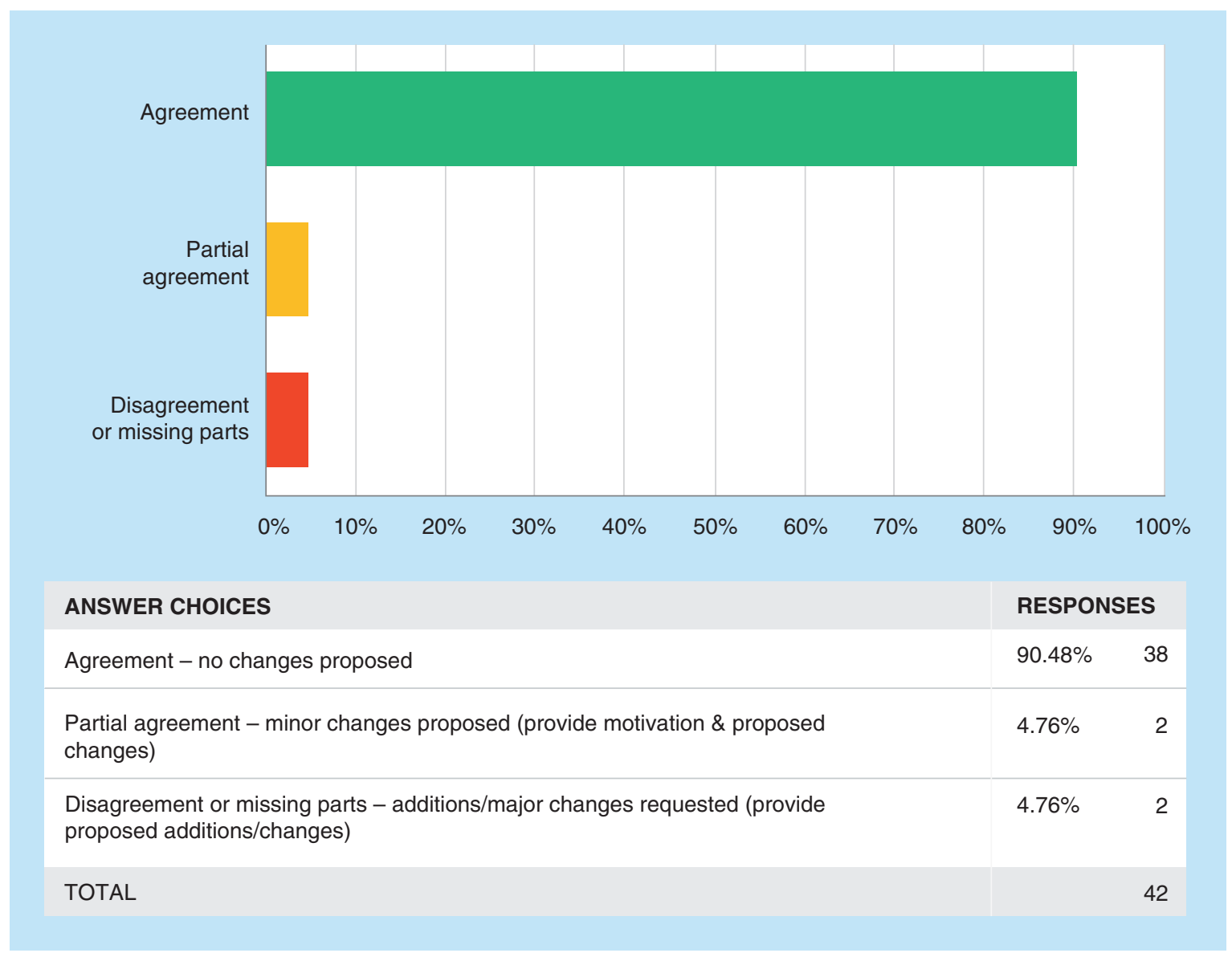

Partial agreement

Three comments asked for further clarification on the rationale for the need to include the inspection list. 
T7Q20: Table 1; Line 1263: "Table 1 describes the recommended documentation for the Validation and Bioanalytical Reports."

Item - Chromatographic System Suitability; Line 1264

Item - Synopsis, Overview of Method Evolution; Line 1264

Item - Reference Standards; Line 1264

Item - Internal Standard; Line 1264

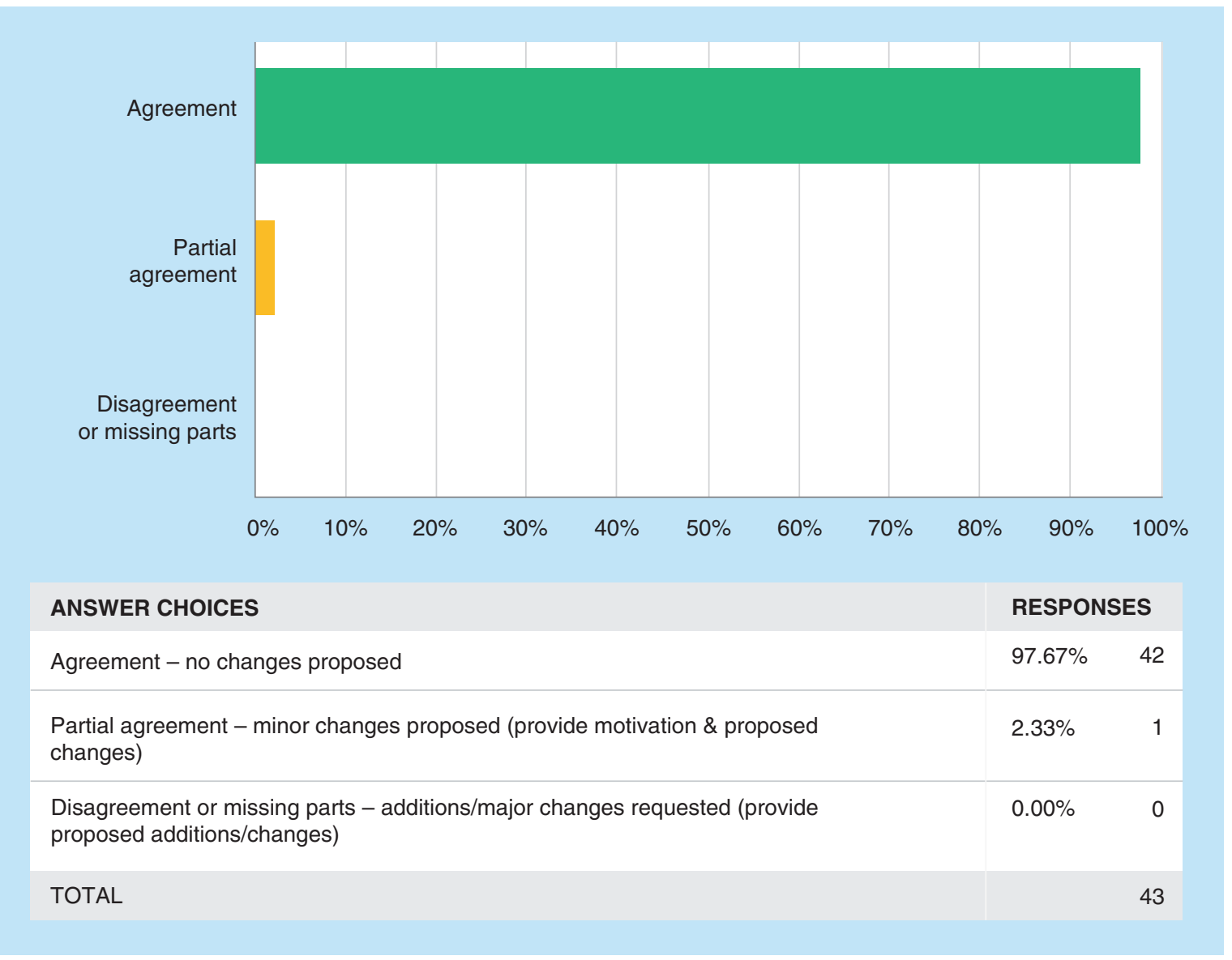


T7Q21: Item - Critical Reagents; Line 1265

Item - Stock Solutions; Line 1265

Item - Blank Matrix; Line 1265

Item - Calibrations Standards and QCs; Line 1265

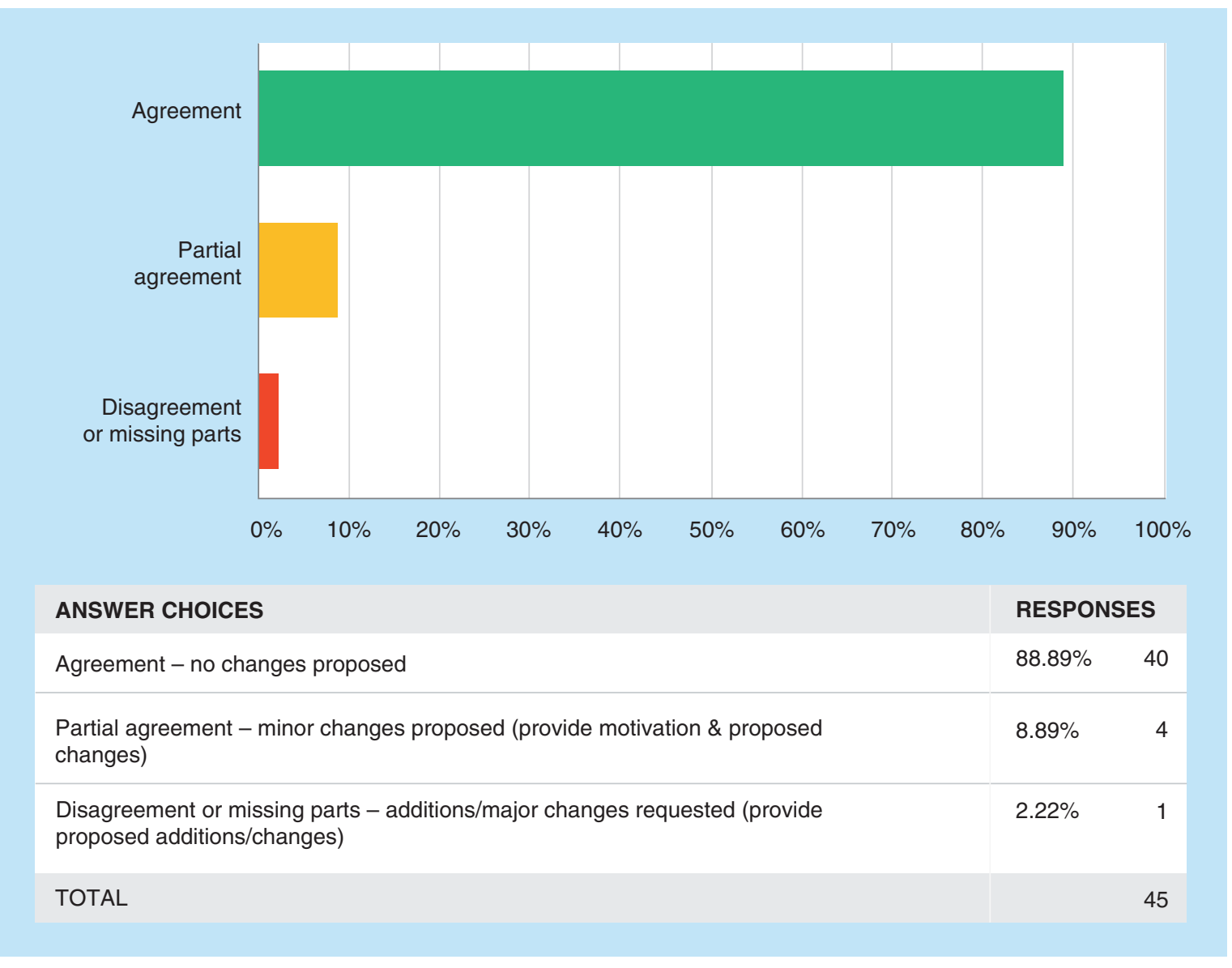

Partial agreement

No trend was found in these comments. 
T7Q22: Item - SOPs; Line 1266

Item - Sample Tracking; Line 1266

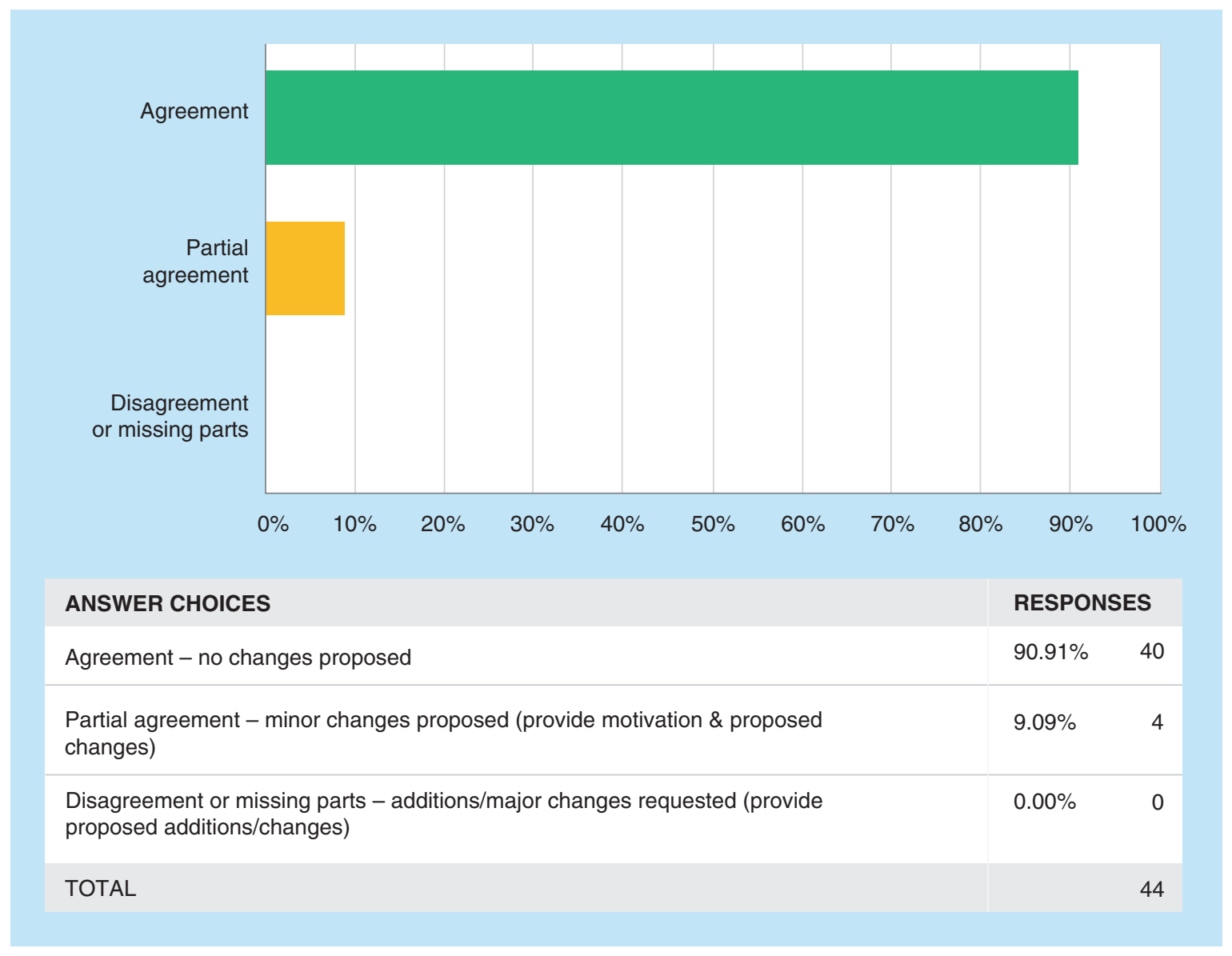

Partial agreement

No trend was found in these comments. 
T7Q23: Item - Analysis

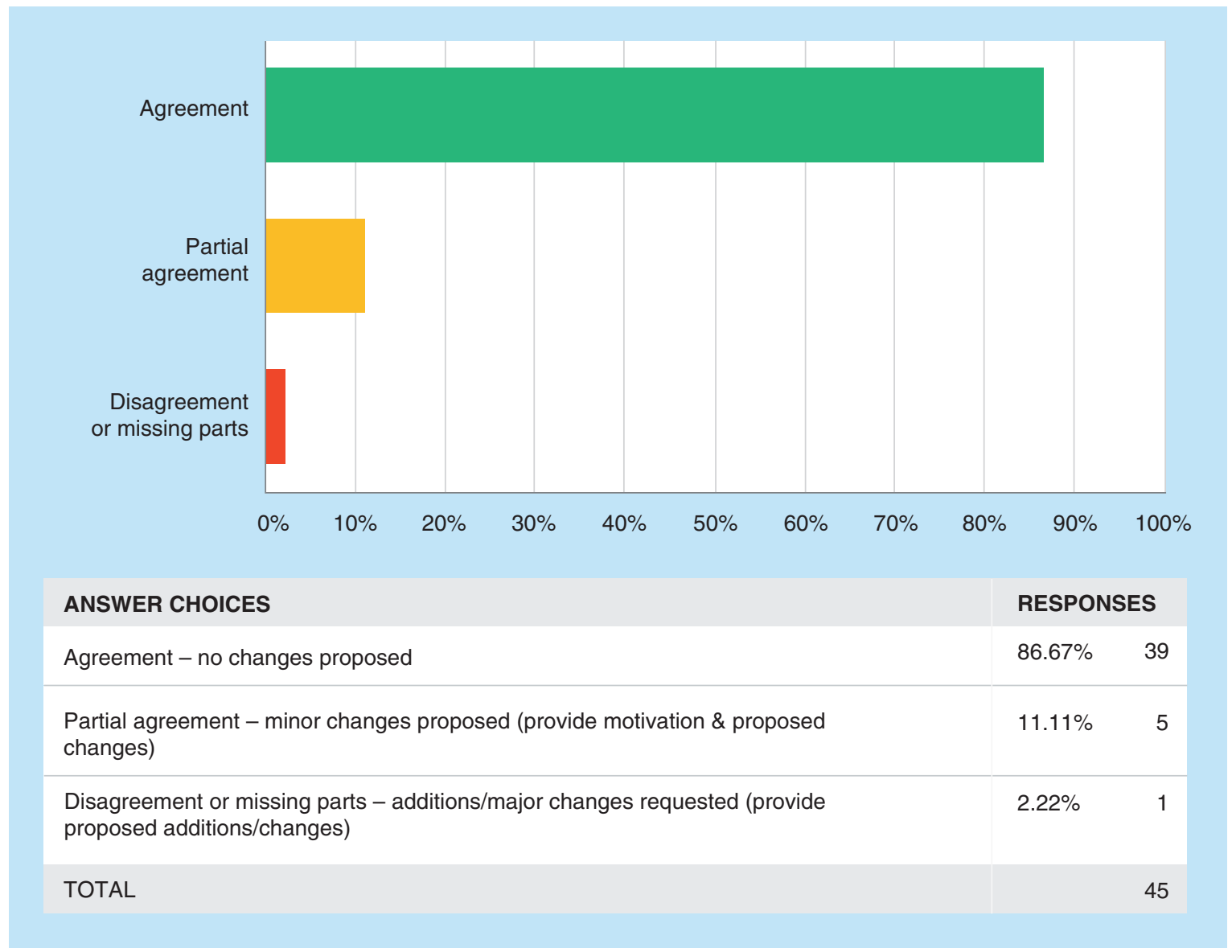

\section{Partial agreement}

It was suggested that instrumentation IDs should only be required in the onsite documentation rather than in the report.

\section{Additional comments from the 13th GCC:}

It was suggested that if the requirement to report original values for reinjected runs was kept, then "if an original result was obtained" should be added. 
T7Q24: Item - Chromatograms and Reintegration; Line 1267

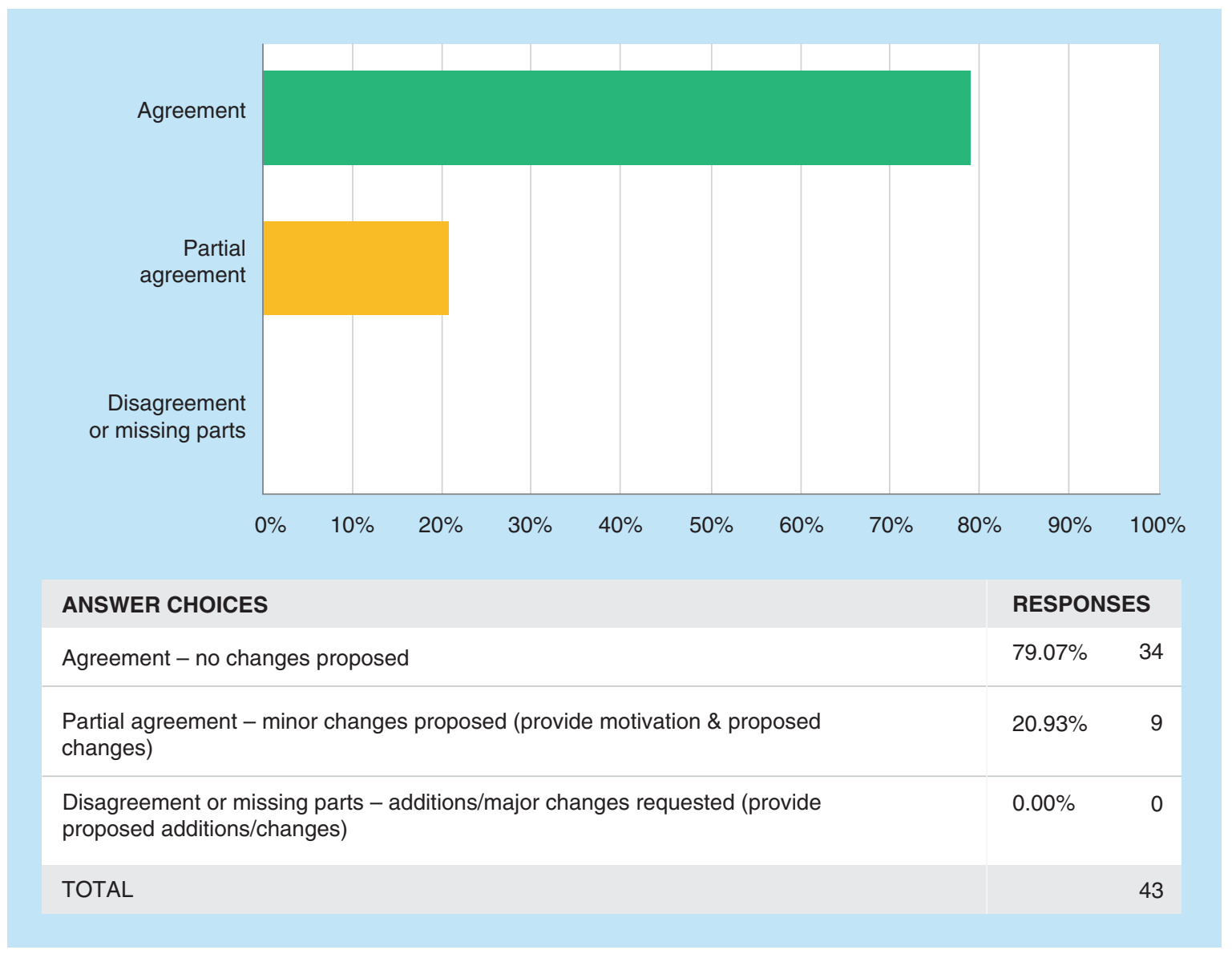

\section{Partial agreement}

There were multiple comments in this section. However, only the below trends were found:

- The reference to BA/BE studies in the Validation Report section was unclear.

- Clarify the need to present $100 \%$ chromatograms.

Additional comments from the 13th GCC:

It was suggested that "in electronic format" should be added to all reporting points requesting chromatograms. 
T7Q25: Item - Deviations from Procedures - Repeat Analysis - ISR - Communication - Audits and Inspections

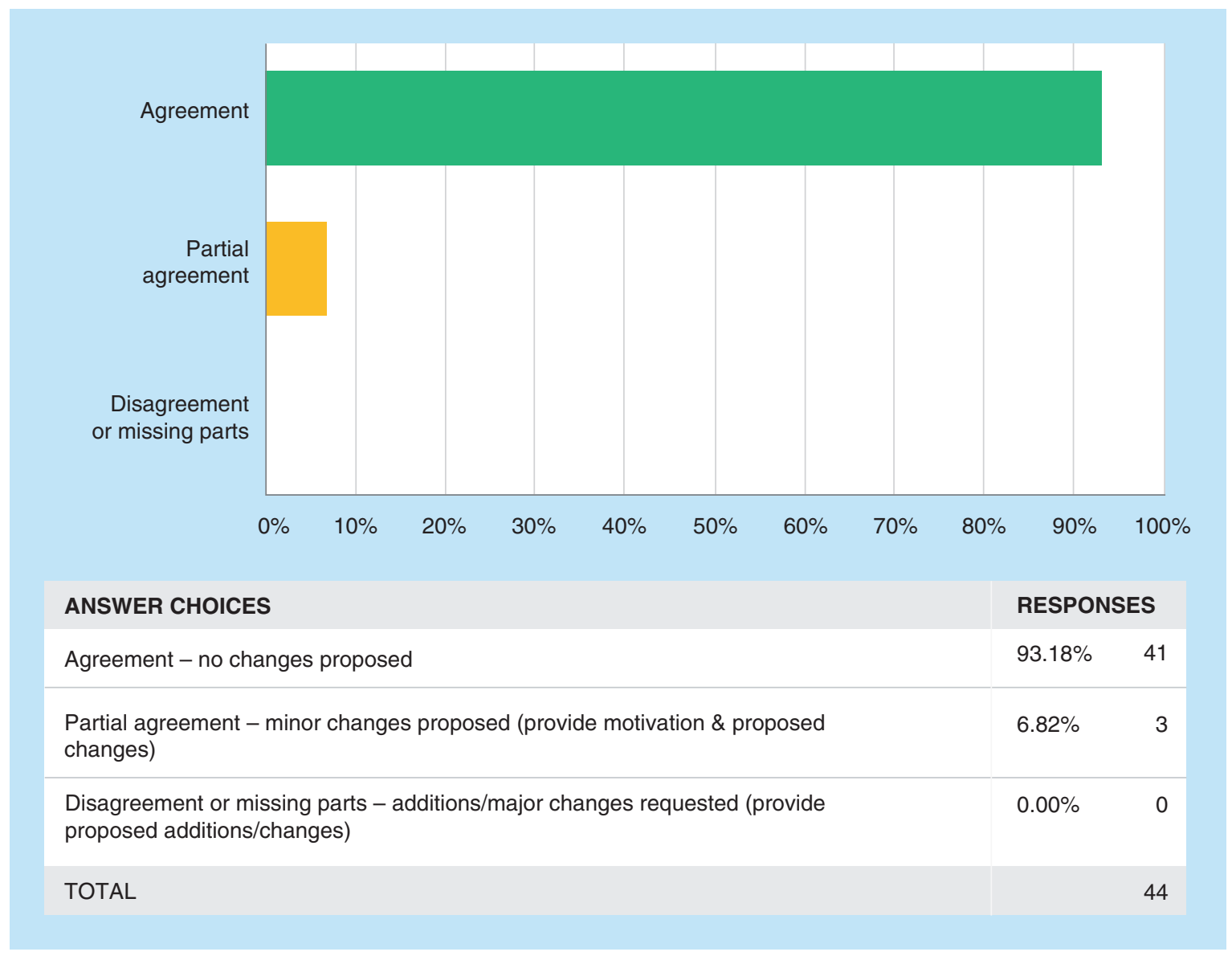

Partial agreement

No trend was found in these comments.

Additional comments from the 13th GCC:

It was suggested that only significant deviations need to be reported in both reports. 
T7Q26: Table 1 footer page 48; Line 1268: “*The applicant is expected to maintain data at the analytical site to support summary data submitted in Validation and Bioanalytical Reports."

Table 1 footer page 48; Line 1269: "Validation and Bioanalytical Reports should be submitted in the application."

Table 1 footer page 49; Line 1270: "†May append or link from Validation Report."

Table 1 footer page 49; Line 1271: "††Submit either in Validation Report or in Bioanalytical Report."

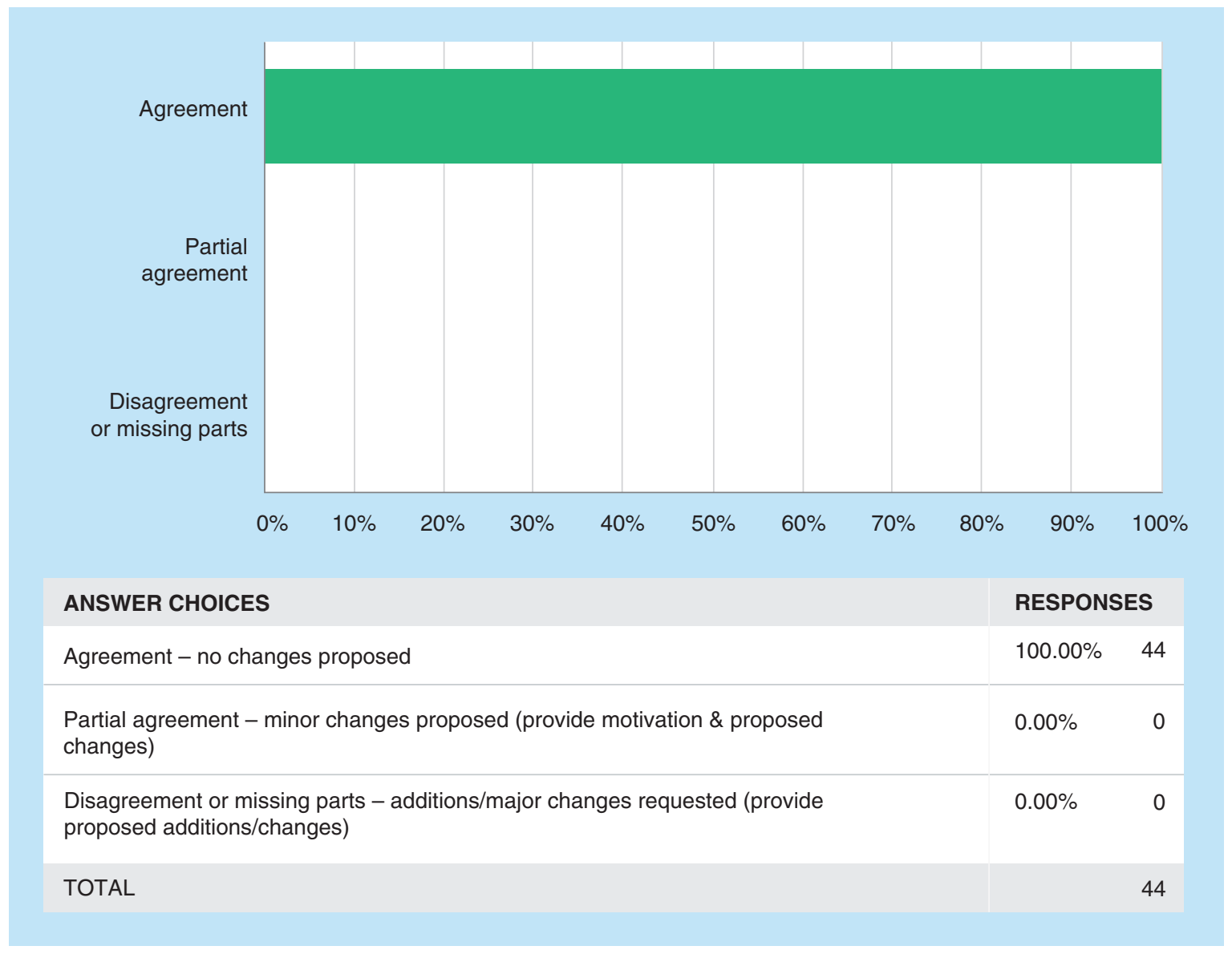

\section{Future perspective}

The GCC will continue to provide recommendations on hot topics in bioanalysis of global interest and expand its membership by coordinating its activities with the regional and international meetings held by the pharmaceutical industry. Please contact the GCC [28] for the date and time of future meetings and for all membership information.

\section{Acknowledgments}

The GCC would like to thank the following:

- Corey Nehls, Michael Buonarati, Stephanie Cape, Rafiq Islam, Christina Satterwhite, Chad Briscoe, and Roger Hayes for leading each team discussion.

- All the GCC member companies who filled in the numerous surveys, participated and contributed to the discussions at the 13 th GCC Closed Forum for Bioanalysis;

- Natasha Savoie (GCC) for taking the minutes of the 13th GCC Closed Forum and drafting the first draft of this document;

- Wei Garofolo (GCC) for organizing the logistics of the meeting and coordinating the review of this document. 


\section{Financial \& competing interests disclosure}

The authors have no relevant affiliations or financial involvement with any organization or entity with a financial interest in or financial conflict with the subject matter or materials discussed in the manuscript. This includes employment, consultancies, honoraria, stock ownership or options, expert testimony, grants or patents received or pending, or royalties.

No writing assistance was utilized in the production of this manuscript.

\section{References}

1 Premkumar N, Lowes S, Jersey J et al. Formation of a Global Contract Research Organization Council for Bioanalysis. Bioanalysis 2(11), 1797-1800 (2010).

2 Breda N, Garofolo F, Cruz Caturla M et al. The 3rd Global CRO Council for Bioanalysis at the International Reid Bioanalytical Forum. Bioanalysis 3(24), 2721-2727 (2011).

3 Lowes S, Jersey J, Shoup R et al. 4th Global CRO Council for Bioanalysis: coadministered drugs stability, EMA/US FDA Guidelines, 483s and Carryover. Bioanalysis 4(7), 763-768 (2012).

4 Nicholson R, Lowes S, Cruz Caturla M et al. 6th GCC focus on LBA: critical reagents, positive controls and reference standards; specificity for endogenous compounds; biomarkers; biosimilars. Bioanalysis 4(19), 2335-2342 (2012).

5 Rocci M, Lowes S, Shoup R et al. $7^{\text {th }}$ GCC: use of incurred samples; fit-for-purpose validation; solution stability; electronic lab notebooks; hyperlipidemic matrix testing. Bioanalysis 6(20), 2713-2720 (2014).

6 Hayes R, LeLacheur R, Dumont I et al. 9th GCC closed forum: CAPA in regulated bioanalysis; method robustness, biosimilars, preclinical method validation, endogenous biomarkers, whole blood stability, regulatory audit experiences and electronic laboratory notebooks. Bioanalysis 8(6), 487-495 (2016).

7 Cape S, Islam R, Nehls C et al. The 10th GCC Closed Forum: rejected data, GCP in bioanalysis, extract stability, BAV, processed batch acceptance, matrix stability, critical reagents, ELN and data integrity and counteracting fraud. Bioanalysis 9(7), 505-516 (2017).

8 Islam R, Briscoe C, Bower J et al. 11th GCC Closed Forum: cumulative stability; matrix stability; immunogenicity assays; laboratory manuals; biosimilars; chiral methods; hybrid LBA/LCMS assays; fit-for-purpose validation; China Food and Drug Administration bioanalytical method validation. Bioanalysis 10(7), 433-444 (2018).

9 Briscoe C, Hughes N, Hayes R et al. 12th GCC Closed Forum: critical reagents; oligonucleotides; CoA; method transfer; HRMS; flow cytometry; regulatory findings; stability; and immunogenicity. Bioanalysis 11(12), 1129-1138 (2019).

10 Lowes S, Jersey J, Shoup R et al. Recommendations on: internal standard criteria, stability, incurred sample reanalysis and recent 483s by the Global CRO Council for Bioanalysis. Bioanalysis 3(12), 1323-1332 (2011).

11 Sangster T, Maltas J, Struwe P et al. Recommendations on ISR in multi-analyte assays, QA/bioanalytical consultants and GCP by Global CRO Council for Bioanalysis (GCC). Bioanalysis 4(14), 1723-1730 (2012).

12 Boterman M, Doig M, Breda M et al. Recommendations on the interpretation of the new EMA Guideline on Bioanalytical Method Validation by Global CRO Council for Bioanalysis (GCC). Bioanalysis 4(6), 651-660 (2012).

13 Lowes S, Boterman M, Doig M et al. Recommendations on bioanalytical method stability implications of co-administered and co-formulated drugs by Global CRO Council for Bioanalysis (GCC). Bioanalysis 4(17), 2117-2126 (2012).

14 Hougton R, Gouty D, Allinson J et al. Recommendations on biomarker bioanalytical method validation by GCC. Bioanalysis 4(20), 2439-2446 (2012).

15 Lowes S, LeLacheur R, Shoup R et al. Recommendations on incurred sample stability (ISS) by GCC. Bioanalysis 6(18), 2385-2390 (2014).

16 Islam R, Kar S, Ritzén H et al. Recommendations for classification of commercial LBA kits for biomarkers in drug development from the GCC for bioanalysis. Bioanalysis 11(7), 645-653 (2019).

17 ICH M10, Draft Bioanalytical Method Validation (February 2019). https://www.ich.org/fileadmin/Public_Web_Site/ICH_Products/Gu idelines/Multidisciplinary/M10/M10EWG_Step2_DraftGuideline_2019_0226.pdf

18 US FDA. Guidance for industry: Bioanalytical method validation. US Department of Health and Human Services, US FDA, Center for Drug Evaluation and Research, MD, USA.

19 European Medicines Agency. Committee for medicinal products for human use (CHMP). Guideline on bioanalytical method validation. EMEA/CHMP/EWP/192217/2009, London, UK (2011).

20 MHLW. Guideline on bioanalytical method validation in pharmaceutical development. Japan (2013). http://www.nihs.go.jp/drug/BMV/250913_BMV-GL_E.pdf

21 ANVISA. Resolution RDC 27 minimum requirements for bioanalytical method validation used in studies with the purpose of registration and post-registration of medicines. Agencia National de Vigilancia Sanitaria, Brazil (2012).

22 SurveyMonkey Inc., CA, USA. www.surveymonkey.com

23 Garofolo W, Savoie N. The decennial index of the White Papers in bioanalysis: 'A decade of recommendations (2007-2016)'. Bioanalysis 9(21), 1681-1704 (2017). 
24 Neubert H, Olah T, Lee A et al. 2018 White Paper on Recent Issues in Bioanalysis: focus on immunogenicity assays by hybrid LBA/LCMS and regulatory feedback (Part $2-$ PK, PD \& ADA assays by hybrid LBA/LCMS \& regulatory agencies' inputs on bioanalysis, biomarkers and immunogenicity). Bioanalysis 10(23), 1897-1917 (2018).

25 Neubert H, Song A, Lee A et al. 2017 White Paper on recent issues in bioanalysis: rise of hybrid LBA/LCMS immunogenicity assays (Part 2: hybrid LBA/LCMS biotherapeutics, biomarkers \& immunogenicity assays and regulatory agencies' inputs). Bioanalysis 9(23), 1895-1912 (2017).

26 Piccoli SP, Sauer JM. Points to consider document: Scientific and regulatory considerations for the analytical validation of assays used in the qualification of biomarkers in biological matrices biomarker assay collaborative evidentiary considerations writing group, critical path institute (C-Path) (2017).

https://healthpolicy.duke.edu/sites/default/files/atoms/files/cpath_ptc_biomarker_qualification_assays_20170623.pdf

27 Jones BR, Schultz GA, Eckstein JA et al. Surrogate matrix and surrogate analyte approaches for definitive quantitation of endogenous biomolecules. Bioanalysis 4(19), 2343-2356 (2012).

28 Global CRO council for bioanalysis. http://www.global-cro-council.org 
